\title{
Street perceptions: \\ A study of visual preferences for New Zealand streetscapes
}

\author{
by \\ Morten Tor Gjerde
}

\begin{abstract}
A thesis submitted to
Victoria University of Wellington

in fulfilment of the requirements for the degree of

Doctor of Philosophy
\end{abstract}





\section{Abstract}

City leaders often make reference to their built and natural environments when they compete domestically or internationally for financial investment, tourism spending and high quality workers. These leaders are aware that people in the workforce, investors and tourists would prefer to be associated with vibrant and attractive places. Research has confirmed the important role the appearance of the built environment plays in people's physical, financial and psychological wellbeing, not the least of which is helping to foster a sense of individual and community pride. However, there is also literature critical of the appearance of many individual buildings and urban streetscapes, particularly those that have arisen through the well-intentioned but uncoordinated efforts of those involved in the development of individual sites.

Recognising that wider public interests have not always been well-served by private development, governments and local authorities become involved to control development outcomes. One aspect of development control is design review, which aims to improve the quality of urban places by influencing the design of individual buildings. However, given that design review is administered by professional experts and that design guidance is based on normative expressions of what good design should be, what assurances are there that urban transformation meets with public expectations? The research reported in this thesis addresses this question. 
This research seeks to identify those streetscape design characteristics that are best liked by people and those that they dislike. A methodology based on mixed research methods was developed. An initial study sought people's preferences for six different urban streetscapes, as depicted photographically. Analysis of nearly 200 responses to the survey questionnaire identified several building and streetscape characteristics that were consistently liked and disliked. To explore these and other responses from another perspective, a second study was designed that would examine people's preferences in more detail and on the basis of their actual experience of the streetscapes. Study Two was developed around three separate case studies and two focus group discussions. Demographic information about the 156 survey respondents was collected, along with their aesthetic perceptions about individual buildings, relationships between buildings and overall streetscapes. This enabled comparisons to be made on the bases of gender, age and occupational background. Of particular interest was to understand the streetscape preferences of lay members of the public, those whose interests design review aims to ensure, and change professionals, who make the design and planning decisions. Two focus group discussions were convened, one for change professionals and the other for lay people, to explore findings from the survey in more detail.

The results indicate that people prefer older buildings whose façade designs are based on more traditional composition patterns, and that the activities with which a building is associated have considerable influence on perceptions. These are two matters about which design control of new building development is not interested. In general, people prefer moderate variations in height between buildings along the length of a street and change professionals seem to tolerate/prefer bigger variations than others. Abrupt differences in height between adjoining buildings were viewed negatively by lay people, in large part because blank walls on internal boundaries become evident. On the other hand, change professionals were less sensitive to such differences, in part because they understood the nature of change and anticipated that future change would help heal such conflicts.

In addition to exploring people's perceptions of New Zealand streetscapes the thesis discusses several of the best-liked and the least-liked buildings in the context of design control processes in order to speculate about which methods might hold the greatest promise for creating well-liked urban streetscapes. While this discussion is relevant it sits 
outside the main thrust of the project and is necessarily brief. In anticipation that this discussion will continue the thesis concludes with a summary of the matters around which design control could, and perhaps should, be interested, based on the collected evidence. The findings of the research help us to better understand how people perceive urban streetscapes and therefore these become a platform for future work, one aspect of which could explore how people's preferences can be better integrated with development control.

Keywords: Urban streetscape; Environmental aesthetics; Design control; Aesthetic preferences 


\section{Contents}

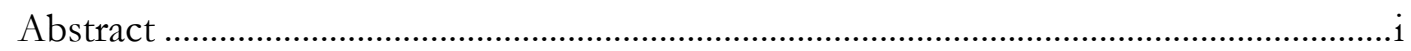

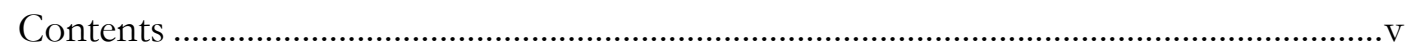

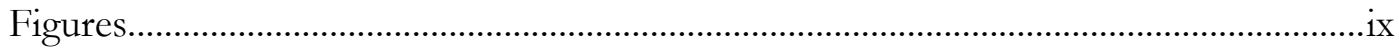

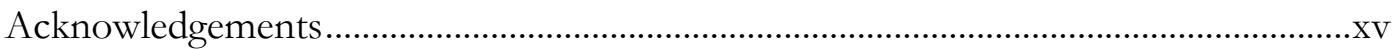

\section{Chapter 1 - Introduction}

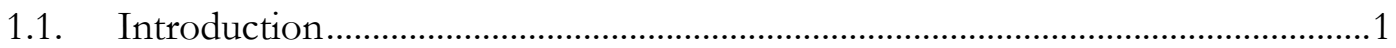

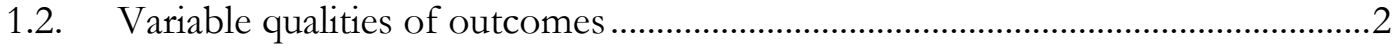

1.3. Relevance of environmental aesthetics ...................................................................

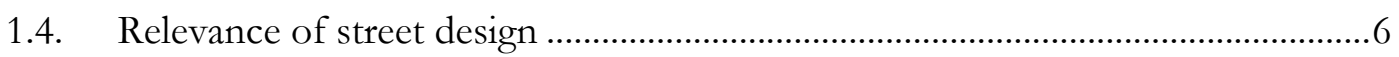

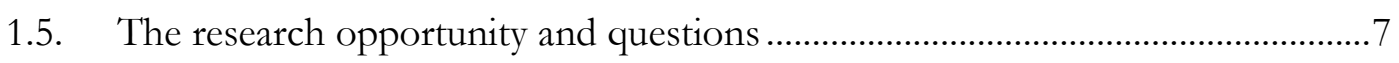

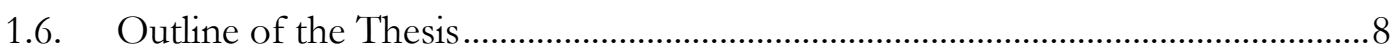

\section{Chapter 2 - Design Guidance and Control}

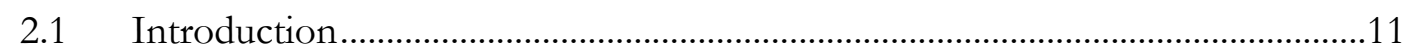

2.2 Context: design control in Britain ...............................................................12

2.3 Context: design control in the United States .........................................................16

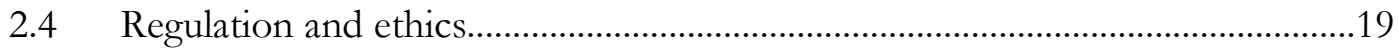

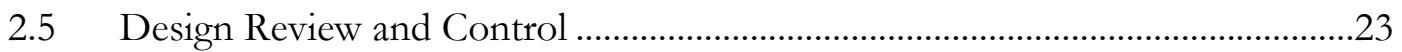

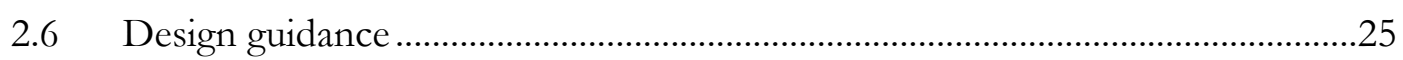

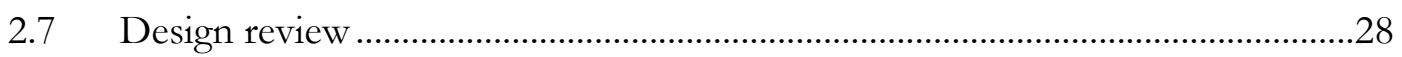

2.8 Theories and principles to inform design guidance ...........................................29

2.9 Other design control methods ..................................................................................33 
2.10 The cases for and against design review ……………......................................... 34

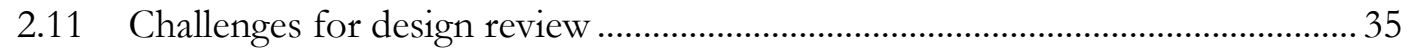

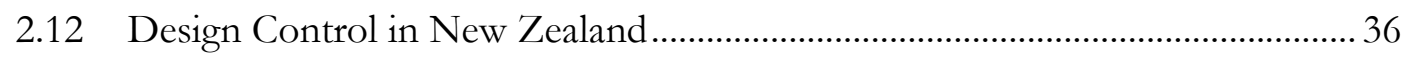

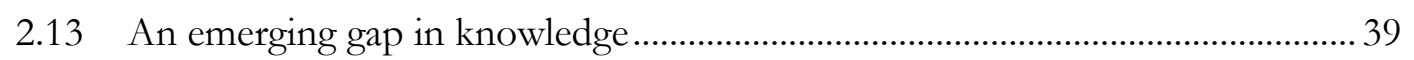

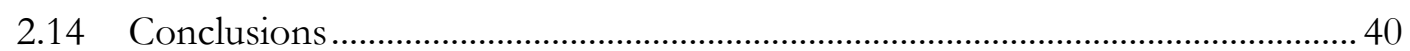

\section{Chapter 3 - Environmental Aesthetics}

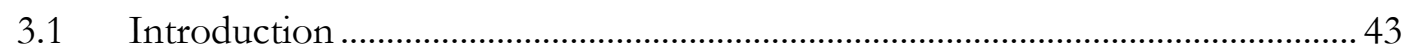

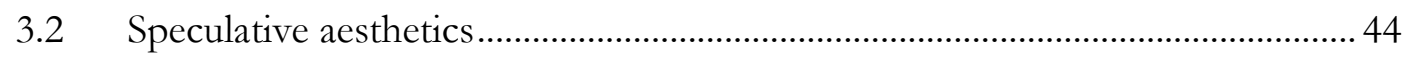

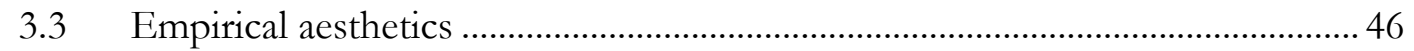

3.4 Response to sensory stimulation........................................................................... 49

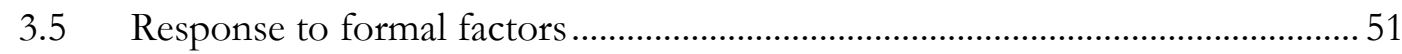

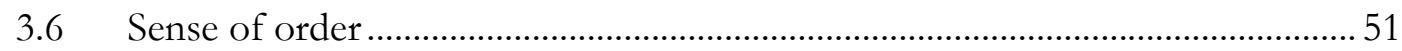

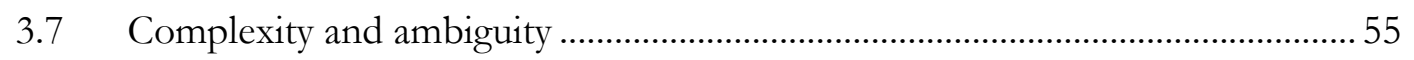

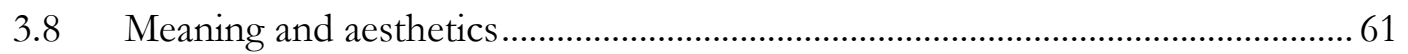

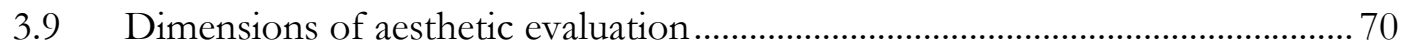

3.10 Lay and professional aesthetic preferences ........................................................... 72

3.11 Theoretical framework and knowledge gap ....................................................... 74

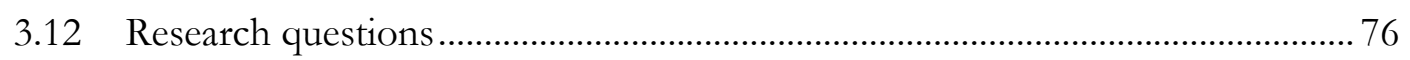

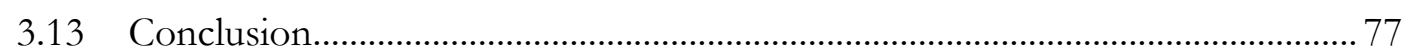

\section{Chapter 4 - Research Design}

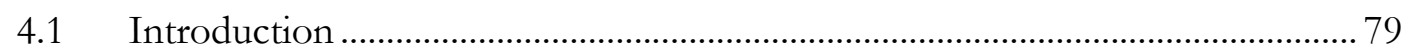

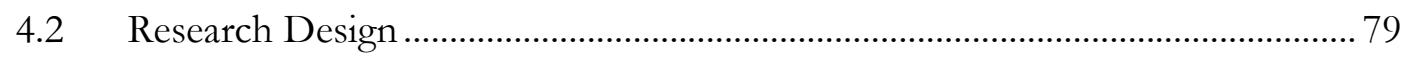

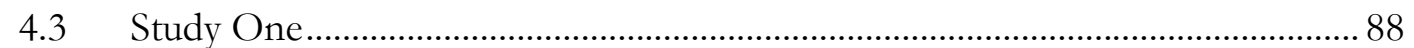

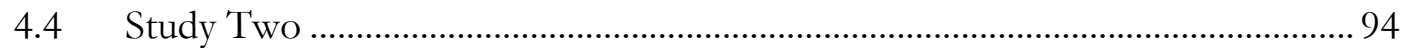

$4.5 \quad$ Focus Group Discussion ………………….................................................... 101

4.6 Evaluation of Built Form Characteristics ............................................................ 103

4.7 Human Ethics Committee considerations............................................................... 106

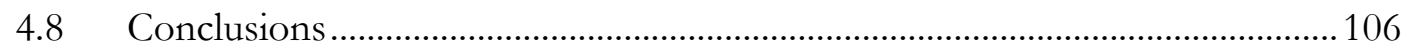




\section{Chapter 5 - Study One}

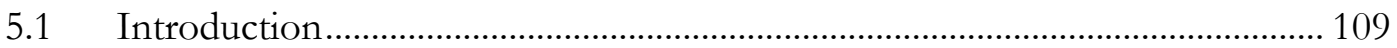

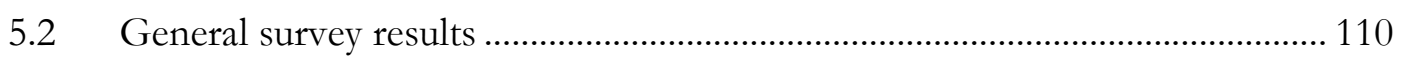

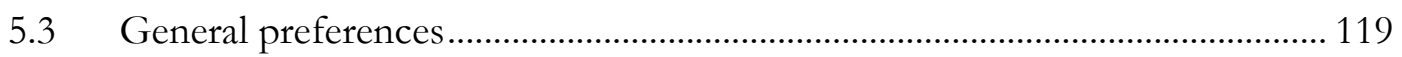

5.4 Comparisons based on demographic groupings ............................................... 129

5.5 Summary of Study One findings ……………….............................................. 140

\section{Chapter 6 - Study Two - College Street}

\begin{tabular}{|c|c|}
\hline 1 & .... 14 \\
\hline 6.2 & College Street Wellington.. \\
\hline 6.3 & General preferences..... \\
\hline 6.4 & 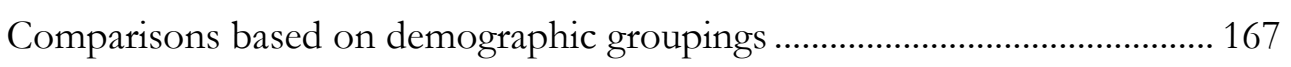 \\
\hline 65 & Focus group discussions ..... \\
\hline & Summary of the College Str \\
\hline
\end{tabular}

\section{Chapter 7 - Study Two - Tyler Street, Auckland}

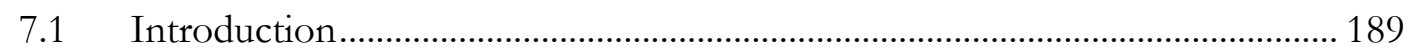

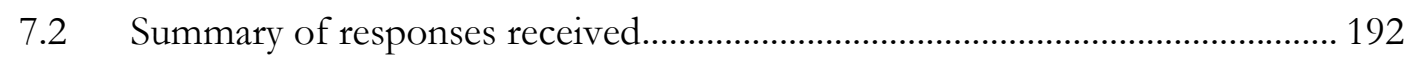

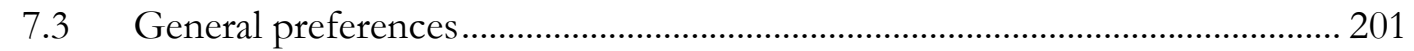

7.4 Comparisons based on demographic groupings ................................................ 210

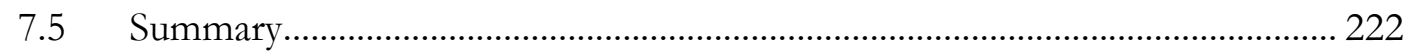

\section{Chapter 8 - Study Two - Kingston \& Wyndham Streets, Auckland}

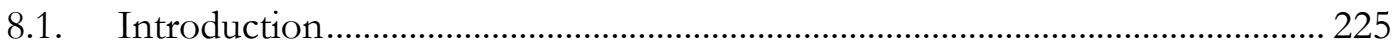

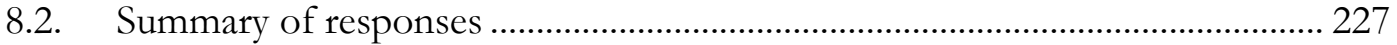

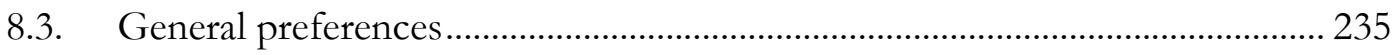

8.4. Comparisons based on demographic groupings ............................................... 249

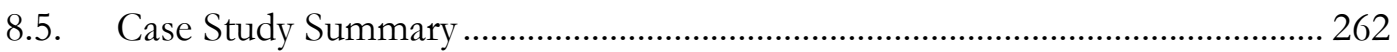

\section{Chapter 9 - Research findings and discussion}

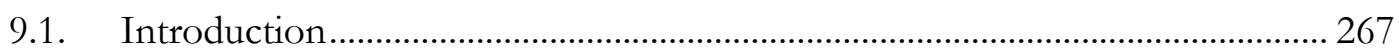

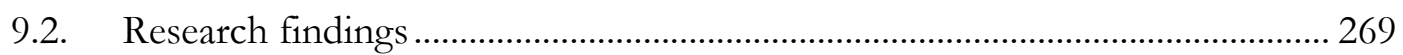

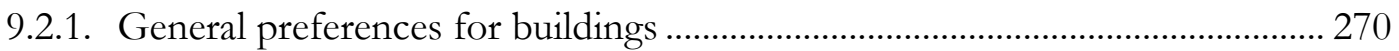

9.2.2. General preferences for urban streetscapes and relationships between

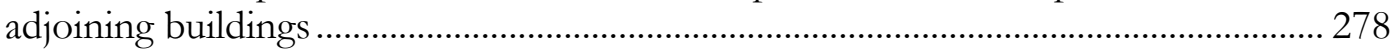

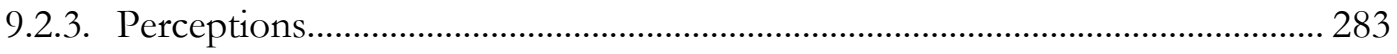

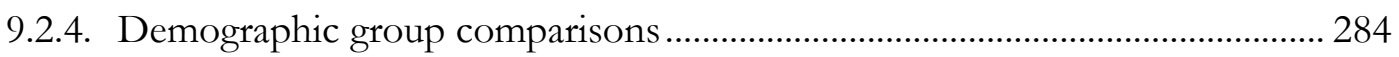

9.2.5. Comparison of presentation stimuli......................................................................... 289

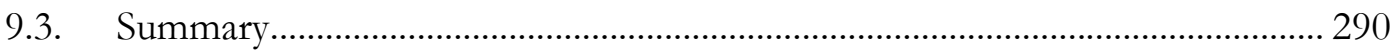




\section{Chapter 10 - Conclusions}

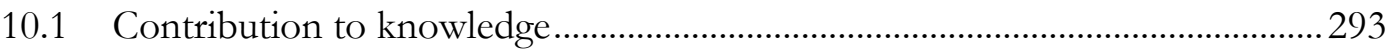

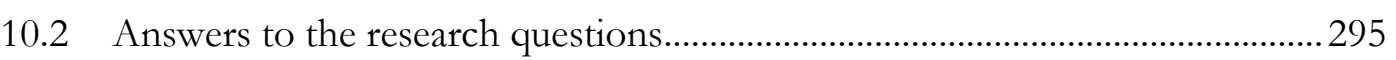

10.2.1 Question 1: what are the characteristics of well-liked buildings and streetscapes

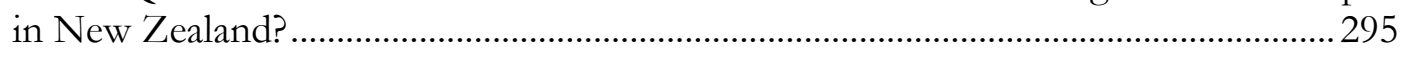

10.2.2 Question 2: how do the aesthetic preferences of professionally trained architects and planners compare with those of lay members of the public?....

10.2.3 Question 3: what are the differences between preferences expressed in relation to photographic representation and real life experience................................................22

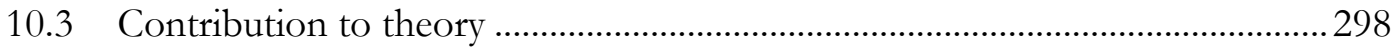

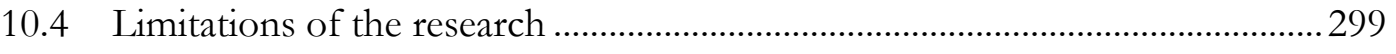

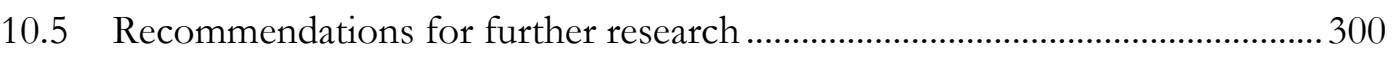

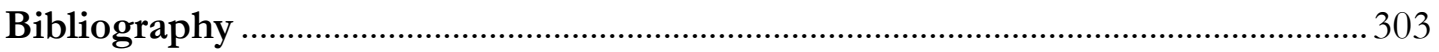

Appendices 


\section{Figures}

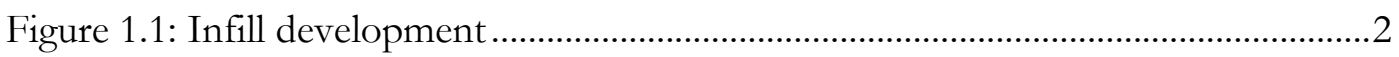

Figure 1.2: John Hancock Tower ..................................................................................

Figure 1.3: Moving from having to being......................................................................6

Figure 2.1: Edwards' example of a disharmonious streetscape .......................................14

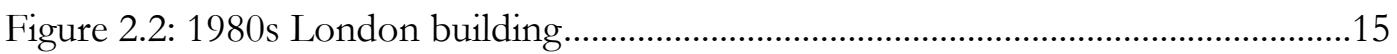

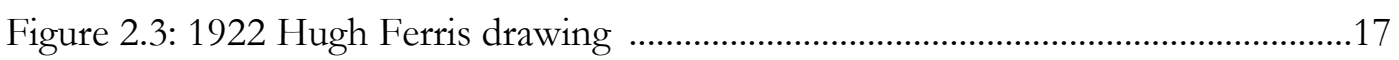

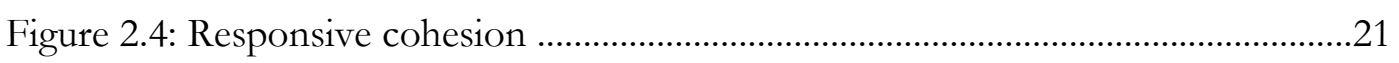

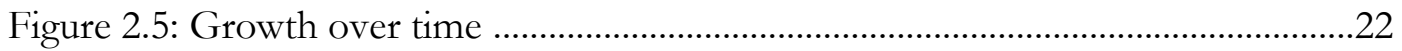

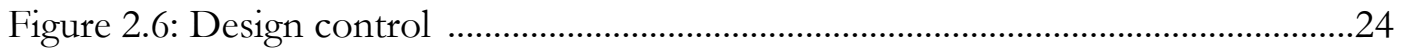

Figure 2.7: Edward's human scale relationships...............................................................

Figure 2.8: Spanish Colonial style in Santa Barbara, California........................................33

Figure 3.1: The Golden Mean proportioning system ....................................................4

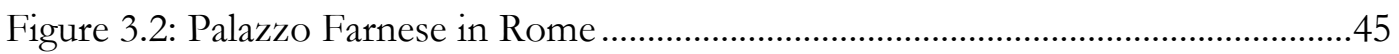

Figure 3.3: Characteristic pattern of aesthetic response .................................................47

Figure 3.4: Framework for aesthetic response to the environment................................48

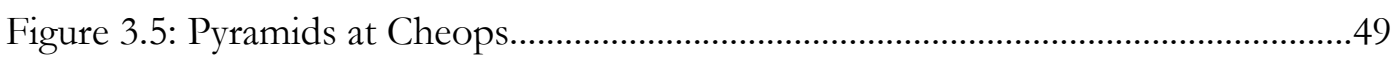

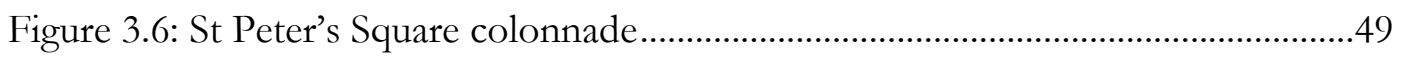

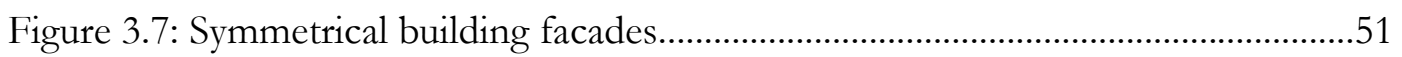

Figure 3.8: Rhythmic pattern of openings and details .....................................................52

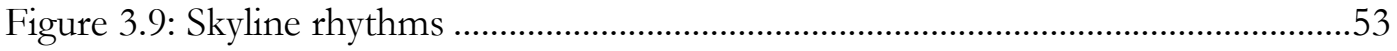

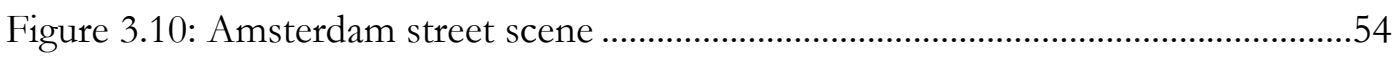

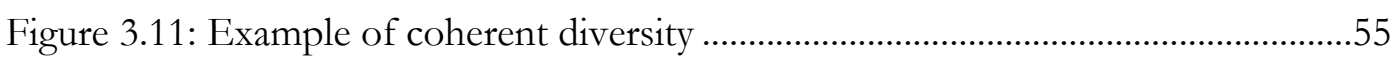

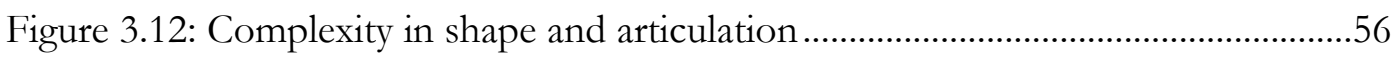

Figure 3.13: Stamps III's studies of building shape complexity ......................................58

Figure 3.14: The Spanish hillside town of Mojacar...........................................................59

Figure 3.15: Three dimensional form articulation ...............................................................59 


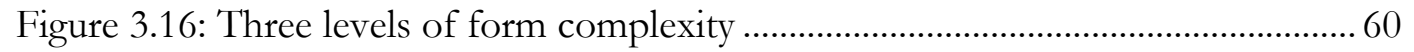

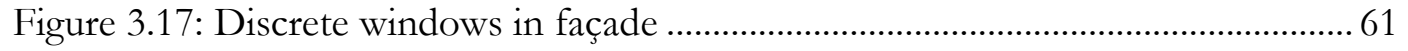

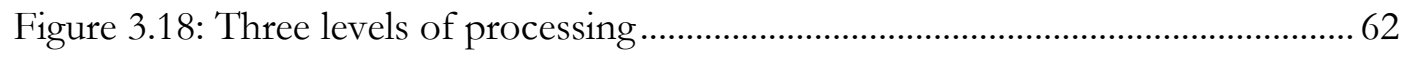

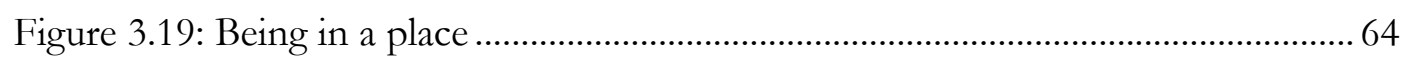

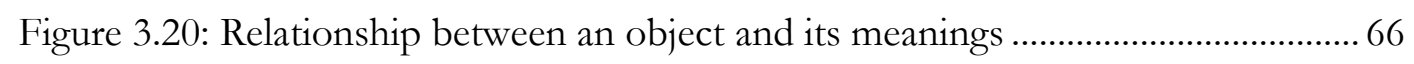

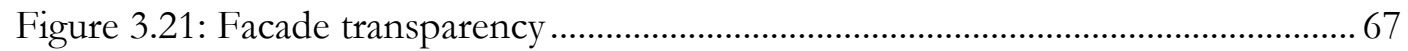

Figure 3.22: Dimensions of environmental effect..................................................... 70

Figure 3.23: Stamps III model of aesthetic response ........................................................ 71

Figure 3.24: Theoretical framework for the research...................................................... 75

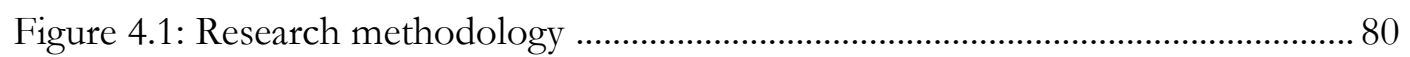

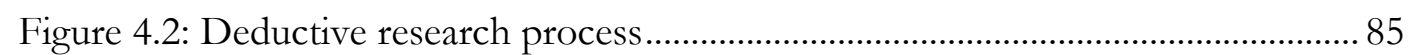

Figure 4.3: Study One street scenes ................................................................................. 91

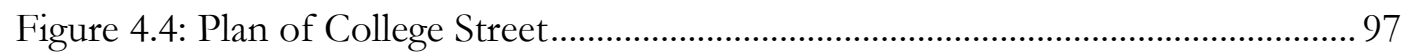

Figure 4.5: College Street survey form ........................................................................... 99

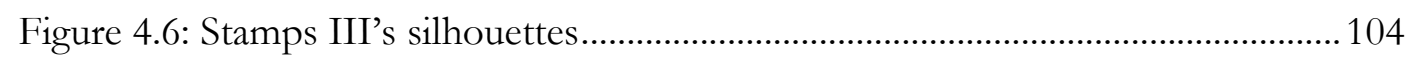

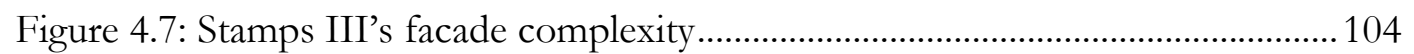

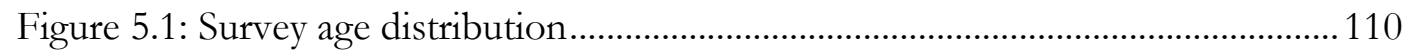

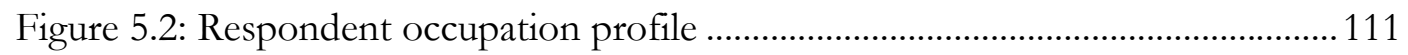

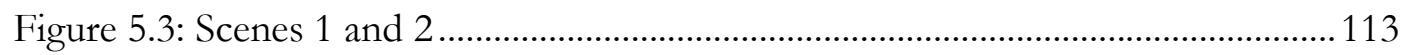

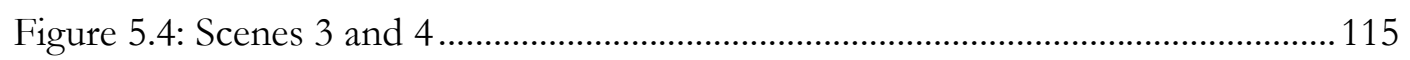

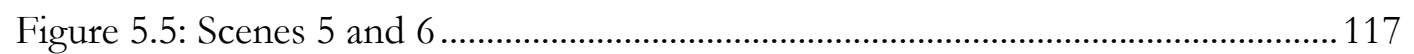

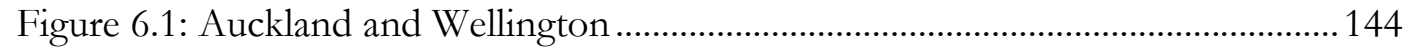

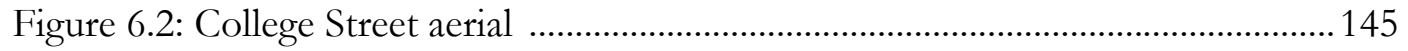

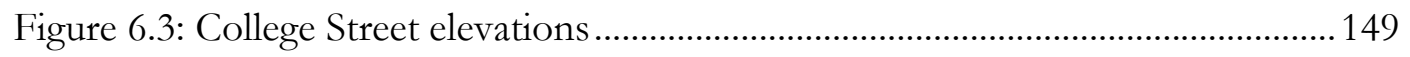

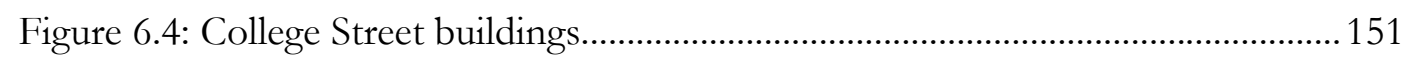

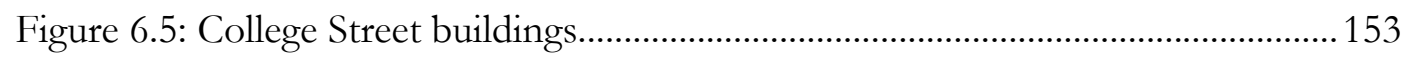

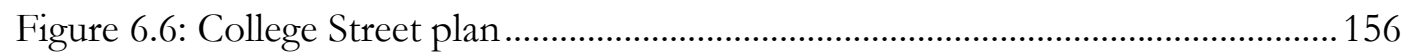

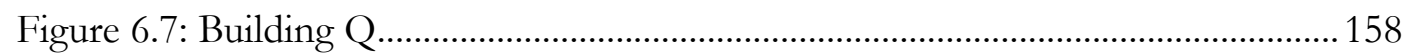

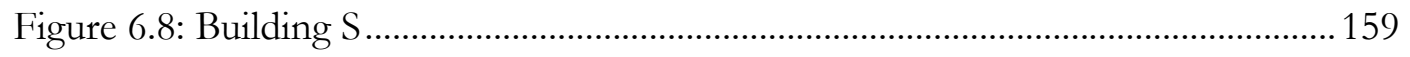

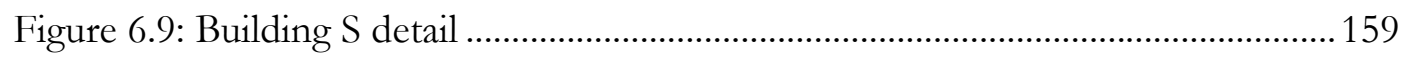

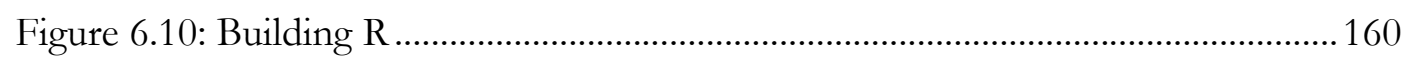

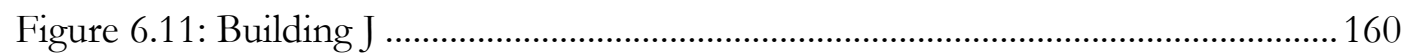

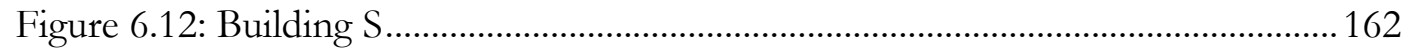

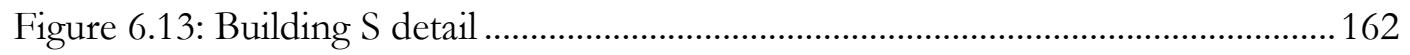

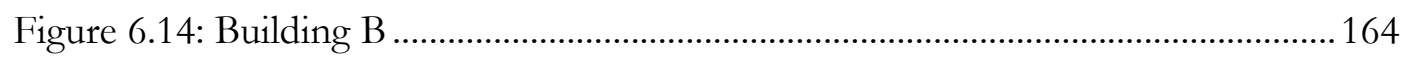

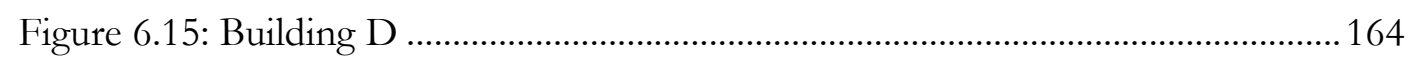




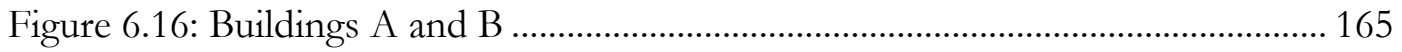

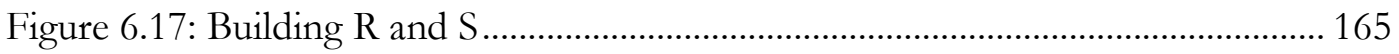

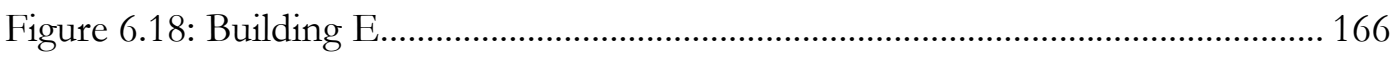

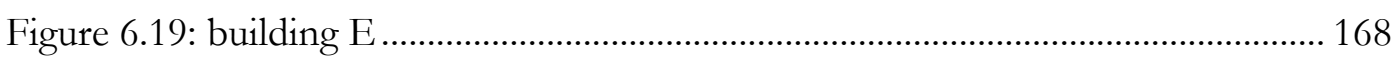

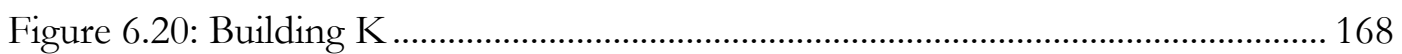

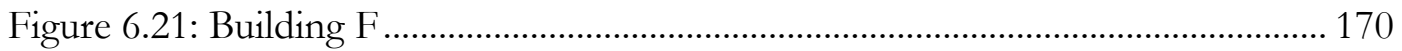

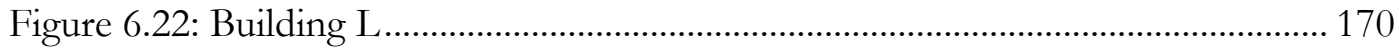

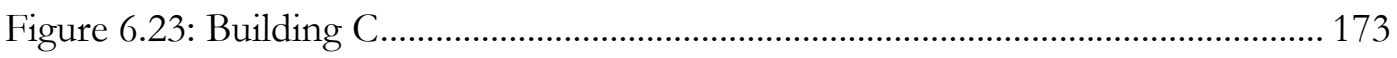

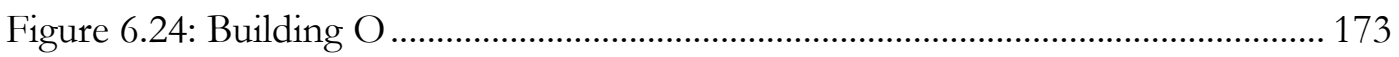

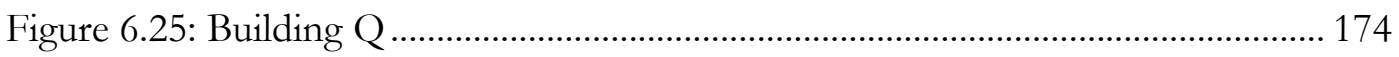

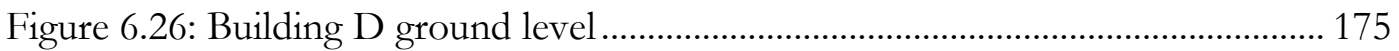

Figure 6.27: South side of College Street........................................................................ 177

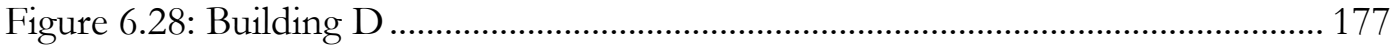

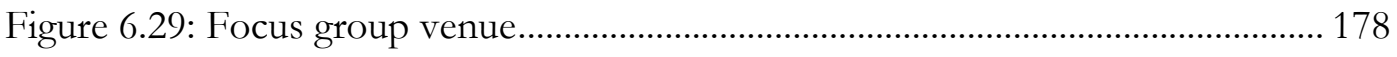

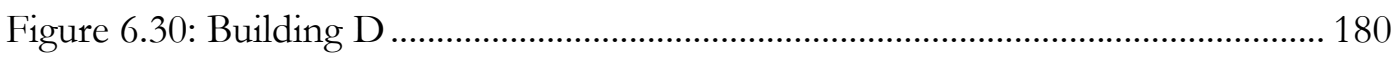

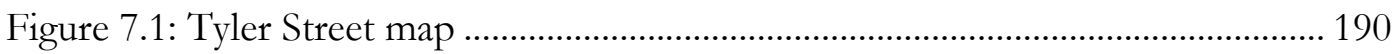

Figure 7.2: Tyler Street aerial......................................................................................... 191

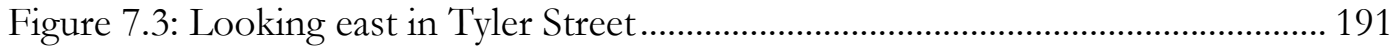

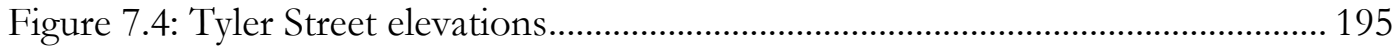

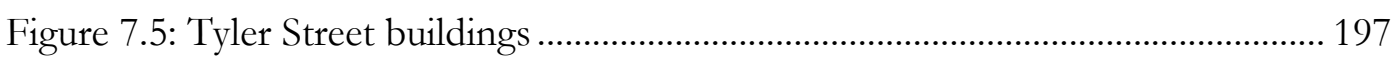

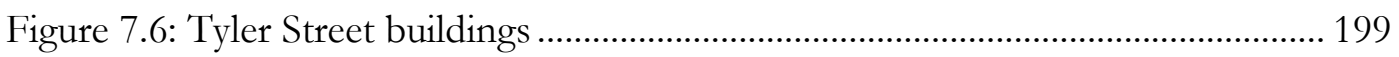

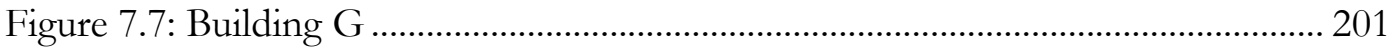

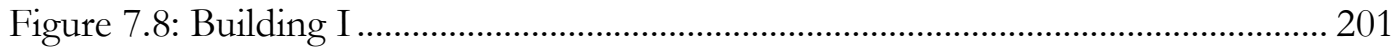

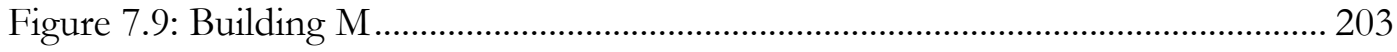

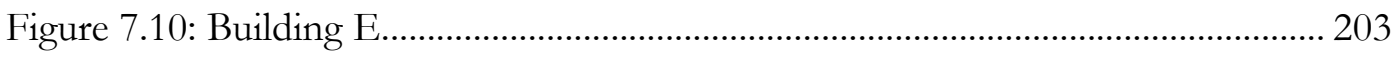

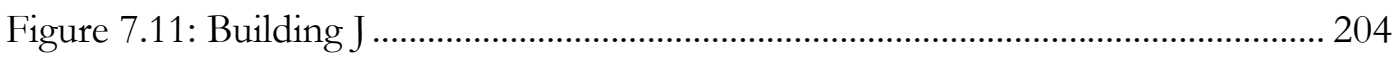

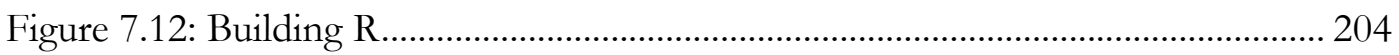

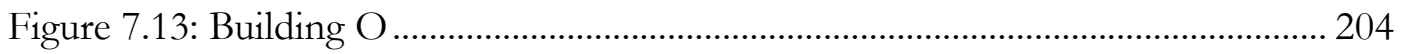

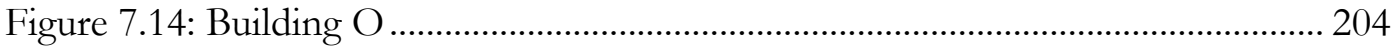

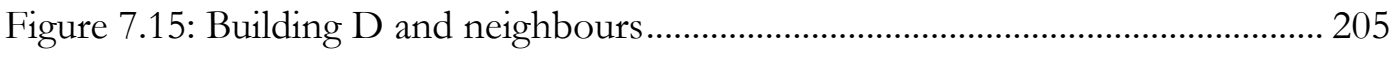

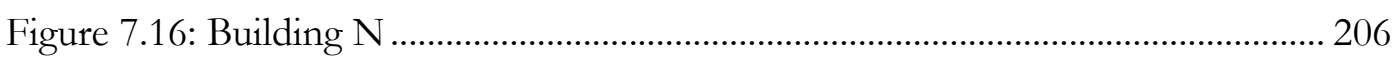

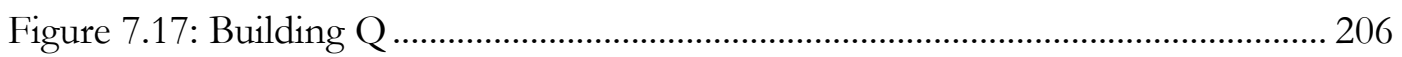

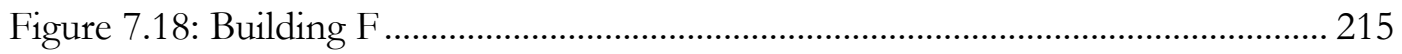

Figure 7.19: Building N detail..................................................................................... 217

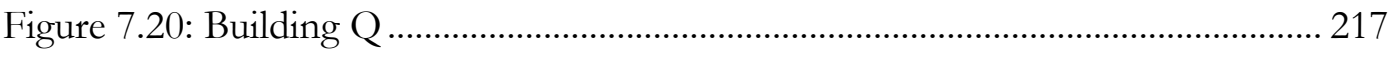

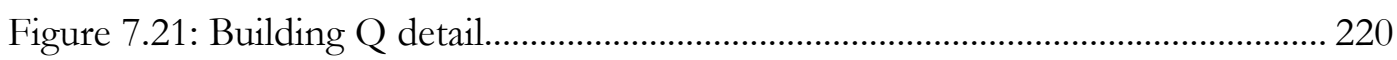


Figure 8.1: Kingston/Wyndham Street aerial view ……………………………...........226

Figure 8.2: Kingston/Wyndham Street elevations ..........................................................222

Figure 8.3: Kingston/Wyndham Street buildings ...........................................................231

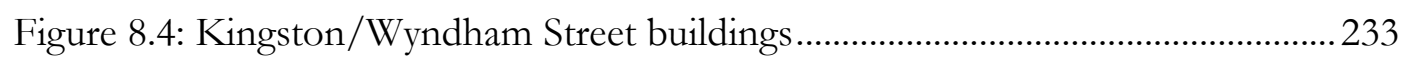

Figure 8.5: St Patrick's Cathedral _...............................................................................225

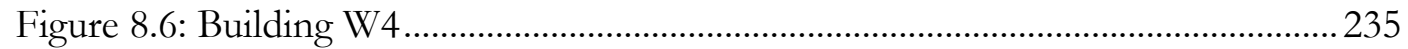

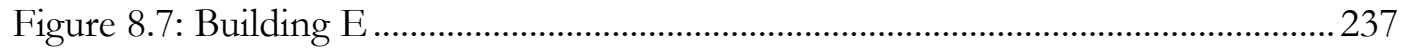

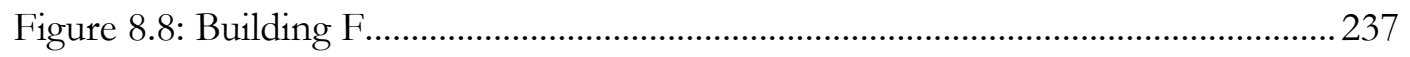

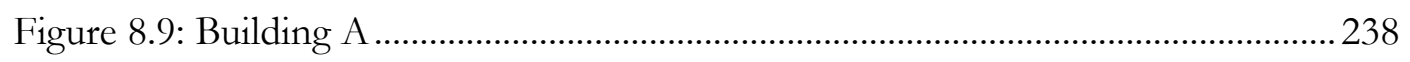

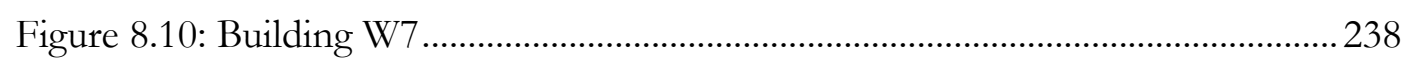

Figure 8.11: Building W7 street level ........................................................................240

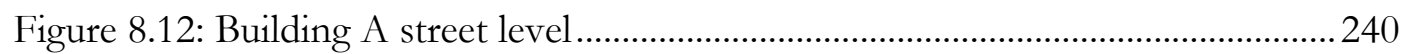

Figure 8.13; Building W3 colonnade …………............................................................ 242

Figure 8.14: Building H street level............................................................................ 242

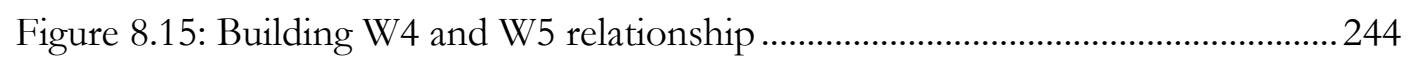

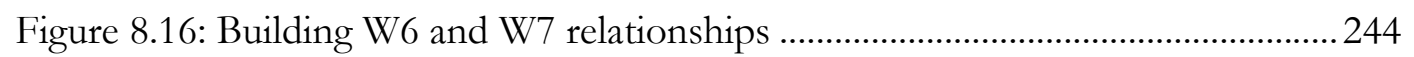

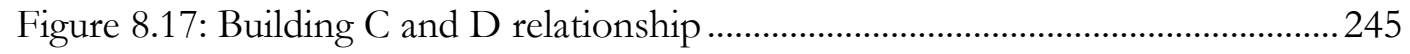

Figure 8.18: North side Wyndham Street alignment......................................................248

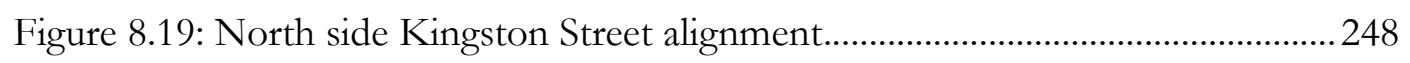

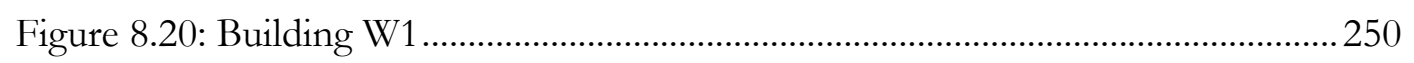

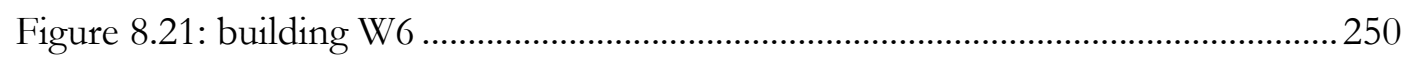

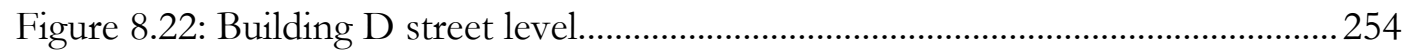

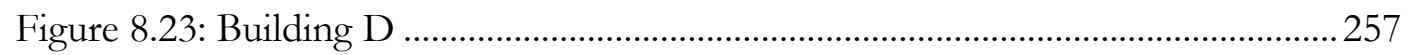

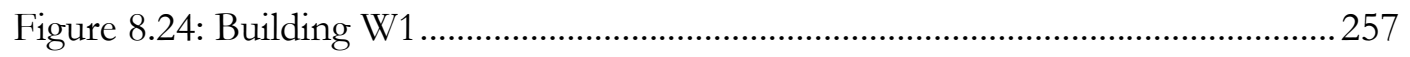

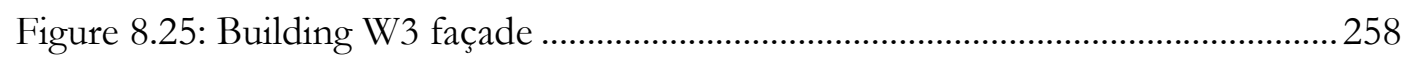

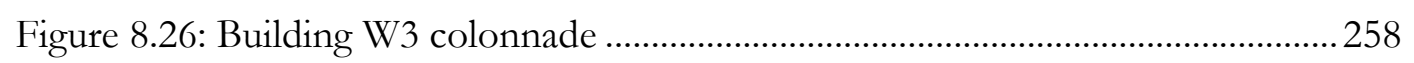

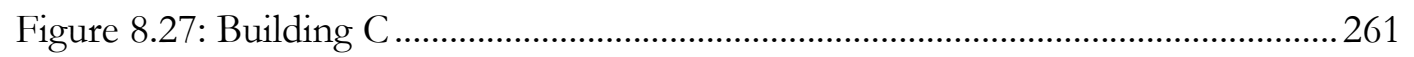

Figure 8.28: South side of Wyndham Street....................................................................261

Figure 9.1: Study One; scene 3 ....................................................................................2 273

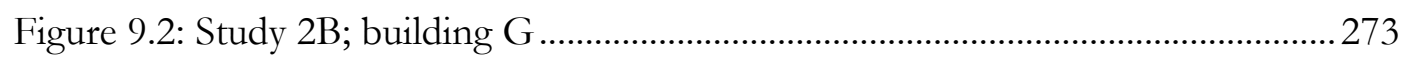

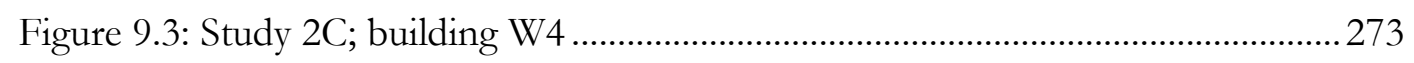

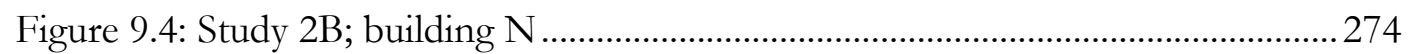

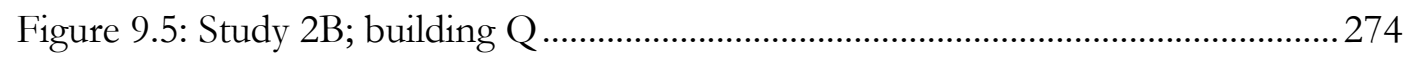

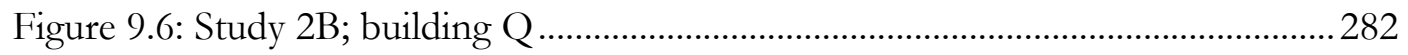

Figure 9.5: Harsh façade at street level .......................................................................22 


\section{Tables}

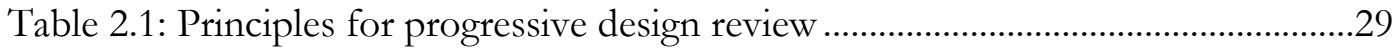

Table 2.2: Development of principles for design control .....................................................

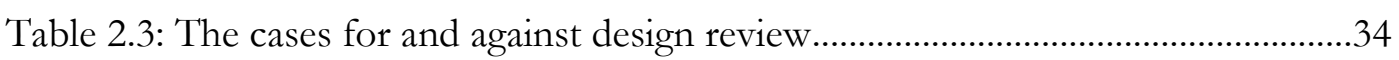

Table 4.1: Key considerations of quantitative and qualitative research paradigms ......84

Table 4.2: Research methodology, linking research methods to research objectives ..87

Table 4.3: Groups targeted for participation in Study Two........................................... 101

Table 5.1: Preference scores for the six scenes.................................................................... 119

Table 5.2: Preference scores for heights in the six scenes ........................................... 122

Table 5.3: Correlation coefficients for respondents' overall preferences with their scores for the relationships of height, materials and colours ....................................... 123

Table 5.4: Mean preference scores for pleasantness and the relationships between

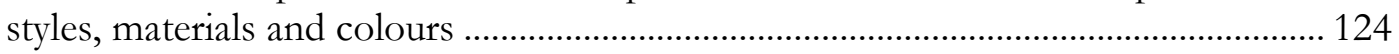

Table 5.5: Responses to question "do any of the buildings in the scene not fit?" .... 125

Table 5.6: Comparison of the mean scores by men and women for scene 3 ............ 129

Table 5.7: Men's and women's responses to streetscape characteristics of

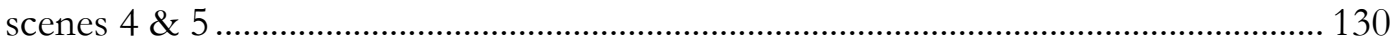

Table 5.8: Comparison of mean scores by age (5 categories) for height relationships

Table 5.9: Comparison of mean scores by age (3 categories) for height relationships

Table 5.10: Comparison of mean scores by age (3 categories) for colour relationships

Table 5.11: Comparison of mean scores by change professionals and lay people for pleasantness and preference .............................................................................................. 136

Table 5.12: Responses to scene 3 by occupational groupings ........................................ 138

Table 5.13: Mena preference scores by occupational group for streetscape

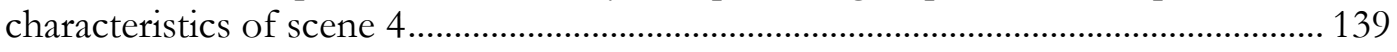

Table 6.1: Distribution of sample in College Street case study ...................................... 147 
Table 6.2: Mean preference scores for streetscape characteristics 155

Table 6.3: Mean preference scores on 3-point scale for individual buildings.

Table 6.4: Mean preference scores for the four buildings rated positively by all age groups

Table 6.5: Mean preference scores by professional grouping for streetscape characteristics

Table 6.6: Mean preference scores by professional grouping for individual buildings

Table 6.7: Mean scores for streetscape preferences for professional focus group participants and other groups.

Table 7.1: Ages, occupations and residential circumstances of the Tyler Street sample.

Table 7.2: Comparison of preference scores for buildings A-F and their relationship scores

Table 7.3: Mean preference scores for streetscape characteristics in Tyler Street.....208

Table 7.4: Mean preference scores for buildings along Tyler Street..............................214

Table 7.5: Mean responses for design characteristics of building F .............................216

Table 7.6: Mean preference scores for buildings $\mathrm{N}$ and Q .............................................218

Table 7.7: Comparison of mean preference scores for Tyler Street streetscape characteristics

Table 8.1: Distribution of responses for age, occupation and residential circumstances in the Wyndham \& Kingston Street case study .............................................................228

Table 8.2: Mean scores for buildings A and W7 ........................................................2239

Table 8.3: Preference scores for buildings $\mathrm{H}$ and W3 ................................................... 241

Table 8.4: Mean preference scores for streetscape characteristics of case study $3 . . .246$

Table 8.5: Mean preference scores for building W6 by gender .................................... 251

Table 8.6: Age group preferences expressed for buildings D and W6 ........................253

Table 8.7: Mean preference scores for buildings in the Kingston/Wyndham study area 256

Table 8.7: Mean scores for streetscape characteristics in the Kingston/Wyndham

Street area for lay people and change professionals ......................................................2. 260

Table 9.1: Comparing the average of the mean preference scores for buildings in each

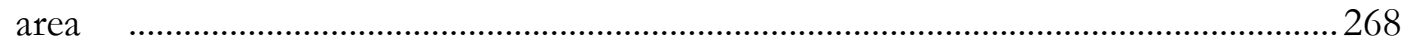

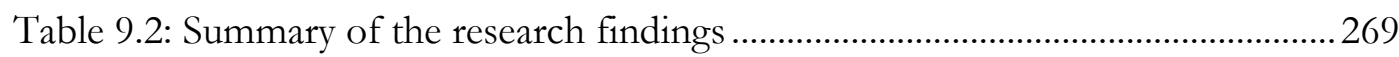

Table 9.3: Mean preference scores for the relationships between building heights in

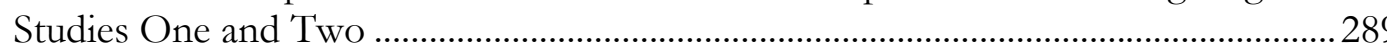




\section{Acknowledgements}

This thesis is dedicated to the memories of Kari and Torbjørn Gjerde, who helped me develop the tools I needed to get the job done! Mange tusen takk, far og mor!

Brenda, thank you for your guidance over the past three years, this project is so much better for your help. More importantly, you've shown me what great supervision can be like and I hope to make use of this with my own students. Sophie, your input has also been invaluable.

I feel a debt of gratitude to Victoria University of Wellington, who have supported my efforts throughout. I couldn't ask for a more accommodating employer.

Finally, I wish to acknowledge the love and enthusiastic support you've shown me, Jo, particularly over the final few weeks. You're amazing, I'll love you always and let's get back to enjoying our weekends together!

Morten

7 July 2015 


\section{Chapter 1}

\section{Introduction}

\subsection{Introduction}

Around the world, people continue to flock to cities to live, work and play. Increasingly, people come to cities to enjoy civic and social amenities as well as economic opportunities that arise when people live more closely together. This phenomenon, which began with the advent of the Industrial Revolution, show no signs of abatement (Carter 1995: 17). Indeed, the proportion of people living in towns and cities internationally has recently passed $50 \%$ and will increase to $67 \%$ by 2050 (United Nations 2012). Urbanisation rates in developed countries are even higher, with $90 \%$ of the United Kingdom's population currently living in cities and $85 \%$ of Australia classified as urbanised (Statistics New Zealand 2004). Even New Zealand, a country that has been settled by humans for less than a thousand years and that continues to trade on its image as a rural wilderness, has evolved into a nation of city dwellers. Some $86 \%$ of New Zealanders were living in cities and towns in 2006 , a rate that is likely to have increased in the period since (Hunt 2008, Ministry of Social Development 2010).

To accommodate the growing numbers of people, the built environment is also changing. While a considerable amount of growth takes place around the edges of cities, expanding the area of land they occupy, transformations are also reshaping existing built-up areas. The changes take place over varying scales and timeframes, ranging from market driven, incremental steps comprising single buildings to large scale redevelopment of entire blocks. This thesis is concerned with the effects these changes have on the visual appearance of cities and towns. 


\subsection{Variable qualities of outcomes}

Responding to economic opportunities, changes are generally undertaken to increase value to the landowner and to others who have direct interest in the project. However, the outcomes affect the setting and people more widely. Where designs are well considered, the outcomes can have positive effects on surrounding public spaces. In Melbourne, for example, the physical and social dimensions of several of Melbourne's inner city suburbs have been transformed through discrete and seemingly uncoordinated projects by landowners over the past 40 years (figure 1.1). Together the changes have given these suburbs more than increased residential and workplace capacity; the quality of the public realm has also clearly been enhanced in the process (O'Hanlon and Sharpe 2009). At a larger and more coordinated scale, the London Docklands area was transformed into an attractive and vibrant part of the city through application of an urban design framework and has set a new standard for design-led development (Carmona, Heath et al. 2010: 70).

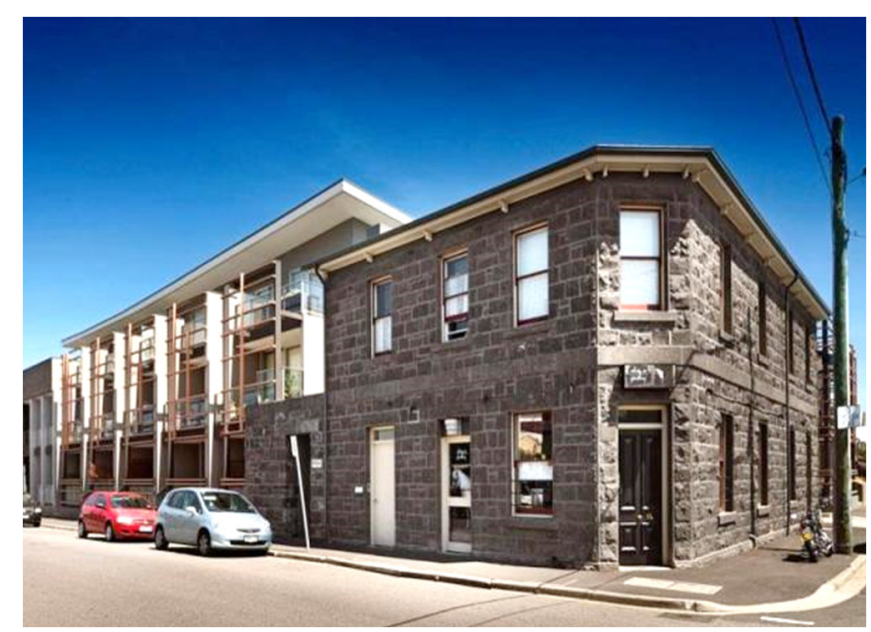

Figure 1.1: A recent infill residential development alongside an historic hotel in Yarraville, Australia. This project is typical of those that continue to transform the inner city suburbs around Melbourne.

Source: realestate.com.au

On the other hand, rapid rates of change in some cities have led to outcomes that several writers have found problematic. For example, wholesale redevelopment of city centres according to Modern architectural and planning principles have in many cases led to austere and placeless environments (Jacobs 1961, Relph 2007). Smith (1977: 9) argued that such places have left people starved of 'visual nourishment'. Although visually stimulating from a distance, the John Hancock Tower in Boston (figure 1.2) is an example of a single building, inserted into its setting without reference to that setting, that has deteriorated the visual and 
spatial qualities of the area around it (Trancik 1986). John Punter, commenting on the direction Sydney's built environment had evolved since the Second World War, stated that it had succumbed to "spectacularly ordinary commercial development" and that the poor aesthetic outcomes are only excused by the discerning public because of the city's spectacular natural setting (Punter 2004: 406). Closer to home, Layla Dawson of the Architectural Review recently discussed the state of architecture in New Zealand and expressed surprise that Auckland appeared so much like an unplanned jumble of high-rise blocks. Why, she asked, would the public put up with such poor architecture (Dawson 2010)? For too long now people have generally felt that environmental aesthetics have been neglected by those who produce the built environment - architects, planners, developers and politicians - and the outcomes are increasingly ugly and unpleasant environments (Cold 2001: 3). Writing in a British context, Bentley (1999: 2) wrote that when the public had been consulted, it was generally found to dislike the ways their cities had been changed in the period following WWII.

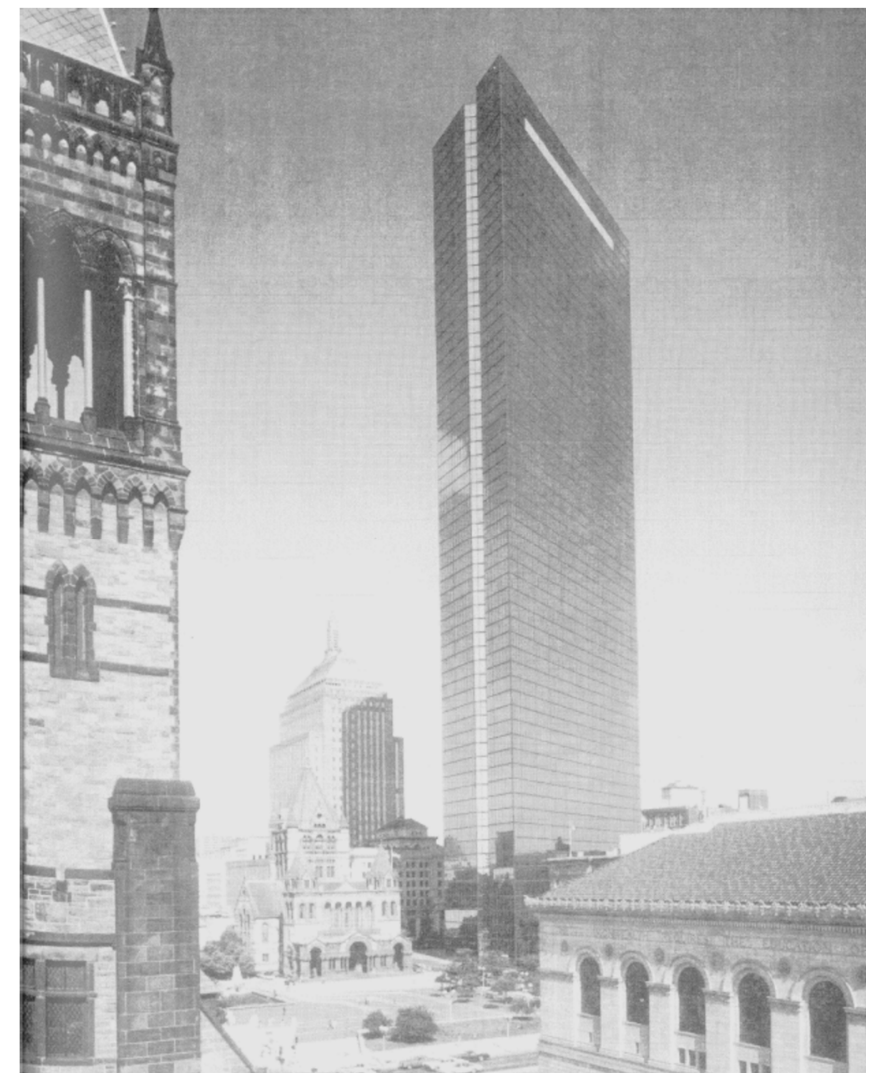

Figure 1.2: The John Hancock Tower is an exquisite sculptural object when viewed from a distance. At street level the building is poor as it makes no references to the space and buildings around it. 
To summarise, while many places are continuing to evolve and change to the satisfaction of interested people, other urban places have been redeveloped in ways that people have been critical of. A question that follows on from this circumstance is, 'why does it appear to be difficult to consistently build places that people will like?' Addressing this question, Bentley (1999) noted several constraints that can affect contemporary development. It seems that property development in the Western world adheres to the principles of capitalism, which in turn leads to efforts on the supply side aimed at lowering building costs. While lowering costs these same actors seek to increase market values, all in an effort to enhance margins of profit. Lower financial investment in the production phase of development leads to buildings that are flatter, less interesting and prone to ageing poorly (Bentley 1999: 87). To distinguish their product from the competition and make them more attractive to the end buyer, developers encourage visually exciting designs from their architects (: 92). Often making use of shiny materials to make up for lack of interest in the details (which cost money to produce), these designs can struggle to fit in with others around them. As noted above, this is often the effect sought by the designer and their client.

The outcome of successive projects undertaken in this manner is a visual cacophony, with each adjoining building vying crassly for attention (Habraken 1994). Numerous other factors such as globalisation, lack of appropriate skills and imposition of time constraints also conspire to diminish the visual qualities of new buildings and therefore the whole of the built environment (Ellin 2006: 98-99). The problem that this research tackles lies in the tensions that exist between the different interests of those who initiate urban transformation and those who have to live with the consequences.

\subsection{Relevance of environmental aesthetics}

A question that must be addressed if this research is to have any relevance is whether the appearance of the built environment really matters to people. In answer to this question, aesthetic perceptions of the built environment have been linked to mental, physical and economic wellbeing. On the one hand, poor urban environments can induce higher levels of emotional and psychological stress in people and have been shown to diminish selfesteem, both of which lead to lower states of wellbeing (George and Campbell 2000, Pallasmaa 2001). On the other, people's wellbeing can be enhanced through the experience of aesthetically pleasing places. Such places can help generate, celebrate and sustain life by making a person feel more complete and satisfied (Porteous 1996: 5, Dovey 2001). 
Smith (2003: 95) connected aesthetic pleasure with processes of natural selection in human evolution. He cited empiric studies that have shown how sensory pleasure, including positive visual experience of a person's environment, can help raise immunoglobulin levels, a key antibody that bolsters the human immune system which in turn increases chances of survival. He also observed that aesthetic perception provides for higher level ordering of environments, which in turn can enhance their legibility people's wayfinding abilities. Both are factors in natural selection (Smith 1980, Smith 1987) Lynch (1960) agreed, pointing out that structuring and identification of the environment, which are core aspects of aesthetic perception, are also vital tools for the survival of all animals that move about.

The appearance of the built environment can have tangible influence over economic values, directly affecting the financial well-being of those who own property or conduct a business in an area. The choices people make every day usually include an aesthetic dimension. These may include deciding which city to live in, which house to buy and even which street to walk along (Madanipour 1996: 164). It seems that visually attractive places are more sought after by the public, helping to increase demand and therefore economic values (Tiesdell, Oc et al. 1996). Increasingly, the built environment is being used as a tool in battles fought between cities for business investment, tourism and high-calibre workers (Carmona, Magalhaes et al. 2002, Cuthbert 2006). People regularly make choices around matters of aesthetics and it is clear that the visual attractiveness of cities affects the financial well-being of individuals as well as the wider community.

There is a sense that the design dimension and the aesthetics of the built environment have been neglected as areas of academic enquiry and professional development, as well as by society more widely, for some time (Punter and Carmona 1997: 2, Cold 2001: 3). As Lynch (1976: 68) found, 'aesthetics is often considered a kind of froth, difficult to analyse and easy to blow away'. How can this be, when there is compelling evidence to suggest that environmental aesthetics has relevance to people in many important ways? In answer to this question, Porteous (1996: 6-8) explained that interest in aesthetics has largely been exhibited at personal, rather than at societal, levels. In their personal lives and as they become increasingly affluent, people move from having a standard of living to enjoying a quality of environment and ultimately having quality of life. See figure 1.3. This progression is seen to parallel a movement from having to being in the manner described in much more detail by Maslow (cited Porteous 1996) in his still relevant framework of human needs. 
Although the hierarchical relationships described in figure 1.3 could also apply more widely across social structures, as they embody the interests of individual members, they do not at present. The ever-optimistic Porteous believes that increasing affluence and resolution of economic, ecological and other problems in Western societies could enable them to place more emphasis on environmental aesthetics in the future (1996: 7).
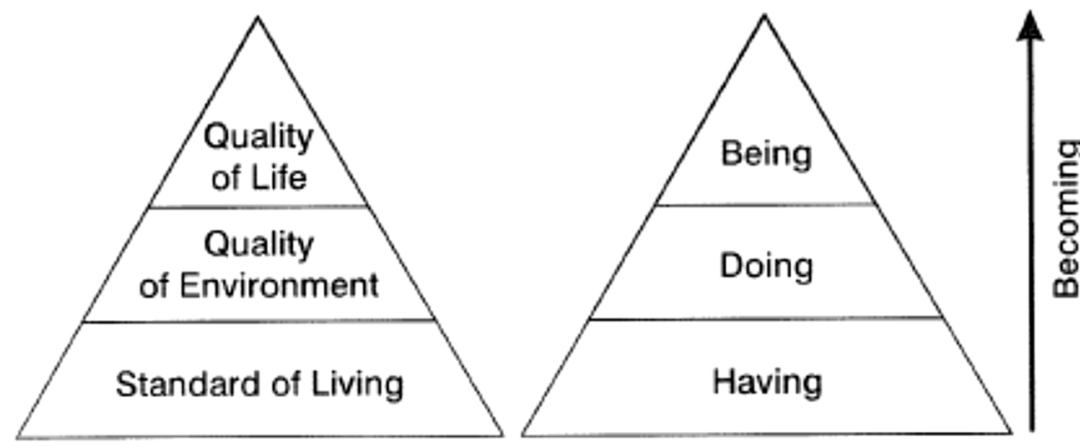

Figure 1.3: Individuals aspire to move from having a standard of living to having quality of life. This corresponds with moving from having to being.

Source: Porteous 1996

\subsection{Relevance of street design}

"Think of a city and what comes to mind? Its streets. If a city's streets look interesting the city looks interesting; if they look dull, the city looks dull". With this statement, Jane Jacobs (1961: 29) articulated the importance of streets in the mental images held of cities around the world and across time. It is the streets and buildings that line their sides that provide people with the most enduring memories of a city. Indeed, without streets there would be no city (Kostof 1992: 194). They moderate the form and structure of cities and have featured prominently in the findings of research into the ways people form mental images of their environments (Lynch 1960). There is little doubt that streets are the primary form of public space in a city (Jacobs 1993: 3, Carmona 2010).

Streets also feature prominently in the social and political histories of most cities (Kostof 1992: 194-200) but it is the physical characteristics of the buildings that define street spaces that are of interest in this research. Jan Gehl (2011:150) discovered that people's behaviour is readily influenced by the quality of the pedestrian environment, including the facades of buildings lining the route. Diversity is a critical success factor for urban streets, a matter that Modernist urban planning principles supporting large uninteresting building facades, seemed 
to overlook (Jacobs 1961: 148-149). Both Gehl (2011: 181) and Allan Jacobs (1993: 6-8) discussed the important role that aesthetic qualities play in making streets memorable and attractive places to visit. However, neither scholar provided detailed suggestions about which characteristics designers should target in order to make their interventions attractive to the public.

Researchers have shown a growing interest in the relationships between urban planning and health, asking questions about design characteristics that might encourage people's healthy behaviour. The literature is unequivocal that significant improvements in public health can be generated through increased levels of moderate physical activity (Carmona, Heath et al. 2010: 233). Walking and cycling are two forms of activity that lead not only to better health outcomes but also reduce the need to travel short distances by automobile. It is in all societies' interests to enhance the uptake of cycling and walking. For most people, actual and perceived safety is the top priority for them to choose to walk and recent research suggests that the form and structure of streets can also have considerable influence on their decisions. Streetscape features such as sense of enclosure, transparency, human scale, visual complexity and imageability have all been found to increase people's walking behaviour (Pikora, Giles-Corti et al. 2003, Hansen 2014).

\subsection{The research opportunity and questions}

This thesis is concerned with perceptions of individual urban buildings and the streetscapes they create. Such perceptions are increasingly relevant as they can influence people's decisions on where to go and how to spend their time. The appearance of buildings and streetscapes can also affect people's physical and mental health, and it continues to inform imageability and wayfinding in ways that have influenced human evolution. Research has identified links between environmental aesthetics to people's wellbeing. Accordingly, streetscape appearances do matter. However, it appears that environmental aesthetics at a societal level have been neglected for some time. This neglect has led to a gap in knowledge about which design characteristics people find pleasant, a matter that is discussed in section 3.11. The gap in knowledge is also made manifest in the variable opinions people have about the ways cities have changed over the past 60-70 years.

As societal circumstances change, the opportunity to put more energy into aesthetic matters is now with us (Porteous 1996, Cold 2001). The present research addresses this opportunity. If society is serious about wanting to create better loved places (CABE and DETR 2000), 
can broadly held aesthetic preferences be identified and harnessed in order to influence the ways cities transform in the future?

Arising from this opportunity and the challenges around it, the primary research question is: what are the characteristics of well-liked buildings and streetscapes in New Zealand? Section 3.11 identifies the gap in current knowledge that this question aims to address.

As is discussed in section 3.10, there is also a gap in knowledge about how the aesthetic preferences of experts, the people who are directly involved in the design and management of urban change, compare with those of the general public. Out of this discussion, a second research question is developed; namely how do the aesthetic preferences of professionally trained architects and planners compare with those of lay members of the public? Answers to this question could inform the way decisions about change are made in the future.

The research design also creates the opportunity to compare effectiveness of two different ways of presenting information about urban buildings and streetscapes to survey respondents. This is addressed by the question: what are the differences between preferences expressed in relation to photographic representation and real life experience of urban streetscapes?

The aims of the research are to answer these three questions.

\subsection{Outline of the thesis}

The thesis is organised in 10 chapters. Chapter 1 introduces the research and provides context to the problem being investigated. This chapter also introduces the research questions. The literature that provides the foundations for the research is presented and discussed in chapters 2 and 3. Chapter 2 discusses key literature in the area of design control, presenting at first a brief historic background and progressing on to consider the potential of design control as well as the challenges that must be confronted in its application. Chapter 3 tackles environmental aesthetics, the study of how humans perceive the environment around them. Theories that help explain how people see and evaluate the physical world are discussed and the chapter relates built form characteristics to the different levels of aesthetic perception. This chapter also develops the theoretical framework that informs the research design and data analysis. The research questions that precipitate from this gap in knowledge are presented at the end of chapter 3. 
Chapter 4 situates the strategy adopted for this research within a system of inquiry and describes the various methods used to generate data. The chapter provides the rationale for the design of two studies, one based on photographic stimuli and the other based around real life experience of urban streets. Chapter 5 discusses the results of Study One. This study invited responses to photographic representation of street scenes and provided input to the detailed design of Study Two. The results of Study Two are discussed in chapters 6 to 8 , with each devoted to a single case. Respondents were invited to record their feelings about individual buildings, the relationships between them and the overall streetscapes as they moved along the street. Chapter 6 includes discussion about the two focus group meetings. The findings that arise through analysis across the two studies are discussed in chapter 9. The findings describe characteristics of buildings and streetscapes that people found attractive and several that they did not. Chapter 10 concludes the thesis by outlining the contributions made by the research to knowledge about people's perceptions of urban buildings and streetscapes. It then returns to the research questions, answering each in turn before making suggestions for further research. 
Chapter 2

\section{Design Guidance and Control}

In order to get good architecture you have to allow for the possibility of bad architecture

John Hiltscher, cited (Delafons 1990: 81)

\subsection{Introduction}

The first chapter introduced the problem that this research investigates, namely that contemporary urban transformation practices in New Zealand lead to uncertain aesthetic outcomes and that in many cases the outcomes are poor. Given that the relevance of the appearance of the built environment in people's lives has been established (refer sections 1.3 and 1.4), what can be done to improve the visual quality of urban buildings and streetscapes? This research aims to identify the building and streetscape design characteristics that people do and do not like. The research starts with a review of the design control literature because, where societies have registered concerns with the design quality of development outcomes, many have turned to regulatory planning and design control to address the problems identified.

In the past some notable proactive efforts have been taken to control the design of buildings and facades. For example, Louis XIV asked his architects to design the perimeter walls of Place Vendome in Paris by constructing the facades around all four of its sides before inviting the individual owners to erect buildings to their own designs behind them. Similarly, the facades surrounding the Royal Crescent and the Circus at Bath were created as urban infrastructure before the individual houses were built (Kostof 1992: 152, Habraken 1998: 51-53). For many reasons, such tightly orchestrated streetscapes are 
not in the interests of governments today; instead they rely on regulation to direct and control the individual efforts of landowners.

The chapter begins with an overview of how design control became established as part of the statutory planning systems of the United Kingdom and the USA. These two countries have extensive cultural and socio-political ties with New Zealand and are also discussed frequently in the English language literature on design control. To provide a context for understanding practices in New Zealand it is therefore relevant to consider how design control has evolved in Britain and America. The chapter proceeds with a discussion around ethics and how ethical approaches invite society, through its government agencies, to regulate development of private land. Design control is then defined in the context of this research and some of the theories and principles that underpin its application are presented and compared. Several of the tensions that can arise in the administration of design control are also highlighted. Finally, an overview of design control practices in New Zealand is presented, along with a critique of its effectiveness.

The chapter concludes by foreshadowing a gap in knowledge around the most appropriate building and streetscape characteristics to be targeted by design control. In light of the research aims (section 4.2.4), chapter 2 presents the circumstances around which the designs for development in urban settings have been regulated. The conclusion also anticipates the research question (see section 3.12), which considers the extent to which buildings and urban streetscapes meet the aesthetic aspirations of the public.

\subsection{Context: design control in Britain}

Design control was initiated in Britain through legislation that addressed a particular problem with the built environment, namely the visual and spatial quality of residential subdivisions in the late 1800s. With the introduction of the Housing and Town Planning Act in 1909, the British government first invited local authorities to plan for healthy and attractive residential dwellings, in pleasant and dignified towns and salubrious suburbs (Cherry 1974: 63-64). The Act's authors were reacting to the grim and depressing housing that had been built according to bylaws that had been written to curb the unsanitary conditions created during the rapid expansion of cities in the $19^{\text {th }}$ Century (Ashworth 1954: 91). Planning practice at that time was strongly influenced by Ebenezer Howard's 
book Tomorrow: a peaceful path to real reform, which provided a model for a coherent, attractive and well-connected towns (Ashworth 1954: 141). Raymond Unwin (1911) had also collected many examples of good town planning practices in his career. These were published to guide the new profession and to suggest what their efforts could lead to. Although industrialisation and growth were considered positive, Unwin lamented the increasing diversity of architectural styles and materials being used in local buildings. He advocated for development to be carefully regulated and for design guidance from planners to help ensure "harmonious and consistent buildings" (Unwin 1911: 360). Establishment of scalar relationships between the parts and details of adjacent buildings was considered to be a key consideration for designers and planners to work with.

As the legislation targeted new housing areas and was rather timid in its language, it was somewhat unexpected then that the Ruislip-Northwood urban district in Middlesex prepared a scheme that required developers to submit full plans to the council for evaluation of the design (Punter 1986). Heeding to Unwin's call, the Ruislip-Northwood scheme was written to enable council control over the character of new buildings and to require changes if the design of the proposal was seen to compromise amenity. Amenity was a catchall phrase that was never clearly defined by Ruislip-Northwood or any other council (Punter and Carmona 1997: 16), effectively setting up the conditions around which design control tensions would develop over the following century.

With successive revisions to the planning legislation, the government's objectives for design control were reaffirmed, although the means for doing so were not always strengthened. Legislators remained cautious about inviting local authorities to become involved in design matters and tended to urge restraint. A planner's scope of influence was narrowly defined as one of preventing design 'outrages' and of asking for changes to only those proposals that could be 'offensive to their surroundings' (Punter 1986). Essentially, design control efforts were aimed at lifting design quality at the bottom end but not necessarily at lifting the overall standard. This approach was supported by the Royal Institute of British Architects (RIBA), who continued to lobby government to limit the extent to which their members' work was subjected to control measures by planners. Their view was that detailed design decisions should be left to the experts, the architects who had been charged with this task by their clients. To architects it was inconceivable 
that planning staff, who had generally little training in design, would be invited to comment on aesthetic matters (Punter and Carmona 1997: 18).

The case for the superiority of architects' aesthetic taste was undermined through the stern words of 'Trystan Edwards, an architect who felt that Modernist urban interventions were in danger of unravelling the beautiful urban streetscapes that had been developed through the considered work of builders and architects over several generations. While contemporary architects had been working to perfect their designs for the detached country villa, Edwards (1944: 44-47) felt that they had lost their way with urban buildings, judging by the negative effects of their contemporary work was having on the streetscapes of cities (figure 2.1). Writing initially in 1924, Edwards advocated for architectural interventions that fitted with their settings, which then, to varying degrees, became the approach for design control in the ensuing period (Punter 1996).

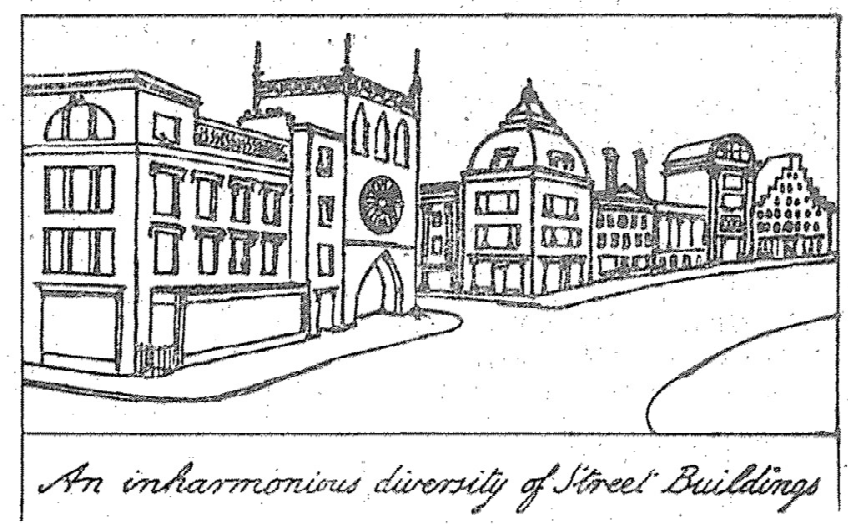

Figure 2.1: Edwards' example of a disharmonious streetscape, where each building asserts its own presence, to the detriment of the street

Source: Edwards 1944

However, making good decisions about design was an on-going issue that frustrated land owners and designers alike. In their efforts to improve their skills and knowledge about design, local councils enlisted the help of experts, many of whom volunteered their services as an adjunct to their professional occupations. In 1924 the central government set up the Royal Fine Art Commission (RFAC) to advise decision makers on the relative design merits of development proposals. However, its involvement was limited to only those cases that were of national importance and to those that could, if built, threaten 
public character (Punter and Carmona 1997: 16). The RFAC was charged with commenting on proposals for monuments and civic art as well as buildings.

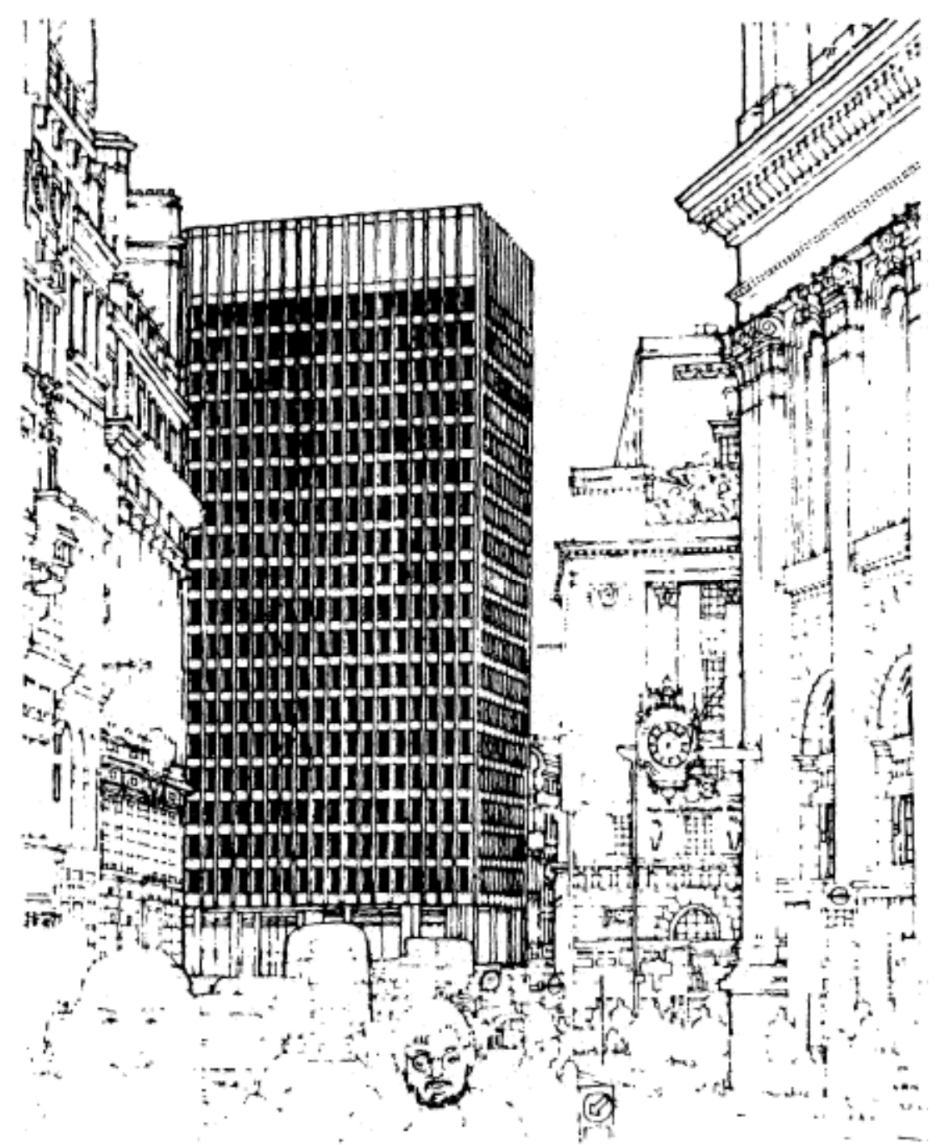

Figure 2.2: A 1980s contentious example of aesthetic control in London. The building had merit but also a deleterious effect on the setting.

Source: Punter 1987

The RFAC was modelled on the American civic art advisory committees that were prevalent at the time and comprised four leading architects and two Lords who would powerfully represent the views of the lay public (Punter 1986: 354). It was largely ineffectual until the 1960s, during which time it began to advocate for better guidance to be provided by government to planners in the form of three dimensional planning policies (Punter 1987). Eventually the commission published its own advice on the qualities that help make a good building (see section 2.8) (RFAC 1994).

As with most social issues, commitment to development control in Britain has risen and fallen with the political tide. After two decades of Conservative rule, in which 
development of the built environment was freed up through removal of development controls, a new government came to power in 1997 intent on creating an urban renaissance (Punter 2011b). With urban design high on its agenda the RFAC was transformed into the Commission on Architecture and the Built Environment (CABE) and its role expanded to include advocacy, research, education and design review (Paterson 2011). Government also developed policy statements and introduced a national urban design framework to help support high quality design outcomes. It seemed that if local authorities had the political will to raise their design standards they would again be supported by government.

In 2010, not long after another change back to a conservative government, CABE was dismantled and many of the important roles it served were abandoned or passed on to others. Although design control remains a part of the British planning system it no longer enjoys the high level of commitment it enjoyed during the first decade of the $2000 \mathrm{~s}$ (Paterson 2011). Punter (1987) and Delafons (1990: 71) were both independently critical of the evolution of design control in Britain, citing the weak policies and even weaker guidance that successive governments had provided. While Parliament had provided for design control for over a century, both authors felt there had been consistent ambivalence, inhibition and conflict shown toward design control during that time.

\subsection{Context: design control in the United States}

Whereas statutory planning in Britain provides considerable discretion in decision-making and is authorised at a national level, planning in the United States is different (Cullingworth 1993: 1). The British approach to land use planning, carried out with reference to nested structure and development control plans, is largely invisible in America. The zoning system that is the basis of statutory planning in American cities sets up the rules and conditions by which land can be put to use (Scott 1969: 193). Zoning ordinances are established independently by local authorities in the absence of any central or state government framework. That all cities appear to rely on zoning has more to do with a shared culture than with political will. The cultural drivers for a rules based form of land use regulation lie, on the one hand in the nation's reliance on the legal system to resolve disputes (Delafons 1969: 16-24, Cullingworth 1991) and on the other in a desire for certainty (Boyer 1983: 161-169). 


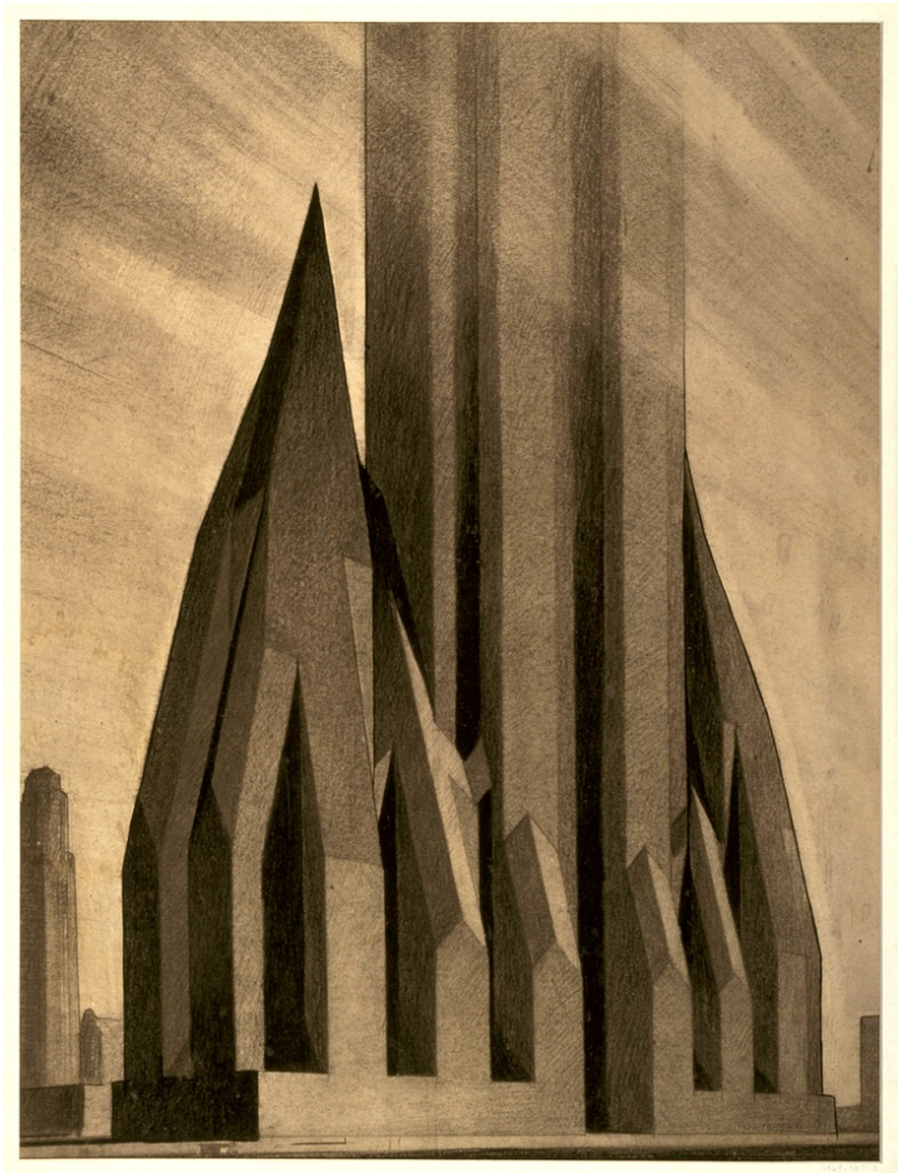

Figure 2.3: 1922 drawing by Hugh Ferris to illustrate the potential form of a building adhering to the 1916 New York City Zoning Ordinance.

Source: https://collection.cooperhewitt.org/objects/18468713/

Zoning was initially devised as a way of separating incompatible land uses within the wider administrative area. Zoning ordinances that describe the uses to which land can be put were also used to prescribe a wide range of requirements and restrictions. Many of these influence built form characteristics, such as rules that set maximum building heights and those that define the areas of a site that can or cannot be built upon (Lang 1994: 85). One example of this is the country's first zoning ordinance, written in 1916 for New York City (Cullingworth 1997: 59). Developed to address public concerns about the harmful effects tall buildings were having on the city's streets, the zoning plan set a height limit of 14 storeys and prescribed a raking line, struck from the centreline of the adjoining street, that taller buildings would need to fit within. For similar reasons, buildings with profiled roofs were also favoured (Boyer 1983: 93) (figure 2.3). Although not aimed at aesthetics per se, 
this and all following zoning instruments have had an influence on design outcomes, such as the stepped shape of the Waldorf Astoria Hotel and the Empire State Building.

Zoning is a much blunter instrument than the British approach, primarily because the zones usually cover large areas of a city and, to ensure fairness, the bulk and location rules generally apply across the whole of a zone (Scott 1969, Boyer 1983: 157-163). The regulations are therefore unable to deal with the specific nature of a particular site within the zone. While inherently rigid, Cullingworth (1993: 4-6) argued that zoning regulations could become more flexible with the application of bonuses, granted to favour the incorporation of public benefits into a project, and through private agreements with adjoining landowners.

By the 1970s many communities had decided that zoning could not achieve the quality of design outcomes their residents wanted, particularly in relation to heritage areas (Case Scheer and Preiser 1994b). This was evidenced by the number of cities that began to introduce specific design control measures into their regulatory systems (Habe 1989). Case Scheer explained that cities were motivated to review design outcomes in order to improve the quality of life for their citizens, to promote vitality, to protect property values, and to generally improve their appearance. Uptake of design review was very rapid. Case Scheer and Preiser (1994a) found that by 1990 up to 83\% of all local authorities in the U.S. had established discretionary forms of design review alongside their zoning regulations and by the end of the decade more than $90 \%$ had some form of appearance control regime (Nasar and Grannis 1999: 425).

The public strongly advocated for design control in American towns and cities, and remain closely involved in its administration through participation in design review panels and preparation of design guidance. Absence of directives on design control from central government has ensured flexibility at local level and ease of access for citizens. People expect design review to be able to deliver more than aesthetically pleasing individual structures. They also see design control as a way of reinforcing community character and enhancing psychological well-being (Habe 1989). 


\subsection{Regulation and ethics}

The field of ethics provides a useful link between aesthetics and regulation (Punter 1994, Taylor 2000). In terms of the relationship between ethics and aesthetics, Porteous (1996: 263-264) argued that the two fields are "irrevocably intertwined". He called on Western societies to refocus their collective consciousness on the beautiful and the morally good in order that their citizens could live fuller lives. For too long, he claimed, the focus had been on political and economic exploitation of the environment.

Another leg of the relationship is that which exists between regulation and ethics. This relationship can be discussed with reference to the Other, a term used in the ethics literature to encompass society as a whole. "Assuming an ethical stance means to assume responsibility for the Other" according to Radford (2010: 381). Development regulation can by this concept be rationalised as a measure taken by government to look out for the interests of - or assume responsibility for - society as a whole. At the smaller scale of individual actions within such a context, the expectation is that regulators would be guided by an ethical viewpoint that incorporates the needs of the Other when discharging their professional responsibilities.

George Santayana provided a more philosophical perspective on the relationship between ethics and aesthetics that could also be seen to invite regulation as a means to enhance the visual appearance of urban places.

Not only are the various satisfactions which morals are meant to secure aesthetic in the last analysis, but when the conscience is formed and right principles acquire an immediate authority, our attitude to these principles becomes aesthetic also. Honour, truthfulness and cleanliness are obvious examples......It is kalokagathia, the aesthetic demand for the morally good, and perhaps the finest flower of human nature. (Santayana 1896 cited Porteous 1996: 264)

It is clear that Santayana believes that the pursuit of aesthetic satisfactions can in itself have an aesthetic dimension. This arguments link clearly the aesthetic outcomes external appearance of buildings - and the pursuit of those outcomes by regulatory planning - in a morally right context.

Silva (2008) describes three principal ethical dilemmas planners must confront. The first is the tension between individual freedoms and regulation. He argues that an appropriate 
middle ground should be established, one that respects the rights of the individual while at the same time limiting the negative effects that exercise of these rights might have on the environment and on others. The middle ground lies on a continuum of approaches that extend between a paternalistic preservation of existing character, effectively curtailing private rights, and complete freedom for property owners.

Planning decisions must also navigate the tensions that arise through the influence of the various interests held by groups and individuals. Marxist theory takes a cynical view of interest, arguing that for the capitalist state there is no such thing as a public interest. Instead, there is only the interest of capital that works in complicity with the state to create the illusion of public control through planning (Allmendinger 2009: 79). Modernist planning principles hold that different interests can be distilled in a process that identifies the public interest in any planning decision (Allmendinger 2009: 183). Indeed, the Royal Town Planning Institute (RTPI) has as its objective the advancement of the art and science of town planning for the benefit of the public (Taylor 1994: 88). A narrow view of public interest is one that requires the interest of every citizen to be met, a seemingly impossible goal to achieve in any meaningful way. Instead, planning must navigate the various interests to arrive at an appropriate decision based on moral or ethical priorities. Reference to an ethical compass, which may in some circumstances favour the interests of a particular group - socially, physically or economically disadvantaged people can be cited as examples - enables reconciliation of the various tensions into an appropriate planning decision (Taylor 1994).

Finally, according to Silva, there is the tension between environmental protection and development. Many project outcomes affect existing built and natural environments inappropriately in order to create benefits for some people. Environmental issues such as pollution and resource depletion can, if unchecked, threaten the very survival of the planet and everyone on it. Taylor (1994) described such matters under a sustainability heading and argued that this is now of such a magnitude and importance that considering the potential effects of development on the environment should be at the heart of all planning decisions. The 1991 New Zealand Resource Management Act, which requires the broad environmental effects of development to be evaluated notwithstanding what may be provided for in a local plan, is an example of a framework within which this tension is played out (Higgins 2010). 
Fox $(2000,2011)$ reorganised Silva's three spheres of interest and tension into three ethical contexts; the biophysical, the social and the built. The three spheres are nested, with the built environment considered to be a subset of the social environment and both fitting within the biophysical domain (figure 2.4). Fox argued that any decision regarding the built environment should acknowledge and respond to these contexts according to responsive cohesion, "the foundational principle that is at work in a wide range of evaluative domains" (Fox 2000: 219). Responsive cohesion is the quality of the relations between the internal qualities of a thing and between the thing and its setting (Radford 2010). An example of this might be the internal relationships between the parts of a town and the external relationships between the town and its contexts, e.g. physical, social. Another example, taken in the context of this research, might be a new building that exhibits an aesthetically pleasing internal composition and is also considered to enhance the wider street setting. Such a building could be said to exhibit the quality of responsive cohesion in the built environment context.

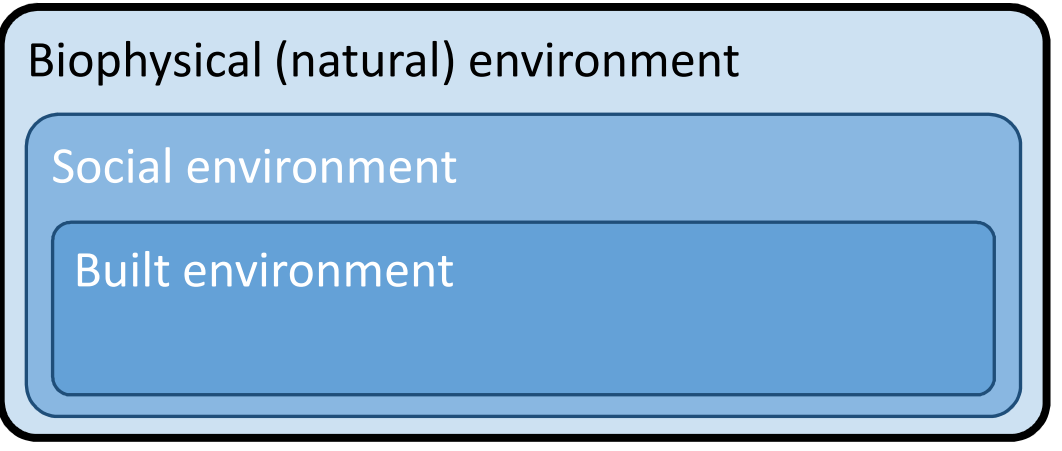

Figure 2.4: Responsive cohesion is conceived as a nested relationship between the natural, social and built environments. Changes to the built environment must be responsive to and coherent with the existing built environment and with the social and natural systems of which it is a part.

Source: Author, after Radford 2010

Edwards (1944) discussed responsive cohesion as a guiding principle for urban change, arguing that new development should be well-mannered and take its cues from the existing setting. An example of how this principle has been used to direct change is found in Christopher Alexander's New Theory of Urban Design. In his view, each successive project in an urban context should be seen as an opportunity to heal the setting and work toward a more complete whole (Alexander, Neis et al. 1987: 20-23) (figure 2.5). 
While each project should exhibit responsive cohesion within the physical context of the built environment, it should also demonstrate the same responsiveness with the social and natural environments. The principles of responsive cohesion provide tools that help advocate for a holistic approach to change management. Therefore, it would not be appropriate to advocate for a building that, despite being responsive and coherent with the street environment, exhibits untenable, negative environmental or social outcomes. Responsive cohesion offers a basis for judgement, not the outcome of judgement and the circumstances of each situation including the context must be interpreted (Radford 2010). Unfortunately, there is currently little evidence that additions to the built environment are evaluated for the effect they have on the local society. Design review is generally undertaken by experts who, although part of the social context, do not necessarily hold the aesthetic views of the wider public (Lightner 1992). This points to a gap in knowledge that this research will address.
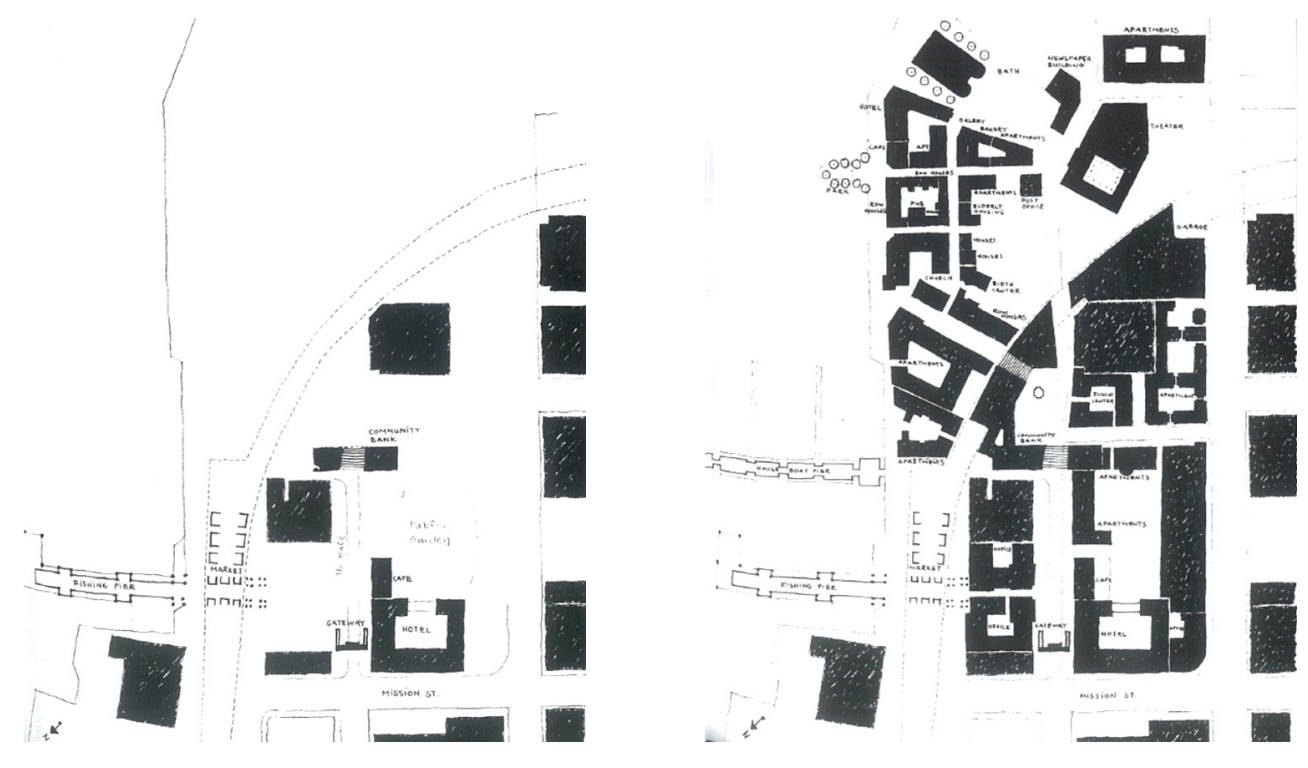

Figure 2.5: Plan showing how growth over time through individual projects can complement and help create a stronger 'whole'. Alexander's New Theory of Urban Design can be considered to follow the responsive cohesion approach to change.

Source: Alexander Neis et al 1987 


\subsection{Design Review and Control}

Although every project undergoes internal forms of design review in the normal course of designer/client/consultant negotiations, government administered design control processes are generally invoked to look after the interests of the public, which can easily be marginalised in the private review process (Jones 2001, Imrie and Street 2011: 16). George and Campbell (2000: 163) noted that "aesthetic controls are based on the belief that there is a collective good in their application (that is) greater than the sum of their cost to each individual." Once a government decides to become involved on behalf of the public in regulating the appearance of the built environment, it can implement design policies by making use of a range of tools. These tools can include direct ownership and operation of buildings and public spaces, regulation of proposals undertaken by private interests, and offering incentives to encourage good outcomes and education (Schuster 2005, Carmona, Heath et al. 2010: 298).

Although design control is a regulatory tool, it is increasingly recognised that those working in this field also play an important role as educators (Kim and Forester 2012). At the local level, design control processes create opportunities for the public and those directly involved in development to learn from each other, particularly around the issues and concerns that motivate each. More formally, large scale design review providers such as $\mathrm{CABE}$ work actively to improve design skills and understanding between professionals and the wider community (CABE 2006).

Nasar \& Grannis (1999) and Punter (2007) advised that design control practices can be either regulatory or discretionary. Regulatory (also referred to as administrative) systems are an add-on to zoning controls, where design outcomes are controlled by rules and objective measures. Examples of this are maximum height limits, prescriptions for location of buildings in relation to boundaries, and floor area ratios. These systems provide high levels of certainty for all parties and regulation can be conducted largely through administrative procedures, reducing demand on local authority resources. However, they are also criticised for being coarse in nature with a tendency toward creating monotonous environments, as new projects within an area are all built to the same prescribed limits (Delafons 1994, Madanipour 1996: 155-158). 
Discretionary systems are more 'pragmatic' as they allow regulation to refer to the immediate setting and other variable factors that cannot be accounted for in most rules based regimes. The more successful discretionary systems, in terms of meeting the needs of different stakeholders, are informed by design guidelines or briefs that provide both a target for designers and a reference for those assessing the proposal (Nasar and Grannis 1999). While discretionary systems often allow local conditions to be taken into account they are also criticised by the development industry and designers for their arbitrariness (Case Scheer 1994: 6).

In the context of this research, design control has a broader, more all-encompassing meaning than design review, although both refer to the processes by which three dimensional design proposals are presented for independent scrutiny at local government level in connection with determination of an application for planning permission (Schuster 1997). In her definition of design review, Case Scheer (1994: 2) excludes the influence of zoning regulations. However, the three-dimensional volumetric controls embodied in zoning ordinances do have considerable influence on design outcomes (Punter 2011a). Hall (1990) and Madanipour (1996: 160-161) consider a wider definition of design control that includes zoning to be more relevant to urban design. When used in this thesis, the term design control incorporates design review methods with the administrative measures embodied in zoning (figure 2.6).

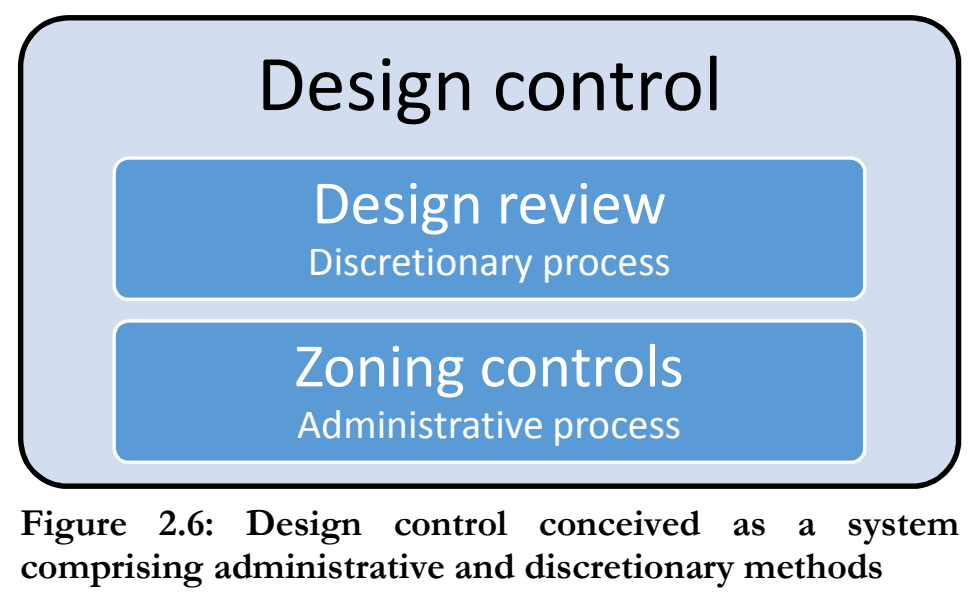

Source: author

About two-thirds of American cities that have adopted discretionary design review as an adjunct to zoning regulation (see section 2.3) rely on the expertise of their staff for 
considering the aesthetic qualities of a proposal (Lang 1996). The remainder convene review panels to advise planning officers (Case Scheer 1994, Hinshaw 1995: 17). While membership of these panels vary, most comprise lay members of the community and some experts (Delafons 1990: 66). In England, design review has traditionally been conducted by planning staff, who can be assisted by experts for proposals in conservation areas. Increasingly however, lay and specialist panels have been convened at local level to assist the decision-making (Punter 2011a). Since 1999, projects of sensitive or strategic nature could be referred to regional panels established and operated by CABE, and since CABE's 2011 amalgamation with the Design Council this independent review function has been provided under their auspices.

Over the past 30 years there has been a tendency for design control to become more discretionary to create scope for development design quality to be scrutinised more closely. Likewise, design guidance informing discretionary systems has become more prescriptive in response to calls for more certainty around the process for applicants. In short, design control systems appear to be converging as they are modified to include both discretionary and regulatory characteristics (Lang 1996, Punter 2007).

\subsection{Design guidance}

Design review would be difficult without an agreed reference for discussions and eventual decision-making. Design guidelines are the most common method of describing the outcomes expected of a development proposal to designers and those undertaking review on behalf of local government. These can have more or less authority depending on the statutory powers under which they were prepared (Carmona, Heath et al. 2010: 298), and one of the ongoing challenges in the British context is that they are often presented as supplementary guidance, sitting outside the statutory framework (Hall 1996: 13, Madanipour 1996: 174). Prompted by legal review, most cities in the United States that make use of design review have adopted design guidelines that help to "improve the objectivity, consistency and predictability of the development evaluation process" (Habe 1989: 196).

Design guidelines sit in the gap between broad planning policies and the inflexible rules associated with zoning (Hinshaw 1995: 21). Three factors are seen to limit the effectiveness of design guidance, which has the broad aim of fostering better built 
outcomes than would otherwise be produced. Firstly, there is a tendency for guidance to favour the status quo design character of the buildings in an area (Hall 1990). Lightner (1992) found that 100 percent of the cities she consulted promoted development outcomes that were consistent with their settings. Whether required explicitly by guidance or through conservative interpretation by reviewers, this has tended to generate architectural uniformity, even when the existing patterns were not particularly desirable. Highly critical of such context based guidance, Lightner advocated design approaches that could generate the equivalent of jazz music along the length of a street. She argued that moments of flamboyance and architectural expression could be accommodated against a stable backdrop of buildings that are consistent with the desired character. One of the positive aspects of such an approach is that it would enable different cultures to be expressed in the buildings they produce. Although she presented two examples that had emerged without the help (constraint) of design guidance, Lightner offered little advice for how such an approach could be provided for in current processes. On what basis could some projects be constrained to serve as background buildings and others encouraged to be flamboyant?

Secondly, there remains little evidence based theory in the field of environmental aesthetics upon which the appropriateness of design outcomes can be considered. This gap in knowledge is identified in chapters 2 and 3 of this thesis, to then become a research aim. Instead, most guidance simply repackages normative, broadly accepted, principles of good design (Punter and Carmona 1997: 201). Broad, principles-based guidance arises because policy makers remain tentative about controlling design and wish to accommodate increasingly pluralistic design cultures. Unfortunately, principles-based guidance is unable to direct outcomes effectively, as it lacks sufficient focus and direction (Cullingworth 1997: 111). As a consequence, interpretations must be made, some of which will be good and others poor. This view is supported by the RFAC (1994: 80-81) who noted that even their own criteria for a good building were insufficient to serve as guidelines.

Finally, Hall (1990) raised the issue of a piecemeal control system that is ill equipped to allow individual proposals to be assessed in relation to a comprehensive vision for the setting. He noted an absence of explicit design policy statements for individual streets and smaller areas of cities and towns. This is problematic, in that it is the total assemblies 
of buildings along the length of a street that are of concern to the public. In the absence of explicit policies, design guidance and review tend to be very general, addressing two principal questions; “is the building design good enough?” (RFAC 1994: 7-15) and, “does it fit with the others in the setting?” (Nasar and Grannis 1999). This leads to designs that are consistent with the existing setting and to uniformity in the designs are offered. This can be seen as a missed opportunity to guide new development in a manner that reinforces a shared vision for the area around the site.

A substantial literature has been developed to assist writers of design guidance. Carmona, Heath et al. (2010: 311-317) noted that guidance can be written to suit a range of scales, conditions and objectives, from site specific design briefs to guidelines that apply over a broad area. Design guides can be tailored to meet the anticipated needs of the end user. For example, in areas beset by poor design quality it may be important to establish a minimum design threshold by writing more specific standards. The negative side of such a prescriptive approach is that it may also limit design quality by prescribing a certain standard or approach. On the other hand, where local stakeholders are already committed to a high quality of design, a looser approach may be appropriate. Guidance written through less prescriptive approaches can enable more creative design solutions, although this is very dependent on the abilities of the designers and quality of the brief (RFAC 1994: 80).

American design guidelines, as the predominant form of guidance provided to reviewers there, are written in very specific terms as those written vaguely or in a manner inviting multiple interpretations cannot be easily defended in court (George and Campbell 2000). To assist interpretation, writers need to link guidelines to relevant planning policy statements (Hinshaw 1995: 21-23). This can also enhance understanding and appreciation of the guidelines by those using them.

Lang (1996) noted two challenges to be confronted when preparing guidance for designers. One concerns the conversion of design principles into specific guidelines. There is a growing base of empiric knowledge about urban design that has been used to develop broad design principles (for example, Bentley, Alcock et al. 1985, Alexander, Neis et al. 1987). However, in order to turn these principles into meaningful guidance it becomes necessary to filter them through the local context and community values. 
Identification and articulation of these qualities can only be made with the help of empiric studies, which can be difficult to resource.

The second challenge is to write guidance that can accommodate changes in taste over time. Lang (1996) noted a greater tolerance of complexity in formal aesthetics today than 70 years ago. Similarly, neo-traditional forms of guidance, such as that prepared for the Seaside community in Florida USA, envisage a physical form reminiscent of a very different time from the present (Case Scheer and Preiser 1994a). How relevant can these be to contemporary society and indeed to aesthetic tastes in the future? To address these questions at the local level, where design should be controlled, Lang called for more research in order to understand local ambitions and motivations.

\subsection{Design review}

To acknowledge and navigate between various interests, George and Campbell (2000: 171-173) described four criteria that a sound design review process should address:

1. A clearly articulated and demonstrable public interest

2. Demonstrable links to the stated intent

3. Application early in the design or decision process

4. Encouraging a variety of acceptable decisions.

Punter (2007) incorporated these criteria into a comprehensive framework for design review and development management (table 2.1). The twelve principles are grouped under four headings; community vision, design planning and zoning, substantive design principles and due process. The principles under the first heading aim to capture the views of the community as a step in generating a comprehensive view to guide development and control. The community vision becomes the reference for all decisions. Secondly, three principles inform development of a suitable process that includes incentives as well as the requirements necessary to regulate for high quality design outcomes. The next three principles address the nature of the relationship between the comprehensive plan and the development industry charged with its implementation. Punter argued that the relationship should be pluralistic and not overbearing in order to allow creative solutions to emerge. Under the final heading, four principles address issues of fairness in administering the design control regime. 
Table 2.1: Principles for Progressive Design Review

\section{Community vision}

Committing to a comprehensive and coordinated vision of environmental beauty and design

Developing and monitoring an urban design plan with community and development industry support and periodic review

\section{Design Planning and Zoning}

Harnessing the broadest range of actors and instruments (tax subsidies, land acquisition) to promote better design

Mitigating the exclusionary effects of control strategies and urban design regulation

Integrating zoning into planning and addressing the limitations of zoning

\section{Broad, Substantive Design Principles}

Maintaining a commitment to urban design that goes well beyond elevations and aesthetics to embrace amenity, accessibility, community, vitality and sustainability Basing guidelines on generic design principles and contextual analysis and articulating desired and mandatory outcomes.

Not attempting to control all aspects of community design but accommodating organic spontaneity, vitality, innovation, pluralism: not being over-prescriptive.

\section{Due Process}

Identifying clear a priori roles for urban design intervention

Establishing proper administrative procedures with written opinions to manage administrative discretion, and with appropriate appeal mechanisms.

Implementing an efficient, constructive and effective permitting process Providing appropriate design skills and expertise to support the review process.

Source: Punter (2007)

\subsection{Theories and principles to inform design guidance}

Architectural commentators and planning regulators have not yet created a theoretical basis for architectural design policy (Lang 1996, Punter and Carmona 1997: 201). One reason for this is the ever increasing range of aesthetic tastes, reflected in the diversity of people and cultures. Indeed, pluralism and choice are considered by many to be positive attributes that arise through the different ways in which individuals and groups express themselves. Yet in relation to the built environment, and noted in sections 2.2 and 2.3, many communities have signalled their unwillingness to allow unbridled pluralism when 
it comes to buildings (Habe 1989). It is therefore problematic that there is little agreement at a theoretical level about the most appropriate forms and characteristics of buildings.

Peter Buchanan (1988) wrote a general critique of building facades in the wake of Modernism, where he noted the banality of so many "fiscal facades" that designers had been willing to justify on grounds of time and cost efficiencies. He was also critical of pastiche compositions that mindlessly reference historical images. Based on these criticisms Buchanan (1988) outlined four design characteristics that all well designed facades should include:

1. Observe an appropriate cornice line and overall height and be built to the street boundary such that they work with the general patterns around the site to reinforce the street wall.

2. Use tactile, decorative materials that reveal their physical nature and that will weather gracefully

3. Include windows in walls that will add visual interest and enable inside/outside connection

4. Make use of appropriate decoration to delight, intrigue and otherwise hold the eye

Punter and Carmona (1997: 203) stated that facades should convey a sense of image and identity to the building and street and contribute to the area's sense of place. They went on to argue for facades that exhibit character and coherence by adhering to design conventions, and that relate well to the neighbouring buildings. Facades should help define and animate the street spaces they abut and should act as a filter, not a barrier, between the street and interior spaces.

Others who have discussed design principles helpful for this research effort include Edwards (1944), Bentley, Alcock et al. (1985), Young (1986) and the RFAC (1994). In Responsive Environments, one of the seminal urban design texts of the $20^{\text {th }}$ Century, Bentley and Alcock et al. outline seven essential qualities of well-designed places. The explanations offered and the examples presented serve to reinforce that these qualities can apply at a range of scales, including the individual building. Buchanan's four preferred architectural characteristics are also evident in the Responsive Environments list of headings. Bentley, Alcock et al. (1985: 42-55) noted the quality of legibility, which they argued is made more difficult in the Modernist city because buildings fail to create positive, well 
defined space around them and because there is no discernible distinction made between publicly important buildings and those that support private land uses (see also section 3.8.3). When aiming to generate enhanced legibility of buildings in urban areas, the relationships of building heights and footprints to each other are important considerations.

In the context of criticising his professional colleagues (section 2.2), Edwards (1944) outlined a number of architectural qualities that can help make a well-mannered building. Perhaps not unsurprisingly, he advocated for consistency of style between adjacent buildings and his preferred style was the classical and its close variants (Punter and Carmona 1997: 16). Development of appropriate scale relationships between buildings, between the building and its various components and with people were also matters that concerned Edwards (figure 2.7).

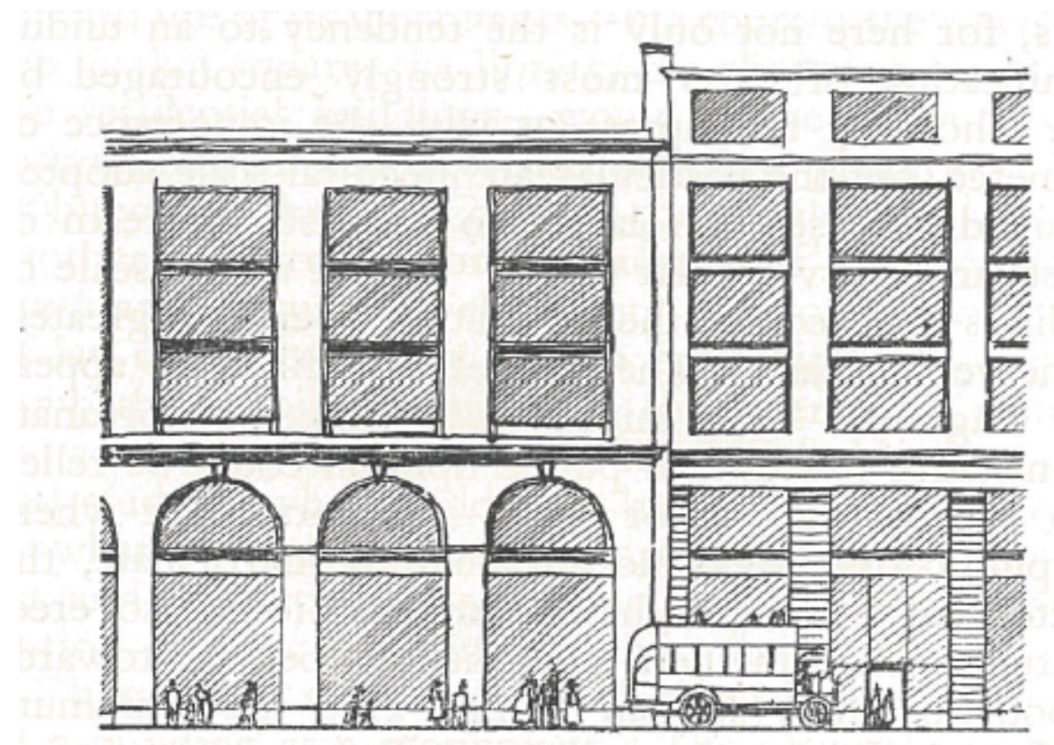

Figure 2.7: Edward's example of poor human scale relationships in buildings, typical of those going up in London at the time.

Source; Edwards 1944

Table 2.2 sets out the principles of appropriate urban buildings according to four key texts. The table relates similar attributes where they have been called for by more than one author. The final column provides a summary of the principles that could be used as a basis for design guidance or, in the context of this research, to inform the theoretical framework and development of methods to examine the research question (see also sections 3.6 to 3.8$)$. 
Table 2.2: Development of principles for design control

\begin{tabular}{|c|c|c|c|c|c|c|c|c|c|c|}
\hline 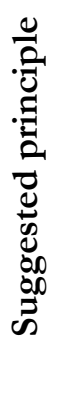 & \multicolumn{2}{|l|}{ 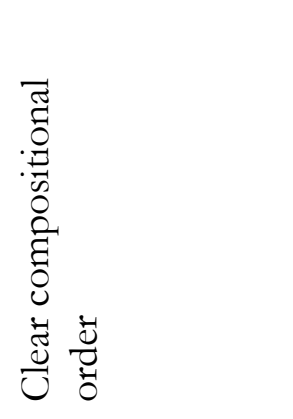 } & 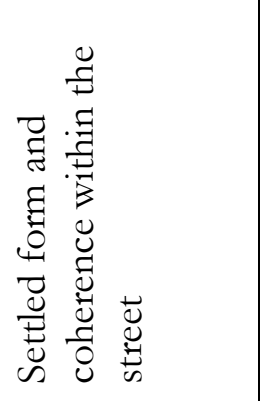 & \multicolumn{2}{|c|}{ 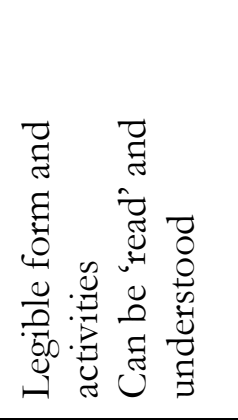 } & \multirow[t]{2}{*}{ 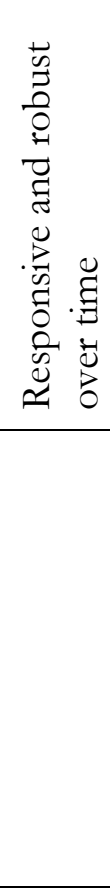 } & \multirow{2}{*}{ 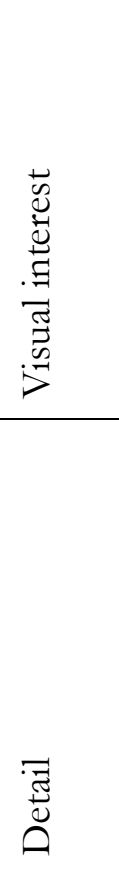 } & \multirow{2}{*}{ 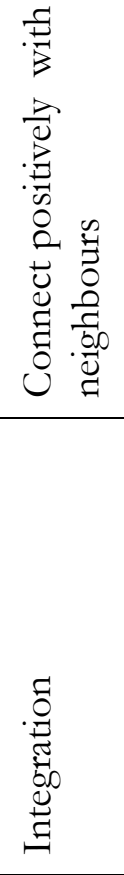 } & \multicolumn{2}{|l|}{ 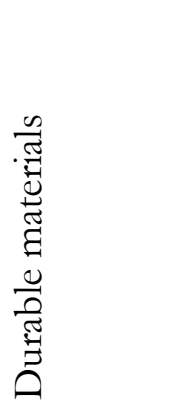 } \\
\hline 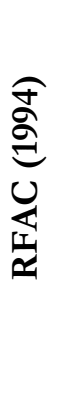 & 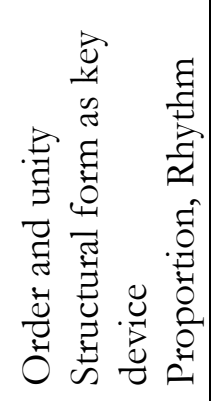 & 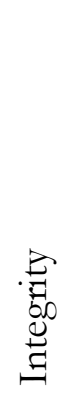 & 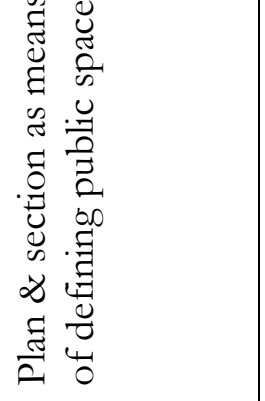 & 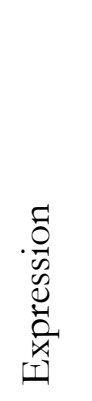 & 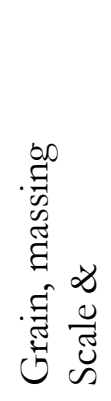 & & & & 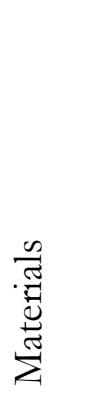 & \\
\hline 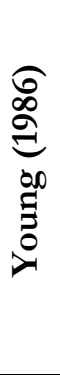 & 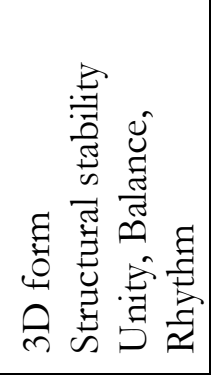 & 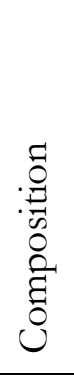 & & 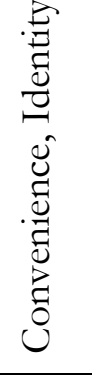 & & & 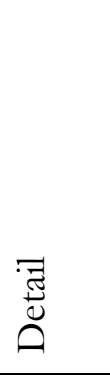 & 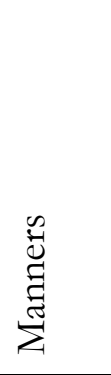 & 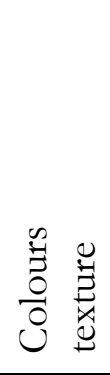 & \\
\hline 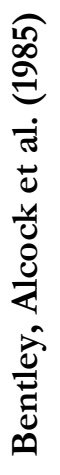 & 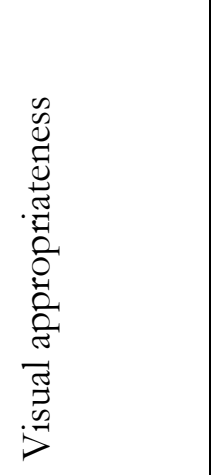 & & 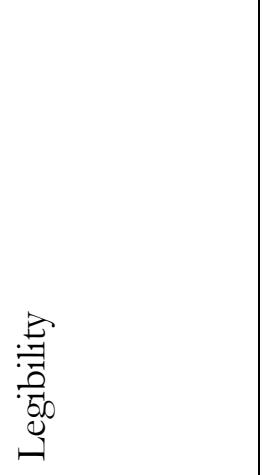 & 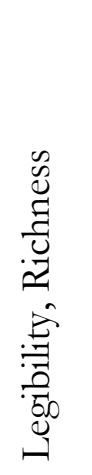 & 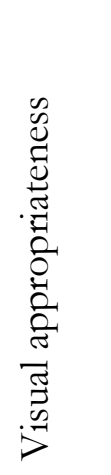 & 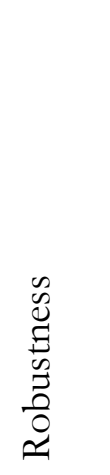 & 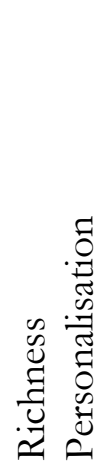 & 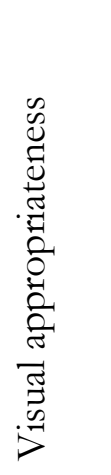 & & 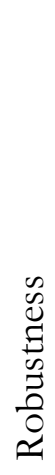 \\
\hline 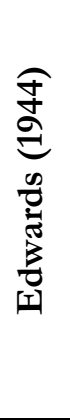 & 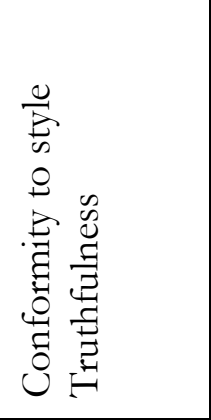 & & 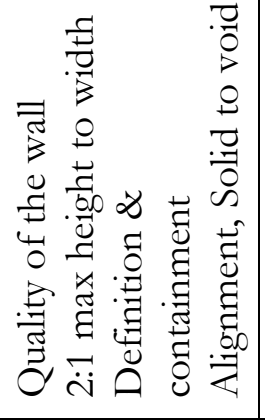 & 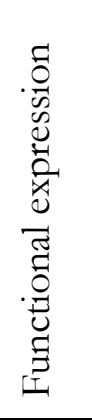 & 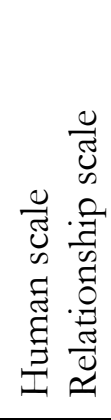 & & & 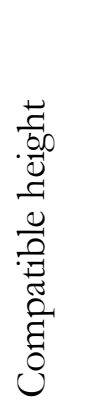 & 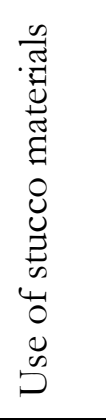 & 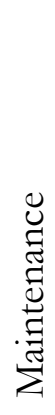 \\
\hline
\end{tabular}




\subsection{Other design control methods}

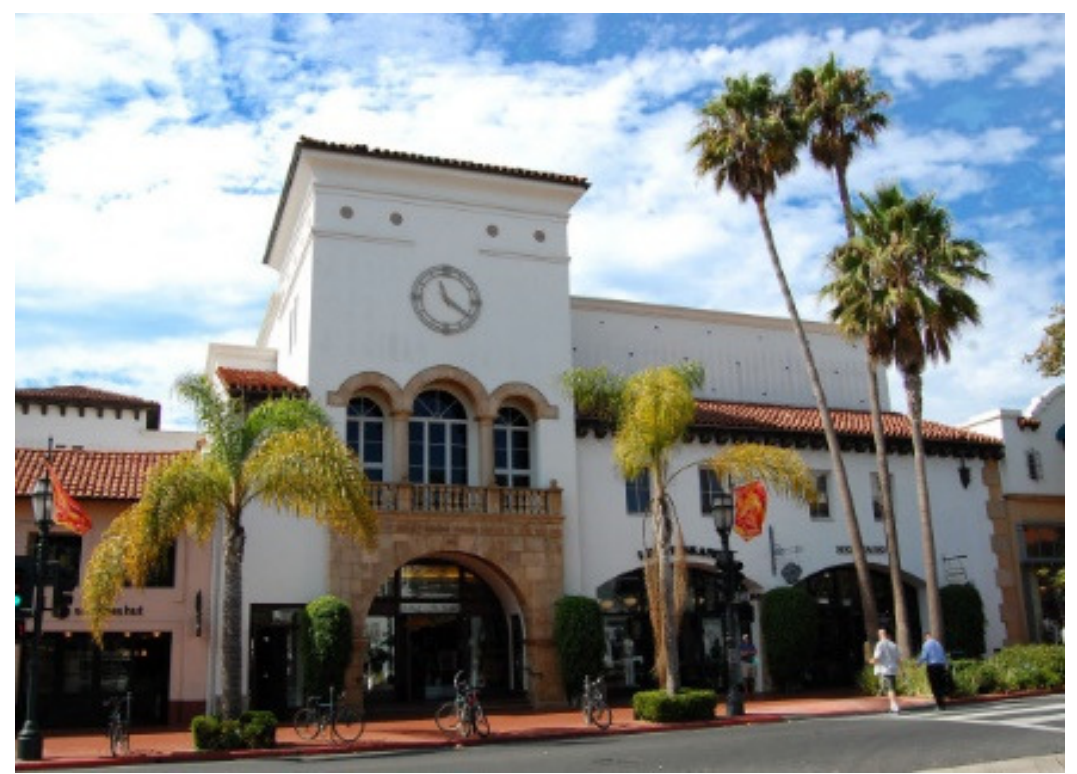

Figure 2.8: The stylistic imperative of design control requires new buildings to adopt the Spanish Colonial style in Santa Barbara, California.

Source: Edwards Pittman Architects

Delafons (1994) discussed other, less common, methods to regulate design outcomes. The Stylistic Imperative requires that a particular architectural style or idiom be used in new projects to reinforce patterns that may already exist in the area. While less common, it is perhaps the method most people equate with design control. An example of this is the imperative for buildings in the city of Santa Barbara to adopt the Spanish colonial style (Pouler 1994) (figure 2.8). The Proprietorial Injunction is a means by which developers of private subdivisions can establish their own design criteria (Baab 1994). These are generally more stringent than those imposed by the public regulator. Finally, many European jurisdictions make use of design competitions, enabling them to choose between alternatives for the development of a site. Delafons has labelled this method the Competitive Alternative. A benefit of competitions is that they often generate a good architectural solution without necessarily conforming to all regulations, allowing for a special outcome that might otherwise be difficult to manage through design review (George and Campbell 2000). 


\subsection{The cases for and against design review}

The underlying aim of design review is that regulation will lead to a better built environment for all (George and Campbell 2000). Although this is sound there is disagreement amongst scholars, design practitioners and regulators about how efficiencies in the development industry are affected, the extent to which freedom of expression is stifled, and whether really excellent design outcomes can emerge out of a process that seems to favour continuity (Habe 1989, Case Scheer and Preiser 1994a). Table 2.3 below sets out the various promises of design review against the challenges it must overcome to achieve these.

Table 2.3: The cases for and against design review

\begin{tabular}{|c|l|}
\hline \multicolumn{1}{|c|}{ Case for } & \multicolumn{1}{c|}{ Case against } \\
\hline Raises the standard of development by & Impedes the quality of building design \\
ensuring more thought goes into its & Is merely cosmetic \\
design & Encourages imitation \\
Prevents outrages & Designers develop schemes they know \\
Encourages designers to stand up to & will win approval \\
clients & Lacks fair predictability \\
Can provide a bridge between lay and & Violates property rights \\
professional tastes, depending on & Violates freedom of speech \\
methods & Is difficult to resource [adding to cost] \\
Seeks to enhance unique qualities of the & For the most part it is reactive, \\
place & depending on applicant's vision, \\
Seeks to enhance vitality through & capabilities and resources \\
commercial viability] & \\
Protects property values & \\
Makes new development compatible or & \\
unified & \\
\hline \multicolumn{2}{|c|}{ Adapted from: George \& Campbell 2000, Carmona 1996, Scheer 1994 } \\
\hline
\end{tabular}

George \& Campbell (2000) highlighted the need for variety in the built environment from a physiological perspective. As human wellbeing is affected by the environment, the case for regulation suggests that new designs should avoid inducing unnecessary stress, anxiety or fear in people. However, as people also learn and develop their senses through experience, it is also important that they are challenged (Smith 2003). More complex 
settings, which arise through unfettered design decisions, could create such opportunities. It is on this basis that control methods advocating for high levels of contextual compatibility should be challenged. In addition, by pushing the current boundaries of design, opportunities for development of new theories, new methods and general advancement of the field of design can be created (George and Campbell 2000).

An extensive study in the US, comprising two separate surveys, found that planners and members of the public were reasonably satisfied with design review and collectively could only suggest two things to improve the process. These were the provision of clearer design guidance and the allowance for greater autonomy by decision makers. Architects, on the other hand, were largely dissatisfied with design review (Case Scheer 1994). Their key concerns related to the capabilities of the local government staff making design review decisions and the subjective nature with which many decisions were made.

However, about the time Case Scheer undertook her study, Schuster (1997) also surveyed members of the Boston Architectural Society. One of his key findings was the "markedly positive" views that architects have of design review. Several years later the same survey was repeated in Portland, Oregon with similar results (Jones 2001). Schuster confronted the discrepancies between his and Case Scheer's surveys and found that she had widely circulated a memo inviting responses through the American Institute of Architects. Schuster claimed that because of this her survey was not impartial. He argued that architects with particularly negative views about design review were more likely to take the time to respond (Schuster 1997). With these conflicting outcomes it seems that architects' opinions about design review are still unclear.

\subsection{Challenges for design review}

To improve the processes and the outcomes of aesthetic review as administered through local government regulations, a number of challenges must be confronted. Design review is time consuming and expensive, it is easy to manipulate through persuasive and pretty pictures, and it is administered by overworked and inexperienced staff. Case Scheer (1994) identified these as the key issues plaguing design review processes. However, these could also be easily solved with the addition of financial or human resources, through education, or by auditing the process for political involvement. 
Case Scheer (1994) then went on to identify four endemic problems that are more difficult to diffuse, as they are precipitated by tensions between competing social, political or legal interests. The first is power and questions around who should be entitled to make key decisions in the process. Pointing to the law, she argued that design review is the only field in which lay people (those not specifically trained in design) are left to rule over professionals in their areas of expertise. Freedom of expression in the built environment is another matter that troubles design review. Concerns about freedom are triggered by societal values aligned with property ownership, particularly in new world countries. This concern is pronounced in the United States, yet the courts there have persistently upheld the rights of government to be in control of design outcomes (Lai 1994). There is then the matter of justice in design review. Working on behalf of the regulator, the reviewer is seen to hold the balance of power and this limits the extent to which a fair hearing is possible. While this issue might be minimised through the use of written guidelines, it is also considered to limit freedoms of individual expression. Developers and designers tend to opt for a path of least resistance when negotiating design review (Jones 2001). As developers want a favourable decision regarding their project, they become unwilling to test the limits of guidelines, nor are they willing to appeal unfavourable decisions because of the time and cost of doing so. A final troublesome area is that of aesthetics and questions around whose aesthetic values should be referenced. Lang (1996) noted that in many cases guidance remains unhelpfully abstract, which pushes projects toward soft solutions and mimicry in order to navigate the review process.

\subsection{Design Control in New Zealand}

Design control is a relatively recent addition to the regulatory planning context in New Zealand. Regulatory town planning was itself slow to establish in New Zealand, given the certainty of colonial settlement plans and prevailing societal attitudes that valued freedom of the individual and exploitation of the environment (Memon 1991). In addition to carving up the land for sale efficiently, gridded street patterns gave residents easier access to open spaces within and around the settlements (Memon 1991, Hamer 1995). The philosophies and practices that underpinned town planning in New Zealand were strongly influenced by British planning discourse in the areas of health and wellbeing. In 1926 the Government introduced the Town Planning Act, almost 20 years after similar legislation in Britain. This Act required towns and cities in New Zealand to develop plans by which 
land use could be regulated in their jurisdictions. The plans that emerged were largely based on Euclidean zoning, adopting methods that were being developed at the same time in the United States. Miller (2002) attributed the growing enthusiasm for town planning during this period to the many civic beautifying associations established around the country. However, critics also claimed that the profession was not delivering visually attractive or functionally sound cities. Martin (1949) attributed this to the general public's lack of discernment in matters of design as well as a shortage of skilled town planners.

The New Zealand context for regulating urban planning and design changed dramatically in 1991 with adoption of the Resource Management Act (RMA). Emerging out of a period during which planning fell out of favour with the professions and government, the RMA has its roots in a period of economic liberalisation (Dixon 2003). In stark contrast to the inflexible and prescriptive planning paradigm it replaced, the RMA supported a performance-based planning approach (Baker, Sipe et al. 2006). Weaving statutory landuse planning with high level discussions around natural resource management, the legislation outlined matters to be considered when determining a planning application more than it discussed specific strategies for resource use (Jackson and Dixon 2007, Higgins 2010, Miller 2011). The Act required local governments to prepare district plans for their jurisdiction, against which planning approvals would be evaluated. The plans set the generally objective development standards for different zones and land-use classifications, describing the expected physical outcomes, land use characteristics and traffic related outcomes that land owners and affected parties could expect as of right. Applications seeking planning approval (resource consent) are then considered by comparing the anticipated effects against those that would arise through a development that complies with the stated development standards. This process is referred to as effects based planning (Dixon 2003).

\subsubsection{Effectiveness of design control in New Zealand}

There is a growing literature on planning and, to a lesser extent, urban design in the aftermath of the RMA. Hunt (2008) and Higgins (2010) each express a widely held view that urban development planning has not been well served by the RMA. This is largely because of its focus on the biophysical realm, its emphasis on measuring effects rather than the nature and scale of development, and it being overly reliant on market forces. Despite the potential of performance-based planning to foster innovative development 
outcomes (Miller 2003), local authorities have mainly fallen back on fixed rules as the basis against which proposals are judged (Baker, Sipe et al. 2006). This has led to design issues being marginalised in the vast majority of planning decisions because, where building form and location are controlled by clear standards, local authorities do not believe it is their role to comment on aesthetic matters (Hunt 2008).

In Wellington, concerns about the poor visual quality of many contemporary buildings in the city led local politicians to question the effectiveness of the local design control processes. Addressing these concerns at the residential scale, McIndoe audited the effectiveness of two character area design guides that ten years earlier had been introduced into the Wellington District Plan. The report reviewed the resource consent process and monitored the architectural merits of completed projects. Following an assessment of 16 projects, the report concluded that the planning rules and associated design guidelines had been effective in achieving desired character outcomes (McIndoe 2003).

These results could have been predicted however, as the study investigated the two character areas with the most prescriptive design guides in the District Plan. The report went on to note a positive correlation between the involvement of a registered architect and a successful design outcome. Similarly, there appeared to be strong correlation between the absence of a skilled professional and a poor design outcome. The findings of the McIndoe Report thus questioned the chances of achieving high or even passable outcomes in projects that do not involve skilled professionals and that are being undertaken in areas with no design guidance.

A year later Rae (2004) reviewed 20 completed projects in Wellington's central area in a similar, subjective evaluation. The study concluded that the guidelines were not effective when used in conjunction with controlled activity status under the District Plan. Controlled activity status is where the proposed building or activity essentially cannot be refused, regardless of the aesthetic qualities of the built form. While the earlier study found pre-application consultation to be widespread and beneficial for project outcomes, the second study found early consultations to be ineffective. The architects consulted by Rae noted that the advice given by Council officers in the early stages was inconsistent and conservative. He also found that when it was undertaken, consultation did not take place at a critical, site-specific analysis stage but only once preliminary design proposals and economic budgets had been formed. As a consequence, Council urban design input was 
unable to prompt significant changes, should they be needed, to improve outcomes. Finally, the research suggested that the discipline specific approach to evaluating projects could have a detrimental effect on projects when compared to assessment of proposals in a more holistic manner. Rae cited changes to a project made to satisfy singular and conflicting advice from heritage, traffic, wind, and urban design advisers, as he felt that many of these had served to diminish the design quality of the outcomes.

While interesting, both of these earlier studies relied on the expertise of the respective authors to evaluate the design outcomes. Neither study sought input from others, including members of the public. This would please Case Scheer and Preiser (1994a), who argued that design review should be the exclusive domain of experts trained in the visual

arts. In particular, they advocated for the design review process, if undertaken at all, to be fully entrusted to architects as the experts in this field. There is however, little evidence to suggest that experts speak on behalf of the wider population. This is one of the gaps in knowledge this research addresses.

\subsection{An emerging gap in knowledge}

The discussion of earlier studies in section 2.12.1 begins to suggest a gap in knowledge around how the general public feel about the buildings produced as a result of planning and design review. Accepting that the appearance of the built environment matters to people and that this entitles governments to become involved in regulating design outcomes, it follows that any evaluation of the methods used to regulate and control design outcomes should incorporate the views of all people, not just the experts. How can the effectiveness of design control be gauged without this information? Moreover, accepting Hall's (1990) argument that it is the streetscape that is of greater relevance to people than individual buildings, evaluation of the outcomes of change should also refer to the effects created in the wider streetscape.

At section 3.10, this thesis discusses earlier studies of people's aesthetic preferences for the built environment. It is clear that there have been few published studies addressing people's perceptions of tightly spaced buildings in an urban context. This research aims to understand the opinions of the lay public, as well as those trained in the design and planning fields, about individual buildings and streetscapes. 


\subsection{Conclusions}

Chapter 2 has discussed literature in the areas of statutory planning and design control. Stimulated by the fact that the appearance of the built environment is recognised as a matter of concern for people, the chapter discussed ways in which several governments have historically elected to manage change on behalf of their citizens. While design control has been authorised and supported in Britain for over a century, successive central governments up until the end of the $20^{\text {th }}$ Century have directly and indirectly advocated for a hands off approach. Direct advice sought to limit their activities to the prevention of design outrages. Indirectly, the failure to provide supporting policy and guidance at national level also curtailed activities at local level. In the United States, design control has been driven at local government level in the absence of any national or state level efforts to do this. In addition to controlling design through volume controls attached to zoning regulations, USA cities have eagerly taken up more discretionary methods of control, administered as design review.

A number of tensions arise through design control. Land owners, developers and the public have generally favourable opinions of design review, as they consider that it improves the design quality of buildings, helps to maintain property values and provides certainty around future change. Architects and designers, on the other hand, appear to dislike design review because it provides the public with opportunities to get involved in their design proposals. Critics of design review point out that it is often based on very broad design principles that are open to subjective interpretation and that it is focussed on the singular building rather than the wider streetscape. To this end, few local authorities have prepared strategic visions for their street environments that could serve as a reference for design review. As a consequence, new developments tend to repeat existing design language or are self-referential in design, sometimes causing them to conflict with the existing patterns.

The chapter concluded with a discussion of two studies that evaluated completed designs in Wellington, NZ. One study found that prescriptive design guidance for character residential areas effectively steered new development to continue and enhance the recognised architectural character. A second found that design review methods for development in the city's urban core were ineffectual because they were not sufficiently authorised by the planning process. Although interesting, these studies were undertaken 
by experts who simply evaluated the outcomes in the same manner as a design reviewer in assessing the application. The views of the public were not sought and in the urban core study the developments were not analysed in terms of their effects on the streetscape. Hall (1990) noted that it is the overall collection of buildings along a street that matter to people.

This chapter has foreshadowed the research question, which will be outlined toward the end of chapter 3. Following confirmation in chapter 1 that the appearance of the built environment is of interest to people, chapter 2 has presented and discussed methods by which government has regulated design outcomes. Design guidance is generally prepared by experts on the basis of broadly held principles of good design. Where these principles are interpreted for use in the local context, which is rare, this work is also done by staff with backgrounds in planning or design. Although lay members of the public can be involved in review panels, the vast majority of review is undertaken by planning staff. This suggests that the views of the public are not well represented in the administration of design control by local authorities. Chapter 3 looks at environmental aesthetics literature in order to further inform this research into people's aesthetic perceptions of urban streetscapes. 
Chapter 3

\section{Environmental Aesthetics}

\subsection{Introduction}

Chapter 2 discussed design control, described as a suite of methods mainly used by local governments to control the design outcomes of projects before they are built. The purpose of design control is to make the design of individual buildings better - mainly from the perspective of the public - than they might have been without such intervention. However, there is little evidence on which to evaluate the effectiveness of design control, which in turn has led to this research. The chapter concluded by suggesting a gap in knowledge about what people think about the design of individual buildings and streetscapes in an urban context. Chapter 3 traverses literature in the field of environmental aesthetics, creating a knowledge platform from which people's perceptions of urban streetscapes can be investigated.

There is general consensus that there are significant gaps in evidence based knowledge about people's aesthetic experience of cities. As a consequence, most of the literature has been developed on the basis of normative theory (Cuthbert 2006: 171-173). As a prelude to discussing environmental aesthetics, the chapter introduces the two principal approaches to research on aesthetics, the speculative and empiric (Lang 2003). The chapter then looks into the literature on environmental psychology and environmental aesthetics to understand how people process and evaluate sensory information. Architectural literature is consulted in order to relate built form characteristics to the different categories of aesthetic response. Before outlining a theoretical framework for the research by bringing the literature discussed in chapters 2 and 3 together, the metrics of aesthetic 
response are discussed. With reference to the previous chapter, the theoretical framework that helps articulate the gap in knowledge, is developed.

\subsection{Speculative aesthetics}

Throughout history, aesthetics in the visual design fields from architecture to the fine arts has generally been the domain of philosophers and theoreticians (Wohlwill 1976). Philosophical or speculative aesthetics generates theories of beauty and pleasure through introspection and analysis of personal belief systems (Lang 2003). Speculative approaches to aesthetics are based on the belief that if beauty can be perceived and understood, then it can also be designed into a building or work of art.

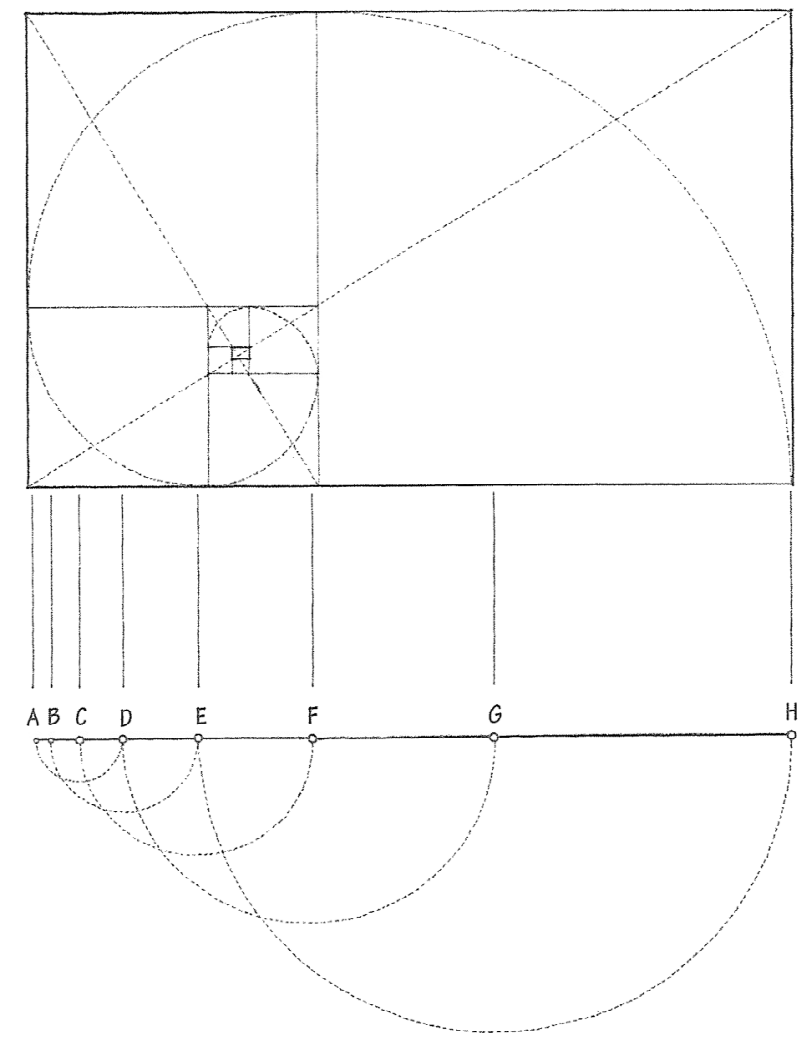

Figure 3.1: The Golden Mean proportioning system, developed by Greek mathematicians more than 2,000 years ago

Source Ching 2007 
Although different cultures have independently speculated over beauty, there are similarities in their bases for it. Speculative aesthetics aims to make general, normative statements about the entities, concepts, terms and values connected with art and beauty (Berlyne 1974). Throughout history, certain proportional relationships have been argued as key to aesthetically successful form and spatial arrangements. The Euclidean Golden Section (figures 3.1 and 3.2) is one such proportioning system, whose use is meant to create a sense of order and harmony among the elements of a visual design (Ching 2007: 300). The Golden Section is an example of a geometrical proportioning system, which Padovan (1999: 46) distinguishes from arithmetic methods of generating such relationships. The Fibonacci series is an example of the latter, developed to describe the spiralling growth pattern of plants. Other well-known theories of proportion include Le Corbusier's Le Modulor and the Greek Classical Orders (Padovan 1999: 10-13, Cuthbert 2006: 175-179).

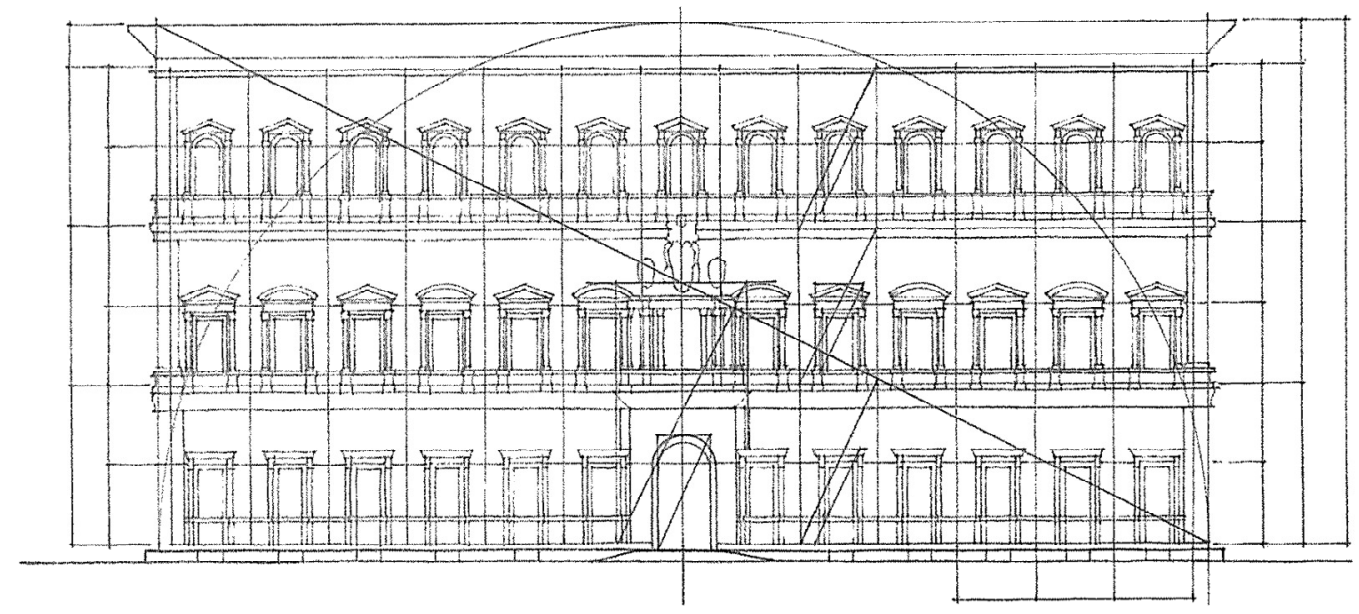

Figure 3.2: Analysis of the facade of Palazzo Farnese in Rome shows how the diagonals of all rectangular shapes, including the overall façade, have similar proportions of width to height.

Source Ching 2007

The main branches of speculative aesthetics are bermeneutic, existential, political and phenomenological; with the first and last associated most closely with architecture and environmental aesthetics (Lang 2003). Phenomenological study of aesthetics seeks intuitive insight into how people sense the physical world (phenomena of objects and situations) and processes this material to find meaning. Phenomenological study of the built environment arose in reaction to the emptiness of Modern Movement environments, looking at concepts such as character, identity and place (Norberg-Schulz 
1980: 6, Lang 2003). Phenomenological analysis is held to be akin to, but distinct from, introspection because it seeks to understand the basis for socially held aesthetic truths. It is a highly subjective field which solicits personal thoughts and experiences about a place in order to determine essential truths, with truth being the collective of those whose experiences are being considered (Scruton 1979: 78-79).

Hermeneutics, on the other hand, is an interpretive examination of the object, akin to reading the object as a text. This process is deductive, relying extensively on conceptual definitions, broadly accepted propositions and the writer's own beliefs and experience. As theories of speculative aesthetics are expressed as normative statements, the ultimate criterion of validity is whether or not the reader is imbued with a sense of conviction through the arguments put forward by the writer (Berlyne 1974:2).

Philosophers have grappled for centuries with the issue of whether contemplation of aesthetics should focus on the object or on the person (Weber 1995: 4). Scruton argued that the aesthetic experience is independent of the object. Citing the existential philosophies of Immanuel Kant, Scruton argued that our experience of a work of art or urban space does not refer to the properties or characteristics of the object (Scruton 1974, cited Cuthbert 2006: 172). He rejected the relevance of meaning, where architecture is conceived of as a language, to the aesthetic experience. Instead he aligned aesthetic judgement to notions of moral judgement (Cuthbert 2006: 172). However, as Weber (1995: 5) noted, if indeed the aesthetic experience is wholly determined by the viewer's own disposition then it follows that the object triggers aesthetic response and its properties are therefore also relevant to study. Ittelson (1978) agreed, describing perception as dependent not only on the environment but also on the person.

While this may be useful in terms of learning to appreciate art, aesthetic philosophies not grounded in science reveal little about how people actually experience the environment (Lang 2003). Nevertheless, progress can be made by first forming theories and then testing their validity through empirical analysis (May 2011).

\subsection{Empirical aesthetics}

Toward the end of the 19th Century Gustav Fechner established a field of enquiry that applies scientific methods to gaining an understanding of patterns of perceptions and 
social behaviour. Empirical aesthetics brings together experimental psychology and the behavioural sciences to examine how individuals and groups of people perceive and process environmental stimuli in the course of making judgements about the world around them (Cuthbert 2006). The aim of empirical aesthetics is to measure hedonic, or value based, responses to different patterns of the built and natural environment, such that empirically based theories can be stated (Lang 2003).

Although researchers in empirical aesthetics have followed a number of different approaches, one characteristic is common to all; the need to identify causality between the object and the human experience of it. It is widely accepted that as stimulation varies, so too does the perception of the aesthetic quality of the object (Lang 2003). The psychobiological approach introduced the notion of arousal. Patterns of stimuli that lead to moderate levels of arousal in the viewer are generally considered pleasurable. Where levels of arousal are very high the experience will be perceived as unpleasant (Berlyne 1974) (figure 3.3). This unpleasant response corresponds to notions of sensory overload and unpleasant sensations of chaos (Rapoport and Kantor 1967). Berlyne also observed that aesthetic experience can be enhanced by reducing the arousal stimulus.

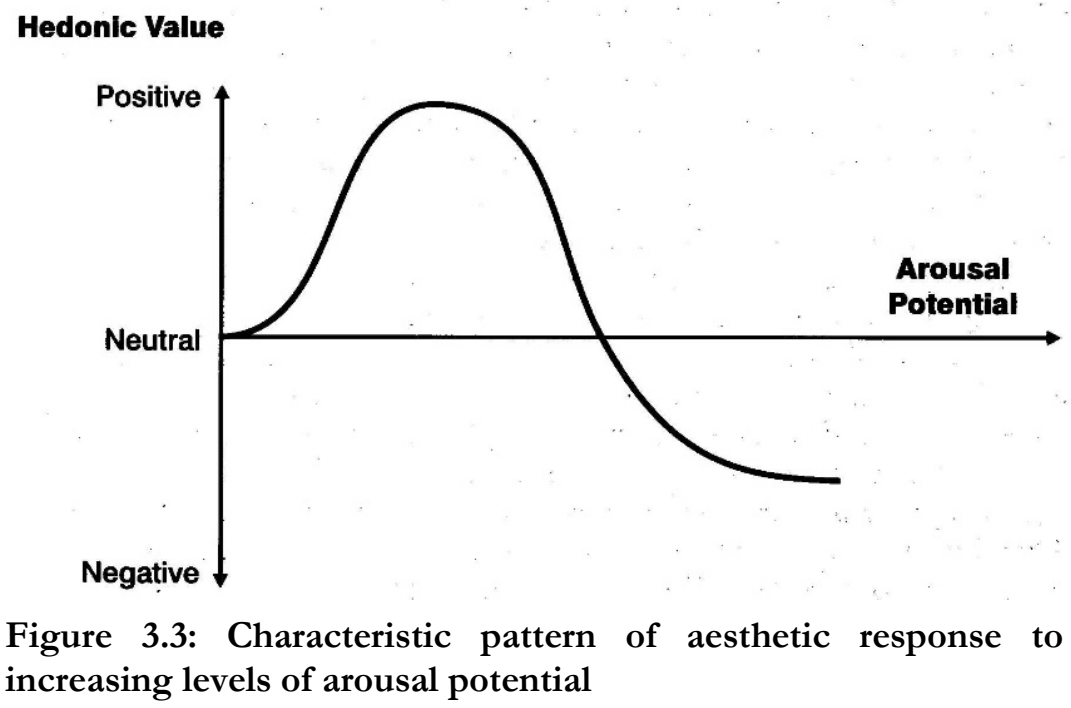

Source: Stamps III 2000

Following social science and psychology methodologies, a number of studies since the 1960s have examined people's perceptions and judgments of their environments to confirm the validity of theories that explain the processes of perception (Berlyne 1974; 
Ittelson 1978; Kaplan 1988; Nasar 1998; Stamps III 2000). Using mapping techniques developed by Lynch (1960) in his classic study of people's mental images of cities and survey methods, Nasar (1998) consulted residents of and visitors to two American cities. He set out to identify the general characteristics of likeable places, which were revealed as naturalness, openness, upkeep, historical significance and order. Critics have observed that Nasar's research simply confirms what most people would already assume (Chapman 1999). Nevertheless, findings such as Nasar's that are based on empiric data provide regulatory planners with evidence on which to base design policy and guidance. Unfortunately, Porteous (1996: 233) believes that translation of research findings into useful planning procedures remains problematic for planners.

Cuthbert (2006: 174) posits that an aesthetically pleasing experience is one that provides pleasurable sensory experiences, a pleasing perceptual structure and pleasurable symbolic associations. This definition provides a useful guide to the different levels of aesthetic perception that are necessary for judging a scene or setting. Aesthetic experience can therefore be conceptualised in three levels; sensory perception, cognition and meaning. Nasar's framework (figure 3.4) for aesthetic response is similar to Cuthbert's. Each of the three response levels that stimulate these responses, along with architectural design characteristics, is discussed in turn in the following paragraphs.

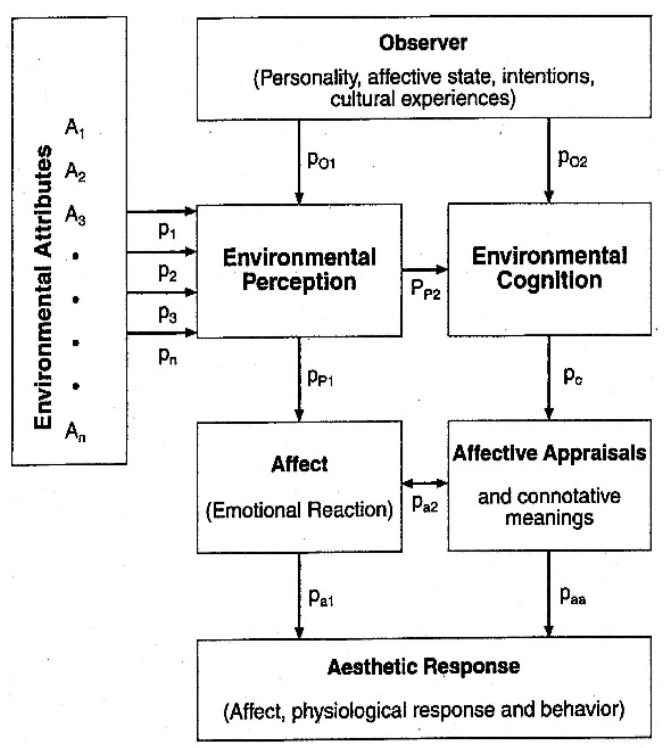

Figure 3.4: Framework for aesthetic response to the environment 


\subsection{Response to sensory stimulation}

Sensory aesthetics is concerned with the sense of pleasure associated with direct sensory experience and stimulation of our perceptual system (Lang 1988). In the second half of the 20th Century the medical field shed new light on the functions of the brain, enabling better understanding of how environmental stimuli are processed. The hippocampus is an ancient part of the brain, located near the stem. It is responsible for emotional arousal, which is often manifest in perceptible changes in the body such as increased heart rate or spontaneous weeping (Smith 1977). The rest of the brain is organised in two halves, with the left neo-cortex excelling in logical processes and language. The left brain comes to understand stimulation by breaking it down into constituent parts for analysis. The right neo-cortex handles complex spatial images and concepts. This side of the brain can manipulate complex visual patterns and reassemble the fragmented information analysed by the left into the image of the whole. Smith (2003) suggests that this arrangement is ideal for making aesthetic decisions and is reflected in the characteristics of a pleasing aesthetic experience, these being sensible whole patterns in combination with appropriate complexity.

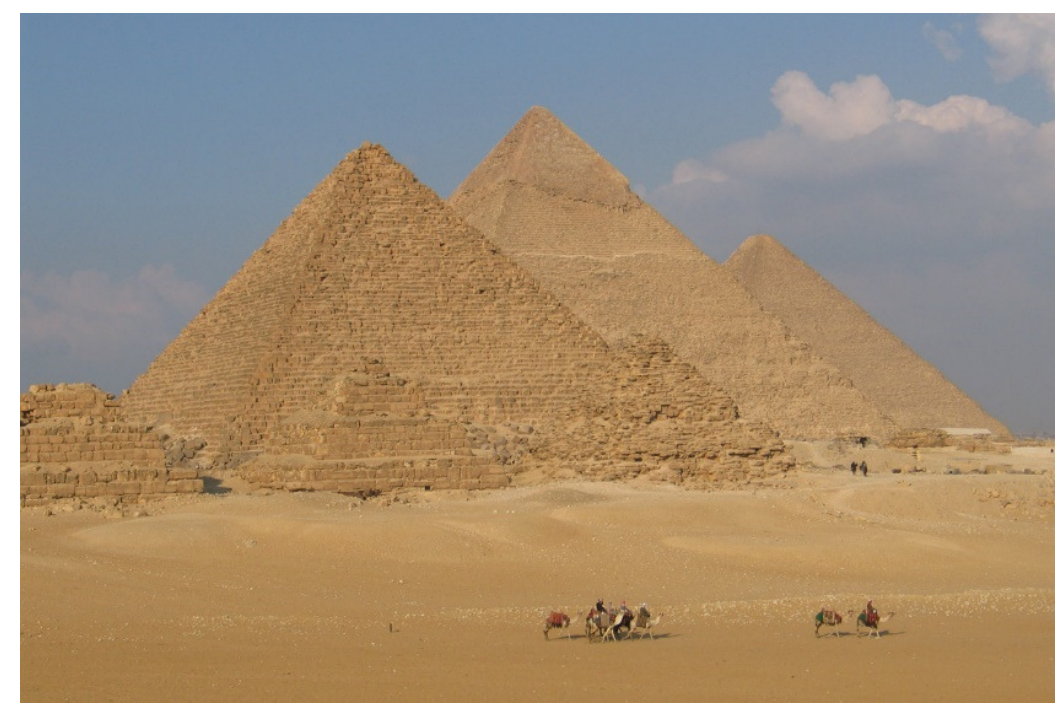

Figure 3.5: The three great pyramids at Cheops

Source: http:/ / famouswonders.com/great-pyramids-of-giza/

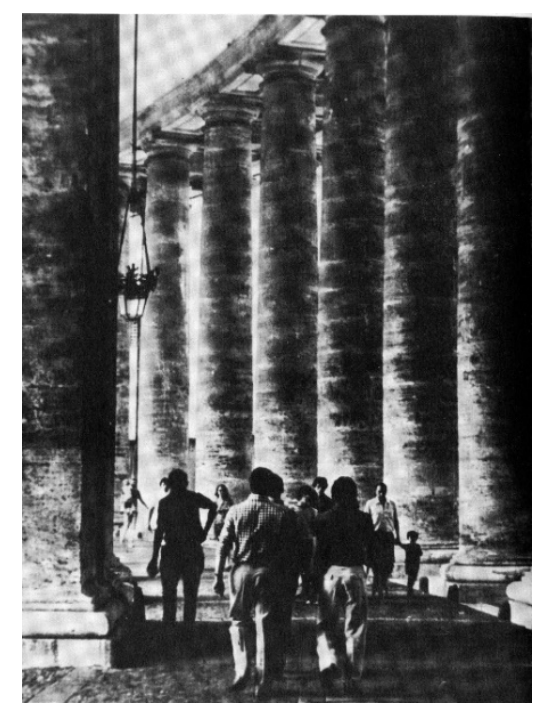

Figure 3.6: The colonnade around St Peter's Square in Rome

Source: Smith 1987 
The hippocampus appears to be easily stimulated by the garish and loud, basic rhythms and shapes, the gigantic, and by bright colours (Smith 1974, Weber 1995, Smith 2003). Smith (1980) comments that the limbic system, centred in the hippocampus, has an appetite for sensory stimulation at or near saturation level and that this is one of the reasons places like Las Vegas have appeal for many people. However, such sensory overload cannot be endlessly tolerated and more rational, dominant parts of the brain react by creating ordered spatial and temporal boundaries.

The Egyptian pyramids (figure 3.5) are an example of forms that appeal to the limbic system, as they are spectacularly large and simple in shape. Similarly, the colonnade at St. Peter's Basilica in Rome (figure 3.6) provides stimulation in the form of super scale columns in a strong, simple rhythmic pattern. The image appeals to the emotions, particularly when presented graphically and highlighting the strongly contrasting light and shade.

\subsubsection{Colour response}

People are known to respond to colour at a subconscious and emotional level and in ways that are influenced by biological and cultural factors (Janssens 2001). Although many psychological studies of people's aesthetic responses to colour have been conducted, few have investigated response to façade colours (O'Connor 2006). Nevertheless, speculative theories circulate. One prominent theory links people's own personal colour preferences to their preferences for colours on artefacts and environmental colour (Janssens 2001). However, studies carried out by Janssens (2001) and O'Connor (2006) did not support this theory and both researchers found that aesthetic response to environmental colour takes account of the overall setting and the relationship of colours to one another. Personal colour preferences did not influence preferences at this scale to any great extent. Janssens also found that preferences for façade colour schemes along a street conformed to Berlyne's (1974) theory regarding arousal potential. Those façade colours that appeared indifferent, because they blended inconspicuously with others, and those that appeared bold were preferred less than those colours that appeared distinct at the same time as fitting in. 


\subsection{Response to formal factors}

The two most important formal factors affecting aesthetic preference are order and visual interest, where visual interest is heightened through increased levels of ambiguity and complexity (Rapoport and Kantor 1967). This advice is qualified by Nasar (1994), who determined that moderate levels of complexity, together with a clear sense of visual order, evoke the highest preference levels. Consistent with Berlyne's advice (see section 3.3), Smith (2003) made comparisons with poetry to suggest that rhythms of architectural features seen in a building façade or across the facades of several buildings are similar to rhymes and couplets in a literary piece. He then went on to suggest that knowledge, or the comprehension of new truths, is simulated through being able to see ordered patterns in the built environment (Smith 2003: 11). The following section discusses these two formal factors and the built form characteristics that help generate order and visual interest.

\subsection{Sense of order}

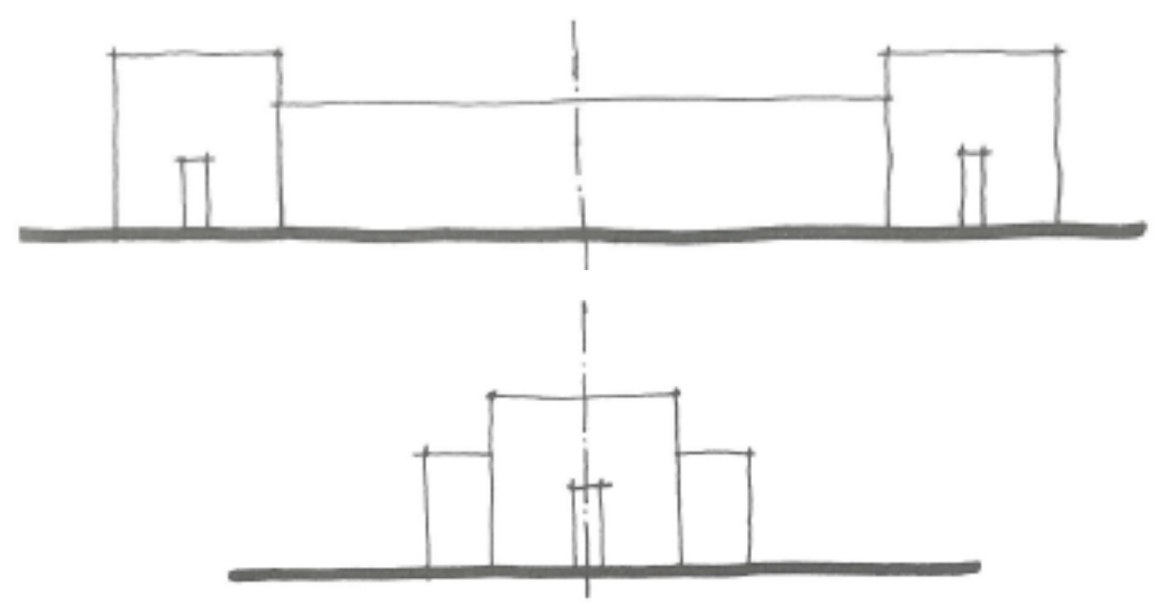

Figure 3.7: Two examples of symmetrical building facades.

Source: Young 1986

It seems all people respond positively to environments in which they can see patterns, rhythms, balance and harmonic relationships. In this respect, aesthetic experience appears to rise above any differences between people that might relate to social or cultural background (Smith 1980). One of the most fundamental ordering devices is symmetry, 
which is the balanced arrangement of elements on either side of a median axis. A sense of order is generated by the ability to divide the symmetrical element into two identical halves (Ching 2007: 348) (figure 3.7).

The concept of redundancy, or duplication of discrete elements within a scene, is the principal factor influencing how people perceive order as there is a tendency for the brain to group elements that have common properties. Groupings based on physical similarities become more apparent with continued processing over time. In line with Gestalt philosophy, the mind perceives the whole at the outset and this overall image is then scanned and processed in search of perceptible order (Weber 1995: 58). Sense of rhythm in a scene creates the most distinct opportunity for perceiving order (figure 3.8). Ching (2007: 382) notes that almost all building types incorporate elements that are by nature repetitive and which can potentially generate rhythmic patterns. Opportunities to create order may be found in the shape and arrangement of elements such as openings, structure and spatial modules, and even down to the repetition of small units such as bricks.
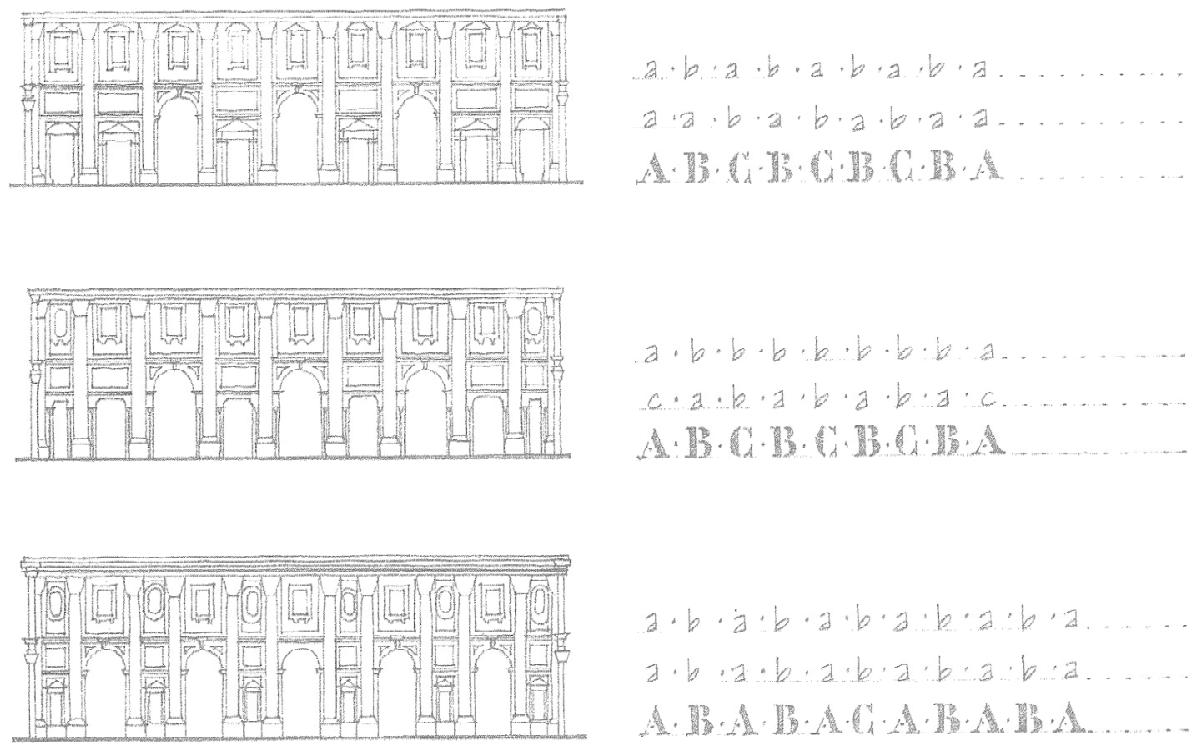

Figure 3.8: Analysis of the internal facade of a basilica, revealing the rhythmic pattern of openings and details.

Source: Ching 2007 


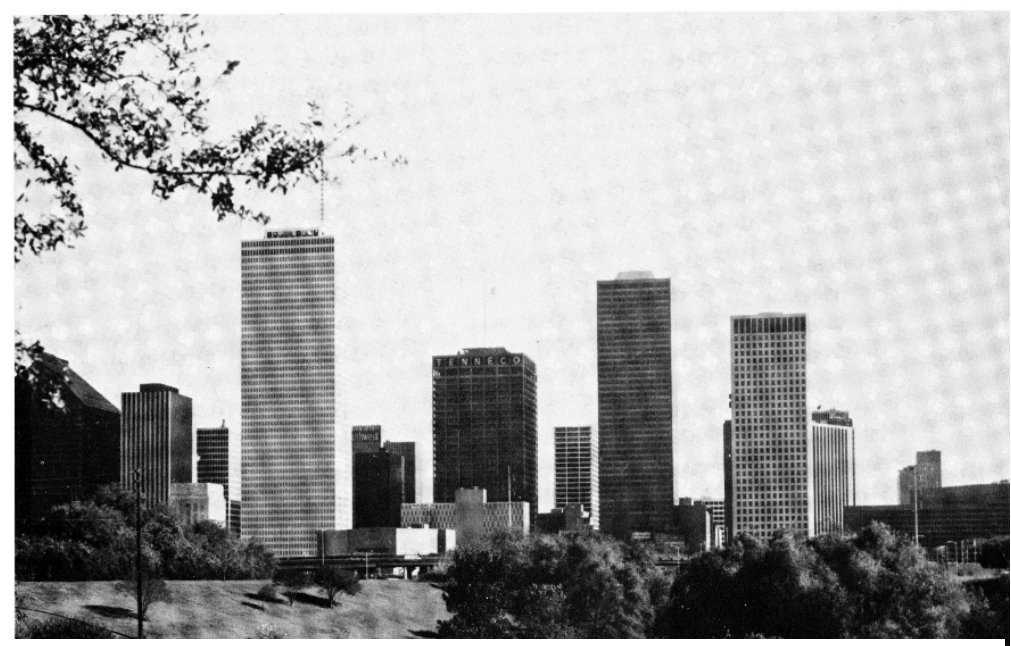

Figure 3.9: At a distance, rhythms can become evident across a setting of freestanding high rise buildings.

Source: Attoe 1981

At the broad scale of the skyline, a rhythmic arrangement of individual towers will largely be perceived as pleasant (Attoe 1981) (figure 3.9). Perception of order is thus not limited to that of a single building façade, as it can also be observed in a collection of spatially contiguous elements, such as a street scene. Apparent rhythms are not limited to horizontal dimensions and these can also be perceived over the height of a building façade, particularly where floor levels are expressed, and more subtly where elements such as window openings have similar shapes and vertical spacings.

\subsubsection{Rhyme and pattern}

Rhyme, described earlier as an amalgamation of order and complexity, is discussed here in terms of the perceptual order it can facilitate. Smith (2003) believes a second form of ordering occurs in the drive to harmonise between contrary elements. Figure 3.10 illustrates a collection of narrow buildings with articulated facades. While at first the scene appears overly complex, Smith's graphic analysis reveals the underlying patterns that many people find pleasant. The diagram identifies the vertical rhythms of building widths, the horizontal rhythms in floor levels and projections while the pattern of windows completes the rhyme. 

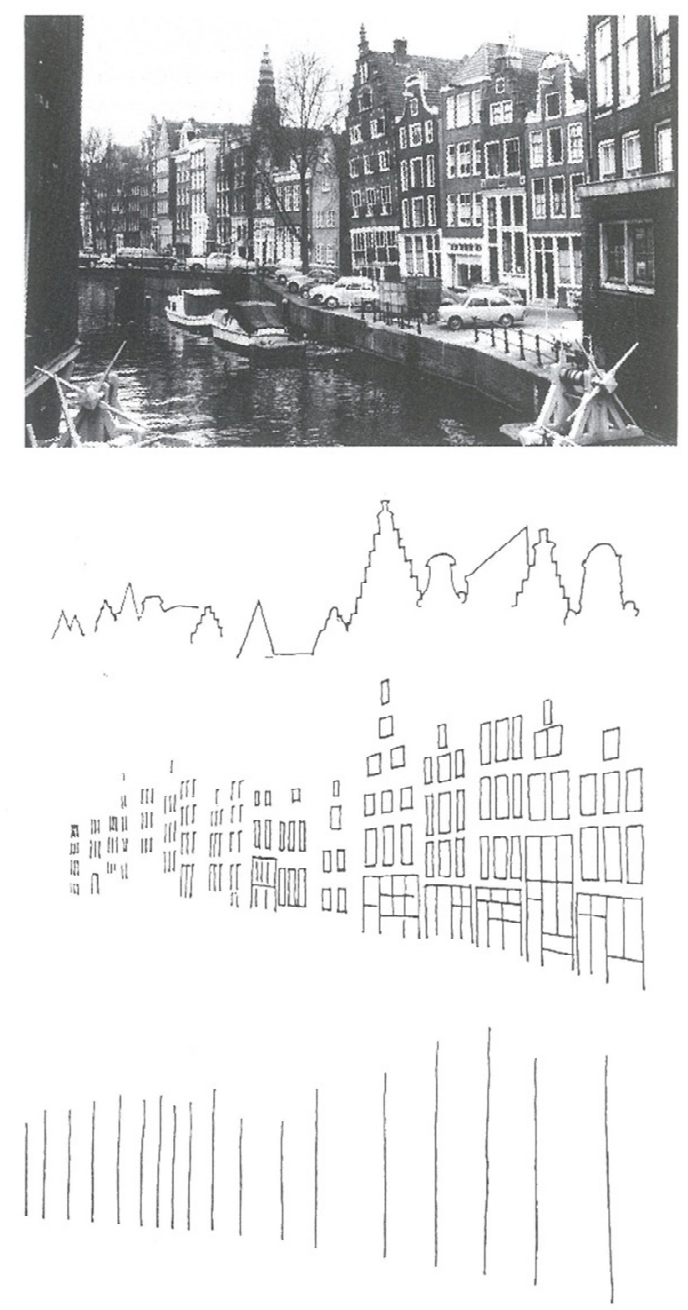

Figure 3.10: A graphic analysis of the underlying rhythmic patterns in this Amsterdam street scene.

Source Smith 2003

Proportion is a particular rhyming pattern that occurs when constituent parts are related to other parts and/or the whole in a harmonious mathematical relationship (Smith 1980, Ching 2007: 294). This has earlier been discussed under the speculative aesthetics heading (section 3.2). As a very subtle example, Smith (2003: 77-80) referred to the sunflower. It seems that nature exhibits patterns and regularities, the details of which have only come to light through the emergent science of biomathematics. The spiralling layout of seeds in a sunflower head meticulously follows the mathematical proportions of the Fibonacci series. Indeed, the spiralling arrangement of the leaves on the stem of the plant, as well as 
the petals surrounding the seed head, on analysis demonstrate conformity to this fundamental geometric relationship. As a consequence Smith argued naturally occurring forms in the biological world have inherent and everlasting beauty (figure 3.11).

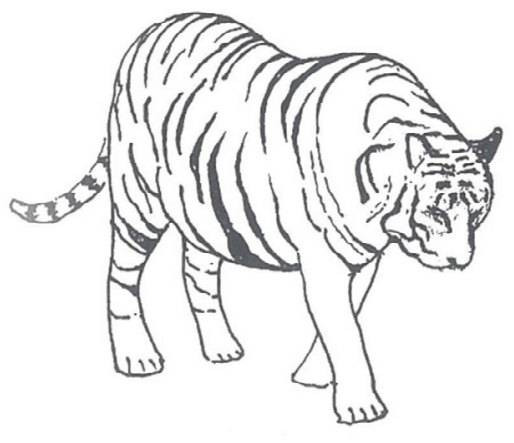

Figure 3.11: Smith cites the tiger as an example of coherent diversity, a key theme in the study of aesthetics

Source: Smith 2003

The earliest and still most common architectural proportioning device is the form of the building material (Rasmussen 1959: 121). Many forms are similar across cultures, such as the clay brick, and have historically been tied to the structural properties or naturally occurring forms of the material itself (Ching 2007: 295). Building elements can be related through expression of their forms to the overall shape and size of the building. Proportions may emerge by manipulating dimensions and arrangement of fenestration and doors in a façade or through expression of floor level relationships and structural elements. Overall heights and widths of facades can also be proportionally related.

\subsection{Complexity and ambiguity}

Wohlwill (1976) researched the ways people visually explore a scene and found that the extent of exploration was relative to the levels of ambiguity and uncertainty they encountered. It seems that ambiguous and complex scenes piqued people's interest, causing them to spend more time engaging with these. Yet despite consistent acknowledgement of the attractiveness of visual complexity and the importance of 
underlying visual order in experimental psychology literature, the two are rarely discussed together in architectural literature. Based on the references consulted in this research there appears to be a tendency for architectural researchers to emphasise order and proportional relationships in their writing, with few willing to venture into the area of ambiguity and complexity and fewer still able to recommend how complexity can be achieved using contemporary practices. One exception is the American architect Robert Venturi.

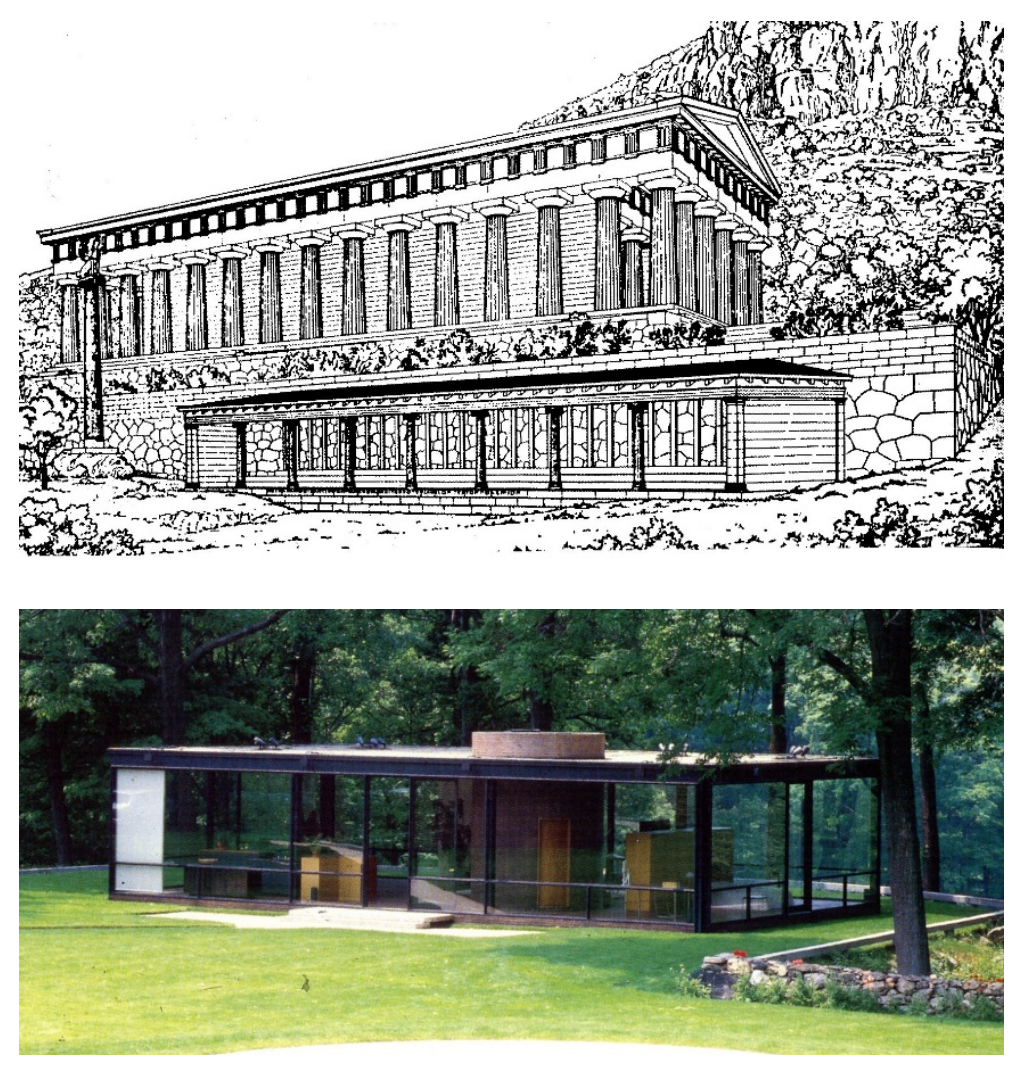

Figure 3.12: Venturi found the complexity in shape and articulation of the Doric temple (top) more satisfying than the Modern pavilion (below).

Source: Fletcher 1987 (top) and Neal 2000

Venturi (1977) wrote about the merits of formal complexity and uncertainty in urban settings, provoked by the monotonous environments that emerged through application of Modernist principles in the redevelopment of North American cities following the Second World War. Discussing the pavilions of Mies van der Rohe, Venturi suggested that their compositional strength, derived from the restricted scope of architectural 
language and content, were also their limitations. He argued that higher levels of satisfaction can be derived from inner complexity. In arguing his point, Venturi compared the characteristics of a Modern pavilion to those of a Doric temple, noting that the older structure exhibited contradictions and tensions through subtlety and distorted geometry, which served to increase their attractiveness to viewers (figure3.12). Bentley, Alcock et al. (1985: 90) also noted the importance of a rich sensory environment, making the observation that visual richness depends on the presence of visual contrasts in the surfaces.

To develop a conceptual framework for this research it is useful to classify the characteristics of built form that can be manipulated to generate visual interest. Stamps III $(1994,2000)$ did this, approaching the task as a reviewer and controller of design outcomes, with the objectives in most cases being to avoid visually chaotic outcomes at one end of the spectrum and utterly monotonous ones at the other. He used a scientific approach, whereby measurement of complexity is made under the three headings of overall shape, complexity of form, and complexity of surface. In the urban context these three hierarchical levels of complexity correspond to silhouette, three-dimensional modelling, and surface articulation. Each is discussed below in turn.

\subsubsection{Silhouette}

The two hemispheres of the human brain apprehend visual stimuli in complementary fashion, with the right side concentrating on the overall shape of an object and the left seeking to fill in the detail. Smith (2003: 40) noted that both work concurrently and yet separately to form a comprehensive image. Whereas the left hemisphere starts with the detail and builds up to an overall view, the right embraces the global. Accordingly, overall shape is an important factor in the aesthetic experience of a building or collection of buildings. Although perception of a building façade is influenced by the angle of view, its overall shape will always appear as a silhouette (Stamps III 2000: 39). Shape perception is also influenced by the level of contrast between the contour and the surrounding field (Ching 2007: 96). Most buildings exhibit straight bottom edges and sides. When buildings are tightly spaced the visual contrast along these contours is reduced. Top edges are however, seen against the sky and are therefore important factors in overall perceptions. 


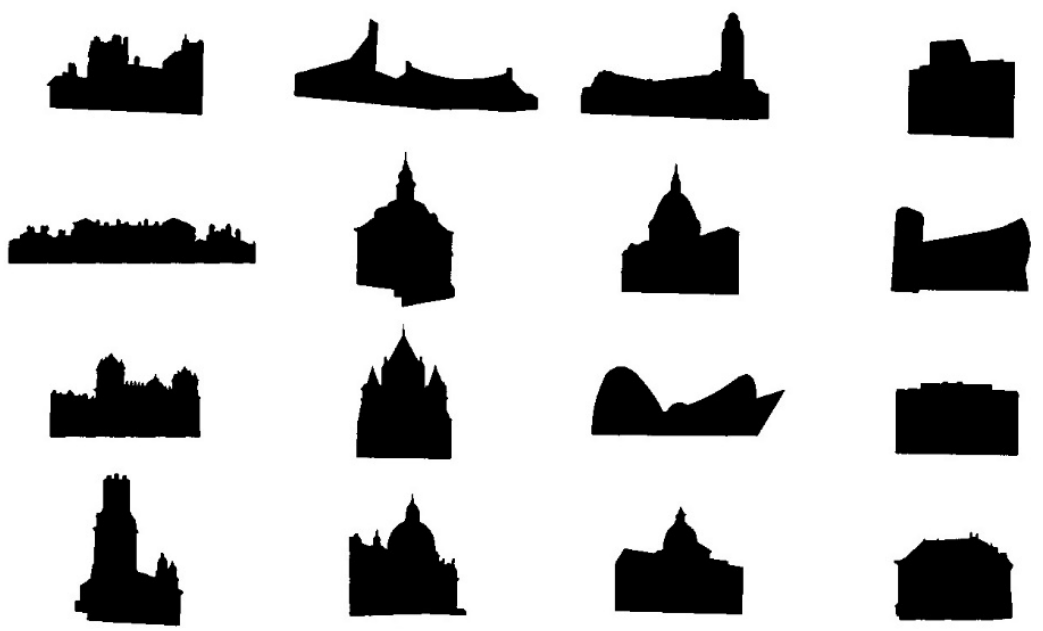

Figure 3.13: Stamps III's studies of building shape complexity led him to conclude that the silhouette of the top edge is a first order consideration.

Source: Stamps III 2000

Stamps III (2000: 39-42) discovered that the number of vertices in the top edge was the most important determinant for the complexity of an object (figure 3.13). While each building outline generally falls under the control of one designer, along the length of a street the differences in height between buildings is less often the product of conscious design. Not only do differences in height potentially enhance complexity, at a distance such differences can also net the sky, the term coined by Cullen (1971) to describe how tall buildings spaced apart reach up to bring the sky down to the street.

\subsubsection{Three-dimensional form complexity}

Within the overall building shape, the next level of perception corresponds to the manner in which the form is articulated in three dimensions. Ching (2007: 88-89) noted that modelling a form can influence the ways visual weight and scale are perceived. Nasar (1988) used massing to describe the object's volumetric composition, which is one of the three key physical attributes that can affect compatibility of a building with its setting. Along with frontage setback and surface detail, massing formed the framework for Groat's study of people's preferences for different contextual design approaches. While the present research adopts the approach of Stamps III (2000) and distinguishes between the perceptual attributes of overall shape, or silhouette, and form complexity, Groat's framework combined these two levels of complexity under the single heading of massing. 
The rationale for the approach taken in the present research is provided by Smith (2003), who described how the human brain processes information about three dimensional forms (discussed in section 3.7.1). Although he challenged the effectiveness of form articulation as a means of reducing perceptions of bulk (figure 3.14), Stamps III (2000: 53) noted it to be one of the most common design features regulated by local governments (figure 3.15). Design guidance typically aims to reduce mass through articulation of the façade or by breaking up overwhelming volumes, which in turn will also increase visual interest by increasing complexity (Berlyne 1974).

Using empiric methods to measure form complexity, Cooper and Oskrochi (2008) sought to understand how people perceived visual variety in residential streetscapes. Their research found that strongly articulated shapes were perceived positively and that shape complexity was closely correlated to character. On this basis, preservation and extension of architectural character in residential areas is increasingly managed through design control (Stamps III 2000: 58). Manipulation of form becomes even more effective for generating visual interest in changing conditions of light. Shadows and highlights are most effective at animating an object.

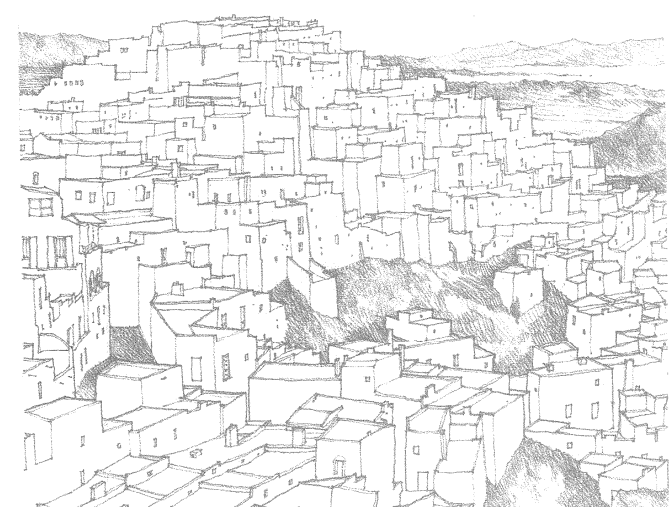

Figure 3.14: View of the Spanish hill town of Mojacar. The articulation of the overall form of the township has occurred naturally over time and helps to reduce the apparent visual bulk of the agglomeration.

Source: Ching 2007

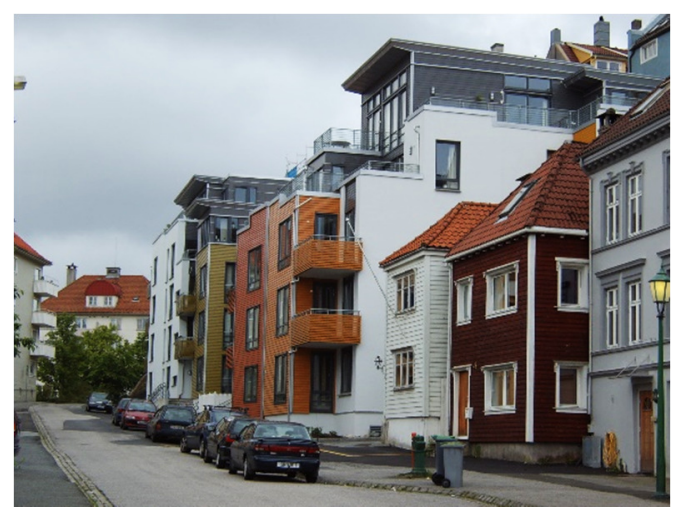

Figure 3.15: The form of the new building (centre) has been articulated in three dimensions in an effort to make it more compatible with those on either side. The effect has been enhanced through the use of colour.

Source: author 


\subsubsection{Detail and surface}

The surfaces of building facades precipitate the next level of perceptions. Introduction of details and treatment of surfaces can generate interest, but the effectiveness of this also depends on the size of interventions in relation to viewing distance. Bentley, Alcock et al. (1985: 89-91) noted that to be effective, details and textures should be coarser and more pronounced when viewed from longer distances.

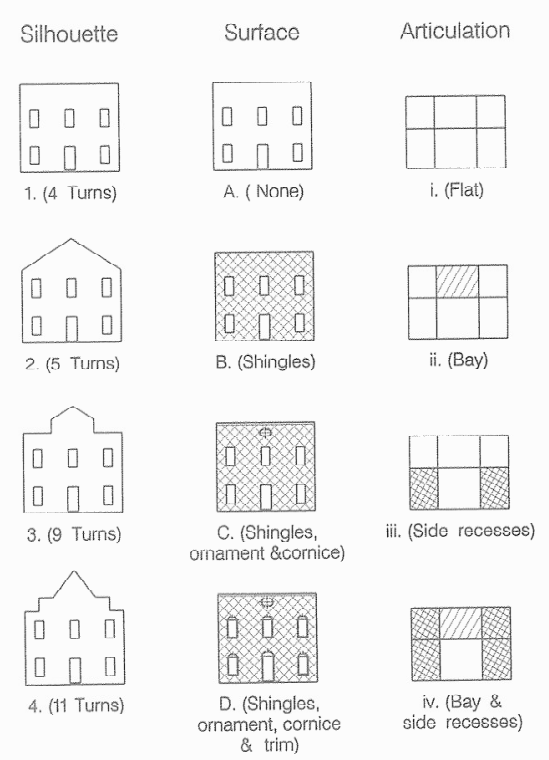

Figure 3.16: Stamps III's studies of perceptions of built form have led him to argue that complexity can be measured at three levels, silhouette, form articulation and surface texture.

Source: Stamps III 2000

The primary method of articulating the external surfaces of built form is by manipulation of the door and window openings. These also provide useful indication of scale and building use patterns, which in turn convey associational meaning. Stamps III (2000: 58) found that an arrangement of openings in a facade can be much more effective at reducing its apparent bulk than three dimensional modelling is. Cooper and Oskrochi (2008) also noted the additional benefit of visual interest enhancement that comes with surface textures and patternmaking. Weber (1995: 228-240) discussed facades that comprise discrete, punched openings (windows), as distinct from expansive openings like curtain wall facades, noting that the former type assumes the status of figure against the ground cast 
by the surfaces of the façade. Weber (: 229) argued that of the many façade properties, the organisation of its component elements into figure and ground determines the extent to which it will appear orderly and this is of "primary importance" in how it will be perceived by people.

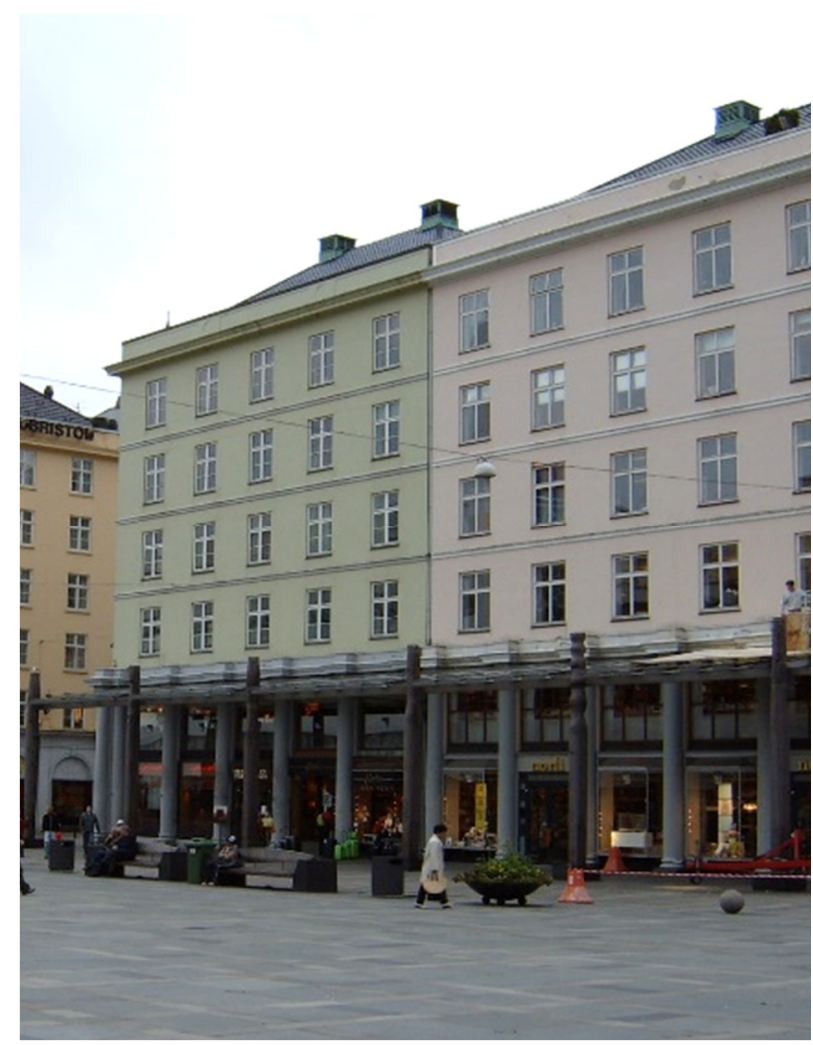

Figure 3.17: The figure:ground effect of discrete windows in the the building facade is key in how the building will be perceived.

Source: author

\subsection{Meaning and aesthetics}

"Aesthetic pleasure is not immediate in the manner of the pleasures of the senses, but is dependent upon and affected by processes of thought" (Scruton 1979:72). Contrary to Smith (2003), Scruton argued that perceptions of the built environment cannot lead to pure, unmediated sensuous pleasure. Instead, it is in the nature of humans to ascribe meaning to sensory stimulation and it is meaning that ultimately mediates between the stimulus properties of the environment and response to it (Rapoport 1982: 28). While aesthetic response may have direct sensory and formal dimensions, the meanings associated with an object can lead to the deepest sense of pleasure (Lang 2003). Through 
the work he did on cognitive mapping in the 1950s and 60s, Lynch (1960) developed a conceptual framework for environmental image that comprised three aspects; identity, structure and meaning.

Later, Nasar (1998) extended this concept to consider the extent to which people liked different parts of the city and why. Nasar's conceptual framework for evaluative image used Lynch's dimension of meaning to define three different levels of meaning that influence people's evaluations of the spaces around them. Lower order denotative meanings are those that allow people to recognise objects for what they are. By applying knowledge gained through life experience people are able to recognise, for example, that a hole in a wall with particular characteristics is a door. This meaning level is not particularly helpful in determining people's preferences, which was Nasar's interest. His research focussed on connotative meanings, which are those that associate emotional values with the object under consideration. Judgements around likability and being able to make inferences based on the physical appearance of an area are both enabled by connotative meanings. The highest level meanings are abstract, which require values to be assigned to connotative meanings (Rapoport 1990: 221). Abstract meanings are formed in the context of personal, cultural and social values (Nasar 1998: 7).
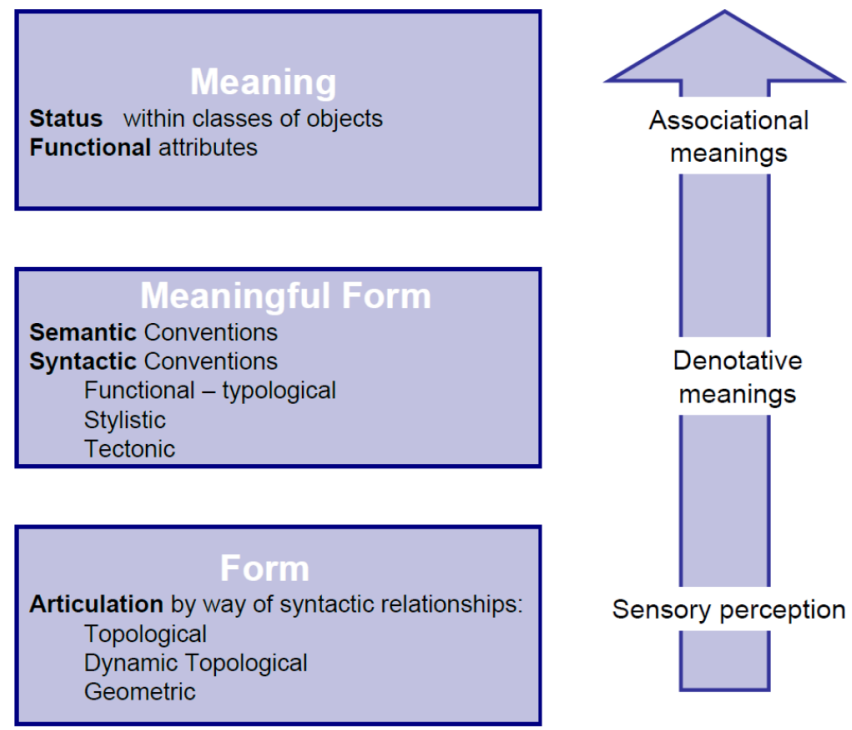

Figure 3.18: Aesthetic judgement involves three levels of processing, with the higher dependent on those below

Source: author after Weber 1995 
Weber (1995: 86-93) developed a model (figure 3.18) for aesthetic perception that accommodates the neurological processes discussed by Smith (2003) (see sections 3.4 and 3.6.1) and the meaning levels described by Nasar (1998). Like Smith (2003), whose discussion of perception follows his understanding of the relevant neurological processes, Weber (1995) set out and discussed the levels of perception from the bottom up. No level can be experienced in isolation and each higher level is dependent on the information processed in the levels that sit below it. At the most basic level, people register form as physical relationships between the constituent parts, which informs a sense of orderliness, and the similarities between the different parts, informing the sense of complexity. At the second level, meaningful form is understood by comparing the physical form to widely understood conventions or schema; for the built environment these could include the different elements such as doors and windows, the architectural style, the functional type or typology and the way the building is put together or its tectonics. The conventions against which the form can be compared may be widely held and socially/culturally defined or may be personal in nature. Conceptually this level of perception corresponds closely with Nasar's cognitive meaning level. Finally, Weber advised that meaning requires the viewer to assign value to the meaningful form, corresponding with Rapoport (1990) and Nasar (1998: 6-7). Weber (1995: 89) suggested that this highest order of aesthetic perception is abstract in nature, having less to do with the properties of the object and more to do with the broader values it represents to the viewer. The model outlines a hierarchy of thought in forming attitudes of meaning in relation to the object and the matters considered are essentially architectonic in nature.

Another view of how meaning enters the experience of a place or setting is illustrated in figure 3.19, which was developed around Jungian philosophy. Porteous (1996: 8-10) suggested that the notion of being in the world, or total experience of the place, is supported equally by the intangible qualities of mind (thought), soul (intuition), heart (feeling) and senses (bodily sensation). He then postulated that a purely aesthetic experience does not reference meanings, in the sense described above. He argued that the experience is solely based on sensory stimulation. He went on to suggest that meanings are relevant to each of the other three experiential dimensions of his model. For example, ethical experience makes reference to meanings in the sense that issues of moral good are deliberated. He 
also noted that a sense of attachment to a place derives in part through the meanings one associates with it. However, Porteous (1996: 8) considered that the role meanings play in aesthetic experience is indirect. Nevertheless, after consulting a broad range of literature on this topic it is clear that Porteous' concept of aesthetic experience is a minority view. Other writers have clearly established that meaning is directly embedded in processes of aesthetic judgement (Rapoport 1982, Weber 1995, Nasar 1998, Carmona, Heath et al. 2010). There are also two principal paths by which meanings are communicated; associational and symbolic (Nasar 1988).

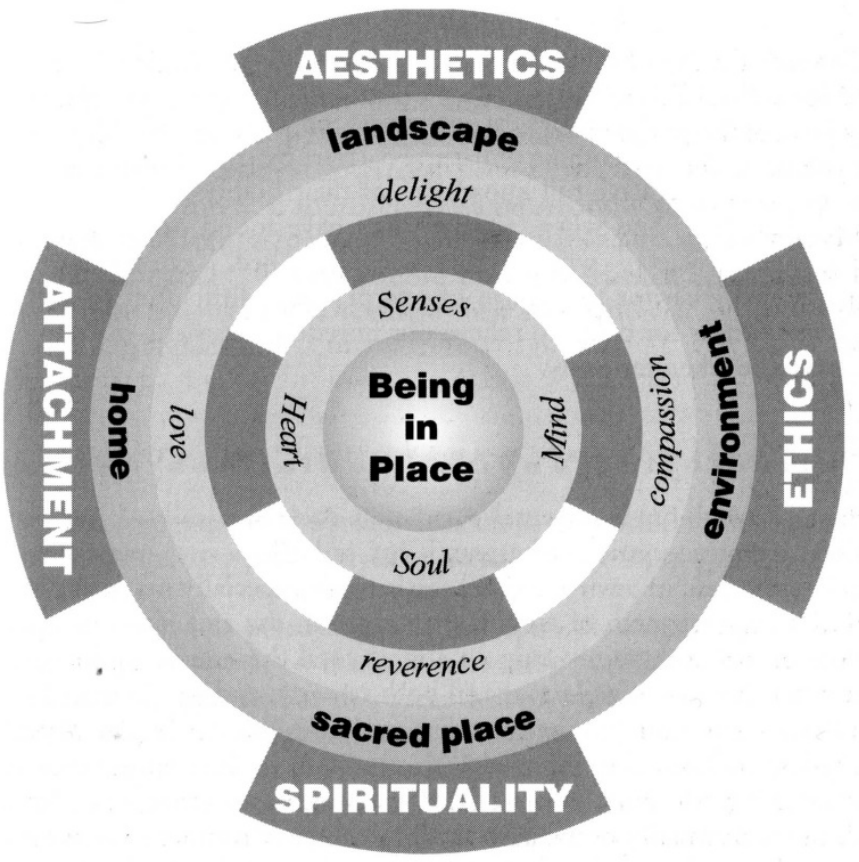

Figure 3.19: Porteous' diagram represents his concept of being in a place. Meaning is an essential component of three of his four dimensions.

Source: Porteous 1996

\subsubsection{Associational meaning}

Meanings attach to environments through use or association. Rapoport (1982: 35) referred to affordance, a term borrowed from psychology, which he defined to be the potential uses of objects and the activities they can afford. Notwithstanding the physical characteristics that influence aesthetic evaluation, association of use can create positive or negative feelings about an object or a setting. This is particularly evident in buildings that 
are associated with public, ceremonial or spiritual activities. It would be impossible to think of a concentration camp as aesthetically pleasing no matter how elegant the physical proportions. Perceptions of uses or activities that take place within a building have a clear effect on how people perceive it (Nasar 1989, Nasar 1994).

Aesthetic meaning may also derive from non-physical associations, such as connections with a historically significant event or an important person. Lynch (1960: 108) discussed the importance of place names in conjuring up the mental image of a place and found that associational meanings can also reinforce the identity or structure that may be latent in the physical form itself. Lang (1988) found that associational values establish over time and are therefore largely out of the hands of designers, yet the importance of and potential for associational values to affect aesthetic experience is clear. Smith (1980) lamented the fact that associational values in old towns often bias aesthetic response in ways that limit the potential for them to change.

\subsubsection{Symbolic meaning}

Signs and symbols that are explicit in a building or a spatial setting become powerful catalysts for the formation of meanings. In terms of aesthetic experience, meanings can derive through a culturally learned association between the object and an idea, a relationship that may be literal or abstract (Lang 1988). In a broad sense, cultures are said to comprise systems of symbols and meanings that influence, and in many cases determine, human social activity (Rapoport 1982: 38). Semiology is the study of symbols and their meanings, a concept that Lang (1988) describes as the triangular relationship between a symbol, a referent and the associated meaning it conjures up in the viewer (figure 3.20). The symbol, or signifier, is the physical form or space under a person's gaze. Lang noted that because individuals and groups of people vary, so too does the referent. Accordingly, the thoughts or meanings associated with an object can vary between people and different cultural groupings. 


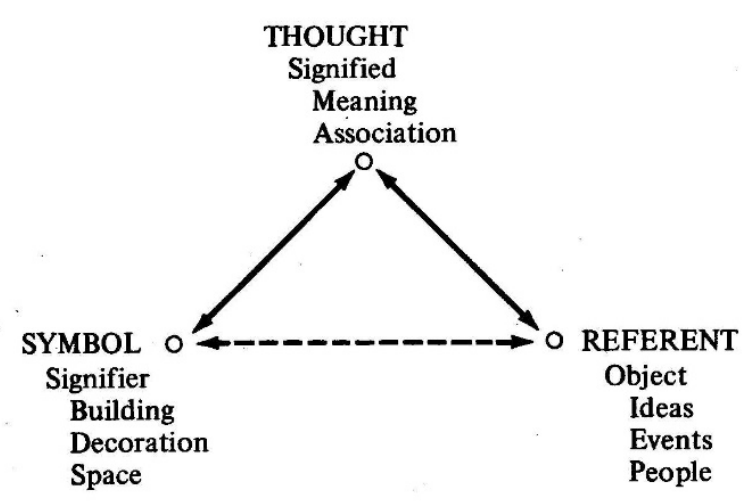

Figure 3.20: The effect and relationship between an object, the symbols it exhibits and the meanings that are derived is known as semiotics.

Source: Lang 1988

Symbols have meanings attached in the local culture and these are susceptible to change over time (Carmona, Heath et al. 2010: 117). Rapoport (1982) distinguished between signs, the meanings of which should be unequivocal, and symbols, where greater flexibility of interpretation is possible. The relevance here is that in less traditional cultures, as typified by those of the globalised Western world, the use of symbols is fraught, as their meanings are not necessarily shared between different cultural groups. The same form may be interpreted differently by different individuals and correspondingly, it is conceivable that similar meanings can be deduced from different forms (Lang 2003). Because of this, intended meanings are often lost in translation in Western societies. This is of particular concern when designers and others who participate in the production of the built environment misinterpret or do not concern themselves with the symbolic lexicons of others (Carmona, Heath et al. 2010: 117).

In a quest to create pure surface and space, Modernism rejected ornamentation, including use of colour. Environments that lack decoration prevent personalisation, conceptually as well as literally, and this has been widely read as an attack on users' meanings (Rapoport 1982). In reaction to placeless Modern settings, Norberg-Schulz (1980: 195, 2007) argued for architects and others involved in making buildings to concretise the spirit of a place through design. It was his contention that connecting a building to its setting through its spatial and material characteristics would help communicate meanings more clearly than the abstract mental constructs that were typified in Modernist buildings. 


\subsubsection{Built form characteristics related to meaning}

\subsubsection{Land use activities}

Variety of use correlates with variety of interpretations and meanings (Bentley, Alcock et al. 1985: 78). From this concept it can be inferred that the land use or activity associated with a setting conveys meaning, which in turn influences aesthetic perception. Indeed, meanings associated with a particular building or environment are closely linked to the social, cultural or personal values that attach to the activity that is understood to take place there (Rapoport 1982). While internal building usage can potentially be conveyed through the facade, perceptions are often confused by aperture size, viewing distance/angle, differences in light level and by the reflective qualities of many contemporary glazing materials. The building in figure 3.21 is completely transparent, allowing the internal functions to be clearly perceived. Activity and use are more commonly read in messages conveyed by building typology, relying on social or cultural conventions (Nasar 1994) or by signage. Most importantly, meanings refer to the values that individuals hold for the represented activities.

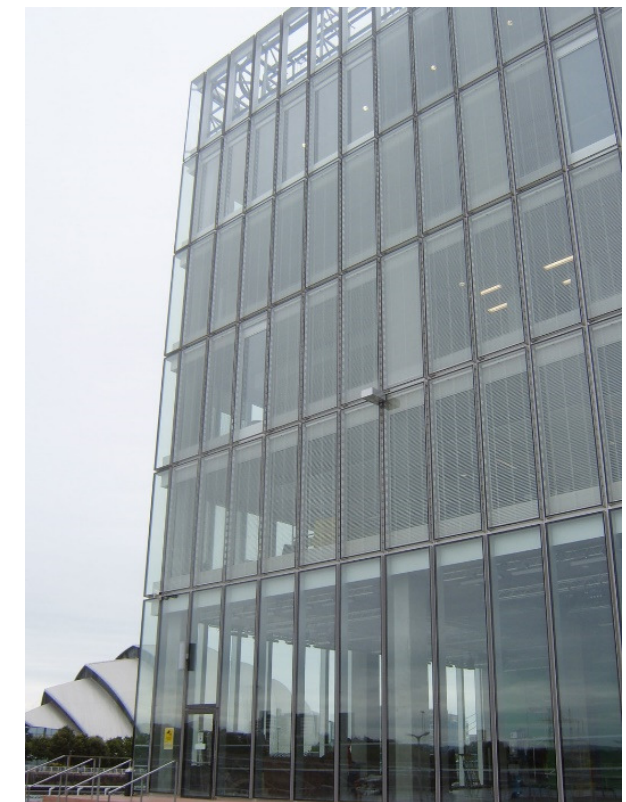

Figure 3.21: Facade transparency can facilitate public perceptions of building use and activity.

Source: Author 


\subsubsection{Scale}

The notion of scale is often discussed in relation to buildings and space, most commonly with reference to the scalar relationships struck between different components of a form or space (Ching 2007: 329). Punter and Carmona (1997: $166 \& 216$ ) on the other hand note that the relationship between the dimensions of a building and those of the human form is the primary factor that establishes the apparent scale of a building or setting. In their view, the scale relationships between the viewer and built form refers particularly to whether the environment feels comfortable to use. As human scale is considered a critical urban design success factor (Rasmussen 1959, Lynch 1981), it seems important to enable buildings to be easily read in this way. Vernacular architecture and earlier limits of technology both helped ensure that these scale relationships were easy to comprehend. However, advances in technology, the use of sophisticated materials and the advent of motorcars have all made these relationships more difficult to establish. Where constituent parts relate comfortably to human size, this provides another avenue for people to project themselves into the building. This can in turn enrich their level of understanding about it and enhance the meanings they hold for the building.

\subsubsection{Architectural style}

The architectural style of a building or space also represents a significant variable in terms of meaning. Nasar (1994) identified the ability of people to distinguish between different styles as a consequence of previous encounters. Through repeated experience of examples with similar formal characteristics people become able to categorise the similarities and differences between styles. Connotative meaning and aesthetic response to particular styles will vary with the setting, with personal experience and with cultural schema, which in today's world may be strongly influenced by the popular media.

\subsubsection{Heritage}

Buildings perceived as historic are generally preferred over those that are not, not only because of generally higher levels of complexity but also because they represent a tangible connection with the past. Nasar (1998: 70) speculated that this arises through favourable associations between the building and a past or current status as well as other positive meanings attaching to a history. This is an important factor to be taken into account when selecting the scenes to be investigated in this research. 


\subsubsection{Tectonics}

Prior to the Industrial Revolution, choice of materials was largely limited to those that could be sourced locally. Vernacular styles, materials selection and building methods were therefore grounded in the local setting and culture (Habraken 1998: 2-3). In contrast, important buildings were those accorded special status in the local setting, often by making use of exotic materials (Rapoport 1982: 29-30). Although these circumstances changed with industrialisation, materials continue to convey important meanings in relation to occupant status, the care and attention bestowed on the building by those who built it, and even how it might feel to be inside the building (Bentley, Alcock et al. 1985: 91, Nasar 1998: 77). It appears that buildings that do not fit with their surroundings very quickly acquire negative meanings. Material usage in new urban projects is one of the most highly regulated aspects of consent processes in the United Kingdom (Punter and Carmona 1997: 218). This is largely in reaction to the poor aesthetic outcomes of new development that were conveniently attributed to material use. In Western cultures, natural materials generally have higher status and are perceived more positively than processed ones, an affective response that is inverted in developing cultures (Rapoport 1982, Nasar 1998: 77).

The way in which an object is put together and the conscious detailing of the fabric also influence cognitive processes. Alcock (1993) makes the point that elaboration is a direct reference to the labour spent to engender a surface or object with visual interest and that this is taken into account when people ascribed meaning. He notes this is a symbol not requiring a lexicon, as the meaning appears to be held universally.

\subsubsection{Maintenance}

People are known to respond favourably to settings that appear well maintained and negatively to those that exhibit untidiness and general lack of care (Rapoport 1982, Nasar 1998: 65-67). Rapoport believes this to be the most important factor in perceptions of American residential areas. Many factors underlie affective response to how places are managed and maintained. Levels of upkeep are closely linked with perceptions of status, affluence and power. Poorly maintained settings also convey a sense of disorder, tending toward chaos, which plays on the aesthetic notion of ordered complexity (Nasar 1998). In addition, poorly maintained areas are associated with bad people and affect sensations of 
personal safety (Rapoport 1982). People tend to have strongly positive evaluations of new places, in part associated with perceptions of cleanliness.

\subsection{Dimensions of aesthetic evaluation}

While the field of aesthetics remains highly subjective to those who associate it with normative theories couched in terms of philosophy, empiric research has helped shed light on the ways humans process visual stimuli in the process of making an aesthetic judgment. Other research has also identified a number of built form characteristics that figure prominently in people's aesthetic experiences. In order to generate a workable model for examining aesthetic response, a rational method of expressing preference is required. Weber (1995: 86-98) provided little guidance on how preferences could be structured. Midway through his examination of how architectural form and space are perceived and assigned meaning, he conceded that judgements are subjective and that feelings and preferences for, or aversions to, an object will differ based on experience and learning.

Berlyne (1974) advised that aesthetic judgements can be measured on scales of hedonic value (figure 3.3). Visual experiences revolve around variables such as preference, pleasure and utility. Non-verbal expressions of judgement are those that are manifest in behaviour patterns such as making positive choices. There are evidential links between fluctuations in arousal and hedonic value, both positive and negative.

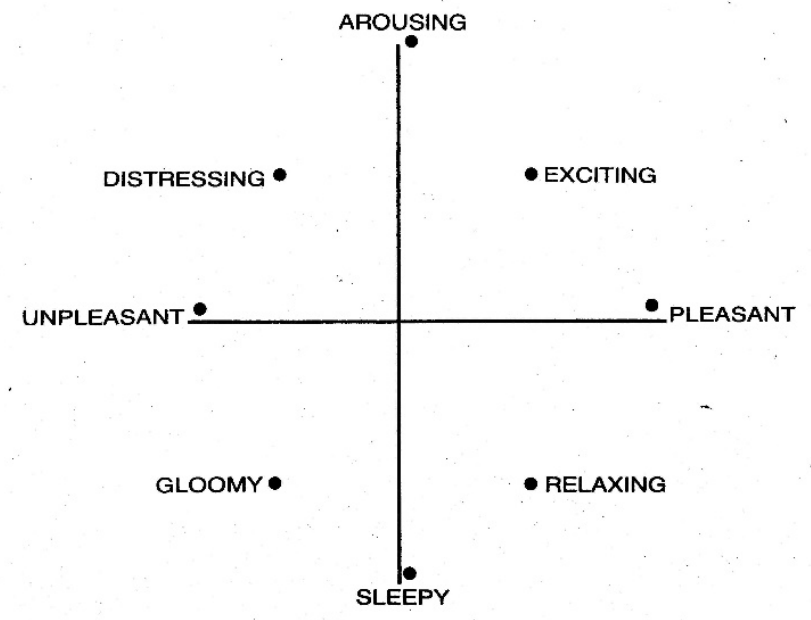

Figure 3.22: Dimensions of environmental affect

Source: Nasar 1998 
Nasar (1994) developed Berlyne's notion of hedonic levels in more detail and arguably in more pragmatic terms when framing recommendations to improve design review processes. He argued that aesthetic response occurs along two dimensions, arousal and pleasantness (figure 3.22). For Nasar, pleasantness is a pure response, corresponding with Berlyne's definitions some years earlier. Excitement and relaxation are two expressions for pleasantness; the first taken in conjunction with a positive level of arousal and the second in conjunction with negative arousal. As shown in Nasar's diagram, this concept assumes that negative arousal levels align with sleepiness. The model is of interest here as it was derived using research on expressions of preference for the built environment and appears to corroborate Berlyne's model derived from psychology.

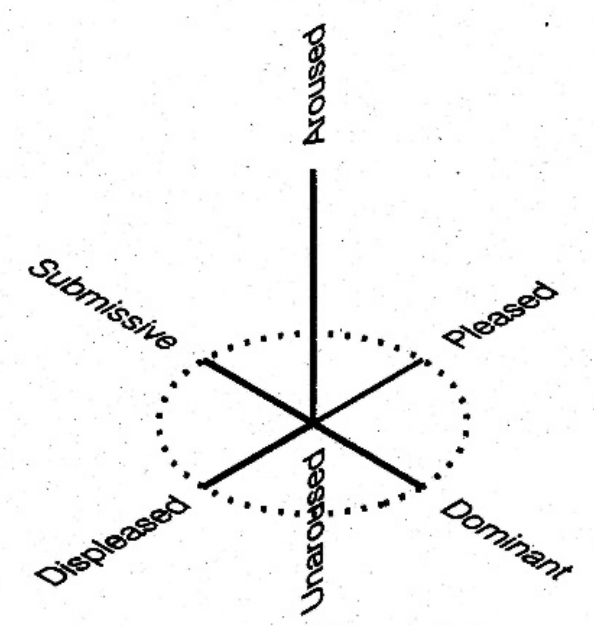

Figure 3.23: Stamps III's model of aesthetic response, differs from Nasar's model by including sensations of submission/dominance arising in the experience of an object or space.

Source: Stamps III 2000

Stamps III (2000) referred to Kant's philosophy on aesthetics to develop a more descriptive model for feelings, the term Kant used to communicate core aesthetic response. Feeling is a vague term because it is general and Kant proceeded to describe this concept in terms of other, more concrete ideas. He considered aesthetic judgements to be centred on feelings of pleasure and displeasure, but because all experience is idiosyncratic he was not able to make any general statements about aesthetics. However, he reasoned that 
underlying all individual experience is the structure of the understanding and the structure of the will. The will and understanding provide the lenses for individual aesthetic experience and Kant argued that when the geometric properties of an object align with the logic of its understanding, then the corresponding pleasure is one of beauty. On the other hand, when an object's geometric properties align with the will, the viewer's experience is sublime (Stamps III 2000: 73-74).

Stamps III developed Kant's descriptions further to create his model for aesthetic response, which is illustrated in figure 3.23. First he established three core dimensions of feelings. Two of these are arousal - corresponding to Kant's idea of understanding and dominance - corresponding to Kant's concept of the will. Stamps III then introduced the dimension of pleasure a term also found in Nasar's model (figure 3.22). While this and other terms are consistent between the models, there are two obvious differences that invite explanation. Firstly, Stamps III's model includes the additional dimension of dominance, consistent with Kant's aesthetic response model. Ascribed to the will, dominance is a relationship between two sources of power, the person and the social or physical environment. Stamps III investigated people's experiences and found that dominance and its corollary submissiveness emerged in their experience of an environment and that this affected their response to it. The second difference is that Stamps III did not consider arousal to have a negative dimension, arguing that lack of arousal is not unarousal but lethargy. In his model the scale for arousal begins at zero.

\subsection{Lay and professional aesthetic preferences}

This research seeks to explore reported differences in the aesthetic preferences of those who participate in the production of built form and those who use it. The broadly held view is that there are differences in the way lay people and design professionals evaluate buildings, based on normative arguments (Case Scheer 1994: 4) as well as on a number of empiric studies (Groat 1994, Stamps III 2000, Pugalis 2009). In his studies comparing aesthetic opinions of planners with those of the lay public, Hubbard $(1994,1997)$ found there to be common lineaments and values shared by the two groups. After noting that design control is often accused of stifling creativity and design excellence, his research found that decisions around design were often influenced by professional interests external to personal response mechanisms. Hubbard concluded by recommending that 
planners become better able to use and rely on their own aesthetic preferences. On the understanding that there are differences in aesthetic perception, Case Scheer (1994) argued that professional views should be privileged when deciding how the built environment is to change.

Despite the strength of evidence for differences, several studies have also found there to be similarities. In an analysis of design review studies undertaken over an eight year period, Stamps III (1994) found better consensus between designers and non-designers in his home town of San Francisco than he had assumed before he undertook the study. Concerned that the results may have been a local phenomenon, he went on to examine similar data taken from other cities around the U.S. and found these to corroborate his earlier findings. Several years later Stamps III (1999) found alignment between the groups when they were asked to evaluate ordinary architecture and natural landscapes, basing his findings on 404 responses collected in three separate studies. However, differences in opinion arose when the stimulus changed to avant-garde forms of architecture. On the strength of these results, Stamps III declared that any differences in opinion between lay people and professionals could be attributed to the nature of the aesthetic stimulus, not demographic differences.

In a study of people's responses to contextual compatibility, Gjerde (2011) found that the views of professional architects and planners were aligned with those of the lay public. While aesthetic judgements aligned significantly in terms of absolute preference (whether or not the streetscape scene was liked), the study also found that professional respondents expressed their opinions more confidently.

Evidence for differences can be seen in the high number of failed projects carried out during the height of the Modern era. The famous Pruitt-Igoe complex in the United States that was ultimately demolished failed because of the differences in aesthetic, as well as social, values between the designers and occupants (Newman 1972 cited Nasar 1988: xxiv). Intuitively, an appreciation gap might arise from a professional education, which has been focussed on speculative aesthetics (Devlin 1990, Hubbard 1994). Studying the development of aesthetic preferences amongst students Wilson (1996) found that those of architecture students progressed toward those of their professional tutors, and away from their student peers in other disciplines, with each year of study. Patterns of 
socialisation of preference were clearly evident, particularly in response to architectural style.

Rapoport (1982) suggested that differences in opinion between lay and professional people could be traced to what each group takes into account when making aesthetic judgements. Lay people react to and evaluate the environment in associational terms; to them meanings are of primary importance. Conversely, designers are known to respond in perceptual terms as their meanings, learned and reinforced by the profession, lie in the physical composition. This may also help explain differences in preference for colour in the built environment. Janssens (2001) found that architects generally prefer colour schemes with higher levels of unity and coherence while lay people prefer those with greater complexity and variation.

The matter is far from clear-cut, as Bentley (1999) conceded that any differences in perception could be traced to the innate need by design professionals to stay in business. He argued that this often causes them to propose and justify designs that are based in their clients' interests of maximising profits rather than by following their own personal or professional aesthetic sensibilities. After consulting the literature comparing professional aesthetic tastes with those of the lay public, the present study has shown considerable misalignment and the topic remains open for further investigation. Given the influence professionally trained designers and planners have on the development of tools for design control and on design review decisions, this matter takes prominence in this investigation of people's aesthetic opinions about urban buildings and streetscapes.

\subsection{Theoretical framework and knowledge gap}

There appears to be a basis for examining people's aesthetic responses to the visual appearance of urban streetscapes. Not only is aesthetics a factor in many of the decisions people make, including for example which street to walk along, but the literature notes that the appearance of the built environment is linked to people's physical, mental and financial well-being. The importance of the appearance of the built environment can be attested by the number of local and central governments that have adopted design control as part of their regulatory planning systems. Governments undertake planning regulation to protect the interests of their citizens, the vast majority of whom have no direct influence on the projects that incrementally change the built environment. It would 
therefore seem appropriate for designers and design reviewers to have knowledge about which building and streetscape design characteristics people find most and least appealing. This knowledge could then be referenced by designers and regulators in order to improve design outcomes.

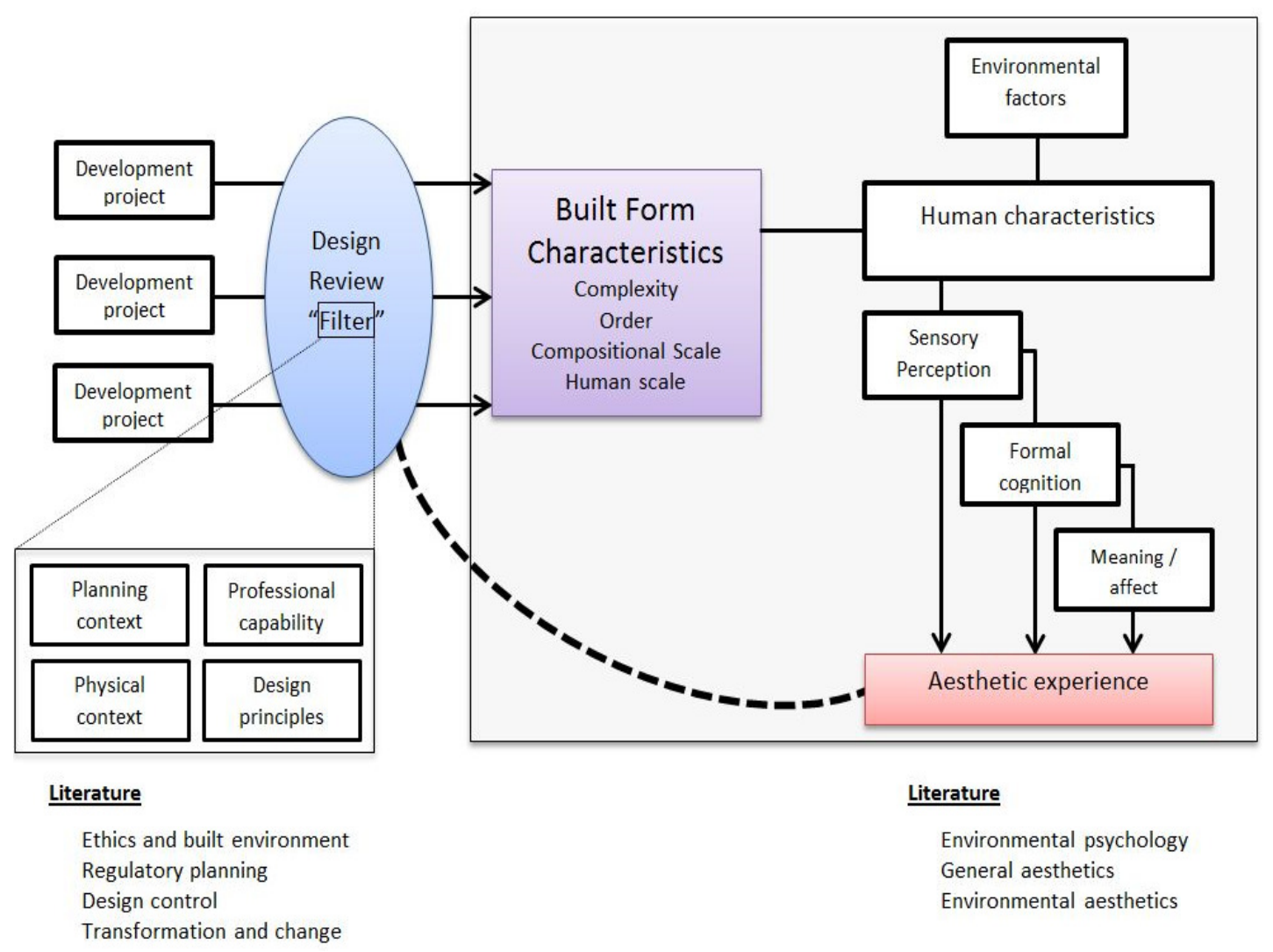

Figure 3.24: Diagram of the theoretical framework for the research. The right side of the diagram draws on (Nasar 1998), (Lang 2003) and (Cuthbert 2006). The literature fields that support each side of the framework are listed.

Having traversed literature in the fields of design review, environmental aesthetics and aesthetic preference studies, a theoretical framework to direct the research effort can be defined (figure 3.24). The left side of the diagram represents how the built environment is created and changed through individual projects. Since the introduction of regulatory planning, each discrete development has been through a design control process. This process will vary between different locations and over time as motivations and capabilities change. 
On the right side of the diagram, literature in the field of environmental aesthetics shows that aesthetic experience is influenced by the characteristics and experiences of the individual as well as environmental conditions, which may vary with season, time of day, levels of vehicular traffic and similar changing conditions (Kaplan 1987, Nasar 1998). Following Smith (2003) and Cuthbert (2006), aesthetic experience has sensory, cognitive and affective dimensions. An individual's response in each of these dimensions is shaped by personal experience and by prevailing social and cultural structures. However, when considering large groups of people, consistencies in aesthetic preference begin to emerge (Berlyne 1974, Stamps III 2000).

The bold dashed line highlights the existing gap in knowledge. This gap was foreshadowed in section 2.13, where the scarcity of evidence based understanding of how members of the general public feel about urban buildings and streetscapes was noted. Such information is important if design control is to advocate for buildings and streetscapes that appeal to the aesthetic sensibilities of all people. In the absence of such information, most guidance is today based on design principles arrived at through speculative aesthetics. In addition, design and design control activities appear to be undertaken by experts (architects, other designers and planners). The literature is inconclusive about whether expert opinions align with the aesthetic opinions of the public (see section 3.10). If the objective of design control is to ensure that development meets with the expectations of the public, how are those expectations made known in the process? This is the key issue for this research.

\subsection{Research questions}

Design control methods are applied inconsistently by local governments in New Zealand. Moreover, there is little empiric evidence on which to base design guidance and control of inner city (re)development. In light of this, the primary question is: what are the

\section{characteristics of well-liked buildings and streetscapes in New Zealand?}

The contextual design characteristics that this research investigates are more pronounced in tightly spaced patterns. A number of earlier aesthetic studies (Nasar, 1998, Groat, 1988) have included landscaped areas between the buildings. Stamps III $(1994,2000)$ and Stamps, Nasar et al. (2005) have carried out a significant number of studies in this area, several of which have been meta-studies combining the findings of research by others. 
These have tended to concentrate on the effects arising from discrete interventions, in the manner of a design review evaluation. In other words these studies have sought mainly to measure the effect created by an addition into an existing setting by focussing on perceptions of that intervention. When the studies ventured into measurement of responses to townscape per se, the scenes were of a residential scale. The present investigation will address a gap in knowledge about the visual effects arising from tightly spaced structures of moderate scale in urban settings, typical of the streetscapes in the towns and cities of New Zealand and, by extension, other new world countries.

A secondary question is: how do the aesthetic preferences of professionally trained architects and planners compare with those of lay members of the public? This question was foreshadowed in section 2.11 in reference to Case Sheer's (1994) four endemic problems for design review. She asked "whose aesthetic tastes should be considered in design review?" This was asked somewhat rhetorically, as she was of the firm view that only those trained in visual design should be invited to review designs for development proposals. Section 3.10 also showed that the literature is inconclusive on whether professional designers hold similar aesthetic views to the public. As the purpose of design control is to create well-liked environments it is also important to ensure that those who make design control decisions are acting in the interests of the public. The second research questions addresses this current gap in knowledge.

\subsection{Conclusion}

This chapter has developed a theoretical framework for examining people's perceptions and preferences for urban buildings and streetscapes. The framework has been developed from consideration of the design control theories and practices discussed in chapter 2 and the general theories of aesthetic perception in chapter 3. The framework diagram has been annotated to identify the gap in knowledge addressed by this research, namely that there is insufficient understanding of people's aesthetic preferences for urban buildings and streetscapes. As a consequence, design control is informed by general design principles that have emerged through literature around speculative aesthetics. As aesthetic preferences for urban buildings and streetscapes are not known it is impossible to gauge whether current design control practices are achieving their aim of creating well-liked urban places. 
The chapter has distinguished between research about aesthetics that is speculative in nature and that which develops empiric evidence using methods consistent with environmental psychology. More than a century of research findings enable the general processes of aesthetic response to be well understood. Responses are generated in different parts of the brain, with the most basic coming through stimulation of the limbic system. The brain then processes the sensory information for conscious and subconscious comparisons with personal, socially and culturally held schemas and value systems. Nasar's (1998) framework describing sensory, connotative and affective levels of environmental perception provides a useful method for first organising the discussion around how perception takes place at each of these levels and then describing built form characteristics that stimulate these processes. This information will be useful during the fieldwork and data analysis stages of the research.

The chapter also discussed several measures of aesthetic preference developed by researchers in the field of environmental aesthetics. Aesthetic responses are generally considered to lie along the axes of pleasure and arousal although Stamps III (2000) has added the dimension of dominance to account for Kant's structure of the human will. Having identified a knowledge gap and described a theoretical framework, this chapter outlined two key research questions to drive the remaining investigation. In chapter 4 a methodology will be developed for pursuing this and answering the research questions. 


\section{Chapter 4}

\section{Research Design}

\subsection{Introduction}

Having developed a theoretical framework with reference to literature in the fields of environmental aesthetics and design review, the thesis now outlines the process that will drive collection and analysis of data in order to answer the research questions. As research is the systematic inquiry of a topic or problem, the research design provides a framework for collection and analysis of data. Bryman (2008: 4) has noted the need to position the research enterprise in its wider theoretical context to facilitate selection of appropriate methods of data collection and make the research relevant.

The first part of this chapter discusses the general research strategy, situating the study within a range of possible approaches. The second part outlines the methods used to collect and analyse the data. Finally, a summary of the research design is presented as the lead-in to chapter 5, where results of a first study of people's aesthetic preferences for streetscapes represented as photographic elevations are discussed.

\subsection{Research Design}

Prior to discussing the methods for collecting and analysing data it is useful to position the research in its broader context. The reference here is to the theories, philosophies and belief systems that help frame a research design. This research adopts and adapts the conceptual framework described by Groat and Wang (2013: 9-13), who suggested that research can be conceived of as a set of nested frames, the outer one being concerned with the researcher's own belief system about the nature of knowledge and how it can be 
apprehended (figure 4.1). The design of this research is discussed following this fourlevel conceptual framework.

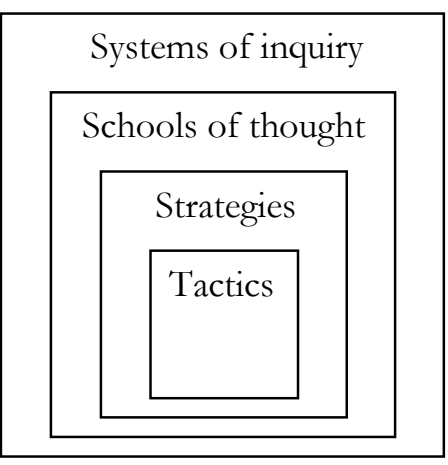

Figure 4.1: The nested levels of a research methodology, linking the strategies and methods to the broader system of inquiry.

Source: Author, after Groat and Wang (2013)

\subsubsection{System of inquiry}

The social world, which is the domain of this research, is a complex and varied human construct. There appear to be two principal schools of thought on the nature of knowledge about the social world. One sees humans and their institutions as fundamentally different to the natural world, with a consequential view that using scientific methods to learn about the social world is inappropriate. Interpretivism invites the social scientist to understand human behaviour empathetically rather than try to explain it (Bryman 2008: 15). One of the best known interpretivist traditions is phenomenology. The philosophical basis for phenomenology is that there is no objective world separate to ourselves (Barnacle 2001: vi-viii, Carmona, Heath et al. 2010: 120). Instead, the environment is whatever the individual experiences it to be, and stems from the individual's subjective construction of it (Seamon 2000). Taking a phenomenological approach, the social scientist aims to "gain access to people's common sense thinking" in order to interpret their actions and social world from their point of view (Bryman 2008: 16). Because its principal source of information is people's own experiences, it is subject to criticism on the grounds of subjectivity (Groat and Wang 2002: 95). Dovey (1991: 44) argued that the usefulness of a phenomenological approach for studying physical environments is limited because it disregards the influences that social structure and ideology may have on people's day-to-day experiences. 
The second social research school of thought is positivism. A positivist epistemological position, along with the closely aligned realist approach, advocates for the application of natural sciences methods to create knowledge about the social world. Bryman (2008: 13) identified five principles of positivism, the most important of which is that true knowledge can only be based on phenomena that can be confirmed by the senses. In other words, normative statements about reality are not acceptable from a positivist perspective; there must be evidence to support the laws that describe how the social world operates. The five principles Bryman ascribes to positivism are:

1. Knowledge can only derive through phenomena that can be apprehended by the senses

2. The purpose of theory is to generate hypotheses that can be tested, which in turn enable these explanations to be tested

3. Knowledge is arrived at through the gathering of facts

4. Science must and can be conducted in ways that are value free

5. Normative statements and scientific statements are distinct from one another. Scientific statements are the domain of the true scientist. This is because normative statements are unable to be confirmed as true (or otherwise) by the senses.

The realist approach shares with positivism a need to explain the world but goes on to envisage a reality that can exist on a number of levels.

The present research sits within the realist reference approach and adopts an objectivist ontological perspective. Accordingly, the research is framed in a system of inquiry that considers culture and society, the social world, to be entities that are separate from the individual actors.

\subsubsection{Field of inquiry}

Nested within the systems of inquiry frame, the next frame of reference situates the research within one or more discipline areas. This research sits in the field of environmentbehaviour studies, which broadly speaking, seeks to illuminate relationships between people, their behaviour and the physical world (Proshansky 1990). The field is multidisciplinary and involves theories from the fields of environmental psychology, architecture, planning and urban design (Portella 2007: 152). 
Although most of the disciplines that contribute to this broad field have been established for some time, environment-behaviour study is itself relatively young, having emerged in the 1960s and 70s in the context of political and social upheaval. This was a period when societies around the world, galvanised by a growing recognition of social and environmental problems arising from human development and the unequal distribution of wealth and resources, began to question the status quo. People with an interest in the built environment and with growing awareness of the social and psychological dimensions implicit in the architectural endeavour, turned to the behavioural sciences for answers (Holahan 1978: 5). There were few answers to be found in the knowledge existing at the time, as behavioural scientists and psychologists were more interested in studying relationships between people and social environments or people's responses to extreme environmental conditions.

As the field began to develop, new ways of studying human-environment interaction were also needed. Up until then the methods had been largely static and linear and were unable to cope with the increasing complexity that researchers encountered (Holahan 1978: 1011, Proshansky 1990: 22-23). The traditional focus of psychologists, which was on people's needs in a particular setting, did not account for the mutual and reciprocal relationship that takes place between people and the places they inhabit. Furthermore, Proshansky noted that these interactions appear to take place at three distinct scales or levels, a point that had not been recognised by earlier researchers. He described the fundamental relationship as the one between the individual person and his/her environment. Beyond this he noted that relationships could be classified at the level of the small group and its environment and also at a third level, which is the interactions that take place between large groups and the spaces they occupy. Recognising the changing nature of environment-behaviour research, Altman and Christensen (1990: 2) noted that the diversity of people now pursuing this type of research have brought a healthy openness to new directions.

Although people experience the world through all five senses, vision is the primary method of knowing the environment (Cullen 1971: 8). More than $80 \%$ of sensory input is visual (Porteous 1996: 31), to the point that the term perception is often used when referring to visual perception. Vision is a highly complex phenomenon and is not just pictorial in nature but is active and searching. That is to say, we seek out views with 
roving eyes and make conscious effort to focus on certain (pleasurable) scenes, whereas sounds and smells come to us indiscriminately (Rapoport 1977: 208). While this researcher accepts that aesthetic experience is multi-sensory (Lynch 1960: 3, Scruton 1979: 72, Porteous 1996: 31, Carmona, Heath et al. 2010: 111), the aim is to identify preferred forms and spatial characteristics in urban settings. In an effort to focus the research and limit its scope, this study addresses the visual components of aesthetic experience.

\subsubsection{Research strategy}

Having situated the inquiry within an overall philosophical frame and briefly described the field of inquiry that will provide the theories to both inform the research and be subjected to examination through the findings, it is then possible to develop the strategy and to design the various tactics, or research methods, that can be deployed to address the research questions (Groat and Wang 2002: 87).

As noted (see section 4.2.1) the frame of reference in this investigation is objective realism. Accordingly, the view is that it is possible to create knowledge about the social world through methods similar to those used to investigate the natural sciences. Adopting this perspective, society is considered an entity distinct from any individual actor. Adopting an objective stance enables dispassionate engagement with society in the effort to answer the research questions.

Bryman (2008: 21-24) suggested that research strategies can be clustered under two distinct headings based on the ways each considers the relationship between research and theory, and on the ontological and epistemological considerations of each. The distinctions between quantitative and qualitative research strategies in social science research are articulated in a dichotomous comparison in table 4.1. While adoption of a realist system of inquiry would typically lead to a quantitative research strategy, the questions that drive the present research invite consideration of alternative theories, in addition to developing findings that can refine existing theories.

Distinctions between quantitative and qualitative research strategies are useful in terms of understanding their key characteristics. However, Bryman (2008: 23) cautioned against driving a wedge between them. He argued that distinctions are not hard and fast and 
cited examples of research strategies that have the characteristics of both. He also noted that methods that adhere to each strategic approach can be effectively combined.

Table 4.1: Key considerations of quantitative and qualitative research paradigms.

Adapted from Groat \& Wang (2013: 71) \& Bryman (2008: 22)

\begin{tabular}{|l|l|l|}
\hline \multicolumn{1}{|c|}{ Question } & Quantitative paradigm & Qualitative paradigm \\
\hline $\begin{array}{l}\text { Ontology: what is the nature } \\
\text { of reality? }\end{array}$ & $\begin{array}{l}\text { Reality is objective and } \\
\text { singular }\end{array}$ & $\begin{array}{l}\text { Reality is subjective and } \\
\text { multiple, as witnessed by } \\
\text { the study participants }\end{array}$ \\
\hline $\begin{array}{l}\text { Epistemology: what is the } \\
\text { relationship of the researcher } \\
\text { to that being researched? }\end{array}$ & $\begin{array}{l}\text { Researcher is } \\
\text { independent from that } \\
\text { being researched }\end{array}$ & $\begin{array}{l}\text { Researcher interacts with } \\
\text { that being researched }\end{array}$ \\
\hline $\begin{array}{l}\text { Role of theory in relation to } \\
\text { research }\end{array}$ & $\begin{array}{l}\text { Deductive: cause and } \\
\text { effect } \\
\text { Testing theory }\end{array}$ & $\begin{array}{l}\text { Inductive: mutual } \\
\text { simultaneous shaping of } \\
\text { factors } \\
\text { Generating theory }\end{array}$ \\
\hline
\end{tabular}

A mixed method approach adopts quantitative and qualitative research methods. The use of multiple methods creates opportunities to triangulate the data, a characteristic that can help overcome the weaknesses of each methodological approach by utilising the strengths of the other (Jick 2006). Some social researchers argue that quantitative and qualitative research methods belong to two separate and incompatible research paradigms (Bryman 2008: 604). An example of this is the view that participant observation methods are linked closely to an interpretivist epistemological position and that these are consequently incompatible with a positivist position. While other researchers acknowledge that quantitative and qualitative methods are closely linked to epistemological positons, they also consider the methods to have degrees of autonomy meaning they are therefore able to be pressed into the service of the other (Bryman 2008: 606).

Groat and Wang (2013: 71) distinguish between a truly quantitative approach, which employs experimental methods to isolate variables of interest by way of experimentation, and correlation. Correlation approaches recognise that patterns of subjective preference may not necessarily be explained causally and instead seek to identify the strength of tendencies. As a supplement to methods that enable quantitative or correlation analyses, qualitative methods can generate non-measurable data to enrich and help reinforce the 
research findings (Portella 2014: 132). Use of the two methods in this research acknowledges the complexity of aesthetic perception at a social scale and the difficulty of capturing the full range of meanings derived from the built environment using any one method.

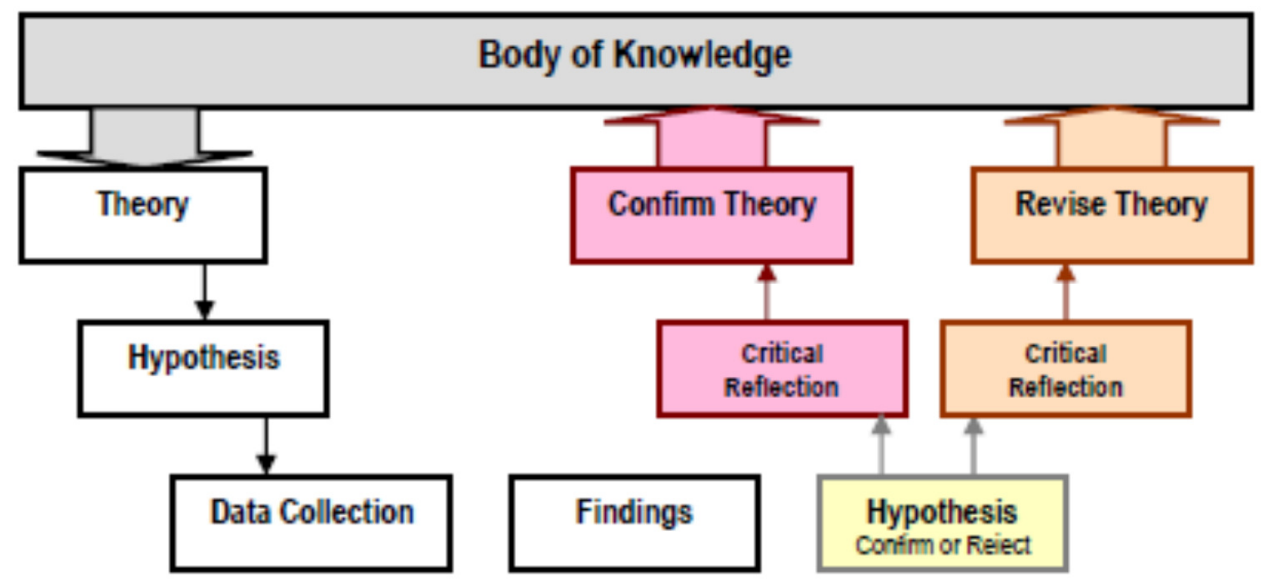

Figure 4.2: The deductive research process relies on induction in order to critique the theories that have informed the hypothesis and collection of data.

Source: author after Bryman 2008

This research seeks to answer the research questions by way of a deductive process (figure 4.2). The theoretical stance, developed from the literature review, is discussed in Chapters 2 and 3. Rather than simply confirming or rejecting the hypotheses, deduction invites researchers to return to the theory to revise, refine and help explain. In this sense, the deductive process also allows for induction, a useful quality in terms of the adopted system of inquiry.

According to Zeisel (2006: 92-94), if the aim of the research is to understand (as opposed to refine a particular theory) then the investigator can choose between diagnostic studies, characterised by breadth of study and favouring precision, and descriptive studies, which attempt to measure one or more characteristics of interest precisely and then reflect on how these relate to the question. Although the present study is guided by a theoretical framework, the necessarily broad topic of people's aesthetic responses to urban streetscapes and the decision to make use of real environments for the fieldwork (see section 4.4) together mean the research does not facilitate the extent of experimentation necessary to be considered descriptive in Zeisel's terms. Accordingly, a diagnostic study 
is contemplated in order to develop "insight into the structure and dynamics of the whole situation, possibly also setting the scene for further research" (Zeisel 2006: 93). Future research in this area could delve more into theory testing.

\subsubsection{Tactics or research methods}

It is useful to break down the research question and formulate objectives around which a plan of work can be developed (Portella 2007). The overall aim of the research, in terms of the question, is to identify the design characteristics of individual buildings and of urban streetscapes in New Zealand that are well-liked and the converse. This information could then be used to influence design control tools and methods in order to achieve better liked buildings and streetscapes. To achieve this aim a number of focussed objectives are targeted, as set out in table 4.2, where they are aligned with the research method(s) that are used. Taken together, this outlines the methodology for the research. The methods are each described in the sections that follow. The final stage of the research design is to develop the methods by which data can be collected for analysis. The plan of work falls neatly into three stages corresponding to

1. development of the theoretical framework

2. development and application of the methods to generate data

3. analysis of the data in the context of the research questions

Chapters 2 and 3 reviewed relevant literature in the field leading to formation of the theoretical framework, corresponding with the first stage of the methodology. The knowledge gained through the literature enables the research tools to be authoritative and their application effective. However, the existing body of knowledge also infuses the whole of the project, enriching the interpretation of data. Ultimately, the body of knowledge will be extended by the findings of this research (Groat and Wang 2002: 47).

The tactics are organised around two discrete studies. Study One principally employs correlational (quantitative) methods to gain insight into people's preferences for streetscape characteristics, using photographs to stimulate the responses. This study aims to attract a relatively high number of respondents, which should add to the robustness of the findings. Study One aims to probe people's broad preferences for the relationships between buildings within a street, which in addition to forming useful findings will inform development of Study Two. 
Table 4.2: Research methodology, linking research methods to research objectives

\begin{tabular}{|c|c|}
\hline \multicolumn{2}{|c|}{$\begin{array}{l}\text { Research aim: } \\
\text { To identify the design characteristics of individual buildings and of urban streetscapes } \\
\text { in New Zealand that are well liked and those that are not well liked. }\end{array}$} \\
\hline Research objective & Research method \\
\hline $\begin{array}{l}\text { Development of a theoretical framework that can } \\
\text { support the breadth and depth of the research, taking } \\
\text { account of: } \\
\text { i. Factors that affect the way urban streetscapes } \\
\text { are produced and change over time } \\
\text { ii. Factors that affect aesthetic perception of the } \\
\text { built environment. }\end{array}$ & Literature review \\
\hline $\begin{array}{l}\text { Understand preferences for different design } \\
\text { characteristics observed in individual buildings and in } \\
\text { streetscapes comprising individual buildings }\end{array}$ & $\begin{array}{l}\text { Study One collects quantitative } \\
\text { data in relation to photographic }\end{array}$ \\
\hline $\begin{array}{l}\text { Understand differences, if any, between aesthetic } \\
\text { preferences expressed by professionals and lay } \\
\text { people. }\end{array}$ & $\begin{array}{l}\text { representation of streetscapes } \\
\text { Study Two collects quantitative } \\
\text { and qualitative data on basis of }\end{array}$ \\
\hline $\begin{array}{l}\text { Identify differences between preferences expressed } \\
\text { in relation to photographic representation and real } \\
\text { life experience }\end{array}$ & $\begin{array}{l}\text { in-situ experience of } \\
\text { streetscapes }\end{array}$ \\
\hline
\end{tabular}

Study Two aims to understand people's aesthetic preferences for urban streetscapes based on their experiences of them (Zeisel 2006: 104). Acknowledging the impossibility of studying society as a whole, even one as small as New Zealand, the case study can create a point from which to draw broader conclusions about the preferences of the wider population. May (2011: 221) notes that the case study offers a "concentration of the global in the local". In the context of the research aims, the question then turns to whether a single case study can cover people's responses to the wide range of architectural design characteristics seen in contemporary cities. As an alternative, multiple case studies can generate more robust data and consequently more compelling findings (Yin 1989: 52). Recognising the potential value of this approach, Study Two is developed around three separate urban places. Yin also felt the need to warn researchers who adopt multiple cases about the collected data being suitable only for within-case analysis. As each case is 
unique, they cannot be combined in an effort to generate larger numbers of data for comprehensive analysis. Instead, each part of a multiple case study should be conducted and analysed independently before a cross-case analysis is carried out to illuminate higher level, generalisable findings (Groat and Wang 2013: 430). The manner in which the cases are replicated is also critical.

The two classifications of case replication are theoretical and literal. Theoretical replications are those where differences between the cases can lead to contrary results for predictable reasons (Yin 1989: 59). If cross-case analysis can link differences in people's responses to the differences between the cases, this understanding can be used to identify design characteristics that are critical for people's perceptions. On the other hand, where perceptions are consistent across the cases despite clear differences between them, this can lead to discovery or confirmation of high-level principles of perception.

Literal replications are those where the cases are similar, leading the researcher to expect that the results will also be similar. The present research evaluates the responses people have to urban streetscapes, no two of which are identical. Accordingly, replication between cases is theoretical. Nevertheless, in order to minimise the chance that peripheral issues might influence people's responses and cloud the analysis, the cases have been carefully selected. The cross-case analysis should also consider the extent to which the characteristics of each differ, including the influence any peripheral issues may have had.

An underlying aim of the research is to enable the findings to be used for further comparison of different design control methods used in New Zealand. Accordingly, the three cases have been selected to represent different design review processes.

\subsection{Study One}

Study One was designed to gauge people's general preferences for the visual characteristics of different urban street scenes. A secondary aim was to look for differences between the perceptions of those trained in design and planning and the lay public, who are not. In addition, comparing the results of Studies One and Two would enable comparisons between preferences based on photographic representation and insitu experience of urban streetscapes to be made. 
An earlier study undertaken by the author in the United Kingdom sought answers to similar questions (Gjerde 2011). While the methods of that research are relevant here, the findings are not transferrable as a considerable dimension of environmental perceptions is socially and culturally based (Rapoport 1982, Portella 2014: 19). Despite a shared heritage, the cultures of the United Kingdom and New Zealand have developed independently over the past 175 years. It is likely that the broadly held perceptions of people in each country differ. The methods used to discover the perceptions of participants in Study One are summarised below.

\subsubsection{Street scene selection}

As is noted in the development of the research question (see section 3.12) there is a gap in knowledge about how people evaluate collections of tightly spaced buildings in urban streets, typical of the centres of cities and towns around the world. Of interest here is not only how people respond to streetscapes but how they justify why they like some and not others. The aim is to understand the streetscape characteristics that are well-liked and those that people dislike. Actual streetscapes were selected and represented in photographic elevations to present the survey respondents with six different typologies of streetscape condition and inter-building relationships.

As discussed at section 3.7, the perceptions of complexity are a key factor in people's aesthetic evaluations. Stamps III (2000) outlined the three scales at which people perceive complexity in the built environment, which correspond to the outline or silhouette, the manner in which the forms are modelled in three dimensions, and the patterns and textures in the surfaces. Complexity and its corollary, visual order, thus guided the search for appropriate street scenes. Along with variations in these two perceptual dimensions, there also needed to be differences in scale and apparent maintenance, two other key factors that influence aesthetic perception. Having regard for the matters of complexity and order, and using experience as a design reviewer, the following six conditions were established.

1. High levels of variation in silhouette, form and articulation. This would be seen in a street that had experienced change over an extended period.

2. Consistent silhouette and 3D form with some variations in surface articulation. This would occur where there had been some control over development that had occurred over an extended period. 
3. Variations in silhouette and 3D modelling with some consistencies in surface articulation.

4. Large scale, single redevelopment of an entire block where there had been an attempt to create variety at one or more perceptual level

5. Large scale, contemporary building forms with wide variety at all three form complexity levels. In effect, this scenario would reflect the current development paradigm.

6. A highly ordered collection of buildings, perhaps reflecting high levels of design control.

The six conditions informed a search for street scenes that could be presented as photographs to survey respondents. Using existing street scenes could facilitate future longitudinal studies of the scenes as they change over time. The alternative approach of fabricating street scenes, using images of buildings from different settings stitched together by computer software, was contemplated but disregarded for lack of relevance in future work.

\subsubsection{Street scene representation}

While acknowledging that experience of the world is three dimensional, it was decided to present the streetscapes as photographic elevations. This would enable individual buildings to be represented fairly in terms of the space each occupies, without the biases created by perspective viewing angle. Moreover, as elevations are used to communicate the characteristics of a proposed development during design control it was reasoned this could help align this part of the research with future crossovers into practice.

A wide search was needed to find street scenes that could portray each of the six scenarios described in section 4.3.1, with streets from four cities of northern Europe - three British and one Norwegian - selected. As the emphasis in Study One was on the broader streetscape characteristics it was important to present people with greater variety than currently found in New Zealand. 

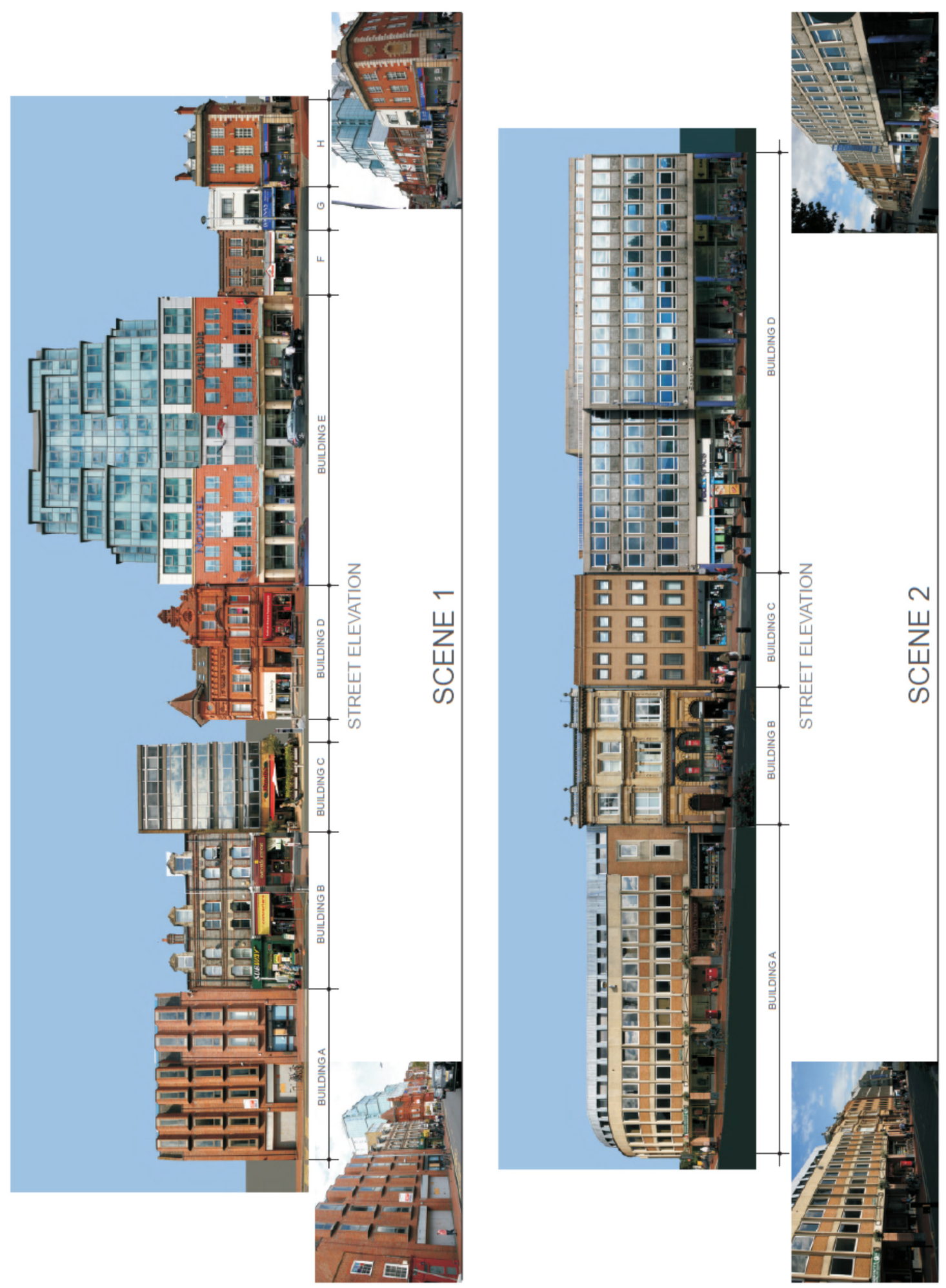

Figure 4.3: : Street scenes 1 and 2 from Study One represented as photographic elevations, supplemented by street level photographs taken from each end of the street

Source: Author 
An appropriate way of presenting the street scenes as photographic elevations was required. An earlier study by Portella (2007) described a technique of presenting streetscape elevations using Adobe Photoshop software, and this was adopted. Two key features of the process are that perspective can be taken out of each image and that many individual images can be stitched together into a comprehensive elevation of all buildings along a single side of the street. However, while the principal presentation of each scene was a photographic elevation, perspective photographs taken at each end of the street at a natural eye level were also included to provide supplementary information about the three dimensional characteristics of the buildings. An example of the images presented is shown in figure 4.3.

The decision to stage the first study using photographs was based on compelling evidence presented by Nasar (1988), Stamps III (1990), Sanoff (1991), and Stamps III (2000) concerning the effectiveness of colour photographic media in environment-behaviour studies. For example, Portella (2007: 177) stated that "user perception and evaluation of streetscapes observed through slides and coloured photographs are very similar to user perception and evaluation of the same streetscapes observed on-site". Stamps III (2000) confronted concerns about the validity of photographic montages, demonstrating experimentally that they serve as excellent predictors of preference. However he went on to caution that preferences given in relation to different media cannot be directly compared. As representation and actual experience are both used in this research, the findings raise discussion on the validity of using photographic representation to stimulate people's environmental aesthetic responses.

\subsubsection{Survey questionnaire design}

Study One pursues a quantitative research strategy. To generate the desired data, a selfcomplete questionnaire was developed. This asked people to give their opinions on the different characteristics of the six selected street scenes. The questions derive from the theoretical framework for environmental aesthetics discussed in section 3.11. The survey is classified as attitudinal (May 2011: 94-95), as the research seeks to understand subjective responses to the built environment, and a five step Likert response scale incorporating the dimensions of aesthetic evaluation was used.

The survey asked people to describe their feelings for different characteristics and relationships between buildings in each scene. Working with concepts outlined by 
Immanuel Kant, Stamps III (2000) described the three core dimensions of aesthetic response as arousal, dominance and pleasure (see section 3.9). According to Stamps III (2000: 80) aesthetic pleasure incorporates positive pleasure with positive feelings of arousal and/or of dominance. For example, a pure, disinterested aesthetic response is one in which the person is both pleased and aroused but feels neither dominant over nor submissive to the environment in the process. Such a response might arise when viewing high art in a public gallery or museum. Extending this, a feeling incorporates all three sensations of pleasure, arousal and dominance. Adopting the terminology of Stamps III, this research uses the term feeling to describe the preference response sensation.

Referring respondents specifically to the pleasure dimension, the survey asked them to rate the visual pleasantness of a scene on a five-point Likert scale. The same response scale was also used to record people's feelings about the height, architectural style, materials and colour relationships in each scene. A final question was open-ended, inviting people to indicate which, if any, of the buildings in the scene did not fit with the others and to articulate the reasons for their opinions. The Likert scale provided inherent coding of the answers, facilitating entry into the SPSS version 21 statistical analysis software. The pro forma list of survey questions that was tailored to each of the scenes is included in Appendix A.

\subsubsection{Sampling}

A key focus of the research is to understand the aesthetic preferences of the public. As such, administration of the survey sought to attract responses from a representative cross section of the wider population, all drawn from the Wellington region for convenience. As the research also aims to compare the preferences of expert designers and planners with those of the lay public it was important to attract useful representation of each group. To address these aims, invitations to participate in the survey were made in public places known to attract members of the public with varied backgrounds, as well as in places with high proportions of planning and urban design professionals. The invitation was made through a display of the six street scenes printed at A1 and arranged on a pinboard behind a table. The table had supplies of the survey forms, pencils and instructions on how to complete them, and a box for the completed forms.

This display was placed in the Wellington and Lower Hutt city libraries for 10 day periods, because libraries were thought to attract people with a range of ages and backgrounds. 
Libraries are also destinations for people who intend to spend time there. Each library provided a central, highly visible location for the display. In order to attract professional experts to participate the display was also placed in the planning departments of Wellington and Lower Hutt Cities and the central atrium space of Victoria University of Wellington Te Aro Campus. An incentive was also offered as participants were invited to enter a draw for an $\$ 80.00$ meal voucher at a local restaurant. The findings of this stage of the research are discussed in chapter 5.

\subsection{Study Two}

The first study was designed to provide insight to New Zealanders' preferences for the relationships outlined in section 4.3 .1 as represented in the selected static streetscapes. The results were also used to inform selection of New Zealand street scenes for Study Two, with the intention of obtaining more detailed information about people's preferences for relationships between individual buildings in a street and for the overall streetscape.

To fully appreciate a physical setting it must be experienced dynamically (Scruton 1979: 96), an idea eloquently captured in Gordon Cullen's classic text Townscape (Cullen 1971). Cullen's arguments were that visual pleasure is enhanced through drama created by juxtaposition of forms and space, revealed through movement. Not only is the city three dimensional in form, but views and experiences of the setting unfold kinetically. Sharp (1968: 43) observed that movement causes buildings and other elements of the townscape to change in their internal relationships as well as in relationship to one another.

The relatively permissive planning rules common to New Zealand cities do not discourage misalignment of heights between neighbouring buildings. It is therefore common to have highly articulated silhouettes in New Zealand streets and it can be argued that three dimensional views of the buildings are the only way to gain full understanding of these relationships. It was, therefore, considered appropriate to design Study Two around people's experiences of streetscapes. Study Two also provided the opportunity to comment on, if not directly compare, responses made in relation to photographic media and for real life streetscapes. 
As discussed in section 3.10, the literature is inconclusive over whether professional aesthetic tastes align with the preferences expressed by those not trained in design or urban planning. This issue invites further investigation and, moreover, none of the earlier studies in this area had been undertaken in New Zealand. Study Two aims to fill this knowledge gap by specifically targeting members of both groups as participants. Not only does this enable direct comparisons within the second study but comparisons could also be made with the findings of Study One, where opinions were based on photographic representations. It has been suggested that viewing a picture or photograph enables people to study it and to adopt a detached viewpoint. Detachment is in turn closely associated with evaluative processes prevalent in Western cultures. Heft and Nasar (2000) discussed this phenomenon as a possible explanation for why static images were preferred over dynamic displays of the built environment in their study of aesthetic preferences. Can a person who is trained in design or urban planning enhance their ability to read the static image? If so, does this in turn lead to higher preference ratings being given to the static images?

In summary then, Study Two was designed, using knowledge gained in the first study, to enable more detailed understanding of how people evaluate typical New Zealand urban streetscapes. Study Two also examined similarities and differences in the aesthetic preferences expressed by professionals and lay people. Comparing the results of the two independent studies enabled further discussion around the effectiveness of photographic representation of street scenes as a means of soliciting people's aesthetic preferences.

\subsubsection{Case selection}

Study Two draws findings on the basis of three independent cases. The merits of a multiple case study research method have been discussed in section 4.2.4. Multiple cases enable a broad range of streetscape relationships to be considered in the overall project. Comparison of results across the cases can help highlight the streetscape characteristics that have broad appeal to people and those that are consistently disliked. This can in turn provide the platform on which theories relating to streetscape preferences and design control can be built or modified (Bryman 2008: 60).

Using multiple case studies would also enable future comparisons between the outcomes of different planning and design control methods. Since 1994, Wellington has made extensive use of design review when determining an application for planning permission 
for sites in the central city area (Wellington City Council 2012). Design review there is carried out by experts informed by a design guide while the form of development in terms of height and setback from boundaries is also influenced by zoning rules. New development is controlled differently in Auckland, where it is largely evaluated in relation to rules attached to zoning controls (Auckland Council 2004: Pt 6). With one exception in the city's central area, the appearance of new buildings has not been controlled beyond the rules addressing building height, floor area ratio (FAR) and location on the site ${ }^{1}$. The exception was created with the introduction of a design guide for the Britomart redevelopment area, near Auckland's waterfront (Auckland City Council 2006). Design review of new development in this precinct is carried out by experts in the process of determining the planning application. Driven by an aim of developing three case studies around streets that had developed under different design control methods, the Wellington and Auckland CBDs were identified and the search for suitable streets in these begun.

Several other criteria narrowed the number of potential sites. Environmental noise and intrusion from vehicular traffic, weather conditions, time of year/day and the patterns of social usage can influence people's perceptions (Nasar 1989). As vehicular traffic in particular can have a negative influence on people's perceptions of the environment streets with relatively low traffic volumes were sought. On the other hand, it was felt that fully pedestrianised streets would not be representative of urban streets in the country. New Zealand has few pedestrianised streets and those that are closed to vehicular traffic have had special treatment in their settings.

Streetscapes with numbers of older and/or heritage buildings were also avoided as people tend to prefer such buildings (Carmona, Heath et al. 2010: 245). The focus of this research is the ordinary and contemporary forms of building that form the majority experience in New Zealand cities. Street orientation was considered a relevant factor in order to ensure that different shading patterns could not be cited as a factor in people's opinions. Similarity of grid orientations in the two CBDs being considered meant that

\footnotetext{
${ }^{1}$ Since 2003 Auckland has operated a multi-disciplinary urban design panel that advises on projects in the central area. The urban design panel is only invited to comment on large, complex or significant projects and the scope of its review is not limited to District Plan matters. The Panel's recommendations sit outside the statutory planning process and are only advisory (Auckland Council (2012). Terms of reference: Auckland urban design panel. Environmental Strategy and Policy. Auckland, Auckland Council: 19p.). While acknowledging the existence and influence of the Panel on some projects, the majority of projects do not come under its scrutiny.
} 
satisfying this criterion was not difficult. It was also important to select cases in locations that had recently seen relatively high levels of development activity and that would be readily accessible. After giving consideration to these key criteria three case study sites were selected. College Street is in the Te Aro area of Wellington, Tyler Street is in the Britomart Precinct of Auckland and the third site comprises two adjacent streets in Auckland, Kingston and Wyndham Streets. The characteristics of each is discussed in the opening paragraphs of chapters $6-8$, where the findings of Study Two are presented.

\subsubsection{Survey Questionnaire}

Investigating people's perceptions of the built form characteristics of buildings lining both sides of the selected streets needed a suitable instrument to gauge preferences. One option considered was the guided sensory walk, which Sanoff (1991: 117-118) described as a way of gauging people's impressions of townscape characteristics. This can generate rich qualitative data in the form of people's sensory responses during the walk as well as quantitative data by administering a coded survey simultaneously or soon after the event. However, this method was discarded due to the relatively low numbers of respondents it was likely to generate. Burns (1997) noted difficulties in finding people willing to commit the necessary time to his guided walks in Oxford, UK. As this research seeks to generate sufficient response numbers for data analysis using statistical software, the decision was made to give respondents a survey for them to complete at each of the case study sites. Self-complete questionnaires are a convenient and effective method of procuring

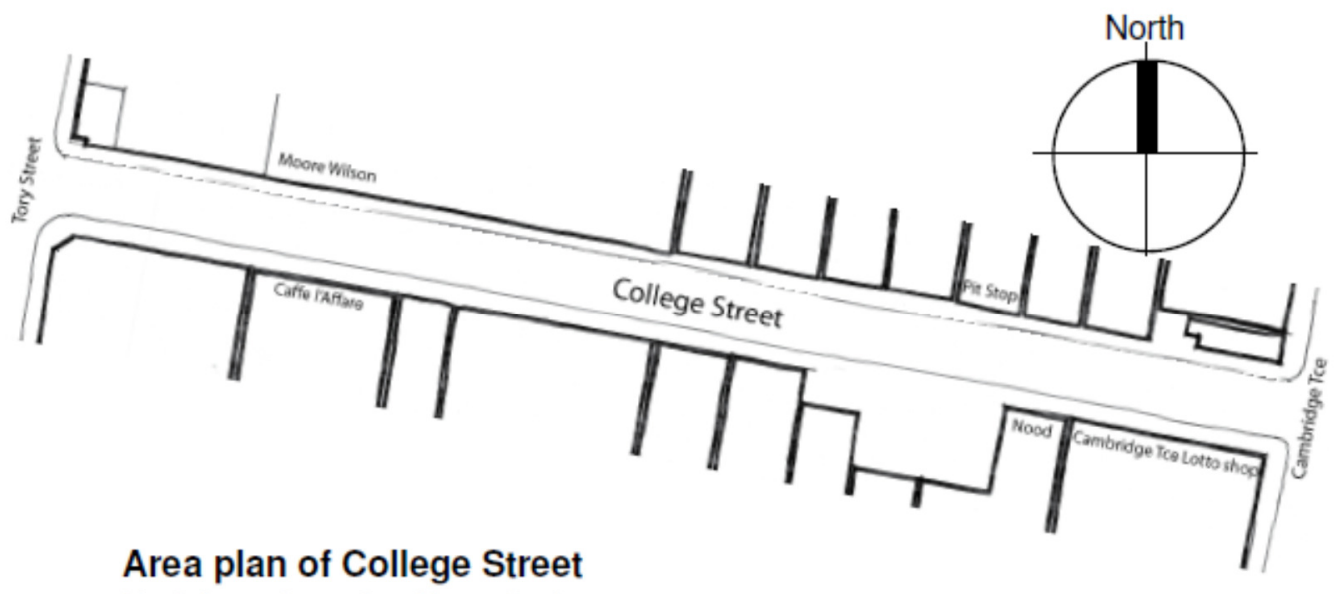

For information only Not to Scale

Figure 4.4: Example of plan provided in the survey form to guide respondents. This plan is for the College Street Case study.

Source: Author 
comparative quantitative data in the social sciences field (Bryman 2008: 195 \& 217), and a higher response rate might be achieved if people were allowed to complete the survey in their own time. The potential for interviewer bias that might affect the validity of data collected through direct contact and discussion is also minimised when the self-complete questionnaire is used (Bryman 2008: 218).

The theoretical framework provided a basis for constructing the questionnaire. As the survey seeks to understand subjective responses to the built environment, a five step Likert response scale incorporating the dimensions of aesthetic evaluation outlined in section 3.9 was used. The aim was for people to give their responses based on their feelings about the buildings while in the street space. To guide them a map was provided and the survey questions were organised around a progressive walk along both sides of the street (figure 4.4). As further guidance, simple drawings were provided of each building relevant to the questions, and of the overall streetscape. The aim was to provide sufficient information to enable respondents to navigate their way along the street but not so much information that it would influence responses.

Respondents were also asked the reasons for some of their aesthetic judgements. For individual buildings, a list of 11 possible reasons was prepared to aid time efficiency and enable quantitative analysis (figure 4.5). Coding of this aspect of the Study One survey had been difficult as people used a range of terms to describe reasons for their opinions. Nevertheless, an open ended question was left for respondents to complete in relation to overall streetscape. The rationale for this was to not limit the possible motivations people might like to cite for this broader subject. The pro forma questionnaire for each of the three cases is in Appendix B. 


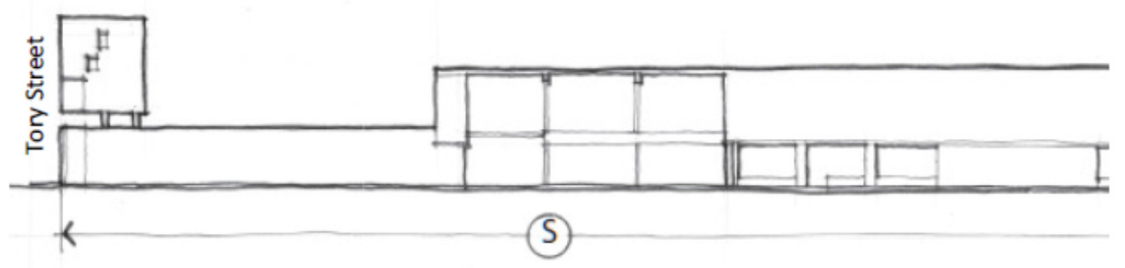

Building S - North Side

18.1

$\begin{aligned} & \text { How do you feel about the relationship } \\ & \text { between Building S and the one to its } \\ & \text { right? }\end{aligned}$
$\begin{aligned} & \text { 18.3 } \\ & \text { How do you feel about the façade } \\ & \text { shape[s] above ground floor level? }\end{aligned}$
$\begin{aligned} & \text { How do you feel about the surface fin- } \\ & \text { ishes of the façade above ground floor } \\ & \text { level? }\end{aligned}$

Figure 4.5: Example of the survey form for one building in the College Street case study. The full survey for each case is included in Appendix B

Source: Author

\subsubsection{Pilot of the survey methods}

A pilot of the proposed method was launched approximately one month before the full fieldwork was scheduled to commence. The purpose of a pilot study is to test the methods for suitability, including testing whether the data format would lend itself to analysis in the context of the research question (Yin 1989: 59). The target group for the pilot study was a selection of staff and students at the VUW School of Architecture. Four 
members of the administration team were contacted by direct email. Approximately 140 students studying urban design theory and practice were invited by way of a message posted to the digital teaching platform for the course. The pilot attracted 14 responses after 30 survey forms were issued. The $47 \%$ response rate was promising.

The pilot was conducted on the College Street case study area. Of particular interest was to learn whether respondents understood that they were to evaluate the buildings in-situ and not through the sketch elevations. It was also a check on the clarity of the language used, and how much time respondents needed to complete the circuit of both sides of the street, compared to the estimated 30-45 minutes. It was anticipated that, if the pilot did not require significant changes to be made to the questionnaire, these responses could be included as data in the full case study. This proved to be the case, as the pilot study did not reveal any significant concerns about the methods or the way they were being utilised. Respondents were asked to provide feedback on these and any other issues with their completed survey form. Two participants who had agreed to this in advance were contacted to tease out any specific concerns. As no matters were seen to be a concern in the pilot study the full survey could proceed as planned.

\subsubsection{Sampling}

A key focus of this research is to understand the aesthetic preferences of the public. As such, it was important that the survey attract responses from a cross section of the wider population. As the research also aims to compare preferences between expert designers and planners and the lay public a useful representation of the expert groups was also needed. To satisfy these aims, invitations were sent to a number of community groups and organisations (table 4.3).

Survey forms were left in cafes close to the study areas in each city, with small signs advertising the survey and inviting customer to participate. In Wellington this worked well, particularly because of the enthusiastic support of one café operator and his staff. In Auckland the responses to the invitations were more muted, and this was exacerbated by the fact the researcher was not based there. As a consequence, armed with survey forms, approaches were made to people in the streets asking if they would be willing to participate. On the two days spent recruiting participants in this manner, the uptake seemed positive. 
Table 4.3: Groups targeted for participation in Study Two, arranged by study site.

\begin{tabular}{|c|c|}
\hline Case study site & Groups invited by direct contact \\
\hline College Street, Wellington & $\begin{array}{l}\text { 1. Three chapters of the University of the Third Age } \\
\text { 2. Kelburn Playgroup } \\
\text { 3. Wellington City Council Planning and Urban } \\
\text { Development }\end{array}$ \\
\hline Tyler Street, Auckland & $\begin{array}{l}\text { 1. Two chapters of the University of the Third Age } \\
\text { 2. Auckland Council Urban Design and Planning } \\
\text { teams }\end{array}$ \\
\hline $\begin{array}{l}\text { Wyndham \& Kingston } \\
\text { Streets, Auckland }\end{array}$ & $\begin{array}{l}\text { 1. Two chapters of the University of the Third Age } \\
\text { 2. Auckland Council Urban Design and Planning } \\
\text { teams } \\
\text { 3. First year student cohort at the University of } \\
\text { Auckland School of Engineering } \\
\text { 4. St Patrick's Church staff }\end{array}$ \\
\hline
\end{tabular}

The completed survey forms were collected in several ways. For the College Street study a collection box was left with the play group and in each of three cafes, where respondents were offered a free hot drink for their efforts. This small reward was advertised with the survey forms to encourage these to be completed and returned. Similarly, in Tyler Street the forms were collected at a local café with the same hot drink reward. In the Kingston and Wyndam Street site all survey forms were distributed with postage paid return envelopes. The reward for participants in this survey was the chance to win one of two $\$ 100$ vouchers to a local restaurant. Respondents had the choice of going into the draw or not.

\subsection{Focus Group Discussion}

In order to generate qualitative data to supplement the quantitative data generated through surveys, two focus group discussions were convened. Discussion group participants were invited from a larger number of survey respondents who had indicated their willingness to participate. The principal value of focus groups is to enable the 
reasons for certain choices or feelings expressed by respondents to be probed (Bryman 2008: 475). Other potential benefits of bringing a focus group together include enabling participants to bring forward issues they think are important in relation to the area of research, enabling participants to work through differing viewpoints, and giving the researcher the chance to analyse the way participants make sense of the phenomena (Portella 2014: 143).

However, Bryman (2008: 475) also warned that some group dynamics tend toward a conservative expression of views. This can arise through socialising factors within the group and he suggested that personal interviews and individual open ended survey questionnaires generally provide a broader range of views. On the other hand, the dynamics within a focus group can also lead to a wider range of issues being raised. The two-pronged approach (see section 4.2.4) of survey and focus group discussion was pursued in order to generate a rich tapestry of data for analysis.

It was decided to convene two separate focus groups, with the catalyst for each being the preliminary findings from the College Street case study. It was anticipated that presentation of the results would lead to discussion of other issues important to the participants. The two sessions were arranged as separate discussions between lay people and design control experts. The rationale for separating the groups was to encourage freer conversation around aesthetic preferences and design review matters within each. It was thought that experts might seek to exert their expertise and so squash the input from lay people. On the other hand there was the potential for lay people to use the opportunity to lecture the experts on what the public really want.

The first discussion took place between lay people in a School of Architecture seminar space. Targeting six participants, seven people were invited with the expectation that one or more would withdraw. The second discussion took place a fortnight later in the same space. Both discussion groups were provided with the same background information via a short, illustrated talk by the researcher. The presentation concluded with several questions to stimulate the conversation, as follows:

1. Do any of the preliminary results stand out to you?

2. Thinking about the way streetscapes change over time, are you happy with the way things happen? 
3. Do you think designers have the public's interests at heart when they design new buildings?

4. Could planning regulation work better?

5. Does any of this matter?

\subsection{Evaluation of Built Form Characteristics}

As the research seeks to correlate people's perceptions of streetscapes with the characteristics of the buildings that help form them, it seems important to evaluate these characteristics. Several methods have been developed for this purpose. In his efforts to address "vague notions of a physical object" in design review, Stamps III (2000: 38-64) described numeric and geometric methods that could be used to measure concepts such as shape complexity, façade articulation, detail richness and character objectively. For example, the shape complexity of a building or streetscape could be described by measuring its outline. The lengths of each of the edges that comprise the outline, the number of turns and the angles of those turns and the degree of symmetry in the perimeter shape can all be measured to enable comparison with others (figure 4.6). At another level, how detailed a façade is could be measured by the pixel counts in a line drawing made of the building (figure 4.7). Stamps III's discussion of the relationships of wholes and parts, an important consideration in visual order, focussed on a seven-step proportional relationship. This, however, seems better suited to prescribing how to achieve more suitable whole:part relationships in a building and not as useful for analysing existing conditions. All of the studies cited by Stamps III made use of line drawing representations of the visual stimuli in order to precipitate people's responses and to measure the characteristics about which opinions were sought objectively. He was also able to identify strong correlations between the objective measures of variety and people's more subjective perceptions of variety. 


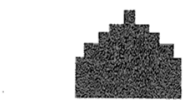

A

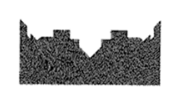

D

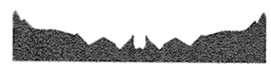

G

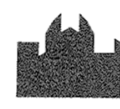

B

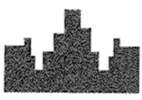

$E$

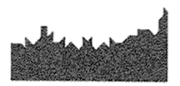

H

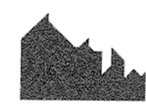

C

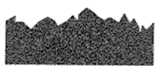

$\mathrm{F}$

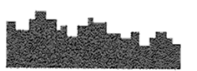

1

Figure 4.6: Silhouettes of individual buildings and streetscapes can be measured for comparisons in a study of people's preferences.

Source: Stamps III 2000

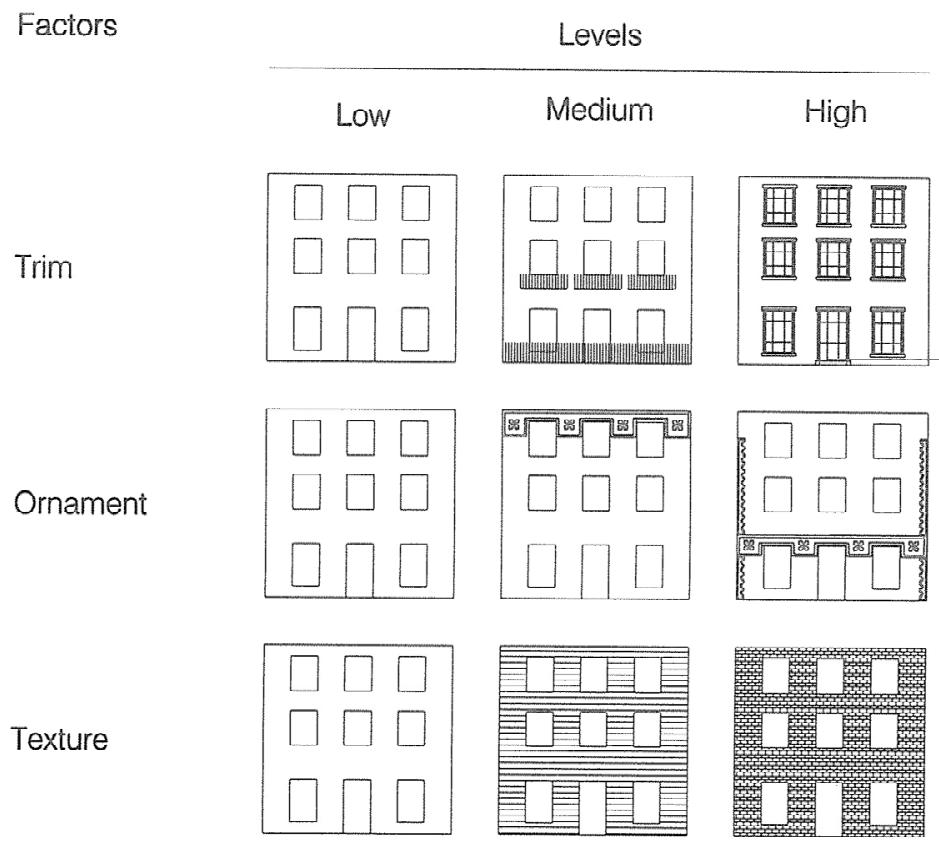

Figure 4.7: Pixel counts in two dimensional line drawings of buildings can be used to classify façade complexity. 
Cooper and Oskrochi (2008) were also interested in shape complexity and developed a method of determining visual variety using fractal analysis of photographic representations of suburban street scenes. Like Stamps III, they identified strong correlations between their objective measures and respondents' perceptions of visual variety.

Other researchers have worked with expert panels to help classify or rate particular characteristics of buildings and environments. For example, Groat (1988) invited four expert judges to rate contextual design strategies in 25 different projects using photographs. She then went on to study people's preferences for each of these strategies. In his study of preferences for different urban street scenes, Nasar (1988) reported that his research assistants, each with formal architectural training, rated the characteristics of the urban street scenes as a step in the research methodology. Heffernan, Heffernan et al. (2014) consulted experts to help them rate the levels of active frontage evident in the buildings that were represented in a set of photographs. The experts made their evaluations against a rating scale that had been developed earlier in the study. The expert opinions enabled the researchers to articulate people's preferences for particular street frontage activation methods.

Oostendorp and Berlyne (1978) suggested that many studies in the environmental aesthetics field are devised to test specific formal attributes, selected in part for theoretical reasons but also because of the researchers' own interests or intuitions. This research does not seek to test specific streetscape design attributes. Instead of a top down approach that tests responses to a defined set of design characteristics, the research invites a bottom $u p$ analysis of a full range of built form characteristics that vary from scene to scene and case study to case study. The findings are therefore prompted by analysis across the individual cases of the study. In this sense, the research follows the approach that led Nasar to be able to describe the key environmental characteristics that inform people's evaluative images of the city (Nasar 1990, 1998). He and his research colleagues did not rate or characterise the two cities in advance of studying people's preferences. Instead they sought to identify the key environmental factors that elicited favourable responses from people.

Researchers in two later visual preference studies undertook their own analyses of the environmental stimuli as experts (Nasar and Hong 1999, Heft and Nasar 2000). With 
that in mind, the author evaluated the characteristics of the street and individual buildings in the process of interpreting the survey results, relying on 30 years of professional experience as an architect and urban designer in the process.

\subsection{Human Ethics Committee considerations}

Victoria University of Wellington requires all research involving human subjects to follow strict guidelines that help ensure members of the public are protected while participating in the research and that ultimately the University is also protected from any untoward research activities. The main concern has to do with protection of people's right to privacy. Studies One and Two asked respondents to give their opinions in confidence. While most responses were provided anonymously some names and contact details were supplied in relation to the incentive or to indicate their willingness to participate in the follow-up focus group in Study Two. The two focus groups were also convened and steps were taken to ensure confidentiality of the opinions expressed by individuals during those two meetings. Human Ethics Committee approval was granted for both studies (approval numbers RM19953 and RM20362).

\subsection{Conclusions}

Chapter 4 has set out the research design and methods that were employed in the collection of data. As a lead to the development of the research methodology, a brief discussion of the philosophical contextual framework for the investigation was presented. The research is aligned with a realist philosophy, which envisages a reality of several levels. While the findings of this research provide evidence of aesthetic preferences that are time and culture specific, realism provides for interpretation of the results to find deeper mechanisms that may be driving environmental perceptions. One of the goals of the thesis is to speculate on the nature of these deeper mechanisms.

The research has been conducted through a mixed methods approach. Quantitative data has been collected by way of self-complete surveys and qualitative data through the focus group discussions. The methodology revolves around two field investigations. Study One seeks people's perceptions of urban streetscapes presented in the form of photographic elevations. The nature of this first study has been to scope people's perceptions broadly. Their responses to six different streetscape conditions provided 
information about their preferences for height, colour and architectural style relationships between the buildings along the depicted streets. While this information generated useful data in terms of the research questions, the results have also been used to inform selection of the three streets that are the subject of Study Two.

Study Two was designed to obtain people's aesthetic responses to individual buildings, relationships between adjoining buildings and to the overall streetscapes. The principal data collection method is the survey instrument, with respondents completing these while they were in the streets being studied. The cases were selected to enable deeper investigation of several results of Study One and also to reduce the likelihood that peripheral issues such as vehicular traffic and sun shading could be cited as factors influencing the responses. Nevertheless, data analysis needs to be sensitive to such matters. The research methods also included two focus group discussions. These were convened with willing respondents to the College Street survey, with one for lay persons and one for professional designers and planners. The logic behind the decision to organise separate sessions was to foster more open discussion in each.

After having outlined theories relating to aesthetic perception, the levels at which perceptions takes place in the human mind and the built form characteristics that are taken into account during these mental processes in chapter 3 , chapter 4 has provided the context for the investigation and presented the methods used to collect and analyse the data. With a view to answering the research questions, the thesis can now proceed to discuss the findings of Study One in chapter 5. 
Chapter 5

\section{Study One}

\subsection{Introduction}

Chapter 5 presents and discusses the key findings of Study One, which set out to understand how people perceive and evaluate urban streetscapes presented through photographic media. Chapter 4 has discussed the relationship of Study One to other research methods and its relevance in the overall research methodology. The findings of Study One are used to inform detailed development of the second study, through possibly revealing built form relationships and characteristics that invite further examination. The second study, which is conducted across three urban streetscapes that respondents experience in person, provides the ideal opportunity to examine such characteristics. As it records preferences in relation to photographic stimuli, Study One also provides a reference for comparing the two methods of gathering aesthetic response, representation and real experience.

The chapter begins with a brief summary of the demographics of the 191 survey respondents. It then goes on to discuss general findings from analysing the responses of the entire, undifferentiated respondent group. The discussion then moves to consider which, if any, individual buildings are considered out of place in their contextual scene. This set of questions targets the nature of design review, which tends to centre on the question of the suitability of a proposed development to fit with its surroundings (section 2.6). Following this, comparisons are made between the responses of demographic groups such as lay people and design/regulatory professionals, men and women, and different age groups. The chapter concludes with a general summary of the findings and their implications for Study Two. 


\subsection{General survey results}

The Study One survey was conducted over a six week period and returned 191 responses. Slightly more than half $(56 \%)$ of the respondents were male. Compared with age distribution nationally (Lohr and Pearson-Mims 2006, Statistics New Zealand 2013), the profile of respondents has more people in the middle age bracket $(40-65 / 69)$ and correspondingly lower numbers in the highest age bracket $(65+/ 70+)$ (figure 5.1). This can in part be attributed to a five year difference in the upper limit demarcation age (65 for the 2013 census and 70 in survey) but also reflects placement of the survey in work places.

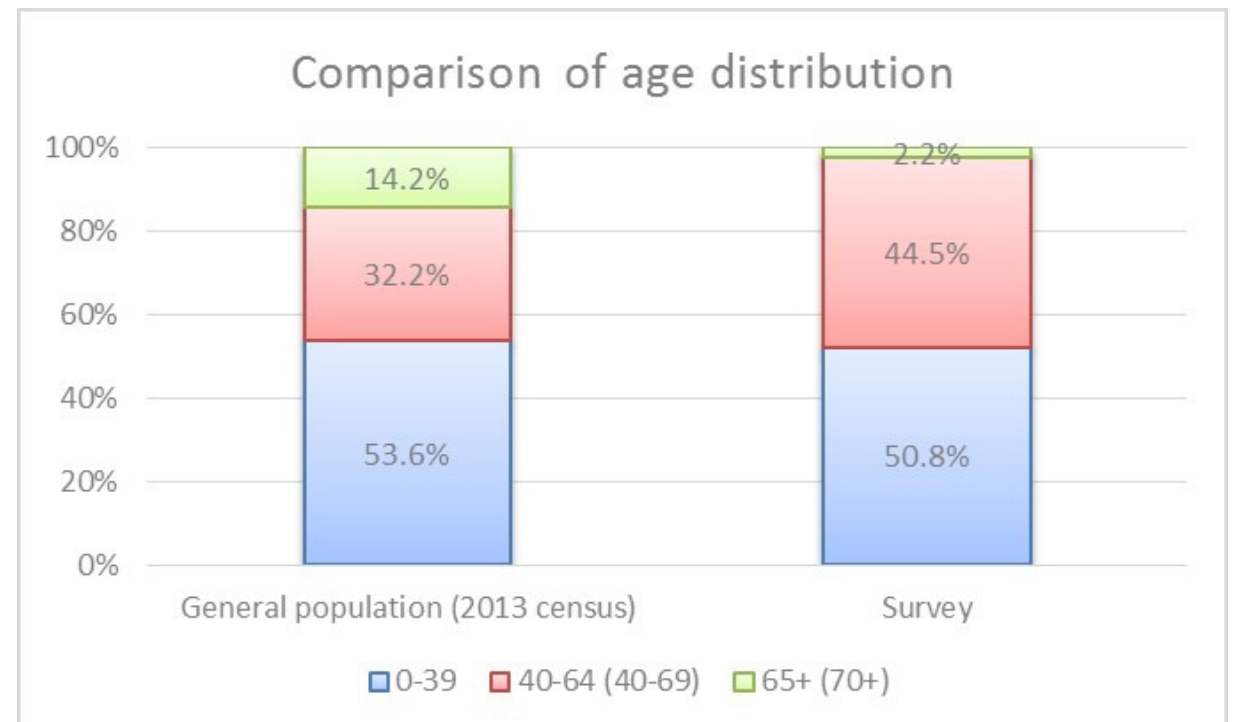

Figure 5.1: Age distribution of survey respondents compared with New Zealand's general population.

Source: Statistics NZ 2013

Some $44 \%$ of respondents had academic qualifications up to Bachelor's degree level and $26.7 \%$ had some form of post-graduate qualification. Only $14 \%$ of respondents had secondary school as their highest qualification level. These figures differ considerably from the general New Zealand population in 2006, the most recent year for which statistics are available. At that time only $11 \%$ of the NZ population had a Bachelor's degree and 5\% a higher degree, although these proportions may have increased over the ensuing years (Statistics New Zealand 2006). The relatively high education level across the sample reflects the research aim of obtaining survey responses from design and planning professionals and the general population. It would appear that placement of the 
displays in city council planning departments and at Victoria University of Wellington led to a bias toward higher education levels. It was hoped that by placing the exhibit in two public libraries that it would attract a representative sample of the general population, but this appears to not have happened. Other tactics would be necessary to attract members of the public with other qualification levels in future research efforts.

The 191 respondents have a range of occupational backgrounds including professional $(25 \%)$, student $(11 \%)$, non-architecture design professional $(5 \%)$, retired $(2.2 \%)$ and unemployed $(1.1 \%)$. Suitable numbers of architecture and planning professionals were sought in order to compare their opinions with those of the lay public and figure 5.2 shows the distribution of architects, planners and lay occupations in the sample. The non-architect design professionals have been included with the architects, as there is evidence to suggest that urban designers also identified with this grouping. The numbers include 54 architects and designers and 35 planning professionals.

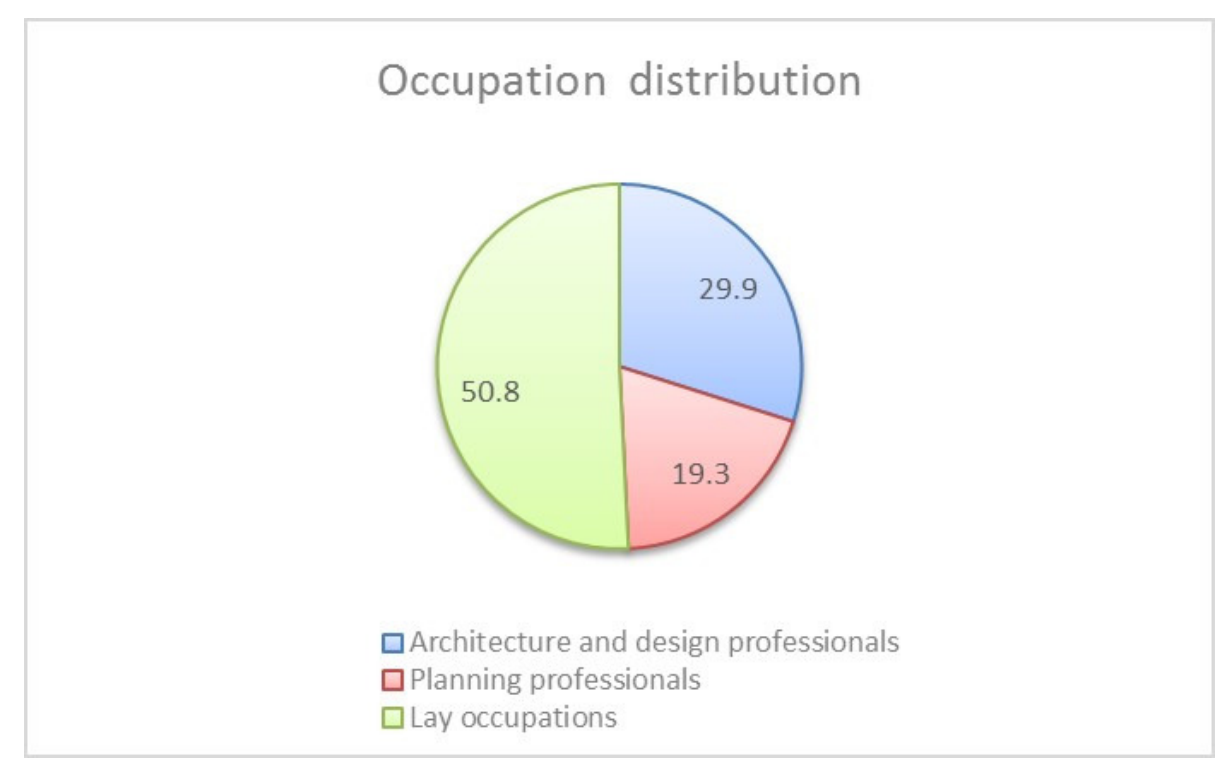

Figure 5.2: Percentages of respondents by occupation.

\section{Source: Author}

Overall, the sample is roughly representative of the general population in terms of age profile but not in the areas of education levels and occupation. These anomalies will have arisen through the sampling techniques, which deliberately sought the views of planning and design professionals. Analysis and interpretation of the data will keep these differences in mind. 

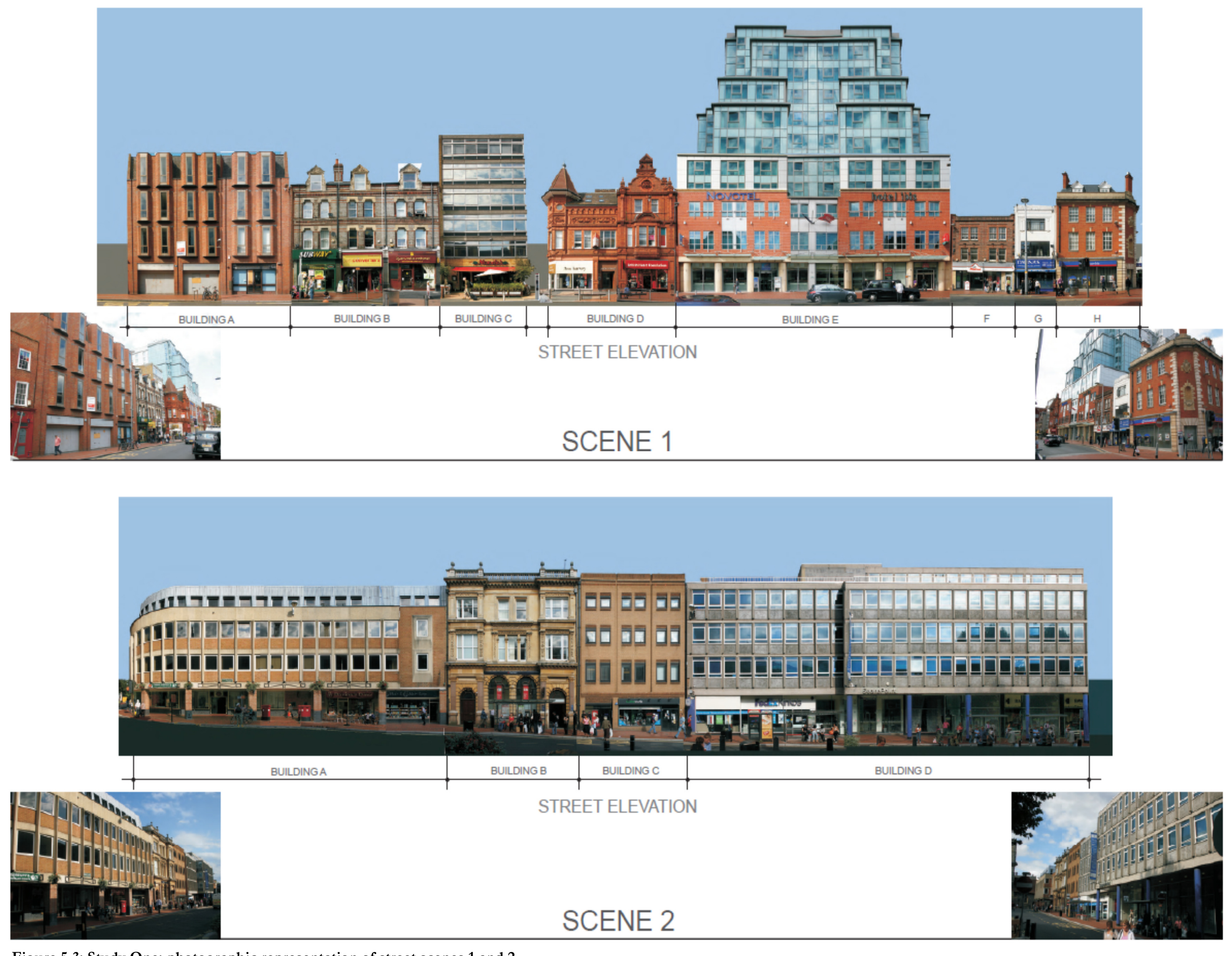

Figure 5.3: Study One: photographic representation of street scenes 1 and 2. 


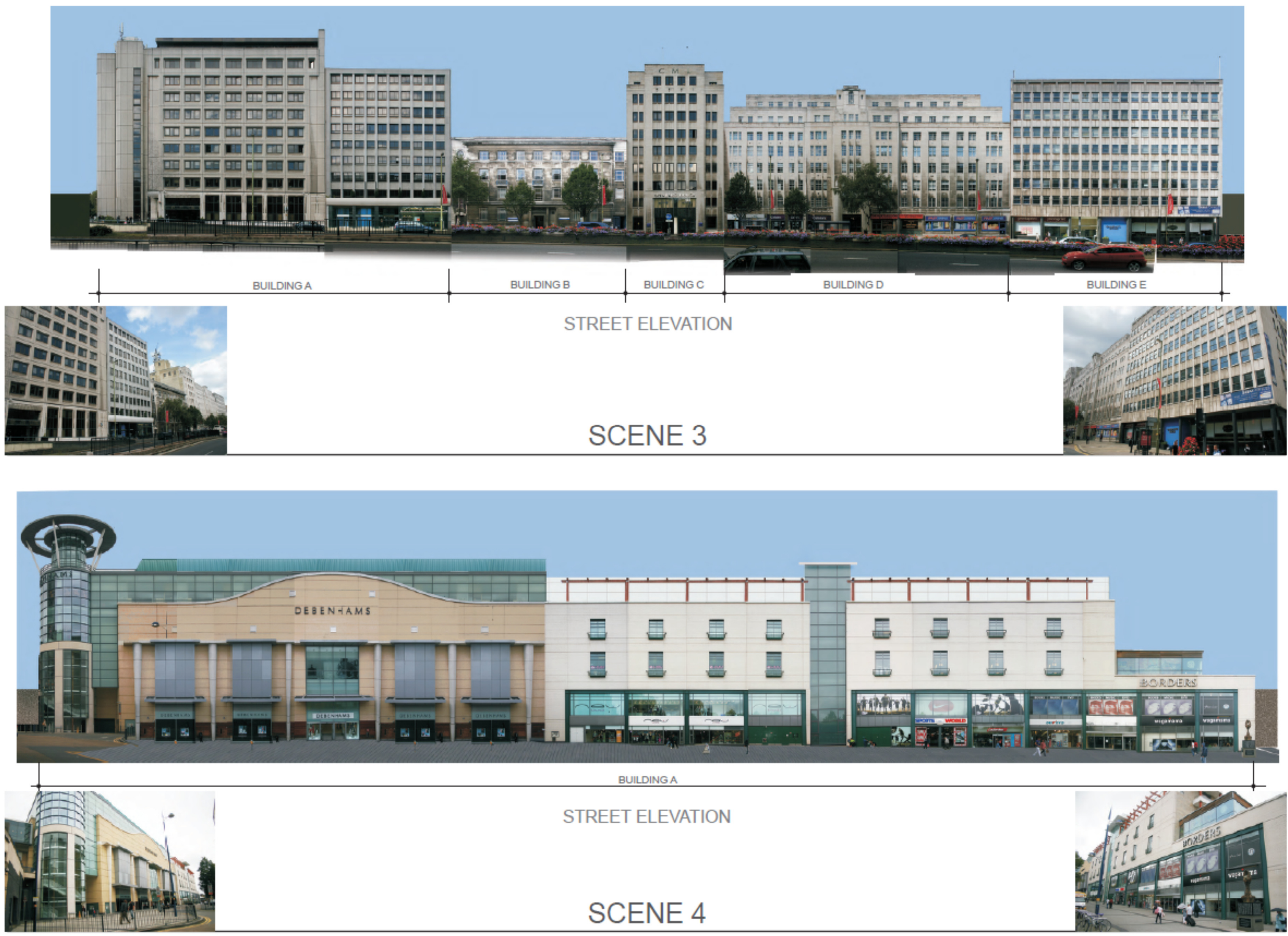

s)monga

STREET ELEVATION

SCENE 4

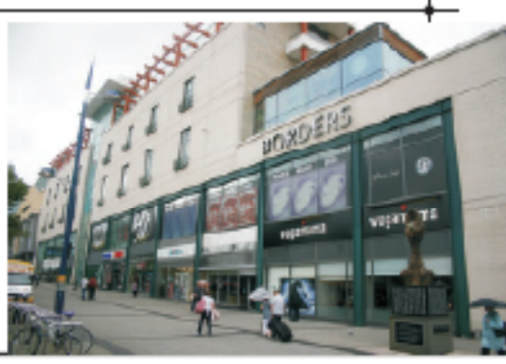




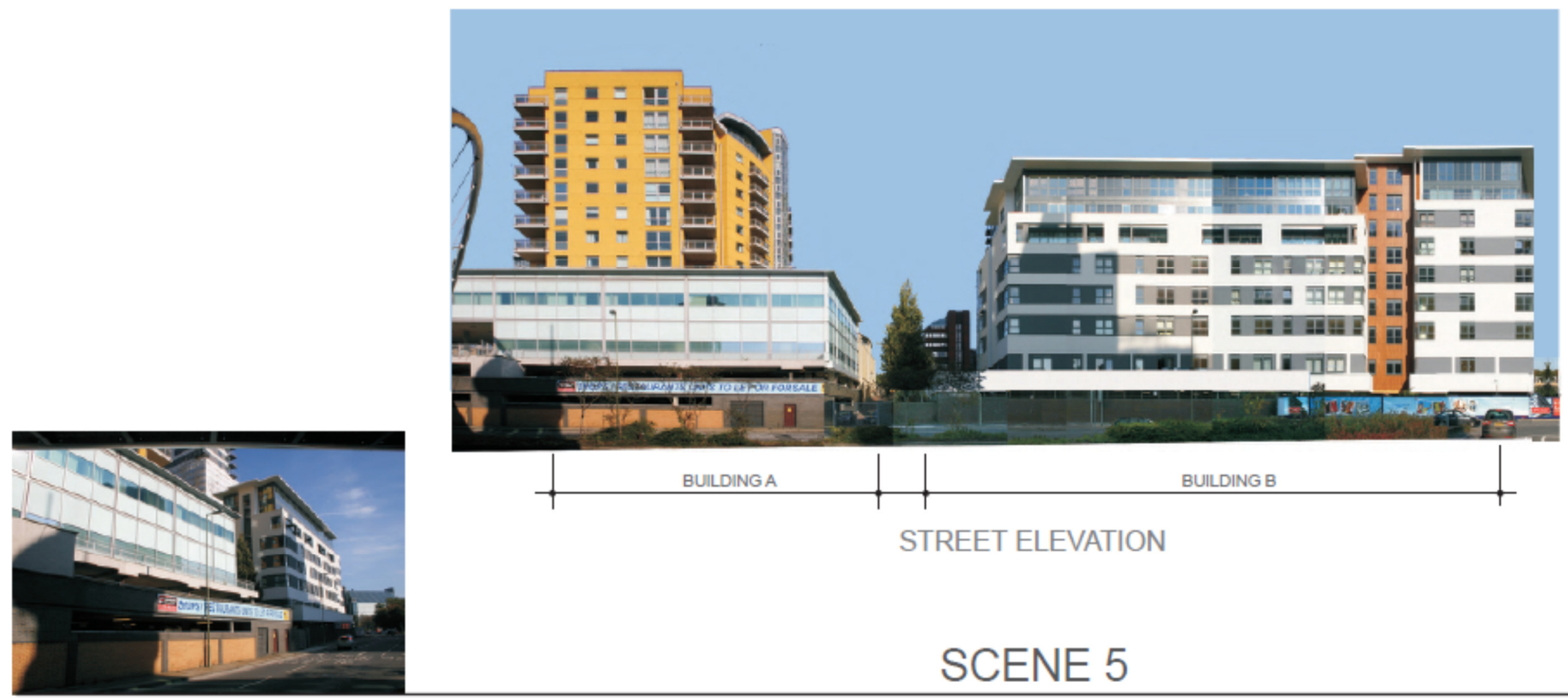

SCENE 5
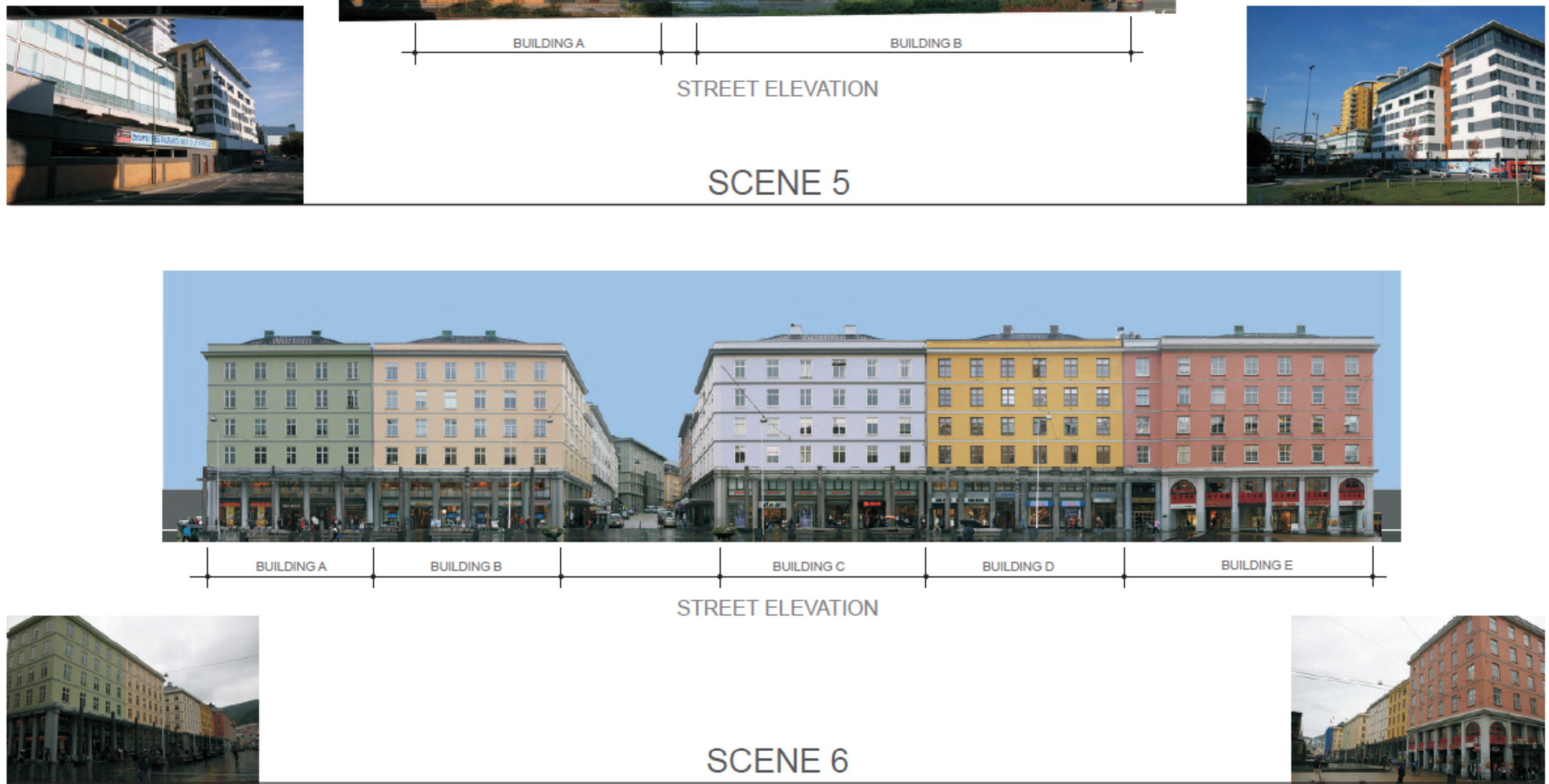

STREET ELEVATION

SCENE 6

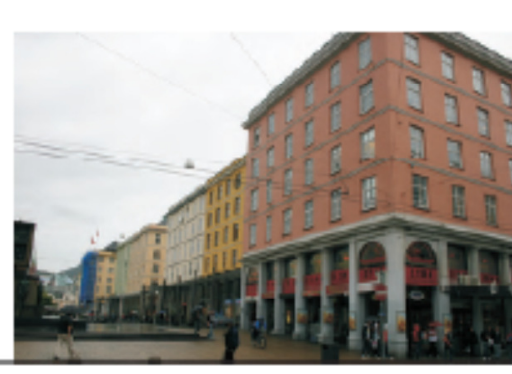

Figure 5.5: Scenes 5 and 6 of Study One 



\subsection{General preferences}

Scene 6 was the most preferred streetscape in response to the question "please indicate your overall preference", with a summary of the rating scores for all scenes shown in table 5.1. The mean response from the five point Likert scale is 3.44, with a standard deviation of 1.224 . More than $56 \%$ of all respondents liked or strongly liked this scene and only $16 \%$ had no opinion. The buildings in scene 6 (figure 5.5) are highly regular and it is the most ordered of the streetscapes in the study. Consequently the positive responses will have come from people who find visual order an aesthetic virtue. While the collection of buildings do not exhibit high levels of variation between them in terms of overall silhouette or the three dimensional modelling of form, there is considerable visual interest generated by the fenestration patterns and colonnade at street level.

Table 5.1: Preference scores for the six scenes, with the mean and distribution of positive, neutral and negative ratings for each

\begin{tabular}{|c|c|c|c|c|c|}
\hline & Mean & $\begin{array}{c}\text { Std } \\
\text { Deviation }\end{array}$ & $\begin{array}{c}\text { Positive } \\
\mathbf{( \% )}\end{array}$ & $\begin{array}{c}\text { Neutral } \\
\mathbf{( \% )}\end{array}$ & $\begin{array}{c}\text { Negative } \\
\mathbf{( \% )}\end{array}$ \\
\hline Overall preference 1 & 3.11 & 1.170 & 45.5 & 15.3 & 38.2 \\
\hline Overall preference 2 & 2.63 & 1.024 & 22.7 & 26.2 & 51.1 \\
\hline Overall preference 3 & 3.23 & 1.051 & 47.1 & 25.9 & 27.0 \\
\hline Overall preference 4 & 3.10 & 1.273 & 45.7 & 19.2 & 35.1 \\
\hline Overall preference 5 & 2.47 & 1.106 & 23.2 & 19.4 & 57.4 \\
\hline Overall preference 6 & 3.44 & 1.224 & 56.4 & 16.0 & 27.6 \\
\hline
\end{tabular}

Somewhat surprisingly, scenes 3, 1 and 4 are not far behind the preferences expressed for scene 6. Although lacking the uniform parapet line of scene 6 , scene 3 is effectively ordered by the fenestration patterns that extend across all buildings (Smith 2003: 41-42). The façade claddings, all of concrete or a similar material, also provide a level of consistency.

The strongest relationship between overall preferences and preferences expressed for a design characteristic in the same scene was found to be architectural style. In other words, 
preferences expressed by individuals for the relationship between architectural styles in a scene correlated most strongly with the score given for the scene overall in every case. For example, the correlation between architectural styles and overall preference for scene 6 is 0.803 ( $\mathrm{p}<0.01)$, a relationship that is significant and one that was examined further through regression analysis. This confirmed that a person's evaluation of architectural style relationships in a scene was found to be the strongest predictor of their overall preference. Scene 6 has $56 \%$ and $57 \%$ positive ratings for overall preference and architectural styles respectively.

People were generally positive about the architectural style relationships in scenes 3 (61\% of responses positive) and 6 (57\% positive). Both scenes are modulated by the individual buildings and all buildings in each scene also have consistency of cladding materials, fenestration patterns and generous, visually accessible ground floor facades. Scene 6 is strongly consistent, with a ground level arcade linking all buildings, matching floor levels, and matching fenestration proportions and arrangement. The order this consistency generates appears to be valued by respondents. However, a lack of variation may have limited the level of positive response. This can be supported by the higher response ratings for scene 3, which is ordered but also offers viewers greater levels of variation between the buildings (figure 5.4). Variety is generated not only in silhouette (height relationships) but also frontage width, façade composition and window proportions. From this it would seem that the public prefer variety within a limited range. This analysis can be supported through reference to the responses for scene 1, which features architectural style relationships that are more vigorously varied. The median score for scene 3 is higher (3.56 versus 3.44), the positive response rate is higher (61\% versus $57 \%$ ) and there are fewer negative responses (16.5\% versus $28 \%$ ).

The strong relationship between overall preference and the general preference scores for relationships of architectural style may be explained with reference to the high aesthetic value that places with strong architectural character have for many people. This is evidenced by patterns of real estate value. Strength of architectural character is a measure of the consistency of physical patterns across an area (Stamps III 2000: 59, McIndoe 2008). Places exhibiting higher levels of such consistency are generally more sought after by people (Tiesdell, Oc et al. 1996: 16-17). Where places have strong character based on 
historic patterns, such places are often protected from change that would diminish the consistency.

It is useful to look at the style relationship ratings for scene 2 in light of clear preferences for consistency of architectural style in a street. Scene 2 (figure 5.3) has a mean preference score just under 3 and this reflects the equal split between those having a negative response (35.3\%) and a positive opinion (38\%). Like scenes 3 and 6 , the buildings in scene 2 have rhythmic windows above ground floor and open, visually accessible ground floor areas. However, it is less preferred. Regression analysis indicates that relationships between finishes, heights and colour (in that order) are the strongest predictors of the score given for style in this scene. Ambivalence toward the relationship between surface finishes in scene 2 equalled that expressed toward architectural styles. The predominant cladding materials appear to be tan coloured bricks, tan stone/plaster and grey concrete, each used in a traditional manner for the period of the building.

The styles of the buildings in scene 2 appear to be consistent except for the neo-classical building 'B', which is also perhaps the oldest building in the streetscape. Respondents were encouraged to indicate which, if any, buildings in a scene did not fit with the others. For scene 2, 67 people indicated that at least one building did not fit, and $43 \%$ of those cited building B. Reasons for this were many and varied, the most consistent being style. Arguably, the design of buildings A, C and D have been influenced by B and it could be argued that, in design terms, the efforts have been successful. However, other relationships between building $\mathrm{B}$ and its immediate neighbours clash, such as the alignment of floor levels and levels of detail/decoration. Windows, although of similar proportions, are clearly smaller in the newer buildings. It is anticipated that the relevance of these relationships, both where they contrast and where they align, will be explored in more detail in Study Two.

When it comes to building heights in a street scene, the respondents favoured the relationships between buildings in scene 3 (figure 5.4 and table 5.2). Scene 3 exhibits a moderate variation in height between buildings, falling somewhere between the strong variation of scene 1 and the consistency of scenes 4 and 6 . On the basis of mean scores, the preference for scene 3 was not conclusive ( 3.55 compared with 3.49 for scene 6 ) nor was the dislike of the height relationships in scene 1 overwhelming (mean score 2.82, not far below the median score of 3). While more people were negative about the height 
relationships in scene 1 (51\% negative and 36\% positive) this scene had the lowest level of non-committed responses (13\% neutral scores). This compares with 32\% neutral preferences for the relationship of heights in scene 5 (figure 5.5). It seems people had an opinion about the strongly articulated height relationships in scene 1, either positive or negative, more so than for the other scenes.

Table 5.2: Preference scores for height relationships between buildings in the six scenes, with the distribution of positive, neutral and negative ratings for each.

\begin{tabular}{|c|c|c|c|c|c|}
\hline & Mean & $\begin{array}{c}\text { Std } \\
\text { Deviation }\end{array}$ & $\begin{array}{c}\text { Positive } \\
\mathbf{( \% )}\end{array}$ & $\begin{array}{c}\text { Neutral } \\
\mathbf{( \% )}\end{array}$ & $\begin{array}{c}\text { Negative } \\
\mathbf{( \% )}\end{array}$ \\
\hline Heights 1 & 2.82 & 1.090 & 35.8 & 13.2 & 51.0 \\
\hline Heights 2 & 3.34 & 1.115 & 53.7 & 18.4 & 27.9 \\
\hline Heights 3 & 3.55 & 0.971 & 61.2 & 21.8 & 17.0 \\
\hline Heights 4 & 3.47 & 1.036 & 54.2 & 26.6 & 19.1 \\
\hline Heights 5 & 2.88 & 0.980 & 30.0 & 31.6 & 38.4 \\
\hline Heights 6 & 3.49 & 1.190 & 56.9 & 18.1 & 25.0 \\
\hline
\end{tabular}

The preferences expressed for the height relationships in scene $3(61 \%$ liked) contrast with the general dislike of the height relationships in scene 1 (51\% disliked). The fact that scenes 6, 4 and 2 in rank order fell behind scene 3 for preference, together with the general dislike of heights in scenes 1 and 5 , seem to confirm participant preferences for silhouette line consistency in a collection of urban buildings. However, it seems that more than consistency, variety is preferred as long as it is tempered by underlying order. In this case, moderate levels of height variation provide the visual interest while fenestration patterns, cladding materials and building styles all help to create visual order across the scene.

Although still strong, preferences expressed for height relationships in each of the scenes had generally lower correlations with people's overall preferences than other architectural factors, such as colour and finishes. Indeed, table 5.3 reveals that people's responses to the relationship of architectural styles in each of the six streetscapes corresponded most closely with their overall preferences for the scene. As scene 6 is well liked (table 5.1) and as the correlation between responses to its architectural style relationships and overall 
preferences (correlation $=0.806, \mathrm{p}<0.01$ ) is considered very high (Bryman 2008), it appears that consistency of architectural style could be a desirable streetscape characteristic. A regression analysis confirmed the strong influence that the style relationships had on people's preferences for scene 6. However, further investigation is needed to confirm this finding. To what extent were the responses influenced by the particular style of the buildings? Correspondence between the style relationships and overall preference for scene 3, another well liked (mean=3.23) streetscape where the buildings exhibit consistency of style, was lower at $0.619, \mathrm{p}<0.01$.

Table 5.3: Correlation coefficients for respondents' overall preferences for a scene and their scores for the relationships of building height, style, cladding materials and colours. The strongest correlation for each scene is highlighted.

\begin{tabular}{|c|c|c|c|c|c|}
\hline & Heights & Styles & $\begin{array}{c}\text { Materials/ } \\
\text { Finishes }\end{array}$ & Colours & $\begin{array}{c}\text { Sig. of each } \\
\text { correlation in row }\end{array}$ \\
\hline Overall 1 & .565 & $\underline{\mathbf{. 7 6 4}}$ & .735 & .671 & $\mathrm{p}<0.01$ \\
\hline Overall 2 & .588 & $\underline{\mathbf{. 6 4 4}}$ & .524 & .481 & $\mathrm{p}<0.01$ \\
\hline Overall 3 & .556 & $\underline{\mathbf{. 6 1 9}}$ & .615 & .608 & $\mathrm{p}<0.01$ \\
\hline Overall 4 & .662 & $\underline{\mathbf{. 7 8 2}}$ & .752 & .767 & $\mathrm{p}<0.01$ \\
\hline Overall 5 & .660 & $\underline{\mathbf{. 6 7 9}}$ & .667 & .650 & $\mathrm{p}<0.01$ \\
\hline Overall 6 & .687 & $\underline{\mathbf{. 8 0 6}}$ & .753 & .748 & $\mathrm{p}<0.01$ \\
\hline
\end{tabular}

The survey also asked people to rate the pleasantness of each scene and to express their preferences for the relationships between the claddings, the colours and the architectural styles. The urban street scenes were selected with both questions in mind and the finishes/materials range between ordinary brick and coloured concrete to glass and exterior plaster. Similarly, a range of colour relationships was sought, from a consistent grey (scene 3 - figure 5.4) to varied and colourful (scene 6 - figure 5.5). Table 5.4 sets out the mean preference scores for each of these streetscape characteristics.

The responses suggest that people liked variety of colour in a scene. The two most colourful scenes are 1 and 6 and they received the highest scores (mean $=3.48$ and mean $=3.62$, respectively). The responses to the colours of scene 4 (figure 5.4) are interesting in this context. The colours of this single, comprehensive development are relatively 
muted and clearly coordinated. As the mean scores for these three scenes are relatively close and separated from the other three, it seems appropriate to consider whether any generalisations about them can be taken forward in the study. The colours of each appear to vary within narrow limits, in the case of scenes 4 and 6 this appears to have been by design and in the case of scene 1 strongly influenced by the colour of the red brick masonry claddings.

The responses to scene 3 suggest that while the respondents liked the consistency of façade cladding materials cross the scene, because the material was predominantly grey they disliked the colour relationships. To what extent were they responding to the predominantly grey colours and to what extent was this due to a lack of variation across the scene?

Table 5.4: Mean preference scores for pleasantness and the relationships between architectural styles, cladding materials and façade colours in each scene. The highest rated score in each column is highlighted

\begin{tabular}{|c|c|c|c|c|c|}
\hline & & Pleasantness & Styles & $\begin{array}{c}\text { Materials/ } \\
\text { finishes }\end{array}$ & Colours \\
\hline \multirow{2}{*}{ Scene 1 } & Mean & 3.10 & 2.92 & 3.05 & 3.48 \\
\cline { 2 - 6 } & Std. Deviation & 1.196 & 1.214 & 1.080 & 1.008 \\
\hline \multirow{2}{*}{ Scene 2 } & Mean & 2.71 & 2.97 & 2.94 & 2.84 \\
\cline { 2 - 6 } & Std. Deviation & .998 & 1.049 & 1.014 & 1.049 \\
\hline \multirow{2}{*}{ Scene 3 } & Mean & 3.14 & $\underline{\mathbf{3 . 5 6}}$ & 3.43 & 2.87 \\
\cline { 2 - 6 } & Std. Deviation & 1.061 & .922 & .981 & 1.123 \\
\hline \multirow{2}{*}{ Scene 4 } & Mean & 3.16 & 3.21 & 3.26 & 3.41 \\
\cline { 2 - 6 } & Std. Deviation & 1.296 & 1.190 & 1.103 & 1.046 \\
\hline \multirow{2}{*}{ Scene 5 } & Mean & 2.52 & 2.79 & 2.85 & 2.94 \\
\cline { 2 - 6 } & Std. Deviation & 1.172 & 1.115 & 1.058 & 1.167 \\
\hline \multirow{2}{*}{ Scene 6 } & Mean & $\underline{\mathbf{3 . 5 3}}$ & 3.44 & $\underline{\mathbf{3 . 5 9}}$ & $\underline{\mathbf{3 . 6 2}}$ \\
\cline { 2 - 6 } & Std. Deviation & 1.204 & 1.193 & 1.066 & 1.275 \\
\hline
\end{tabular}




\subsubsection{Which buildings do not fit and why}

Respondents were invited to critique each scene by indicating whether or not there were any buildings that did not fit with others and nominating these. This is one of the important determinations of the design control process when deciding the fate of a redevelopment proposal. Table 5.5 summarises the results for these questions, which are then discussed below.

Table 5.5: Summary of the responses in relation to the question: "Do any of the buildings in the scene not fit"

\begin{tabular}{|c|c|c|c|c|}
\hline Scene & All fit & $\begin{array}{l}\text { One or more } \\
\text { does not fit }\end{array}$ & Which does not fit & $\begin{array}{l}\text { Principal reasons (first ranked } \\
\text { building) }\end{array}$ \\
\hline 1 & 21 & 162 & $\begin{array}{l}\mathrm{E}(104), \mathrm{C}(77), \\
\mathrm{A}(31)\end{array}$ & $\begin{array}{l}\text { Height, domination, } \\
\text { architectural style }\end{array}$ \\
\hline 2 & 99 & 73 & $\mathrm{~B}(29), \mathrm{D}(29), \mathrm{A}(18)$ & $\begin{array}{l}\text { Architectural style (B) } \\
\text { Colour, façade design (D) }\end{array}$ \\
\hline 3 & 114 & 56 & $\mathrm{~B}(33), \mathrm{E}(13), \mathrm{A}(10)$ & Height \\
\hline 4 & 138 & 28 & $\mathrm{~A}($ all $)$ & Façade scale \\
\hline 5 & 81 & 86 & $\mathrm{~A}(56), \mathrm{B}(14)$ & $\begin{array}{l}\text { Colour, lack of engagement } \\
\text { with street }\end{array}$ \\
\hline 6 & 144 & 20 & Not clear & $\begin{array}{l}\text { The few respondents who } \\
\text { provide a reason suggested the } \\
\text { buildings in the scene are too } \\
\text { similar }\end{array}$ \\
\hline
\end{tabular}

Across all six scenes, the strongest negative reaction was to building $\mathrm{E}$ in scene 1 (figure 5.3). Building E illustrates many of the issues facing contemporary urban redevelopment and how designers confront these issues in order to enhance integration with the setting. Building E clearly extends across the frontages of three or more amalgamated sites to create the economies of scale needed to meet development aims (Bentley 1999: 77-78). Development potential is often dependent on higher allowable heights than those restricting earlier buildings. To address the potentially overwhelming scale of the new building in this setting the designers have modulated the façade in both horizontal and vertical dimensions. Along its length, the façade is broken into three distinct modules. 
The two equal ends are separated by the two central bays that appear as a 'negative margin' between them. Vertically, the design uses building materials and architectural modelling to minimise visual contrasts as the building extends up above the general height of neighbouring buildings. The fact this building exists in this street is testament to a design control process that supports this approach.

The public response $(89.6 \%)$ is that, despite the intentions, the design does not fit with others around it. This differs from 16\% of architects and 9\% of planners who thought all buildings in the scene fitted well. It would seem architects are more willing to see merit in the design efforts made to integrate such a large intervention with its setting. Nevertheless, of the 104 people who indicated that building E does not fit, more than half (60) thought building E was one of several that did not fit. Specific reasons noted by respondents in defence of their opinions include the height of building $\mathrm{E}$ and its architectural style. Others were critical of the extensive use of glass in the upper levels. Several respondents suggested that building $\mathrm{E}$ has a dominant effect because of its height and overall dimensions, despite the design efforts to step the form of the building back from the street above the podium level.

A high number of respondents were also critical of the effects of building $\mathrm{C}$, which is perhaps the clearest example of Modernist architecture in the streetscape scene. This building has façade glazing banded at each floor level, reflective properties and minimal areas of solid wall. Some 77 of the 162 people who nominated a building that did not fit with the others in scene 1 cited building $C$. Colour, window configuration and suggestions that the design included too much glass were the principal reasons for this mismatch.

Although scene 3 was highly regarded, 33\% felt it could have been improved if one or more buildings could be omitted. Architects were more closely aligned with lay people, as $67.5 \%$ of architects thought that all buildings in the scene worked well together compared with $69.7 \%$ of lay people. Only $58 \%$ of planning professionals thought the buildings all fitted well with one another. Building B was the most popular misfit choice, with 33 of 56 people nominating it. While most people gave the reason for the mismatch as being too low in height, one respondent suggested it did not fit because it was the only building in the street that exhibited a human scale. 
For scene $6,12 \%$ of respondents thought that the buildings (figure 5.5) did not all fit well with each other. This is certainly curious, as all five buildings in the scene have very similar architectural language and heights. Moreover, an identical street level arcade links buildings $\mathrm{A}$ to $\mathrm{D}$ together, with building $\mathrm{E}$ stepping out to intercept this unifying feature. A greater percentage of architects (17.5\%) felt that one or more building did not fit in scene 6 than lay and planning professionals (10.5\% each) but only two of those responding went on to identify the offending building(s). Overall, of the 20 people who felt there was a mismatch, only 4 identified the offending buildings. Reading between the lines of the responses made by these 20 people, it appeared they were most frustrated by the high level of consistency between the buildings. Variation between them is largely limited to colour and, in the case of building E, façade alignment.

Responses made in relation to scene 2 (figure 5.3) suggest there are differences in the way change professionals view building fit within the streetscape compared with lay people. Some $61 \%$ of architect and $60 \%$ of lay respondents considered all buildings in scene 2 to fit, despite the generally low regard they had for the scene overall (mean score 2.63, and $5^{\text {th }}$ ranked overall). A lower number of planners (45\%) felt that all buildings fitted well. Looking then at the respondents who felt that at least one of the buildings did not fit with the others it seems that planners, like architects, saw building D as the misfit. While the façade proportions and design details of building $\mathrm{D}$ align very closely with building $\mathrm{A}$ and to a lesser extent building $\mathrm{C}$, the trained professionals considered the contrasting colour of building $\mathrm{D}$ to be the problem. Lay people, on the other hand, generally saw building $\mathrm{B}$ as a poorer fit with the others in the scene. The obvious difference between building $\mathrm{B}$ and the others is its façade composition, which adopts a neoclassical approach. The view of lay people on older, heritage buildings and their settings will be explored further in Study Two.

Reactions to scenes 4 and 6 are interesting, as both scenes exhibit high levels of consistency and in scene 4 , the individual parts are inseparable. Scene 4 is a single building created by the comprehensive redevelopment of a large, amalgamated site. The designers have sought to break the scale of this large form down through the use of colour, cladding materials and modelling techniques. As a consequence the building reads as being more than one, a fact attested to by the $17 \%$ of respondents who felt that one part does not fit with the rest. Architects and planners felt more strongly about this $(27.5$ and $27.6 \%$ 
respectively) than lay people $(9.3 \%)$. Men were also nearly twice as likely as women to hold this view. Reasons for the mismatch centred mainly on the scale and domination of the building as well as a negative reaction to the large fashion and houseware store, Debenhams. However, respondents giving this as a reason did not elaborate, so it is unclear whether their reactions were directed at the architecture or the business, again something to be explored in Study Two. Also interesting is that one respondent, a planner, suggested that the building height at the right side was too low. It is not clear whether the image was being considered as an abstract composition when the respondent made this assessment or whether it was made with reference to the height and scale of a person. This could be one of the potential difficulties of evaluating aspects of the built environment on the basis of photographic representation.

\subsubsection{Issues to carry forward to Study Two}

This part of the study has generated results for further consideration. Firstly, it appears that the public is not convinced by design efforts made to disguise mismatches of scale and form between adjoining buildings in a streetscape. Evidence of this is seen by the clear indication that building $\mathrm{E}$ in scene 1 does not fit with its neighbours. This is further supported by the reasons people gave to back up their responses, the primary ones being bulk - the overall height and width dimensions - and dominance, a consequence of scale relationships.

A second result is the strength of influence architectural style appears to have on people's preferences, as architectural style relationships in a scene are the key predictor of overall preference. A considerable number of respondents also gave architectural style as the reason for a particular building or buildings not fitting with the scene generally. However, it was not just contrasts in style, for example building B in scene 2, that influenced responses but also conformity in styles, as in scene 6. Although not a majority, a number of respondents appear to have wanted variation in architectural styles in scene 6. Overall, there is a sense arising from the responses to the question of overall preference that people seek variety within controlled means. 


\subsection{Comparisons based on demographic groupings}

\subsubsection{Gender comparisons}

Although the number of published findings is low, research into gender-specific responses to the built environment is unanimous in concluding that there are no differences. This research was set up to enable comparisons between different demographic groupings, including gender. This is relevant for several reasons. Firstly, the research referred to above is old, with the most recent paper (Stamps III) published in 1999. Secondly, the findings are supported by only three research efforts and none consider responses to urban streetscapes, as all are based on responses to individual, detached buildings. Gender streetscape preferences will help fill knowledge gaps in this area. This information is relevant because those involved in decision-making about urban change are predominantly male. Despite making up half the general population, women comprise only $21 \%$ of the architects and designers and $48 \%$ of the planners in the country (New Zealand Registered Architects Board 2013, Statistics New Zealand 2013).

Consistent with the earlier studies, the present research reveals that there are considerable areas of overlap in the aesthetic evaluations made by men and women. When considering urban streetscapes, opinions appear to be consistent around all evaluation categories for a particular scene. For example, the median scores given by men and women vary by between 0.01 and 0.10 for scene 3 (Table 5.6). It can be noted that none of these differences are statistically significant.

Table 5.6: Comparison of the mean scores given by men and women for the different streetscape characteristics of scene 3

\begin{tabular}{|l|c|c|c|c|}
\hline & Females & Males & Difference & Sig. (2-tailed) \\
\hline Pleasantness 3 & 3.14 & 3.13 & 0.01 & \\
\hline Heights 3 & 3.53 & 3.56 & 0.03 & \\
\hline Styles 3 & 3.56 & 3.55 & 0.01 & \\
\hline Materials \& finishes 3 & 3.41 & 3.46 & 0.05 & \\
\hline Colours 3 & 2.85 & 2.88 & 0.03 & \\
\hline Overall 3 & 3.19 & 3.29 & 0.10 & \\
\hline
\end{tabular}

Similar alignments between the genders were seen in the responses to scenes 1, 2 and 6. For scene 1 the differences in means varied between 0.03 and 0.19 . Women were slightly 
more positive about the scene overall but a little more negative about the relationship between building colours. One of the key concerns raised by the total respondent group about scene 1 was the variation in heights. This is reflected quantitatively in the mean score sitting below the neutral line and qualitatively in the reasons given to support respondents' opinions about buildings that did not fit. Although not reflected in the comparison of mean scores, it appears that women were more decisive when evaluating height relationships. Only $7.4 \%$ of women expressed a neutral opinion about the heights in this scene whereas $18.6 \%$ of men were neutral. Women were both more negative and more positive than male respondents; in this case it seems they had stronger opinions to express about the height relationships.

Table 5.7: Comparison of men's and women's responses to the streetscape characteristics of scenes 4 and 5

\begin{tabular}{|c|c|c|c|c|}
\hline & Females & Males & Difference & Sig. (2-tailed) \\
\hline \multicolumn{5}{|c|}{ Scene 4} \\
\hline Pleasantness & 3.35 & 3.03 & 0.32 & \\
\hline Heights & 3.61 & 3.39 & 0.22 & \\
\hline Styles & 3.38 & 3.05 & 0.33 & \\
\hline Materials \& finishes & 3.32 & 3.19 & 0.13 & \\
\hline Colours & 3.58 & 3.27 & 0.31 & \\
\hline Overall & 3.26 & 2.99 & 0.27 & \\
\hline \multicolumn{5}{|c|}{ Scene 5} \\
\hline Pleasantness & 2.30 & 2.70 & 0.40 & $\mathrm{p}<0.05$ \\
\hline Heights & 2.72 & 3.04 & 0.32 & $\mathrm{p}<0.05$ \\
\hline Styles & 2.62 & 2.92 & 0.30 & \\
\hline Materials \& finishes & 2.23 & 3.09 & 0.41 & $\mathrm{p}<0.05$ \\
\hline Colours & 2.68 & 3.09 & 0.41 & $\mathrm{p}<0.05$ \\
\hline Overall & 2.27 & 2.65 & 0.28 & $\mathrm{p}<0.05$ \\
\hline
\end{tabular}

Looking across all six scenes, women generally held more positive opinions about scenes with consistent height relationships and more negative opinions where heights varied. The scene providing common ground for both genders is scene 3, where the buildings have moderate height variations (mean scores: $\mathrm{F}=3.53$ and $\mathrm{M}=3.56$ ). Women gave their 
lowest scores to scenes 1 (mean $=2.75)$ and $5($ mean $=2.72)$. Men were generally positive about the height relationships in scene 5 (table 5.7) although they aligned reasonably well with women's views on height in scene 1 (mean $=2.84)$.

As set out in table 5.7 , the responses to streetscapes in scenes 4 and 5 had the strongest correlations for gender. The responses to questions about scene 5 were statistically significant for all questions except that focussed on style relationships. Women were consistently more negative than men about this streetscape, expressing scores for all questions that averaged well below the neutral line. Men, on the other hand, were positive (in terms of mean score) for the relationships of height, materials and finishes and colours in this scene. On the basis of this analysis it would seem there are gender based differences.

Scene 5 comprises large, contemporary buildings with ground level car parking that limits visual and physical connection between the building and adjoining footpath. The massing design of building A creates a podium/tower arrangement and colour is used to differentiate parts of the composition. It was picked out by $57.7 \%$ of women and $47.8 \%$ of men as not fitting. Colour was cited more often by both genders as the reason for this. Despite this, the mean score for the colour relationships in the scene from men was 3.09, a positive response. On the other hand, more than half of all female respondents gave the colour relationships a negative score. Another colourful scene is number 6. Both genders were positive about colour relationships here, with the mean scores for each falling within 0.07 points of each other. Both scenes employ a yellow shade, raising the issue of why the response to one is so negative and the other positive. One possible explanation could be that colour is used in isolation in scene 5 and that the majority of the facades of both buildings is white. There is a lack of coordination, even within the same site. Colour has been used at street level but in a way that is unrelated to the colours used elsewhere. On the other hand, scene 6 appears to have been composed and the colours are similar in hue and intensity, which is another dimension to the ordered interest that this scene appears to generate.

Men and women were equally divided over Scene 4 but in this case it is women who were more positive in their opinions. The consistently higher scores expressed by women for 
characteristics of this scene may be attributable to the wider meanings they associate with the buildings and activities within them. This is a commercial shopping centre.

Although only the mean score comparison for colour relationships can be classified as statistically significant $(\mathrm{F}=3.58, \mathrm{M}=3.27, \mathrm{p}<0.05)$, the others are all approaching this level of significance. The response to the colour relationships in scene 4 is interesting. In 5 out of 6 cases men and women were reasonably aligned in their views regarding these. The exception for scene 5 has been discussed above. The cladding colours in scene 4 appear to be inherent in the materials, being coloured, exposed aggregate precast concrete panels and tinted glass, with a limited palette of accent colours. The colours appear to have been consciously chosen, which would be consistent with the nature of the development process, and all appear muted in hue and intensity. Women's responses to colour in this scene are ranked second behind scene 6 , whereas it is ranked third for men. The responses given to the colours of scenes 4 and 6 have the second ranked correlation $(0.236, \mathrm{p}<0.01)$ of all colour responses. Only the correlation between scenes 2 and 3 is stronger $(0.421, \mathrm{p}<0.01)$, with the ratings of colours in these scenes generally negative.

It appears that both men and women like to see colour in the built environment, whether applied or inherent in the materials. Where there is absence of colour (scene 2) or the colours appear dirty (scene 3) responses were generally negative. Both genders like to see coordinated colour schemes in muted pallets (scenes 4 and 6). Opinions begin to diverge when strong colour is used. Men have a higher preference rating for the colours in scene 1 , and when colour appears to be used as an accent (scene 5) men remain generally positive but women are negative. These observations will be checked further in Study Two.

\subsubsection{Age comparisons}

The built environment has to be for people of all ages. A rich urban life attracts people from many different backgrounds with a diversity of ages. People of different ages may perceive the environment differently, and perceptions may change with age. However, there is very little literature comparing people's perceptions of the environment based on age. Stamps III (1999) produced a meta-analysis of demographic effects in environmental aesthetics, referencing 107 independent sources. Only one of his reference studies, dating to 1989 , looked at differences in relation to buildings. Even then, that study distinguished only between people under and over the age of 12 . Four others looked at the effects of 
age on perceptions of landscape scenes. It seems that further examination of demographic differences on people's perceptions of urban streetscapes could help designers and other researchers better understand the influence of age on preference.

Five age categories were defined in the survey form with the number of respondents falling into each shown in parentheses: under 25 (22), 25-39 (70), 40-54 (62), 55-69(23) and $70+(4)$. The number of $70+$ respondents is less than ideal and it would be difficult to argue validity of the findings for this group.

Comparing mean scores for height relationships across the six scenes revealed that people aged 55-69 were less positive about consistency, as they gave lower preference ratings for scenes 2, 4 and 6 (table 5.8). These scenes each exhibit nearly consistent height relationships across them.

Table 5.8: Comparison of mean scores given by age (5 categories) for the height relationships in each scene

\begin{tabular}{|l|c|c|c|c|c|c|}
\hline & $<\mathbf{2 5}$ years & $\mathbf{2 5 - 3 9}$ & $\mathbf{4 0 - 5 4}$ & $\mathbf{5 5 - 6 9}$ & $\mathbf{7 0 +}$ & $\begin{array}{l}\text { Sig.(2- } \\
\text { tailed) }\end{array}$ \\
\hline Scene 1 & 2.73 & 2.80 & 2.74 & 3.00 & 2.75 & \\
\hline Scene 2 & 3.77 & 3.49 & 3.13 & 3.22 & 4.25 & $\mathrm{p}<0.05$ \\
\hline Scene 3 & 3.55 & 3.43 & 3.67 & 3.57 & 3.75 & \\
\hline Scene 4 & 3.67 & 3.53 & 3.41 & 3.22 & 4.25 & \\
\hline Scene 5 & 2.95 & 2.83 & 2.81 & 3.13 & 3.25 & \\
\hline Scene 6 & 3.77 & 3.54 & 3.58 & 3.17 & 3.25 & \\
\hline
\end{tabular}

The score this age group gave to scene $6($ mean $=3.17)$ was nearly half a point lower than scores given by younger people. On the other hand, this same group gave higher preference ratings for the height relationships in scenes 1 and 5, which have considerable variation. For scene 3, people in this group had views consistent with all other age groups. The height preferences expressed in relation to scene 2 were statistically significant $(p<0.05)$, indicating that preferences for these uniform height relationships diminish with age. This is confirmed by the responses to scene 6 , although the significance of these mean scores is not as high.

It was then decided to create new age data by changing the intervals. The purpose of this was to enhance the clarity of groups by age difference, and assimilate the small number 
of 70+ respondents into a larger group. Additionally, the reorganisation accounted for the age when a person might have influence over urban transformation. The three categories that resulted from this reorganisation are; under 25, 25-54 and 55+, with the large middle group, representing probable working age.

With the new groupings the preferences for height variation expressed by those 55 years and over is maintained, although the mean score differences are slightly lower (table 5.9). The lower preference ratings by group for scenes with low levels of height variation is also clearer. On the other hand, those under 25 appear to have held contrasting views. Younger people were more positive about consistent height relationships (scenes 2, 4 and 6) than either of the other two groupings. This is not however, complemented by lower preference ratings for strongly articulated height relationships, as young people aligned with the preferences of the 25-54 age group. Those who are nearing the end of their working lives $(55+)$ were more tolerant of height variations and less of scenes with uniform height. For scene 3, where the variations in height are moderated by the order generated by architectural styles, colour and finishes, preference scores for height relationships were close for all three age groups.

Table 5.9: Comparison of mean scores given by age (3 categories) for the height relationships in each scene

\begin{tabular}{|l|c|c|c|}
\hline & $<\mathbf{2 5}$ years & $\mathbf{2 5 - 5 4}$ years & Over 55 years \\
\hline Scene 1 & 2.73 & 2.77 & $\mathbf{2 . 9 6}$ \\
\hline Scene 2 & 3.77 & 3.32 & 3.37 \\
\hline Scene 3 & 3.55 & 3.55 & 3.59 \\
\hline Scene 4 & 3.67 & 3.47 & 3.37 \\
\hline Scene 5 & 2.95 & 2.82 & 3.15 \\
\hline Scene 6 & 3.77 & 3.56 & 3.19 \\
\hline
\end{tabular}

Responses to the question of colour relationships for scene 1 (figure 5.3) deserves further investigation with the new age groupings. Table 5.10, comparing the means scores for this question, shows the range is 0.82 and that each group is distinct from the others. The comparison is significant at $\mathrm{p}<0.01$. Although the colour relationships in scene 1 have not been designed in the manner of scenes 4 and 6 , there is colour coordination between buildings arising from use of a limited materials palette. Variations in brick tone provide 
visual interest across the scene and brick buildings help create a sense of order that allows buildings $B, C$ and $G$ to vary. Other factors, such as scale relationships between the colour areas and the positive heritage meanings that may be associated with brick structures may also be at play. For colour issues in the other five scenes, there is a reasonable consistency between the new age groupings.

Table 5.10: Comparison of mean scores given by age (3 categories) for the colour relationships in scene 1

\begin{tabular}{|l|c|c|c|}
\hline & Under 25 years & 25-54 years & Over 55 years \\
\hline Scene 1 colours & 3.18 & 3.42 & 4.00 \\
\hline
\end{tabular}

\subsubsection{Comparing change professionals and lay people's opinions}

This section examines the preferences expressed by professional designers, planning professionals and those not trained in these fields, which form a particular interest for this research, because professionals administer design control on behalf of the lay public. Respondents were given nine occupation choices, including architecture/urban design and planning. The data were later manipulated to create different categories for comparison. For example, architects/urban designers were grouped with planners to create a category called change professionals, recognising these are the key professions deciding how urban streetscapes and other parts of the built environment change.

In his extensive analysis of earlier empiric studies, Stamps III (1999) described similarities and differences in aesthetic evaluation for different demographic groups. He found a strong correlation $(\mathrm{r}=0.89)$ between the evaluations of designers and non-designers when they viewed natural elements and architecture, apart from when the buildings fell into the category of avant-garde. The present research provides an opportunity to re-examine the relative preferences of each group, albeit in relation to urban streetscapes presented in photographic format.

The first set of comparisons is made between change professionals and the lay public. Table 5.11 sets out the mean value scores for pleasantness and overall evaluation of the six scenes. These comparisons suggest that lay people tend to be more positive in their views than change professionals, who may be more critical when evaluating examples from their own field of expertise. Planners, for example, are entrusted with critical 
evaluation of development proposals and their work is often subjected to intense scrutiny in quasi-legal and legal settings. Architects are educated to think critically about their own work and about the built environment generally (Wilson 1996). Beyond this general observation, views are aligned reasonably well for scenes 1-3. The mean scores are nearly the same for both groups. Scene 3 ranks second most preferred for professionals and third for lay people. The generally positive response to scene 3 has been discussed in section 5.3.

Table 5.11: Comparison of mean scores given by change professionals and lay people for pleasantness and overall preference for all scenes

\begin{tabular}{|l|c|c|c|}
\hline & Change Professionals & Lay People & Sig. (2-tailed) \\
\hline Pleasantness 1 & 3.04 & 3.15 & \\
\hline Overall 1 & 3.09 & 3.12 & \\
\hline Pleasantness 2 & 2.65 & 2.75 & \\
\hline Overall 2 & 2.66 & 2.61 & \\
\hline Pleasantness 3 & 3.13 & 3.15 & \\
\hline Overall 3 & 3.23 & 3.24 & \\
\hline Pleasantness 4 & 2.77 & 3.45 & $\mathrm{p}<0.005$ \\
\hline Overall 4 & 2.71 & 3.39 & $\mathrm{p}<0.005$ \\
\hline Pleasantness 5 & 2.39 & 2.61 & \\
\hline Overall 5 & 2.36 & 2.55 & \\
\hline Pleasantness 6 & 3.39 & 3.63 & \\
\hline Overall 6 & 3.24 & 3.59 & $\mathrm{p}<0.05$ \\
\hline
\end{tabular}

Although scene 6 has the highest preference rating for both groups, closer inspection reveals that designers and planners are divided in their opinions. Planners (mean=3.57) and the lay public (mean=3.59) are quite close, both having reasonably positive overall ratings. Not only do architects give an overall rating well below the other two groups, but on average they give it a negative (mean $=2.91)$ evaluation. Clearly designers do not see sufficient merit in the highly ordered arrangement of the five buildings in scene 6 . Only $40 \%$ of the designers liked the scene either somewhat or strongly, whereas $62.8 \%$ of planners and $61.1 \%$ of lay people gave it positive overall evaluations. 
Architects' overall preferences were linked most closely to the responses they gave to the question on style relationships $(\mathrm{R}=0.867)$. Lay people align with the designers in this case, as styles, followed by colour relationships, are the two strongest predictors of their overall preference scores. Architects had a generally negative response to the style relationships in scene $6($ mean $=2.89)$, possible because the low level of variation, tending toward monotony, in architectural styles across the scene displeased them. Scene 3, which appears to have the second most consistent pattern of architectural styles, was rated most highly by architects in response to this question. It seems the threshold between ordered visual interest and visual monotony is a very narrow one for architects. They rated scene 6 poorly and, as a group, they would seem to prefer greater differences in individual building designs. In contrast, the non-architects rated the relationship of architectural styles in scenes 3 and 6 very highly with virtually the same median score. The mean score comparisons of the overall preference and style questions for scene 6 are significant at $\mathrm{p}<0.01$ and $\mathrm{p}<0.005$ respectively. It seems that while non-architects appear to be pleased with perceptible similarities of style, they are not dissuaded by virtual repetition of style along the length of a street.

The visual characteristics of scene 3 provide common ground for positive preferences from change professionals and lay people. The scoring of both groups is closely aligned for pleasantness and overall preference (table 5.11). Generally both groups also expressed positive opinions about the buildings in this scene fitting with each other, at $67.1 \%$ of all respondents, $63.4 \%$ of change professionals and $69.7 \%$ of lay people.

Looking further at the results for scene 3 , there is very close alignment of mean scores for pleasantness and relationship between styles for change professionals and lay people (table 5.12), although architects seemed more willing to express an opinion, positive or negative, than the other groups. This is witnessed by the fact $15.9 \%$ of architects had a neutral opinion, whereas nearly double that percentage were neutral in the other two occupational groupings. This led to a slightly higher percentage of negative opinions (36.3\%) but also led to $47.7 \%$ of architects having a somewhat or very pleasant response. This comparatively low level of ambivalence was matched only by the responses to scene 6 , where architects had a much lower pleasantness response (mean=3.16 compared to mean=3.64, $\mathrm{p}<0.05$ ) and overall opinion of the scene (mean=2.98 compared with mean=3.64, $\mathrm{p}<0.01)$. 
Table 5.12: Responses to scene three by occupational groups

\begin{tabular}{|l|c|c|c|c|}
\hline & Architects & Planners & Lay People & Sig. (2-tailed) \\
\hline Pleasantness 3 & 3.11 & 3.15 & 3.15 & \\
\hline Heights 3 & 3.68 & 3.66 & 3.47 & \\
\hline Styles 3 & 3.55 & 3.65 & 3.54 & \\
\hline Finishes 3 & 3.26 & 3.54 & 3.46 & \\
\hline Colours 3 & 2.75 & 2.56 & 3.01 & \\
\hline Overall 3 & 3.30 & 3.14 & 3.24 & \\
\hline
\end{tabular}

The opinions of change professionals and lay people differed for colour response in scene 3 , with its areas of unpainted concrete (table 5.12). Both architects and planners considered the colour relationship to be negative while the mean score for lay people was just above neutral (mean=3.01). Once again architects, more than any other group, were able to form an opinion as only 13.6\% had neutral responses. Across the colour relationships of all scenes, planners returned their lowest mean score for scene 3. Concrete is often given negative connotations (Forty 2012: 9-10) but these results suggest a different story. Looking at the mean responses for finishes and materials, the public response average is highest, and the other groups are positive. Preliminary findings arising from Study One suggest lay people are not only tolerant of grey, natural concrete building facades, but may well be attracted to them.

Scene 3 is the only one of the six in this study that includes soft landscaping, in this case taking the form of street trees and flower beds in the foreground. The softening effect and colours provided by each of these may have influenced the responses of all groups. It may also be that professionals were better able to set aside the influence of landscaping and concentrate their responses on the built form. The effects of landscaping are outside the scope of this thesis, although there is a considerable literature confirming the attraction scenes with trees and other landscape features hold for people (Nasar 1990, Lohr and Pearson-Mims 2006). Orians (1986: 10-15) also discussed human fascination with freestanding trees, referring to human evolution on the African savannah as a background factor in this attraction. 
Table 5.13: Mean preference scores of the three occupational groups for the streetscape characteristics of scene 4

\begin{tabular}{|l|c|c|c|c|}
\hline & Architects & Planners & Lay People & Significance \\
\hline Pleasantness 4 & 2.52 & 3.09 & 3.45 & $\mathrm{p}<0.005$ \\
\hline Heights 4 & 2.98 & 3.66 & 3.61 & $\mathrm{p}<0.005$ \\
\hline Styles 4 & 2.63 & 3.09 & 3.47 & $\mathrm{p}<0.005$ \\
\hline Finishes 4 & 2.80 & 3.11 & 3.50 & $\mathrm{p}<0.005$ \\
\hline Colours 4 & 2.89 & 3.43 & 3.61 & $\mathrm{p}<0.005$ \\
\hline Overall 4 & 2.45 & 3.03 & 3.39 & $\mathrm{p}<0.005$ \\
\hline
\end{tabular}

Scene 4 (figure 5.4) presents a point of departure between the professional and lay groupings. The stark differences between change professionals and lay people for pleasantness (mean $=2.77$ and 3.45 respectively, $\mathrm{p}<0.005$ ) and overall preference (mean=2.71 and 3.39 respectively, $\mathrm{p}<0.005$ ) deserve further analysis (table 5.13). Comparing the mean scores for responses to each question addressing scene 4 , architects consistently rated it lower than lay people did. Planners tended to be somewhere in the middle, although there is alignment between lay people and planners over height relationships. These comparisons are all statistically significant.

Two factors may help explain the reasons for such strong and consistent differences of opinion. The scene represents a comprehensive redevelopment of an entire urban block and is built as one building designed to appear as two. Jane Jacobs, referring to this form of development stated that "cataclysmic money pours into an area in concentrated form, producing drastic changes" (Jacobs 1961: 293). She felt large scale redevelopment destroys diversity, which she held to be a fundamental characteristic of good streets. The dis-benefits of comprehensive redevelopment and the benefits arising through incremental change are supported in the literature (see for example Carmona, Heath et al. 2010: 258-265). Architects' responses may well have been influenced by such sentiments, which have become part of the professional culture (Hubbard 1994, Wilson 1996). This suggests the architects' negative opinions of the shopping centre depicted in scene 4 may in part be attributed to broadly held professional opinions against this type of development. 
A second factor that may have influenced lay people's opinions of scene 4, is the positive associations people form for shopping and consumption (Foxall and Greenley 1998). The images provided to respondents clearly indicate, by way of signage, the nature of the activities that take place within the building. Arguably, the form and façade design are also redolent of the large shopping centre typology. These factors could have positively influenced people's aesthetic responses. While all respondents may have felt this positive association (table 5.4 shows women were more positive about all aspects), the negative associations some architects have with the large format typology may have affected their otherwise positive responses to the activities. This result raises the matter of meanings associated with people's experience of the urban environment. The influence such meanings have on people's aesthetic responses is well established in the literature (see for example Rapoport 1982: 13, Lang 1988). Whether this dimension of aesthetic perception can be accounted for in design review will be addressed further in chapter 7 .

\subsection{Summary of Study One findings}

Study One collected people's aesthetic responses to six street scenes. The scenes were selected to portray a range of typical spatial relationships that design review must address in urban redevelopment. The streetscapes were presented as photographic elevations and responses sought from a wide demographic range. The responses included a high proportion of planners and designers, the two key professions active in design control.

There were generally strong preferences for coordinated visual interest in the building facades. The strongest sense of visual interest was generated through repetition of windows, creating coherent patterns and visual texture, with scenes 3 and 6 presenting the most consistent and coherent displays. Scenes 1 and 2 also incorporated buildings with window openings in building facades but the patterns were not as consistent. Other sources of visual interest include strongly expressed vertical mullions (scene 3) and horizontal mouldings and cornice lines (scene 6).

While consistency of height is highly valued it seems this is not consistent across all occupational groups. In particular, architects appeared to dislike the consistency of height in scene 6. It seems some variation is preferred and again, scene 3 provides the common ground in this respect. The buildings of scene 3 varied between two and three storeys in height and these relationships were clearly favoured. 
As the visual stimuli were presented as two-dimensional elevations it was difficult to gauge preferences for the actual three-dimensional modelling of the streetscape. The latter is the second of three 'levels' of streetscape perception identified by Stamps III (2000: 39) that this research also adopts. Preferences at this perceptual level will be recorded in the second study. Of the design characteristic relationships between buildings examined in Study One (height, colour, styles, materials and finishes), the strongest indicator of overall preference was the relationship between the architectural styles. This correlates with widely held preferences for places with clear architectural character, as spatial character is understood to derive through consistency (refer section 5.3).

Many people had negative reactions to buildings that are large in the overall pattern of the streetscape. Such size problems arise from contemporary redevelopment practices, where economies of scale are sought in part by aggregating smaller properties. It seems the efforts made by designers to break down visual bulk were unsuccessful. Techniques such as articulation of the façade along its length, modelling of a form over its height and use of visually lightweight materials did not convince the public that a large building is compatible with its adjoining neighbours, when such a building stands out in a collection of much smaller structures. Whether two such buildings in a street scene would have the same effect remains unknown

In terms of demographic bases for aesthetic response, with a few exceptions, Study One confirmed earlier studies showing no difference in the preferences expressed by men and women. Age group comparisons revealed a slight tendency for the 55+ group to prefer greater visual variety, whether through colour or height relationships. Whether these differences reflect a change in opinion as people get older or are the preferences of a specific age-related culture are again unknown.

Data analysis also filtered responses by occupation, especially between change professionals (the architects and planners groups), who are those involved in design review, and lay people. It seems architects have low tolerance of uniformity, even when a scene is otherwise visually interesting. This finding is made on the basis of this group's generally low scoring of scene 6, the most consistent of all those presented. Similarly, architects appeared to be more tolerant of variations, such as between the heights of buildings, their architectural styles and the materials and finishes. Their generally positive responses to questions concerning scene 1 provide further evidence of this. Despite these 
differences with planners and the lay public it appears that all three groups converge in their positive opinions of scene 3 . This suggests that moderate variations in height, tempered by clear consistency in the architectural styles, can produce a well-liked streetscape.

However, the preferences expressed for scene 3 should be checked further, as this is the only scene in which soft landscaping appeared. The literature is clear that there are strongly held preferences for soft landscape elements in depictions of the built environment. Study Two provides an opportunity to look more closely at this.

Finally, the results provide evidence of the strong influence meanings have on preference. The literature notes that buildings or scenes with strong associational meanings for a group, or for an entire population, will be perceived more positively. The large shopping centre in scene 4 was evaluated more positively by women, possibly due to the pleasant associations with shopping as a leisure activity. On the other hand, designers and planners felt more negative about this scene, a response perhaps associated with the strong negative meanings the built environment professional culture attaches to large, comprehensive redevelopment.

Study Two builds on knowledge gained from Study One about people's preferences and how they are expressed. The results of the fieldwork, undertaken as a set of three separate case studies, are presented in chapters 6 to 8 . Study Two attempts to shed further light on the matters raised in Study One by asking people to record their experiences of real urban streetscapes. 
Chapter 6

\section{Study Two - College Street}

\subsection{Introduction}

Chapter 6 introduces the follow-up to Study One, the first of three independent case studies investigating people's experiences of comparable urban streetscapes. Study One invited people to respond to photographic representations of urban street scenes. The different streets were presented as elevations of the building facades along one side of the street, depicting particular circumstances and building relationships that were relevant to the problem underpinning the research questions. These views, although unnatural in the ways people experience urban space, enabled efficient investigation of the streetscapes. To supplement the data collected in Study One, Study Two was designed to allow people to report on their experience in the selected street.

Three cases were selected following a process that sought to eliminate or at least reduce a number of potentially distracting variables such as vehicular traffic, vacant sites and differences in orientation. The rationale for selection of each case study site is outlined in section 4.4.1. Chapters 6 to 8 discuss the three streets in the order the fieldwork was conducted.

College Street in Wellington was first used for the pilot study of the survey methods before being investigated in the spring of 2013 for Study Two. Following a general analysis of the data collected, two focus groups were convened to discuss the findings. These discussions are reported in section 6.5. 
Then, in the summer of 2013-14, people in Auckland were surveyed about their perceptions of Tyler Street in the Britomart area. Overlapping with this, the second Auckland study was launched to survey opinions of the buildings along Wyndham and Kingston Streets in the city's central business district. A brief summary of the results of each case study is provided at the end of each chapter. Chapter 9 analyses and discusses the results across Studies One and Two in an effort to identify the key research findings and answer the research questions.

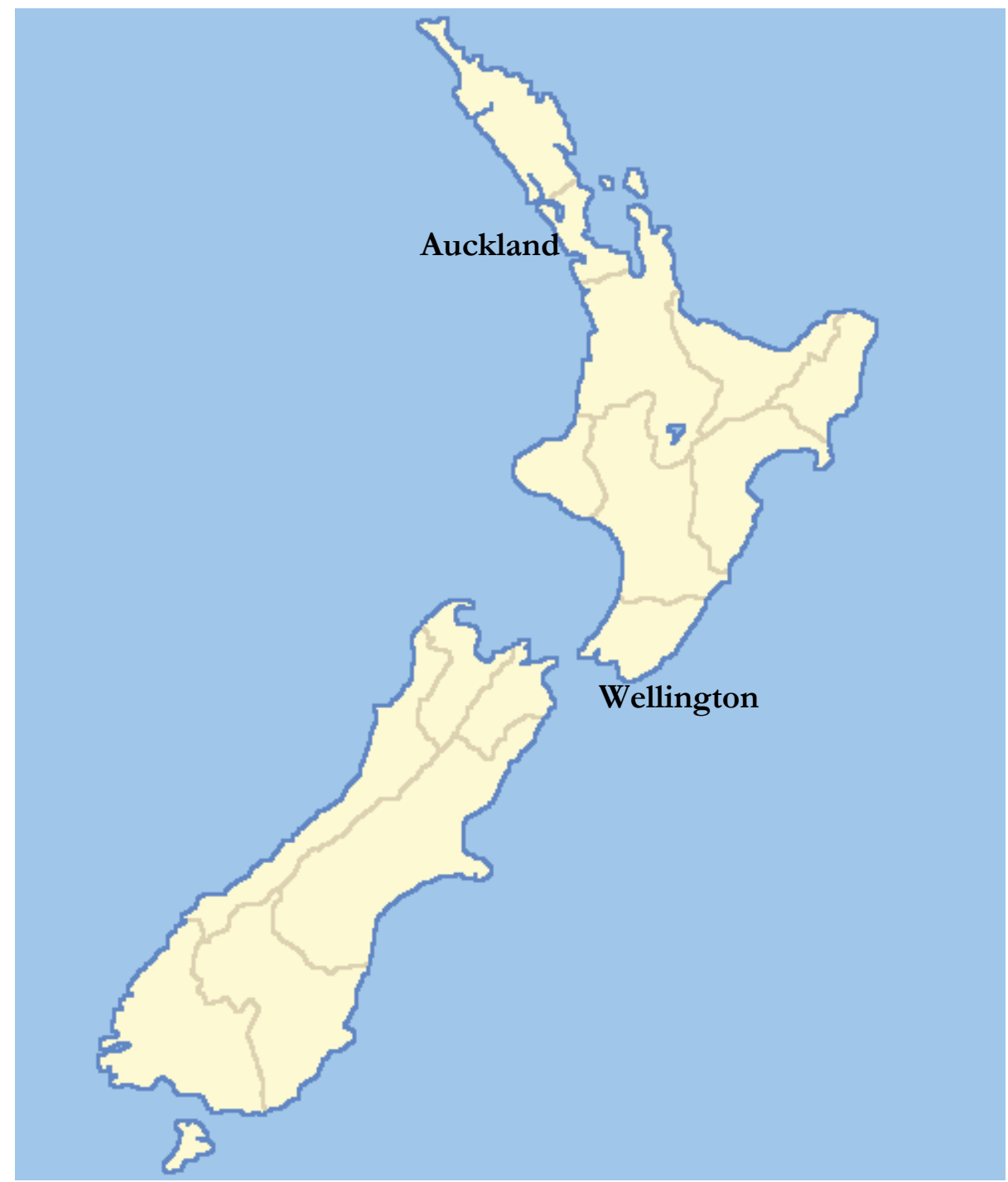

Figure 6.1: Location of Wellington and Auckland, the two cities where the three case studies are located.

Source: www.mapsof.net 


\subsection{College Street Wellington}

\subsubsection{Case study site description}

The 265 metre long College Street runs between Tory Street to the west and Cambridge/Kent Terraces to the east in the Te Aro area of Wellington (figure 6.2). Te Aro is a large and generally flat part of the city, where ongoing changes are stimulated by demand for residential accommodation close to the city centre. The increasing residential population in turn helps stimulate demand for other services. College Street is an example of this transformation, where new residential and retail activities are seen alongside light industrial and warehouse activities.

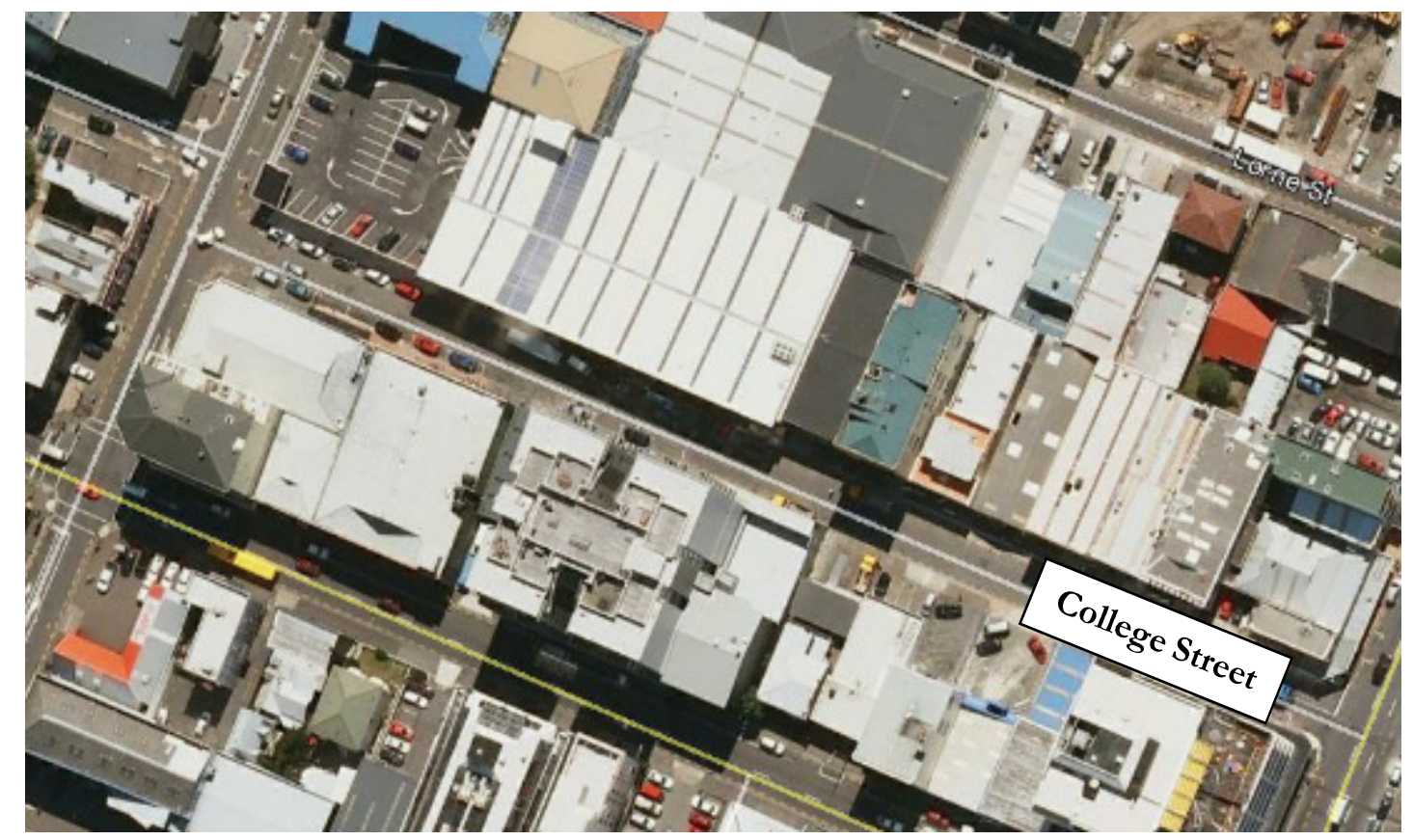

Figure 6.2.: Aerial view of College Street, running between Cambridge Terrace in the east and Tory Street in the west.

Source: GoogleEarth.com

Although building typologies are similar, the ranges of building heights and frontage widths on the two sides of College Street are quite different. Figure 6.3 presents the drawn elevations of both sides of the street. These elevations were used to orient respondents as they completed the surveys. The eastern end of the north side - buildings $\mathrm{K}$ to $\mathrm{R}-$ has a steady rhythm of narrow frontages up to building $\mathrm{S}$, a structure that takes up nearly half the length of the street itself. The heights along this side change by no more than 2 storeys at each boundary and most variations are less than this. Building $\mathrm{S}$ is a key 
destination in this street, being a food and consumer goods market with a regional profile. The southern side has much greater variation in building widths and heights. The most abrupt change in height is the 7 storey differences between buildings $C$ and $D$ and between buildings $\mathrm{F}$ and $\mathrm{G}$.

\subsubsection{Summary of responses}

The College Street survey was conducted between 19 September and 30 October 2013. Invitations to participate were left with the Kelburn Play Centre, Raglan Roast coffee shop and the Wellington City Council offices. Notices inviting participation were sent to staff members of the Wellington School of Medicine and the Wellington branch of the University of the $3^{\text {rd }}$ Age through their administrators. These efforts were made to generate responses from a broad range of demographic backgrounds, particularly targeting young parents, older people and professionals working in the planning field. Invitations were also sent to a number of architectural practices to target that group of change professionals. In total 75 survey responses were received and the data entered into SPSS software for analysis. It is difficult to discuss a response rate as survey forms were generally only collected by those people who intended to complete the survey. Respondents were rewarded with a prepaid coffee or hot drink at one of three coffee shops in the city, where the completed forms were also collected. Respondents to the College Street survey were then invited to indicate willingness to participate in the focus group discussions that would be arranged to discuss the preliminary findings. There were 29 participants (39\%) who volunteered for these.

The demographic profile of the respondents was broad. In terms of gender, $52 \%$ of the respondents were male and $48 \%$ female, with $26 \%$ change professionals and $74 \%$ lay members of the public. In terms of formal education $14 \%$ had secondary school as their highest qualification and $49 \%$ of the respondents had a post graduate degree. Table 6.1 sets out additional demographic details of the sample. 
Table 6.1: Distribution of sample by age, occupation and residential circumstances in the College Street case study.

\begin{tabular}{|l|r|}
\hline & Distribution (\%) \\
\hline Age & 27.4 \\
Under 25 & 31.5 \\
$25-39$ & 27.4 \\
$40-54$ & 13.7 \\
$55-69$ & 0 \\
Over 70 & 100.0 \\
\hline Occupation & \\
Planner & 10.8 \\
Designer & 14.9 \\
Other professional & 35.1 \\
Trade & 2.7 \\
Home unpaid & 2.7 \\
Student & 28.4 \\
Other & 5.4 \\
Total & 100.0 \\
\hline Live & \\
Rural & 3.3 \\
Suburban & 45.0 \\
Inner suburb & 25.0 \\
Townhouse & 8.3 \\
Suburban apartment & 6.7 \\
Central apartment & 11.7 \\
Total & 100.0 \\
\hline
\end{tabular}




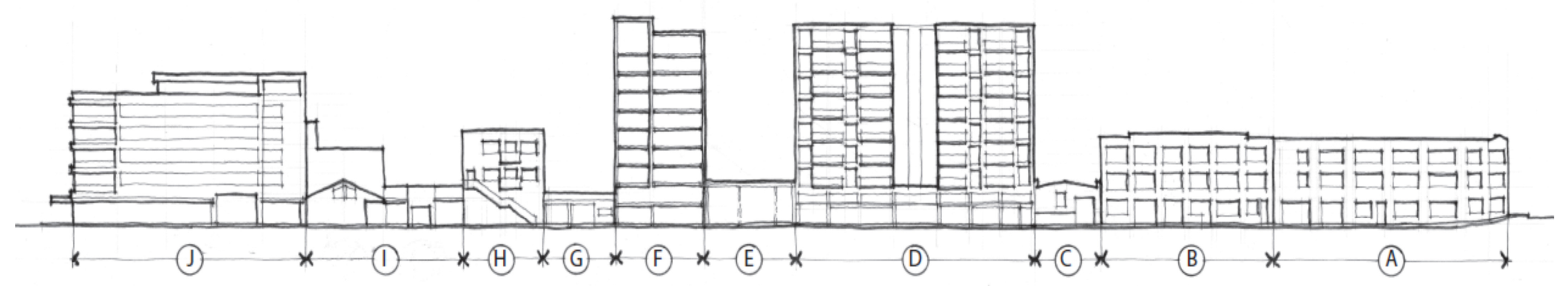

College Street South Elevation: no scale

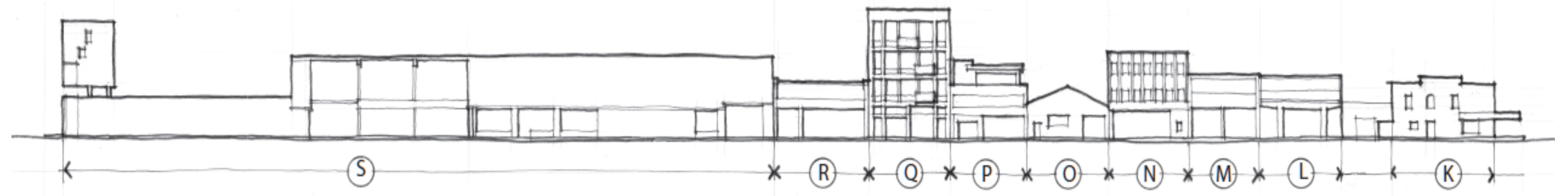

College Street North Elevation: no scale

Figure 6.3.: Outline elevations of all buildings along College Street were provided to help survey respondents orient themselves during the survey 



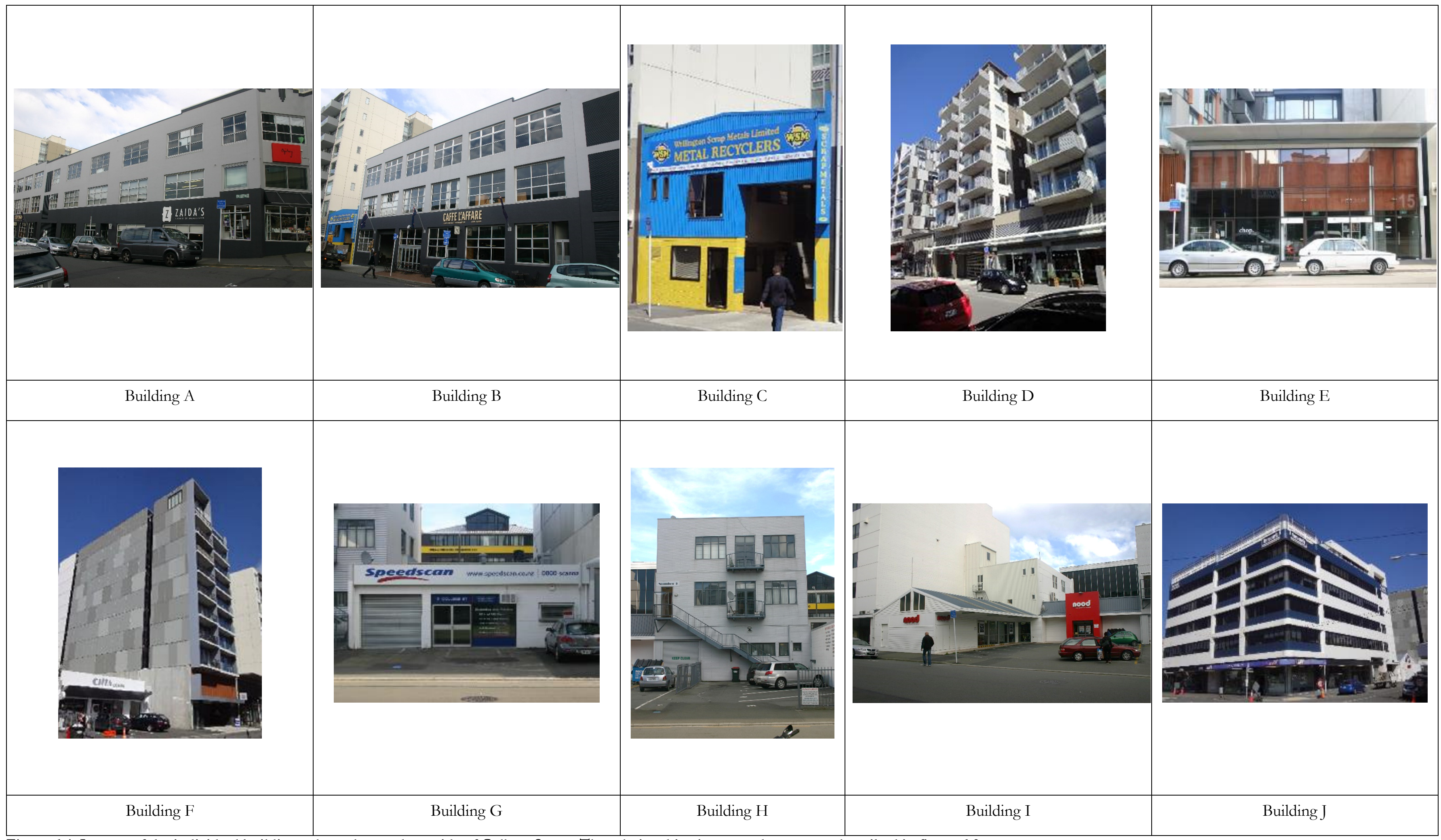

Figure 6.4: Images of the individual buildings along the southern side of College Street. The relationships between them are as described in figure 6.3

Source: author 



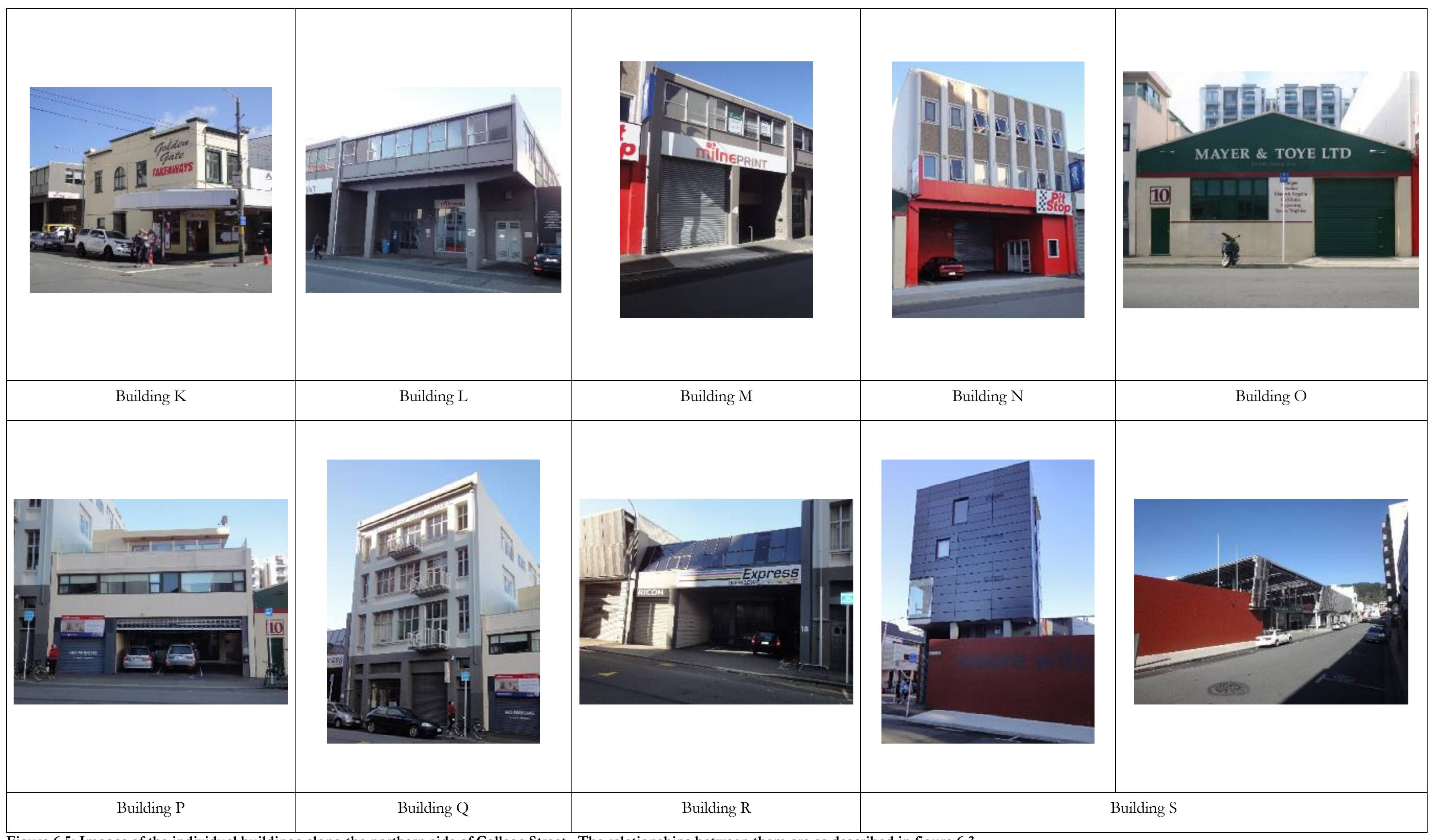

Figure 6.5: Images of the individual buildings along the northern side of College Street. The relationships between them are as described in figure 6.3.

Source: author 



\subsection{General preferences}

\subsubsection{Overall streetscape}

People generally preferred the relationships of building heights on the northern side of the street $($ mean $=3.43)$ to the southern side $($ mean=2.81) (table 6.2). Two people rated the height relationships on the northern side very pleasant (score $=5)$ and both indicated that the variations in height added interest. One of these thought the similarities in height at the western end - buildings $\mathrm{A}$ and $\mathrm{B}$ - were disappointing. With the mean score falling below the neutral line, $52 \%$ of the respondents felt the height relationships on the south side were unpleasant. Reasons given by respondents for their negative views centred on the extent of the height variations.

For building heights on the northern side, $51.3 \%$ of respondents thought these were pleasant and only $17.6 \%$ unpleasant. One female respondent noted that she liked the "variation within a low range". This comment was typical of those given by others in support of their positive preference scores for this side of the street.

Table 6.2: Mean preference scores for streetscape characteristics

\begin{tabular}{|c|c|c|}
\hline & Mean & Std. Deviation \\
\hline South side streetscape & & \\
\hline Building Heights & 2.81 & 1.099 \\
\hline Facade Alignment & 2.57 & 1.016 \\
\hline Facade Styles & 2.71 & .969 \\
\hline North side streetscape & & \\
\hline Building Heights & 3.43 & .938 \\
\hline Facade Alignment & 3.18 & .887 \\
\hline Facade Styles & 2.70 & .989 \\
\hline
\end{tabular}

The difference between the mean scores for alignment of facades (mean diff $=0.61$ ) is similar to that for height relationships (mean diff=0.62), although scores for the former are lower. Buildings A to F help establish a strong alignment at street level on the south side of the street (figure 6.6). Above this, the alignment is only broken by differences in building height and by the design of building $\mathrm{D}$, which is stepped in plan at the upper floor levels to create light wells, and E, which is single storey. However, at the eastern end of the street buildings $\mathrm{G}, \mathrm{H}$ and I are set back to different extents and each has an area of car parking between it and the street edge. Perhaps this poor edge development 
in combination with differences in building height over the whole length, has led the majority of respondents to dislike the southern side of the street, as only $22.7 \%$ of respondents found the alignment pleasant.

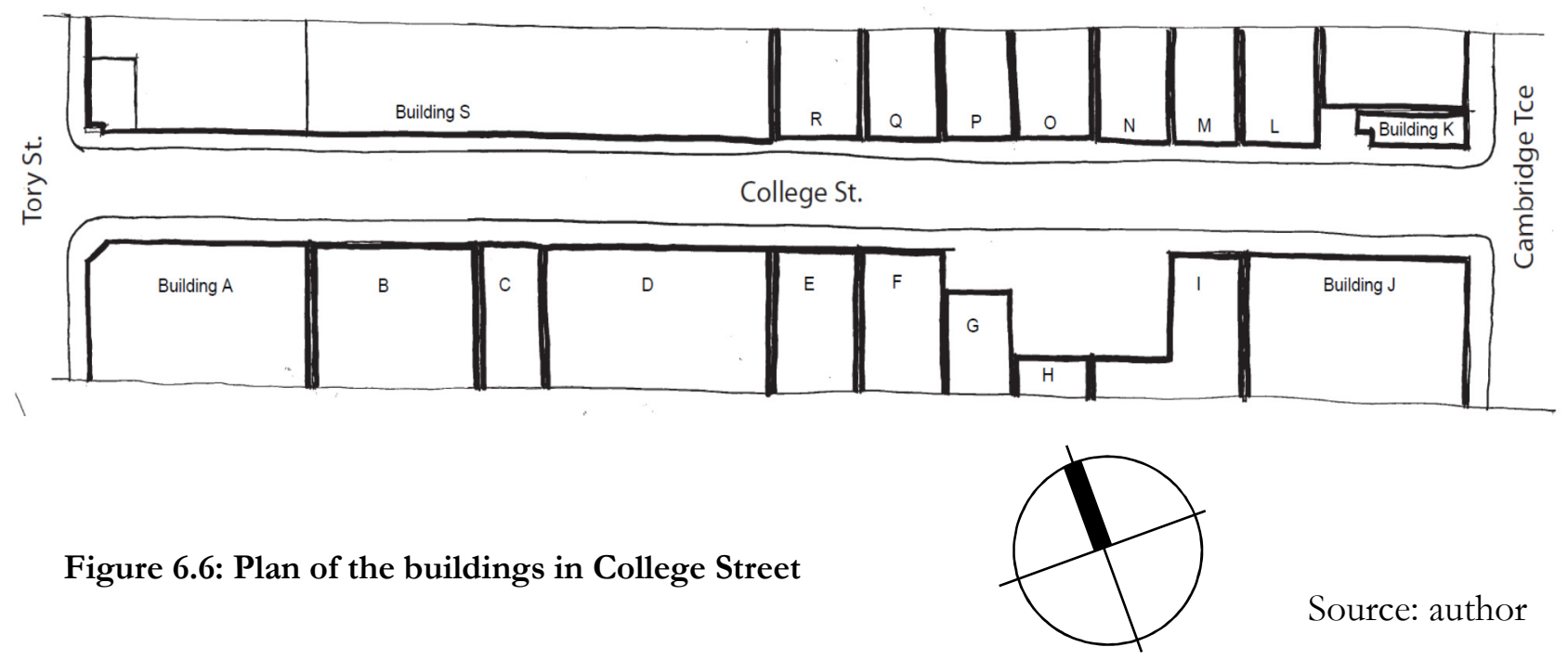

Façade alignment on the north side received an overall mean score of 3.18 , and $37 \%$ of respondents felt positively about this, although there was a high number $(40 \%)$ of neutral scores. One factor that may have lowered this mean score is the poor edge definition provided by the buildings at street level. Many buildings, for example building $\mathrm{L}$ in figure 6.5, have been set back at ground level to allow for vehicle parking, consistent with the light industrial building typology. While this may have practical benefits for building users, it also appears to have negatively influenced preferences for this streetscape.

The mean scores for relationships between façade design styles is consistent for both sides of the street. People were equally dismissive of the relationships on the south side $($ mean $=2.71)$ and the north side (mean=2.70). The distribution of scores is also similar with $23 \%$ (north) and $22.7 \%$ (south) recording a neutral opinion. Further analysis of the two variables reveals there is a low correlation $(\mathrm{r}=0.153)$. This in turn suggests that although the respondents considered the style relationships to be equally poor, there was little consistency in the way individuals felt about the relative aesthetic merits of each side of College Street. 


\subsubsection{Individual buildings}

Respondents were asked to indicate whether or not they liked each building viewed as they moved along the street in both directions and to provide two reasons for their answer from a list of eleven form or design attributes (table 6.3). Buildings Q (mean=2.45) and S (mean $=2.32$ ) were the two best and buildings $\mathrm{R}($ mean=1.77) and $\mathrm{J}($ mean $=1.79)$ the two liked least by the respondents.

Table 6.3: Mean preference scores on 3 point scale for individual buildings

\begin{tabular}{|l|c|c|}
\hline & Mean & $\begin{array}{c}\text { Std. } \\
\text { Deviation }\end{array}$ \\
\hline A & 2.22 & .647 \\
\hline B & 2.28 & .676 \\
\hline C & 2.05 & .928 \\
\hline D & 2.08 & .748 \\
\hline E & 2.19 & .770 \\
\hline F & 2.26 & .741 \\
\hline G & 1.89 & .890 \\
\hline H & 1.84 & .810 \\
\hline I & 1.95 & .852 \\
\hline J & 1.79 & .827 \\
\hline K & 2.19 & .854 \\
\hline L & 1.95 & .809 \\
\hline M & 1.81 & .817 \\
\hline N & 1.98 & .877 \\
\hline O & 2.05 & .820 \\
\hline P & 1.86 & .833 \\
\hline Q & 2.45 & .740 \\
\hline R & 1.77 & .803 \\
\hline S & 2.32 & .825 \\
\hline
\end{tabular}

Building Q is a 4 storey, building approximately 21 metres high, on the north side of College Street (figure 6.7). Although named the Fletcher Trust Building, it is currently used for residential purposes, and seems to have been converted from its former commercial purpose. When this took place, a small balcony was added to each of the three upper floors. Of the respondents, 45 of the 75 liked the building and 11 disliked it. The former cited façade details (28 people) and height (15 people) as their key reasons for 
liking it, while those who disliked the building also cited façade details and windows/glass as their main reasons.

The public were enthusiastic about other aspects of building Q, as 64 respondents liked the design above ground floor level with only 3 people giving it a score below neutral. This led to a median score of 4.07 for design above street level on a scale of 5. Similarly, people liked the surface finishes above ground, ranking building Q second best in the street in this category, and the design at street level, ranking it first with a median score of 3.75. This positive appreciation of building Q warrants further analysis.

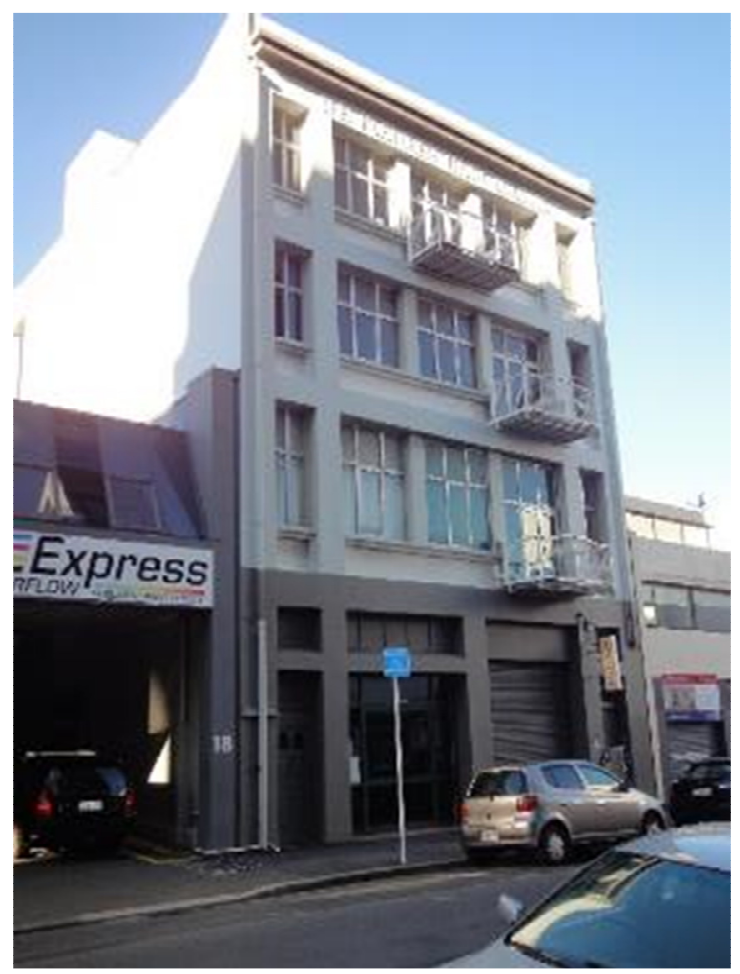

Figure 6.7: Building Q, liked best by the respondents in the survey of College Street.

Source: author

The street façade of building Q is modulated both vertically and across its width. The rhythms vary slightly in both directions and the columns and spandrels that help this modulation have considerable depth, giving the façade a three dimensional quality. Highlighting the façade details through colour also enhances the sense of modelling. The surface finishes are modest and in certain light conditions reveal the handmade nature of the plaster render. The three balconies provide additional visual interest and clues as to 
the primary use of these upper levels. Although one of the bays at ground level is covered by a security door at night, the façade is extensively glazed and supports retail activities. This helps generate visual interest and engagement with passers-by. The west facing side wall is blank but only two storeys can be seen above building $\mathrm{R}$. The side wall has generous window openings on the eastern boundary shared with building P. Overall the building is of a modest scale and construction that has acquired a patina that comes with age. Its functional uses can be easily read by people in the street. These may have been factors in the positive opinions about building Q.

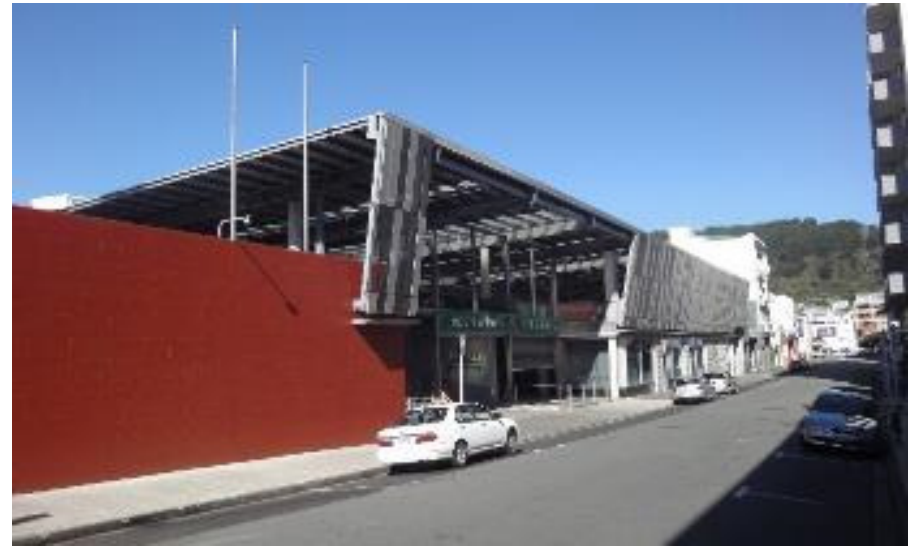

Figure 6.8: Building S seen from the west.

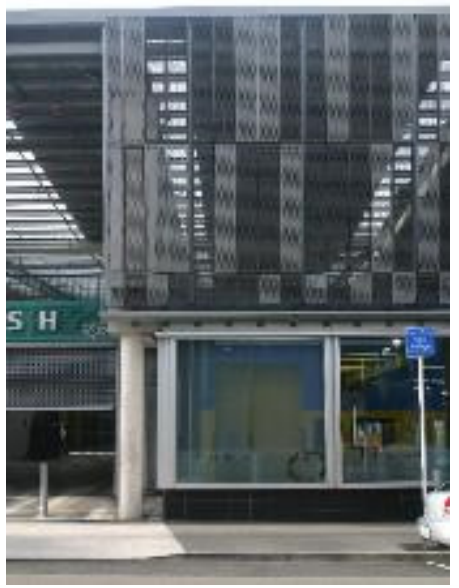

Figure 6.9: Detail of the building $S$ street façade

Over the past decade the site of building $\mathrm{S}$ has been redeveloped in several stages, although less than half of its College Street frontage has been changed during that time. The building is approximately 130 metres long and varies in design over that length (figures 6.8 and 6.9). This building was liked by 41 respondents and disliked by 17 . Those liking it cited the façade details and the overall shape as the main reasons for their opinions. These were also the two principal factors cited by those who disliked it.

Buildings $\mathrm{R}$ (mean=1.77 on 3 point scale) and $\mathrm{J}$ (mean $=1.79)$ were the two buildings liked least by respondents. Building $\mathrm{R}$ is a two storey commercial office building, with a relatively narrow site frontage, on the north side of the street (figure 6.10) and is between the two most well liked buildings ( $\mathrm{S}$ and $\mathrm{Q}$ ). This building creates no apparent relationship with the street as the ground level has a blank shutter over part of the frontage and three car parking spaces across the remainder. The windows at first floor level are 
tinted and sloped back, limiting the ability to look inside from the street. With the tinted windows and other colourings, the building is mostly dark grey. The way respondents felt about the design at street level is the strongest predictor of whether or not they liked the building overall. The 18 people who said they did not like the building indicated that overall shape was a factor and 16 people indicated the façade details (or seemingly the lack of these). While most people rated this building poorly, those under 25 years rated it positively. Additionally, professionals (mean=1.10) rated this building poorly while the lay members of the public (mean $=2.00)$ were significantly $(\mathrm{p}<0.005)$ less harsh in their views.

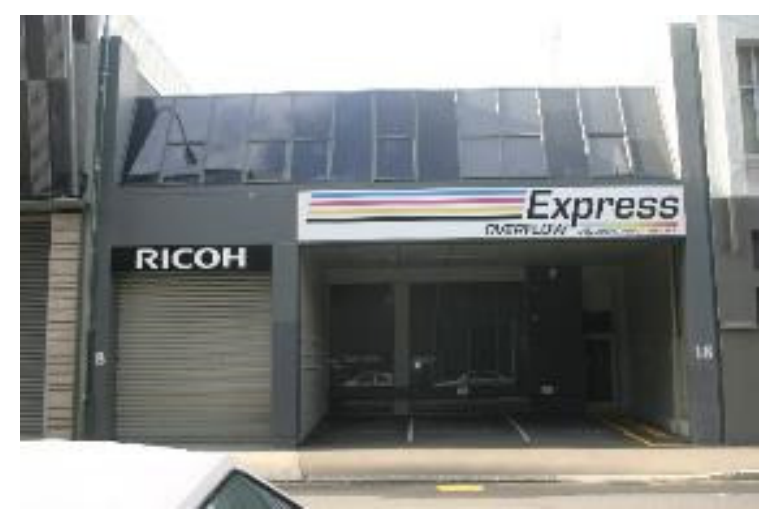

Figure 6.10: Building R

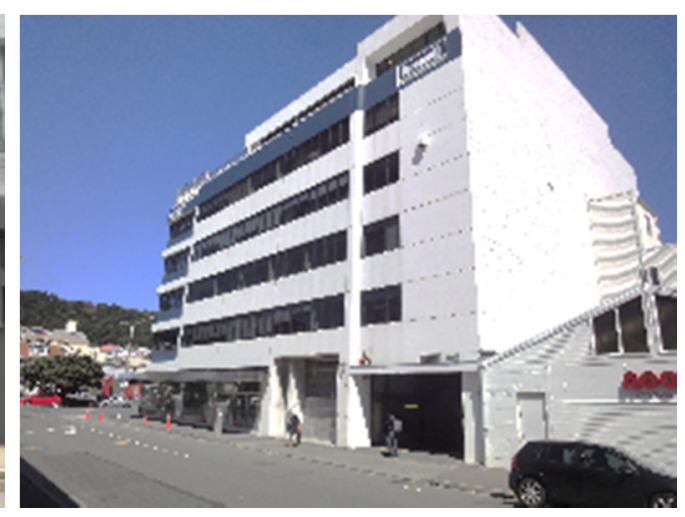

Figure 6.11: Building J

Source: author

Source: author

Building J is a multi-storey commercial office building at the eastern end of College Street. It faces the other direction onto a major boulevard/public space along Cambridge and Kent Terraces (figure 6.11). The façade, which is typical of its period of construction, employs horizontal strip glazing between solid spandrel panels of equal depth. Poor façade details and the overall shape of the building were cited most frequently as reasons people disliked building J. Through regression analysis, how people felt about the surface finishes above ground was the strongest predictor of people's overall opinion of building J. Again, professionals (mean $=1.26$ ) liked this building far less than the general public (mean=1.96, $\mathrm{p}<0.005)$. Students and those under 25 years felt positively about the building and nearly a third (28\%) of all respondents neither liked nor disliked it. Despite the low mean score the building was not absolutely disliked. As with the responses to building $\mathrm{R}$, the strength of the negative opinion of the change professionals brought the overall score for building $\mathrm{J}$ down. 
Respondents were asked to rate façade design characteristics for each building on a fivepoint Likert scale. The first was the overall design of the façade and the second the surface finishes. People were asked to record their feelings about the overall design at ground level. This was done to test whether different parts of a facade could elicit different responses. At ground level the emphasis is on the close relationship with pedestrians, often giving access to commercial activities, and on motor vehicles, where off street car parking car parking is provided. Floors above ground have traditionally had higher proportions of solid wall area, perhaps reflecting the more private nature of the activities on upper levels. Upper levels are also perceived from greater distance, such as across the street, and make a greater contribution to the overall image of the building. Where design review of urban buildings is undertaken in the regulatory planning process, these are aspects it should consider (Carmona, Heath et al. 2010: 316, Wellington City Council 2012).

It did not seem relevant to consider responses to design and surface finishes above ground floor for single storey buildings. Of those that were rated, the perceptions of the design quality above ground level for buildings $\mathrm{J}$ and $\mathrm{R}$ were still the lowest, however in this case the order was reversed. People's responses to the design of building $\mathrm{J}$ above ground correlated very strongly with their responses to the finishes $(r=0.847, p<0.01)$ and, as noted above, façade details were the reason cited most frequently amongst those who disliked the building. The façade is very flat and the glazing and spandrels are in virtually the same plane. Although the façade has a clear sense of order established by the alternating pattern of the strip glazing, it appears that because the surfaces are flat there is insufficient visual interest to generate a positive response. Change professionals were more negative about both the design and surface finishes than lay people, a result that may reflect the negative opinions of these characteristics pervading the professional culture (Bourassa 1991: 104).

Responses to the design of building Q above ground, which was rated highest by the sample group, have been discussed above. The design of building $\mathrm{S}$ was also rated highly, and its finishes above ground level were liked the best (mean=3.87). The lay public (mean=3.95) were much more positive about the design than change professionals, who rated it just above neutral (mean=3.16, $\mathrm{p}<0.05$ ). The development has two main areas above ground floor, a car parking level at the eastern end and a small apartment anchoring 
the western end at the corner with Tory Street (figures 6.12 and 6.13). Both are clad in different forms of metal, the car parking area using sloping metal sheets, perforated and coloured to create a pattern. These parts of the building are distinguished from the level below, not only by surface finishes but also through a step in plan or a small gap. Both designs could be described as novel and yet professionals were less comfortable with the outcomes.

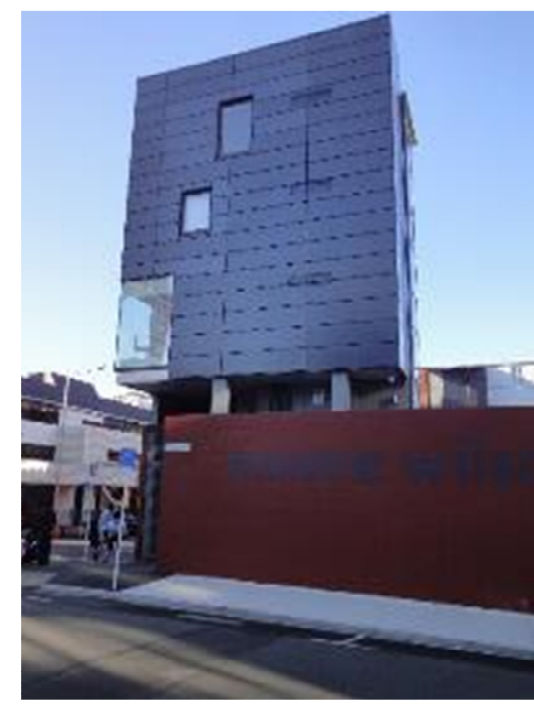

Figure 6.12 A recently completed residential unit at the western end of building $\mathrm{S}$.

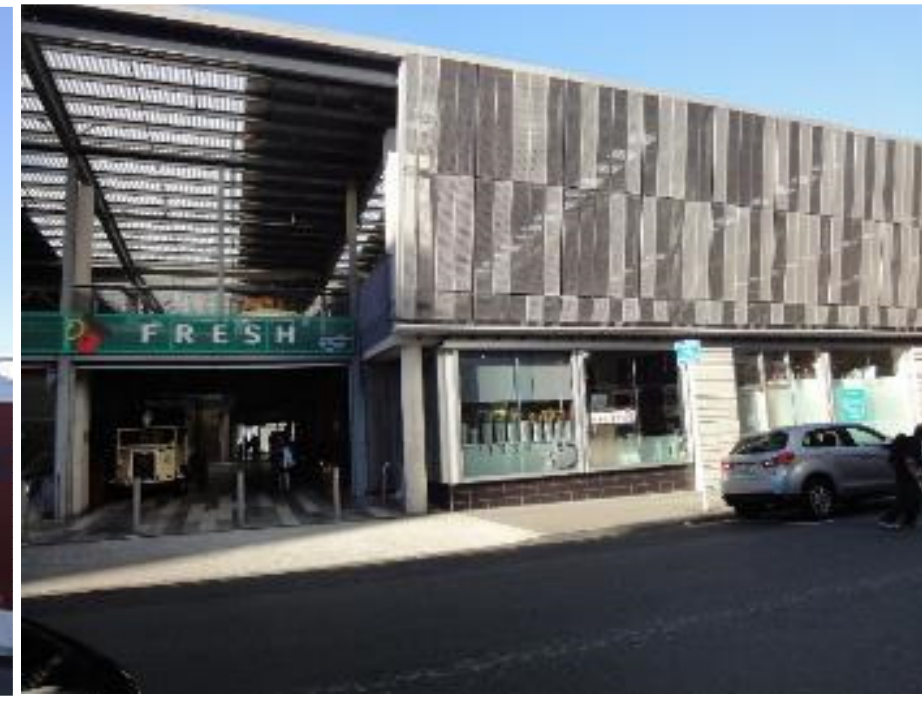

Figure 6.13: Sloping metal screen conceal the first floor car parking area of building $S$. Late in the day in the summer the sun illuminates the translucent screen, creating patterns.

The business occupying the building is a specialist food and homeware retailer. This and the fact the building is relatively new and well maintained may also have influenced perceptions. It appears that the weather conditions and time of day the survey was taken may also have had an influence. Contrary to the pattern elsewhere in the street, respondents felt more positive about building $S$ in overcast conditions than they did when the weather was sunny. This response may relate to the predominantly grey metal screen over the car park and the black of the apartment cladding. In sharp light conditions, this façade is mostly shaded during the day, which makes the detailing more difficult to perceive. This also may have helped to push preference ratings down when respondents visited on sunny days. 
As noted above, street level is where the passing public have the closest contact with urban buildings. As this is an area in transition from light industrial land uses, the ground levels of many buildings in College Street were not designed to facilitate interchange with a passing public. This is reflected in the fact that the ground level designs of 13 of the 19 buildings in the street were disliked (mean $<3)$. Again, buildings $\mathrm{Q}$ and $\mathrm{S}$ achieved the highest mean scores. Both have areas of glazing, so the passing public can see the activities inside ground level. However, the glass areas are not extensive and, in the case of building Q, the shopfront to a contemporary fashion shop is concealed behind a roller shutter after hours.

Buildings E, K and Q (figures 6.4 and 6.5) are the only buildings in the street without accommodation for motor vehicles along their ground level facade. With a mean score just under neutral, the ground level design of building $\mathrm{K}$ is the least liked of the three, while the other two received strongly positive scores. Asking about facades allows preferences for contemporary approaches of near complete openness to be compared with more traditional forms of discrete punched openings within a wall or an expressed structural frame. The ground levels of buildings $\mathrm{A}$ and $\mathrm{B}$ have been modified to accommodate cafés (figure 6.14). The modifications have retained the expression of columns running through and this limits the visual openness of the interior. Nevertheless, the sample preferred these ground level designs to those of buildings $D$ (figure 6.15) and E (figure 6.19), which feature expansive areas of glass. These responses may well have been influenced by factors other than the building design, such as the nature of the internal activities and the meanings they convey, as well as use of the footpath by café customers. Weather may also have been a factor, although only in the responses to buildings $\mathrm{D}$ and $\mathrm{E}$. Responses on a sunny day were more favourable toward the ground floor of building $\mathrm{D}$ and less favourable to that of building $\mathrm{E}$ than on an overcast day. Responses to buildings A and B did not vary with weather conditions. 


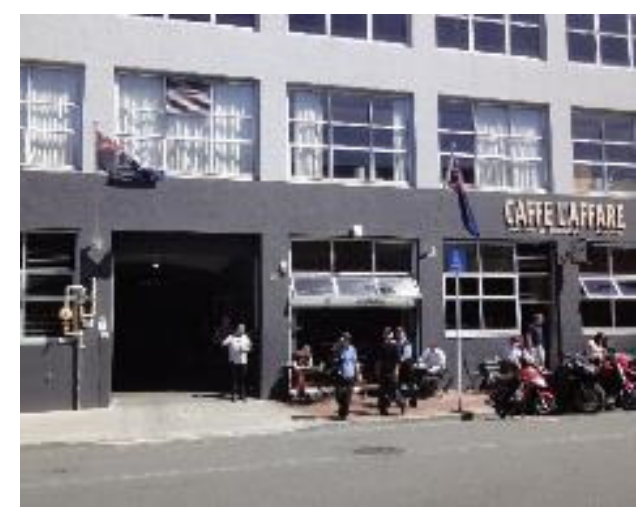

Figure 6.14: Building B has been modified at ground floor level to allow the cafe activity spill out onto the street.

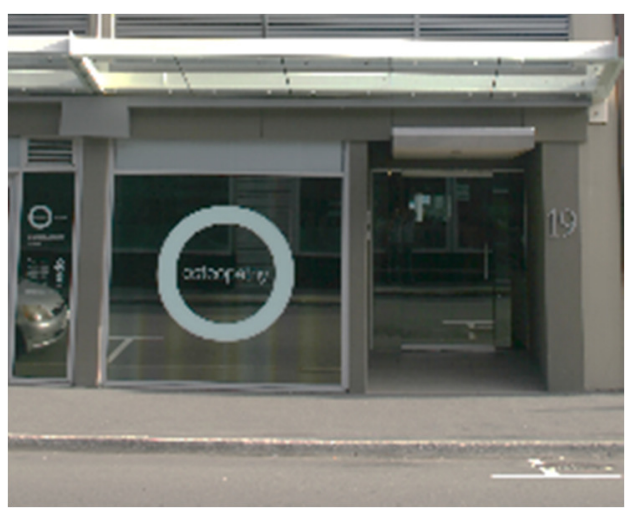

Figure 6.15: Building D has an extensive glass shopfront at street level.

Source: author

\subsubsection{Relationships between adjacent buildings}

The best liked relationship between adjacent buildings was that between buildings $A$ and B (figure 6.16). These buildings are hard to distinguish from one another as they were repainted in matching colour schemes less than two years ago. Their heights are very similar, particularly where the two parapets meet, and both adopt a similar design language, based around a commercial/warehouse typology. Only $7.4 \%$ of respondents were negative about the relationship, although $35.6 \%$ were non-committal, which tempers the enthusiasm for this relationship that was otherwise liked by $57 \%$ of respondents. Another very well-liked relationship is between one of the best liked buildings $(\mathrm{S})$ and the least liked (R) (figure 6.17). While R's relationship to building Q, a taller building to the east, is not well liked, the R/S interface seems to have found favour for a number of reasons. Firstly, the two buildings are of similar height and, in certain light conditions, also similar in colour. The roof edge of building $\mathrm{R}$ leans back from the street edge while the top edge of building $\mathrm{S}$ leans forward. Perhaps the older and less liked building $\mathrm{R}$ was considered to defer to the better liked characteristics of building S. Strong horizontal lines in both buildings between ground floor and upper level also appear to relate these two buildings to one another. 


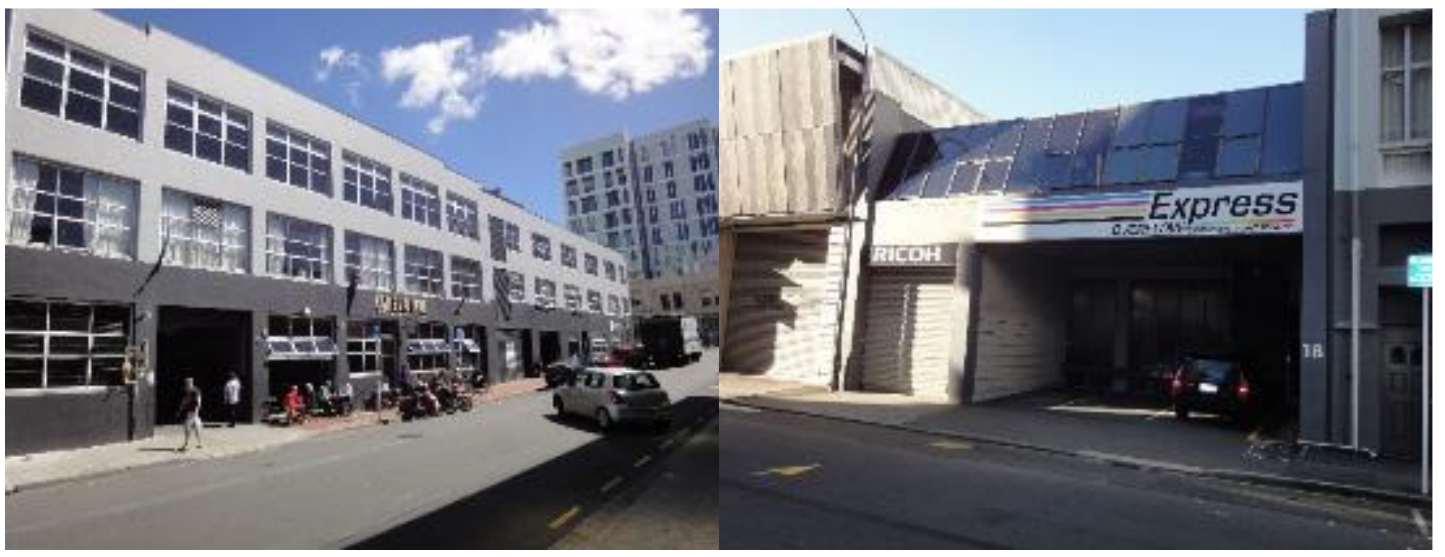

Figure 6.16: The relationship between building A (right) and B was judged best in College Street.
Figure 6.17: The relationship between buildings $S$ (left) and $R$ was also liked.

Source: author

Source: author

The least liked relationship is between buildings $F$ and $G$ (mean=1.97), followed closely by that of $\mathrm{C}$ and $\mathrm{D}$ (mean=2.09). These two relationships incorporate large differences in overall building height and in the case of $\mathrm{C} / \mathrm{D}$, strong differences in colour. Change professionals judged the building $F / G$ relationship more harshly than lay people but the two groups were closely aligned in their judgments of the $\mathrm{C}$ and $\mathrm{D}$ relationship. People were also more ready to form an opinion on this latter relationship, as only $10 \%$ of the respondents were neutral rating while such responses for most other cases varied between $24-36 \%$.

The relationship between buildings $F$ and $G$ creates steps in plan, as building $G$ is set back from the street edge, and in elevation, as the difference in height is $8+$ floor levels. Although the designers of the more recent building F have sought to create visual interest with colour and texture on its boundary wall, this does not overcome the poor form relationship. To its west, building $\mathrm{F}$ also forms a relationship with building E. Building $\mathrm{E}$ is only two storeys and yet forms positive relationships with building $\mathrm{F}$ (mean=3.16) and building $\mathrm{D}$ (mean=3.00) (figure 6.18). 


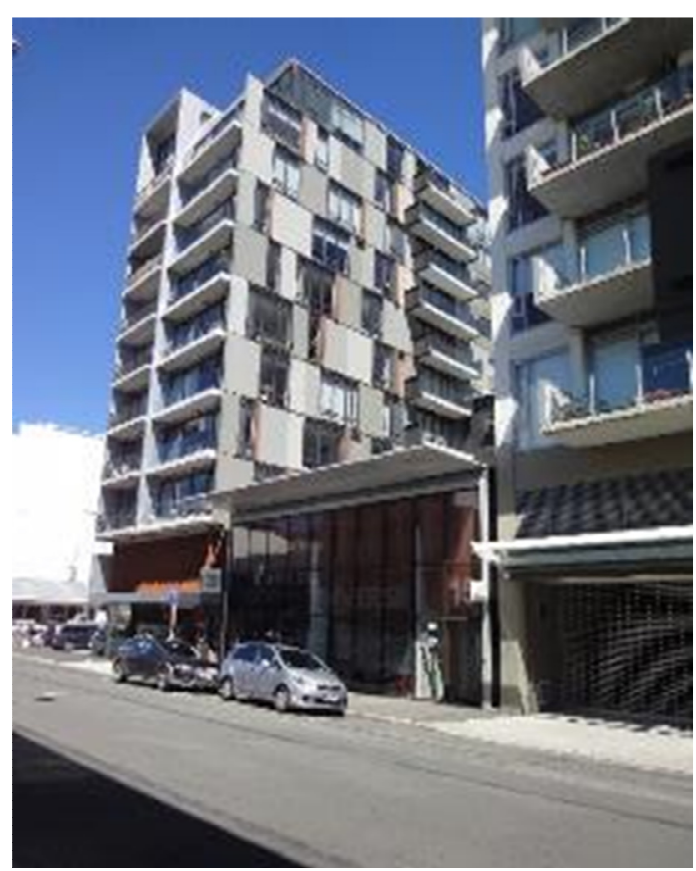

Figure 6.18: Building $\mathrm{E}$, in the middle, has positive relationships with building F (left) and building D (right).

Source: author

A significant difference is seen in the way the boundary wall of the taller building is treated, which looks more like a street façade than a fire rated side wall. It has window openings and what appear to be balcony projections toward, if not over, building E. The period of construction looks similar to building E, noticeable in the way glass is used, the colours and the detailing. Although clearly separate structures, these buildings share a common design language. This, together with the more positive way the side wall of building $\mathrm{F}$ is treated, can help explain why respondents rated the relationship between them positively.

While the building C/D relationship is stepped in elevation only (both buildings are built to the street edge), a significant area of the boundary wall of the taller building is visible. In this case the designers of the building have created a narrow recess over the full height of the elevation with windows at its back at every level. While the mean response to the relationship between $\mathrm{C}$ and $\mathrm{B}$ is negative, it was rated higher that the building $\mathrm{C} / \mathrm{D}$ relationship. It seems the negative responses to differences in building height may be a relative concern, and in this case the seven floor level difference in height has been judged more harshly than the two storey difference. 


\subsection{Comparisons based on demographic groupings}

\subsubsection{Gender comparisons}

Men and women have closely aligned preferences for the south side of College Street. Comparing means, it appears both genders have similar levels of dislike for building height relationships and façade alignment. There are however, strong differences of opinion over the appearance of the north side. Women (mean=3.68) are more positive about the building height relationships than men (mean=3.23) and the difference is significant at $\mathrm{p}<0.05$.

Men generally found the façade alignment to be unpleasant (mean=2.74) while women thought this aspect was pleasant $($ mean $=3.67, \mathrm{p}<0.01)$. More than half the men were neutral, being unable to form an opinion on façade alignment. Men also had a poor opinion of the relationship of façade design styles on the north side, rating these lower than the design relationships on the southern side of the street. Women, on the other hand, rated the northern side design relationships higher than they rated the southern side and overall were more positive than men. While $40 \%$ of women found the relationships to be pleasant only $10 \%$ of men did.

Out of nine individual buildings on the north side, men only rated buildings $\mathrm{Q}$ and $\mathrm{S}$ positively. Women liked six of the nine buildings, which may also help explain why they were positive about the relationships between them. When it comes to building design and streetscape relationships, women seem easier to please than men. Although this would require more investigation, it may be useful to consider which factors could appeal more to women. Of the 19 buildings in College Street, seven have ground floor business activities encompassing cafes, fashion/clothing shops, homeware/furniture shops and premium groceries. Women rated each of these buildings the same or more positively than men. The comparison of mean response scores for building E (figure 6.19) $(\mathrm{men}=2.03$, women $=2.41)$, on a 3 point scale, is significant at $\mathrm{p}<0.05$. Women also scored the design of the various buildings at street level for all but one building higher than men. This pattern of response appears to support evidence in the literature that the activities known to take place within a building have an influence on how it is perceived (Rapoport 1982: 14-15, Lang 1988). This gender based difference in perception was also found in Study One results (section 5.4.1). 


\subsubsection{Age comparisons}

Study One also suggested that age may influence streetscape preferences (section 5.4.2). In particular, older people preferred more variety in the setting, especially in terms of building height relationships. Consistent with this, the College Street case study suggests that young people were generally more critical of the variations observed between buildings. Respondents under the age of 25 disliked the alignment between buildings and the relationships of architectural styles on both sides of the street. They also disliked the more varied height relationships on the southern side. Of the six questions asking about preferred features of the overall streetscape, young people were only positive about one; the heights of buildings on the north side of the street. In contrast to this they liked 17 of the 19 buildings in College Street. This compares with people aged 25-54 liking only 8 and those over 55 liking 9.

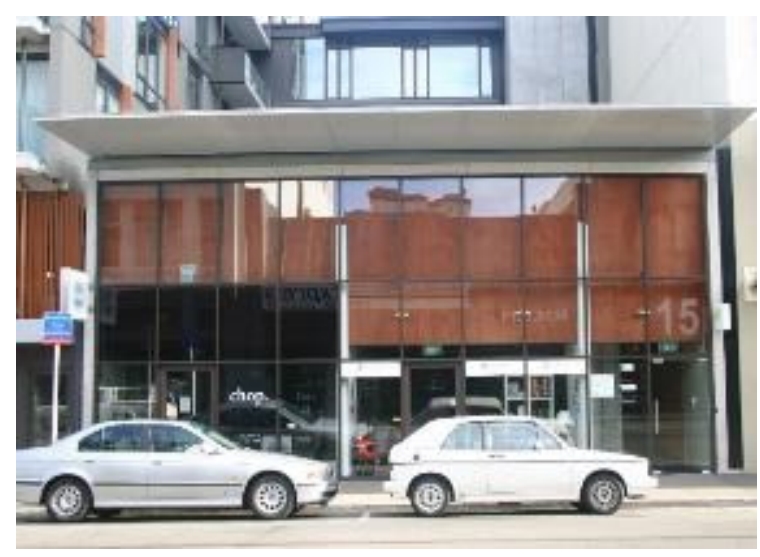

Figure 6.19: Ground level shopfront of building $\mathbf{E}$

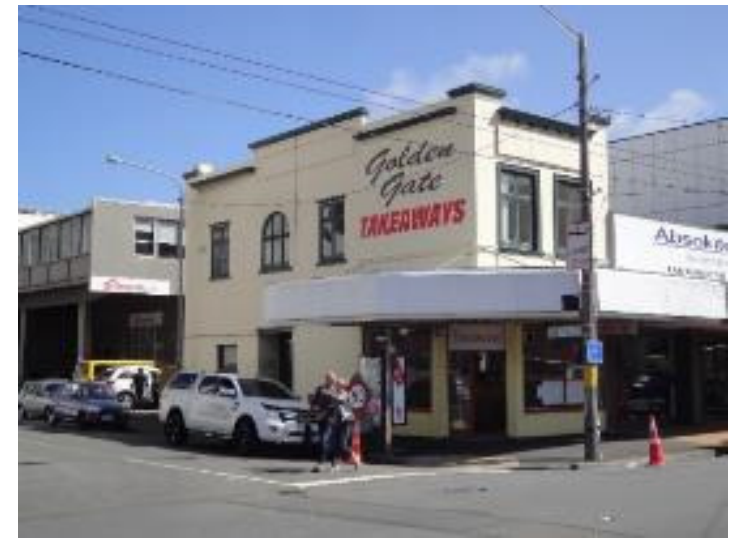

Figure 6.20: Building K

Source: author

Source: author

The two buildings disliked by young people were $\mathrm{E}$ and $\mathrm{K}$, which have different styles.

Building E (figure 6.19) is relatively new, single storey and largely glazed at street level.

Building K (figure 6.20) is two storey and representative of the traditional corner shop.

Its upper floor appears to have a different use and it has been designed to address Cambridge Terrace, as the facade facing College Street is secondary. For people over 55 this was their second favourite building (mean $=2.70$ on 3 pt. scale). Young people were marginally displeased with its design at street level, although this displeasure was less than their dislike of the building overall. 
The favourite building of those under 25 was building $\mathrm{D}$ (mean=2.50). People between 25 and 54 liked building Q (mean=2.56) while those over 55 preferred building $\mathrm{S}$ $($ mean $=2.80)$. Comparison across the top five rated buildings by age reveals that not one is common to all three age groups. In a search for buildings that all age groups felt positive about, four emerged and these were (in rank order) buildings $\mathrm{Q}$ (mean=2.45), S (mean=2.33), B (mean=2.26) and F (mean=2.25). There appears to be little to link these four buildings. Two are mixed-use residential buildings, three are of moderate height (building $\mathrm{F}$ is the tallest in the street) and two are repurposed older, characterful buildings. The ground level interest varies across them, from building Q, having a large shop front, to building $\mathrm{F}$, which has a large entrance to car parking that dominates the street level façade (figure 6.21). This poor design characteristic is reflected in the ratings given by all age groups (table 6.4).

Table 6.4: Mean scores of the four buildings rated positively by all age groups.

\begin{tabular}{|l|c|c|c|c|c|c|}
\hline & \multicolumn{2}{|c|}{ Under 25 } & \multicolumn{2}{c|}{$25-54$} & \multicolumn{2}{c|}{55 and older } \\
\hline & $\begin{array}{c}\text { Overall } \\
\text { (3 pt. scale) }\end{array}$ & $\begin{array}{c}\text { Street level } \\
\text { design }\end{array}$ & $\begin{array}{c}\text { Overall } \\
\text { (3 pt. scale) }\end{array}$ & $\begin{array}{c}\text { Street level } \\
\text { design }\end{array}$ & $\begin{array}{c}\text { Overall } \\
\text { (3 pt. scale) }\end{array}$ & $\begin{array}{c}\text { Street level } \\
\text { design }\end{array}$ \\
\hline Building Q & 2.20 & 3.65 & 2.56 & 3.91 & 2.50 & 3.60 \\
\hline Building S & 2.00 & 4.05 & 2.37 & 3.63 & 2.80 & 3.90 \\
\hline Building B & 2.05 & 3.47 & 2.37 & 3.63 & 2.20 & 3.10 \\
\hline Building F & 2.35 & 2.90 & 2.19 & 2.52 & 2.30 & 2.70 \\
\hline
\end{tabular}

The one thing that seems to link these buildings is that they all seem well looked after, either having been built recently or been recently maintained. This aspect will also be considered in the following two case studies to help determine its relative importance in perceptions of urban streetscapes.

\subsubsection{Comparing change professional and lay people's opinions}

This next section of the analysis considers how the views of change professionals compare with those of the lay public.

Change professionals appear to be more tolerant of large differences in height along a street and less positive about height relationships that vary within a narrow band of difference. Nevertheless, both groups returned negative mean scores for the building 
heights on the southern side of the street (change profs mean $=2.95$, lay mean $=2.77$ ) and positive mean scores for those on the north (change profs mean $=3.16$, lay mean=3.53). In terms of the relationships of facades to each other and the street edge, both groups had negative opinions about the alignment along the southern side of the street. The change professionals $($ mean $=2.42$ ) tended to be more negative than lay people (mean=2.63).

Opinions about the building alignment along the northern side differed. Here, change professionals were negative about the alignment relationships while lay people were positive, with $44.4 \%$ of the latter positive compared to only $15.8 \%$ of change professionals. The comparison was statistically significant at $\mathrm{p}<0.05$. Every building on the northern side is built to the street edge, which is recommended practice for buildings in urban settings such as College Street, as street edge definition is a desirable characteristic (Wellington City Council 2012). However, the change professionals' scores may have been influenced by four buildings where the ground level is open for car parking, despite the building above and associated structural elements being aligned to the street edge (figure 6.22).

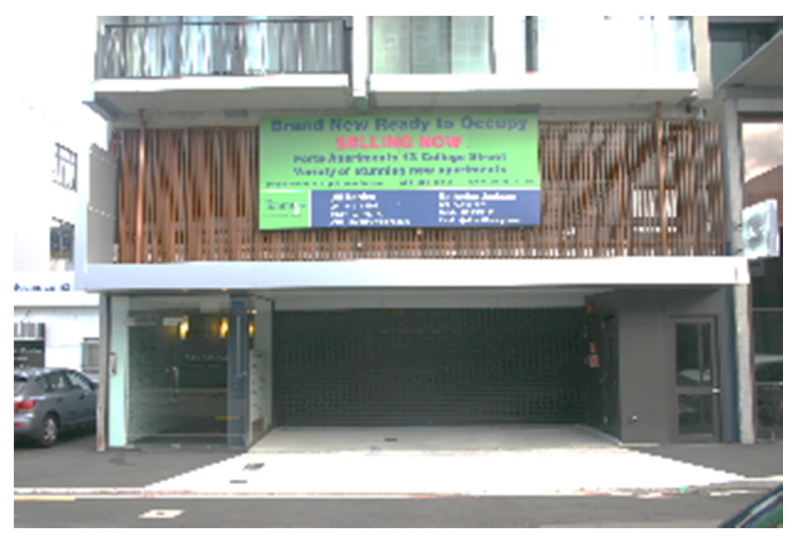

Figure 6.21: The street façade of building $F$ is dominated by a wide entry to the car parking area within.

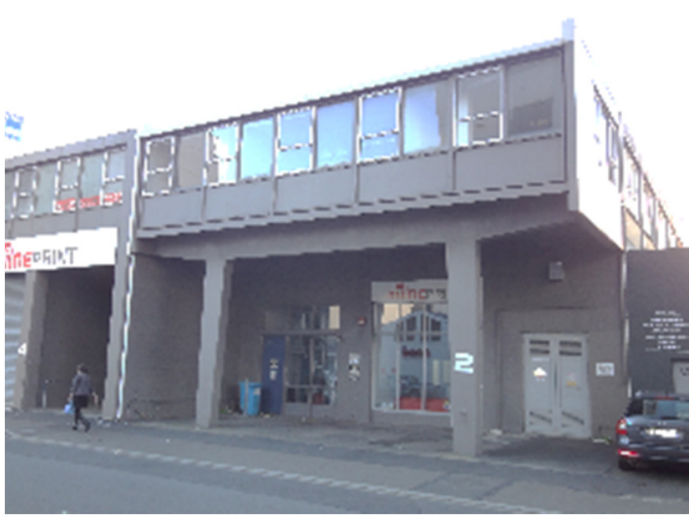

Figure 6.22: Building L, one of four buildings along the northern side of College Street hollowed out at ground floor level to make space for car parking.

Source: author

Source: author

The aggregate scores of planners and architects were aligned regarding height relationships but less so for the façade alignments on the two sides of the street (table 
6.5). Dividing the sample into lay people, planners and architects revealed that each group had a different level of dislike for the facade styles along the street. The lay public were closest to being neutral about these relationships, but nearly half of that group felt they were unpleasant to view (46.4\% south side and $47.3 \%$ north side). Planners were very opinionated about these relationships. Only $12.5 \%$ of this group registered neutral opinions, with $75 \%$ of planners disliking the façade styles to the south and $87.5 \%$ those to the north.

Table 6.5: Mean scores by professional grouping for streetscape characteristics

\begin{tabular}{|c|c|c|c|}
\hline & Planners & Architects & Lay public \\
\hline South side streetscape & & & \\
\hline Building Heights & 3.00 & 2.91 & 2.77 \\
\hline Facade Alignment & 2.63 & 2.27 & 2.63 \\
\hline Facade Styles & 2.25 & 2.64 & 2.79 \\
\hline North side streetscape & & & \\
\hline Building Heights & 3.13 & 3.18 & 3.53 \\
\hline Facade Alignment & 2.63 & 2.91 & 3.31 \\
\hline Facade Styles & 2.00 & 2.55 & 2.84 \\
\hline
\end{tabular}

These levels of dislike for the whole streetscape can be compared to the opinions of planners for individual buildings (table 6.6). Planners liked 5 of 10 buildings on the south side and only 2 of 9 on the north. The lower rating for the north streetscape seems to correspond to the lower ratings they gave to individual buildings. The lay public gave higher scores on average than the other two groups for both sides of the street, as $31 \%$ were positive about the northern side and $30.4 \%$ about the south. This group also liked the individual buildings more than the other groups, as 8 of the 10 buildings on the south were liked by lay people and all buildings on the north.

In light of these opinions about individual buildings it is somewhat surprising that lay people did not rate the façade style relationships higher. However, the results suggest that it is not only important to consider the quality of individual buildings but also how they fit together in the street. The results also highlight the difficulties that arise when a street or wider urban area is in transition from one type of economic activity to another. In College Street, the change is from light industrial to high end commercial and residential. All groups appear to have had expectations of greater coherence between 
buildings. However, the final outcome of a period of transition cannot be predicted, as it is driven by private initiatives. This suggests tensions could arise over whether design review should target the uncertain future form of the area or seek to work with the existing character. It is clear from the responses to questions about the design relationships of College Street that lay people and change professionals alike are interested and have an opinion. This is one of many tensions around which design review and regulation must operate.

Table 6.6: Mean scores by professional grouping for individual buildings

\begin{tabular}{|c|c|c|c|}
\hline Building & $\begin{array}{c}\text { Change } \\
\text { Professionals }\end{array}$ & Lay public & Sig (2-tailed) \\
\hline A & 2.42 & 2.15 & \\
\hline B & 2.44 & 2.22 & \\
\hline C & 1.47 & 2.25 & $\mathrm{p}<0.005$ \\
\hline D & 2.00 & 2.11 & \\
\hline E & 2.37 & 2.13 & \\
\hline F & 2.39 & 2.21 & \\
\hline G & 1.31 & 2.09 & $\mathrm{p}<0.005$ \\
\hline H & 1.58 & 1.94 & \\
\hline I & 1.47 & 2.11 & $\mathrm{p}<0.005$ \\
\hline J & 1.26 & 1.96 & $\mathrm{p}<0.005$ \\
\hline K & 2.05 & 2.24 & \\
\hline L & 1.50 & 2.09 & $\mathrm{p}<0.01$ \\
\hline M & 1.26 & 2.00 & $\mathrm{p}<0.005$ \\
\hline N & 1.42 & 2.18 & $\mathrm{p}<0.005$ \\
\hline O & 1.36 & 2.29 & $\mathrm{p}<0.005$ \\
\hline P & 1.26 & 2.07 & $\mathrm{p}<0.005$ \\
\hline Q & 2.74 & 2.36 & \\
\hline R & 1.10 & 2.00 & $\mathrm{p}<0.005$ \\
\hline S & 2.21 & 2.36 & \\
\hline & & & \\
\hline
\end{tabular}

Buildings A and B (figure 6.16) are conversions of warehouse buildings, appearing to date from the first half of the $20^{\text {th }}$ Century, and were reasonably well liked by lay people and change professionals alike. On the basis of mean preference scores both groups also liked buildings E, F, K, Q and S, although not in that rank order. Change professionals rated buildings B (mean=2.44 on 3-pt scale) and A (mean=2.42) second and third highest after building Q (mean=2.74). Buildings $\mathrm{B}$ and $\mathrm{A}$ were ranked $6^{\text {th }}$ and $11^{\text {th }}$ respectively by lay 
people, thus both were ranked lower than building C (figure 6.23). Buildings A and B have large windows both at ground floor and the two levels above, all with clear glazing. As mentioned earlier, a common design language links the two buildings as do the identical colour schemes.

A regression analysis was made to establish which, if any, of the architectural design characteristics was the strongest predictor of preference for building A. Looking first at the whole sample, the design of the building at street level linked most closely to whether a person liked the building. However, when change professionals were analysed independently, the best predictor was the design of the building above ground level. This same pattern was observed in the responses to more than half of the individual buildings in College Street. This could indicate that lay people and change professionals view the environment differently and the results of the other two case studies may help shed further light on this.

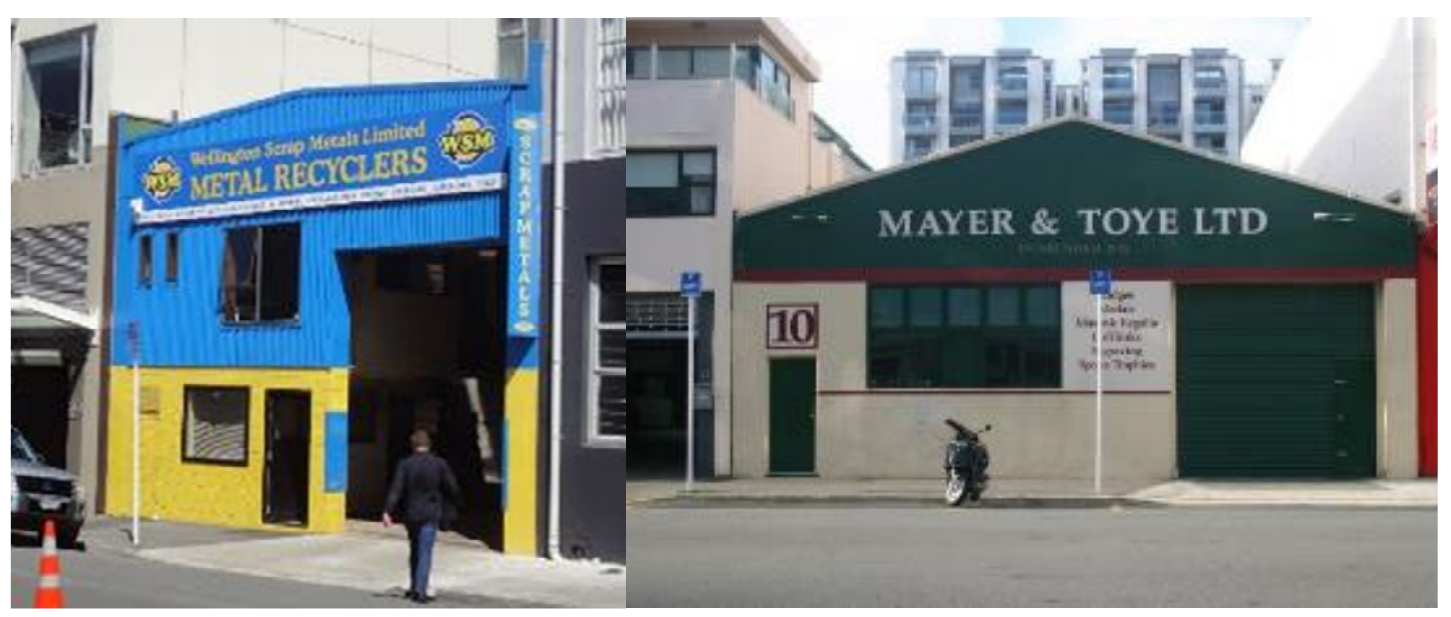

Figure 6.23: Building C, which was rated more positively than buildings $\mathrm{A}$ and $\mathrm{B}$ by lay people
Figure 6.24: Building O, which lay people also liked strongly.

Building C (figure 6.23) divides opinion between change professionals and lay people. The lay public is moderately positive about this building that is a clear remnant of the original light industrial character of the street. The same results were also observed for buildings $\mathrm{I}$ and $\mathrm{O}$ (figure 6.24). All three are single storey buildings, although $\mathrm{C}$ has a 
mezzanine level, with gable ends to the street. The preferences for these three buildings may reflect differences of opinion about roof shapes in this context.

It also seems that lay people referenced more than just the physical form of these three buildings in their preference ratings. Scores for the design above ground level, finishes above ground level, and design at street level all fell below the neutral line but the overall preferences for each were positive. Indeed, $\mathrm{C}$ was the $4^{\text {th }}$ most liked building, based on mean scores. Each building is small in scale and two of the three are still used for activities associated with a light industrial area. Each is aligned to the street edge and after hours, when the roller shutters are down, this industrial character is maintained. If lay people associate positive meanings with the gable form, daytime business activities within the buildings, or the way these buildings stand at the street edge, these same factors do not appear to influence the opinions of change professionals to the same extent.

The façade designs and finishes above ground floor level of six buildings in College Street were well liked by change professionals and the lay public. These six were the only ones in the street that garnered positive opinions from both groups. Of the six, the façade design and finishes of building Q (figure 6.25) were liked the best by both groups. Within the change professional group, architects and planners were equally positive about this building with mean scores around 4 on a rating scale of 5 . Change professionals liked the design of building $\mathrm{R}$ above ground level the least (mean=1.74) followed by building I (mean=1.79). Lay people liked the design of building J (mean=2.39) least above ground level. This building is perhaps the antithesis of building Q. Building J (figure 6.11) has strip windows with tinted glazing so activities inside are invisible and the scale of the building is significant for both height and width.

Only six buildings were well liked at ground level by both change professionals and lay people. Within this general result the planners' opinions of building D (figure 6.26) differed. Planners rated this ground level design at mean=2.63, somewhat below the neutral line. This dislike could be due to the tinted glass in most parts of the ground floor shop fronts, presumably used to reduce solar gain, in a virtually all glazed façade facing north, and where the canopy, a feature of most streets in New Zealand cities, is also glazed. Another factor could be the simple design at this level, as the façade is composed of glass between structural elements with vertical divisions (mullions) at seemingly random intervals. 


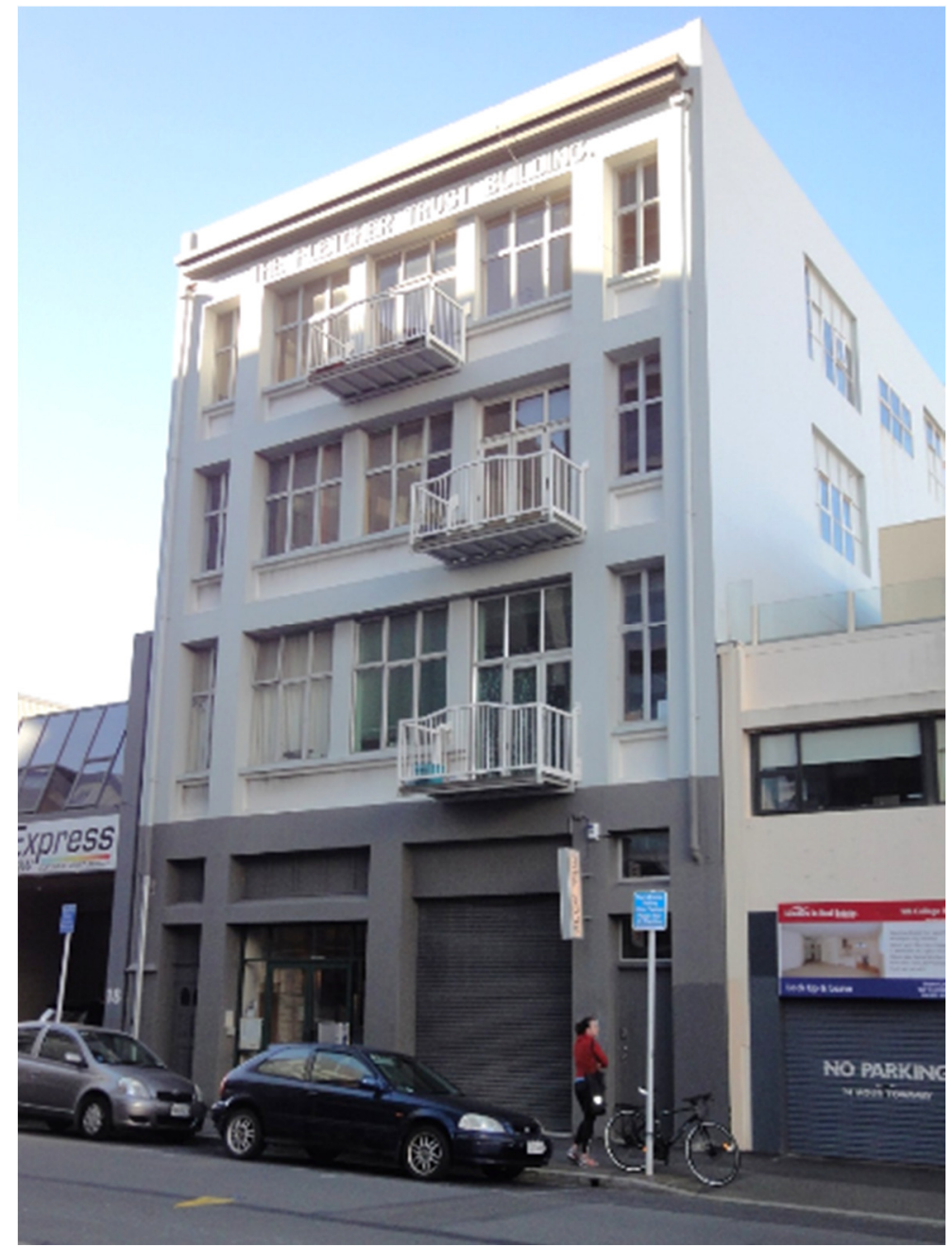

Figure 6.25: Building $Q$ is not only the best liked building but also has the most attractive design and finishes above ground floor level.

Source: author

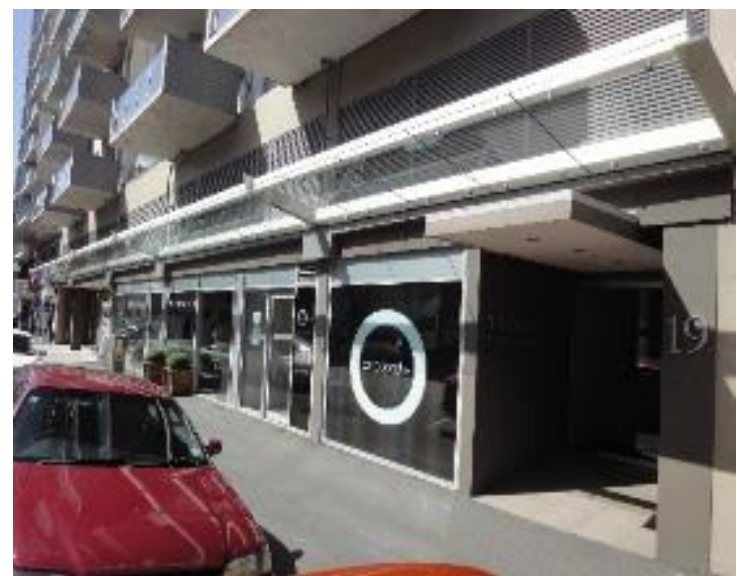

Figure 6.26: The ground level shop front of building $\mathbf{D}$ was disliked by planners.

Source: author 
On the basis of mean score comparisons, only three of the 17 relationships between adjacent buildings were liked both by change professionals and lay people. These were the relationships between buildings A and B (figure 6.16 and discussed in section 6.3.3), $\mathrm{E}$ and $\mathrm{F}$, and $\mathrm{L}$ and $\mathrm{M}$. Lay people also liked the relationships between $\mathrm{M}$ and $\mathrm{N}$ and between $\mathrm{R}$ and $\mathrm{S}$. Not only were the two distinct groups within the change professionals grouping positive about the relationship between building A and B but planners had a mean score of 4.13 , indicating a very strong preference for it. The $\mathrm{L} / \mathrm{M}$ relationship was liked second best of all the relationships, despite the individual buildings not being well liked (figure 6.22). This relationship has several characteristics similar to the A/B relationship. The buildings have a common colour scheme, they match in overall height and the floor levels align across the shared boundary. The façade height and width proportions, as well as the fenestration arrangement at first floor levels, are similar in both buildings. Although the ground floor designs differ, they have a consistent overall height and both buildings house the same business with a consistent signage that also serves to link the buildings.

The overall positive rating of the relationship between buildings $\mathrm{R}$ and $\mathrm{S}$ has been discussed earlier (section 6.3.3). However, change professionals did not like it as much as lay people and their mean score $(=2.94)$ fell below neutral. Underlying this is the fact architects strongly disliked the relationship while planners and lay people liked it. Across all 17 relationships, lay people and change professionals had similar views for 14. Bearing in mind that 13 of the 17 relationships were disliked by all respondents it seems that despite differences in the strength of views, change professionals disliked similar characteristics in relationships between buildings as did the public.

Looking at these opinions more closely by comparing those of the two change professions (architects and planners) with the lay public does not reveal any real differences, as there were only notable deviations of opinion in three instances. The difference in relation to buildings $\mathrm{R}$ and $\mathrm{S}$ has been noted. At the $\mathrm{D} / \mathrm{E}$ interface, planners thought this relationship was good while lay people and architects disliked it. Although all three groups disliked the relationship between buildings $\mathrm{P}$ and $\mathrm{Q}$, planners were considerably more negative about it. 
Examining the building $\mathrm{D} / \mathrm{E}$ relationship in more detail, a similarity of design language and materials seems to occur across buildings D, E and F (figures 6.27 and 6.28). All three groups liked the relationship between buildings $\mathrm{E}$ and $\mathrm{F}$ but lay people and architects disliked that between $\mathrm{E}$ and $\mathrm{D}$, while planning professionals had a positive view of the relationship. It may be that planners saw a positive relationship forged on the basis of materials, details and design overcame the effects of pronounced height differences.

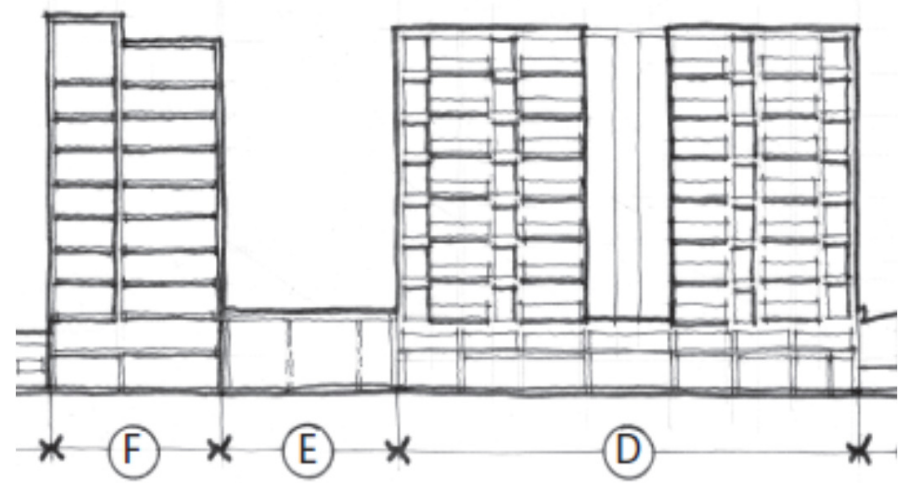

Figure 6.27: Elevation of relationship between buildings D, E and $F$ on the south side of College Street.

Source: author

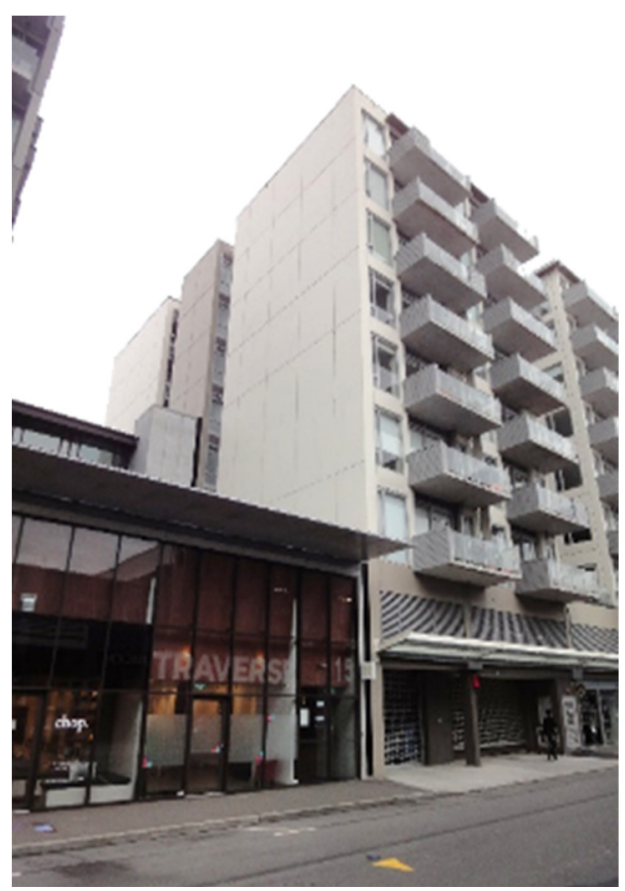

Figure 6.28: Side wall of building $\mathrm{D}$ above roof of building $\mathbf{E}$.

Source: author 


\subsection{Focus group discussions}

To probe the initial College Street case study findings further, two focus groups were convened. Section 4.5 provides a brief description of the design of the focus groups and how they fit with the overall research design. In addition to providing a forum for indepth discussion of the issues each group considered important, they also generated useful information for interpreting other data. The participants in the discussions were recruited from the 29 survey respondents who had indicated their willingness to participate. Accordingly, all had prior knowledge of the street and nature of the survey. Nevertheless, as a reminder to participants and to help stimulate discussion, a short presentation of the initial findings of the College Street case study, accompanied by visual images, was made at the beginning of each session.

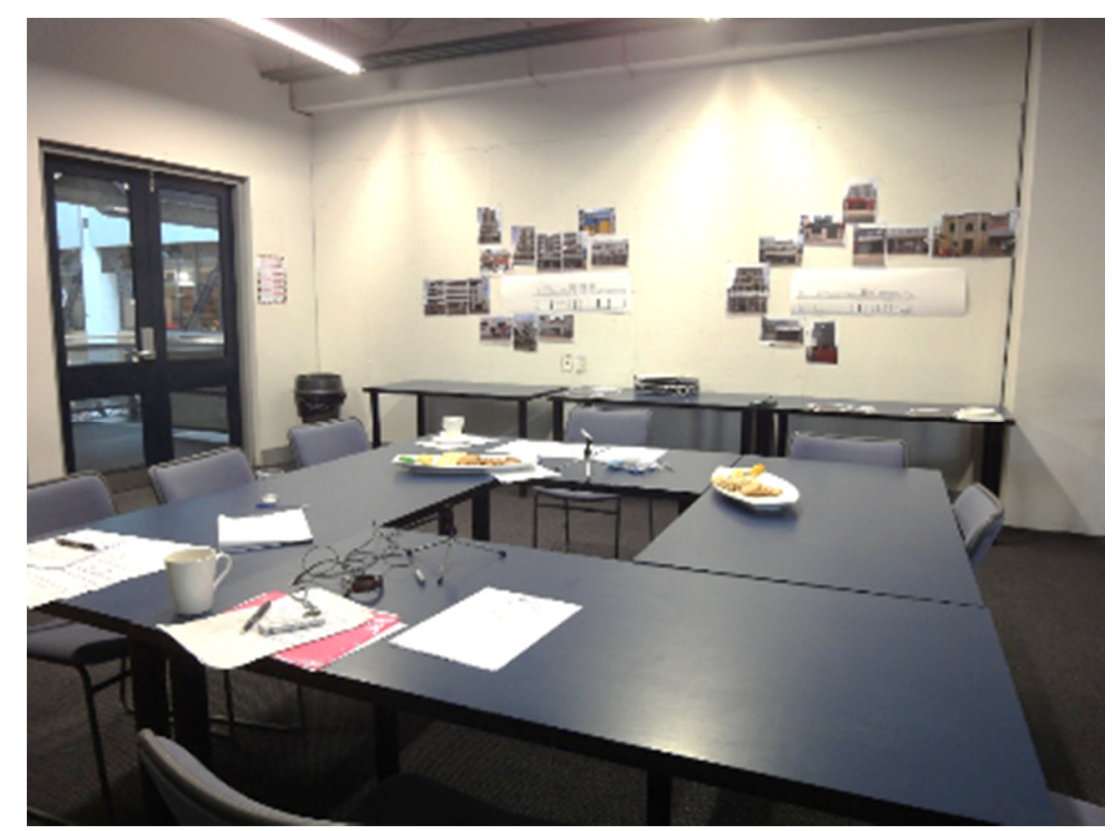

Figure 6.29: The focus group discussion room. All participants were invited to sit round the table to make use of the images on the wall during the discussion.

Source: author

Participants were seated around a large table in a seminar space with the researcher and a student recruited to take notes. Each person was provided with an A3 copy of the elevation drawing of each side of the street. Larger versions of the same drawing with photographs of all the buildings were mounted on the walls of the room (figure 6.29). 
Participants were encouraged to use these drawings and photographs when discussing their perceptions. In addition to the notes recorded by the assistant, each session was recorded for future reference.

\subsubsection{Lay persons' focus group}

The lay persons' focus group, comprising five women and one man, took place on a weekday in the late afternoon. From the outset, the discussion was lively and everyone contributed. As a consequence, the brief introduction continued over the entire one and a half hour period as it was interrupted on numerous occasions by discussion. Although the discussions were wide ranging, several key themes were developed by the participants.

At the overall scale of the street, comparisons were made with a small street recently comprehensively redesigned part of a large-scale redevelopment in the heart of Wellington's CBD. Chew's Lane, which is much shorter than College Street and fully pedestrianised, was well known to all participants. There was a strong preference for the coordinated paving along the length of Chew's Lane and for the way buildings open out onto the street to create more intimate spaces for people to sit outside the various cafes along it, which offer those walking through something to observe. Chew's Lane was referenced when several participants critiqued the disjointed relationships between buildings in College Street and the fact there are widely varying standards of private development and public space development along its length. It seems people were unsympathetic to the fact College Street is an area in transition and that they expected the public realm of the street to be improved through design details such as found in Chew's Lane.

Another key issue for this group was that so much of the recent development appears large in comparison with the older, smaller scale buildings. As a consequence several participants felt these newer buildings and their owners were being disrespectful to the street. Buildings $\mathrm{D}$ and $\mathrm{F}$ were offered as examples of this trend and other recent projects from other parts of the city were also cited. Building height in relation to the heights of older buildings was the main influence on this view, while others included the balconies extending out across the front boundary over the street and the blank side walls of tall buildings seen above the lower ones. While the impact of the blank side walls was cited as a visual aspect, it became clear that the height relationships and extension of balconies over the street had strong, negative meanings for those who spoke about these. The term 
'greed' was used more than once and by more than one participant. One participant in relation to Building D said “they've ruined the whole street by going so high”. Another matter raised on several occasions in the 90 minutes was the need for trees and foliage in College Street to make it more attractive.

Apart from $\mathrm{H}$ and I, all buildings on the southern side of College Street were mentioned during the conversations by at least one person whereas only buildings $\mathrm{R}$ and $\mathrm{S}$ from the north side were discussed. Buildings $\mathrm{D}$ and $\mathrm{S}$ were discussed most. Building $\mathrm{S}$ was generally viewed positively. Opinions about it ranged from how little it dominates over the others in the street even though it is long, how it has an attractive appearance even though it is only a warehouse, and how the façade treatment and planning both help to conceal car parking from the road. Although people were surprised to hear it was one of the top two rated buildings in the street, they were also quick to suggest that this was probably because of the positive meanings people have for its use. This interpretation was reinforced when they heard that women were more positive about building $S$ than men and that older people were more positive than younger people.

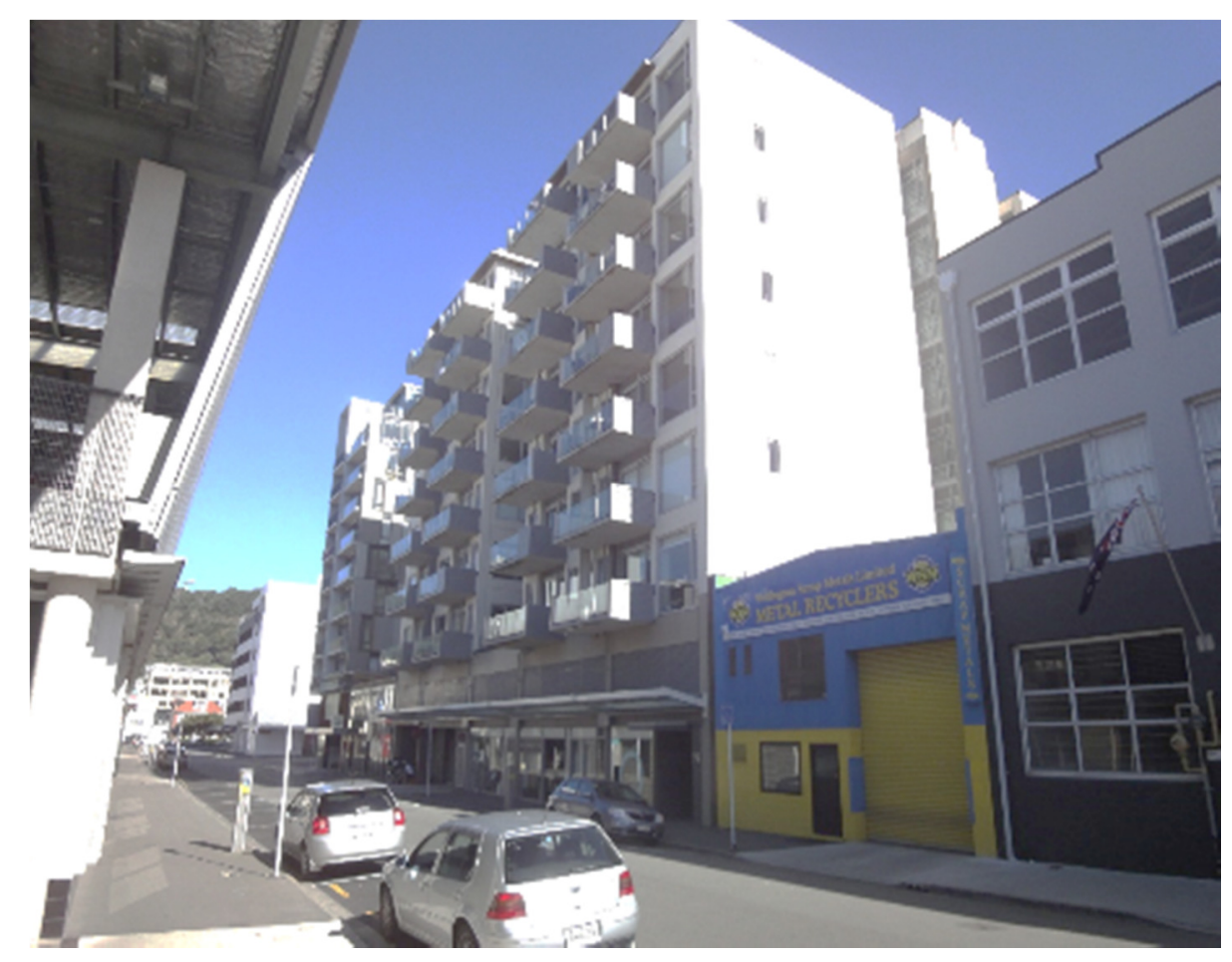

Figure 6.30: Building $D$, in the middle of the image, from the west. Building $\mathrm{C}$ painted blue and yellow houses a metal recycling business.

Source: author 
Building $\mathrm{D}$ was in the main discussed negatively. As noted, participants had poor opinions of the side boundary wall (figure 6.30). After an explanation about fire and why the wall could not incorporate large windows, several people suggested that the wall should not have been built on the boundary. When it was noted that all other buildings in the street would also have similar blank side walls for similar reasons, respondents were quick to say that the other ones could not be seen. These discussions took place in the context of the image presented in figure 6.30, with the sun highlighting the west-facing side wall. The possible influence this could have had on the discussion should be acknowledged when the findings of the research are being formed. The negative reaction to private balconies extending across the street boundary into College Street was also discussed and appears to have been a factor in the general dislike of building D.

Another factor discussed at length was the effect of the large windows into the apartments of building $\mathrm{D}$ above street level. This discussion centred mainly on how these windows would lead to problems for the residents. The first concern was the lack of privacy for apartment owners and the second was the possible build-up of heat during the day. The participants agreed that these two factors may have been reasons why so many of the windows were covered by blinds during the day. One person noted it would be difficult for residents to place any furniture in the space and that this was evidence that designers and developers did not have the project users in mind. This was how participants interpreted building $\mathrm{D}$, although they had no way of knowing whether these were the residents' experiences.

What became very clear through this discussion was the extent to which people were able to project themselves into the apartments and understand the implications of the large windows for those living there. Indeed, when the question "do you project yourself into the space in the process of evaluating the aesthetic appearance of the building?" was put to the group a female participant said she did so regularly and that doing so was critical to her ability to understand building D.

The lay persons' focus group also raised general matters affecting perception. One concerned the perceived quality of materials and construction of the buildings, which arose in connection with a wider discussion of why contemporary buildings are often perceived to be of low quality. The materials used in many new buildings have a reputation for deteriorating quickly, thereby conveying a sense of cheapness. One 
participant noted that how a building ages is a key factor in how she evaluates buildings and others nodded agreement. Traditional materials like copper would look better with age according to one group member and others noted that the way buildings are constructed can add to their visual appeal. Glass was thought to be a cheap material that was used far too much in newer buildings.

The group, responding to a prompt from the researcher, resoundingly validated the importance of design and appearance. Several participants linked their own feelings of pride and satisfaction to the appearance of the city or of particular streets. However, no participant could offer concrete examples of this. The focus group concluded after about one and a half hours, with the participants thanked for their time and contribution to the research.

\subsubsection{Change professionals' focus group}

The second focus group took place three weeks later in the same space and was set up in a similar manner. Invitations were issued to 7 people involved in some aspect of managing urban change, either as designers, policy-makers or regulators. The discussions took place between the two men and two women who were able to attend.

Very early in the discussion it became clear that members of the group applied their knowledge of how urban change takes place when they make an aesthetic judgement, allowing them to smooth over certain shortcomings in the process. As an example, the group agreed that the poor spatial relationship between buildings $C$ and $D$ could be overlooked because the situation was temporary. They felt the site of building $\mathrm{C}$ would be redeveloped in the foreseeable future, even though no one knew of any plans to do this. This possibly enabled participants to minimise the effects of the sharp differences in building height, the visible side wall of building $\mathrm{D}$ and the strong contrast in colour between the two sites when they evaluated the streetscape. There was a shared view that building $\mathrm{D}$ could have been lower to fit better with its neighbours but that requiring this may have meant the project was not economically viable. All agreed that the street was better for having the site redeveloped, although no one remembered what it was like before building $\mathrm{D}$ was constructed.

This same approach appeared to influence participants' views of other buildings and relationships, with several recent changes in the street cited as examples of how the 
streetscape was incrementally changing for the better. They were unwilling to judge certain characteristics of the current streetscape poorly because they anticipated the problem would reduce or disappear with further change. Indeed, during the course of the survey building $G$ had begun to change, although when completed the changes were largely cosmetic and did not rectify the differences in height with building F.

In a similar vein, all participants were able to consider how certain design characteristics provided for, or were evidence of, activities that could enhance the public realm. Thus, the balconies on building $\mathrm{D}$, which one urban design professional in the group conceded were poor in appearance and were 'cheeky' in the way they cantilevered into the street, were considered to be positive because they were a sign of residential activity. The participants agreed that this is good for the city and they understood that Council was working hard to increase. Although the architect in the group felt the lack of privacy for those using the balconies would cause people to evaluate them negatively, he also felt this could easily be overcome with the addition of a screen. The fact that he could foresee a change that would enhance the privacy levels enabled him to view the balconies favourably.

The group reacted when notified of the lay group's clear preferences for the alignment between buildings on the north side of College Street. The professional group acknowledged a more consistent alignment of facades along the street edge above ground floor but were concerned about the 'hollowed out' ground level appearance of many buildings. As noted in section 6.3.1, this had arisen because the light industrial land uses required convenient parking and loading in front of their buildings. In some cases, the light industrial activities and parking remain, as with buildings $\mathrm{L}, \mathrm{M}, \mathrm{N}, \mathrm{P}$ and $\mathrm{R}$. This made the north side unpleasant to walk along and all professionals indicated their preference for walking along the southern side of the street. In addition to having a street edge with shops and other visual interests, the southern side was generally in the sun and seemed more spacious. This discussion culminated with the architect member suggesting there could be merit in having a lower allowable height for buildings on the northern side of a street in order to ensure better solar access and outlook for the southern side. The inconsistent alignment along the southern side of the street also led to a short discussion about using this to create a public square in front of buildings $\mathrm{G}, \mathrm{H}$ and I. There was general agreement this would be a positive use for the space, rather than the current car 
parking, but acknowledged that this would be difficult to achieve as the sites were in private ownership.

Despite prompts to return to a discussion of individual buildings and the streetscape as defined by this research, the group was clearly in a design mode and continued to discuss street trees, paving design and green space generally. There was consensus among the participants that these were important matters that needed to be addressed by local government. There were strong suggestions around the table that these mattered much more to people's perceptions than the design of any single building.

Two other key issues discussed by the professional group are relevant to streetscape quality. One is the importance of windows, particularly at street level but also across the whole façade of a building. The group felt that an awareness of how a building is being used is important. College Street was particularly attractive because of the small businesses, with the focus for this comment being the metal recycling business trading from building C. Activities such as this helped create a rich mix that was particularly attractive to the participants in this focus group. The second matter was building materials. All four participants agreed that the way materials age is more important than how they appear when the building is just finished. The urban designer in the group stated she always considered this when undertaking a design review. Building maintenance was also considered essential for ongoing visual appeal. The policy planner in the group lamented the difficulty of bringing maintenance requirements into the planning framework, if only for the pragmatic reason that it would be difficult to administer.

The discussion was concluded after one and a half hours. The matters raised for discussion by the professionals seemed strongly tempered by their knowledge of design, planning policy and planning regulation practices. It seems the views of this small group were closely aligned to those of their professional colleagues when judging the relationships of architectural styles on both sides of College Street (table 6.7). Overall, they preferred the height and façade alignment relationships on the north side, as did their colleagues. However, this small group was more negative about the southern side heights and façade alignments and more positive about those on the north side of the street. The four members were unanimous in their dislike of the façade alignment along the southern side. 
Table 6.7: Mean scores for streetscape preferences (on scale 1-5) for professional focus group participants and other groups

\begin{tabular}{|l|c|c|c|}
\hline & $\begin{array}{c}\text { Professional focus } \\
\text { group participants }\end{array}$ & $\begin{array}{c}\text { Change } \\
\text { professionals }\end{array}$ & Lay people \\
\hline South - heights & 2.50 & 3.00 & 2.78 \\
\hline South - façade alignment & 2.00 & 2.50 & 2.64 \\
\hline South - styles & 2.50 & 2.44 & 2.80 \\
\hline North - heights & 3.00 & 3.31 & 3.50 \\
\hline North - façade alignment & 3.25 & 2.81 & 3.28 \\
\hline North - styles & 2.50 & 2.38 & 2.81 \\
\hline
\end{tabular}

This conflicts somewhat with the discussion in the group, where the participants noted they were willing to be more forgiving of height or alignment mismatches because of the potential for this to be repaired through future redevelopment. The difference between the survey scoring and the discussion may well be the extent of what was considered, as the survey questions asked respondents to look at the whole streetscape rather than one particular part.

The focus group was consistent in their ratings of building D. All groups neither liked nor disliked the building on average. However, the professional focus group was more negative than both groups about buildings $\mathrm{C}$ and $\mathrm{S}$ and this may help explain some of the discussion around each of them. Early in the discussion, when it was mentioned that the whole of the respondent group had rated building $S$ the second most liked, one focus group participant expressed surprise that it was liked at all; in her view "it is a terrible building, absolutely terrible." During the discussion about the height relationship between buildings $\mathrm{C}$ and $\mathrm{D}$ it was generally agreed that building $\mathrm{C}$ was the odd one out. In the preferences expressed for other buildings the focus group members were aligned more closely to the larger group of change professionals.

\subsection{Summary of the College Street Case Study}

College Street in Wellington is in an area of transition, moving from light industrial uses and building typologies to activities associated with people living in the city including residential accommodation, high-end retail and cafes. This has led to considerable 
variation in the physical form of the street, particularly as newer buildings seek to maximise the development envelope.

There appears to be a general preference for more settled height relationships along the length of the street and for consistency in the way buildings align with each other and with the street edge. This is evidenced by the stronger preferences for these aspects, which are found along the north side of the street but less so along the south side. Nevertheless, the opinions of some subgroups deviated from this general tendency. Although aligned with men in their dislike of vigorous height and alignment changes on the south side of the street, women had a more positive view of the settled relationships on the north side. Women were also more positive about the relationships between design styles on both sides of the street, and this corresponded with liking more of the buildings. A strong factor in these positive opinions about buildings may be the retail, café and other activities, all of which appear to be increasing in number as the street changes in nature.

In addition to having well-liked physical characteristics, the best liked individual buildings were also clearly legible and supported activities that people felt positively towards. That is, where the purpose of the building was obvious to people in the street and where these activities were considered pleasant, people's opinions of a building were enhanced. This theme became evident through analysis of the survey results and was articulated during the focus group discussions. Nevertheless, physical characteristics remained the most important consideration for lay people when evaluating buildings. This became evident in the discussions around building $\mathrm{D}$, where participants considered that the large physical form of the structure had a negative influence on the street.

Professionals involved in the processes of urban change seemed more willing to overlook stark differences, such as variations in building height along the south side and the awkward relationships that arise as new projects are built to more permissive height restrictions. This is evidenced by the higher median preference scores this group returned for the relationship of heights of buildings on the south side of College Street. Change professionals also prefer more variation in streetscapes than lay people. Lower preference scores for relationships that are close and higher preference scores for relationships that are more varied provide evidence for this. The professionals' focus group discussion also helped articulate this as a difference between the professional and lay groupings. Chapter 
7 discusses the Tyler Street case study and a discussion across the results of Studies One and Two will be found in Chapter 9. 
Chapter 7

\section{Study Two - Tyler Street, Auckland}

\subsection{Introduction}

The second case study is Tyler Street in the Auckland precinct known as Britomart. In the last 15 years this area, which is close to the city centre, has undergone a rapid transformation, initiated by the development of the Britomart underground railway station. The precinct, highlighted in the map of the area (figure 7.1), was built on reclaimed land (Auckland City Council 2014) close to Auckland's commercial harbour. In the first decades of the $20^{\text {th }}$ Century the area was developed by trade and mercantile businesses for their head offices and warehouses. Many of the older buildings, particularly those along Customs Street East and Quay Street, date from that period.

Tyler Street runs between Queen Street to the west and Britomart Place to the east (figure 7.2). It is narrow, perhaps reflecting its origins as a service lane behind the buildings facing the wider Quay Street to the north. This is also evident in the facades of the buildings on the north side of Tyler Street, several of which read as rear or side elevations. Its 400 metre length is broken by the extensions of Commerce and Gore Streets. The building heights vary significantly on both sides of the street (figure 7.3). Whereas buildings consistently define the north side along its length this is not the case on the south side, in part because buildings P and Q are set back from the street. However, the major factor in this less well-defined street edge is the large public open space known as Takutai Square, between buildings $\mathrm{N}$ and $\mathrm{O}$. The cross streets and the low height of building $\mathrm{O}$ also play a part in creating a sense of openness along this side. The area is busy throughout the day, with people attracted to its cafes and bars as well as the high- 
end retail shops. There are a number of international fashion and consumer good outlets along Tyler Street and the ground floor of building N, with its internal mall that bisects the building between Takutai Square and Britomart Place. Accordingly, the facade facing onto Tyler Street is less interactive than those of the majority of buildings along the length of the street.

There are many commercial offices in the upper floors of the buildings along Tyler Street and only building B appears to contain residential activities. A number of the older former warehouse or administrative buildings have been adaptively reused. One of these is the former Chief Post Office, building R, which is now part of the Britomart Transport Centre.

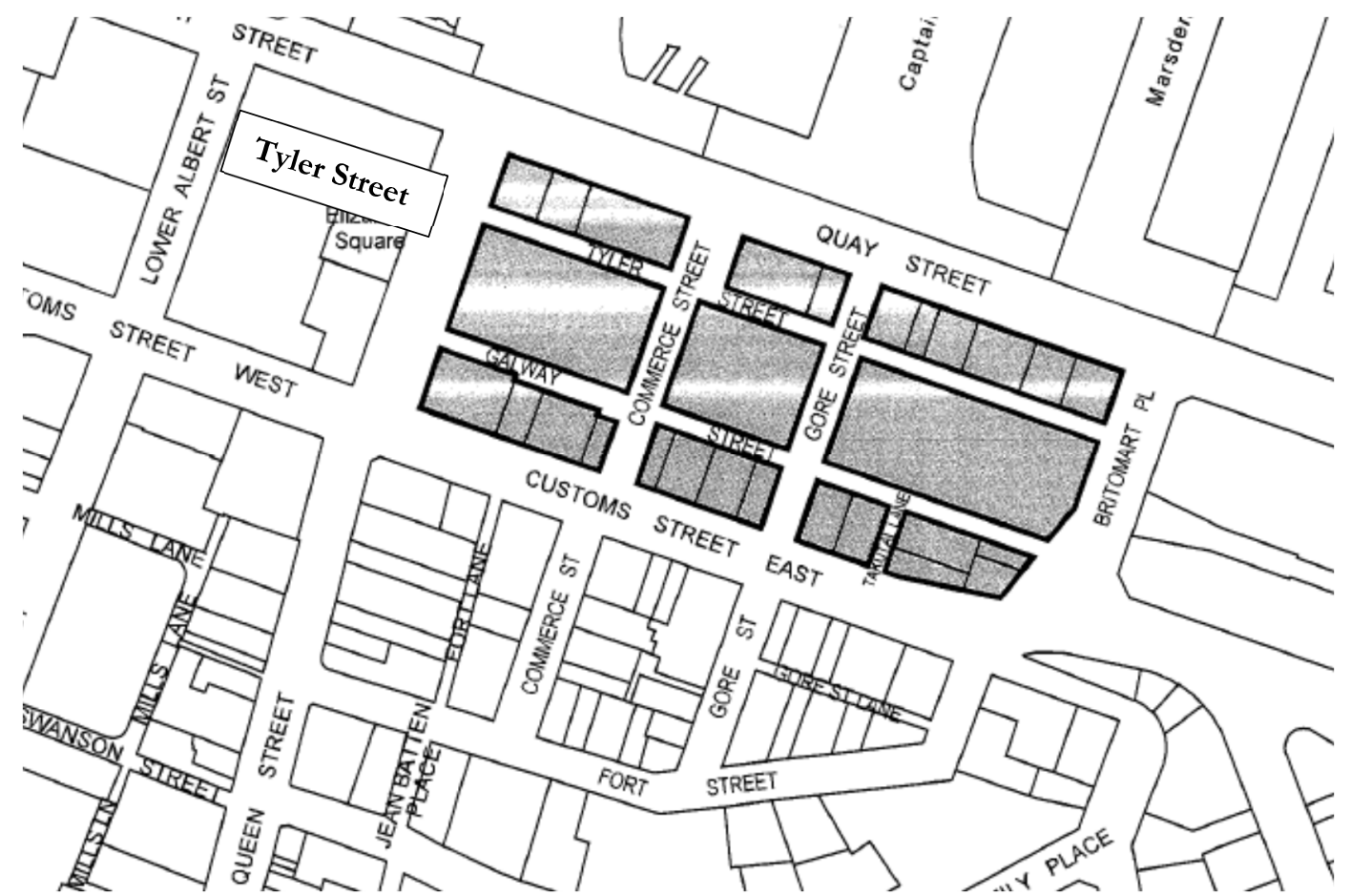

Figure 7.1: Map of the area around the Tyler Street case study site, beside the commercial waterfront in the Auckland CBD

Source: Auckland Council 2014 


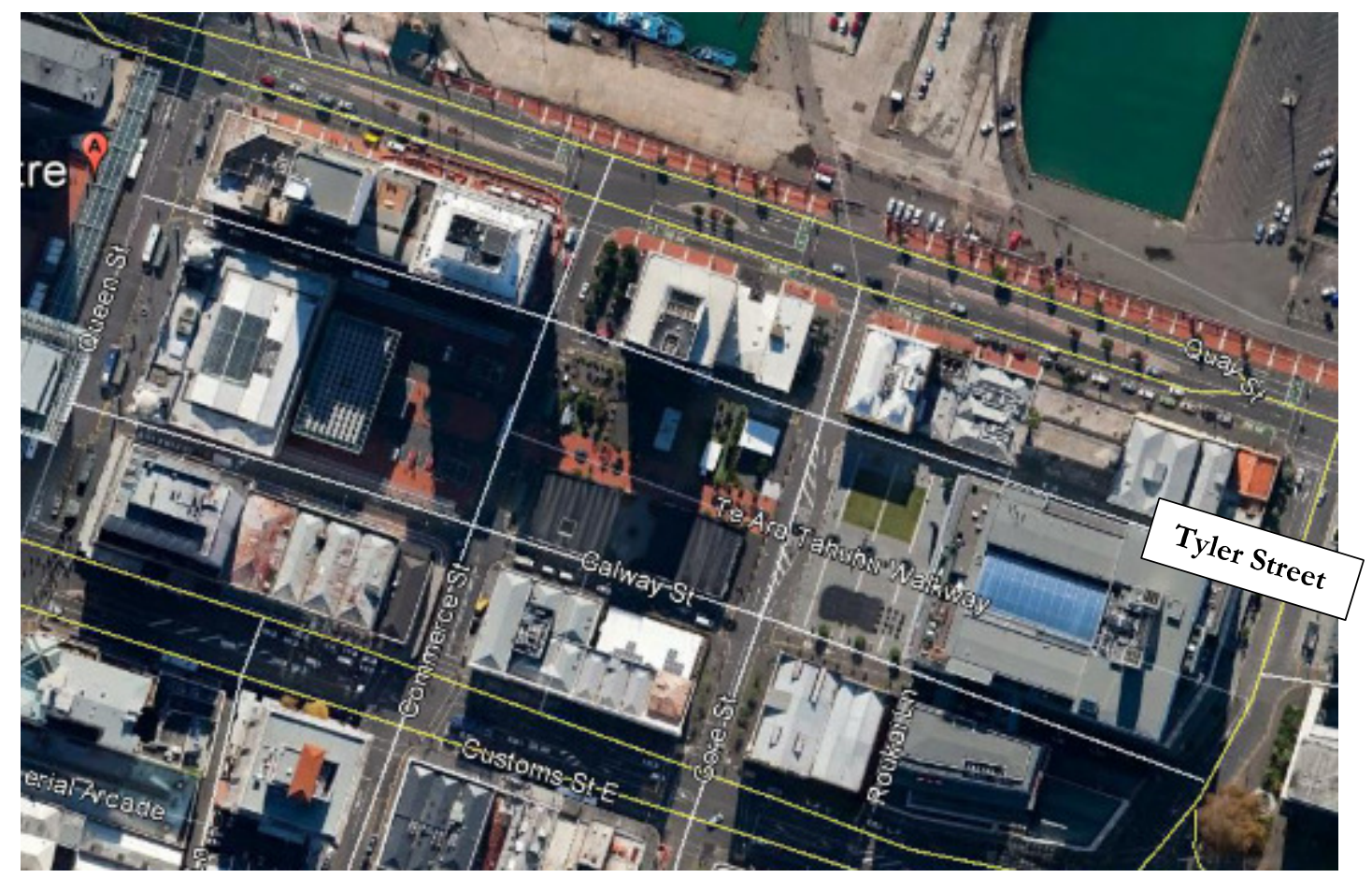

Figure 7.2: Aerial image of the Britomart precinct. Running parallel to Quay Street and the waterfront, Tyler Street extends between Queen Street and Britomart Place

Source: Google Earth

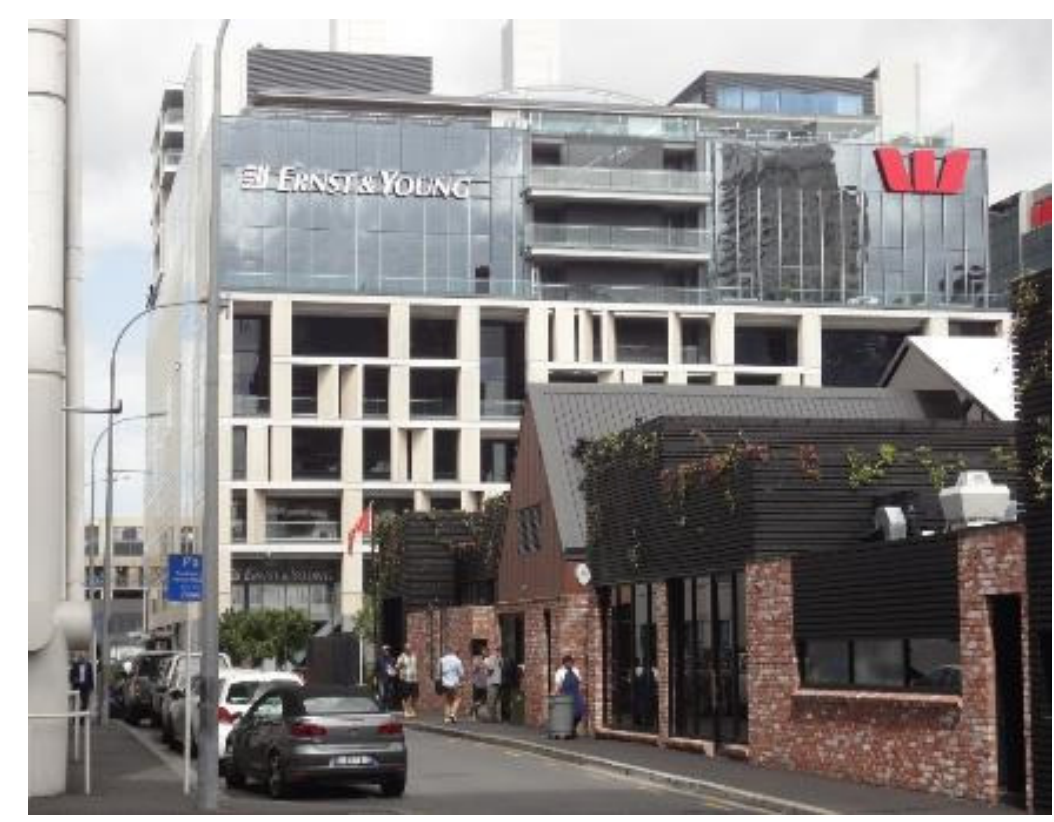

Figure 7.3: View east along Tyler Street from the intersection with Commerce Street. Building $\mathbf{O}$ is in the foreground and building $\mathbf{N}$ in the distance. 


\subsection{Summary of responses received}

The Britomart survey was conducted between 22 October 2013 and 15 January 2014 (see also sections 4.4.4 and 6.1). Efforts were made to recruit a wide range of people. Arrangements were made to leave copies of the survey form in central locations at six local businesses, two university departments and with the Auckland Council urban design team. The staff and students of these organisations were then invited by email to collect and complete the surveys at their leisure. In addition, notices were placed in the newsletters of two branches of the University of the $3^{\text {rd }}$ Age. Over a December weekend several hours were spent walking around the area, offering the survey forms to interested members of the public. All who completed the survey were offered a pre-paid hot drink at a local café, where the forms were also collected. These efforts returned 40 survey forms, although several were incomplete.

Approximately $51 \%$ of the respondents were female and $49 \%$ male. Just over $20 \%$ were involved in occupations involved with change in the built environment. The respondents were more highly educated than the general population with $31 \%$ holding a university bachelor's degree and 51\% a post-graduate degree as their highest qualification. This reflects the types of location where the survey forms were placed. Although several trade based businesses were approached when distribution of survey was being planned, all refused to house the forms. The nature of the businesses in the area suggests that they cater to people with a higher than average socio-economic status. The findings, therefore, need to be considered in terms of the high education level of respondents. Table 7.1 sets out the other demographic details of the Tyler Street sample. 
Table 7.1: Ages, occupations and residential circumstances of the Tyler Street sample

\begin{tabular}{|r|r|}
\hline & Distribution (\%) \\
\hline Age & \\
Under 25 & 28.6 \\
$25-39$ & 31.4 \\
$40-54$ & 20.0 \\
$55-69$ & 11.4 \\
Over 70 & 8.6 \\
Total & 100.0 \\
\hline Occupation & \\
Planner & 5.9 \\
Designer & 14.7 \\
Other professional & 44.1 \\
Trade & 2.9 \\
Home unpaid & 2.9 \\
Student & 14.7 \\
Retired & 8.8 \\
Other & 5.9 \\
Total & 100.0 \\
Rural & 2.9 \\
Suburban & 40.0 \\
Suburban apartment & 11.4 \\
Central apartment & 28.6 \\
Total & 100.0 \\
\hline Inner suburb & 54.3 \\
& 5.7 \\
\hline
\end{tabular}




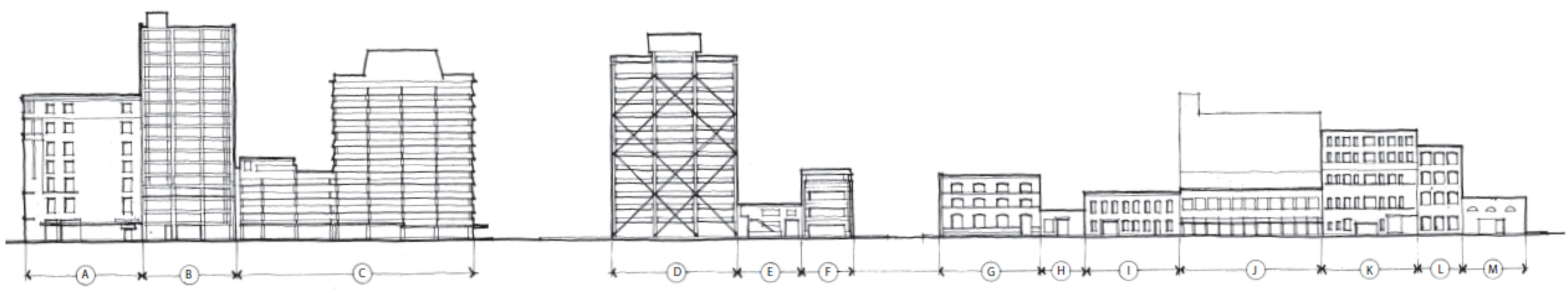

Tyler Street North Elevation: no scale

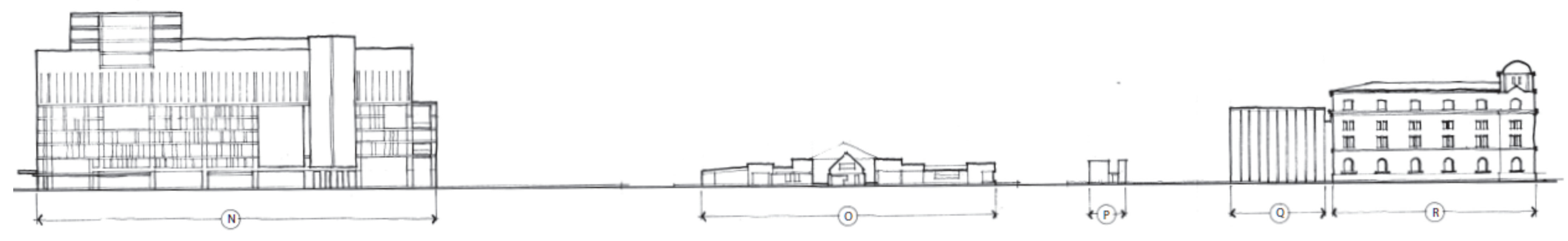

Tyler Street South Elevation: no scale

Figure 7.4: Outline elevations of each side of Tyler Street provided to respondents to help them orient them during their visit. 



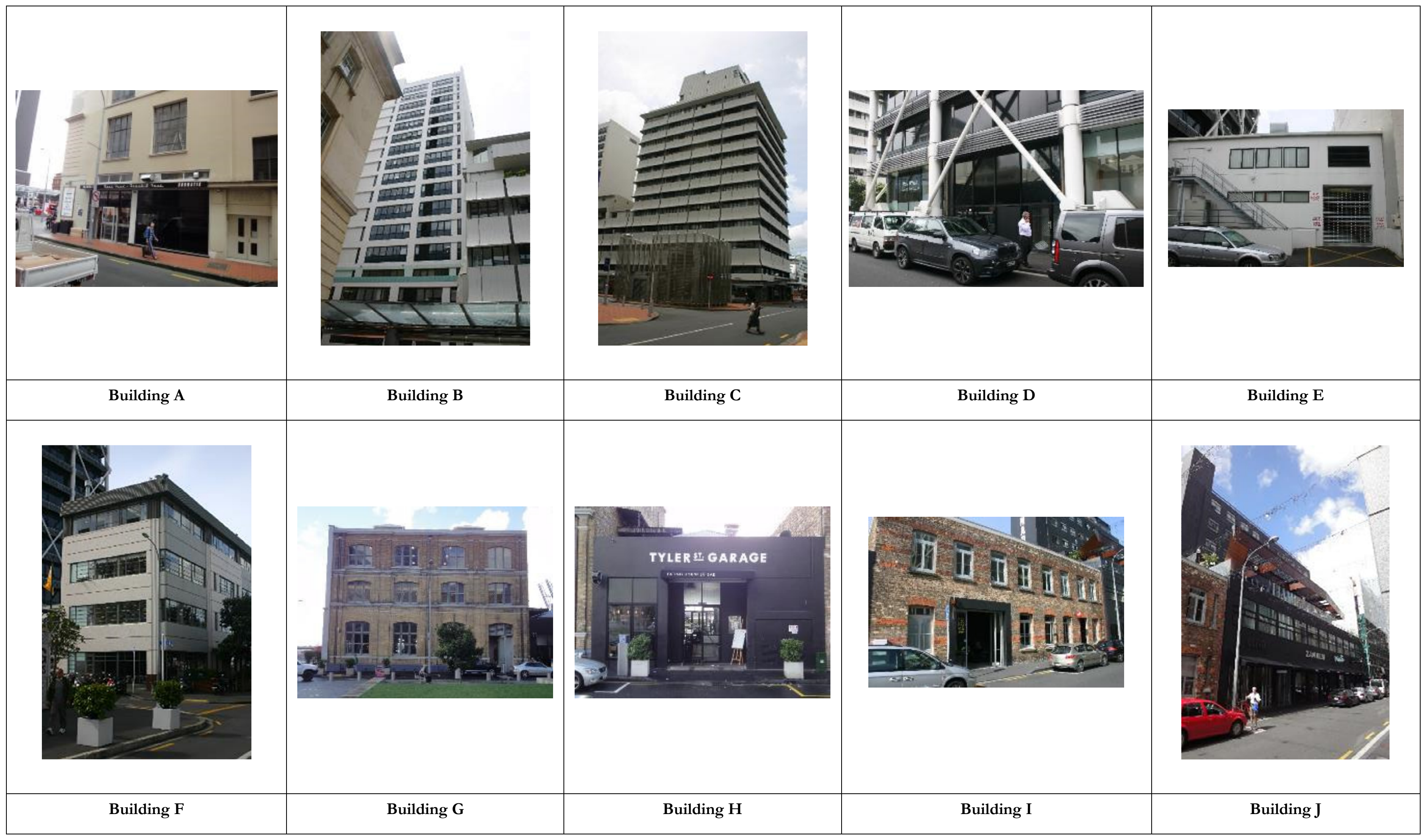

Figure 7.5: Images of each building along the northern side of Tyler Street 



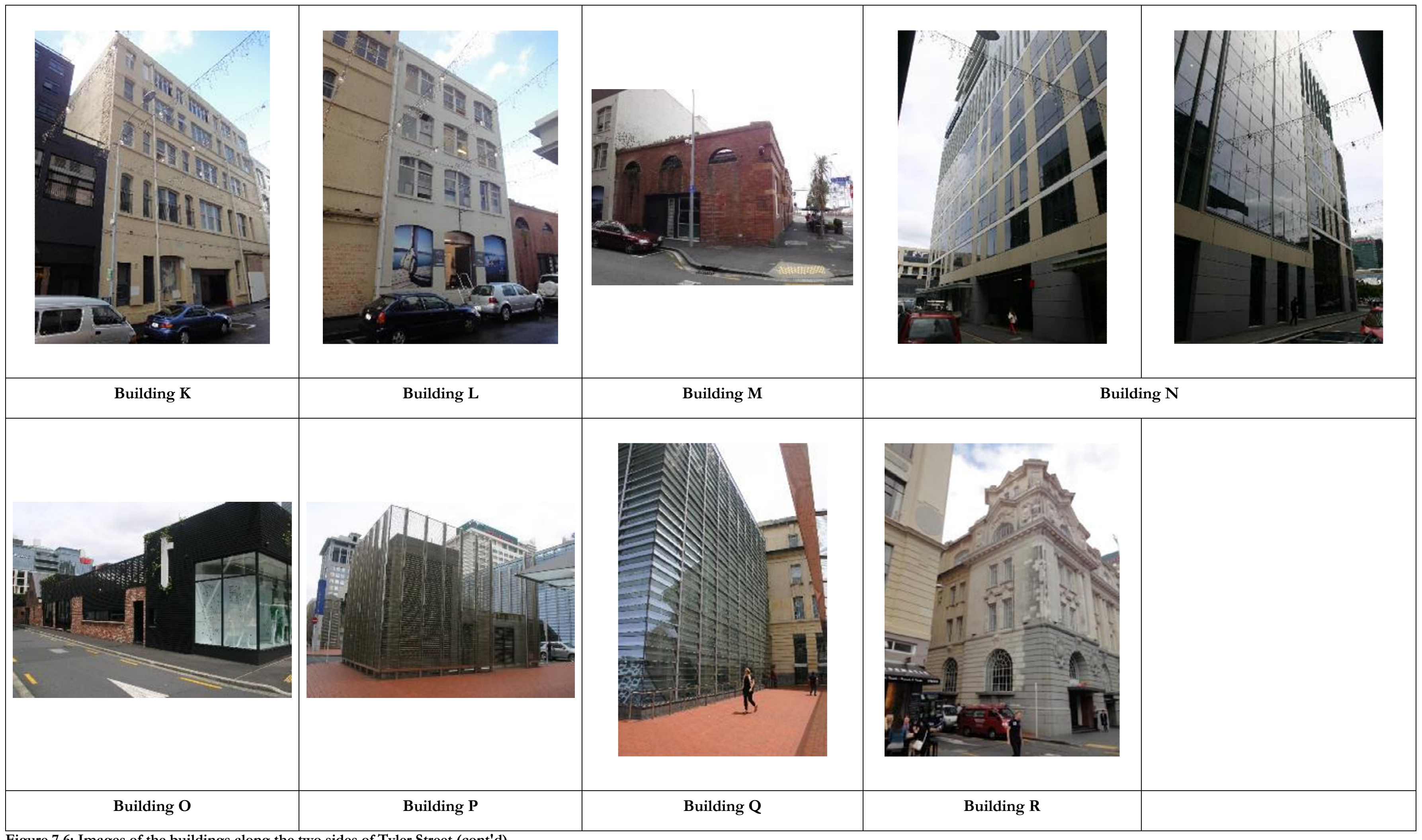

Figure 7.6: Images of the buildings along the two sides of Tyler Street (cont'd) 



\subsection{General preferences}

\subsubsection{Individual buildings}

Overall the buildings in Tyler Street were liked much more than those of College Street. Of the 18 buildings in Tyler Street, 14 (78\%) received positive mean scores and several were extremely well-liked. Three of the buildings that were considered not liked still had mean scores just under the neutral point. In the whole of the street only building $\mathrm{E}$ was roundly disliked.

The area around Tyler Street has been thoroughly transformed from its earlier character and the process is largely complete. The current land use activities appear to be consistently commercial and retail as the warehouse buildings have been converted to other uses. Consequently, there is little evidence of the original warehouse and light industrial activities. These activities are still in College Street and some people responded negatively toward them. While such activities and the buildings that host them can enrich an area through added diversity, they may also confuse perceptions, leading to less satisfactory aesthetic responses.

Another factor in the more positive scoring of Tyler Street could be the degree of landscaping and green space compared with College Street. Takutai Square is a green public open space and elsewhere the street has a number of young trees in front of buildings. How these factors may have influenced aesthetic perceptions of Tyler Street will be discussed further Chapter 9.

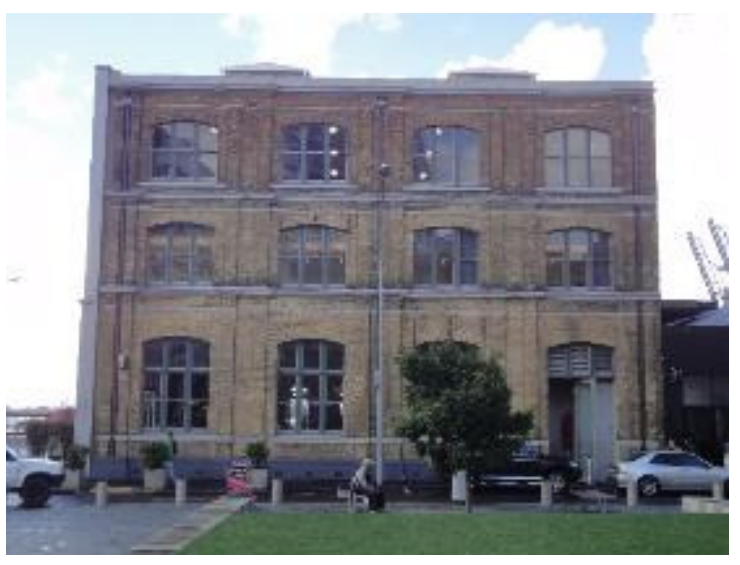

Figure 7.7: Building $\mathrm{G}$ was best liked.

Source: author

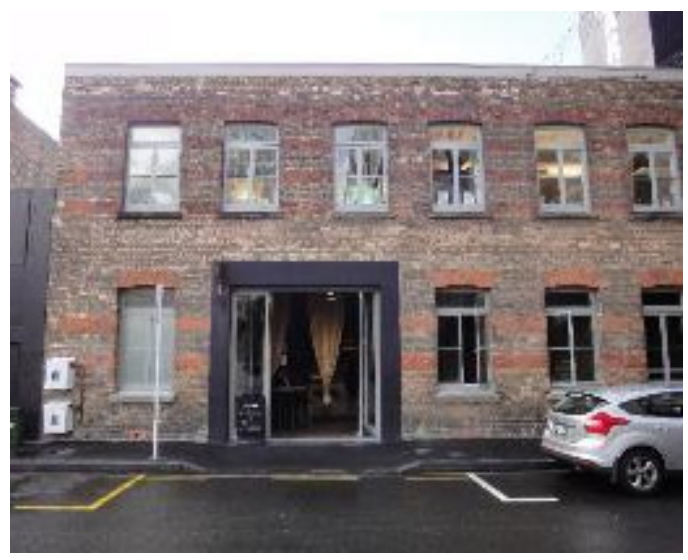

Figure 7.8: Building I was second most liked in Tyler Street.

Source: author 
Opinions of building $\mathrm{G}$ were almost unanimously positive, with $97.3 \%$ of the respondents liking it (figure 7.7), and the other person neither liking nor disliking it. The mean preference score was 2.97 on a scale of 3. Building $G$ is a three-storey former warehouse building with a particularly generous ground floor height. The south elevation onto Tyler Street is made of yellow-fawn brick masonry. The windows all have a shallow arched head and the building has an obvious patina of age. Like other older buildings in the area, it has been adaptively reused and now houses a café/bar on the ground floor with commercial offices above. Those liking the building cited facade details and oldness as the two factors most influencing their opinions. The building also had the highest scores for design above ground level (mean=4.59), surface finishes above ground floor level $($ mean $=4.62)$ and design at street level (mean=4.54).

Located two sites to the east of building $G$, building I (mean=2.92) was second-best liked (figure 7.8). Three people were neutral but no one disliked the building. Like building $G$, facade details and age were the two most common reasons for people liking the building. Following this, building $\mathrm{O}(\mathrm{mean}=2.80$ ) was third preferred (figure 7.3). Building $\mathrm{O}$ appears to be made of recycled brick in combination with stained timber. Taken with earlier preferences, this suggests that the public are attracted to brick masonry, which can simultaneously provide a surface with visual interest and a sense of order. The masonry module is small and well known, making it an ideal material for helping viewers understand the overall scale of a structure.

While brick masonry is the common factor across in the three best liked buildings in Tyler Street, there are other brick buildings, including building $M$ (mean=2.44 ranking it $7^{\text {th }}$ equal in preference) which is now a bar/café. Situated on the corner of Tyler Street with Britomart Place, building $\mathrm{M}$ differs in that its main facade has a limited engagement with the street (figure 7.9). Of the five people who disliked the building, four also disliked its design at street level. However, this perception was not shared by those liking building M. Of the 21 people who liked it, 16 liked the design at street level, which might suggest that the texture and colour of the brick can overcome perceived shortcomings in the way the design relates to the street. 


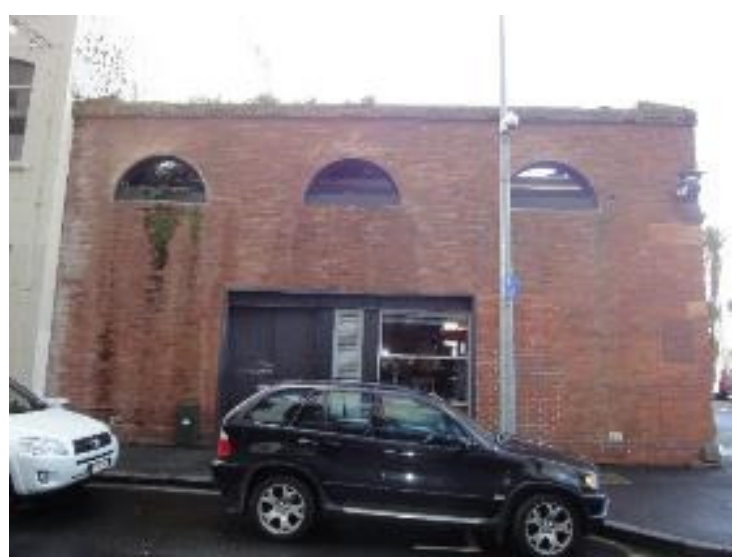

Figure 7.9: Building $M$, which is not liked as well as the other three brick buildings in Tyler Street possibly because of the lack of engagement with the street

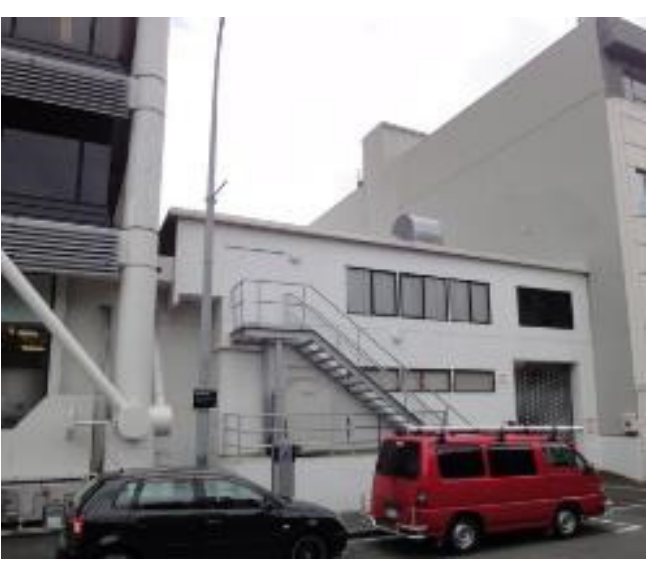

Figure 7.10: Building $\mathrm{E}$, the least liked of all buildings.

Source: author

Source: author

The strongly positive response to the design of building $G$ at street level invites comparison with others along the street. At street level the design of building $G$ maintains the facade modulation above but the windows are taller to correspond with the floor to floor height. The eastern window at this level has been modified to become a covered entry into the building. There are subtle details, such as the engaged columns flanking each window and the considerable wall thickness is evident in the reveals and sill. Despite the floor level being higher than the footpath it is easy to look into the building to observe its interior and activities. These may all be factors in the positive perceptions of this building at ground level.

The ground level design of building $J$ is well-liked (mean=3.81) but not as much as buildings $\mathrm{O}$ (mean=4.26) and $\mathrm{R}$ (mean=4.00). Building $\mathrm{R}$ (figure 7.12) is similar to buildings $G$ (figure 7.7) and I (figure 7.8) in that the ground level comprises discrete openings that relate closely to the pattern of openings above. The solid wall between the openings is rendered to emulate stone construction. Building J (figure 7.11) is perhaps more typical of contemporary urban buildings in that the ground level is largely glazed for display of retail goods. The results of this survey suggest the public prefer to see coherent architectural compositions featuring traditional 'punched' window openings over expansive glazed shop fronts. This interpretation is enhanced by the fact the ground level design of building $\mathrm{O}$ (mean=4.26) is liked more than that of building $\mathrm{J}$. Building $\mathrm{O}$ 
(figures $7.13 \& 7.14$ ) has a long shop front that incorporates a variety of materials and opening shapes. Although some glazing is tinted to limit views into the back, it seems that this design is more attractive to people than one based on transparency but with limited design character.

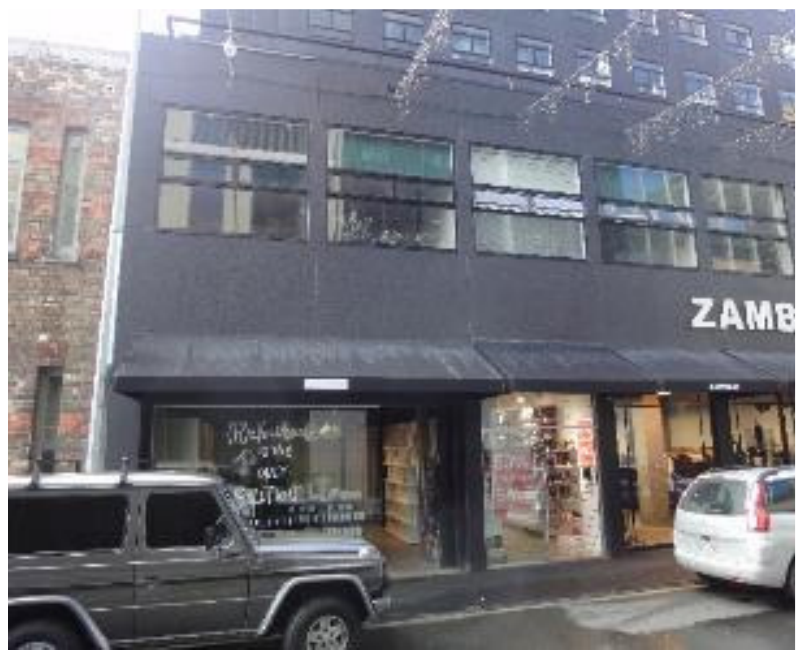

Figure 7.11: Building J, featuring expansive shop front glazing.

Source: author

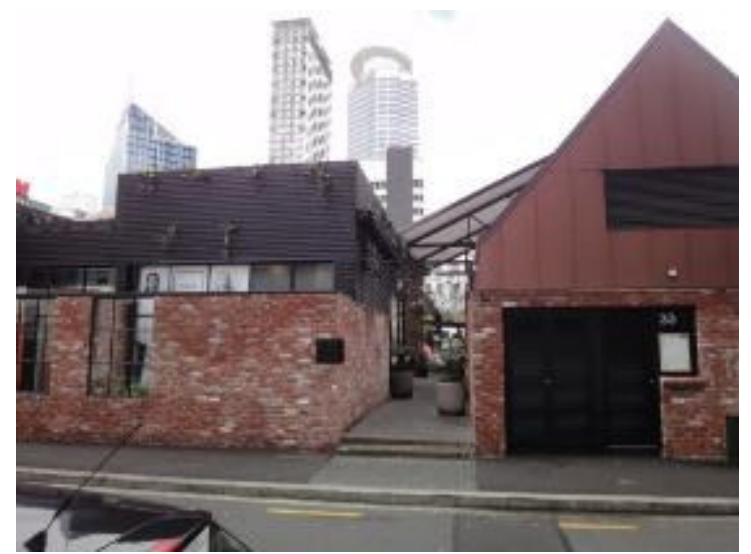

Figure 7.13: Detail of the Building $O$ street façade.

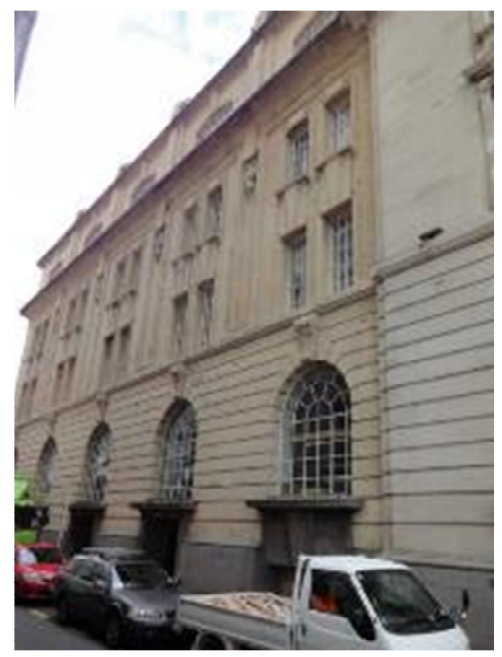

Figure 7.12: Building $\mathbf{R}$

Source: author

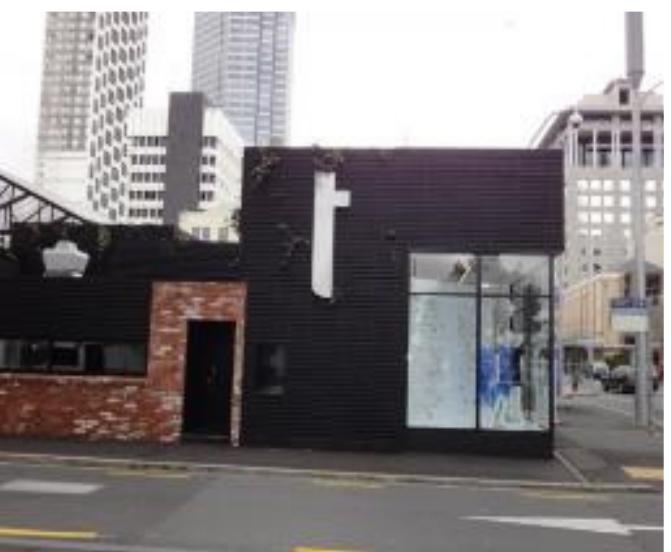

Figure 7.14: Detail of the Building O street façade.

Source: author

Building $\mathrm{E}$ (mean=1.39) was the least liked of the 18 buildings in the street (figure 7.10). This might be expected given its rudimentary, utilitarian design. Nevertheless, three people liked the building each giving its height as a factor for this. Adjacent to this site is building $\mathrm{D}$ (mean=1.84). Although its mean score sits well above that of building $\mathrm{E}$, it 
ranks as the second least liked building in Tyler Street. Of the $42 \%$ of respondents who disliked the building, only three felt its height was a prime factor in their opinion. The majority of those disliking the building did so because of its facade details. On the other hand, $27 \%$ of respondents liked the building and all who did noted the facade details as a reason for this. Building D dates to the early 1980s and its diagonal steel tubes are used as an expressive form of structural bracing, recalling the rigging on the yachts in the nearby harbour (figure 7.15). It would seem that this expressive facade detail polarises opinions and is key to people's feelings about the building overall.

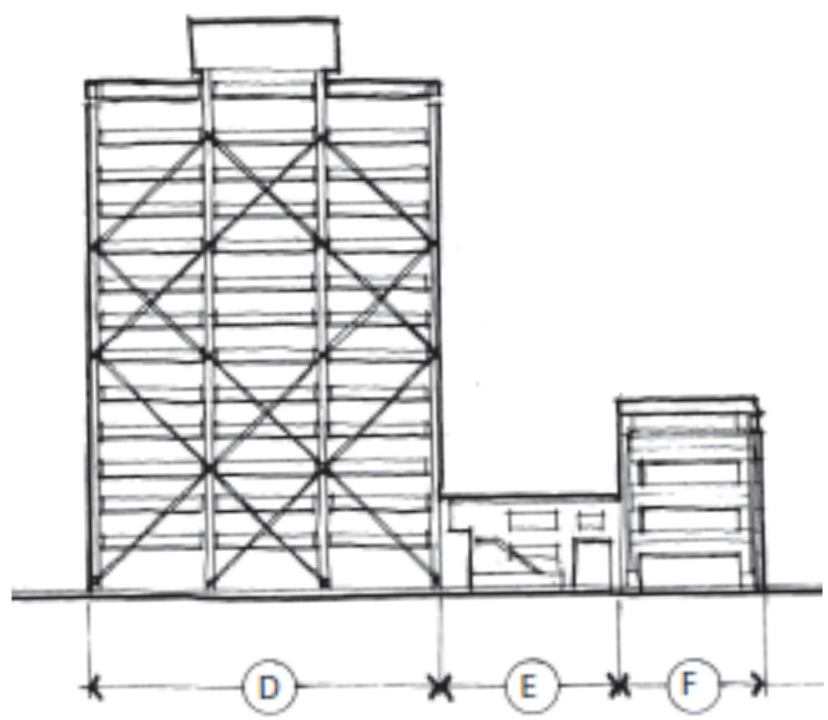

Figure 7.15: Line drawing of building $D$ and its neighbours. The expressive structural bracing was both liked and disliked by survey respondents.

Source: author

With a mean score of 2.56 , building $\mathrm{N}$ was liked by $67.6 \%$ of the survey participants. This ranked it just behind another new intervention, building Q, with mean score of 2.60 (figures 7.16 and 7.17). Building $\mathrm{Q}$ is a largely glazed atrium space that accommodates the vertical circulation associated with the Britomart underground rail station. Both buildings are contemporary designs that make extensive use of glass, steel and concrete. Building $\mathrm{N}$ is the pivotal structure in the Britomart precinct, serving as the backdrop to 
Takutai Square and helping to define its eastern edge along Britomart Place. Glass and windows were cited as a key factor in their decision by 16 of the 23 people who liked building $\mathrm{N}$. The second most popular reason for liking the building was the facade detailing. Similar responses were recorded in relation to building Q.

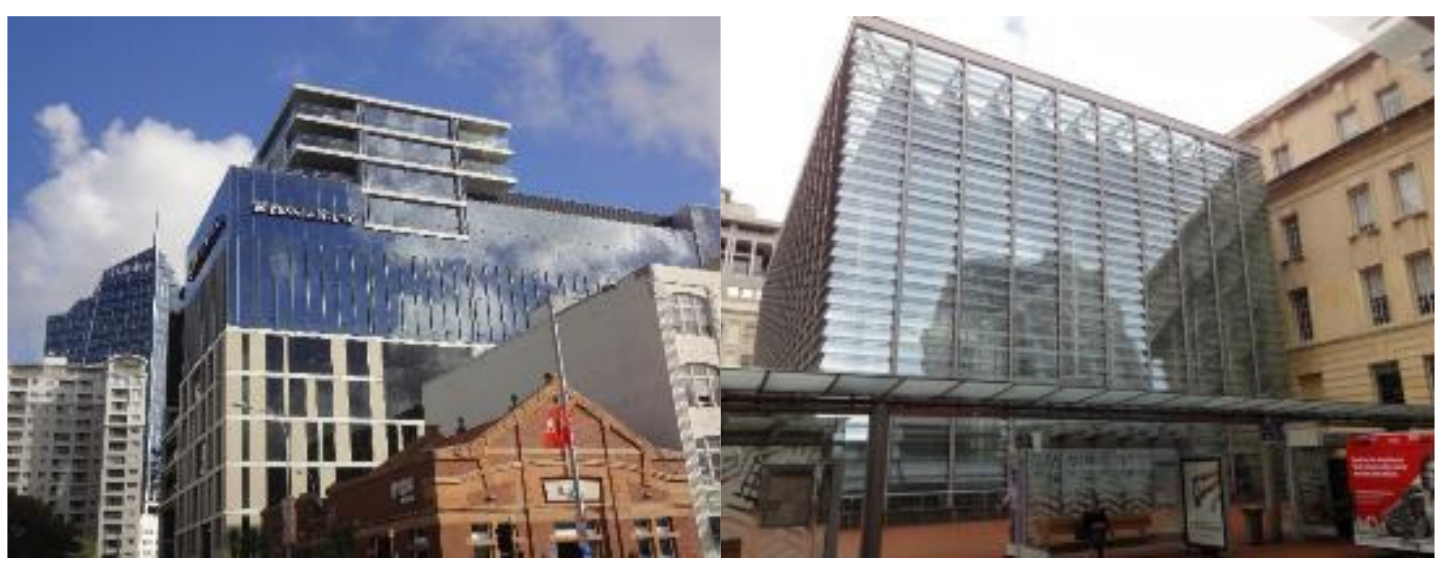

Figure 7.16: Building N, seen across the top of the Quay Street elevations of buildings $M$ (left) and $L$.
Figure 7.17: Building $Q$, built as an annex to building $R$ when the underground rail station was developed early in the 2000s.

Source: author

Source: author

\subsubsection{Relationships between buildings}

The best liked relationship between adjacent buildings was that of buildings $\mathrm{N}$ and $\mathrm{O}$. However, it is probable that the high preference rating for this relationship was influenced by Takutai Square intervening between the two buildings. Not only is this public space attractive but being a wide area it helps buffer the visual contrast between these two very different designs. Building $\mathrm{N}$ is large, shiny and corporate in nature while building $\mathrm{O}$ is small in scale, rustic, and handmade. It seems that these two different design expressions can co-exist successfully with a sufficiently positive open space between them.

The relationships between buildings A to F inclusive along the north side of Tyler Street were not well-liked. These low scores correspond to the preferences expressed for these individual buildings. Building A was liked, as was building $\mathrm{F}$ but those in between were not. Where the relationship in question included a building that was not liked, respondents also judged the relationship to be poor. For example, while building A was liked (mean=2.18), building B was not (mean=1.97) and the relationship between them was not liked (mean=2.83) (table 7.2). An exception was the relationship between 
buildings $\mathrm{C}$ and $\mathrm{D}$. While both buildings were disliked, the relationship between them was seen as positive. Two sets of factors may have influenced this. Firstly, the two buildings are of similar height and typology, being commercial office tower. While respondents may not have liked the aesthetic qualities of the individual buildings they could perhaps recognise the common ground between them and apply that to form a positive opinion about the relationship. Secondly, Commerce Street separates the two buildings and, much like the relief Takutai Square provides between buildings $O$ and $P$, this may have helped mitigate the perception of the relationship.

Table 7.2: Comparison of preference scores for buildings A-F and their relationship scores

\begin{tabular}{|l|c|c|c|}
\hline & $\begin{array}{c}\text { Mean preference score } \\
\text { (3-point scale) }\end{array}$ & \multicolumn{2}{|c|}{$\begin{array}{c}\text { Mean relationship scores } \\
\text { (5-point scale) }\end{array}$} \\
\hline Building A & 2.18 & A to B & \\
\hline Building B & 1.97 & $\mathbf{2 . 8 3}$ & B to C \\
\cline { 1 - 1 } Building C & 1.89 & C to D & $\mathbf{2 . 6 4}$ \\
\hline Building D & 1.84 & $\mathbf{3 . 1 4}$ & $\mathrm{D}$ to E \\
\hline Building E & 1.39 & E to F & $\mathbf{1 . 9 0}$ \\
\hline Building F & 2.28 & $\mathbf{2 . 4 6}$ & \\
\hline
\end{tabular}

The relationships between buildings in the line $\mathrm{F}$ to $\mathrm{L}$ were all well-liked, again coinciding with the generally positive views of the individual buildings. These buildings vary in height between one and five storeys with a maximum height difference between adjacent buildings of three storeys. The best liked relationship in this line was that between buildings K (5 storeys) and L (4 storeys).

\subsubsection{Overall streetscape}

Tyler Street was generally well-liked by survey respondents with the exception of the building heights along the north side (figure 7.4). With a mean score of 2.83 (on a 5-point scale), more people found these relationships unpleasant than pleasant. Although some $27.7 \%$ of respondents liked the height relationships, $47.2 \%$ did not. Many of the latter drew attention to the buildings at the western end of Tyler Street, noting that buildings A to $\mathrm{D}$ were too tall (figure 7.4). Other reasons given included a perceived lack of cohesion between the buildings, mainly because of height mismatches, and that some of the lower 
structures appeared to be temporary gap-fillers. Table 7.3 summarises the mean of scores given to streetscape characteristics along both sides of Tyler Street.

Table 7.3: Mean preference scores for streetscape characteristics in Tyler Street.

\begin{tabular}{|c|c|c|}
\hline & Mean & $\begin{array}{c}\text { Std. } \\
\text { Deviation }\end{array}$ \\
\hline North side streetscape & & \\
\hline Building Heights & 2.83 & 1.124 \\
\hline Facade Alignment & 3.27 & 1.024 \\
\hline Facade Styles & 3.21 & 1.175 \\
\hline South side streetscape & & \\
\hline Building Heights & 3.83 & 1.150 \\
\hline Facade Alignment & 3.88 & 0.977 \\
\hline Facade Styles & 3.60 & 1.193 \\
\hline
\end{tabular}

The building height and alignment patterns differ between the two sides of Tyler Street. People generally liked the height relationships and facade alignments along the southern side, as $68.6 \%$ of respondents liked the heights and $70.2 \%$ liked the alignment between facades. However, one respondent who did not like the southern building heights noted that "it feels like three different streets, there is too much diversity between the parts". All sites along the northern side have been built and, although the heights of buildings vary, the street edge alignment of these structures is relatively consistent. Among the southern buildings $\mathrm{N}, \mathrm{O}$ and $\mathrm{Q} / \mathrm{R}$ establish three strong built edges but the physical containment diminishes between each of these buildings, particularly at Takutai Square between buildings $\mathrm{N}$ and $\mathrm{O}$.

The literature advocates for clearly defined street edges in urban settings (Jacobs 1993: 277, Wellington City Council 2012) and the College Street case appears to confirm people's preferences for consistent building alignment along the footpath, the opposite to the findings at Tyler Street. The southern side of Tyler Street is not well aligned, mostly because of the large gaps that exist between buildings $\mathrm{N}$ and $\mathrm{O}, \mathrm{O}$ and $\mathrm{P}$ and $\mathrm{P}$ and $\mathrm{Q}$. In addition, the facade of building $\mathrm{Q}$ is set back from the facade of building $\mathrm{R}$ (figure 7.17). A key difference between College and Tyler Streets may be the way the resultant spaces from misalignment have been treated. In College Street the spaces are generally paved for parking and manoeuvring of automobiles. With the exceptions of the 
carriageways of Gore and Commerce Streets, in Tyler Street the spaces have been developed for people. The step in alignment between buildings $\mathrm{Q}$ and $\mathrm{R}$ is paved and primarily used by people waiting for buses. Takutai Square is a well-landscaped public space. It would appear the sample has responded well to the open spaces along the length of Tyler Street because they have been planned positively.

As Tyler Street is relatively narrow and several buildings along the northern side are tall, the gaps may also provide spatial relief. If the development pattern evident at the two ends of Tyler Street had continued over its length it would have been overwhelming, as the spatial proportions would have been narrow and deep. In this context, the gaps between buildings on the southern side and the lower heights of buildings $\mathrm{O}$ and $\mathrm{P}$ can be understood as providing spatial relief and 'breathing space', a positive characteristic according to seven of the 24 people who liked these relationships.

The positive preference scores for the relationships of facade design styles on the two sides confirm the generally high ratings people gave to the individual buildings along Tyler Street. All five buildings along the southern side were well-liked, with the mean scores ranging between 2.26 (building $\mathrm{P}$ ) and 2.80 (buildings $\mathrm{O}$ and $\mathrm{R}$ ) on a 3-point scale. The design styles of these buildings are diverse, as are their heights, volumes and ages. The mean score of 3.60 (5-point scale) for the relationship of southern side design styles is underscored by $62.8 \%$ of the respondents liking the relationships and only $11.4 \%$ being neutral or undecided. It appears that people liked the variations in design styles across the length of Tyler Street, noting that there are factors helping to mitigate the incompatibilities between them. In many urban streets where buildings are closely spaced, such as College Street (chapter 6) there is insufficient mitigation of incompatibilities with the result that people dislike the relationships.

The style relationships on the north side of Tyler Street also generated strong responses, both positive and negative. Only $5.9 \%$ of respondents found these relationships neither pleasant nor unpleasant, while $41.2 \%$ found them to be unpleasant, and $53 \%$ liked the relationships between facade design styles.

The design styles of buildings $\mathrm{G}$ to $\mathrm{M}$ share several characteristics, which include discrete door and window openings, generous ground floor heights, moderate building height and an appearance of age. These buildings all appear to have roots in the warehouse typology 
of the area as originally developed. On the other hand, buildings $A$ to $F$ (with the exception of building E) appear to share a commercial/office function. The design styles of these six building are perhaps more varied but the three in the middle (buildings B to D) display similar vertical modulation through expression of the floor levels. Behind this primary modulation a secondary, more subtle, structural horizontal modulation is apparent. Building $\mathrm{F}$ is an earlier example of the same typology.

This analysis suggests that there are underlying patterns in the design of each building that links them, making the collection appear more coherent. There are however two factors that may limit the applicability of this interpretation. Firstly, Gore and Commerce Streets create gaps that separate the buildings into three smaller clusters. The gaps help mediate between disparate building designs, improving perceptions of the relationships between them. This is evidenced in the analysis of the south side of Tyler Street and the responses people gave to questions about the relationships between individual buildings on the north side of the street. The relationships $\mathrm{C} / \mathrm{D}$ and $\mathrm{F} / \mathrm{G}$ were judged markedly higher than those for adjacent buildings. Secondly, the survey questionnaire asked people to evaluate the north side streetscape relationships at the eastern end, around building $\mathrm{M}$. While it was anticipated that respondents would develop their opinions over the length of the street, they may have weighted their perceptions by their responses to the more coherent collection of buildings $\mathrm{G}$ to $\mathrm{M}$.

\subsection{Comparisons based on demographic groupings}

\subsubsection{Gender comparisons}

A comparison of mean scores suggests that men and women have similar preferences for most of the buildings along Tyler Street. The preferences expressed for building $\mathrm{N}$ were identical (mean $=2.56,3$-point scale). For the 18 buildings along the street, the differences in mean preference rating ranged between 0.0 (building $\mathrm{N}$ ) to 0.38 (building J). Men were more decisive with their opinions about both buildings but this level of decisiveness was not part of a pattern. Women were notably more decisive about building $\mathrm{C}$, which they generally disliked; $\mathrm{G}$, which they unanimously liked; and $\mathrm{O}$, which they strongly liked.

The two best liked buildings were also the two that both genders were least ambivalent about. Men and women were in strong agreement about buildings G and I. However, the factors taken into account by each group when evaluating the two buildings overall were 
different. Women's opinions about the design of each building at street level correlated most strongly with their opinions of the building overall, whereas men were more focussed on the design of each building above street level. This pattern was observed in perceptions of $50 \%$ of the buildings in Tyler Street. Most notably, it was evident in the evaluations of buildings D, F, G, I, J and O. These buildings all have restaurant/bar or fashion businesses operating at the ground floor level, a factor that may possibly help explain why women's perceptions of the ground level had such influence on their overall perceptions of the building. Similar observations were made about women's preferences for buildings in the College Street case (section 6.4) and streetscapes in Study One (section 5.4.1). On the other hand, men's perceptions of all 18 buildings were most closely related with their evaluations of either the design or the finishes above ground level. This suggests there may be differences in the characteristics that each gender refers to when evaluating buildings.

Women were consistently more positive about the street level designs of buildings in Tyler Street. In only two cases, those of buildings $\mathrm{F}$ and $\mathrm{O}$, were men more positive. These two buildings share few design characteristics and it is therefore difficult to understand why men were more positive, or women more negative, about each. Two cases where women were markedly more positive in their evaluations of the ground level facades were buildings $\mathrm{J}$ and $\mathrm{L}$. While each building appears to have once been used for warehousing, the ground level designs are very different. The building J shopfront is extensively glazed, revealing the nature of the shops inside. In contrast, two of the three bays of the building $\mathrm{L}$ facade have been blocked up and visitors to the building must ascend a short flight of steps to access a foyer. This ground level facade has more in common with those of buildings $G$ and $I$, which both genders liked equally. However, one factor links the two buildings, in that the ground floors are occupied by women's fashion shops. This corroborates with literature suggesting that meanings play an important role in aesthetic judgement (section 3.8). In this case women may have assigned stronger positive meanings to the businesses operating from these two buildings, lifting their overall perceptions of the ground level design quality.

Men and women expressed largely similar preferences for the designs and finishes of the facades of buildings above ground level, and both groups agreed on their preferences for these characteristics of building G. 


\subsubsection{Age comparisons}

Respondents were once again grouped into one of three age-related categories in order to make the findings more robust (see section 5.4.2). Those under 25 appear to have eclectic tastes when it comes to building styles. The two best liked buildings for this group were $\mathrm{N}$ and $\mathrm{R}$, both generating unanimous scores of 3 on the three-point scale. Building $\mathrm{N}$ is shiny and new, anchoring the southern end of Tyler Street as well as Takutai Square. Building R, situated at the western end of the street, presents a more traditional image with solid, weighty walls, punctuated openings and ornate detailing in comparison with that of building N. These buildings have an imposing presence in the street and they are both publicly accessible, one (building R) as the Britomart Transport Centre and the other as a shopping centre on the lower floors. The next two best liked buildings for the under 25 s were $G$ and $Q$, both with near unanimous mean scores of 2.90. Again, the fabric and design expression of each of these buildings is very different, building $Q$ being transparent and scaleless while building is $G$ a well-used and maintained remnant of the earlier warehouses that stood throughout the area.

The older age groups were perhaps more consistent with their preferences for individual buildings. Those aged 25-54 preferred the older buildings in the street and those of a smaller scale. Their top ranked buildings were $G$ and I, both with 3.00 mean preference scores, meaning that all respondents in this category liked the buildings. The next best liked buildings were $\mathrm{O}(\mathrm{mean}=2.78)$ and $\mathrm{R}$ (mean=2.67). The first three buildings are brick masonry construction and this age group liked their street level design. Other than a reversed ranking of buildings $\mathrm{O}$ and $\mathrm{I}$, those aged 55 and over followed the 25-54 year olds with their top four rated buildings in the street. The two contemporary buildings of interest, buildings $\mathrm{N}$ and $\mathrm{Q}$, were ranked in the middle by both groups aged 25 and above. Although the two buildings were liked by both groups, they liked other buildings better.

While all three age groups disliked building E, each group had a different list of other buildings they disliked. For example, those aged 25-54 disliked buildings B, C and D. The common features here are height and a perceived typology of commercial office towers that rely on the expression of floor levels and other structural elements to provide architectural interest. The generally light colour of each is also prone to show staining. People over 55 only disliked building B in addition to building E while those under 25 
disliked building A. The under 25s generally liked buildings B and D and were neutral about building $\mathrm{C}$.

Respondents aged 55 and over were the most positive about every streetscape characteristic. Each of the other two age groups disliked at least one but the older participants were positive about all. On the 5-point scale they returned mean preference scores of over 4 for each characteristic for the south side of Tyler Street. People in the 55 and over bracket on average liked the height relationships on the north side of the street. The reasons they gave suggest they like the contrast of heights, styles and materials in the streetscape. One participant even suggested there was "a nice balance of appropriate and inappropriate relationships". From this statement it can be assumed that people in this age category like the stimulation offered by strong variations in height and that they have a higher threshold before the variety begins to trigger displeasure.

\subsubsection{Comparing change professional and lay people's opinions}

The change professionals who participated in the Tyler Street survey appear to have been more critical of the individual buildings and streetscapes than lay people. Nevertheless, the overall opinions of both groups are, for the most part, aligned. The following section discusses similarities and differences in the ways lay people and change professionals evaluated the Tyler Street streetscape.

For 13 of the 18 buildings, the change professionals were less positive, or more negative, about them than their lay counterparts (table 7.4). Nevertheless, the differences in mean scores were not large, ranging from 0.09 (building N) to 0.52 (building J). Change professionals liked the small cluster of buildings $\mathrm{F}$ to I more than lay people and their mean score for building F was 0.53 higher. These buildings are all old and have been adaptively re-used to accommodate café/bar activities at ground level and commercial offices/living spaces above. As building $\mathrm{F}$ has the largest difference in mean scores, it presents the best opportunity for further analysis of the way each group approaches the aesthetic evaluation. 
Table 7.4: Mean preference scores for buildings along Tyler Street, with responses were recorded on a 3-point scale.

\begin{tabular}{|c|c|c|c|}
\hline Building & Occupation group & Mean & Std. Deviation \\
\hline \multirow{2}{*}{$A_{-}$} & Change Profs & 2.00 & .816 \\
\hline & Lay people & 2.26 & .712 \\
\hline \multirow{2}{*}{$\mathrm{B}_{-}$} & Change Profs & 1.71 & .755 \\
\hline & Lay people & 1.92 & .828 \\
\hline \multirow{2}{*}{$C_{-}$} & Change Profs & 1.71 & .755 \\
\hline & Lay people & 1.89 & .891 \\
\hline \multirow{2}{*}{$\mathrm{D}_{-}$} & Change Profs & 1.71 & .755 \\
\hline & Lay people & 1.85 & .863 \\
\hline \multirow{2}{*}{$\mathrm{E}_{-}$} & Change Profs & 1.14 & .377 \\
\hline & Lay people & 1.41 & .636 \\
\hline \multirow{2}{*}{$\mathrm{F}_{-}$} & Change Profs & 2.71 & .487 \\
\hline & Lay people & 2.19 & .681 \\
\hline \multirow{2}{*}{$G_{-}$} & Change Profs & 3.00 & .000 \\
\hline & Lay people & 2.96 & .192 \\
\hline \multirow{2}{*}{$\mathrm{H}_{-}$} & Change Profs & 2.43 & .786 \\
\hline & Lay people & 2.37 & .883 \\
\hline \multirow{2}{*}{ I_ } & Change Profs & 3.00 & .000 \\
\hline & Lay people & 2.89 & .320 \\
\hline \multirow{2}{*}{$J_{-}$} & Change Profs & 2.00 & .816 \\
\hline & Lay people & 2.52 & .752 \\
\hline \multirow{2}{*}{$\mathrm{K}_{-}$} & Change Profs & 2.00 & 1.00 \\
\hline & Lay people & 2.50 & .514 \\
\hline \multirow{2}{*}{$\mathrm{L}_{-}$} & Change Profs & 2.00 & .816 \\
\hline & Lay people & 2.26 & .859 \\
\hline \multirow{2}{*}{ M_ } & Change Profs & 2.43 & .534 \\
\hline & Lay people & 2.56 & .697 \\
\hline \multirow{2}{*}{$\mathrm{N}_{-}$} & Change Profs & 2.50 & .836 \\
\hline & Lay people & 2.59 & .693 \\
\hline \multirow{2}{*}{$\mathrm{O}_{-}$} & Change Profs & 2.71 & .487 \\
\hline & Lay people & 2.81 & .483 \\
\hline \multirow{2}{*}{$P_{-}$} & Change Profs & 2.42 & .786 \\
\hline & Lay people & 2.26 & .764 \\
\hline \multirow{2}{*}{$Q_{-}$} & Change Profs & 2.29 & .951 \\
\hline & Lay people & 2.70 & .541 \\
\hline \multirow{2}{*}{$\mathrm{R}_{-}$} & Change Profs & 3.00 & .000 \\
\hline & Lay people & 2.78 & .506 \\
\hline
\end{tabular}


Up to $71.4 \%$ of change professionals liked building F (fig 7.18 ) compared to only $33.3 \%$ of the lay public. More than half the lay public neither liked nor disliked the building. For $80 \%$ of the change professional who liked the building, the facade details were cited as a reason for this. One-third of the lay public who liked building F did so because of the facade details. A much greater percentage of this group was influenced by the building's relationship to the street edge as $56 \%$ of the lay public who liked it stated that its alignment with the street was instrumental in their evaluation. In light of these statements a regression analysis was conducted to investigate which design characteristics was the best predictor of a respondent's overall opinion of the building. The characteristic linked most strongly to the change professional's opinion was the design of the facade above street level. Lay respondents' overall opinions of the building were linked most strongly to their preferences for the street level design.

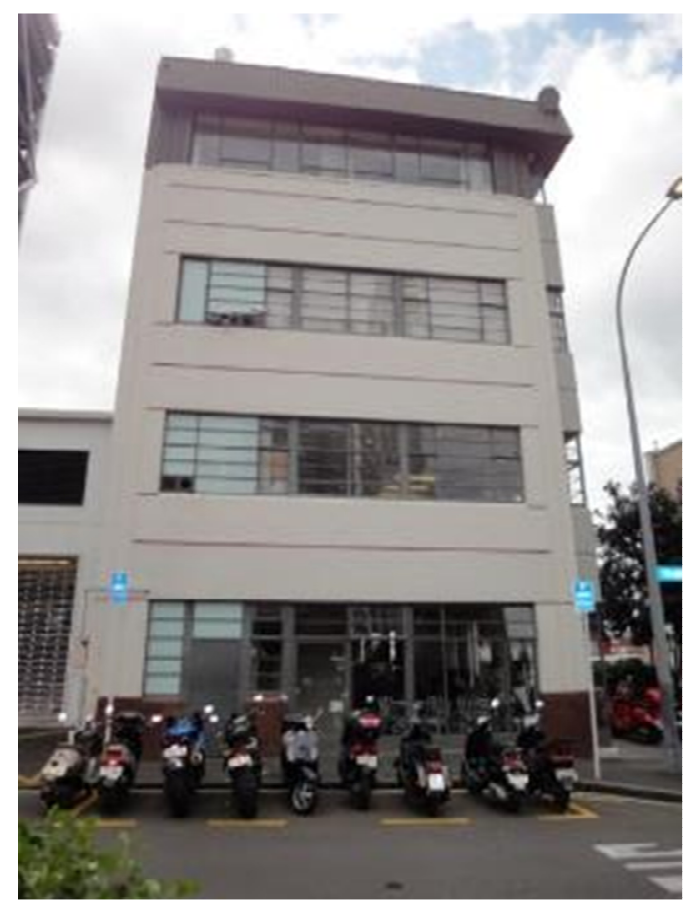

Figure 7.18: Building F, with the largest difference in preference scores between change professionals and lay people.

Source: author 
Table 7.5: Mean responses for design characteristics of building $F$ on a five point scale.

\begin{tabular}{|l|c|c|c|}
\hline $\begin{array}{l}\text { Building F design } \\
\text { characteristics }\end{array}$ & Change Profs & Lay people & Sig (2-tailed) \\
\hline $\begin{array}{l}\text { Facade design } \\
\text { above street level }\end{array}$ & 3.86 & 3.22 & \\
\hline $\begin{array}{l}\text { Surface finishes } \\
\text { above street level }\end{array}$ & 3.57 & 3.04 & \\
\hline $\begin{array}{l}\text { Building design at } \\
\text { street level }\end{array}$ & 4.14 & 3.77 & \\
\hline
\end{tabular}

Change professionals liked each of the different facade design characteristics of building F more than lay people (table 7.5) although the comparisons were not statistically significant. Notwithstanding correlation between lay people's opinions of the street level design and their preferences for the overall building, the reasons given by the lay people who did not like building F were the building's overall shape and the design of the windows. One stated that he disliked the building because it was old while a design professional liked it because of the way it had been maintained. Building $\mathrm{F}$ is four storeys in height with a flat facade onto Tyler Street dominated by horizontal windows. The more visually interesting facade is at $90^{\circ}$, facing onto Gore Street. It may be that the change professionals considered the building comprehensively, taking in the more interesting facade when forming their opinions.

The responses to the small group of older buildings $G$ to I follow a similar pattern to those for building F. For each building the change professionals were not only more positive about the building as a whole but also about the discrete facade design characteristics. While lay public responses to buildings $G$ and I were very positive, change professionals unanimously liked both buildings. Architects and planning professionals, the two constituents of the change professionals group, had similar, positive opinions about these four buildings. It appears that change professionals have consistently positive opinions about buildings of moderate scale that have stood the test of time. These four buildings appear to be well cared for and sensitively converted. The buildings are all considerably lower in height than what appears to be allowable in the area under planning regulations and the construction materials and fenestration patterns contrast with those of more contemporary buildings. The designers of the contemporary building $\mathrm{O}$ have 
understood the attractiveness of these characteristics and have created a building that is the third best liked in Tyler Street, ranking only behind buildings G and I.

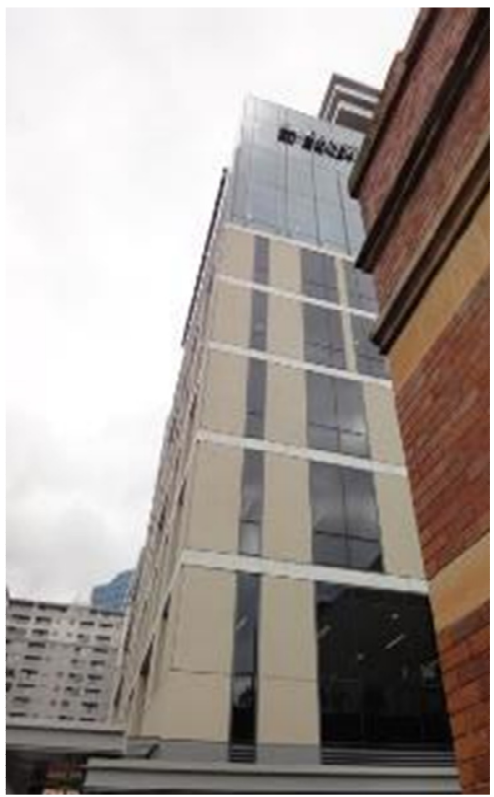

Figure 7.19: The northeast corner of building $\mathrm{N}$, at the corner of Tyler Street and Britomart Place. The facade incorporates large areas of glass, particularly at the upper levels.

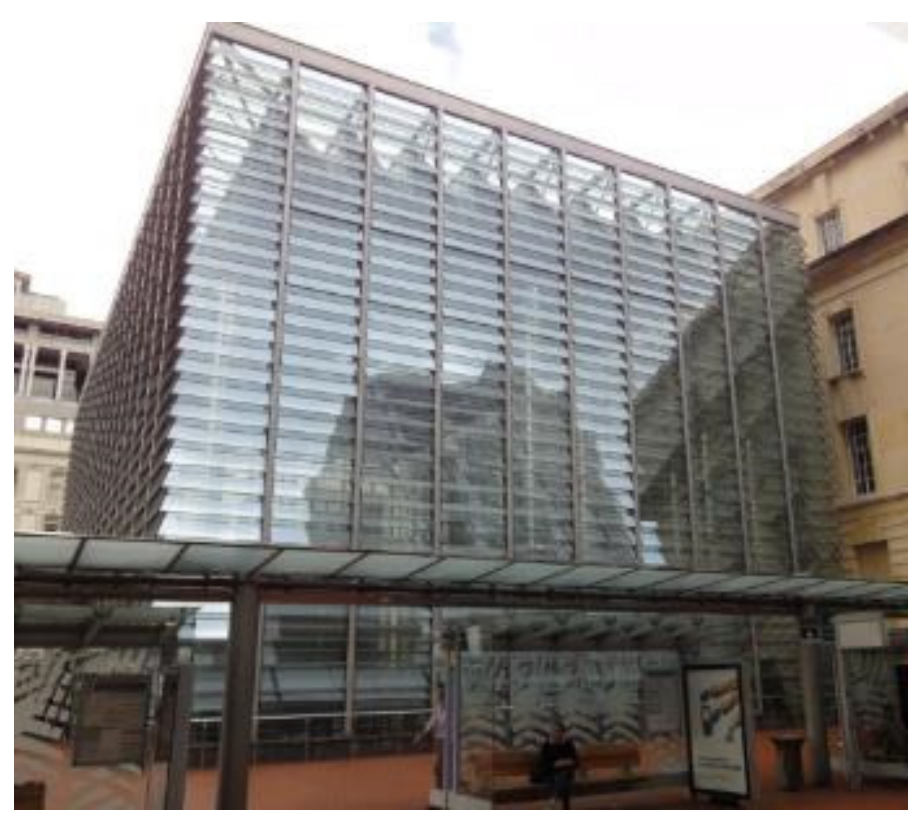

Figure 7.20: Building $Q$, adjacent to building $\mathbf{R}$ to the right. The two buildings are an integral parts of the Britomart Rail Station.

Source: author

\section{Source: author}

Two more recent buildings perhaps more representative of contemporary practices in terms of scale, form and materials are buildings $\mathrm{N}$ and $\mathrm{Q}$ (figures 7.19 and 7.20). Referring to table 7.6, lay people liked both buildings more than change professionals. The two groups were much closer in their opinions about building $\mathrm{N}$ and this is also reflected in the preferences expressed for each of the different facade design characteristics. A similar percentage of each group liked building N (66.7\% of change professionals and $67.9 \%$ of lay respondents), with $16.7 \%$ and $10.7 \%$, respectively not liking the building. Within the change professionals group, architects and planners held similar opinions. Facade details were given as a reason by $75 \%$ of the change professionals for liking building $\mathrm{N}$, with the glazing design second in importance. Nearly three quarters of the lay people who liked building $\mathrm{N}$ were influenced by the glass and glazing design and just under half thought the building's newness was attractive. The upper levels of building $\mathrm{N}$ are extensively 
glazed with highly reflective glass. Although relatively new, the building also appears to be well maintained. The attractiveness of the glass is undoubtedly enhanced by the fact it is clean.

Building Q was built in conjunction with the conversion of building $\mathrm{R}$ to the Britomart Transport Centre. While in effect several storeys tall, the building is only a single level above ground, functioning as a large atrium space. Again, change professionals both liked and disliked the building and its constituent features in a similar pattern to lay people but their scores were generally lower, suggesting they are more critical. The one exception is the differing opinions about the street level design of building Q. Change professionals disliked this aspect whereas lay people liked it.

Table 7.6: Mean preference scores for buildings $\mathbf{N}$ and $\mathrm{Q}$ and the preferences expressed for street facade design attributes.

\begin{tabular}{|c|c|c|c|c|c|}
\hline \multirow[b]{2}{*}{ Building characteristics } & \multicolumn{2}{|c|}{ Building $\mathbf{N}$} & \multicolumn{2}{|c|}{ Building Q } & \\
\hline & $\begin{array}{c}\text { Change } \\
\text { Profs }\end{array}$ & Lay people & $\begin{array}{c}\text { Change } \\
\text { Profs }\end{array}$ & Lay people & \\
\hline $\begin{array}{l}\text { Does the respondent like } \\
\text { the building? }\end{array}$ & 2.50 & 2.59 & 2.29 & 2.70 & 3-point scale \\
\hline $\begin{array}{l}\text { Building design above } \\
\text { street level }\end{array}$ & 3.83 & 4.03 & 3.29 & 3.85 & \\
\hline $\begin{array}{l}\text { Surface finishes above } \\
\text { street level }\end{array}$ & 3.67 & 3.93 & 3.14 & 4.04 & 5-point scale \\
\hline $\begin{array}{l}\text { Building design at street } \\
\text { level }\end{array}$ & 3.17 & 3.79 & 2.86 & 3.93 & \\
\hline
\end{tabular}

Lay people liked building Q's street level design more than that of building N. The street level of building $\mathrm{N}$ features extensive glazed areas between the expressed concrete columns and beams. As building $\mathrm{N}$ is essentially an internal mall, there is little activity along its Tyler Street frontage. Other than access to a loading bay and an unmarked entry/exit door, a single retail tenancy faces onto the street along this frontage. The glazing along this side is also tinted. While the materials are fresh and well maintained and the design coherent, lay people preferred the street level design of building Q, which also exhibits similar features but where the glass is more transparent, enabling activities in the building to be seen. This suggests a possible reason for the difference in mean preference scores but the fact change professionals disliked building $\mathrm{Q}($ mean=2.86) at street level is more difficult to explain. 
Change professionals and lay people appear to dislike similar relationships between adjacent buildings along Tyler Street. The relationship between buildings D and E was liked least by both groups with the next two least-liked were those between buildings $\mathrm{E}$ and $\mathrm{F}$ and $\mathrm{B}$ and $\mathrm{C}$. The $\mathrm{B} / \mathrm{C}$ and $\mathrm{D} / \mathrm{E}$ relationships revolve around significant steps in building height, and are the two biggest steps in the whole street. The buildings themselves were also amongst the least liked by each group, with the exception of building F, which change professionals liked. Although the individual buildings and the relationships between them were not well-liked, there did not appear to be a correlation between preferences for the building and opinions held for the relationships forged with other buildings. Instead it appears that these evaluations were each undertaken independently. This held true for both occupational groupings.

Change professionals preferred the relationships between the buildings they liked best, that is between buildings $\mathrm{I}$ and $\mathrm{J}$, between $\mathrm{I}$ and $\mathrm{H}$ and between $\mathrm{K}$ and $\mathrm{L}$. These relationships are typified by similarities in architectural style, building height and street level land use. Each building in the preferred relationships appears old and adaptively reused for café, fashion retailing and/or commercial office activities. The variations in height between the buildings are slight, and none steps more than two storeys. These relationships were also liked by lay people but not as much as the relationships between buildings $\mathrm{N}$ and $\mathrm{O}$ and $\mathrm{O}$ and $\mathrm{P}$. These two relationships take place across open space and the best liked relationship is between the two buildings on either side of Takutai Square. As discussed earlier, the significant differences in building height, architectural style and materials between buildings $\mathrm{N}$ and $\mathrm{O}$ seem to be overcome the positive public open space between them. Once again, correlations between preferences for the building and the relationships it forms with other buildings are not strong for either group, suggesting that these evaluations took place independently.

A relationship that often arises in transforming urban areas is between a contemporary building and one that is older, perhaps with a sense of character. The design of the new intervention may aim to be compatible with the existing building or contrast with it (Wellington City Council 2012). The relationship between buildings $\mathrm{Q}$ and $\mathrm{R}$ is an example of a contrasting design response. Building $\mathrm{R}$ is a neo-classical composition with a clear tripartite vertical expression and horizontal modulation provided by the windows and facade detailing. The building fabric is massive, with its thickness suggested by 
detailing and at window reveals. The design of building $\mathrm{O}$ contrasts markedly with building $\mathrm{R}$, as the newer building is light, scaleless and transparent. Change professionals (mean=3.50) and lay people (mean=3.18) both liked the relationship between these buildings. Architects (mean=3.70) liked the relationship much more than planners $($ mean $=3.00)$, whose mean score indicated they were neutral about it. This difference in mean score probably reflects the influences of the two different professional cultures. The architectural profession has for some time looked favourably on contrast as an appropriate design response (Punter and Carmona 1997: 209-213). Perhaps the most well-known such project is I.M. Pei's addition to the Louvre (Groat 1994). Specific design features that may also help reconcile the relationship between the two buildings are the setback in plan and lower building height of building Q and the 'negative margin' where the two buildings join (figure 7.21). In relation to design review processes, this example suggests that with appropriate care and attention it is possible to create a well-liked relationship between new and old, where the new design adopts a strategy of contrast.

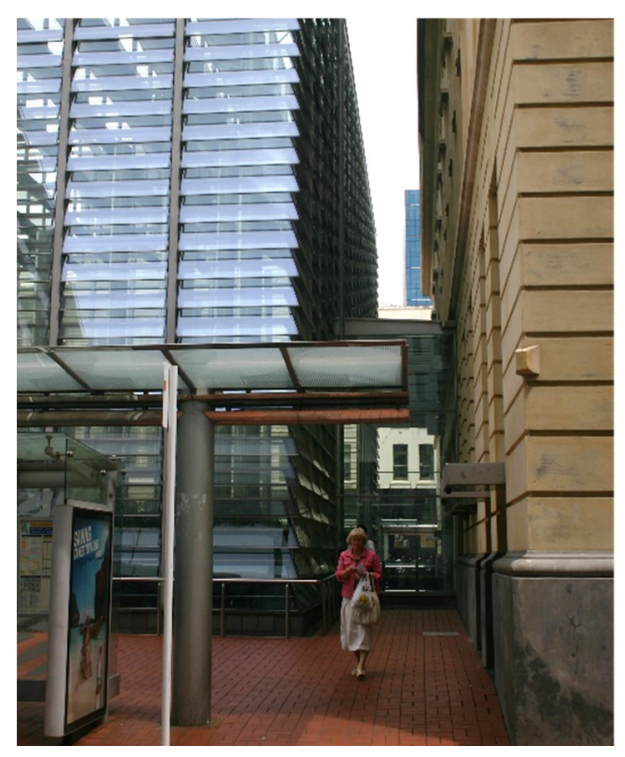

Figure 7.21: The interface between buildings $Q$ (left) and $R$ is a form of negative margin. This separates these two contrasting architectural forms at their junction

Source: author 
Table 7.7: Comparison of mean preference scores for Tyler Street streetscape characteristics

\begin{tabular}{|l|c|c|}
\hline & $\begin{array}{c}\text { Change } \\
\text { professionals }\end{array}$ & Lay public \\
\hline South side streetscape & & \\
\hline Building Heights & 3.29 & 3.96 \\
\hline Facade Alignment & 3.17 & 4.04 \\
\hline Facade Styles & 3.29 & 3.68 \\
\hline North side streetscape & & \\
\hline$\quad$ Building Heights & 2.14 & 3.00 \\
\hline Facade Alignment & 3.14 & 3.30 \\
\hline Facade Styles & 2.43 & 3.41 \\
\hline
\end{tabular}

Table 7.7 reveals that both groups liked the streetscape relationships along the southern side of Tyler Street. Change professionals were less positive than their lay counterparts, as $75 \%$ of lay people liked the height relationships along the southern side compared with $42.9 \%$ of change professionals. Themes running through the reasons given by lay people for liking these height relationships included

- Variety makes for a more interesting skyline

- Space above and around the buildings is attractive

- Attractive relationships between building heights and open spaces

One of the change professionals saw merit in the large buildings at each end of the southern streetscape serving to anchor the street with lower buildings between while another disliked the heights because of the dominance of building N. Within the change professionals group the architects (mean=3.20) had lower opinions of the heights than planners (mean=3.50).

Change professionals disliked the height relationships on the north side of the street, although in fact it was the architects (mean=1.60) who brought down the planners' positive opinions (mean=3.50) of these relationships. Despite their generally positive opinions about many of the relationships between adjoining buildings, the architects disliked the sharp changes in height accompanied by changes in materials and architectural styles. The architect respondents were also responsible for dragging the mean scores of the change professionals below the neutral point for facade design style and facade alignment relationships along the north side. Overall lay people liked the Tyler Street 
streetscape along both sides and the planner respondents followed close behind, being generally positive about the street overall. Other than relating the architects' severe dislike of the north side height relationships to excessive variation, why this group disliked the facade alignment and design style relationships along this side remains unknown. This is particularly puzzling in light of the number of individual buildings and relationships between pairs of buildings the architects liked. Perhaps the group of buildings B to E, which they disliked, dragged their opinions of the street down.

\subsection{Summary}

Forty people spent time evaluating the individual buildings and overall streetscape characteristics of Tyler Street in Auckland's Britomart precinct. A summary of the key results are presented in the following paragraphs.

The buildings and overall streetscape of Tyler Street were generally well-liked by people and the preference scores were on the whole higher that those accorded to the buildings and streetscapes of College Street (chapter 6). The best liked buildings in Tyler Street shared several design and land use characteristics. It seems that the public preferred buildings that were relatively low in height - up to 4 storeys - and those incorporating discrete door and window openings in the solid facades. The three best-liked buildings had public or semi-public ground floors, with retail and café activities. A unique distinction between these buildings was that they were largely constructed of brick. Brick masonry construction provides buildings and surfaces with a sense of scale, visual warmth and evidence of the construction process. The literature supports the fact that these attributes help make it an attractive construction material. This was clearly understood by the designers of building $\mathrm{O}$, which has only recently been built with reused bricks. This building was rated the third best liked in the street.

Well-liked relationships between adjacent buildings typically occurred between buildings that were themselves well-liked. Other factors that made these relationships attractive included similarities in overall height, where differences were limited to two floor levels, and similarities in facade design style. Well-liked relationships also occurred between buildings separated by open spaces, either in the form of Takutai Square or streets. Separation by open space seemed to help mitigate visual effects that, had the buildings been closely adjoined, might be perceived negatively. The sharp contrasts between 
buildings $\mathrm{N}$ and $\mathrm{O}$ produced the best liked relationship, clearly influenced by Takutai Square between them. The designers of building Q, the glazed structure containing the vertical circulation facilities of the Britomart Transport Centre, created a well-liked relationship between this building and the former Chief Post Office, a neoclassical building of substance. This relationship is based on contrast rather than compatibility and the public made it the $5^{\text {th }}$ best rated relationship. In addition to contrasting the design of the pre-existing building with glass, the designers ensured appropriate levels of deference by setting the facade back from and keeping the height of the new structure below that of the older building.

The highly varied building heights on the north side of Tyler Street were not well-liked. Although the buildings toward the eastern end of the street have more harmonious height relationships, many people reacted against the variations in height of the buildings at the western end, which in turn brought the preference scores down. While in physical terms the building heights along the south side of the street are very varied, the public preferred these relationships. This difference in preference could at least in part be attributed to the mitigating effects of the open spaces between disparate buildings and the fact the skyline seemed to have been consciously composed, being higher at each end and lower in the middle of the street. The survey results provide evidence of the positive streetscape effects arising from the open spaces along the street. They have all been designed positively and several respondents acknowledged this. Not only are these spaces attractive, they also provide buffering between buildings with different design characteristics, which helps the relationships between them to be perceived more positively.

Although the facade design styles of the buildings in Tyler Street are sharply varied, these relationships were well-liked by respondents. In addition to the ameliorating effects of the open spaces, including the streets that cross Tyler Street, the grouping of buildings having similar styles was considered an important factor that helped lift perceptions.

Men and women expressed similar preferences for buildings and streetscape characteristics. However, each gender refers to different formal aspects of the building or the street scene when making an evaluation. Women's overall perceptions link most closely to their perceptions of the building's street level design. In addition to the design attributes at street level the land-use activity was also a key factor in aesthetic evaluation. 
The buildings liked best by women all had café or fashion retail activities at ground level. Men, on the other hand, tended to focus on the design or surface finishes above street level when forming their opinions of the building overall. Their preferences were not as closely linked to the ground level design.

There were some differences in aesthetic preference with age. Young people under 25 had quite eclectic tastes when evaluating individual buildings. The top four rated buildings of this group all had different design characteristics and were different to those preferred by older people. This may suggest that young people have not yet formed strong aesthetic opinions, remaining open and considering a wider spectrum of design attributes. Those over 25 were more consistent in their preferences and the older, brick buildings were liked best. While it was reassuring, in terms of design control processes, to understand that people of all ages agreed on the least liked building in the street, there were differences in opinions about which others were not liked. People in the 25-54 age group, those most likely to be making design review decisions, were the most critical group. They disliked four buildings in Tyler Street.

There was general alignment between the preferences for individual buildings expressed by lay people and those involved in urban change. However, the two groups were distinguished by the fact change professionals were more critical, as evidenced by their lower mean scores, compared to lay people. The only exception was in respect of several older buildings along Tyler Street's north side. Building designers and planning professionals rated buildings $\mathrm{F}$ to I higher than lay people, a result that may run contrary to conventional wisdom. Chapter 8 examines the final case study of Kingston and Wyndham Streets in Auckland. 
Chapter 8

\section{Study Two - Kingston \& Wyndham Streets, Auckland}

\subsection{Introduction}

The third case in Study Two, is two short, parallel streets on the west side of Auckland's central business district. Kingston Street extends between Albert Street to the east and Hobson Street to the west, crossing Federal Street along the way. It is about 170 metres long and contains eight buildings. Wyndham Street is one block to the north of Kingston Street, and extends from Queen Street at its eastern end to Hobson Street to the west. Although longer, it is not a through road, which helps keep vehicular traffic volumes down and satisfying an important selection criterion. It also crosses Federal Street (figure $8.1)$.

The area around both streets is busy with pedestrian activity, stimulated by destinations such as the District Court on the corner of Kingston and Albert Streets, St Patrick's Cathedral and Square, where Federal Street terminates at Wyndham Street, and the Sky City Casino and tower to the south along Federal Street. A number of small cafes and eateries have become established in the area over the past twenty years as pedestrian activity has grown.

The buildings in the area support a wide range of activities. In addition to the cafes and District Court noted above, these include permanent and itinerant residential, commercial office, retail and cultural. One building is a multi-storey car park. The buildings also vary 
in style, age and typology. Several buildings occupy sites with frontages extending the whole length of the block, presumably from amalgamating the smaller lots of the original subdivision.

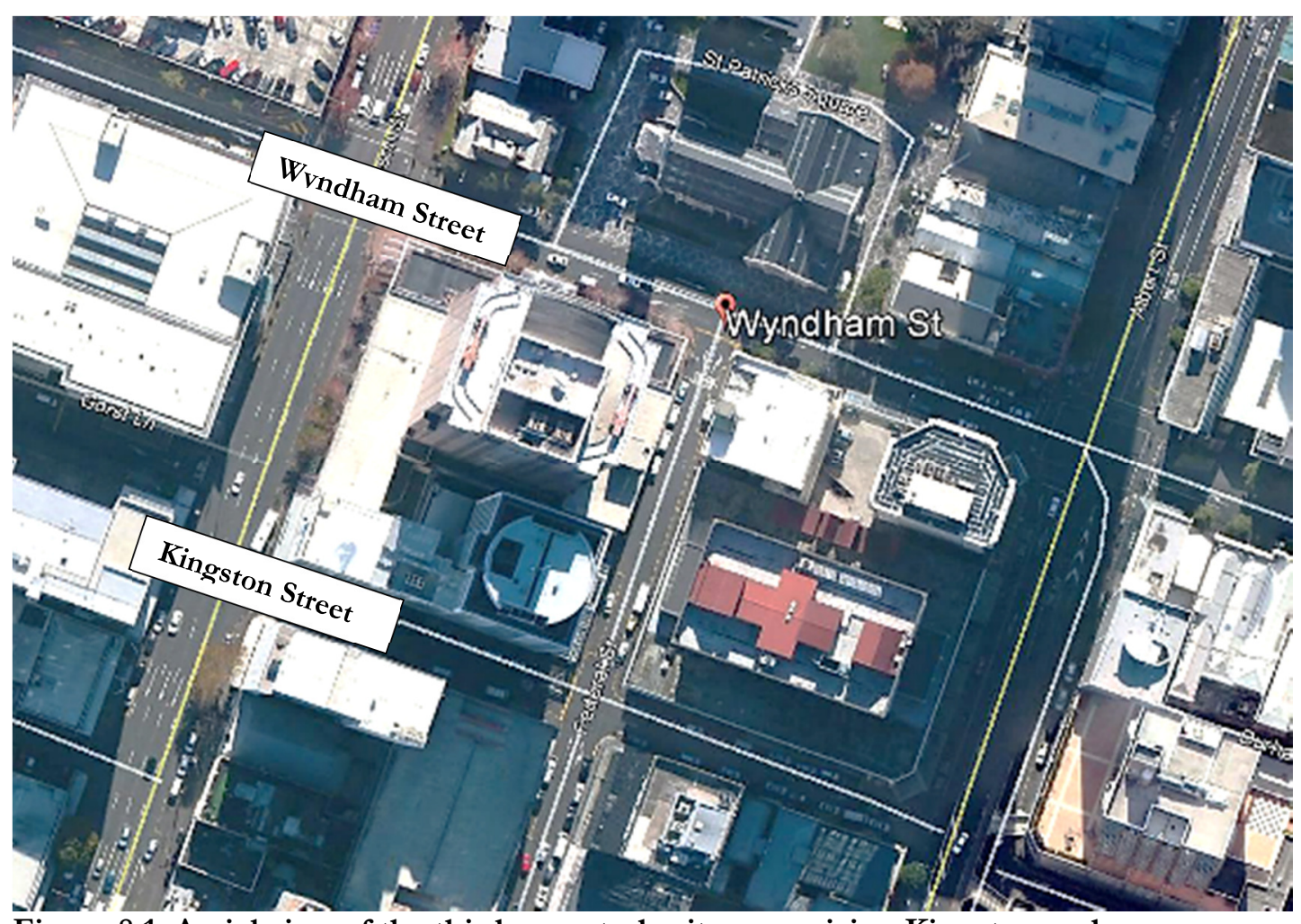

Figure 8.1: Aerial view of the third case study site comprising Kingston and Wyndham Streets

Source: Google Earth.

In addition to the spatial relief Federal Street provides to both Kingston and Wyndham Streets, St Patrick's Square is a key public open space on the north side Wyndham Street. The square extends around all sides of St Patrick's Cathedral, which has been designed to be seen in the round. Most other buildings along both streets have been built to the street edge and consequently help to define a vertical urban space. The western end of Kingston Street is particularly tall, because the street is narrow and the buildings on both sides high. In contrast, Wyndham Street feels more spacious, partly attributable to a greater width but is also as a consequence of the open spaces around St Patrick's Cathedral. Building heights vary considerably along both streets, ranging from the two-storey building W4 to the 20 storey building W3, both in Wyndham Street. Wyndham Street incorporates areas of green landscaping including street trees, while Kingston Street has comparatively little landscaping and what there is occurs on the private side of street boundaries. 
Figure 8.2 projects the elevation views of the four streetscapes of the Kingston/Wyndham Street study area. These views, along with a plan drawing of each street, were included in the survey response booklet to help guide respondents around the study area. Figures 8.3 and 8.4 present photographic images of each building in the study area, as a reference for the reader. As the study area incorporates two streets, the identifier for the buildings in Wyndham Street have been numbered with a preceding 'W' to distinguish them from the buildings in Kingston Street.

\subsection{Summary of responses}

The Kingston/Wyndham Street case study was undertaken concurrently with the Tyler Street study between 22 October 2013 and 15 January 2014. Invitations to participate in the survey were distributed in a manner similar to that of Tyler Street and several organisations were given the opportunity to participate in both surveys. Nine lay people and three change professionals did this. Respondents to the Kingston/Wyndham Street survey were rewarded for their participation by being invited to enter a draw for two separate prizes. Most respondents submitted their contact details for this and, following the draw, two pre-paid vouchers for a local restaurant were sent to the winners. The prize draw incentive helped attract responses from 41 people. While not representative of the general population, the respondents had a diversity of backgrounds as seen in table 8.1.

The group comprised 55.3\% women and 44.7\% men. Only two respondents (5.4\%) were aged 55 and over. This helps explain why only one (2.7\%) respondent listed his/her occupation as retired. Design or urban change occupations were $35.1 \%$ of respondents, $5.4 \%$ were from trade or technical occupations and 10.8\% were in other occupations. Like the previous case studies, this group was highly educated, with $80.5 \%$ holding a tertiary level qualification.

The majority of respondents (59.5\%) undertook the survey between the hours of 10am and $2 \mathrm{pm}$ and $75.7 \%$ visited Kingston and Wyndham Streets when the weather was clear and sunny. 
Table 8.1: Distribution of responses for age, occupation and residential circumstances in the Kingston \& Wyndham Streets case study

\begin{tabular}{|l|r|}
\hline & Distribution (\%) \\
\hline Age & 29.7 \\
Under 25 & 43.2 \\
$25-39$ & 21.6 \\
$40-54$ & 2.7 \\
$55-69$ & 2.7 \\
Over 70 & 100.0 \\
Total & \\
\hline Occupation & 8.1 \\
Planner & 27.0 \\
Building Designer & 13.5 \\
Other professional & 5.4 \\
Trade & 5.4 \\
Home unpaid & 27.0 \\
Student & 2.7 \\
Retired & 10.8 \\
Other & 100.0 \\
Total & \\
\hline Live & 0 \\
Rural & 36.1 \\
Suburban house & 25.0 \\
Inner suburb house & 5.6 \\
Townhouse & 8.3 \\
Suburban apartment & 25.0 \\
Central apartment & 100.0 \\
Total & \\
\hline
\end{tabular}



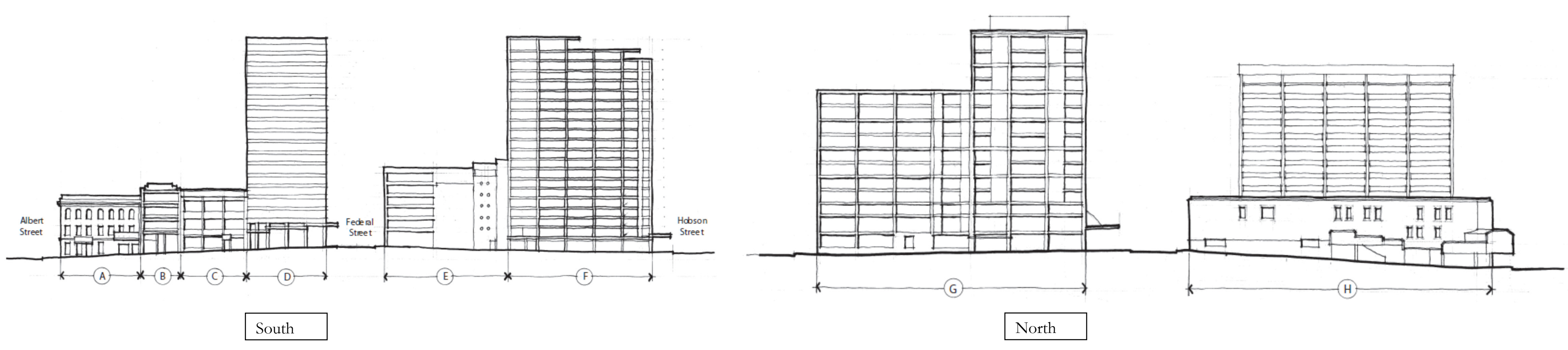

Kingston Street Elevations: no scale
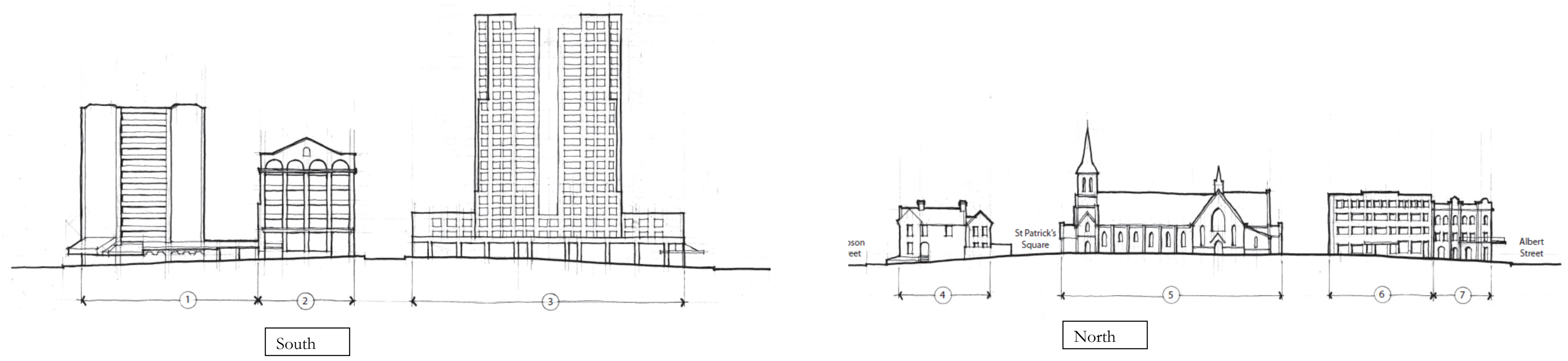

Wyndham Street Elevations: no scale

Figure 8.2: Outline elevations buildings in the Kingston \& Wyndham Street case study provided to help survey respondents orient themselves during their visit. 



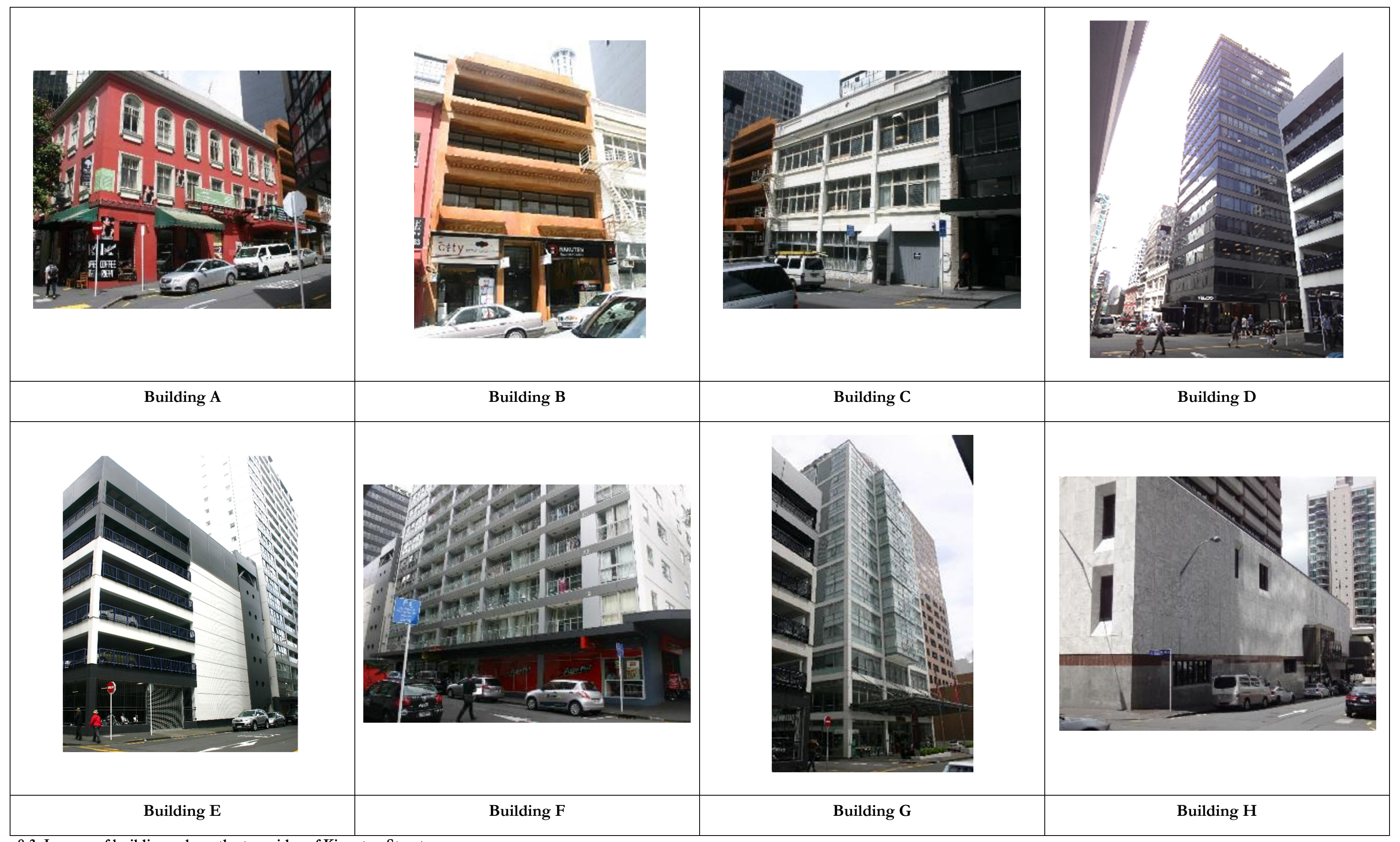

Figure 8.3: Images of buildings along the two sides of Kingston Street 



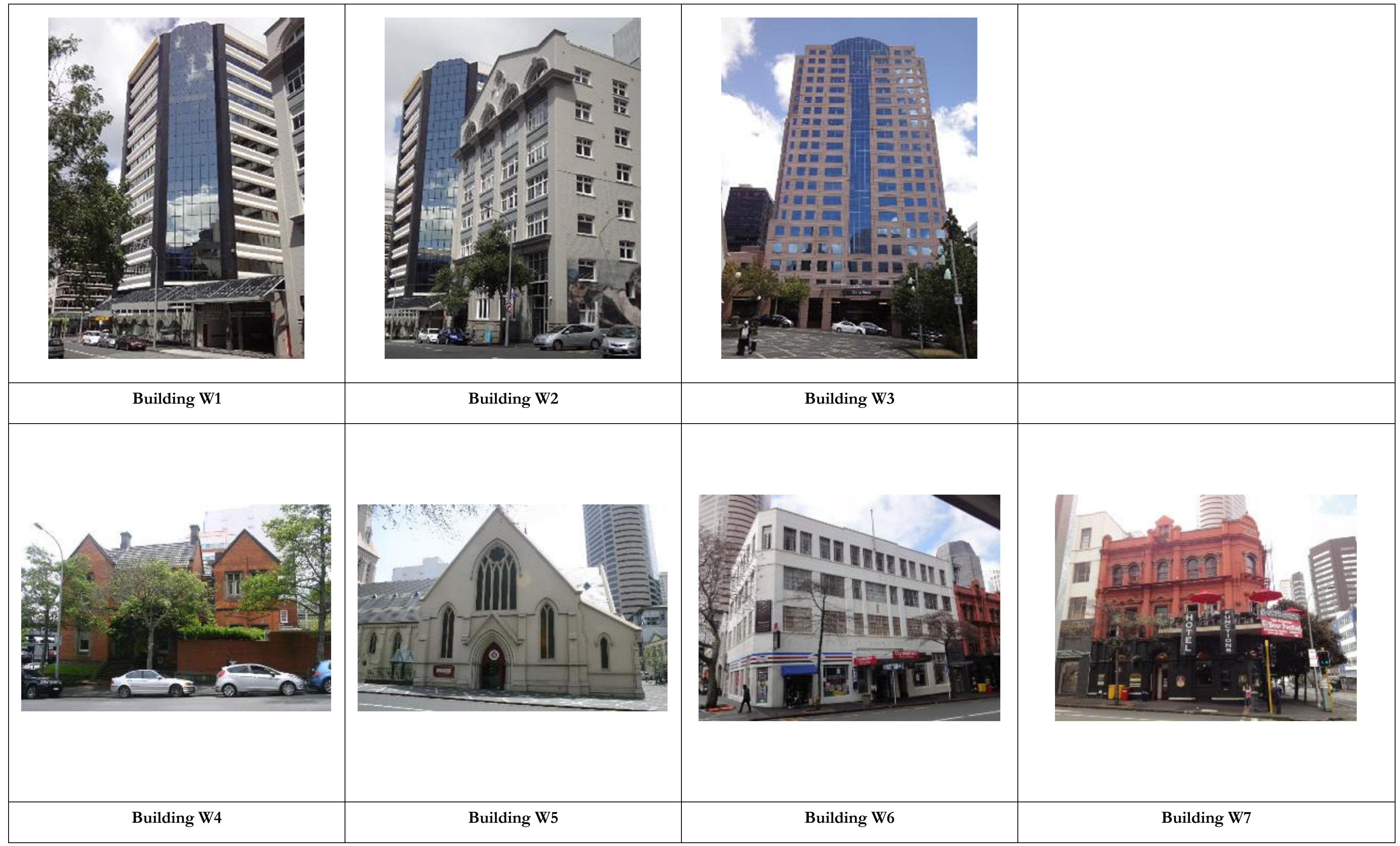

Figure 8.4: Images of buildings along the two sides of Wyndham Street

Source: author 



\subsection{General preferences}

\subsubsection{Individual buildings}

Respondents were asked about the 15 individual buildings in the Wyndham/Kingston study area and a comparison of mean scores revealed they liked nine of them. The average of all mean scores for the buildings in the study area was 2.21 (on a rating scale of 3). This fell between the averages of 2.05 in the College Street study area, and 2.35 for the Tyler Street buildings. Generally people were positive about the individual buildings in the study area.

The two best liked buildings (W4 and W5) were in Wyndham Street, with the same mean preference score of 2.87 on the 3-point scale (figures 8.5 and 8.6). The two buildings are separated by St Patrick's Square, a quasi-public space that is a destination for lunchtime visitors and a thoroughfare between Wyndham Street and the Auckland CBD to the east. Although one person disliked building W5, 89.2\% of the respondents liked it. A key reason for liking it was its façade details, cited by $79 \%$. The second most cited reason $(39 \%)$ was its overall shape. Nevertheless, after analysing the relationships between responses to building design elements it seems the strongest predictor of whether a person liked the building was his/her opinion of the design at street level.

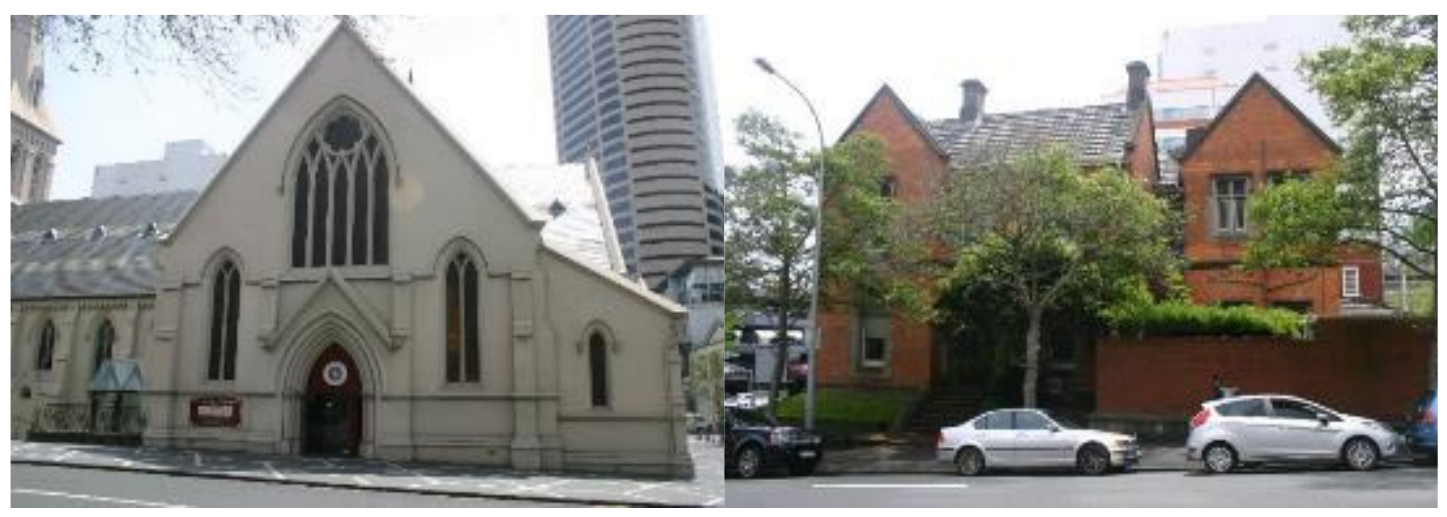

Figure 8.5: Building W5, St Patrick's Cathedral, was equal best liked in the study area with building W4.
Figure 8.6: Building W4, which with building W5 is part of the St Patrick's Cathedral complex.

Source: author

Source: author

Building W5 also received the highest mean preference score for each of the streetscape design characteristics. People found the design above ground floor level (mean=4.66 on 5-point scale) to be highly pleasurable as they did the surface finishes (mean=4.56) and 
the street level design (mean=4.67). The building is an example of Gothic Revival in terms of its proportioning and detailing (St Patrick's Cathedral 2013). The external walls of the building step in plan, with the end of the transept nearest the street edge and the nave set further back. The building is a very generous single storey and the rendered brick walls are decorated with mouldings that highlight the openings and provide vertical modulation over its height. There is also a rhythmic arrangement of buttresses along the nave, seen across a fenced lawn area. A contemporary glazed porch, providing direct access to the transept, faces toward Wyndham Street.

While the overall design is coherent and visually interesting, it does not allow visual connection with the interior, a characteristic common to the best liked street level designs in the other two other study areas. Building W5 does not have the retailing or café activities that featured in other best-liked street level facades. In addition to its design features, characteristics that may have influenced people's strongly positive opinions of this building include the meanings attached to its being a church, its architectural heritage (Rapoport 1982), the design of the adjacent spaces adjacent, the extent to which the building is legible, and the level to which it is maintained.

Sitting first equal with building W5 was building W4. Like building W5, this is a freestanding building and three of its four sides can be seen from public space. Building W4 is domestic in scale, two storeys high and constructed of brick, the only fair-faced brick building in this case study. The building has gabled roofs over articulated subvolumes. Individuals liked and disliked building W4 in similar proportions to W5. The same person disliked both buildings, calling building W4 "a pointless little building in the city". Although the primary reason for liking this building was its façade details, age was the second-ranked reason. A regression analysis revealed that people's preferences for the façade design above street level linked strongest to their response to the building overall. This may in part have been a consequence of a solid wall partially obscuring the ground floor as well as the attractive design of the building's roof line and upper storey. 


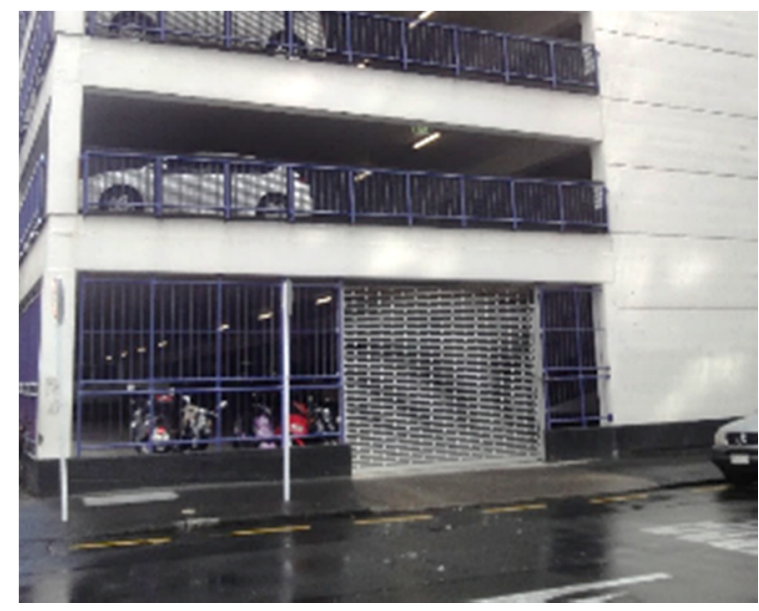

Figure 8.7: Building E, a multi-storey car park, was least liked of all buildings in this case study.

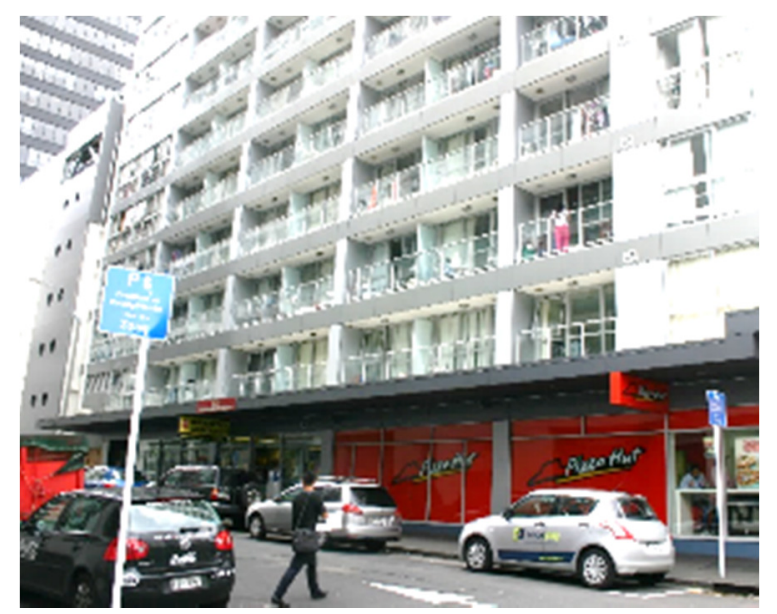

Figure 8.8: building $F$ was the second least liked in the study area.

Source: author

Source: author

Perhaps understandably, building E (mean=1.54), a purpose built multi-storey car park on the corner of Federal and Kingston Streets, was least liked of all in the study area (figure 8.7). While the majority of people (58.5\%) disliked it, some 12.2\% liked it. Most people who disliked it (79\% of 58.5\%) indicated the façade details and overall shape (46\%) were the primary reasons for this. The façade design characteristics of the building were the least liked of all buildings in the study area, with the street level design achieving a mean score of 1.90 on the 5-point scale. Half of the building frontage along Kingston Street is a solid, unadorned wall while the other half is open behind a steel grating, revealing the parked vehicles. While $70.7 \%$ of the respondents found this aspect of the building unpleasant, 9.8\% indicated it was somewhat pleasant.

Occupying the site alongside building $\mathrm{E}$ and extending through to the Hobson Street corner, building F was the second least-liked building (mean=1.78). It was completed in 2010 to an approximate height of 65 metres (Dickson 2014). Although not the tallest in the study area its 21 floors are the highest number. This building has retailing activities at ground floor level and residential above. The latter are somewhat concealed by the arrangement of individual balconies, which are also the main architectural feature of the façade above street level (figure 8.8 ). Some $43.9 \%$ of the respondents disliked building $F$ while $22 \%$ liked it. The best predictor of a person's opinion about the building overall was their opinion of the façade design above street level. In the case of building F, 45\% of the respondents found this aspect unpleasant. 
The street level design of building F was also not well liked (mean=2.51) and it ranked as the second least liked in the study area. The current ground level tenancies are a fast food outlet and a convenience store. Although the entire shop front is potentially clear glazed, each shop has blocked a considerable proportion of their frontage, thereby limiting visual connection from the public footpath to the interior. The manner in which the operators have altered their shop fronts may be a factor in, if not wholly responsible for, the low mean preference rating for the street level design of building $F$.

After buildings W4 and W5, two other well liked buildings were A and W7. These structures are older and, although not officially recognised as having heritage value, could both be described as characterful (figures 8.9 and 8.10). The facades of both are richly detailed and colourful. With mean preference scores of 2.77 (building W7) and 2.44 (building A) they ranked fourth and fifth best liked of the 15 buildings in the study area. Some $84.6 \%$ of the respondents liked building W7 and only $7.7 \%$ had neutral opinions about it. Although $53.7 \%$ of the sample liked building A, some $36.6 \%$ were unable to decide whether they liked it or not.

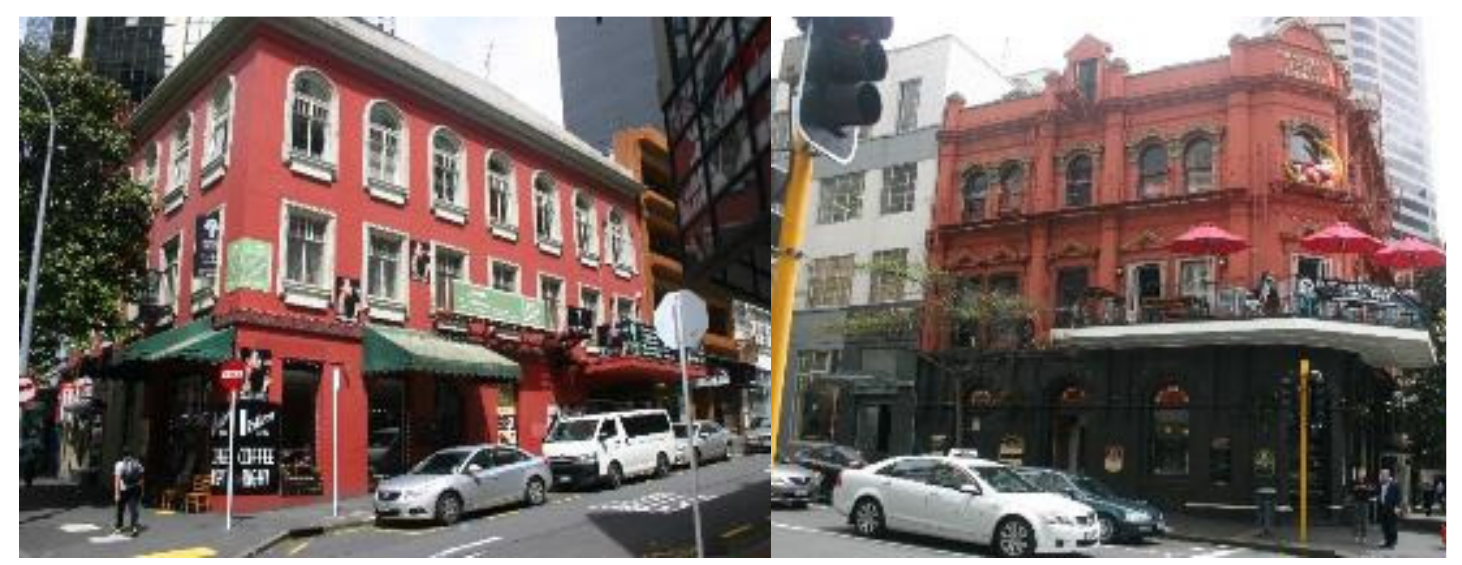

Figure 8.9: Building A

Figure 8.10: Building W7

Source: author

Source: author

Not only was building W7 liked better than building A, its façade design features were also preferred, based on a comparison of mean scores (table 8.2). The two influential factors listed most often by respondents who liked building A were façade details $(86 \%)$ and overall shape (57\%). Those liking building W7 also cited façade details (76\%) and overall shape (39\%). A regression analysis found that the factor that linked most closely to people's overall preferences for the building was the design at street level, which people 
found very pleasant. On the other hand, respondents gave the street level design of building $\mathrm{A}$ a poor rating (mean=2.83), with 53.7\% finding it unpleasant. Despite this, people generally liked building A as their opinions of the street level design were not closely linked to their overall opinions of the building.

Table 8.2: Mean scores for buildings A and W7 for overall preference and different façade design characteristics

\begin{tabular}{|c|c|c|c|}
\hline & Building A & Building W7 & \\
\hline $\begin{array}{l}\text { Does respondent } \\
\text { like the building? }\end{array}$ & 2.44 & 2.77 & 3-point scale \\
\hline $\begin{array}{l}\text { Façade design } \\
\text { above street level }\end{array}$ & 3.90 & 4.15 & \multirow{3}{*}{ 5-point scale } \\
\hline $\begin{array}{l}\text { Surface finishes } \\
\text { above street level }\end{array}$ & 3.76 & 4.00 & \\
\hline $\begin{array}{l}\text { Building design at } \\
\text { street level }\end{array}$ & 2.83 & 4.08 & \\
\hline
\end{tabular}

Figures 8.11 and 8.12 show the street level façade designs of buildings $A$ and W7 are quite different. While there are large areas of solid wall in the building W7 shopfront, the design of these relates to the arrangement of fenestration above the canopy in proportion and detail. There have been few, if any, changes to the design, so the overall appearance has remained coherent. On the other hand, the ground level design of building A bears no relationship to the façade above canopy level, probably as a consequence of having been modified. Through their responses to the survey questions people have articulated which approach they prefer, as the difference in mean score is compelling. 


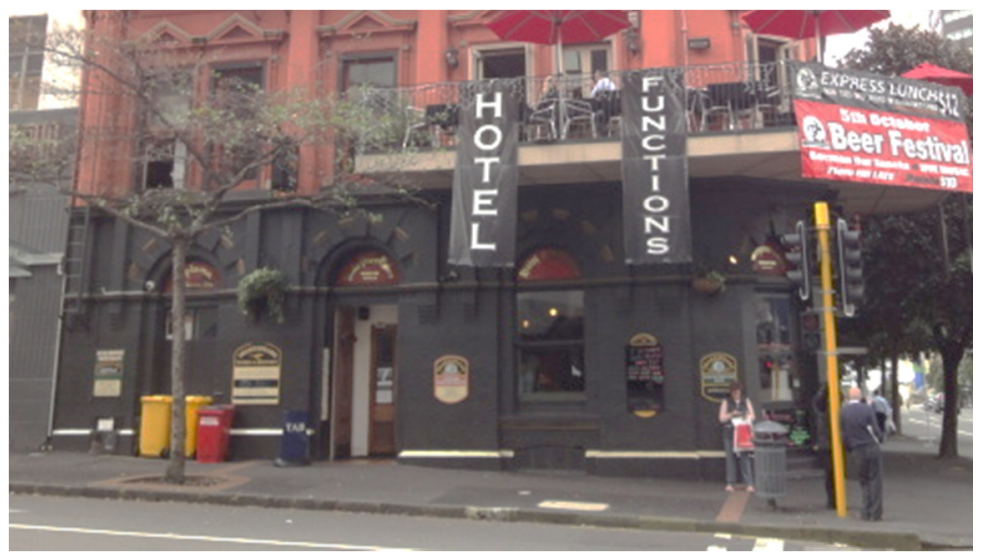

Figure 8.11: The street level design of building W7

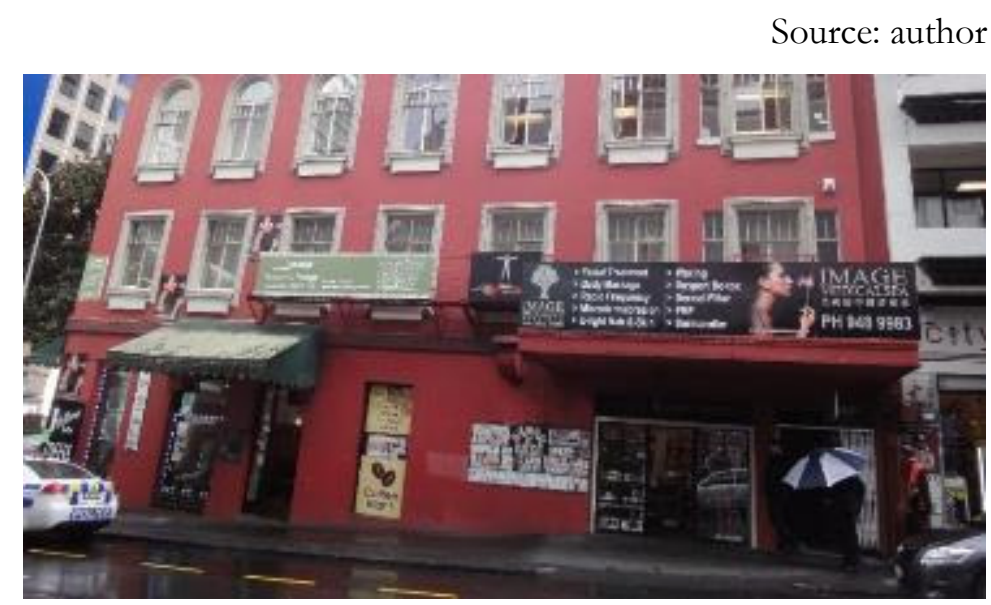

Figure 8.12: The street level design of building A

Source: author

It appears people prefer more traditional façade compositions, rather than those that are Modernist in nature. In more positive terms, such building facades generally have fenestration patterns, base/middle/top vertical design hierarchies, make use of traditional materials, and are ornamented through the use of cornices, mouldings and other surface details. These characteristics apply to the five most liked building facades in the study area. Ranked at number six, building W3 was the highest rated building employing a contemporary or Modernist design approach.

A comparison of the street level designs of three buildings in Kingston Street suggests that people prefer arrangements where the public can see inside. Buildings D, F and G are contemporary with extensively glazed shopfronts along the street edge. The street level designs of $\mathrm{D}($ mean=3.27) and $\mathrm{G}($ mean=3.05) allow people to observe the 
commercial activities before deciding whether or not to enter. However, the ground level tenants of building $\mathrm{F}$ have blocked the majority of their glazed shop front only leaving doorways that allow views from the street inside. While $48.8 \%$ of respondents liked the street level design of building D and 39\% that of building G, 58.5\% disliked that of building $\mathrm{F}$ (mean=2.55). While the differences could be influenced by the nature of the businesses operating from each building they could also to reflect dissatisfaction with the barriers to direct visual engagement with the interior.

The Kingston/Wyndham Street study area has two podium style buildings, one of which was liked and the other disliked based on mean score comparisons. They are different, as are the settings, which may have influenced the responses and it is difficult to draw conclusions about the relative merits of each on the survey alone. Building $\mathrm{H}$ is the Auckland District Court and occupies the north side of Kingston Street between Albert and Federal Streets. Built in the early 1980s, its podium façade cladding uses different types of polished stone. Building W3 is a 1990s commercial office tower, clad in precast concrete panels, coloured and detailed to resemble stone. The same cladding has been applied consistently over the building, podium and tower. A comparison of the mean preference scores is in Table 8.3. A high level of neutral responses may have limited the mean preference score for building W3. Only 7.7\% disliked the building while 35.9\% liked it. However, more than half of the respondents (56.4\%) were neutral. By comparison, $34.1 \%$ of respondents disliked building $\mathrm{H}$ while $24.4 \%$ liked it. Of those who disliked building $\mathrm{H}, 79 \%$ did so because of its façade details.

Table 8.3: Preference scores for buildings $\mathrm{H}$ and $\mathrm{W} 3$, two podium style buildings in the study area

\begin{tabular}{|c|c|c|c|}
\hline & Building $\mathbf{H}$ & Building W3 & \\
\hline $\begin{array}{l}\text { Does respondent } \\
\text { like the building? }\end{array}$ & 1.90 & 2.28 & 3-point scale \\
\hline $\begin{array}{l}\text { Façade design } \\
\text { above street level }\end{array}$ & 2.88 & 3.29 & \multirow{3}{*}{5 -point scale } \\
\hline $\begin{array}{l}\text { Surface finishes } \\
\text { above street level }\end{array}$ & 2.88 & 3.39 & \\
\hline $\begin{array}{l}\text { Building design at } \\
\text { street level }\end{array}$ & 2.59 & 3.64 & \\
\hline
\end{tabular}


The biggest difference in preference scores for these two buildings was for the design of each building at street level. While the mean response to the W3 street level design lagged behind that for building W5 (St Patrick's Cathedral) and other older buildings, it was the highest rated street level design of all contemporary buildings and was higher than many other older buildings in the study area. The design is simple, and generated by the tower's structural grid pattern. What sets it apart from others, including building $\mathrm{H}$, is that the street frontage is a publicly accessible outdoor colonnade, welcoming passing pedestrians onto the site. The area within the colonnade is landscaped, paved and provided with seating (figure 8.13). There are no apparent restrictions on the public using this space. The colours and texture of the cladding materials generate visual interest and warmth and the whole of this frontage appears clean and well maintained.

Building $\mathrm{H}$ presents few of these qualities to the public as they pass along its street frontage. Although seating areas have been incorporated into the design along the Albert Street frontage, along Kingston Street the building meets the back of the pavement abruptly (figure 8.14). Respondents rated this frontage very low, and its mean preference score was the third lowest in the study area. The marble cladding materials used in building $\mathrm{H}$ are more expensive than those of building W3 but have been applied to a façade that is largely solid. A strong correlation was observed between people's opinions about the façade surface finishes of building $\mathrm{H}$ and their overall preference rating for it.

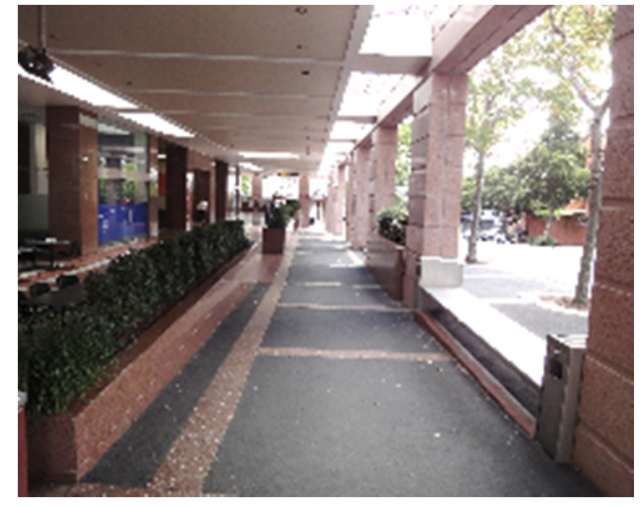

Figure 8.13: behind the colonnade of building W3 at street level.

Source: author

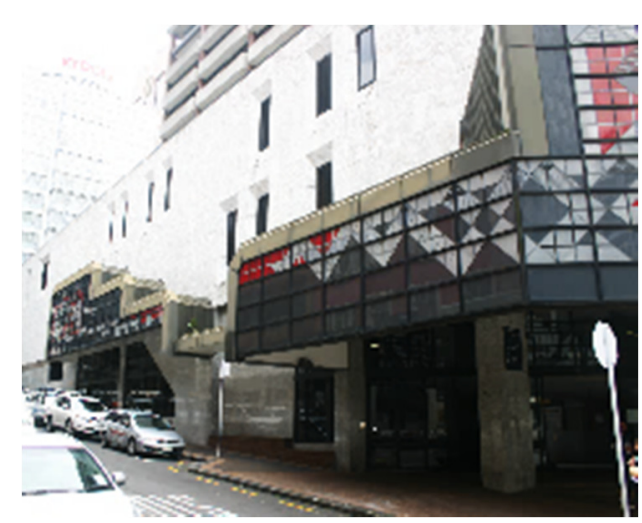

Figure 8.14: The street level design of building $\mathbf{H}$ offers few opportunities to engage with the building interior

Source: author 
Although this comparison was made because both buildings are a podium/tower typology occupying an entire block, it is perhaps unfair to contrast them in this manner, as their street environments are different with one being a commercial office (W3) and the other a court of law $(\mathrm{H})$. As a business, the commercial office building competes for tenants and visitors, a process that is aided by remaining attractive to existing and potential tenants. Building $\mathrm{H}$ looks more worn, an assessment that can in part be explained by its age but possibly also because of a different maintenance regime. Differing standards of maintenance affects the way each building is perceived. Environmental factors that may have lowered liking for building $\mathrm{H}$ include the narrower width of Kingston Street and the fact its street façade is generally seen in the shade, being on the north side of Kingston Street. On the other hand, building W3 faces wider Wyndham Street and toward St. Patrick's Square, both of which help generate a sense of openness. It is also located on the southern side of the street, facing the sun. These factors could have positively influenced opinions of building W3.

\subsubsection{Inter-building relationships}

Only four of the relationships between adjoining buildings were liked, with the mean scores for the other seven relationships falling below neutral. The best liked relationship was that between the two best liked buildings, W4 and W5 (mean=4.43) (figure 8.15). This attracted the highest number of opinions about this relationship; as only $9.1 \%$ expressed a neutral view, the lowest rate for neutral opinions in the Kingston/Wyndham Street study. This relationship is formed across St Patrick's Square, a public space that is both a shortcut for pedestrians and a public open space haven in the city. The two buildings are part of St Patrick's Cathedral and both are heritage listed in the Auckland District Plan. While the finishes are different (fair faced brick and rendered brick), both buildings have articulated volumes, pitched gabled roofs, windows that are taller than they are wide, and facades with decorative detailing. There is a slight height difference between the main bodies of the two buildings, with the tower of the cathedral at their nearest juxtaposition. Wyndham Street also falls away as it passes in front of building W4. 


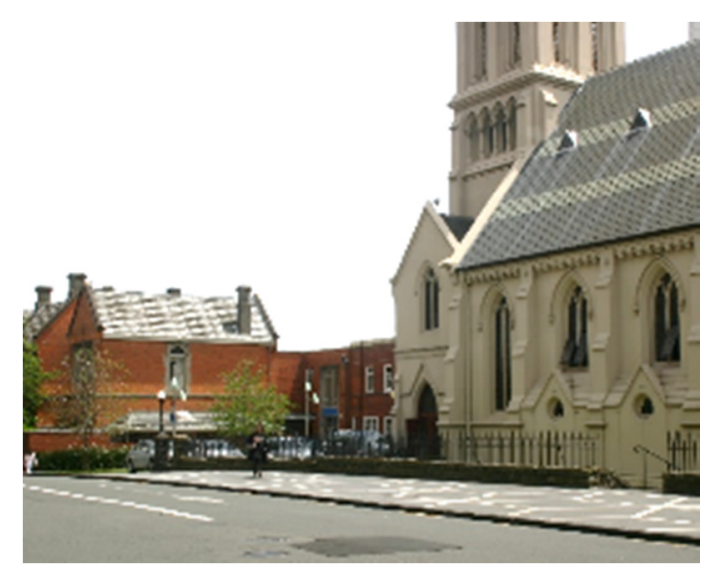

Figure 8.15: The relationship between buildings W4 (left) and W5 was best liked by the respondents.

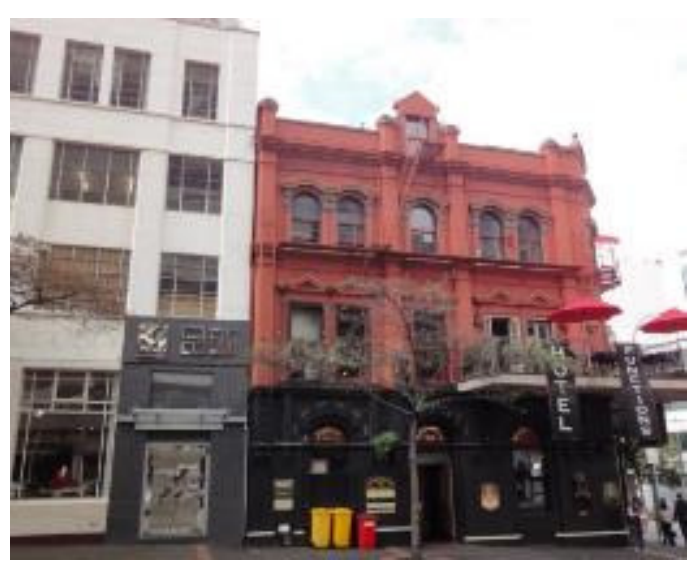

Figure 8.16: The best liked relationship between tightly adjoining buildings was between W6 (left) and W7.

Source: author

Source: author

The second highest rated relationship also takes place across St Patrick's Square, between buildings W5 and W6 (mean=3.32). This is a big drop in mean score from that for the relationship between W4 and W5 discussed above. In contrast W5 and W6 share few similarities, which may be reflected in the mean scores. They are both finished in painted render and both facades have decorative but different details. However, it would appear that the 14 metre gap and characteristics of St Patrick's Square, including the extensive landscaping, are sufficient to mediate between two very different buildings.

The best liked relationship between closely spaced buildings is that of W6 and W7 $($ mean $=3.19)$ (figure 8.16). Sitting slightly above neutral, $43.6 \%$ of respondents liked the relationship while $33.4 \%$ disliked it and 25\% were undecided. Both buildings are low compared to others around them, there is a slight difference in overall height, and neither has been maintained in pristine order and therefore both have acquired a patina with age. Both designs are based on a classical hierarchy, in which ground level is distinguished from those above. Differences in colour, architectural language and detailing are obvious and may have limited the extent to which the relationship was liked.

Unlike the Tyler Street study where roads crossing it appeared to mitigate perceived building differences, as these relationships were generally liked even when the individual buildings were disliked, it appears the gaps created by intersecting streets in this case study were not able to mitigate negative perceptions of some inter-building relationships. The mean preference scores for the relationships between buildings $\mathrm{D}$ and $\mathrm{E}$ (mean=2.36), 
W2 and W3 (mean=2.80) and $\mathrm{G}$ and $\mathrm{H}($ mean $=2.83)$ are all near neutral. This different finding is even more puzzling as buildings W2 (mean=2.82 on 3-point scale) and W3 (mean $=2.28)$ were both well-liked. Perhaps the narrower width of Federal Street meant it was inadequate for mediating between contrasting designs.

The least liked relationship in Kingston and Wyndham Streets was that between buildings $\mathrm{C}$ and $\mathrm{D}$ (mean $=2.03$ ) as only $9.8 \%$ of respondents liked it and $73.2 \%$ disliked it. Building $\mathrm{C}$ is an older three storey building with generous floor to floor heights and large windows, and was generally liked by respondents (mean $=2.05$ on 3-point scale). Building D was generally disliked (mean=1.93) and respondents indicated that its height, glazing and colour were all key factors in their decision. These are also the three areas where the design of building $\mathrm{D}$ contrasts with building $\mathrm{C}$ (figure 8.17).

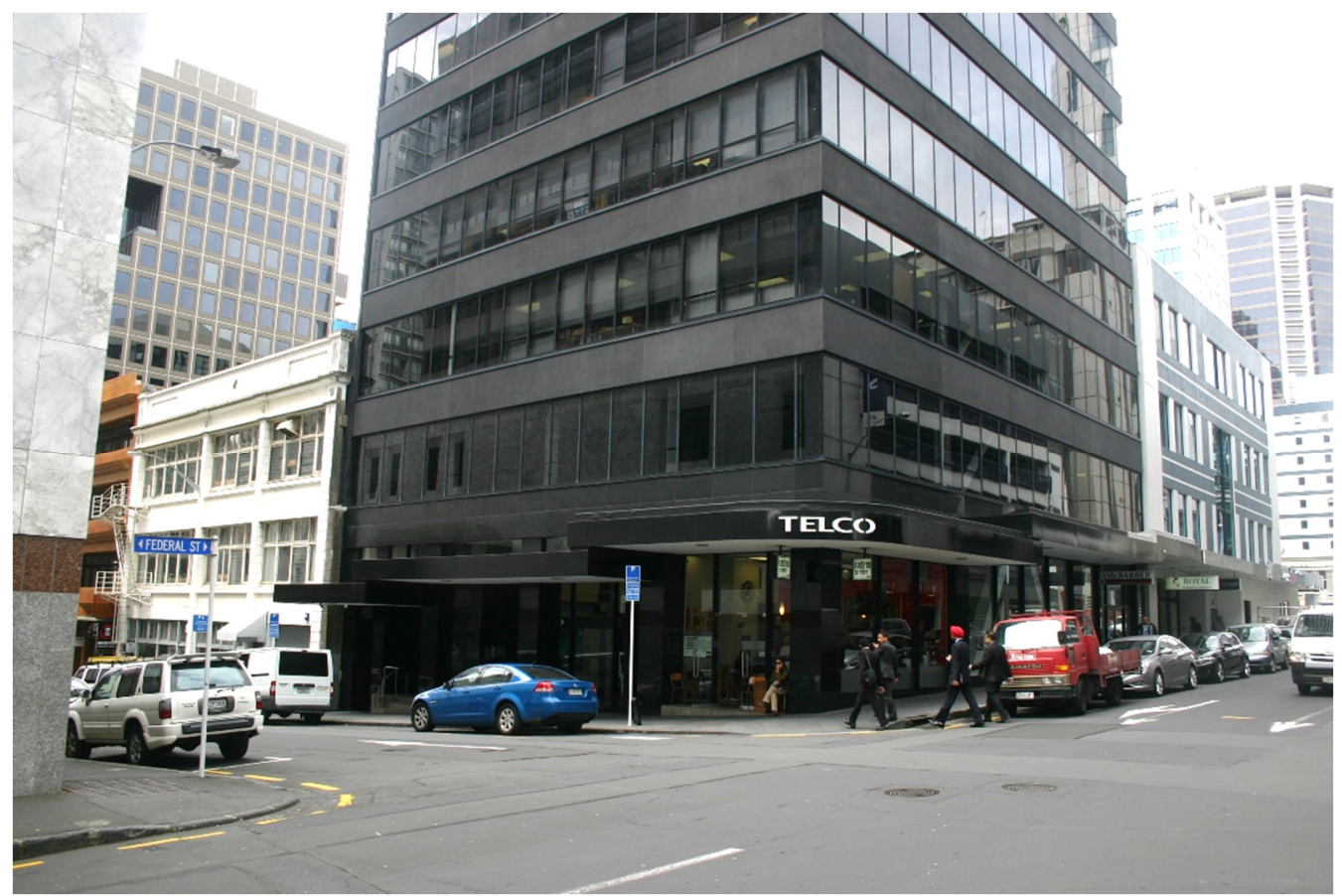

Figure 8.17: The three storey building $C$ is to the left of building $D$. The relationship between them was judged the poorest in the study area.

Source: author

\subsubsection{Streetscape preferences}

The four overall streetscapes in Wyndham and Kingston Streets received mixed responses. Only the north side of Wyndham Street was well liked judging by the mean preference scores for relationships between building heights (positive for $84.7 \%$ ), façade alignment and façade styles. Characteristics of the other streetscapes were both liked and 
disliked (table 8.4). The buildings along the north side appear reasonably consistent in overall height based on the true elevation. Viewed in three dimensions from the street the gabled roof of building W5 recedes and the height of this structure may therefore read differently. Several respondents critiqued the effects of building W6 on the streetscape when articulating why they liked or disliked the height relationships, with one noting the scene was reminiscent of a "rose between two weeds". Another respondent wrote "the height relationships help make the building W6/W7 block appear non-threatening". The majority of respondents however, felt that the relationships were harmonious and attractive and several noted the mediating effects of St Patrick's Square.

Table 8.4: Mean preference scores for streetscape characteristics of case study three.

\begin{tabular}{|c|c|c|}
\hline & Mean & Std. Deviation \\
\hline \multicolumn{3}{|c|}{ Kingston south streetscape } \\
\hline Building Heights & 2.44 & 0.672 \\
\hline Facade Alignment & 3.00 & 0.894 \\
\hline Façade Styles & 2.12 & 0.780 \\
\hline \multicolumn{3}{|c|}{ Kingston north streetscape } \\
\hline Building Heights & 3.07 & 1.081 \\
\hline Facade Alignment & 2.83 & 0.930 \\
\hline Façade Styles & 2.41 & 1.071 \\
\hline \multicolumn{3}{|c|}{ Wyndham south streetscape } \\
\hline Building Heights & 3.00 & 1.076 \\
\hline Facade Alignment & 3.23 & 0.872 \\
\hline Façade Styles & 2.87 & 1.069 \\
\hline \multicolumn{3}{|c|}{ Wyndham north streetscape } \\
\hline Building Heights & 4.21 & 0.767 \\
\hline Facade Alignment & 3.95 & 0.793 \\
\hline Façade Styles & 4.13 & 0.800 \\
\hline
\end{tabular}

In contrast, building height relationships on the south side of Kingston Street were widely disliked, with more than half the respondents finding them somewhat or very unpleasant. The reasons given centred mainly on the inconsistent height relationships leading to "confusion" in the mind of one person, and a "messy" environment for another. Only one of the 41 respondents liked the height relationships but gave no justification for this. 
Buildings $\mathrm{D}$ and $\mathrm{F}$ were highlighted for poor relationships but building $\mathrm{E}$ was also cited by some respondents. The three inter-building relationships between buildings $\mathrm{D}, \mathrm{E}$ and F were ranked the poorest in the whole study area. Because these relationships all occur on one side of Kingston Street this would probably have lowered people's perceptions of the whole streetscape.

The negative response to the south side of Kingston Street was not limited to building height relationships, as a lower mean score $(=2.12)$ was recorded for relationships between façade styles. Two people, $4.9 \%$ of the sample, found the style relationships somewhat pleasant but an overwhelming majority $73.2 \%$ found them somewhat or very unpleasant. The façade styles are diverse, ranging from the ornate and well-liked building A to the utilitarian and poorly maintained building E. The style of the newest addition to the street, building $\mathrm{F}$, is unlike any others along this side, although it does have some connection to building $G$ across the street. This only serves to extend the diversity of design styles on the southern side. Of the six buildings along this side of Kingston Street, only A (mean=3.90) and C (mean=3.04) achieved positive mean preference scores for façade design style. All other scores were below the neutral point and formed the four bottom ranked facades in the whole of this study area.

It seems respondents were also unimpressed with the style relationships along two of the other three streetscapes. Mean preference scores for the north side of Kingston Street (mean=2.41) and the south side of Wyndham Street (mean=2.87) fell below the neutral line. These two streetscapes include buildings that were both liked and disliked, although none of the inter-building relationships were liked based on mean score comparisons. Only on the north side of Wyndham Street did respondents like façade styles (mean $=4.13$ ), and these were well liked by $84.6 \%$ of the respondents. Although the styles are diverse they are also older buildings. Other than building W4, which is brick, the buildings have painted exteriors that can be refreshed by repainting.

The best liked alignment between building facades was the arrangement of buildings along the north side of Wyndham Street (mean=3.95) (figure 8.18). No respondent found the alignment between facades unpleasant and $66.7 \%$ found them pleasant. On the other hand, the alignment between facades on the north side of Kingston Street (figure 8.19) was generally disliked (mean=2.83), although half the respondents were neutral. Nevertheless, the two streetscapes can be compared. 


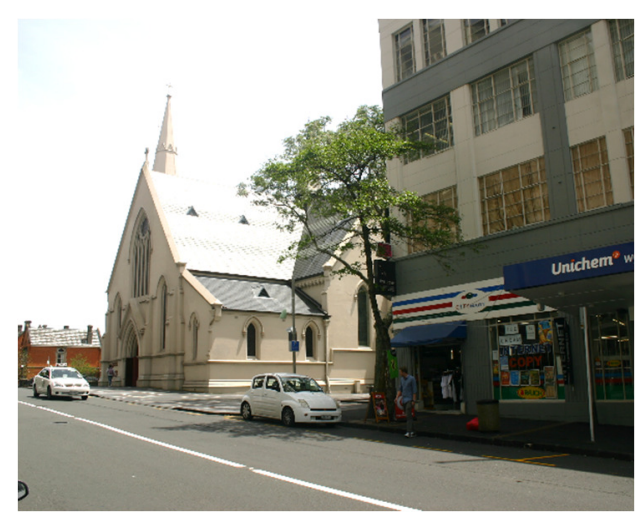

Figure 8.18: Alignment on north side of Wyndham Street.

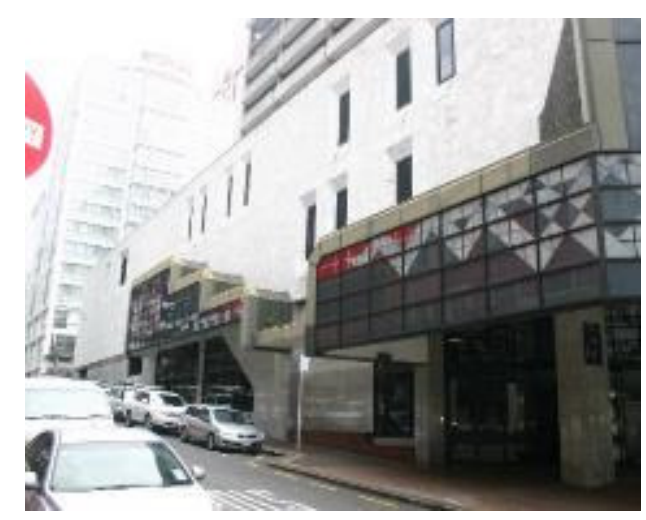

Figure 8.19: The least liked street alignment was the north side of Kingston Street.

Source: author

Source: author

The Wyndham Street north edge is the least spatially defined in the study area, as buildings W4 and W5 are set back from the footpath and St Patrick's Square creates gaps between buildings in two locations. However, both resultant open spaces have been positively designed and managed and people seem to like the outcomes. As seen in figure 8.19, the north side of Kingston Street is consistently defined by the two buildings along that side of the street. Buildings $\mathrm{G}$ and $\mathrm{H}$ both hug the back of the footpath and the facades rise as vertical surfaces. The sense of enclosure, a relevant spatial characteristic, provided by each of these street edges is quite different. Sitte (1979: 55) believed that buildings should clearly define and enclose street spaces, arguing that people's experience of such streets would be greater than their experience of streets where buildings inconsistently define the space. Urban design guidance in Wellington makes reference to this recommendation (Wellington City Council 2012). However, when investigating the qualities that make one street or public space more walkable than another, Ewing, Handy et al. (2006) found that sense of enclosure was not as important as other urban design qualities such as human scale and imageability. Their research findings may help explain why the north side of Kingston Street appears less pleasant to respondents than the north side of Wyndham Street.

Buildings $\mathrm{G}$ and $\mathrm{H}$ conform more strictly to Sitte's recommendations as they establish a clear boundary to the street space. The human scale relationship is strained, but for different reasons. Building $G$ is very tall. Even though at ground level the building is largely transparent, the upper levels are visually impenetrable because of the building height and the reflective glazing. A benefit of podium style buildings, like building $\mathrm{H}$ is 
that the podium element can be designed to foster a suitable human scale. However, the podium of building $\mathrm{H}$ substantially lacks any features that can communicate a sense of human scale. The majority of the façade, at street level and above, is a solid wall that limits, rather than facilitates, visual perception. Despite helping to define the street space positively it may be the visually impenetrable facades of buildings $\mathrm{G}$ and $\mathrm{H}$ that limit the extent to which the space exhibits a human scale and respondents have read this as an unpleasant alignment of street facades.

The openness of the north side of Wyndham Street conflicts with Sitte's recommendations. However, people's strong preferences for this alignment appear to confirm Nasar's findings that people prefer defined openness (Nasar 1998: 67-69).

\subsection{Comparisons based on demographic groupings}

\subsubsection{Gender comparisons}

Male and female respondents in the Kingston/Wyndham Street survey largely had similar preferences for individual buildings. For 11 of the 15 buildings in the study area the mean preference scores for both genders were within 0.20 on the 3-point scale. Not only were the mean scores comparable but the ranking for nearly all the buildings that could be described as characterful was similar for both groups. Both genders rated buildings A, W2, W4, W 5 and W7 highly and the distribution of responses for liking, disliking and neither liking nor disliking followed a similar pattern for each.

The responses to the two disliked buildings were slightly different. Women liked building E the least (mean=1.71) although $42.9 \%$ were neutral. Men were more scathing, awarding it a mean score of 1.35 , as $76.5 \%$ of the male respondents disliked building $E$ and only 11.8\% were neutral. Building W1 (figure 8.20) was also disliked by all respondents but men and women felt differently about it. While women liked this building (mean=2.14) men did not (mean=1.71), with $42.9 \%$ of women stating they liked it against only $11.8 \%$ of men. 


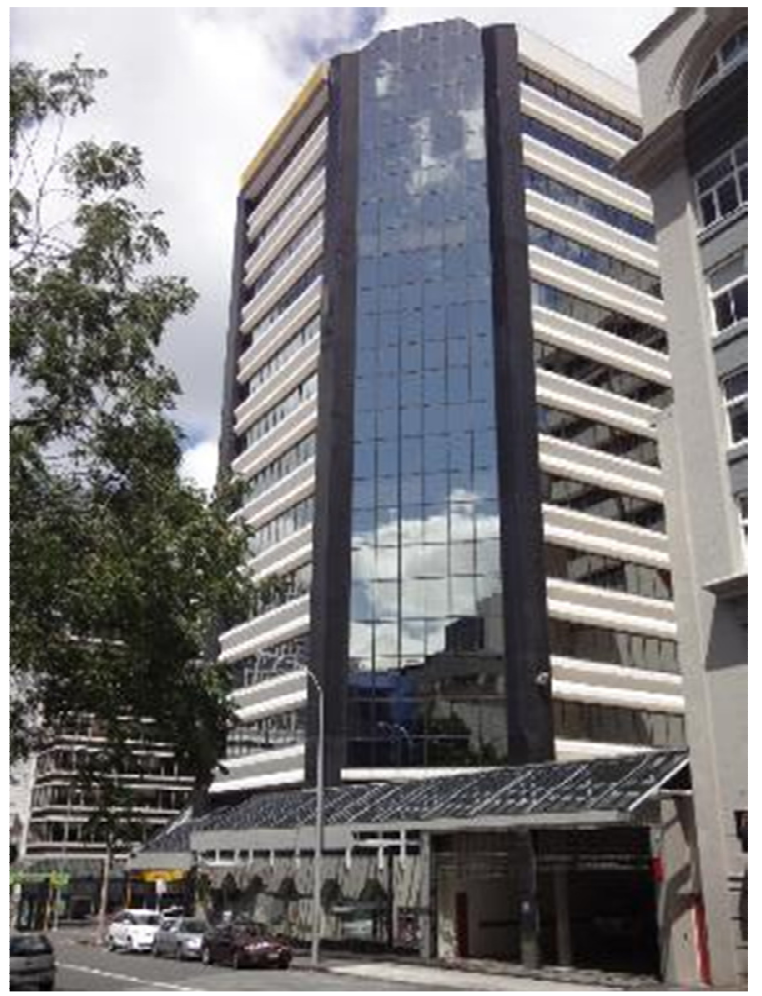

Figure 8.20: Building W1, which women liked and men disliked.

Source: author

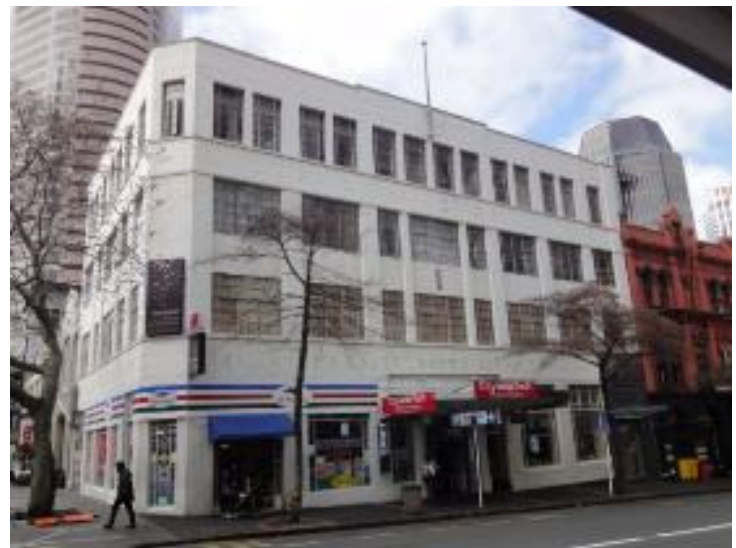

Figure 8.21: Building W6 was liked by men and disliked by women.

Source: author

Men (mean=2.65 on 5-point scale) and women (mean=2.76) agreed that the street level design of this building was poor and also agreed in their responses to the questions regarding design and surface finishes above ground level. The mean scores from both groups for each were around the neutral point with women being a little more positive 
about the façade design of the tower element. This may help explain why their overall response to the building was positive. The opinions women had of the façade design above ground level were the best predictors of their overall responses.

Table 8.5: Mean preference scores for building W6 and its characteristics by gender.

\begin{tabular}{|c|c|c|c|}
\hline & Women & Men & \\
\hline Overall preference & 1.90 & 2.29 & $\begin{array}{l}\text { 3-point } \\
\text { scale }\end{array}$ \\
\hline $\begin{array}{l}\text { Facade design above } \\
\text { ground floor level }\end{array}$ & 2.62 & 3.71 & \multirow{3}{*}{$\begin{array}{l}5 \text {-point } \\
\text { scale }\end{array}$} \\
\hline $\begin{array}{l}\text { Surface finishes } \\
\text { above ground floor } \\
\text { level }\end{array}$ & 2.57 & 3.41 & \\
\hline Design at street level & 2.57 & 3.29 & \\
\hline
\end{tabular}

Building W6 (figure 8.21) produced an even stronger example of differences between men and women. Table 8.5 shows men $($ mean $=2.29)$ liked the building overall while women (mean=1.90) did not. About one third of the respondents in each group were neutral and $38.1 \%$ of women disliked the building. In contrast only $17.6 \%$ of men disliked it and $47.1 \%$ liked it. Similar differences of opinion could also be seen in responses to the questions concerning façade design, surface finishes and the design at street level. The strongest correlation between preference for a façade design characteristic and overall preference for both groups was the façade design above street level. In light of the similar responses to other characterful buildings in this case study, building W6 is unusual in attracting such different responses from men and women.

Building W6 has the least architectural embellishment of the four buildings along the north side of Wyndham Street. Nevertheless, the design reflects an art deco compositional approach and could be considered coherent. Two factors could have generated different responses by gender. The floors above ground feature large windows that enable views into the interior from the street. Approximately half of the windows had curtains drawn throughout the day and for those they were open most views were of an untidy interior space. Discussions during the College Street layperson's focus group, 
which comprised mainly female participants, suggested women were particularly interested in what windows in a building could or could not reveal. Because the windows of building W6 gave a view of a messy interior, women might have felt more negatively about it. Another influential factor could have been the untidy appearance of at least half the ground floor shopfront. Although the eastern shop appears tidy and clean, the minimarket in the western half of the ground floor of building W6 does not. Women rated the ground level of building W6 similar to that of building F, which exhibits similar characteristics. Although other factors may have been responsible for the different responses by men and women to building $\mathrm{W} 6$, the extent to which each gender considers what happens inside a building when making their aesthetic evaluations deserves to be explored further on the basis of this result.

The two groups were generally consistent in their responses to questions about relationships between adjacent buildings, as with one exception, men and women had similar opinions about each building to building relationship in this case study. The exception was the relationship between building W6, a building that men and women had different opinions about, and building W7. Women (mean=2.88) disliked it while men $($ mean $=3.71)$ were very positive about it. The influence a negative opinion about a building can have on the relationship it forms with others was discussed in section 7.3.2. This type of response appears to have been confirmed in this case study, as men held positive opinions of all three buildings and so held positive opinions about the relationships between them. Women on the other hand disliked one of them and this appears to have affected their opinions of the relationships between them.

\subsubsection{Age comparisons}

There were no significant differences in opinions about buildings, relationships between them or the overall streetscapes based on age. Nevertheless, some differences will be discussed. Overall, opinions between the age groups were reasonably aligned for 10 of the 15 buildings in Wyndham and Kingston Streets but for the other five there were differences, mainly between the young (under 25) respondents and those who were older. 
Table 8.6: Age group preferences expressed for buildings $\mathrm{D}$ and W6

\begin{tabular}{|l|c|c|c|c|}
\hline & Under 25 & $\mathbf{2 5 - 5 4}$ & $\mathbf{5 5}$ and over & \\
\hline Building D & & & & \\
\hline Do you like this building? & 2.27 & 1.75 & 1.50 & $\begin{array}{c}\text { 3-point } \\
\text { scale }\end{array}$ \\
\cline { 1 - 4 } $\begin{array}{l}\text { Façade design above ground } \\
\text { level }\end{array}$ & 3.18 & 2.63 & 2.50 & \multirow{2}{*}{$\begin{array}{c}\text { 5-point } \\
\text { scale }\end{array}$} \\
\cline { 1 - 4 } Surface finishes above ground & 3.45 & 2.63 & 3.00 & \multirow{2}{*}{$\begin{array}{c}\text { 3-point } \\
\text { scale }\end{array}$} \\
\cline { 1 - 4 } Façade design at street level & 4.00 & 2.96 & 2.50 & \multirow{2}{*}{ 5-point } \\
\cline { 1 - 4 } Building W6ale
\end{tabular}

Building D was generally liked by people under 25 years of age but disliked by older people. Building D is a tall, slender tower on the Federal and Kingston Street corner and all respondents made the relationship between it and building $C$ to the east (section 8.3.2) the least liked. People under 25 were more positive than the other groups about the façade design of building D and found the street façade particularly pleasant (figure 8.22). This was also the factor that linked most strongly to young people's opinions of the building overall (table 8.6). The street level façade steps down to accommodate the gentle slope of Kensington Street. This creates a high shopfront that is extensively glazed. Along with the main entrance to the building this façade includes a small café. The façade does not follow a straight line as it undulates to accommodate steps and doorways. During each of the visits to the site people were observed standing, leaning and sitting on the steps and in the alcoves along this street frontage. Young people also found the design and surface finishes of the tower to be pleasant whereas the other two age groups did not. Liking these aspects of the building was consistent with the way young people also liked those of buildings W1 and W3, two other commercial towers in the study area. Overall, young people found taller buildings more pleasant than their older counterparts. 
In addition to responding more favourably toward buildings D, W1 and W3, people under 25 also liked buildings $F$ and $G$ more than people in the other two groups.

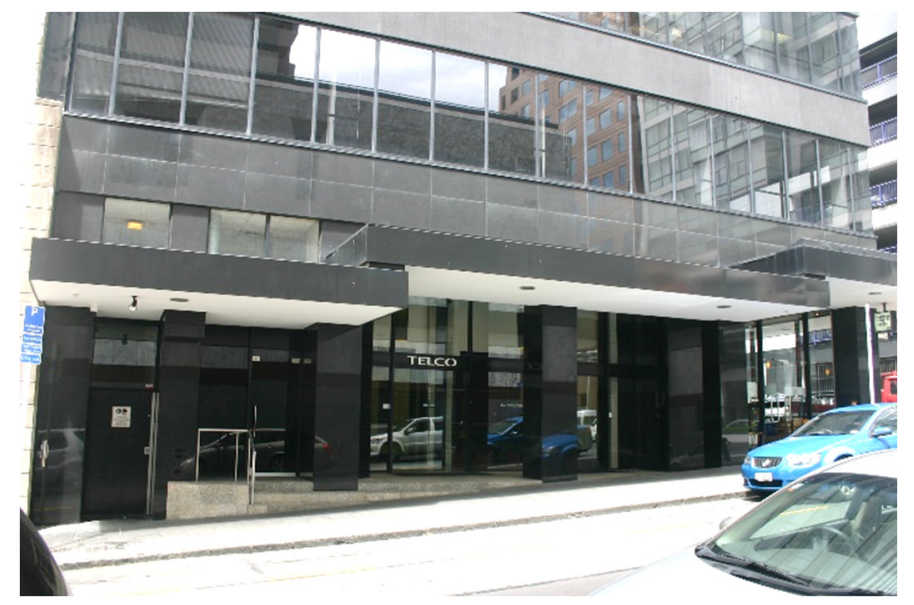

Figure 8.22: The street level facade of building $D$, which young people found particularly pleasant.

Source: author

People under 25 years differed from older people in their opinions of building W6, generally disliking a building generally liked by others (figure 8.21). People under 25 disliked it at street level, as did those aged 25 to 54. Possible reasons for this have been discussed above in relation to observed differences between men and women for this building (section 8.4.1). Young people's opinions about the building overall were linked most closely to their opinions of the façade design above ground level. Young people found both the design and the surface finishes to be unpleasant while those who were older generally found them pleasant. Again, the possible reasons for this have been discussed above and can be summarised as the large windows revealing too much untidiness or the drapes across them being drawn. Because women were also of the same opinion, a check was made to determine whether the young person's group comprised mainly women. This showed $63.6 \%$ of the respondents under 25 were women and people under 25 were $33.3 \%$ of all women respondents. It seems that both young people and women dislike building W6, based on a comparison of mean scores.

General similarities between age groups for relationships between adjoining buildings were observed, with some exceptions. People 55 and older liked building W2 a little more than others and were much more positive about the way it related to those adjacent to it. 


\subsubsection{Comparing change professional and lay people's opinions}

Lay people and change professionals have similar opinions about older, characterful buildings. As noted above, buildings A, W2, W4, W5 and W7 in this case study area could be described as characterful. The two groups like each of these buildings, with fairly close mean preference scores for each (table 8.7). The responses to building A deviate most, with a mean score difference of 0.37 . While the change professionals liked the façade design and surface finishes of this building above ground level more than lay people, they were more critical of the design at street level, which both groups disliked.

In addition to lay people and change professionals having similar preferences for older character buildings in the study area, there were buildings $(\mathrm{B}, \mathrm{E}, \mathrm{F}$ and $\mathrm{H})$ both groups did not like. These disliked buildings do not appear to share traits as they range in frontage width and overall height as well as façade composition and building typology. The buildings are both new and old and in different states of repair and maintenance. The most intriguing is building $\mathrm{F}$, which was completed little more than four years ago. In addition to disliking the building, both groups also found the street level design and the design and the surface finishes of the building above ground level unpleasant. In their responses to all questions about building F, lay people and change professionals were consistently negative, with the change professionals more so.

In addition to creating a watershed for aesthetic preferences on the basis of gender and age building W6 drew different opinions from lay people and change professionals. The latter seemed to like this building whereas lay people did not, a difference of opinion considered significant at $\mathrm{p}<0.01$. No change professional disliked building W6 and 76.9\% liked it. In contrast, $46.2 \%$ of lay people disliked it with only $15.4 \%$ liking it. This division based on occupation was also echoed in the responses to questions about the building façade design and finishes above and at ground floor level. Possible reasons for these differences of opinion have been discussed in section 8.4.1. 
Table 8.7: Mean preference scores for buildings in the Kingston/Wyndham Street study area ( 3 point scale)

\begin{tabular}{|c|c|c|c|c|}
\hline & Occupational group & Mean & Std. Deviation & Sig. \\
\hline \multirow{2}{*}{ A } & Change profs & 2.69 & 0.480 & \\
\hline & Lay people & 2.32 & 0.723 & \\
\hline \multirow{2}{*}{ B } & Change profs & 1.85 & 0.801 & \\
\hline & Lay people & 1.79 & 0.630 & \\
\hline \multirow{2}{*}{$\mathrm{C}$} & Change profs & 2.38 & 0.870 & \\
\hline & Lay people & 1.89 & 0.875 & \\
\hline \multirow{2}{*}{$\mathrm{D}$} & Change profs & 1.23 & 0.439 & \multirow{2}{*}{$\mathrm{p}<0.01$} \\
\hline & Lay people & 2.25 & 0.799 & \\
\hline \multirow{2}{*}{$\mathrm{E}$} & Change profs & 1.23 & 0.599 & \multirow{2}{*}{$\mathrm{p}<0.05$} \\
\hline & Lay people & 1.68 & 0.723 & \\
\hline \multirow{2}{*}{$\mathrm{F}$} & Change profs & 1.77 & 0.725 & \\
\hline & Lay people & 1.79 & 0.833 & \\
\hline \multirow{2}{*}{ G } & Change profs & 1.77 & 0.832 & \\
\hline & Lay people & 2.25 & 0.799 & \\
\hline \multirow{2}{*}{$\mathrm{H}$} & Change profs & 1.69 & 0.751 & \\
\hline & Lay people & 2.00 & 0.770 & \\
\hline \multirow{2}{*}{ W1 } & Change profs & 1.46 & 0.519 & \multirow{2}{*}{$\mathrm{p}<0.05$} \\
\hline & Lay people & 2.19 & 0.801 & \\
\hline \multirow{2}{*}{ W2 } & Change profs & 2.85 & 0.376 & \\
\hline & Lay people & 2.81 & 0.402 & \\
\hline \multirow{2}{*}{ W3 } & Change profs & 2.00 & 0.408 & \multirow{2}{*}{$\mathrm{p}<0.05$} \\
\hline & Lay people & 2.42 & 0.643 & \\
\hline \multirow{2}{*}{ W4 } & Change profs & 3.00 & 0.000 & \\
\hline & Lay people & 2.81 & 0.491 & \\
\hline \multirow{2}{*}{ W5 } & Change profs & 2.92 & 0.289 & \\
\hline & Lay people & 2.84 & 0.473 & \\
\hline \multirow{2}{*}{ W6 } & Change profs & 2.77 & 0.439 & \multirow{2}{*}{$\mathrm{p}<0.01$} \\
\hline & Lay people & 1.69 & 0.736 & \\
\hline \multirow{2}{*}{ W7 } & Change profs & 2.85 & 0.555 & \\
\hline & Lay people & 2.73 & 0.604 & \\
\hline
\end{tabular}




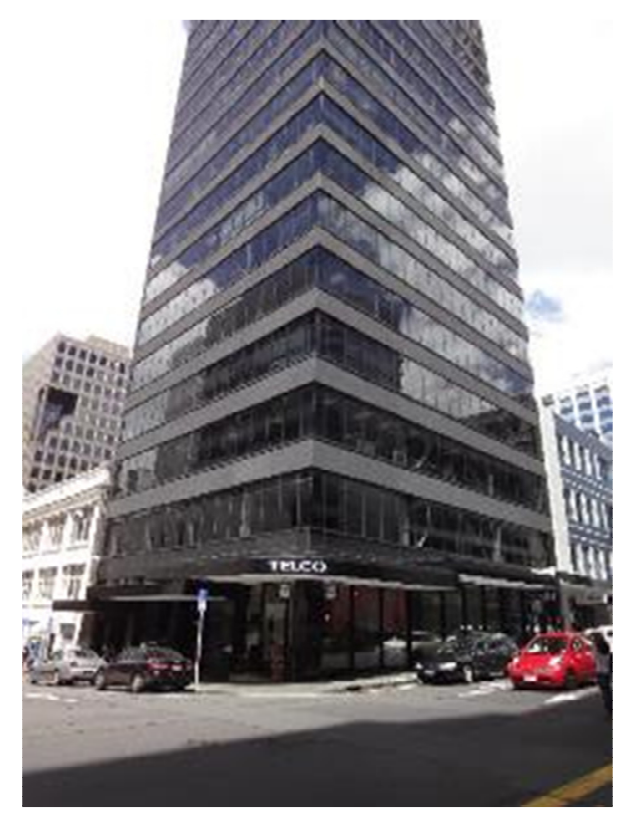

Figure 8.23: Building $\mathrm{D}$ divided opinions between lay people and change professionals.

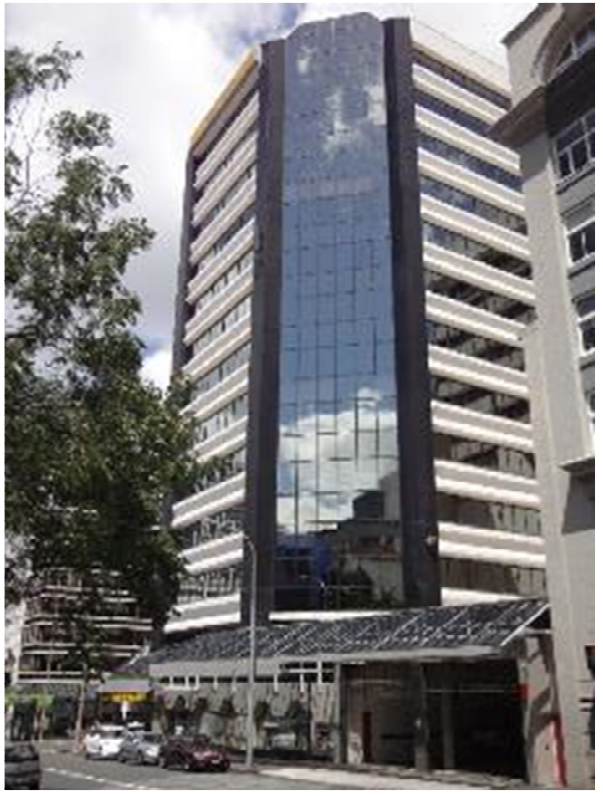

Figure 8.24: Building W1 divided opinions between lay people and change professionals.

Source: author

Source: author

Two buildings that divide opinions between lay people and change professionals are both tall and slender commercial office towers. Buildings D (figure 8.23) and W1 (figure 8.24) both occupy corner sites and have been designed to acknowledge these positions through their massing on site and overall height. The buildings make use of tinted or reflective glass, which in both cases is dark. While lay people were marginally positive about both, change professionals were decidedly negative (table 8.7). Within these mean scores no change professional liked building $\mathrm{D}$ whereas $46.4 \%$ of lay people did. Similarly no change professional liked building W1 while $42.3 \%$ of lay people did. Lay people generally found the street level design of building D pleasant, in contrast to change professionals who did not. This is relevant because even though they also had strongly positive opinions about the design of the façade of building D above ground level, a regression analysis reveals that the street level design was the best predictor of overall preference for the building by lay members of the public. In the case of building W1, the best predictor of a lay person's overall preference was their opinion of the façade design above street level.

So far, comparison between the preferences of lay people and change professionals has revealed building characteristics that both groups disliked and also divided opinions about 
building form. Of greater interest to the aims of this research is identifying design characteristics over which the two groups agree. One clear area of agreement was for older, characterful buildings. Change professionals and lay people liked buildings A, W2, W4, W5 and W7. Two of these are currently heritage listed in the Auckland District Plan. Of the contemporary buildings in the case study area, only W3 appealed to both groups although it may be more accurate to see W3 is a building both groups did not dislike. The mean score for W3 change professionals was neutral.

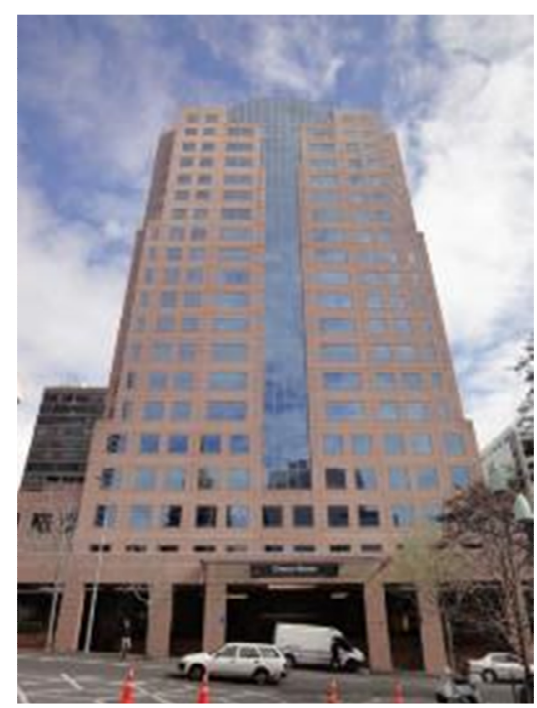

Figure 8.25: Building W3

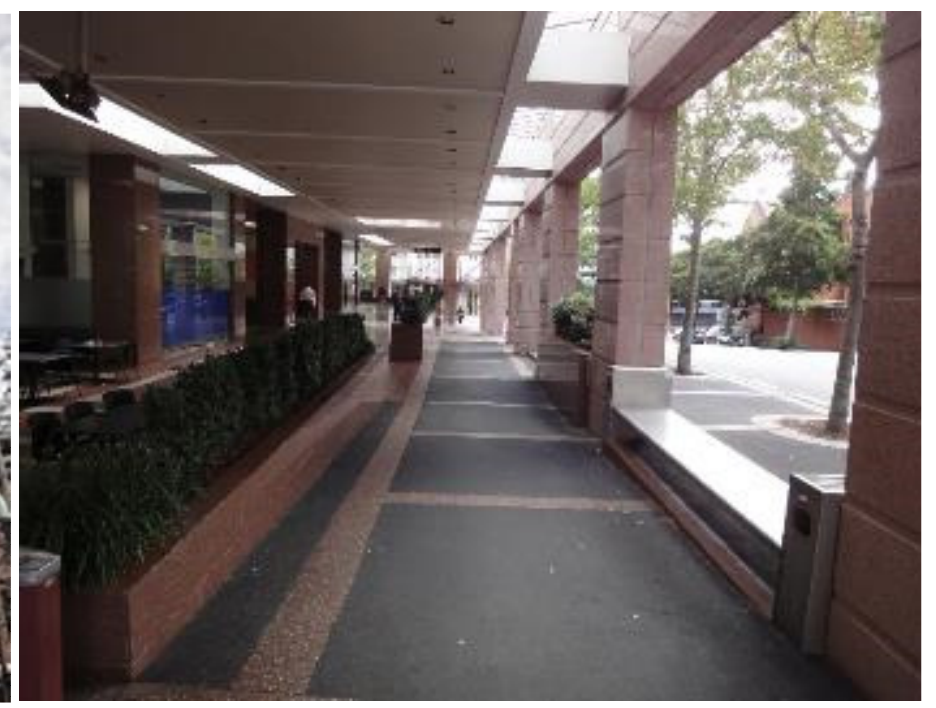

Figure 8.26: The walkway behind the street edge facade of building W3.

Source: author

Source: author

While both groups liked building W3, there was disagreement about the façade design and surface finishes above ground floor level (figure 8.25). Change professionals considered both characteristics unpleasant (mean $=2.67$ and $=2.75$, respectively) while lay people found them pleasant (mean=3.58 and $=3.69$ ). As building W3 is a commercial office tower this result is consistent with the responses to buildings D and W1, described above. There is strong evidence to suggest that change professionals find tower buildings clad in self-finishing monolithic materials and tinted or reflective glazing unpleasant. Conversely, lay people found these characteristics pleasant.

The two groups agreed about the street level design of building W3. Lay people were very enthusiastic with $73.1 \%$ finding it pleasant with a mean preference score of 3.88 . Although change professionals were less enthusiastic, with a mean score of 3.15 , some 
$46.2 \%$ of this group considered it pleasant. The design welcomes engagement by pedestrians and can be considered a significant public amenity in this area. Not only does it provide shelter to pedestrians but it also has a small café and places for the public to sit throughout the day (figure 8.26). Despite finding the street level design pleasant, lay people's responses to this question were not linked as closely to their overall response to the building as their feeling about the façade design above ground level, as the regression analysis revealed. Similarly, whether change professionals liked or disliked the building could be predicted most clearly by their responses to the question about façade surface finishes above ground level. While not perfectly clear which contemporary building characteristics were well liked by both built environment professionals and lay people, the street level atrium space of building W3 was a design that both groups found pleasant. The only other street level designs that appealed to both groups were those of the older character buildings W2, W4, W5 and W7.

There was general agreement between the two groups over the relative pleasantness of two of the four streetscapes (table 8.8). Change professionals and lay people found all streetscape characteristics on the north side of Wyndham Street pleasant. The relationships of building height, alignment between building facades along the length of the street and the relationships of façade style were given mean scores in the region of 4 on a 5-point scale by both groups. This streetscape includes three buildings well-liked by both groups and one (W6) that received contrasting responses. Despite not liking building W6 and the relationships between it and the two neighbouring buildings, lay people liked the overall characteristics of this side of Wyndham Street, although their mean scores were consistently below those of change professionals. To reiterate earlier observations about this streetscape, it features older buildings that, in all but one instance, are separated by positively designed open spaces, such that they provide visual and other amenities to the public. 
Table 8.8: Mean scores for streetscape characteristics in the Kingston/Wyndham Street area for lay people and change professionals

\begin{tabular}{|c|c|c|c|c|}
\hline & & Mean & $\begin{array}{c}\text { Std. } \\
\text { Deviation }\end{array}$ & Sig. \\
\hline \multirow{2}{*}{$\begin{array}{l}\text { Relationship of building heights } \\
\text { Kingston Street South }\end{array}$} & Change profs & 2.46 & 0.660 & \\
\hline & Lay people & 2.43 & 0.690 & \\
\hline \multirow{2}{*}{$\begin{array}{l}\text { Relationships of façade alignment } \\
\text { Kingston Street South }\end{array}$} & Change profs & 3.08 & 0.954 & \\
\hline & Lay people & 2.96 & 0.881 & \\
\hline \multirow{2}{*}{$\begin{array}{l}\text { Relationship of façade styles } \\
\text { Kingston Street South }\end{array}$} & Change profs & 2.00 & 0.816 & \\
\hline & Lay people & 2.18 & 0.772 & \\
\hline \multirow{2}{*}{$\begin{array}{l}\text { Relationship of building heights } \\
\text { Kingston Street North }\end{array}$} & Change profs & 2.54 & 1.050 & \multirow{2}{*}{$\mathrm{p}<0.05$} \\
\hline & Lay people & 3.32 & 1.020 & \\
\hline \multirow{2}{*}{$\begin{array}{l}\text { Relationships of façade alignment } \\
\text { Kingston Street North }\end{array}$} & Change profs & 2.38 & 0.960 & \multirow{2}{*}{$\mathrm{p}<0.05$} \\
\hline & Lay people & 3.04 & 0.854 & \\
\hline \multirow{2}{*}{$\begin{array}{l}\text { Relationship of façade styles } \\
\text { Kingston Street North }\end{array}$} & Change profs & 1.69 & 0.630 & \multirow{2}{*}{$\mathrm{p}<0.01$} \\
\hline & Lay people & 2.75 & 1.075 & \\
\hline \multirow{2}{*}{$\begin{array}{l}\text { Relationship of building heights } \\
\text { Wyndham Street South }\end{array}$} & Change profs & 2.69 & 0.947 & \\
\hline & Lay people & 3.15 & 1.120 & \\
\hline \multirow{2}{*}{$\begin{array}{l}\text { Relationships of façade alignment } \\
\text { Wyndham Street South }\end{array}$} & Change profs & 2.92 & 0.759 & \\
\hline & Lay people & 3.38 & 0.897 & \\
\hline \multirow{2}{*}{$\begin{array}{l}\text { Relationship of façade styles } \\
\text { Wyndham Street South }\end{array}$} & Change profs & 2.23 & 0.599 & \multirow{2}{*}{$\mathrm{p}<0.01$} \\
\hline & Lay people & 3.20 & 1.118 & \\
\hline \multirow{2}{*}{$\begin{array}{l}\text { Relationship of building heights } \\
\text { Wyndham Street North }\end{array}$} & Change profs & 4.54 & 0.518 & \multirow{2}{*}{$\mathrm{p}<0.05$} \\
\hline & Lay people & 4.04 & 0.823 & \\
\hline \multirow{2}{*}{$\begin{array}{l}\text { Relationships of façade alignment } \\
\text { Wyndham Street North }\end{array}$} & Change profs & 4.23 & 0.725 & \\
\hline & Lay people & 3.81 & 0.800 & \\
\hline \multirow{2}{*}{$\begin{array}{l}\text { Relationship of façade styles } \\
\text { Wyndham Street North }\end{array}$} & Change profs & 4.54 & 0.518 & \multirow{2}{*}{$\mathrm{p}<0.01$} \\
\hline & Lay people & 3.92 & 0.844 & \\
\hline
\end{tabular}

Both groups also disliked the south side of Kingston Street, although their mean preference scores for alignment between buildings were around the neutral point. In particular, both groups found the relationship of design styles very unpleasant. This streetscape includes the two most disliked buildings ( $\mathrm{E}$ and $\mathrm{F}$ ) and the three least liked inter building relationships $(\mathrm{C} / \mathrm{D}, \mathrm{D} / \mathrm{E}$ and $\mathrm{E} / \mathrm{F})$, making this overall dislike predictable. Its characteristics are a diversity of building ages, frontage widths and heights. With the exception of the street level design of building $\mathrm{D}$, which lay people liked, the other buildings have street level frontages both groups found unpleasant. Several of the buildings are not well looked after or appear disorganised (figure 8.27). 


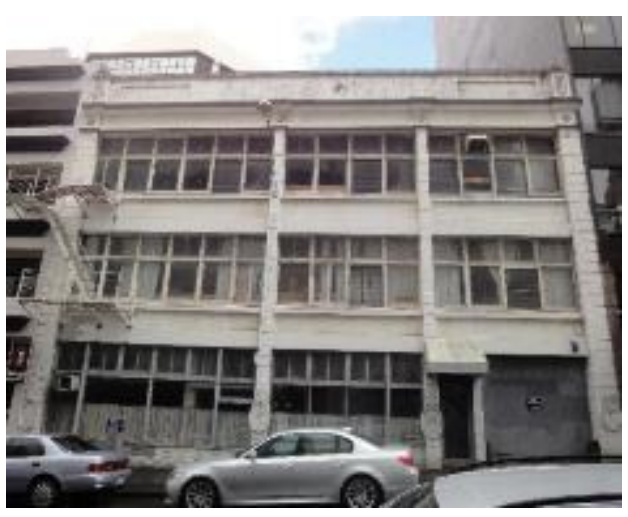

Figure 8.27: Building $C$ along the southern side of Kingston Street. This building, along with others in this streetscape, looks poorly maintained.

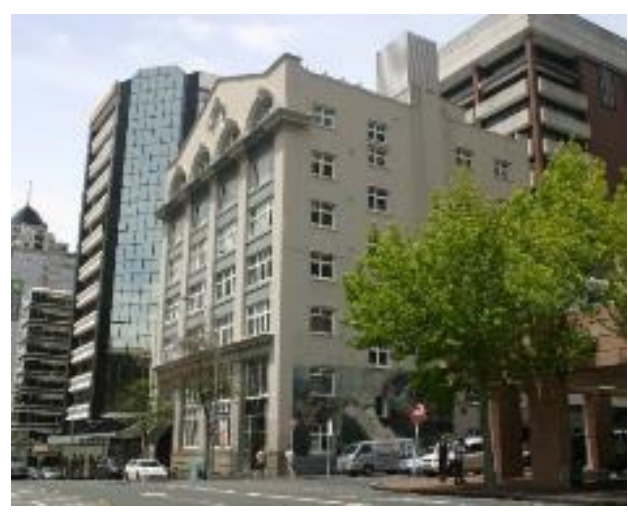

Figure 8.28: Looking east along the southern side of Wyndham Street. A corner of the building $\mathrm{W} 3$ podium is seen on the right with $\mathrm{W} 2$ in the centre of the image.

The southern streetscape of Wyndham Street divided opinions of lay people and change professionals (figure 8.28). While lay people on the whole found the height, alignment and style relationships pleasant, the professional group felt all of these were unpleasant. In contrast to the two sides of Kingston Street, where buildings effectively form continuous walls to contain the street space, the southern side of Wyndham Street is punctuated by three freestanding buildings that are separated from each other. Buildings W1 and W3 are towers that only extend across only a portion of their street frontage while building W2 extends across the full width of its site. The net effect is that each building appears isolated from the others. The change professionals disliked the relationships between these three buildings and this may be reflected in their displeasure with the streetscape characteristics. It seems the contribution made by building W2 was not sufficient to overcome the poor alignment between facades, poor relationship of heights and particularly poor mixture of design styles. While lay people were ambivalent about the relationships between buildings (mean=3.05 and =3.03) they liked each of the buildings individually and the three aspects of streetscape. The spaces between buildings are not planned as positively as those on the opposite side of Wyndham Street and it seems they are insufficient for buffering three very different design styles, at least in the minds of lay people. 


\subsection{Case Study Summary}

A sample of 41 people evaluated the individual buildings and overall streetscape characteristics of Kingston and Wyndham Streets, which lie in the western part of the Auckland central business district. The aesthetic preferences of the sample were analysed to identify which design characteristics, if any, were most liked and which were not. Comparisons were also made between the responses of different demographic groupings within the overall group.

More than half of the 15 buildings in the study area were liked by the respondents and the average of mean preference score for the individual buildings was 2.21 on a 3-point scale. This average score is between those for buildings in College Street (2.05) and Tyler Street (2.35). There was a clear preference for older, more characterful buildings in this study area with the top five ranked buildings, based on mean preference score, falling into this category. These older buildings were all of a moderate height, although building W2, the third highest ranked building in the area, is approximately 36 metres tall. Other characteristics shared by these best liked buildings include traditional/classical façade compositions with clearly expressed base, middle and top sections, monolithic façades with windows, and the fact all were well maintained.

The two best liked buildings convey a sense of history and cultural importance. The influence of such factors in aesthetic responses is well established in the literature (section 3.8). The high preference scores achieved by buildings W4, the St Patrick's Presbytery, and W5, St Patrick's Cathedral, can be explained by these phenomena and these results support prevailing theories that associational meanings carry considerable weight in the processing of aesthetic stimuli. In addition to conveying meaning these buildings are both surrounded by visually attractive public spaces and gardens and are well maintained. Both are factors that enhance people's aesthetic perceptions.

The two least liked buildings stand alongside one another, one being older and used for car parking and the other being the most recent addition to the study area, built for retail and residential purposes. Building $\mathrm{E}$ is a utilitarian, multi-storey car parking structure and presents an unattractive façade to Kingston Street. People found every part of this building unpleasant, a response that could have been predicted. Building F, on the other hand, is new and has introduced residential activities into this part of Auckland. The 
façade design of this building above ground floor level, a gridded arrangement of balconies, was strongly disliked as was the street level design. The floor to floor height of these upper levels is low and the proportions very different to the more generous upper floors of the buildings that were well liked. The street level was designed to be visually open, with expansive areas of glazed shopfront. However, both retail tenants have reduced interior visibility with the design of their fitouts. This building was perceived poorly by the survey respondents.

The inter-building relationships were influenced by the relative attractiveness of the individual buildings and the nature of the spaces between buildings, if any. There were few close inter-building relationships in the study area as a consequence of several large buildings occupying the whole length of their block and the influence of St Patrick's Square in Wyndham Street. Where the buildings were liked the relationships between them were also liked but where one or more was disliked this led to a disliked relationship. The nature of St Patrick's Square is such that it lifted the preferences for the relationships that take place across it. This was clearly evidenced by the responses to the relationship between W5, a well-liked building, and W6, which several subgroups disliked.

Variations in building height were again a factor in preferences for streetscapes. Responses to the big variations in height relationships on the southern side of Kingston Street were negative, leading to low mean preference score as well as negative comments made by individual respondents. Although the spatial definition of Wyndham Street is poor, and ignores some well-respected recommendations, the alignment of buildings on both sides was well liked. Conversely, the two sides of Kingston Street are much better defined in spatial terms but these alignments were disliked. Although these results alone are not sufficient reasons to dismiss the recommendations of Cullen (1971) and Sitte (1979) that street spaces should be well defined, they do serve to highlight the importance of positively designed spaces and street edges. All but one of the gaps and open spaces along Wyndham Street appear to have been positively designed, first to allow visual and physical extension of the street space and at a more detailed level to welcome public engagement. The negative responses to the Kingston Street alignments were probably influenced by people's perceptions of the façade designs and building height relationships, both of which contribute to the three dimensional perception of the street spaces. 
Building W6 was a preference watershed between several subgroupings. Women felt differently about this building from men, young people felt differently to older people and lay people disliked it while change professionals found it quite attractive. It also has characteristics that appeared, in other circumstances, to be attractive to all people in the sample. These include the fact the building is old and has a patina that comes with age, the design has a compositional hierarchy of base, middle and top, it is loosely based on the Art Deco style, and it incorporates discrete window openings with clear glass rather than continuous bands of a tinted or reflective glazing. However, the large window openings enabled the messy interior spaces to be readily observed from the street, or to curtains or blinds being drawn across them. In light of the focus group discussions (section 6.2.5) it was reasoned these large openings led to lowered preferences for the building by some people. In addition, the majority of the street level shopfront is unkempt and restricts visibility into the shop. These were factors that contributed to the varied responses of individuals by subgroup.

Other than in their responses to building W6 there were few differences in the way men and women evaluated the buildings and the relationships between them in the Kingston/Wyndham Street study area. This is one of the reasons why the differences in respect of building W6 are so intriguing. The responses were also grouped by age to enable opinions of those of an age most likely to lead or influence changes to urban streetscapes to be compared with those younger and older. Building W6, discussed above, drew different responses from people under 25 who disliked it and its street level design. On the other hand, this group was far more positive about building D, a tall, slender commercial office tower. An important factor in this was the fact young people found the street level design very pleasant. Young people may have been influenced by the many opportunities this design provided for people to 'hang out' alongside it, in the alcoves, on the steps and on furniture provided by the café tenant. This was one of two buildings, along with W3, in the study area that supported a sense of street life. Young people found this aspect and the whole building attractive in contrast to those who were older.

This third investigation of Study Two also highlighted differences and similarities in the way change professionals and lay people perceive urban streetscapes. People in each of these two groups shared preferences for the older, more characterful buildings in the study area. There were differences in opinion about W6, which lay people disliked and 
change professionals strongly liked. Lay people appeared to like buildings D and W1, both quintessential commercial buildings designed to acknowledge their locations on street corners and primarily clad in dark tinted or reflective glass, whereas change professionals did not.

Change professionals and lay people both found the north side of Wyndham Street pleasant in every respect and the south side of Kingston Street unpleasant. These two streetscapes are very different in nature with Kingston Street strictly defined along its length by a disparate collection of buildings, two of which were the least liked in the study area, and Wyndham Street being visually open as a consequence of buildings being spaced apart. The north side of Kingston Street also contains three of the best liked buildings, all of which are older and characterful. Perhaps of equal importance with the quality of buildings along this streetscape is the quality of the spaces between and around the buildings. The positive responses to the north side of Wyndham Street appear to confirm the positive influence of Takutai Square in the Tyler Street study area. 
Chapter 9

\section{Research findings and discussion}

\subsection{Introduction}

Chapters 5 to 8 discussed the results of the two studies undertaken to address the aims and objectives of the research. Study One invited responses to photographic elevations of urban street scenes with the prospect this would provide initial insight into preferred streetscape design aspects. This study found strong preferences for streetscapes that exhibit visual interest in a coordinated manner across the buildings in the scene. While respondents found a streetscape comprising buildings of identical height to be pleasant, they did not like it as much as one where the building heights varied within a narrow band of difference. The first study thus found that people enjoyed greater visual pleasure in streetscape characteristics that provided moderate variations across the building forms and façade designs.

In addition to generating data for directly answering the research questions, the findings of Study One provided input to the design of Study Two, in which people were asked to give their aesthetic preferences for selected streets. The Study One results raised questions about the nature of people's evaluations of individual buildings, of the relationships between adjoining buildings and what factors most influenced people when making their aesthetic judgements. It raised the matter of how natural elements such as trees and plants might influence perceptions of urban streetscapes. These questions were addressed in the survey design and selection of sites for Study Two. The second study also incorporated two focus groups of willing participants from the College Street, Wellington case. The Study Two results were reported in discrete chapters corresponding 
to each of the three case studies and for convenience are referred to as 2A (College Street), 2B (Tyler Street) and 2C (Kingston/Wyndham Streets).

Chapter 9 discusses the findings, looking at the evidence thematically. Linked to the research questions and objectives, the key issues arising from the ways people perceive and evaluate urban streetscapes are discussed. The discussion traverses the different cases in an effort to identify consistent patterns of response to buildings and urban streetscapes. Where conflicting responses arise, the most notable are discussed.

The 204 individual findings from Studies One and Two were entered into a spreadsheet and linked to one or two themes as appropriate as these emerged during the analysis. The aim was to arrive at a concise and robust list of themes to facilitate a coherent and useful discussion of how different streetscape characteristics were perceived. A full list of case study findings is included as Appendix C.

Table 9.1: Comparing the average of the mean preference scores for buildings in each study area.

\begin{tabular}{|l|c|c|c|c|}
\hline & $\begin{array}{c}\text { Average of the mean } \\
\text { preference scores for } \\
\text { individual buildings }\end{array}$ & $\begin{array}{c}\text { Buildings } \\
\text { that were } \\
\text { liked }\end{array}$ & $\begin{array}{c}\text { Buildings } \\
\text { that were } \\
\text { not liked }\end{array}$ & $\begin{array}{c}\text { Percentage of } \\
\text { buildings that } \\
\text { were liked }\end{array}$ \\
\hline $\begin{array}{l}\text { Study 2A } \\
\text { College Street }\end{array}$ & 2.05 & 10 & 9 & $53 \%$ \\
\hline $\begin{array}{l}\text { Study 2B } \\
\text { Tyler Street }\end{array}$ & 2.35 & 14 & 4 & $78 \%$ \\
\hline $\begin{array}{l}\text { Study 2C } \\
\text { Kingston/ } \\
\text { Wyndham Streets }\end{array}$ & 2.21 & 9 & 6 & $60 \%$ \\
\hline
\end{tabular}

The averages of the mean preference scores for all buildings in each of the three study areas are set out in table 9.1, along with the percentages of individual buildings that were liked in each. While respondents to the Tyler Street case study were clearly more positive about the buildings in that study area than respondents were about the individual buildings in each of the other two sites, this does not necessarily indicate the buildings in Tyler Street hold greater visual appeal in an absolute sense. Aesthetic perception can be influenced by many factors, including culture (Bourassa 1991: 90). Environmental factors such as the weather may also have affected responses in the three locations. As the study 
$2 \mathrm{~B}$ and $2 \mathrm{C}$ surveys were conducted in the weeks over the summer solstice, conditions were probably warmer and sunnier than when College Street was surveyed. Although it is not in the scope of this research to investigate the roles of culture or environmental conditions on perception, these matters may have been factors affecting the ways people evaluated each of the streets and another reason why direct comparisons between them are not appropriate.

\subsection{Research findings}

Discussion of the findings begins with table 9.2 where the evidence provided by the two studies in support of the key findings of this research is noted.

The findings in Table 9.2 are arranged under four headings, in the left column; building design characteristics that appear to be liked and not liked, streetscape characteristics that are liked and not liked, findings about the way people perceive urban streetscapes and similarities/differences in the preferences expressed by different demographic groups. These findings have emerged by analysing the findings from the individual studies. The table indicates the source of evidence for each stated finding.

Table 9.2: Summary of research findings

\begin{tabular}{|l|l|c|c|c|c|c|}
\hline \multicolumn{1}{|c|}{ Research finding } & \multicolumn{5}{c|}{ Evidence } \\
\cline { 2 - 7 } & $\begin{array}{c}\text { Study } \\
\text { One }\end{array}$ & $\begin{array}{c}\text { Study } \\
\text { 2A }\end{array}$ & $\begin{array}{c}\text { Study } \\
\text { 2B }\end{array}$ & $\begin{array}{c}\text { Study } \\
\text { 2C }\end{array}$ & $\begin{array}{c}\text { Focus } \\
\text { groups }\end{array}$ \\
\hline $\begin{array}{l}\text { People preferred traditional cladding } \\
\text { materials, particularly those that can be } \\
\text { repainted }\end{array}$ & N/A & $\checkmark$ & $\checkmark$ & $\checkmark$ & $\checkmark$ \\
\hline $\begin{array}{l}\text { People preferred buildings with discrete } \\
\text { window openings }\end{array}$ & $\checkmark$ & $\checkmark$ & $\checkmark$ & $\checkmark$ & $\checkmark$ \\
\hline $\begin{array}{l}\text { People preferred building facades that } \\
\text { appear clean and well maintained }\end{array}$ & N/A & $\checkmark$ & $\checkmark$ & $\checkmark$ & $\checkmark$ \\
\hline $\begin{array}{l}\text { People preferred street level designs that } \\
\text { enable visual engagement with the activities } \\
\text { inside }\end{array}$ & N/A & $\checkmark$ & $\checkmark$ & $\checkmark$ & $\checkmark$ \\
\hline $\begin{array}{l}\text { People disliked horizontally banded } \\
\text { building facades }\end{array}$ & N/A & $\checkmark$ & $\checkmark$ & $\checkmark$ & $\checkmark$ \\
\hline $\begin{array}{l}\text { The findings were inconclusive about } \\
\text { whether contextual fit is considered when } \\
\text { people evaluate individual buildings }\end{array}$ & N/A & $\checkmark$ & $\checkmark$ & & $\checkmark$ \\
\hline
\end{tabular}




\begin{tabular}{|c|c|c|c|c|c|c|}
\hline \multirow{4}{*}{ 这 } & $\begin{array}{l}\text { Relationships between buildings where } \\
\text { there was coordinated visual interest across } \\
\text { the individual facades were best liked }\end{array}$ & $\checkmark$ & $\checkmark$ & $\checkmark$ & $\checkmark$ & \\
\hline & $\begin{array}{l}\text { People preferred streetscapes where } \\
\text { building heights vary within a narrow band }\end{array}$ & $\checkmark$ & $\checkmark$ & & $\checkmark$ & $\checkmark$ \\
\hline & $\begin{array}{l}\text { People preferred streets where building } \\
\text { façades are aligned consistently }\end{array}$ & $\mathbf{N} / \mathbf{A}$ & $\checkmark$ & $\checkmark$ & & $\checkmark$ \\
\hline & $\begin{array}{l}\text { Open spaces were found to mitigate } \\
\text { potentially negative relationships between } \\
\text { adjoining buildings }\end{array}$ & $\mathbf{N} / \mathbf{A}$ & $\mathbf{X}$ & $\checkmark$ & $\checkmark$ & $\mathbf{N} / \mathbf{A}$ \\
\hline \multirow{2}{*}{ 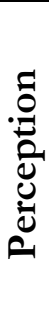 } & $\begin{array}{l}\text { People's understanding of building function } \\
\text { was important in aesthetic evaluation of } \\
\text { buildings and streetscapes }\end{array}$ & $\mathbf{N} / \mathbf{A}$ & $\checkmark$ & $\checkmark$ & $\checkmark$ & $\checkmark$ \\
\hline & $\begin{array}{l}\text { Change professionals' opinions were } \\
\text { influenced by their knowledge of urban } \\
\text { change }\end{array}$ & & $\checkmark$ & & & $\checkmark$ \\
\hline \multirow{3}{*}{ 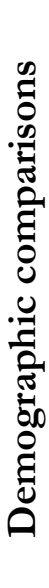 } & $\begin{array}{l}\text { Lay people and change professionals held } \\
\text { similar preferences for buildings and } \\
\text { streetscapes }\end{array}$ & $\checkmark$ & $\checkmark$ & $\checkmark$ & $\checkmark$ & $\mathbf{N} / \mathbf{A}$ \\
\hline & $\begin{array}{l}\text { The aesthetic preferences of planning } \\
\text { professionals and architects differed more } \\
\text { than those of change professionals and lay } \\
\text { people }\end{array}$ & $\checkmark$ & $\checkmark$ & $\checkmark$ & $\checkmark$ & $\mathbf{N} / \mathbf{A}$ \\
\hline & $\begin{array}{l}\text { While there were areas where men's and } \\
\text { women's perceptions differed, there were } \\
\text { no fundamental differences in opinions of } \\
\text { buildings and urban streetscapes. }\end{array}$ & $\checkmark$ & & $\checkmark$ & $\checkmark$ & $\mathbf{N} / \mathbf{A}$ \\
\hline
\end{tabular}

\subsubsection{General preferences for buildings}

While possibly not at the top of agendas that drive urban change, most designers and their clients would probably like to be responsible for creating buildings that are liked by their peers as well as more broadly. This leads to the question, "what are the attributes of wellliked buildings?' The characteristics that have emerged from the analysis of the findings are now discussed.

\subsubsection{People preferred traditional, monolithic wall materials}

People liked 33 of the 52 buildings across all three cases in Study Two. Of the 33 that were liked, 24 (72.7\%) were clad using traditional monolithic materials, with the two most common materials being clay bricks and painted concrete/cement plaster. A critical 
distinction between traditional and non-traditional materials is that the former are significantly thicker than the latter and therefore the resultant facades also appear thicker, a quality most apparent at door and window openings. These materials appear monolithic even though they may or may not be providing load bearing structural support.

For each of the 24 liked buildings with wall surfaces of traditional and paintable materials, the façade details were listed in the top three reasons for this. Additional support for this finding was provided by the fact the majority of the buildings disliked in Study Two were clad in glass, metal sheet or other non-traditional materials.

Although painted concrete/plaster render was the most common traditional material in Study Two, the best liked was brick masonry. Evidence for this comes from the fact all five brick buildings in Study Two were well-liked and four of these ranked at the top of their respective street preference lists. Building $G$ was best liked in Study 2B, followed by buildings I and O. In Study 3C the highest rated building was W4, with a higher mean score than St Patrick's Cathedral, which is immediately adjacent to it. The principal façade of each of these buildings is brick masonry and building $\mathrm{O}$ has recently been constructed using salvaged red clay bricks.

Evidence that people strongly liked brick is compelling but this only emerges from Study 2B and 2C. Study 2A contained no brick buildings and people's feelings for brick facades were unclear following Study One. The first study asked for opinions on the relationships between materials and finishes in a streetscape and the two examples with buildings with brick finishes were rated in the middle of the six. Scenes with exclusively concrete (scenes 3 and 4) and painted plaster facades (scene 6) were rated higher. However, the question was about relationships across the streetscape and those that were more strongly preferred had a stronger consistency of material use. Where respondents identified buildings in a scene as not fitting with the others, facade material was never listed as a reason for this. On this basis the Study One results have neither reinforced nor undermined this preference for brick facades emerging from the research.

Nevertheless, the use of traditional wall materials cannot guarantee a building will be liked any more than their avoidance will mean a building is disliked. Preferences for traditional wall types are not absolute as many other factors contribute to how a building is perceived. For example, building S in Study 2A was second best liked despite the fact that it had 
extensive areas of metal screen cladding at first floor level. The matt, dark grey finish to the metal panels and the overall compositional design of the building, in addition to other factors such as positive meanings associated with its retailing function, may have lifted the preference rating despite the use of metal cladding.

Similarly, respondents liked building $\mathrm{N}$ in Study 2B despite its extensive areas of glass curtainwall cladding. Negative feelings about glass cladding were expressed by the professionals' focus group and came through in the poor mean preference scores for buildings $\mathrm{D}$ and $\mathrm{W} 1$ in Study 2C, the only other buildings in Study Two to use glass cladding. Perhaps respondents overlooked or forgave the extensive areas of reflective curtain wall glazing in the upper levels of building $\mathrm{N}$, as the lower façade levels had variable size, discrete openings in what appears to be stone cladding. In actual fact the cladding is precast, polished concrete with integral colour, resulting in an appearance similar to natural stone. In addition, the retail activities in the publicly accessible lower floors of the building may have enhanced positive responses to it.

The positive responses to buildings $\mathrm{S}$ in Study 2A and N in Study 2B contradicted the finding that people prefer buildings with facades of traditional materials. Both of these buildings were recently constructed and temper the use of non-traditional materials with others that are more traditional. The buildings are well maintained and appear well designed. Both support retail activities that people find attractive. These factors may have influenced what could otherwise have been negative responses to their façade cladding materials.

\subsubsection{People preferred buildings with discrete window openings}

The research findings strongly suggest people are attracted to buildings with discrete window openings. These were found in 24 of the 33 (72.7\%) buildings with mean scores of 2 or above on the 3-point preference scale in Study Two. In Study One, the two streetscape scenes liked best by respondents comprised buildings that only had discrete window openings. Three of the four best liked buildings in Study $2 \mathrm{~A}$ and all four of those best liked in Studies 2B and 2C had façade compositions based around discrete window openings. 
Discrete windows provide buildings and their facades with a sense of scale and human proportion. Ching (2007: 330-335) stated that windows can establish a scale relationship in the façade of a building, enabling viewers to better understand the size of other elements in it and within the building. In addition, windows are well understood compositional elements that help people to gauge the overall size of a building.

In a discussion of how the human mind perceives patterns in the built environment, Smith (2003: 35-47) noted that "the mind derives...pleasure from extrapolating rhythm from situations in which likeness is tempered with difference". The windows of the

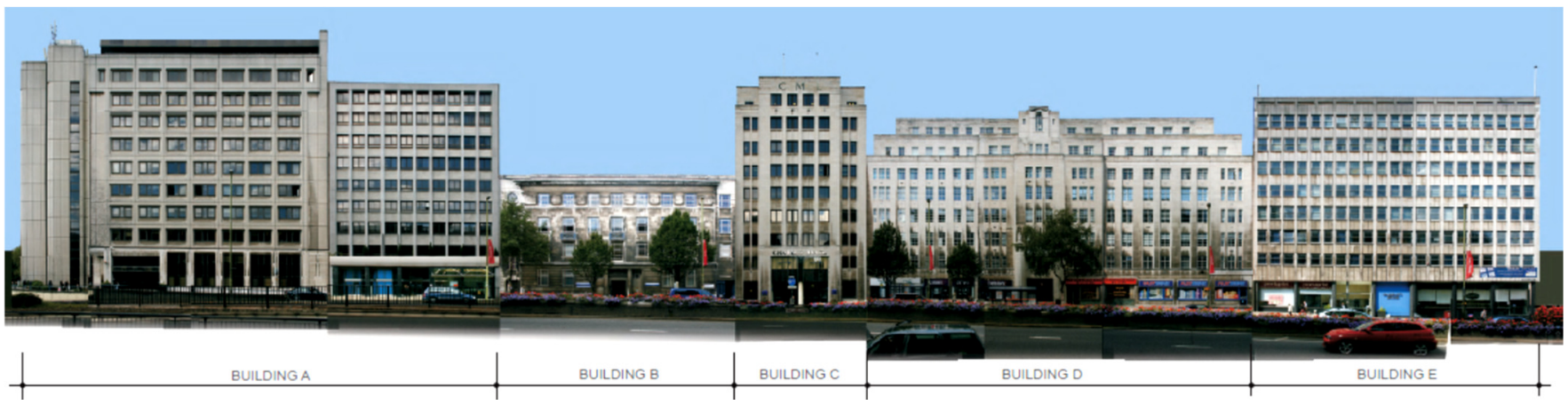

Figure 9.1: All buildings in Scene 3 of Study One have discrete window openings. The pattern of windows is a characteristic that helps link the facades as well as generate desirable levels of visual interest.

Source: author

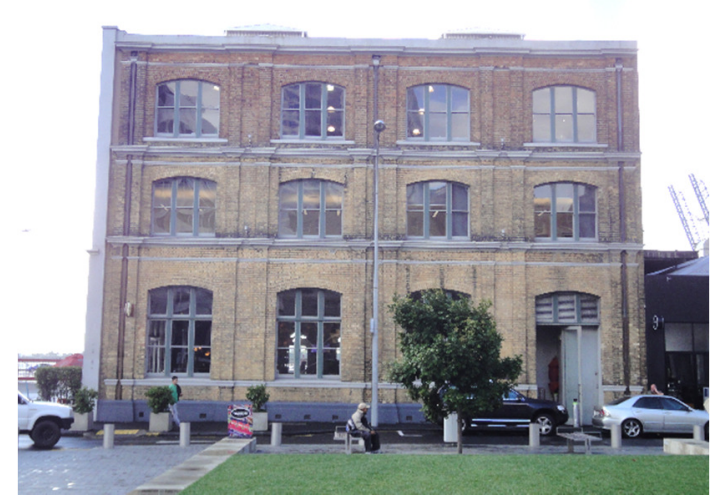

Figure 9.2: Building G in Study 2B with a facade to Tyler Street characterised by large, discrete window openings.

Source: author

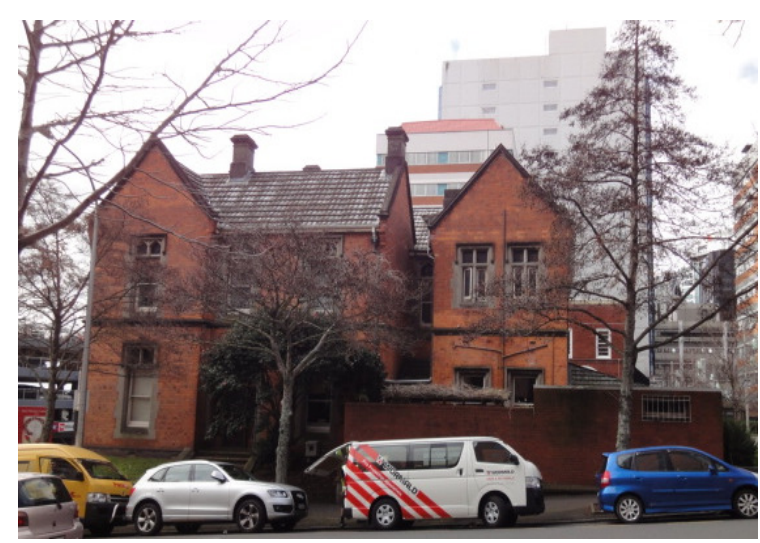

Figure 9.3: the façade of building W4 also features discrete window openings.

Source: author 
buildings in scene 3 of Study One (figure 9.1) set up aesthetically pleasing patterns in the street facades, which could explain why this scene was so well liked. This quality is also seen in the patterns of openings in the facades of individual buildings (figures 9.2 and 9.3).

The findings do not however, suggest that only buildings with discrete window openings are well liked nor that all buildings with discrete windows will be liked. Buildings $\mathrm{N}$ and Q with curtain wall cladding/glazing in part or over the whole of the facade (figures 9.4 and 9.5) were well liked by most respondents in Study 2B. This form of cladding is effectively the antithesis of the more traditional discrete window openings discussed above. The lower six floors of building $\mathrm{N}$ do feature a pattern of discrete openings, so this part of the façade may have influenced its evaluation. Similarly, the vertical mullions and expressed horizontal joints between individual panes of glass in the façade of building Q generate a fine grained pattern that was clearly attractive to respondents.

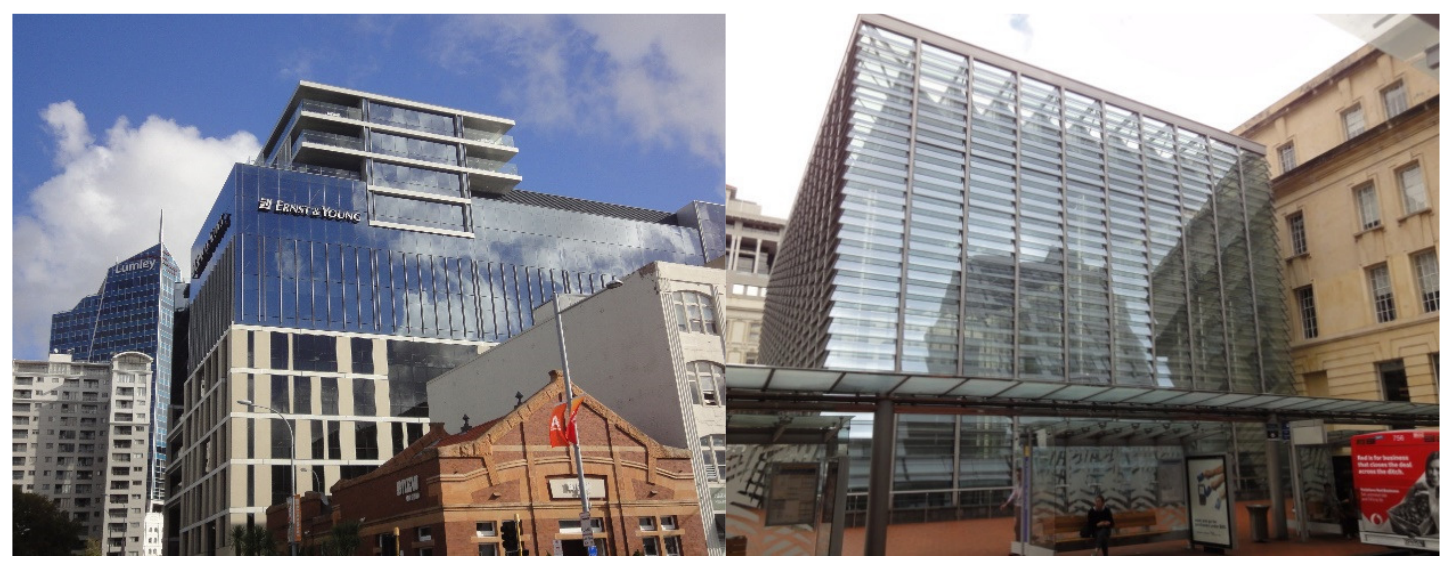

Figure 9.4: Building $\mathrm{N}$ in Study 2B, seen from the east and across building $M$. Tyler Street runs between the two buildings.
Source: author
Figure 9.5: Building Q, which like the upper parts of building $\mathbf{N}$, is primarily clad in glass, although there are no individual windows in the façade.

Source; author

Not only did the best-liked buildings have discrete window openings but the preferred windows also had clear glass. This held true for all buildings with the four best mean preference scores in each part of Study Two. This finding supports another finding that people like buildings that they can understand (section 9.3.1.1). Being able to see through a window enables the function a building to be more readily identified than if the façade has reflective or tinted glass. 


\subsubsection{People preferred clean and well maintained building facades}

The Study Two results confirmed people's preferences for buildings that appeared clean and well maintained, confirming the findings of other researchers around the meanings that influence aesthetic perception in section 3.8.3. The influence of building maintenance on perception was suggested by the results of Study One (section 5.3) and confirmed in each separate case study conducted in Study Two.

The levels of maintenance and cleanliness seen in a façade appeared more important to respondents than its material qualities, as evidenced by responses to building $\mathrm{K}$ in Study 2A. This is a building with many of the characteristics of a well-liked building; it has discrete window openings, is of a reasonably small scale and the façade surface is a painted render. However, the majority of people who felt neutral or disliked this building cited maintenance as the primary reason for their response. Building $\mathrm{L}$ in Study $2 \mathrm{~B}$ and buildings $\mathrm{C}$ and $\mathrm{W} 6$ in Study 2C were experienced in the same manner, with many respondents citing the poor levels of maintenance as a reason for their dislike.

Such responses were not limited to older buildings in the study areas. While building D in Study 2A is relatively new, 9 people were either neutral or disliked it because they perceived it as being poorly maintained. The focus groups confirmed the importance of maintenance. The change professionals' focus group probed the matter of maintenance a little deeper to question the quality of the cladding materials and how the construction was detailed. A member of the group noted the poor weathering characteristics of fibre cement sheet board and the others agreed that this was a material that had a negative influence on their perceptions, in part because of how they thought the material would age.

\subsubsection{People preferred street level designs that enabled visual engagement with the activities inside}

People's responses to individual buildings were partly influenced by what they understood to take place inside, as is discussed in section 9.2.3.1. Related to this is the finding that people were particularly responsive to buildings that afforded views into street level interiors. While the meanings and values people assigned to these activities had a clear influence on their perceptions, this finding is primarily concerned with the physical nature of the interface between the public footpath and building interior. Openings or 
transparency at this level makes the building more visually interesting. Buildings that invite entry, like shops, enjoy an obvious benefit. Having a shopfront that allows the interior to be seen easily can be as effective as signage for shops and other commercial activities that rely on passing foot traffic.

It was predominantly the female and lay respondents who made reference to the street level of a building when evaluating it. These groups liked glazed street level designs that gave views of the interiors, although these areas of transparency did not need to be large. One of the best-liked street level designs was building $S$ in Study 2A. Despite extensive areas of blank wall this building also had well placed openings, revealing the colourful and busy shopping activities inside. The laypersons' focus group confirmed the importance of glazed openings in this and other buildings for this purpose.

Nevertheless, there were a number of exceptions to this finding. Buildings W4, W5 and W7 (older buildings in Study 2C) had relatively small openings along their respective street frontages. All were well liked, particularly their ground level designs, although W4 (the Presbytery) and W5 (St. Patrick's Cathedral) have functions which can be read from the building form alone, without the need to see inside. The street level design of each of these buildings is closely tied to the overall façade design, which together could be read as a coherent whole. In these cases the design attributes appear to have outweighed other factors in their perceptions. This was evidenced by the fact that the ground floor design of W1, also an older character building but one whose ground floor had been severely modified, was disliked.

Also in Study 2C, building F demonstrated that poor management of the shopfront was a factor in how the building was perceived. The street level of this recently completed building had extensive areas of glazing but both tenants had partially blanked these off with an opaque sign in one case and display shelving in the other. These were factors that led to the clear dislike of the street level design of this building. Overall, this research found that people liked street level facades that provided transparency along their length.

\subsubsection{People disliked layered (horizontally banded) building facades}

The finding that people disliked layered or horizontally banded building facades is consistent with the finding that people preferred buildings with discrete windows. Layered facades arise from placing one floor of a multi-storey building onto another and 
the appearance of these can range from elegant to banal (Hanlon 2009: 91). Strip glazing is often used to span between solid horizontal elements (spandrels) and the resultant façade appears as alternating bands of solid and void, whereas facades with discrete window openings can appear more unified, appearing primarily as a solid element extending across the whole of the façade. It seems the respondents felt that the taller buildings with layered facades in all three Study Two streetscapes tended toward the banal, as $70 \%$ of these were found to be unpleasant across all three cases.

Building D of Study 2B is an example. The four façades of this commercial office building are identical and result from the expression of the structural system. Round columns divide each face of the building into three bays and support horizontal beams that have subsequently been fitted with horizontal louvers (see figure 7.15). Each façade has a horizontal emphasis despite the expressed columns, largely because of the bay proportions. As a consequence, the facades appear layered. Some $81 \%$ of the respondents who disliked the building cited the façade details as a reason for this.

The dislike of layered facades was complemented by strong preferences for buildings that adopted more traditional, tripartite compositional strategies. There were 46 buildings of two or more storeys in the three Study Two streets and 29 of these were found pleasant by the respondents. Of these 29 buildings, $21(72.4 \%)$ had a traditional compositional pattern of a base, middle and top. Tripartite compositions, particularly in association with discrete window openings, enhance levels of visual interest in a façade and can help create a sense of order through the principle of hierarchy (Ching 2007: 338).

\subsubsection{Contextual fit as a consideration when people evaluate individual buildings}

Respondents identified several buildings in the six Study One street scenes that did not fit well because they were noticeably larger than the others in the same scene. However, Study One did not seek the answer to whether the mismatch in size influenced people's preferences for the larger building. Contextual compatibility as a factor in people's preferences for individual buildings is a matter of interest, in part because design review decisions are made about individual buildings. While contextual fit may be a factor in perceptions of streetscapes it is not clear how it influences the way people feel about the buildings individually. 
Unfortunately, the results of Study Two are inconclusive on this point. Across the three study areas there were a number of instances where people cited compatibility features such as height and overall shape as reasons why they liked a particular building. On the other hand, some buildings that were liked, and even well liked, were different in height or overall shape to others around them. Building D in Study $2 \mathrm{~A}$ was a particular example of this. Overall, people did not dislike this building, despite the relationships it formed with both neighbouring buildings being disliked, and strongly disliked in the case of the relationship with building $C$. The two focus groups were each invited to discuss contextual compatibility with reference to this building. Both groups acknowledged that building $\mathrm{D}$ was incompatible with those alongside and around it. Several members of the professional focus group felt this incompatibility did not diminish their opinions of the building because they considered the circumstance to be temporary and that future development would help to correct the clashes in building height. This response is consistent with those of change professionals (see 9.3.1.2). The lay focus group strongly disliked the building because of its size and poor relationships with other buildings and the street. Despite agreeing with the focus group about the poor relationships, lay respondents expressed a marginal liking for building D overall. Although the focus group was unable to explain this discrepancy, the building's poor compatibility may have been overlooked by respondents in favour of other positive attributes, such as its function, its design composition or its relative newness.

\subsubsection{General preferences for urban streetscapes and relationships between adjoining buildings}

While urban change generally takes place through successive (re)development of single sites and it is the individual projects that are scrutinised during design review, once implemented they become part of a wider streetscape and so, are perceived in relation to others. This part of chapter 9 discusses the findings about people's preferences for these relationships and for overall streetscapes.

\subsubsection{Relationships between buildings with a coordinated visual interest across the individual facades were best liked}

This finding came to light in the responses to Study One and was confirmed by Study Two, although in the latter there were fewer obvious patterns of architectural features across its streetscapes. Several of the street scenes in Study One comprised buildings that 
had evidently been constructed within a short period of time. A clear example is scene 3 (figure 5.4), where the buildings all appeared to date to the 1960s and 70s. Here the pattern is created by the arrangement of the discrete window openings across the facades. In Tyler Street (Study 2B), buildings G to M inclusive appeared to be linked by the size and arrangement of window openings in their facades. Similar patterns were also observed along the entire north side of Wyndham Street (Study 2C). Examination of other scenes found that potential linking details could also include vertical mullions, mouldings, and cornice lines.

In terms of adjacent building relationships, the one between buildings A and B in Study 2A was well liked. This is a relationship forged by similarity of architectural styles and similar relationships were also noted across the ex-warehouse structures of Tyler Street (Study 2B) and the northern side of College Street (Study 2A). People found these relationships very pleasant.

This finding confirms the attractiveness of areas of architectural character. Character emerges through patterns of consistency in the overall form and arrangement of buildings in an area, or in their façade designs and details (McIndoe 2008). Owing to historic patterns of development, character emerges most often when both these patterns are present. Many design guides have recognised the importance of such patterns and ask for them to be incorporated into contemporary designs. An example is the Cuba Street Heritage Area design guide in Wellington (Wellington City Council 2012: appendix 3).

\subsubsection{People preferred streetscapes where building heights vary within a narrow band}

The two studies examined people's preferences for the relationships between building heights along a street and found that they were judged most pleasant where they varied within a narrow 2 to 3 storey range. In Study One, the most preferred scenes varied within this band while scenes with buildings of consistent heights and those with widely varied building heights were rated lower. Building height consistency will appeal to people's natural, underlying preferences for order (section 3.6) but it seems that a row of five buildings built all to the same height, as seen in scene 6, was excessive order at the expense of interest. People preferred the height relationships of the two scenes with variations of up to three floor levels. 
People's on-site evaluations of urban streetscapes in Study Two confirmed these results and the laypersons' focus group revealed possible reasons for this finding. In College Street (Study 2A) people gave a moderately high preference rating (mean $=3.43$ on $5 \mathrm{pt}$ scale) to the height relationships on the north side of the street. The heights of buildings along this side of the street vary by one or two levels only. Conversely, the much more varied relationships on the southern side received a negative mean score from respondents. The north side of Wyndham Street (Study 2C) was also highly rated for the building height relationships (mean=4.21) although responses may have been influenced by several landscaped open spaces between buildings. The heights along this side of the street varied up by up to two floor levels - including the height of the cathedral tower and perceptions may also have been influenced positively by the gable roof forms of buildings W4 and W5. The reasons given by those who liked the relationships in both cases can be summarised as interesting variations of height within a narrow band of difference.

The big difference between Study One and Study Two is that in the former, people were only asked to rate two dimensional elevations. Noting that relationships between buildings actually have three dimensional characteristics, the laypersons' focus group agreed that where side walls are seen above the height of neighbouring buildings they became a factor in their evaluations. Where these walls are blank they were generally perceived negatively. Several members of the focus group noted that when side walls are particularly large they will have come about through over-development of the site, at least in relation to surrounding development patterns. As such, they were considered evidence of the developer's greed. This was seen as further evidence that the built environment conveys meanings to people, which they then filter through personal value structures.

Height was not necessarily a negative factor when people evaluated individual buildings but it became more significant in their evaluations of streetscapes. Tall buildings along a street, particularly where they occurred in groups, were perceived negatively by respondents. Jacobs (1993: 278) concluded that buildings of up to three storeys in height retain a sense of human scale. Buildings above this height begin to lose this quality. He then argued that achieving an appropriate spatial definition for a street may require taller buildings, depending on its width. Drawing on his analysis of the best streets in the world, he stated that the best proportions (vertical to horizontal) fall between 1:1.1 and 1:2.5 (: 279). The proportions (vertical to horizontal) of the north side of College Street vary 
between 1:1.1 and 1:2. The proportions of the north side of Wyndham Street vary between 1:1.25 and 1:2. Clearly these two sides of their respective streets meet Jacobs' standards for proportions.

Where height had a negative influence it was either because this led to an overbearing presence in the street, or because excessive variations in height meant large, blank side walls were easily seen.

\subsubsection{People preferred streets with building façades consistently aligned along the street edge}

This finding has an obvious link to the finding that people preferred small variations in building height, as aligned facades help also to conceal blank side walls. As streetscapes were presented as elevations, people's responses to relative façade alignment could not be evaluated in Study One.

A key factor in whether stepping between facades of adjacent buildings was seen as unpleasant was the way the ensuing space was treated. In College Street (Study 2A), respondents disliked the stepped alignment between facades of buildings when used for car parking. However, in Tyler Street (Study 2B) the change in façade alignment between buildings $\mathrm{Q}$ and $\mathrm{R}$ create a wider footpath, adjacent to a busy bus stop. This relationship was well liked. The space (figure 9.6) was well integrated with the adjoining footpath and the wider public space network and could, in associational terms, be read as a gesture of respect toward the older building $\mathrm{R}$.

Although aligned to the back of the footpath, the long and poorly detailed façade of building $\mathrm{H}$ in Kingston Street (Study 2C) was disliked (figure 9.7). This suggests that alignment and positive spatial definition were considered alongside other aspects of the façade design, such as the materials or ground floor transparency. How people respond to highly stepped and modelled facades of individual buildings was not investigated, as Study Two had no such buildings. 


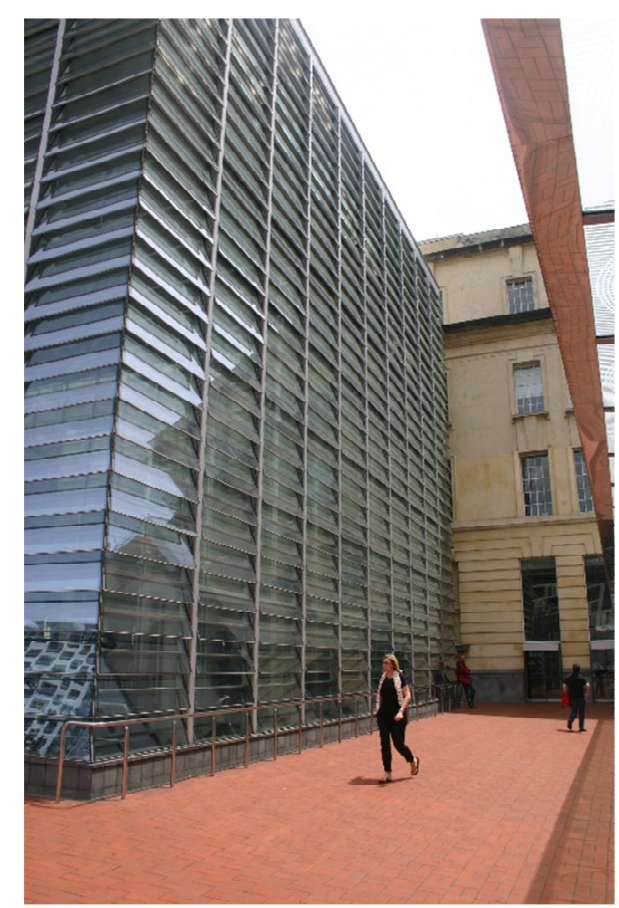

Figure 9.7: The space in front of building $Q$ (left) is developed for pedestrians and the alignment between $Q$ and $R$ was well liked.

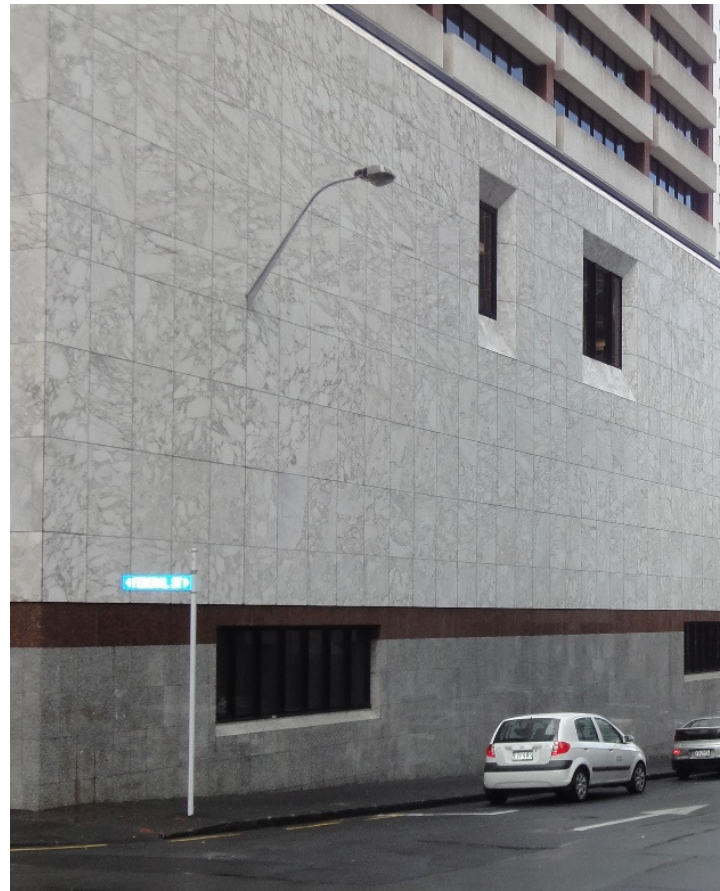

Figure 9.6: Although consistently aligned, this harsh façade was not liked by respondents.

Source: author

Source: author

\subsubsection{Open space mitigates potentially negative relationships between adjoining buildings}

Although this research has concerned itself primarily with built form characteristics that emerge through urban redevelopment, the influence that spaces around buildings also have on perceptions became evident as the data and conditions were analysed. Study One raised the question of whether street trees and flower beds had an influence on people's perceptions (see section 5.5), and Study Two confirmed the importance landscaping and positive open space plays in streetscape perceptions.

It appears that open space provides an opportunity for differences between buildings to be mitigated. This was seen most clearly in responses to the southern side of Tyler Street (Study 2B). As discussed in section 7.3.3, Takutai Square provides a spatial buffer between buildings $\mathrm{N}$ and $\mathrm{O}$, enabling these two very different buildings to coexist in a well-liked relationship. Streets running perpendicular to the street being surveyed also appear to 
create effective spatial buffers, as long as they are wide enough and the space positively designed.

The change professionals' focus group suggested that conversion of the open space on the southern side of College Street, currently used for car parking, into a landscaped space could change the way people see that side of the street. The current perceptions of it are negative. The importance of vegetation was also confirmed by the positive responses to the southern side of Tyler Street and the northern side of Wyndham Street. While the buildings were each well liked, respondents also thought that the relationships between them were enhanced by the landscaped spaces.

\subsubsection{Perceptions}

\subsubsection{Understanding building function was important in people's aesthetic evaluation of buildings and streetscapes}

The two focus group discussions suggested that people project themselves into buildings when experiencing and evaluating them. How much sun a room might receive, whether the space is used for residential or other purposes, and how private a building user might feel all influenced the aesthetic responses of many people. The designs of some buildings enabled this form of user empathy better than others. Clear, glazed openings on upper floor levels aided this process but in two circumstances, where the openings were particularly large, the buildings were liked less in part because of perceived problems of privacy and solar gain for the residents.

The meanings and values people held for building functions were also taken into account during evaluation. As articulated in the literature (Carmona, Heath et al. 2010: 117 \& 122) and during the focus group discussions, the positive evaluations of buildings with retail businesses at ground level and those with cultural significance, like the St Patrick's Cathedral (building W5 in Study 2C), confirmed the importance people place on function. Buildings with uses that appealed to older respondents, such as building $\mathrm{S}$ in Study 2A, were more likely to be rated higher by that age group. The ground level of a building with an activity appealing to a younger demographic group, such as building D in Study 2C, was only liked by that that group. Women rated buildings with ground floor retail businesses catering for a female clientele higher than men. There were several examples 
of this in Study One and Studies 2A and 2B. These results confirm that people responded to function as well as the physical fabric of buildings when making aesthetic judgments.

\subsubsection{Change professionals' opinions were influenced by their knowledge of urban change}

Differences between the ways architects and lay people each evaluate the built environment have been acknowledged (Case Scheer 1994: 4, Stamps III 2000: 120). This research suggests one reason for these differences is the change professionals' detailed knowledge of how places change. This was raised during the professionals' focus group discussion and provided a way of interpreting differences in the survey responses.

Change professionals' understanding of change produced a more forgiving attitude to the streetscapes under review. Firstly, they understood the challenges facing development, and developers in particular. They seemed willing to overlook difficult relationships between adjoining buildings and the broader effects of incompatible development on the streetscape, because they felt that concessions needed to be made in order to stimulate the development process. They reasoned that some form of development, even if not ideally suited to the setting, was better than no development.

The second reason for their willingness to be more forgiving of poor streetscape outcomes from incompatible development was linked to their understanding of the change processes. They were more tolerant of poor streetscape relationships because they perceived these as temporary circumstances that could be corrected with future change. Architects and planners thus incorporated their understanding that urban environments are in a continual states of flux (Greenberg 2011: 79) into their aesthetic judgements about streetscapes.

\subsubsection{Demographic group comparisons}

Comparison of the aesthetic perceptions of lay people with those of change professionals is particularly useful because of the role change professionals play in design and design control processes. This raises the question of whether their views align with those of the wider public they are meant to be serving. 


\subsubsection{On balance, lay people and change professionals had similar preferences for buildings and streetscapes}

In general it seems that change professionals and lay people made similar evaluations of all the buildings and streetscapes. Perhaps it would be more accurate to say 'there were few differences in the preferences held for buildings and streetscapes by change professionals and lay people'. The finding emerged because the majority of opinions of these two demographic groups aligned and where they differed, the differences were small.

Both groups generally disliked streetscapes that exhibited large steps in height between buildings, with change professionals disliking this characteristic more than their lay counterparts. While Study 2A suggested that change professionals were more tolerant of this condition, the other parts of the fieldwork suggested the opposite, as lay people were less negative about large differences in height. The two groups also agreed about recently completed buildings in each of the study areas. Buildings such as D and S in Study 2A, $\mathrm{N}$ and Q in Study 2B and F in Study 2C were consistently liked or disliked by both groups. A near perfect alignment of mean preference scores in relation to building F in Study 2C revealed that lay people and professionals both found the building to be unpleasant. The two groups may have focussed on different aspects of the building when evaluating it, professionals on the composition above the street and lay people on the ground floor, but they arrived at the same conclusion.

In another example, change professionals and lay people both found great pleasure in building $\mathrm{N}$ of Study $2 \mathrm{~B}$. Although this large building dwarfs many of those around it, it was noted in section 9.2.1.6 that people tend to evaluate buildings in isolation and so the scale mismatch would not have been a strong factor. In addition, the building is adjacent to a large public space, which helps ameliorate differences between buildings, as explained in section 9.2.2.4. Indeed, the responses of lay people and change professionals suggest they were equally influenced by such open spaces. Finally, it seems they agreed on liking buildings and streetscapes displaying discrete window openings, and those that were based on traditional base/middle/top façade compositions.

While the overall finding is one of agreement there were some differences. The influence that knowledge of urban transformation processes had on change professionals' preferences was discussed in section 9.2.3.2. Another subtle difference was that change 
professionals consistently referenced the design of the building above ground level whereas lay people's opinions of a building overall were linked more closely to their perception of the street level design. Correlation analyses revealed the strength of these connections.

Study 2C suggested that lay people's evaluations were influenced by a building's state of repair. This is not to suggest that change professionals were disinterested in this aspect and the professional's focus group certainly highlighted maintenance as an important streetscape consideration. However, it seems lay people placed greater weight on poor maintenance when evaluating buildings $\mathrm{C}$ and W6. These buildings had characteristics that lay people consistently found attractive elsewhere, such as glazed shopfronts and traditional façade compositions. However, both buildings were in need of façade painting, which may also be why lay people rated them lower than change professionals did.

Change professionals generally tended to be more critical of a building or streetscape. While mean preference scores given by both groups generally fell on the same side of the neutral line, change professionals tended to rate a building or relationship lower than their lay counterparts. Change professionals were thus less positive about buildings and streetscapes that both groups liked and more negative about those that were disliked. This agrees with findings of an earlier study, which concluded that change professionals express their opinions about streetscapes more confidently (Gjerde 2011).

Studies $2 \mathrm{~A}$ and $2 \mathrm{~B}$ suggested that change professionals held stronger preferences for older, characterful buildings, particularly those that had been adaptively reused, such as the converted former warehouse buildings ( $G$ to $L$ ) in Study 2B. They were less tolerant of street level designs that made provision for cars, unlike lay people, who consistently rated the ground levels of such buildings higher than change professionals. The latter were also more critical of older, utilitarian buildings that were not people centred and that they considered to detract from the potential vitality of the street. Taken together, the clear preferences expressed for converted older buildings and dislike for utilitarian buildings that reflect the original and underlying character of the area suggest that change professionals live in anticipation of the area transforming into one that is more people centred. This would also support the finding that change professionals use their understanding of transformation processes when they evaluate urban streetscapes. 


\subsubsection{Areas where architects and planning professionals disagreed when evaluating buildings and streetscapes}

The aesthetic preferences of planners and architects were not fundamentally different. In many cases where the mean preference scores generated by each group were different, this could be considered a difference of intensity of opinion rather than a difference of absolute opinion. In those cases, one professional group liked or disliked a building or streetscape more than the members of the allied profession did. A few relevant differences of opinion are discussed below.

Study One discovered that architect respondents found streetscapes with greater variations of building height to be more attractive than their planning colleagues and correspondingly, found those that were more uniform to be less pleasant. However, when people responded to real life streetscapes these differences were not confirmed. Indeed, architects and planners held largely similar views. Both groups liked height relationships moderated by the open spaces between buildings in Studies 2B and 2C and both groups disliked the height relationships along the southern side of Study 2A. They found the height relationships along both sides of Kingston Street unpleasant, and in this case planners were more pronounced in their views.

As noted above, change professionals were generally more positive about older character buildings, particularly those that had been adaptively reused. The single exception was building L in Study 2B. This former warehouse building was disliked by architects and liked by planners (as well as lay people). Although architects and planners liked the relationships the building formed with its neighbours, they held very different views on the façade design, street level design and the materials. Comprehensively, the architects disliked the building in contrast to planners. One possible reason for this may have been that the building facades had not been renewed like others in the street and, because the ground floor was set well above footpath level, there was no easy transition from outside to inside. This one building was an anomaly in an otherwise consistent alignment of planners' and architects' opinions about older buildings.

The two professional groups held very different opinions about large scale redevelopment. Large buildings generally happen through amalgamation of smaller sites, leading to a different urban 'grain', or rhythm of frontage widths along a street. Planners held positive opinions about these buildings whereas architects disliked them. These 
results were consistent for Study One and Studies 2A and 2C. The designers may have perceived the façade consistency of a single development to be less interesting than the surrounding pattern. This corresponds with the earlier findings of this research, which suggest that architects tolerate and prefer higher levels of visual stimulation in urban streetscapes than other groups. In addition, the architects may be more familiar with the writings of Jacobs (1961) and Bentley (1999: 77-78), both of whom expressed disdain for the coarse grain and lack of visual interest that comes with large scale redevelopment projects.

\subsubsection{Gender was not a factor in the way people evaluated buildings and urban streetscapes.}

Men's and women's preference scores varied within a small range across Studies One and Two and were considered generally similar. Although the results of Study 2A suggested that women were more positive in their aesthetic judgements than men, this was not upheld in the other parts of Study Two. Study One found that the perceptions of men and women were closely aligned.

Although peripheral to the finding of general alignment, several nuanced differences in the way each gender perceived the buildings and streetscapes were noted. There was a tendency for women to prefer consistency across streetscapes more than men. This was observed in relation to building heights and colour. Women were also more critical of buildings that were in a poor state of repair. Although there were few of these in the whole of Study Two, the mean preference scores of women were consistently lower for these. Women's feelings about poor maintenance were confirmed by the reasons they gave in support of their opinions.

Women's opinions appeared to have been influenced by the way the ground level of buildings were being used. In Studies 2A and 2B it emerged that positive opinions women held for the ground level activities translated into positive feelings about the building. This was confirmed by the two focus group meetings. The uses that influenced women's positive opinions were fashion retailing and the high-end grocery business (building $\mathrm{S}$ ) in Study 2 A. 


\subsubsection{Comparison of presentation stimuli}

The research also provided the opportunity to compare responses to photographic representations of urban streetscapes with those made by people in the street. Although of secondary importance, as this does not directly address either of the research questions, it is relevant to the study in two ways. Firstly, several earlier studies referenced in this thesis have made use of photographic stimuli. These have relied on other research suggesting that responses to photographic and in-situ stimuli correlate, but not directly. While relative ranking of preferences can be compared for these two methods, the scores cannot be interchanged, even if used to study responses to the same buildings. Secondly, as development proposals are presented for design review as representations, it would be useful to understand how well responses to representations correlate with real life experience of buildings and streetscapes.

Table 9.3: Mean preference scores for the relationships between building heights in Studies One and Two.

\begin{tabular}{|c|c|c|c|c|c|}
\hline & & \multicolumn{4}{|c|}{ Building height variations along streetscape } \\
\hline & & Consistent & Slight & Moderate & Large \\
\hline \multirow{6}{*}{ 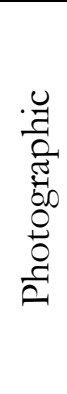 } & Scene 1 & & & & 2.82 \\
\hline & Scene 2 & & 3.34 & & \\
\hline & Scene 3 & & & 3.55 & \\
\hline & Scene 4 & 3.47 & & & \\
\hline & Scene 5 & & & & 2.88 \\
\hline & Scene 6 & 3.49 & & & \\
\hline \multirow{8}{*}{ 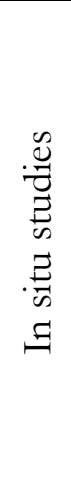 } & College St South & & & & 2.81 \\
\hline & College St North & & & 3.43 & \\
\hline & Tyler St South & & & & 3.83 \\
\hline & Tyler St North & & & & 2.83 \\
\hline & Kingston St South & & & & 2.44 \\
\hline & Kingston St North & & & 3.07 & \\
\hline & Wyndham St South & & & & 3.00 \\
\hline & Wyndham St North & & 4.21 & & \\
\hline
\end{tabular}

This research found that evaluations of building height relationships in a streetscape presented photographically corresponded only moderately with evaluations made on site. 
To arrive at this finding, the height relationships of each streetscape in the two studies was categorised as having absolute consistency or exhibiting slight/moderate/large variations (table 9.3). As Study Two did not contain any streets of absolute consistency, comparisons with scenes 4 and 6 of Study One were not possible. When comparing responses to scenes of moderate building height variations, preferences expressed in Study One aligned with those expressed for College Street North in Study Two. There was also alignment observed between the responses to scenes 1 and 5, exhibiting large variations in building height, with responses to all but one of the Study Two cases with similar characteristics. Where opinions about large height variations did not align, as in the case of Tyler Street South, the differences could be attributed to the generally positive influences landscaped open spaces have on perceptions, as discussed in section 9.2.2.4.

As the two studies were not set up to directly compare responses to photographic representations and personal experience of the same street, these results must be treated as indicative rather the conclusive. Opportunities to examine relationships between these two and other aesthetic response stimuli are discussed in chapter 10 as future research.

\subsection{Summary}

This chapter presents the overall research findings by comparing the results of Studies One and Two. Themes that could help explain the way people visually evaluated the buildings and streetscapes in the two studies were developed. The themes were based on patterns of perception observed across two or more of the individual case studies and are summarised in table 9.2 .

This research into streetscape preferences found that people like buildings with discrete window openings and correspondingly, disliked buildings with facades which are horizontally banded (such as continuous strip windows in commercial building facades). People also liked traditional materials and the most preferred of these was brick. Of the 12 best liked buildings in the three parts of Study Two, 11 had brick facades. Maintenance was a factor in perceptions; people responded favourably to buildings that were well maintained and found those not maintained to be unpleasant. Respondents were also interested in the activities that took place within buildings at street level. Those that enabled visual links to positive activities inside were rated highest. From these findings it can be inferred that people liked urban buildings that 
- Were understandable in terms of function

- Adopted traditional compositional patterns

- Stimulated visual interest and positive associational meanings

- Appeared to be cared for

At the street scale, people preferred height relationships between buildings to vary within a narrow band of difference. This finding was corroborated by the consistent dislike of streetscapes with large height variations. People also liked building façades that are continuous along the street edge. A response underpinning both these findings was the dislike of flank walls on internal site boundaries, which are often left blank and uninteresting. The streetscapes that exhibit coordinated visual interest across the individual facades were well liked, as were those that incorporate landscaped open spaces along their length. Open space designed for use by people was seen as an effective means to help offset negative feelings that could arise from poor relationships between buildings. 


\section{Conclusions}

The central aim of this research has been to identify the building and urban streetscape design characteristics that people like, as well as the converse. The premise for the research, set out in chapters $1-3$, is that the ways in which places change over time lead to streetscapes that have a wide range of design characteristics and that people's perceptions of them also vary widely. The visual characteristics of individual buildings also vary and these are often perceived poorly (Bentley 1999: 205). This research has addressed the problem that individual urban buildings and streetscapes are of inconsistent quality and that little is known about which design characteristics people find appealing. Moreover, as urban change is initiated and managed by experts in the field, it is unclear whether their aesthetic preferences for buildings and streetscapes are consistent with those of lay people. Understanding the aesthetic preferences of these two groups, lay people and change experts, is necessary in order to manage the way design and design control decisions are made. The following sections summarise what has been learned, how this might affect the theories and practices of environmental aesthetics and design control, and suggest areas for future research.

\subsection{Contribution to knowledge}

The research findings have been discussed in chapter 9 and the most salient of these are outlined below (section 10.2) in relation to the specific research questions they answer. The research has created new knowledge about people's preferences for building design 
and urban streetscape characteristics in the cities of Wellington and Auckland. Design characteristics that are not well liked have also been identified. More is known about the differences and similarities of people's perception of the built environment according to their gender, ages and occupational backgrounds. In particular, the aesthetic preferences of people trained in planning and architectural design have been compared with those of the general public. Understanding where preferences overlap and where they differ has been made possible through this research. Knowledge about these matters has direct relevance for the processes of design and urban change management.

By comparing the findings of the two studies, more is now known about the correlation between aesthetic responses to photographic media and the real life experience of streetscapes. This is relevant because the findings of earlier research on this relationship have not been consistent, and two dimensional representation of design proposals are generally used to communicate the characteristics of new development proposals.

The research has also developed a new method for understanding people's perceptions of buildings and urban streetscapes in New Zealand. By inviting people to walk along the streets of interest and record their feelings this ensures that people's experiences of urban space inform their opinions. This is different from other methods that use photographic or three dimensional representation techniques that require the respondents to project themselves into an imagined space. Although the method required considerable time commitment from respondents, it proved effective in generating sufficient responses to facilitate the analysis.

\subsubsection{Dissemination of knowledge}

It has been noted that the findings of this research have relevance to architectural design and urban change management processes. As such, it would seem useful to ensure that the people who make decisions affecting the form and appearance of urban built environments have direct access to the results. It is therefore proposed to disseminate the findings beyond simply lodging the thesis with the Victoria University of Wellington Library.

The research outcomes will be interpreted for professional and academic audiences through papers prepared for presentation at academic conferences and in academic journals published in the fields of urban and architectural design, town planning and 
resource management as well as environmental psychology and aesthetics. The papers that flow out of the research will convey information on the methods that were developed to gather information on people's aesthetic preferences, what those preferences are as well as to discuss the findings in the context of other design and environmental psychology matters that were not specifically addressed in the project. In this way the research can be seen as a catalyst for further examination of environmental aesthetics and design control in other contexts.

The findings will also be conveyed through lectures and other forms of teaching, not only to students of architecture, landscape architecture and urban design but also in professional settings. As the research process has been aided by the author's contacts with the change professions it is anticipated that those same connections will facilitate dissemination of the results.

\subsection{Answers to the research questions}

\subsubsection{Question 1: what are the characteristics of well-liked buildings and streetscapes in New Zealand?}

The primary issue driving the research has been to understand people's preferences for buildings and urban streetscape design characteristics. In respect of individual buildings, the research found that the façade design features people most like are discrete window openings. Correspondingly they dislike buildings with facades that are horizontally banded (such as continuous strip windows). People also preferred facades that are monolithic in nature, where the structural floor levels behind the façade are not expressed in it. Such monolithic facades are generally considered more traditional in composition, with the façade conceptually seen as a wall extending over the height of the building. This finding corresponds well with preferences for discrete windows and a dislike for horizontally banded facades. While the majority of traditional, monolithic facades incorporate surfaces that can be painted, the most preferred surface finish appears to be brick, which is problematic given the earthquake requirements of New Zealand.

People expressed strong preferences for buildings that were well maintained and found those that were not to be unpleasant. Although not specifically a design characteristic, maintenance and the extent to which it can be perceived can be influenced through design. 
The surface qualities of cladding materials, their durability and the way different components of a construction are detailed all influence how a building ages.

Buildings that enable visual links to interior spaces, particularly at ground level, are also liked, as the extent to which buildings were liked was also influenced by the activities people understood to take place within them. An example of a well-liked activity at ground level is a bar or café. Positive perceptions of such activities are enhanced when people can see into the interiors. While in many cases the two factors of well-liked activities and a visually permeable façade treatment worked well together to enhance people's perceptions, they were not mutually dependent. An example of an activity women like is retail but it was not necessary to see the activities taking place inside for the host building to be liked. Signage and building typology also appeared suitable signifiers of the retail activities, which led to the building being liked.

At a broader scale, building height relationships that vary within a narrow band of difference is a well-liked streetscape characteristic. Correspondingly, large variations between buildings are generally disliked. These two answers to the research question support each other. The characteristic that underlies this preference is ordered variety, which is known to elicit a positive aesthetic response.

Continuity of building façades along the street edge is another well-liked streetscape characteristic. However, it appears that spatial definition, in the sense advocated by Sitte (1979) and Jacobs (1993), is not the characteristic people find most appealing. Preferences for façade continuity are linked to a dislike of blank and uninteresting flank walls on internal site boundaries. They are also linked to the notion of positive space, where the space between the building facades and the footpath is developed positively for use by pedestrians.

Another well liked streetscape characteristic is coordinated visual interest across the individual facades. Once again, this feature corresponds to the broadly held aesthetic preferences for ordered variety. Finally, green landscaping and positive open spaces in a street are very well liked streetscape characteristics. These also mitigate the negative visual effects that might arise from other poor relationships between buildings. An example of this is in relation to large differences in building height. Where these are mediated by open 
space, even an intersecting road, the otherwise negative visual effects are reduced and may even become positive.

\subsubsection{Question 2: how do the aesthetic preferences of professionally trained architects and planners compare with those of lay members of the public?}

The second research question links to urban transformation and change processes, including design control. Most decisions about the shape of buildings and streets are made by professionals, charged with this responsibility because of their expertise. This question considers whether change professionals also speak for the wider public when they make decisions that affect the aesthetic characteristics of the built environment. This research found that the preferences of these two groups are largely aligned, but with some differences.

There is a consistent tendency for change professionals to be more critical in their opinions about buildings and streetscapes. Although the preferences expressed by both groups were similar, the opinions of change professionals were less positive when both groups liked a building or streetscape and more negative when they did not. This may reflect a cultural characteristic of change professionals.

While overall preferences between these groups align, there are differences in the way opinions are formed. Firstly, change professionals tend to focus on the design of buildings above ground floor level when making their overall evaluation of a building, whereas lay people tend to emphasise the ground level design. This also reflects closer attention on the activities taking place within a building by lay people; where activities are perceived positively, the building is more likely to be rated positively.

Secondly, change professionals include consideration of how the built environment changes when forming their opinions about buildings and streetscapes. In particular, they appear to be willing to overlook poor design characteristics if they believe these will be short-lived. It seems that lay people evaluate streetscapes in the present whereas change professionals consider future possibilities when making their evaluations.

\subsubsection{Question 3: what are the differences between preferences expressed in relation to photographic representation and real life experience}

The design of the two studies, focussed on questions of aesthetic preference, did not allow for direct comparisons between photographic representation and real life experience of 
the same streets. This third question was somewhat peripheral to the main aims of the research. Nevertheless, responses to relationships of building height appear to have a moderate correlation between these two representation methods. This is useful knowledge, as it is increasingly common for representations of new development proposals to include photographic montages to show the project's visual impact.

\subsection{Contribution to theory}

The questionnaires were designed and the individual scenes and streets of each study selected with background knowledge of how people perceive the urban environment. This knowledge was gained through examination of literature in the field of environmental aesthetics and reference to the key theories of perception. The aim here is to see how the findings of this research align with these theories. One of the clearest findings is that people like ordered variety in a building or streetscape. This can be seen in the preferences expressed for discrete windows in a façade or across a streetscape. Smith (2003) discussed the rhythmic patterns that windows can create and noted how such patterns appeal to an innate desire to order and group elements. The patterns of windows across several buildings in a streetscape can help link these together, enhancing visual coherence. Such patterns also increase levels of interest in a scene. These findings, read in conjunction with the negative responses to streetscapes where buildings vary in height considerably, also confirm Berlyne's (1974) theory of hedonic pleasure (see section 3.3). Patterns of stimuli that lead to moderate levels of arousal are considered pleasurable whereas those that foster high arousal levels are unpleasant.

The findings confirm the important role that associational meanings play in people's perceptions of the environment. As noted by Rapoport (1982), Lang (1988) and Porteous (1996), aesthetic perception involves visual information being processed in the mind of the viewer in relation to the meanings and values he or she associates with it. Such a schema of meanings is developed over the course of people's lives, through personal experiences and in relation to cultural and social norms (see section 3.8). The findings, which indicate that activities taking place within a building and the level of care and maintenance are important to the way people evaluate a building, confirm this aspect of environmental aesthetics theory. 
There is a widely held view that experts trained in architecture and planning perceive the built environment differently from those who are not trained. However, the literature also presents evidence to support a contrasting view (see section 3.10). The findings from this research suggest there are considerable areas of overlap between the preferences held by lay people and change professionals but that the two groups arrive at their evaluations by prioritising different aspects of the design. Change professionals, and particularly architects, also appear to take account of anticipated future changes in their experience of urban streetscapes.

\subsection{Limitations of the research}

The research has been designed to understand the ways that people feel about certain urban buildings and streetscapes in Wellington and Auckland and it would be useful to be able to generalise the findings for the whole of New Zealand. However, there are a number of factors that limit the extent to which this can be done. Firstly, while the research was conducted in two of the country's largest cities, the wider population is diverse and many cultural characteristics are place specific. The respondent groups were not representative of the diversity of the general population, as they have a bias toward people with higher education levels, there were low numbers of older people and an imbalance in the representation of occupational classifications.

Secondly, the number of people responding to the surveys was limited. Study One attracted 192 responses and each of the cases in Study Two attracted a minimum of 40 responses. While these numbers enabled statistical analysis and clear patterns to emerge, they were too small to allow scaling up to the wider population. These factors may have limited the robustness of the findings in terms of fitting with the wider population.

The cases in Study Two were real and therefore naturally complex settings. Case selection and survey administration took steps to limit the influence of as many variables as possible. Examples include selection to avoid differences in weather conditions, vehicular traffic and orientation to the sun. Nevertheless, it is possible that other unforeseen variables between the case study sites may have affected the results.

The number of building design and streetscape characteristics were also limited in Study Two, in part through the objective of using real streets and in part by the need to select 
the streets to limit the influence of other variables. The opportunity to reference streets in other cities and to avoid having to consider environmental influences in Study One enabled a much broader range of streetscape characteristics to be presented.

\subsection{Recommendations for further research}

The research returned findings that have contributed to knowledge in the field and that have stimulated consideration of other research to complement and extend the work. One possible area for this was anticipated in the design of the current project. Selection of the three streets in Study Two will enable relationships between design control methods and building design preferences to be studied. Transformations in each of these streets have, for at least the past 20 years, been subjected to different design control methods. Further research could interrogate the effectiveness of these methods in achieving well liked buildings and avoiding poor outcomes. This work would need to engage with the processes followed to gain planning permission.

As the ability to compare responses to photographic representation and real life experiences of streets was limited in this research, further work could be done to illuminate comparisons. The present project has generated a good understanding of how three real streets are perceived and these same streets could be presented photographically to other respondents for preference ratings to be compared.

Another opportunity for further research is to examine the liked and disliked design characteristics in more detail through the use of hypothetical streetscapes. The methods developed for Study One lend themselves to being used in this way, to portray particular inter-building and streetscape relationships. Experimental approaches can be used to isolate design variables in order to examine aesthetic responses to them more closely. As other researchers have previously done (see for example Groat 1988, Stamps III 2000), hypothetical streetscapes could also be used to examine preferences for particular infill design approaches. Such experimental work would build on the knowledge developed in the present research and could help refine guidelines for design.

Finally, while the method of obtaining people's perceptions by way of the survey instrument was successful, further research could be undertaken to develop other methods. Collecting people's responses to experiences of buildings and streetscapes 
requires a considerable commitment of time and so may limit the number and range of willing participants. Rather than photographic representation, digital technologies could enable participants to walk along virtual streets and this opens up another area for research in streetscape preferences. 


\section{Bibliography}

Alcock, A. (1993). Aesthetics and urban design. In S. McGlynn \& R. Hayward (Eds.), Making better places: urban design now (pp. 42-52). Oxford: Butterworth Architecture

Alexander, C., Neis, H., Anninou, A., \& King, I. (1987). A new theory of urban design. New York: Oxford University Press.

Allmendinger, P. (2009). Planning Theory. Basingstoke: Palgrave Macmillan.

Altman, I., \& Christensen, K. (Eds.). (1990). Environment \& Behavior Studies: Emergence of Intellectual Traditions (Vol. 11). New York: Plenum Press.

Ashworth, W. S. (1954). The genesis of modern British town planning :a study in economic and social bistory of the nineteenth and twentieth centuries. London :: Routledge \& Kegan Paul.

Ataov, A. (1998). Environmental aesthetics. Journal of Planning Literature, 13, 239-257.

Attoe, W. (1981). Skylines: understanding and molding urban silhouettes. Devon, UK: J Wiley \& sons Ltd.

Auckland City Council. (2004). Auckland Council District Plan: Auckland City - Central Area Section. Auckland: Auckland Council.

Auckland City Council. (2006). Britomart Precinct Urban Design Guidelines. Auckland: Auckland City Council.

Auckland Council. (2012). Terms of reference: Auckland urban design panel. Auckland: Auckland Council.

Auckland Council. (2014). Part 14.6 Britomart precinct. Auckland: Auckland Council 
Baab, D. J. (1994). Private design review in edge city. In B. C. Scheer \& W. F. E. Preiser (Eds.), Design review: challenging urban aesthetic control (pp. 187-196). New York: Chapman \& Hall

Baker, D. C., Sipe, N. G., \& Gleeson, B. J. (2006). Performance-based planning: Perspectives from United States, Australia and New Zealand. Journal of Planning Education and Research, 25, 396-409.

Barnacle, R. (2001). Phenomenology and wonder. In R. Barnacle (Ed.), Phenomenology [online] (pp. 3-19). Melbourne: RMIT University Press

Barnett, J. (1974). Urban design as public policy. New York: Architectural Record Books.

Barry Rae Transurban Ltd. (2004). Review of district plan design guides: Wellington City Council (pp. 64). Wellington: Wellington City Council.

Beatley, T. (1989). Environmental ethics and planning theory. Journal of Planning Literature, 4(1), 1-32.

Bechtel, R. B., Marcus, R. W., \& Michelson, W. (Eds.). (1987). Methods in environmental and behavioral research. New York: Van Nostrand Reinhold Company.

Beer, A. R. (1983). Development Control and Design Quality: Part 2: Attitudes to Design. The Town Planning Review, 54(4), 383-404. doi: 10.2307/40113547

Beer, A. R. (1991). Urban design: The growing influence of environmental psychology. Journal of Environmental Psychology, 11(3), 359-371.

Bentley, I. (1999). Urban transformations: power, people and urban design. London: Routledge.

Bentley, I., Alcock, A., McGlynn, S., Murrain, P., \& Smith, G. (1985). Responsive environments: a manual for designers. Oxford, UK: Architectural Press.

Berlyne, D. E. (1974). The new experimental aesthetics. In D. E. Berlyne (Ed.), Studies in the new experimental aesthetics: steps toward an objective psychology of aesthetic appreciation (pp. 1-26). Washington D.C.: Hemisphere Publishing Corporation

Bourassa, S. C. (1991). The aesthetics of landscape. London: Belhaven Press.

Boyer, M. C. (1983). Dreaming the rational city. Cambridge, MA: The MIT Press.

Brolin, B. C. (1980). Architecture in context: fitting new buildings with old. New York, NY: Van Nostrand Reinhold Company.

Bryman, A. (2008). Social research methods (3rd ed). Oxford, UK: Oxford University Press.

Bryman, A., \& Cramer, D. (2011). Quantitative data analysis with IBM SPSS 17,18 and 19: A guide for social scientists. East Sussex, UK: Routledge.

Buchanan, P. (1988). Facing up to facades. Architects' Journal, 188(51), 22-56.

Burns, A. (1997). Private buildings public space: an aesthetic exploration. (unpublished Master's thesis), Oxford Brookes University, Oxford.

CABE, \& DETR. (2000). By design: Urban design in the planning system: towards better practice. HMSO.

CABE. (2006). Design review: How CABE evaluates quality in architecture and urban design. In CABE (Ed.), (pp. 25). London: Commission for Architecture and the Built Environment,. 
Carmona, M. (1996a). Controlling urban design - Part 1: a possible renaissance? . Journal of Urban Design, 1(1), 47 - 73.

Carmona, M. (1996b). Controlling urban design - part 2: realising the potential. Journal of Urban Design, 1(2), 179 - 201.

Carmona, M. (1998). Design control- bridging the professional divide, part 1:a new framework. Journal of Urban Design, 3(2), p175-200.

Carmona, M. (1998). Design control- bridging the professional divide, part 2: a new consensus. Journal of Urban Design, 3(3), 331 - 358.

Carmona, M. (2003). An international perspective on measuring quality in planning. Built Environment, 29(4), 281-287.

Carmona, M., Heath, T., Oc, T., \& Tiesdell, S. (2010). Public places urban spaces. Oxford, UK: Architectural Press / Elsevier.

Carter, H. (1995). The study of urban geography. London: Hodder Headline Group.

Case Scheer, B. (1994). Introduction: The debate on design review. In B. C. Scheer \& W. F. E. Preiser (Eds.), Design review: challenging urban aesthetic control (pp. 1-10). New York: Chapman \& Hall

Case Scheer, B., \& Preiser, W. F. E. (1994b). Introduction. Environment and Behavior, 26(3), 307-311.

Case Scheer, B., \& Preiser, W. F. E. (Eds.). (1994a). Design review: challenging urban aesthetic control. New York: Chapman \& Hall.

Cherry, G. E. (1974). The evolution of British town planning. New York: Halstead Press.

Cherry, G. E. (1982). The politics of town planning. New York: Longman Inc.

Cherry, G. E. (1988). Cities and plans :the shaping of urban Britain in the nineteenth and twentieth centuries / Gordon E. Cherry. London ;New York :: E. Arnold.

Childs, M. C. (2009). Civic concinnity. Journal of Urban Design, 14(2), 131-145.

Ching, F. D. K. (2007). Architecture - form, space and order - 3rd ed. Hoboken, NJ: J. Wiley \& Sons, Inc.

Cold, B. (Ed.). (2001). Aesthetics, well being and health. Aldershot: Ashgate Publishing Limited.

Cook, R. S. (1980). Zoning for downtown urban design. Lexington, MA: Lexington Books.

Cooper, J., \& Oskrochi, R. (2008). Fractal analysis of street vistas: a potential tool for assessing levels of visual variety in everyday street scenes. Environment and Planning B: Planning and Design, 35, p 349-363.

Cullen, G. (1971). The Concise Townscape (Second edition, first published 1961 ed.). London: The Architectural Press.

Cullingworth, B. (1997). Planning in the USA. London: Routledge.

Cullingworth, J. B. (1979). Town and country planning in Britain. London: George Allen \& Unwin Ltd.

Cullingworth, J. B. (1991). Aesthetics in US planning: From billboards to design controls. Town Planning Review, 62(4), 399-413. 
Cullingworth, J. B. (1993). The political culture of planning. New York: Routledge Inc.

Cuthbert, A. R. (2006). The form of cities, political economy and urban design. Oxford, UK: Blackwell Publishing.

Danaci, H. M. (2012). Architectural education and environmental aesthetics. Procedia Social \& Behavioral Sciences, 51(2012), 878-882.

Dawson, E., \& Higgins, M. (2009). How planning authorities can improve quality through the design review process: Lessons from Edinburgh. Journal of Urban Design, 14(1), 101-114.

Dawson, L. (2010). Architecture in the land of the long white cloud suffers from suburban creep and a lack of identity. The Architectural Review, 227(1357), 33.

Delafons, J. (1969). Land-use controls in the United States (2nd ed.). Cambridge, MA: The MIT Press.

Delafons, J. (1990). Aesthetic control: A report on the methods used in the USA to control the design of buildings. Berkeley, CA: University of California.

Delafons, J. (1994). Democracy and design. In B. C. Scheer \& W. F. E. Preiser (Eds.), Design review: Challenging urban aesthetic control (pp. 13-19). New York: Chapman \& Hall

Devlin, K. (1990). An examination of architectural interpretation: architects versus nonarchitects. The Journal of Architectural and Planning Research, 7(3), 235-244.

Dixon, J. (2003). Planning in New Zealand: Legacy of ambivalence and prospects for repositioning. Planning Theory \& Practice, 4(3), 348-353.

Dovey, K. (1991). Framing places: mediating power in built form. London: Routledge.

Dovey, K. (2001). The aesthetics of place. In B. Cold (Ed.), Aesthetics, well-being and health (pp. p. 93-101). Aldershot, Hants: Ashgate Publishing Ltd.

Edwards, A. T. (1944). Good and bad manners in architecture (2nd ed.). London: John Tiranti Ltd.

Ellin, N. (2006). Integral urbanism. New York: Routledge.

Fisher, P. (2008). Is There an Emerging Age Gap in US Politics? Society, 45(6), 504. doi: 10.1007/s12115-008-9152-y

Fishman, R. (2000). The American planning tradition: An introduction and interpretation. In R. Fishman (Ed.), The American planning tradition (pp. 1-32). Washington DC: The Woodrow Wilson Center Press

Forty, A. (2012). Concrete and culture : a material history. London :: Reaktion.

Fox, W. (2000). Towards an ethics (or at least a value theory) of the built environment. In W. Fox (Ed.), Ethics and the built environment (pp. 207-221). Abingdon: Routledge

Fox, W. (2006). A theory of general ethics: Human relationships, nature and the built environment. Cambridge MA: The MIT Press.

Fox, W. (2011). Foundations of a general ethics: Selves, sentient beings, and other responsively cohesive structures. Royal Institute of Philosophy Supplement, 69(-), 4766. 
Fox, W. (Ed.). (2000). Ethics and the built environment. Abingdon: Routledge.

Gehl, J. (2011). Life between buildings: Using public space (J. Koch, Trans.). Washington DC: Island Press.

George, R. V., \& Campbell, M. C. (2000). Balancing different interests in aesthetic controls. Journal of Planning Education \& Research, 20(-), 163-175.

Gjerde, M. (2011). Visual evaluation of urban streetscapes: How do public preferences reconcile with those held by experts. Urban Design International, 16(3), 153-161.

Groat, L. (1988). Contextual compatibility in architecture: an issue of personal taste? In J. L. Nasar (Ed.), Environmental aesthetics: theory, research and applications (pp. p.228-257). Cambridge: Cambridge University Press

Groat, L. (1994). Carbuncles, columns and pyramids: lay and expert evaluations of contextual design strategies. In B. C. Scheer \& W. F. E. Preiser (Eds.), Design review: challenging urban aesthetic control (pp. 156 - 164). New York: Chapman \& Hall

Groat, L., \& Wang, D. (2013). Architectural Research Methods - 2nd Ed (2nd ed.). Hoboken, N.J.: J Wiley \& Sons, Inc.

Gunder, M. (2000). Urban policy formation under efficiency: The case of the Auckland City Council's Britomart project. In P. A. Memon \& H. Perkins (Eds.), Environmental planning \& management in New Zealand (pp. 394). Palmerston North: Dunmore Press

Habe, R. (1989). Public design control in American communities. The Town Planning Review, 60(2), 195-219.

Habraken, N. J. (1994). Cultivating the field: about an attitude when making architecture. Places, 9(1), 8-21.

Habraken, N. J. (1998). Structure of the ordinary. Cambridge, MA: The MIT Press.

Hall, A. C. (1996). Design control. Oxford, UK: Butterworth Architecture.

Hall, T. (1990). Design control: a call for a new approach. The Planner, 76(39), 14-18.

Hamer, D. (1995). The making of urban New Zealand. Journal of Urban History, 22(1), 639.

Hammersley, M. (Ed.). (1993 - reprinted 1999). Social research: philosophy, politics and practice. London: Sage Publications Ltd.

Hanlon, D. L. (2009). Compositions in architecture /Don Hanlon. Hoboken :: John Wiley \& Sons.

Hansen, G. (2014). Design for Healthy Communities: The Potential of Form-Based Codes to Create Walkable Urban Streets. Journal of Urban Design, 19(2), 151-170. doi: 10.1080/13574809.2013.870466

Harrison, J., \& Ryan, J. (2010). Musical taste and ageing. Ageing \& Society, 30(4), 649-669.

Heath, T. F. (1988). Behavioural and perceptual aspects of the aesthetics of urban environments. In J. Naser (Ed.), Environmental aesthetics: theory, research and applications (pp. p.6-10). Cambridge, UK: Cambridge University Press

Heffernan, E., Heffernan, T., \& Pan, W. (2014). The relationship between the quality of 
active frontages and public perceptions of public spaces. Urban Design International, 19(1), 92-102.

Heft, H., \& Nasar, J. (2000). Evaluating environmental scenes using dynamic versus static displays. Environment \& Behavior, 32(-), 301-322.

Higgins, M. (2010). Urban design and the planning system in Aotearoa-New Zealand: Disjuncture or convergence? . Urban Design International, 15(1), 1-21.

Hillman, J. (1990). Planning for beauty. London: HMSO.

Hinshaw, M. L. (1995). Design review (R. Department, Trans.) (pp. 1-33). Chicago, IL: American Planning Association.

Holahan, C. J. (1978). Environment and behavior: A dynamic perspective. New York: Plenum Press.

Holden, G. A. (1996). Epistemology of city centre urban design guidelines: A study of the Australian scene. Newcastle, NSW, The University of Newcastle. Doctor of Philosophy: 405.

Hubbard, P. (1994). Professional vs lay tastes in design control--an empirical investigation. Planning Practice \& Research, 9(3), 271-283.

Hubbard, P. (1997). Diverging attitudes of planners and the public: an examination of architectural interpretation. Journal of Architectural and Planning Research, 14(4), 317328.

Hughes, J., \& Sharrock, W. (1990). The philosophy of social research. London: Longman.

Hunt, J. (2008). Urban design controls and city development in a New Zealand context: Reflections on recent experiences in Auckland's urban core. Paper presented at the International Planning History Society 13th biennial conference: Public versus private planning: themes, trends and tensions, Chicago.

Imrie, R., \& Street, E. (2011). Architectural Design and Regulation. Chichester, West Sussex :: Wiley-Blackwell.

Ittelson, W. H. (1978). Environmental perception and urban experience. Environment \& Behaviour, 10(2), 193 - 213.

Jackson, T., \& Dixon, J. (2007). The New Zealand Resource Management Act: an exercise in delivering sustainable development through an ecological modernisation agenda. Environment \& Planning B: Planning and Design, 34(-), 107-120.

Jacobs, A. B. (1993). Great streets. Cambridge, MA: The MIT Press.

Jacobs, J. (1961). The death and life of great American cities (3rd ed.). New York: Random House.

Janssens, J. (2001). Facade colours not just a matter of personal taste. Nordisk Arkitekturforskning, 2, 17-21.

Jarvis, R. K. (1980). Urban Environments as Visual Art or as Social Settings?: A Review. The Town Planning Review, 51(1), 50-66. doi: 10.2307/40103683

Jarvis, R. K. (1991). Too Many Words? Recent Developments in Studies of the Visual Environment: A Review Article. The Town Planning Review, 62(1), 109-115. doi: $10.2307 / 40112987$ 
Jick, T. D. (2006). Mixing qualitative and quantitative methods: Triangulation in action. In A. Bryman (Ed.), Mixed methods (Vol. II, pp. Vol II; 217-228). London: Sage Publications

Jones, R. A. (2001). Design communication and aesthetic control: Architects, planners and design review. Journal of Architectural and Planning Research, 18(1), 23-38.

Kaplan, S. (1987). Aesthetics, affect and cognition; environmental preference from an evolutionary perspective. Environment \& Behaviour, 19(1), 3-323.

Kaplan, S. (1988). Where cognition and affect meet: a theoretical analysis of preference. In J. Naser (Ed.), Environmental aesthetics: theory, research and applications (pp. p.56-63). Cambridge, UK: Cambridge University Press

Kim, J., \& Forester, J. (2012). How design review staff do far more than regulate. Urban Design International, 17(3), 239-252.

Kolakowski, L. (1993). An overall view of positivism. In M. Hammersley (Ed.), Social research: Philosophy, politics and practice (pp. p 1-8). London: Sage Publications Ltd

Kostof, S. (1991). The city shaped: Urban patterns and meanings through history. London: Thames \& Hudson.

Kostof, S. (1992). The city assembled: The elements of urban form through history. London: Thames \& Hudson.

Lai, R. T. (1994). Can the process of architectural design review withstand legal scrutiny? In B. C. Scheer \& W. F. E. Preiser (Eds.), Design review: challenging urban aesthetic control (pp. 31-41). New York: Chapman \& Hall

Lang, J. (1974). Theories of perception and "formal" design. In J. T. Lang (Ed.), Designing for buman behavior: architecture and the behavioral sciences. (pp. 98-110). Stroudsburg, Pa.: Dowden, Hutchinson \& Ross

Lang, J. (1988). Symbolic aesthetics in architecture: toward a research agenda. In J. Naser (Ed.), Environmental aesthetics: theory, research and applications (pp. 11-26). Cambridge, UK: Cambridge University Press

Lang, J. (1994). Urban design: the American experience. New York: Van Nostrand Reinhold.

Lang, J. (1996). Implementing urban design in America: Project types and methodological implications. Journal of Urban Design, 1(1), 7-22.

Lang, J. (2003). Aesthetic theory. In A. R. Cuthbert (Ed.), Designing cities: critical readings in urban design (pp. 275-284). Oxford, UK: Blackwell Publishing

Lightner, B. C. (1992). Design review: a critical evaluation. Cities, 9 (November), 280 - 287.

Lohr, V. I., \& Pearson-Mims, C. H. (2006). Responses to Scenes with Spreading, Rounded, and Conical Tree Forms. Environment and Behavior, 38(5), 667-688. doi: $10.1177 / 0013916506287355$

Lynch, K. (1960). The image of the city. Cambridge, MA: MIT Press.

Lynch, K. (1981). A theory of good city form. Cambridge, MA: The MIT Press.

Madanipour, A. (1996). Design of urban space: an inquiry into a socio-spatial process. Chichester: John Wiley \& Sons. 
Marshall, S. (2009). Cities design \& evolution. Abingdon, UK: Routledge.

Martin, D. E. B. (1949). A concise introduction to town and country planning: Every citizen's responsibility. Wellington: $\mathrm{AH} \& \mathrm{AW}$ Reed.

May, T. (2011). Social research: issues, methods and process (Fourth ed.). Maidenhead, UK: Open University Press.

McGlynn, S., \& Hayward, R. (Eds.). (1993). Making better places: urban design now. Oxford, UK: Butterworth Architecture Ltd.

McIndoe, G. (2003). Mt. Victoria and Thorndon Residential Character Implementation Audit (pp. 20). Wellington: Wellington City Council.

Memon, P. A. (1991). Shaking off a colonial legacy?-Town and country planning in New Zealand, 1870s to 1980s. Planning Perspectives, 6(-), 19-32.

Memon, P. A., \& Perkins, H. (Eds.). (2000). Environmental planning \& management in New Zealand. Palmerston North: Dunmore Press.

Miller, C. L. (1998). New Zealand's planning history - quo vadis? Planning Perspectives, 13, 257-274.

Miller, C. L. (2002). The origins of town planning in New Zealand 1900-1926: A divergent path? Planning Perspectives, 17(-), 209-225.

Miller, C. L. (2011). Implementing sustainability: the New Zealand experience. Abingdon, UK: Routledge.

Ministry for the Environment. (2002). People + places + spaces: a design guide for urban New Zealand. Wellington: Ministry for the Environment.

Ministry of Social Development. (2010). The social report 2010 (pp. 179). Wellington: Ministry of Social Development.

Moughtin, C. (1999). Urban design street and square (2nd ed). Oxford, UK: Architectural Press.

Nasar, J. L. (1987). Environmental correlates of evaluative appraisals of central business district scenes. Landscape and Urban Planning, 14(0), 117-130. doi: http://dx.doi.org/10.1016/0169-2046(87)90017-X

Nasar, J. L. (1988). Visual preferences in urban street scenes: a cross-cultural comparison between Japan and the United States. In J. L. Nasar (Ed.), Environmental aesthetics: theory, research and applications (pp. 260-274). Cambridge: Cambridge University Press

Nasar, J. L. (1989). Perception, cognition and evaluation of urban places. In I. Altman \& E. H. Zube (Eds.), Public places and spaces (Vol. 10, pp. 31-56). New York: Plenum Press

Nasar, J. L. (1990). The Evaluative Image of the City. Journal of the American Planning Association, 56(1), 41-53. doi: 10.1080/01944369008975742

Nasar, J. L. (1994). Urban design aesthetics: the evaluative qualities of building exteriors. Environment \& Behaviour, 26(No. 3, May 1994), pp 377-401.

Nasar, J. L. (1998). The evaluative image of the city. Thousand Oaks, Calif. USA: Sage Publications. 
Nasar, J. L. (Ed.). (1988). Environmental aesthetics : theory, research, and applications Cambridge: Cambridge University Press.

Nasar, J. L., \& Hong, X. (1999). Visual preferences in urban signscapes. Environment and Behavior, 31(5), 671-691.

Nasar, J., \& Grannis, P. (1999). Design review reviewed. Journal of the American Planning Association, 65(4), 424 - 433.

Norberg-Schulz, C. (1980). Genius loci : towards a phenomenology of architecture London Academy Editions.

Norberg-Schulz, C. (2003). The phenomenon of place. In A. Cuthbert (Ed.), Designing cities: critical readings in urban design (pp. 116-127). Oxford: Blackwell Publishing

O'Connor, Z. (2006). Bridging the gap: facade colour, aesthetic response and planning policy. Journal of Urban Design, 11(3), p 335-345.

ODPM. (2005). Planning Policy Statement 1: Delivering Sustainable Development. HMSO.

O'Hanlon, S., \& Sharpe, S. (2009). Becoming Post-industrial: Victoria Street, Fitzroy, c.1970 to Now. Urban Policy and Research, 27(3), 289-300. doi: 10.1080/08111140903112038

Oostendorp, A., \& Berlyne, D. E. (1978). Dimensions in the perception of architecture. I. Identification and interpretation of dimensions of similarity. Scandinavian Journal of Psychology, 19(March), 73-82.

Orians, G. C. (1986). An ecological and evolutionary approach to landscape aesthetics. In E. C. Penning-Rowsell \& D. Lowenthal (Eds.), Landscape meanings and values (pp. 3-25). London: Allen and Unwin

Padovan, R. (1999). Proportion: Science philosophy architecture. London: E \& FN Spon.

Paterson, E. (2011). Design review in the UK: its role in town planning decision making. Urban Design International, 16(2), 94-104.

Pevsner, N. (1959). New Zealand. Architectural Review, 126(752), 205-206.

Pevsner, N., \& Games, S. (2014). Pevsner : The Complete Broadcast Talks: Architecture and Art on Radio and Television, 1945-1977. Burlington, VT: Ashgate Publishing Company.

Pikora, T., Giles-Corti, B., Bull, F., Jamrozik, K., \& Donovan, R. (2003). Developing a framework for assessment of the environmental determinants of walking and cycling. Social Science \& Medicine, 56(8), 1693-1703. doi: http://dx.doi.org/10.1016/S0277-9536(02)00163-6

Pløger, J. (1999). Aesthetic planning and design control in Norway. Urban Design International, 4(1\&2), 47 - 54.

Portella, A. (2014). Visual Pollution: Advertising, signage and environmental quality. Surrey, United Kingdom: Ashgate.

Portella, A. A. (2007). Evaluating commercial signs in historic streetscapes. (PhD Written ), Oxford Brookes University, Oxford, UK.

Porteous, J. D. (1996). Environmental aesthetics: ideas, politics and planning. London: Routledge.

Pouler, P. J. (1994). Disciplinary society and the myth of aesthetic justice. In B. C. Scheer 
\& W. F. E. Preiser (Eds.), Design review: challenging urban aesthetic control (pp. 175186). New York: Chapman \& Hall

Proshansky, H. M. (1990). In pursuit of understanding: an intellectual history. In I. Altman \& K. Christensen (Eds.), Environment \& Behavior Studies: Emergence of Intellectual Traditions (Vol. 11, pp. 9-30). New York: Plenum Press

Punter, J. (1986). A history of aesthetic control: Part 1, 1909-1953: The control of the external appearance of development in England and Wales. The Town Planning Review, 57(4), 351-381.

Punter, J. (1986). Aesthetic control within the development process: a case study. Land Development Studies, 3, 197-212.

Punter, J. (1987). A history of aesthetic control: Part 2, 1953-1985: The control of the external appearance of development in England and Wales. The Town Planning Review, 58(1), 29-62.

Punter, J. (1994). Aesthetics in planning. In H. Thomas (Ed.), Values and planning (pp. 38 - 67). Aldershot, UK: Ashgate Publishing Ltd

Punter, J. (1996). Urban Design Theory in Planning Practice: The British Perspective. Built Environment (1978-), 22(4), 263-277. doi: 10.2307/23288422

Punter, J. (1999). Improving the instruments, processes and products of aesthetic control in Europe. Urban Design International, 4(1\&2), 79-99. doi: 10.2307/23288422

Punter, J. (2004). From the ill-mannered to the iconic: Design regulation in central Sydney 1947-2002. The Town Planning Review, 75(4), 405-445.

Punter, J. (2007). Developing urban design as public policy: best practice principles for design review and development management. Journal of Urban Design, 12(2), 167202.

Punter, J. (2011a). Design review - An effective means of raising design quality? In S. Tiesdell \& D. Adams (Eds.), Urban design in the real estate development process (pp. 182198). Chichester, UK: Blackwell Publishing Ltd

Punter, J. (2011b). Urban design and the English urban renaissance 1999-2009: A review and preliminary evaluation. Journal of Urban Design, 16(1), 1-41.

Punter, J., \& Carmona, M. (1996). Design policies in English local plans: the search for substantive principles and appropriate procedures. Urban Design International, 1(2), 125-143.

Punter, J., \& Carmona, M. (1997). The design dimension of planning. Abingdon, UK: Routledge.

Radford, A. (2009). Responsive cohesion as the foundational value in architecture. The Journal of Architecture, 14(4), 511-532.

Radford, A. (2010). Urban design, ethics and responsive cohesion. Building Research \& Information, 38(4), 379-389.

Radford, A. D. (1994). Local architectural language and contextualism. In B. C. Scheer \& W. F. E. Preiser (Eds.), Design review: challenging urban aesthetic control (pp. p165 174). New York: Chapman \& Hall 
Rahman, O. M. A. (1992). Visual quality and response assessment: an experimental technique. Envrionment and Planning B: Planning and Design, 19, 689-708.

Rapoport, A. (1977). Human aspects of urban form :towards a man-environment approach to urban form and design / Amos Rapoport. Oxford ;New York :: Pergamon Press.

Rapoport, A. (1982). The meaning of the built environment: a nonverbal communication approach. Beverly Hills, Calif.; London: Sage.

Rapoport, A., \& Kantor, R. E. (1967). Complexity and ambiguity in environmental design. Journal of the Institute of American Planners, 33, p 210 - 221.

Rasmussen, S. E. (1959). Experiencing architecture. Cambridge, MA: MIT Press.

Reeve, A., Goodey, B., \& Shipley, R. (2007). Townscape assessment: the development of a practical tool for monitoring and assessing visual quality in the built environment. Urban Morphology, 11(1), 25-41.

RFAC. (1994). What makes a good building? : an inquiry by the Royal Fine Art Commission. London: RFAC.

Rudlin, D. (1999). The Hulme and Manchester Design Guides. Built Environment (1978-), 25(4), 317-324. doi: $10.2307 / 23288865$

Sanoff, H. (1991). Visual research methods in design. New York: Van Nostrand Reinhold.

Schuster, J. M. (1997). The role of design review in affecting the quality of urban design: The architect's point of view. Journal of Architectural Planning Research, 14(3), 209225.

Schuster, J. M. (2005). Substituting information for regulation: In search of an alternative approach to shaping urban design. In E. Ben-Joseph \& T. Szold (Eds.), Regulating place: Standards and the shaping of urban America (pp. p 333-358). New York: Routledge

Scott, G. (1980). The architecture of humanism. London: The Architectural Press.

Scott, M. (1969). American city planning since 1890. Berkeley, CA: University of California Press.

Scruton, R. (1979). The aesthetics of architecture. London: Methuen \& Co. Ltd.

Seale, C. (Ed.). (2004). Social research methods. London: Routledge.

Seamon, D. (2000). A way of seeing people and place: Phenomenology in environmentbehavior research. In S. Wapner, J. Demick, T. Yamamoto, \& H. Minami (Eds.), Theoretical perspectives in environment-behavior research (pp. 157-178). New York: Kluwer Academic/Plenum Publishers

Sharp, T. (1968). Town and townscape. London: John Murray.

Silva, C. N. (2008). Urban planning and ethics J. M. Rabin \& E. M. Berman (Eds.), Encyclopedia of Public Administration and Public Policy (pp. 2006-2011). doi:10.1081/E-EPAP2

Sitte, C. (1979). The art of building cities :city building according to its artistic fundamentals / by Camillo Sitte; translated by Charles T. Stewart. Westport, Conn. :: Hyperion Press.

Smith, P. F. (1974). The dynamics of urbanism. London: Hutchinson Educational Ltd.

Smith, P. F. (1980). Urban aesthetics. In B. Mikellides (Ed.), Architecture for people (pp. 74 - 
86). London: Studio Vista

Smith, P. F. (1987). Architecture and the principle of harmony. London: RIBA Publications.

Smith, P. F. (2003). The dynamics of delight: architecture and aesthetics. London Routledge.

Southworth, M. (1989). Theory and Practice of Contemporary Urban Design: A Review of Urban Design Plans in the United States. The Town Planning Review, 60(4), 369402. doi: $10.2307 / 40112852$

St Patrick's Cathedral. (2013). St Patrick's Cathedral Heritage Foundation. Retrieved 3 December 2014, from http:/ / theproject.stpatricks.org.nz/

Stamps III, A. E. (1990). Use of photographs to simulate environments: A meta-analysis. Perceptual and Motor Skills, 71(December), 907-913.

Stamps III, A. E. (1991). Public preferences for high rise buildings: Stylistic and demographic effects. Perceptual and Motor Skills, 72(-), 839-844.

Stamps III, A. E. (1994). All buildings great and small: Design review from high rise to houses. Environment and Behavior, 26(3, May 1994), p 402-420.

Stamps III, A. E. (1994). Validating contextual urban design principles. In S. J. Neary, M. S. Symes, \& F. E. Brown (Eds.), The urban experience: A people-environment perspective (pp. 141-153). London: E \& FN Spon

Stamps III, A. E. (1996). People and places: Variance components of environmental preferences. Perceptual and Motor Skills, 82(-), 323-334.

Stamps III, A. E. (1999). Demographic effects in environmental aesthetics: A metaanalysis. Journal of Planning Literature, 14(2 (November 1999)), 155-175.

Stamps III, A. E. (2000). Psychology and the aesthetics of the built environment. Dordrecht, The Netherlands: Kluwer Academic Publishers Group.

Stamps III, A. E., Nasar, J., \& Hanyu, K. (2005). Using pre-construction validation to regulate urban skylines. Journal of the American Planning Association, 71(1), p73-91.

Statistics New Zealand. (2013). National Population Estimates: At 30 June 2013. Wellington: Statistics New Zealand Retrieved from http://www.stats.govt.nz/browse_for_stats/population/estimates_and_projecti ons/NationalPopulationEstimates_HOTPAt30Jun13.aspx.

Taylor, N. (1994). Aesthetic judgment and environmental design: is it entirely subjective? The Town Planning Review, 65(1), 21-40.

Taylor, N. (1994). Environmental issues and the public interest. In H. Thomas (Ed.), Values and planning (pp. 87 - 115). Aldershot, UK: Ashgate Publishing Ltd

Taylor, N. (1998). Urban planning theory since 1945. Sage Publications: London.

Taylor, N. (2000). Ethical arguments about the aesthetics of architecture. In W. Fox (Ed.), Ethics and the built environment (pp. 193-206). Abingdon, UK: Routledge

Thiis-Evensen, T. (1999). Archetypes of urbanism: a method for the esthetic design of cities. Oslo: Universitetsforlaget.

Thorns, D. C. (2002). The transformation of cities. Basingstoke, UK: Palgrave.

Tiesdell, S., Oc, T., \& Heath, T. (1996). Revitalizing historic urban quarters Oxford, UK: 
Architectural Press.

Townshend, T., \& Madanipour, A. (2001). Urban design in the planning system. In M. Roberts \& C. Greed (Eds.), Approaching urban design: the design process (pp. 131-140). London: Taylor \& Francis

Trancik, R. (1986). Finding lost space: theories of urban design. New York: John Wiley \& Sons.

United Nations. (2012). World Urbanization Prospects: The 2011 Revision (pp. 318). New York: United Nations.

Unwin, R. (1911). Town planning in practice. London: T Fischer Unwin Ltd.

Venturi, R. (1977). Complexity and contradiction in architecture (Second ed.). New York: Museum of Modern Art.

Wakeford, R. (1990). American development control: Parallels and paradoxes from an English perspective. London: HMSO.

Weber, R. (1995). On the aesthetics of architecture. Aldershot: Avebury.

Wellington City Council. (2011). Central Area Urban Design Guide. Wellington: Wellington City Council.

Wellington City Council. (2012). Central Area Urban Design Guide. Wellington: Wellington City Council Retrieved from http://wellington.govt.nz/ /media/yourcouncil/plans-policies-and-bylaws/district-plan/volume02/files/v2central.pdf.

Williams, M., \& May, T. (1996). Introduction to the philosophy of social research. London: Routledge.

Wilson, M. A. (1996). The socialization of architectural preference. Journal of Environmental Psychology 16(-): 33-44.

Wilson, M. A. and D. V. Canter (1990). The development of central concepts during professional education: an example of a multivariate model of the concept of architectural style. Applied Psychology 39(4): 431-455.

Wohlwill, J. F. (1976). Environmental Aesthetics: The environment as a source of affect. In I. Altman \& J. F. Wohlwill (Eds.), Human Behavior and Environment: Advances in theory and research (Vol. New York, pp. 37-86): Plenum Press

Yin, R. K. (1989). Case study research: Design and methods. Newbury Park CA: Sage Publications.

Young, M. (1986). Architectural and building design :an introduction. London :: Heinemann.

Zacharias, J. (2001). Pedestrian Behavior Pedestrian Behavior and Perception in Urban Walking Environments. Joumal of Planning Literature, 16(1), 3-18. doi: $10.1177 / 08854120122093249$

Zeisel, J. (2006). Inquiry by design. New York: W. W. Norton \& Company. 


\section{Appendix A}

Study One questionnaire form 

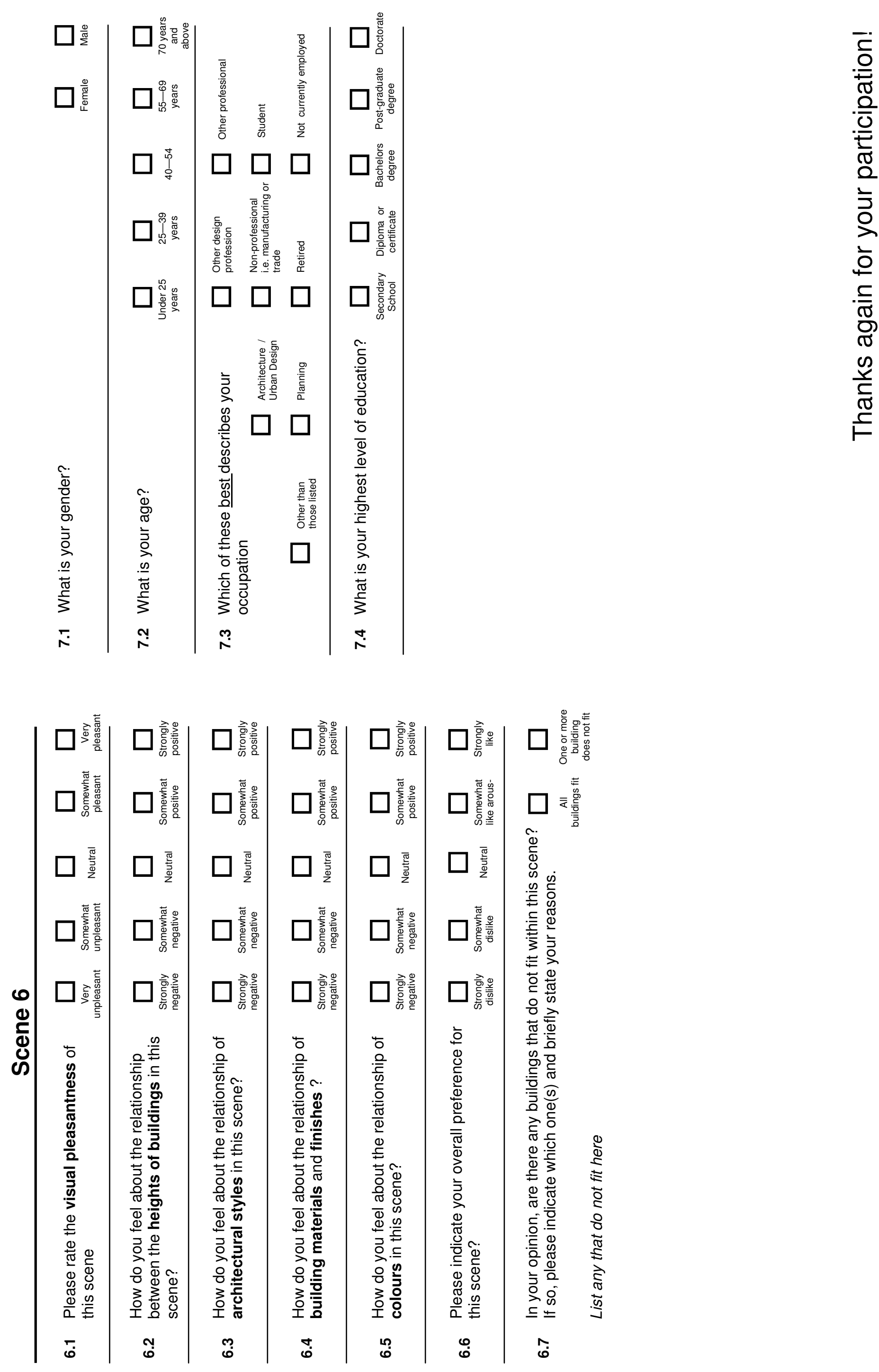


\section{Appendix B}

Study Two questionnaire forms

Study 2A - College Street, Wellington

Study 2B - Tyler Street, Auckland

Study 2C - Kingston and Wyndham Streets, Auckland 


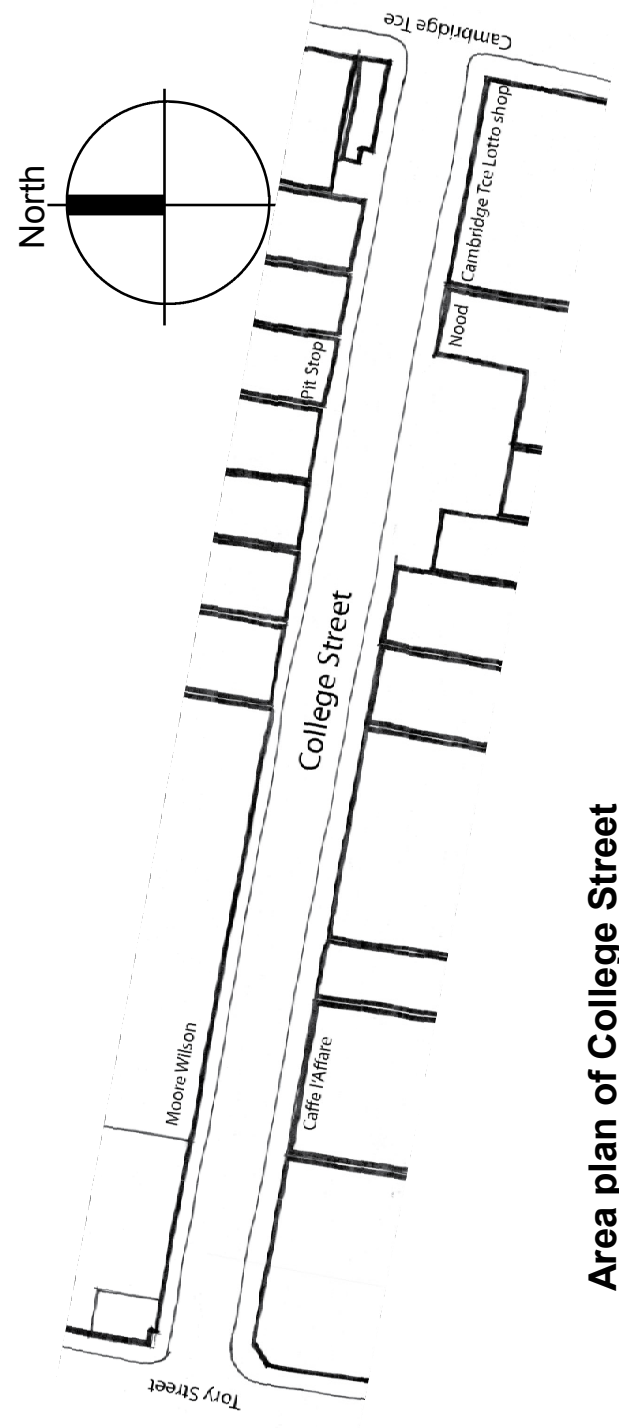

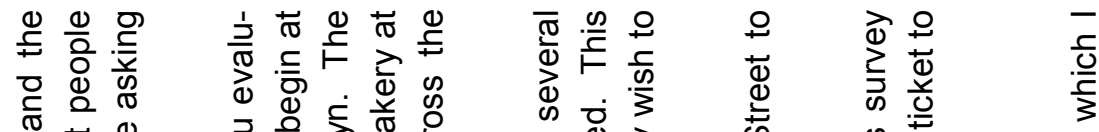

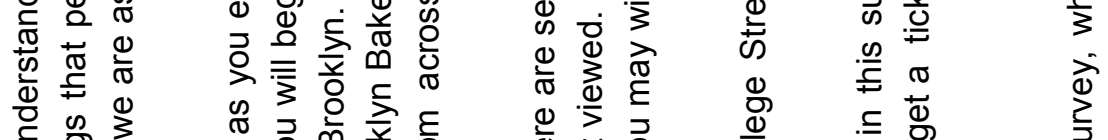

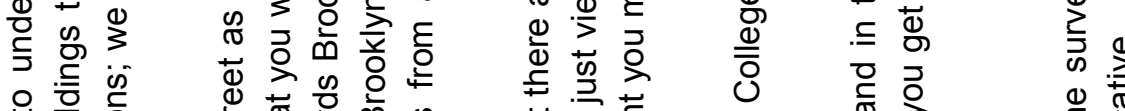

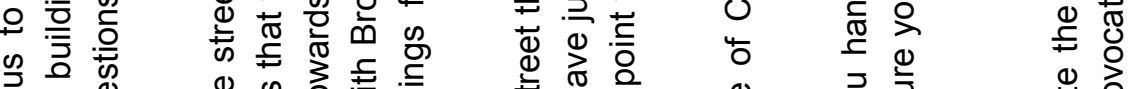

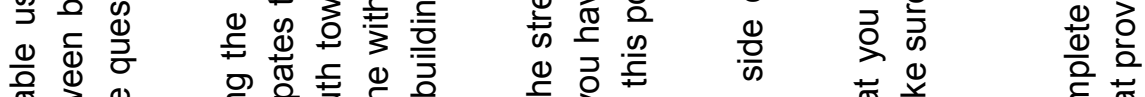

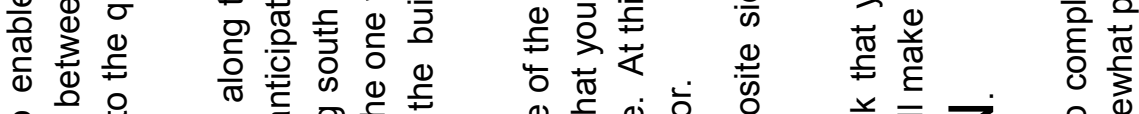

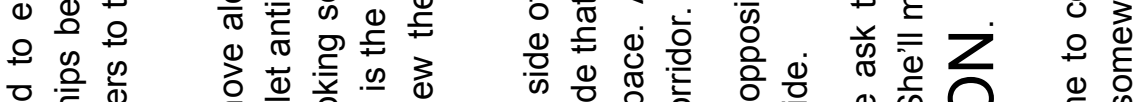

ठ

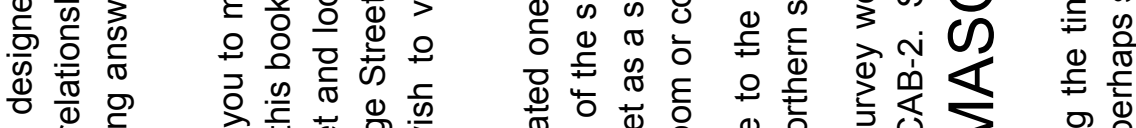

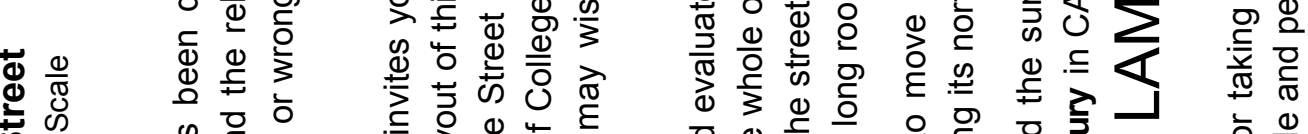

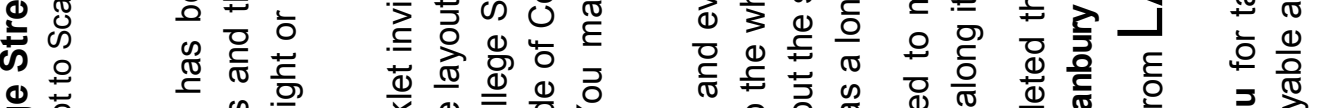

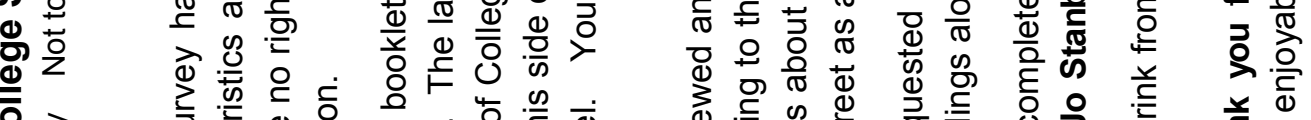

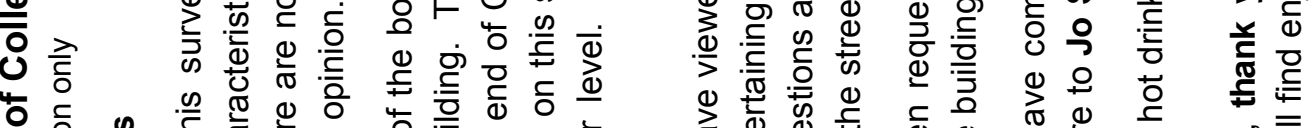

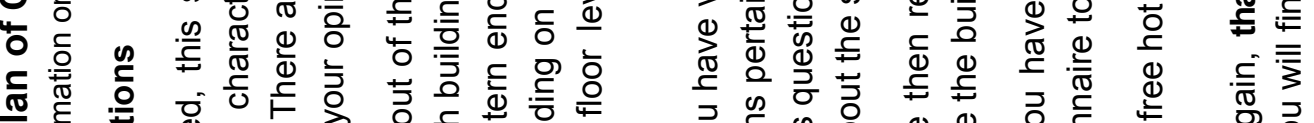

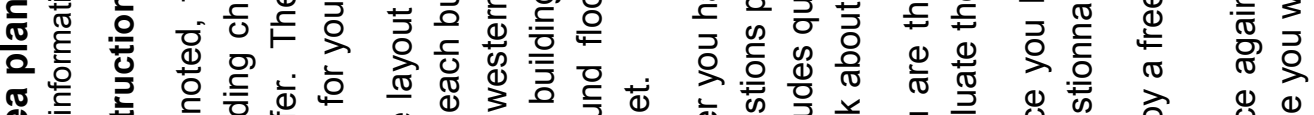

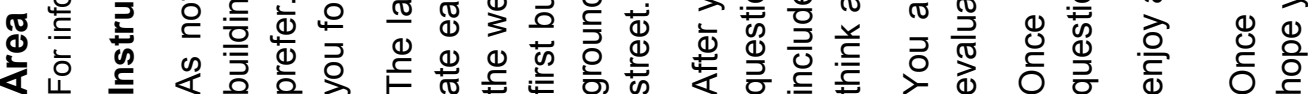

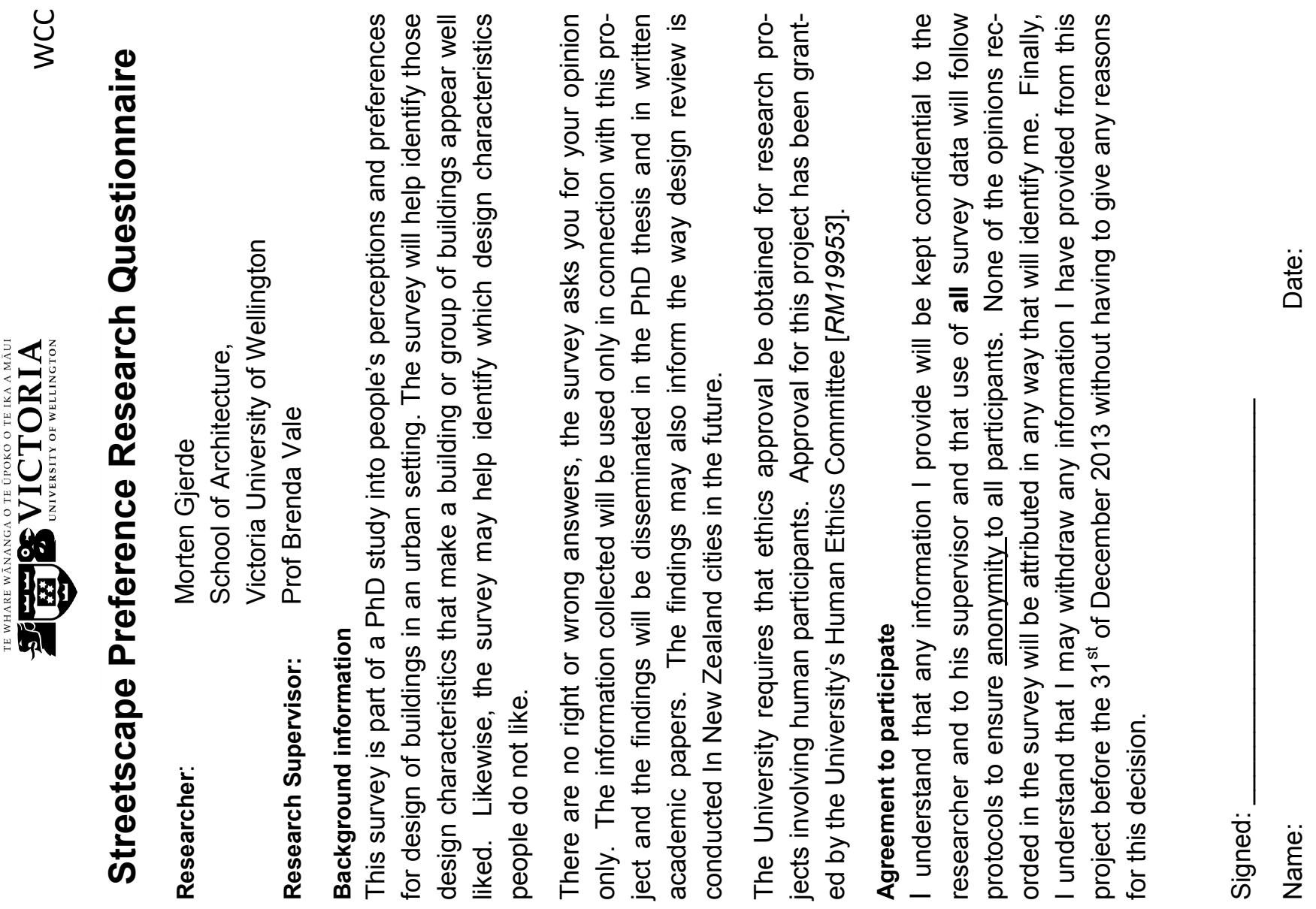




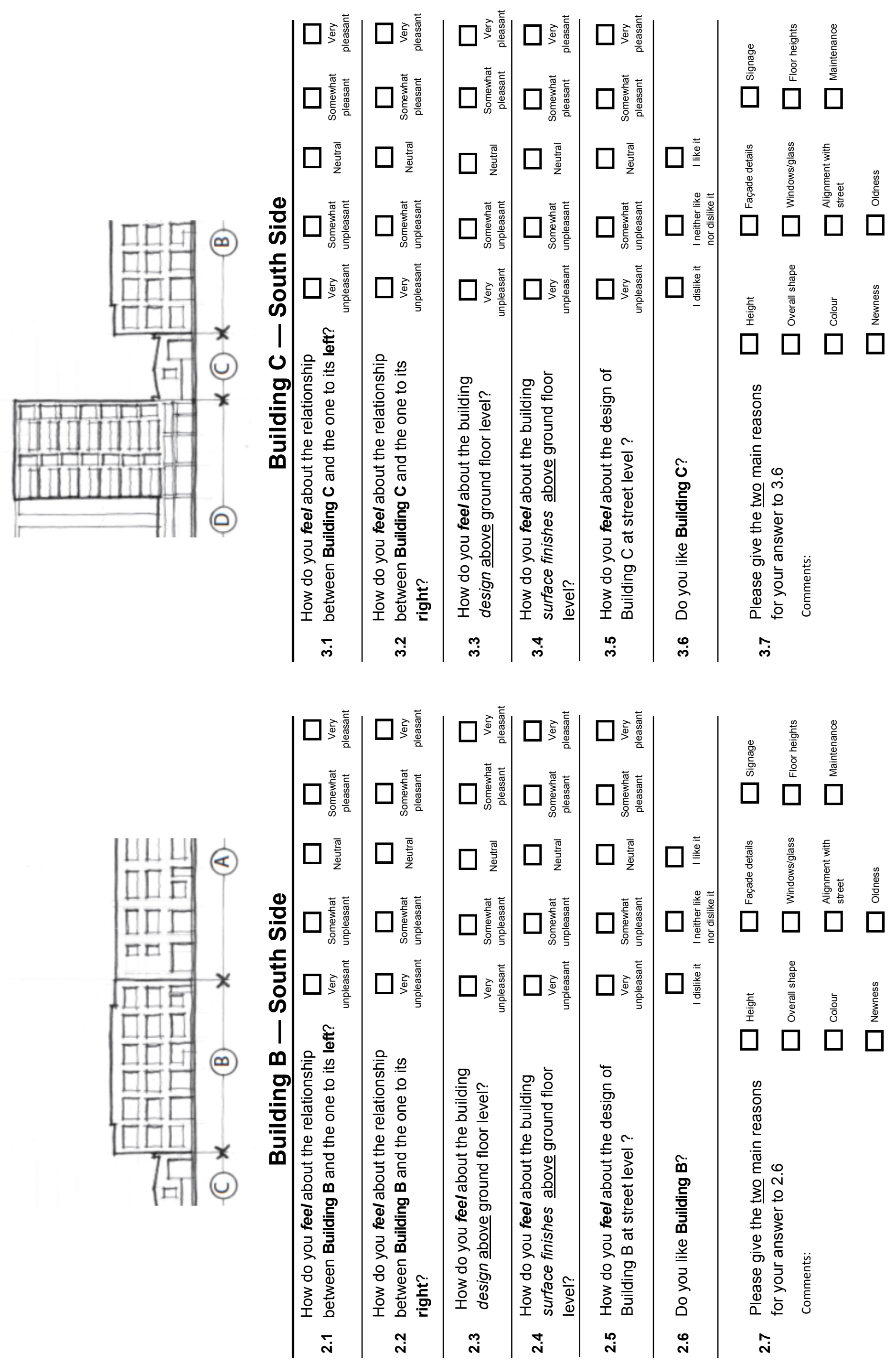




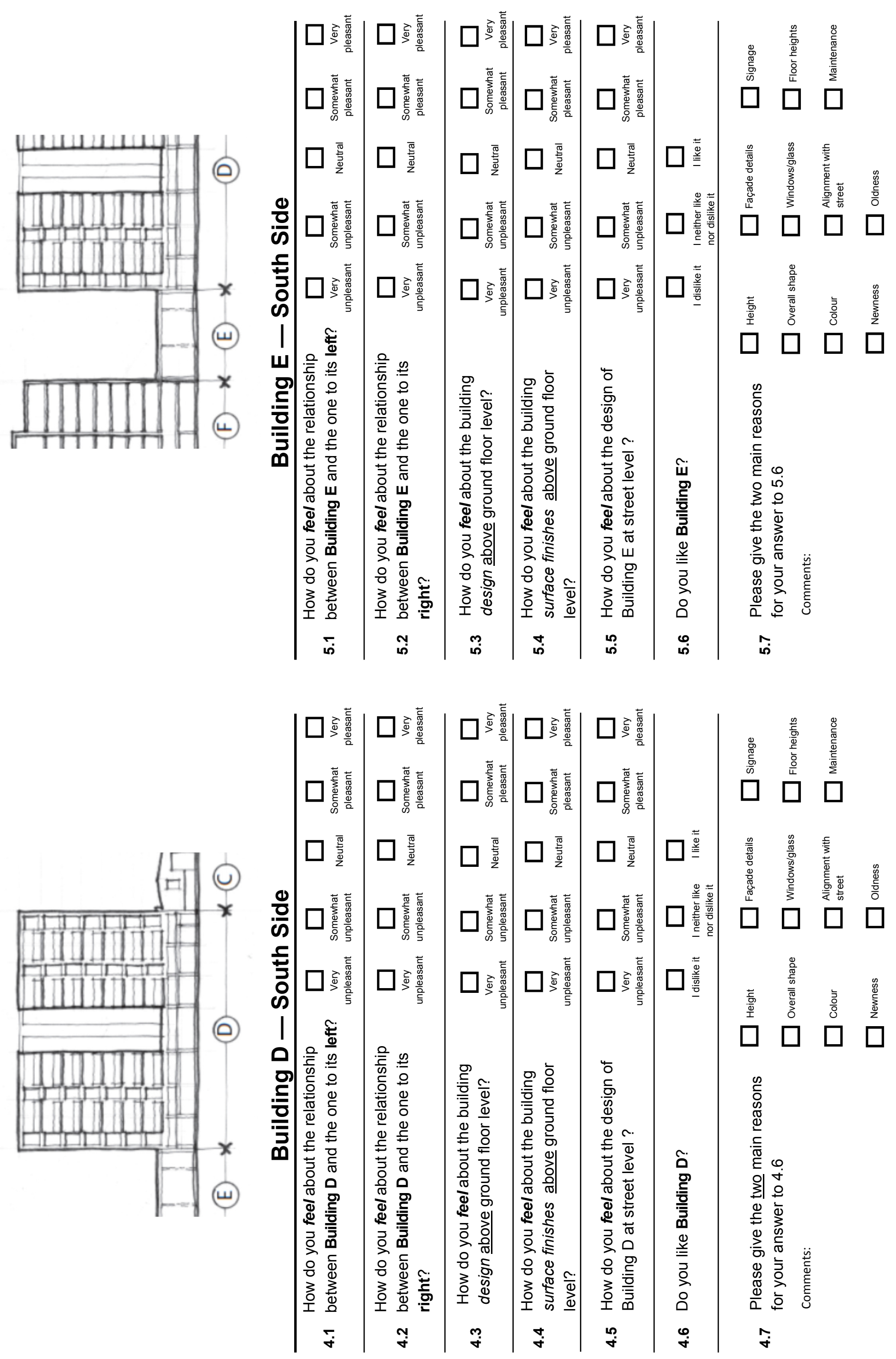




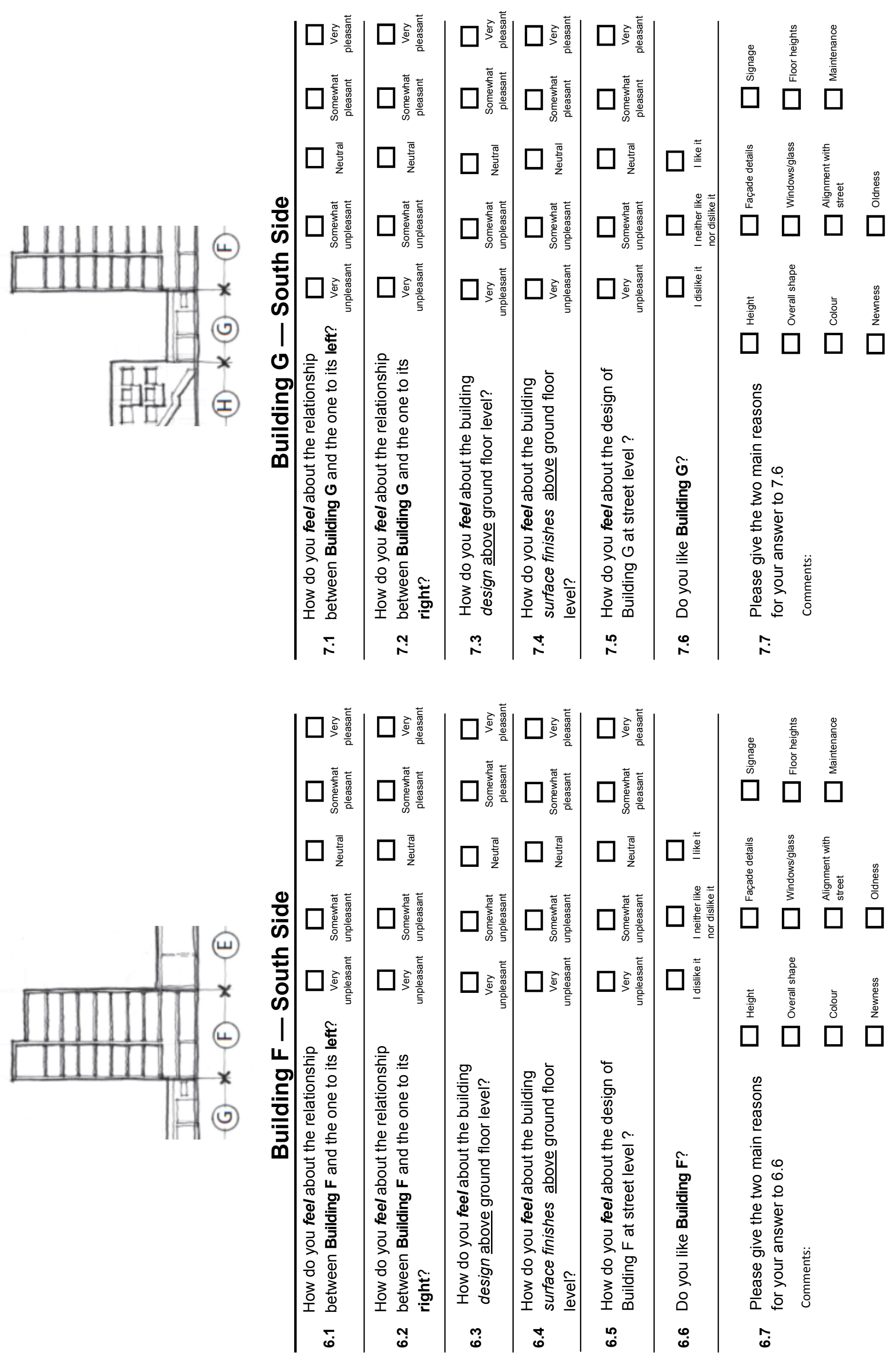




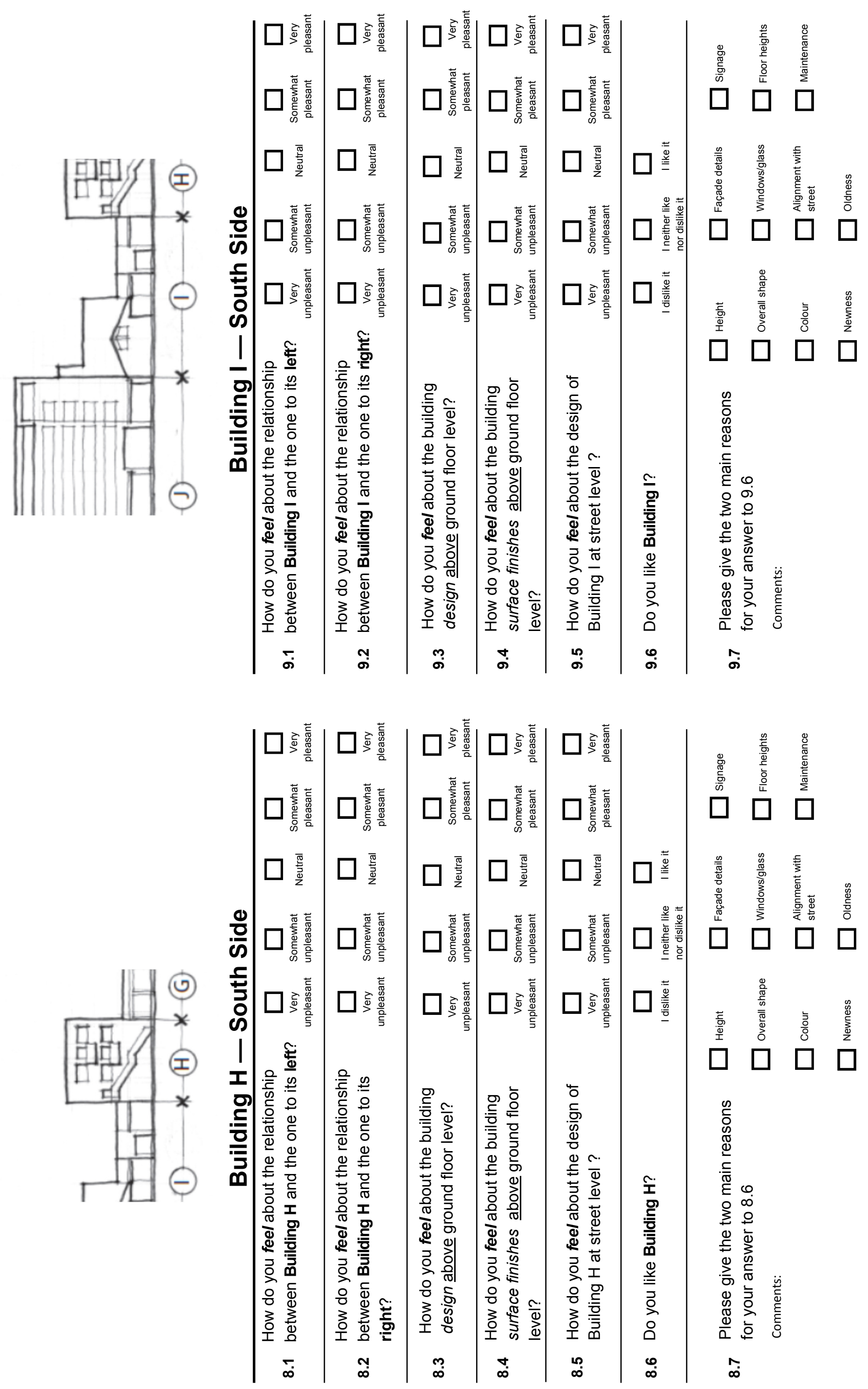




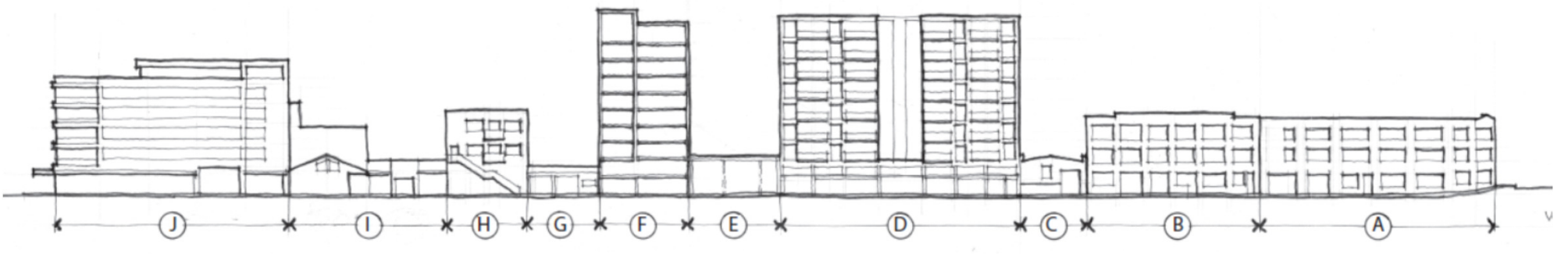

Overall Block-South Side

11.1 How do you feel about the relationship between building heights along this side of the street?

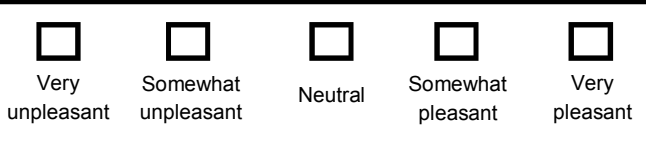

11.2 Please state briefly the principal reason for your response to question 11.1

11.3 How do you feel about the alignment [in and out] between building facades along the length of this side of the street?

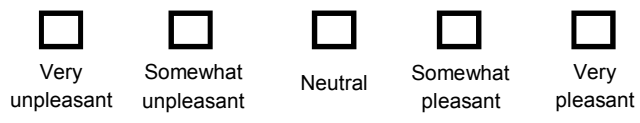

11.4 How do you feel about the relationship between façade design styles along this side of the street?
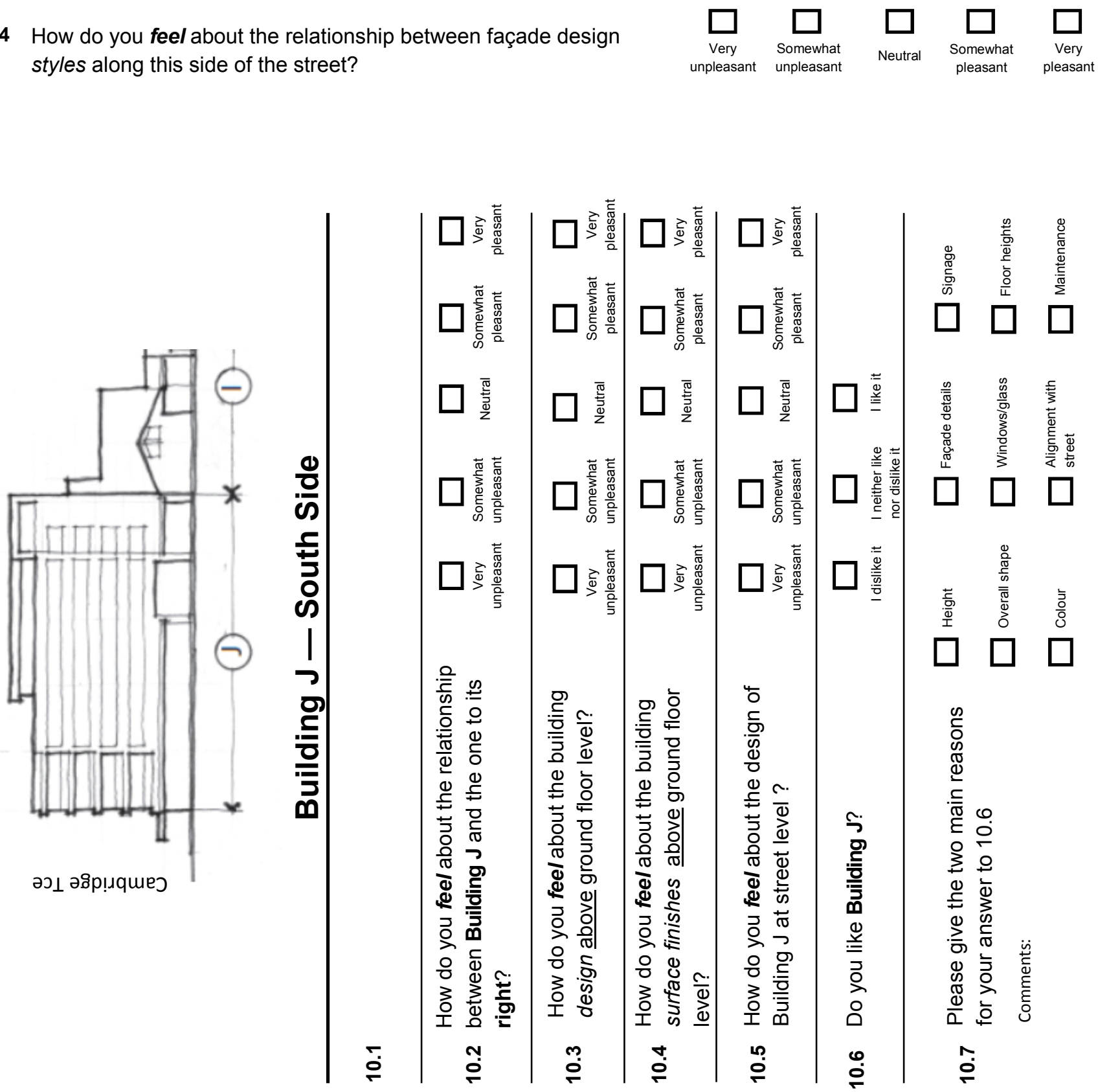

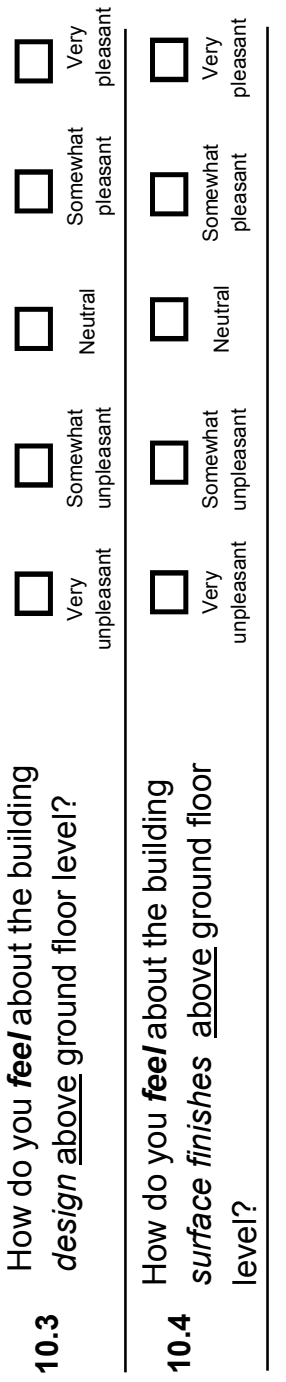

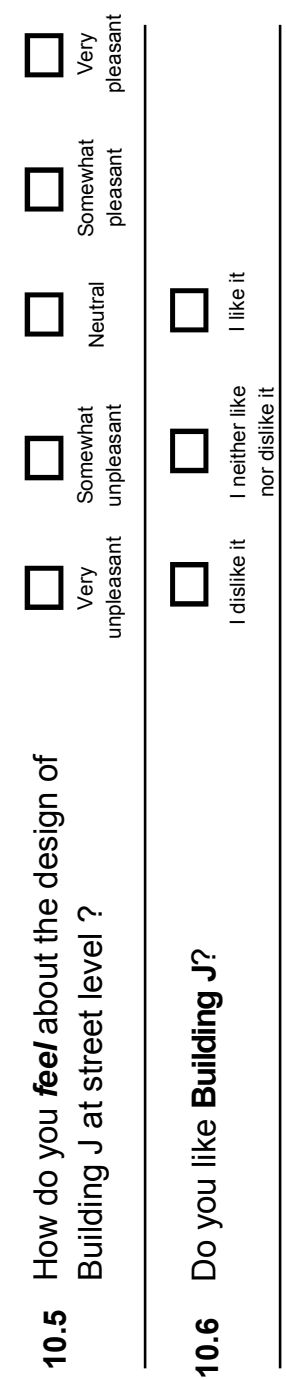
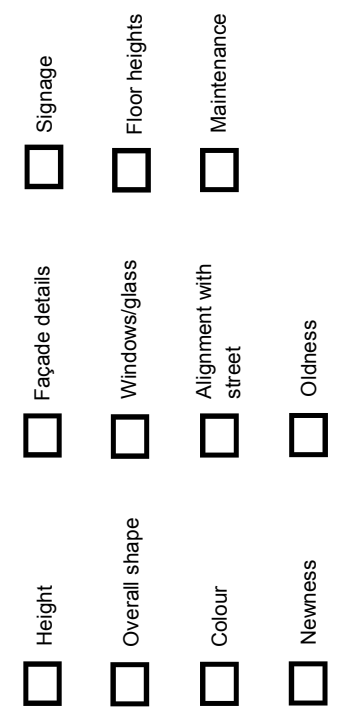

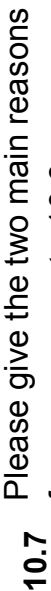



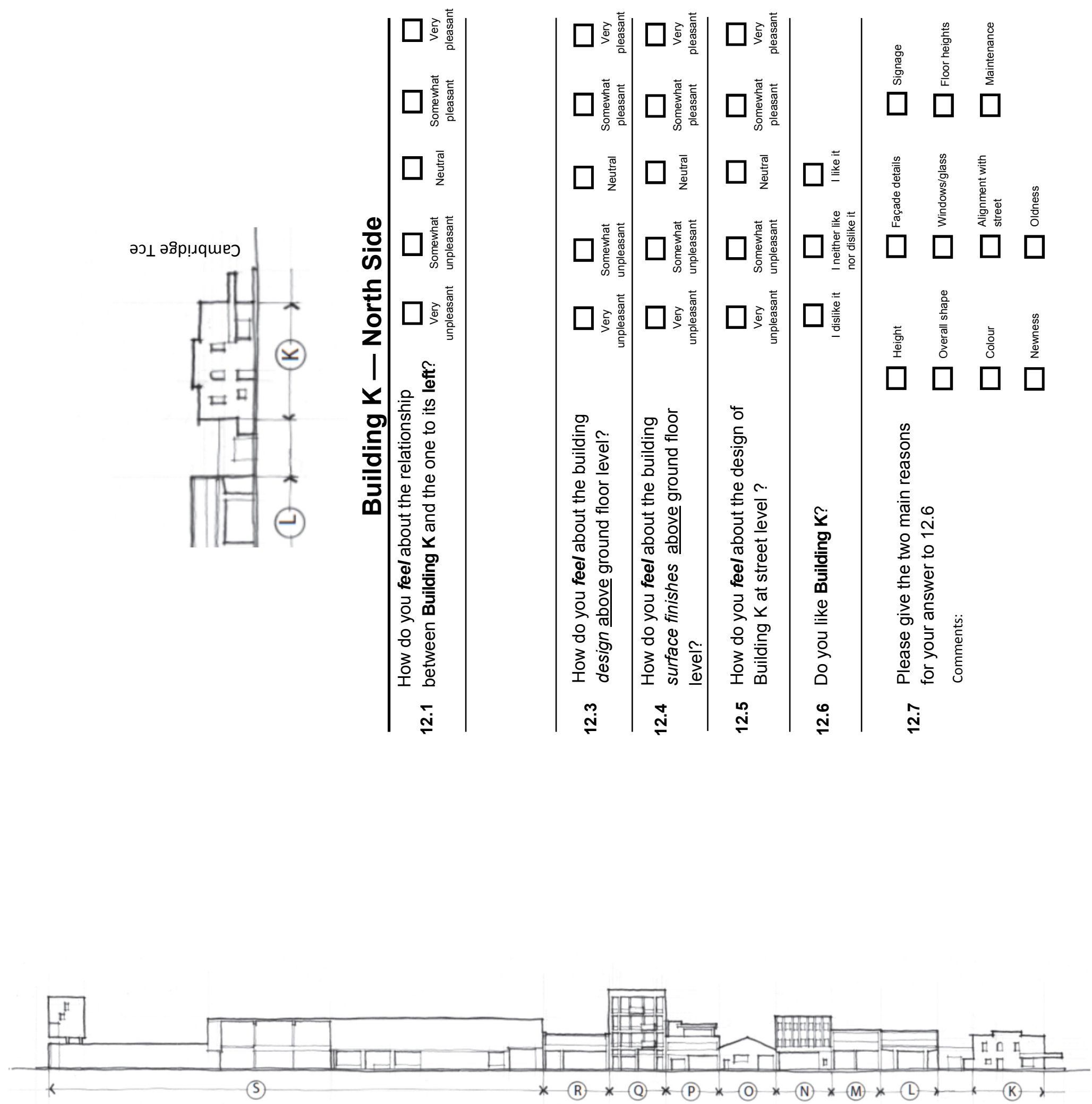

\section{Overall Block-North Side}

As you now begin your return journey along to evaluate the buildings on the northern side of College Street please cast your eye over the whole of the collection of building facades along this line. Think about the collection of buildings as a whole and how you feel about them all together.

Once you have gone through to evaluate each building individually you will be asked to cast your eye back over these buildings and to then answer some questions about them as a group. 

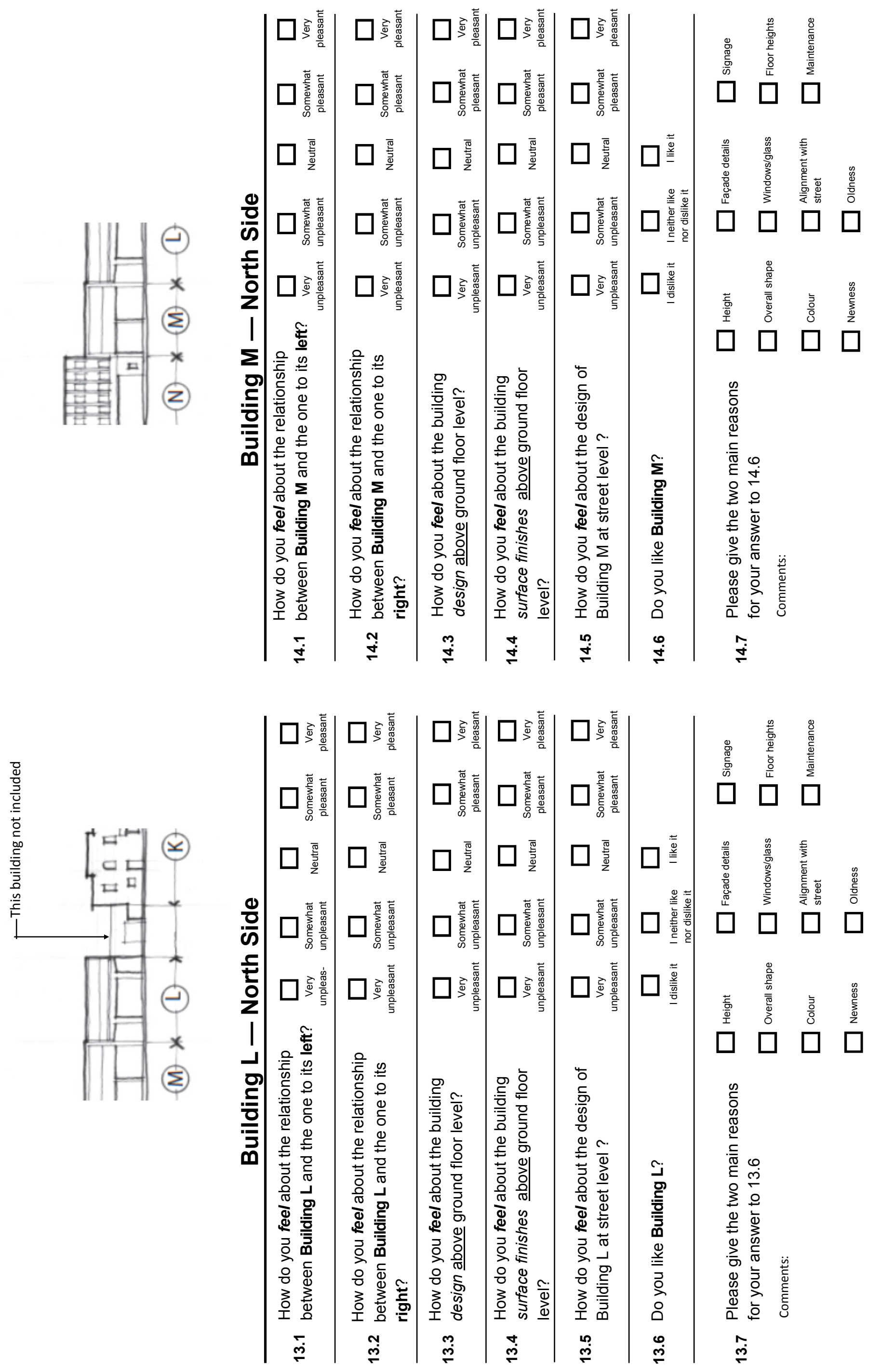


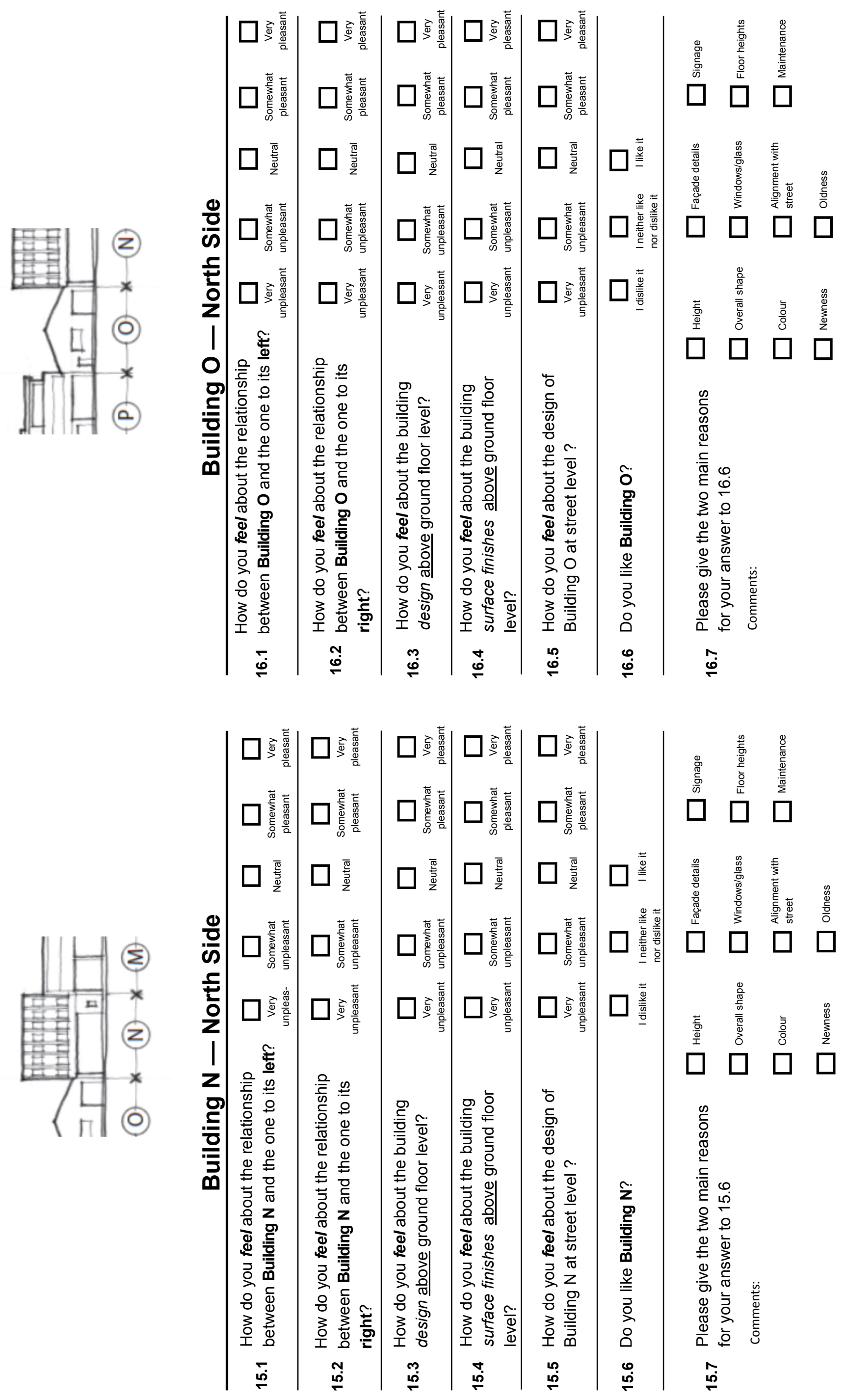




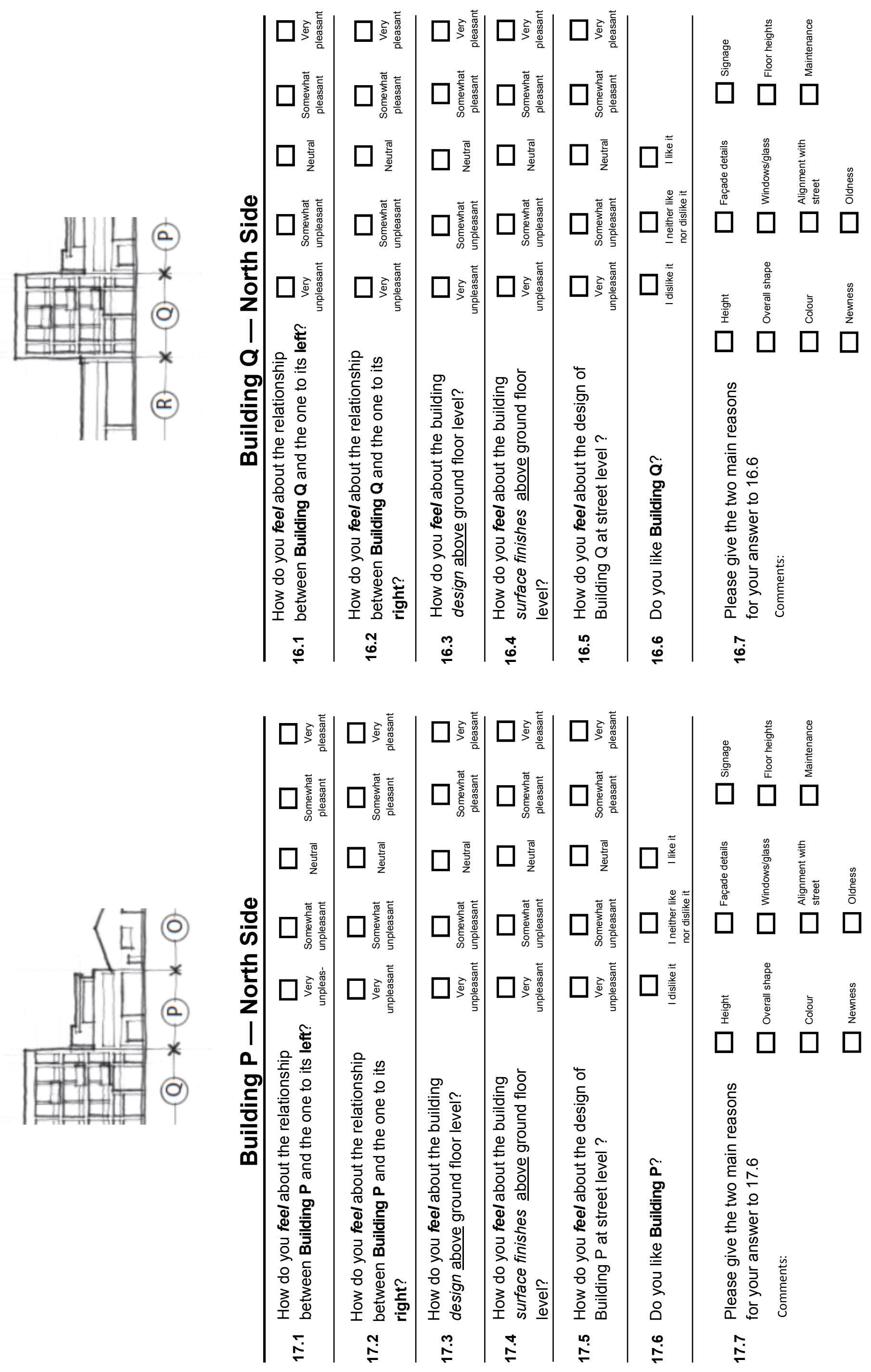



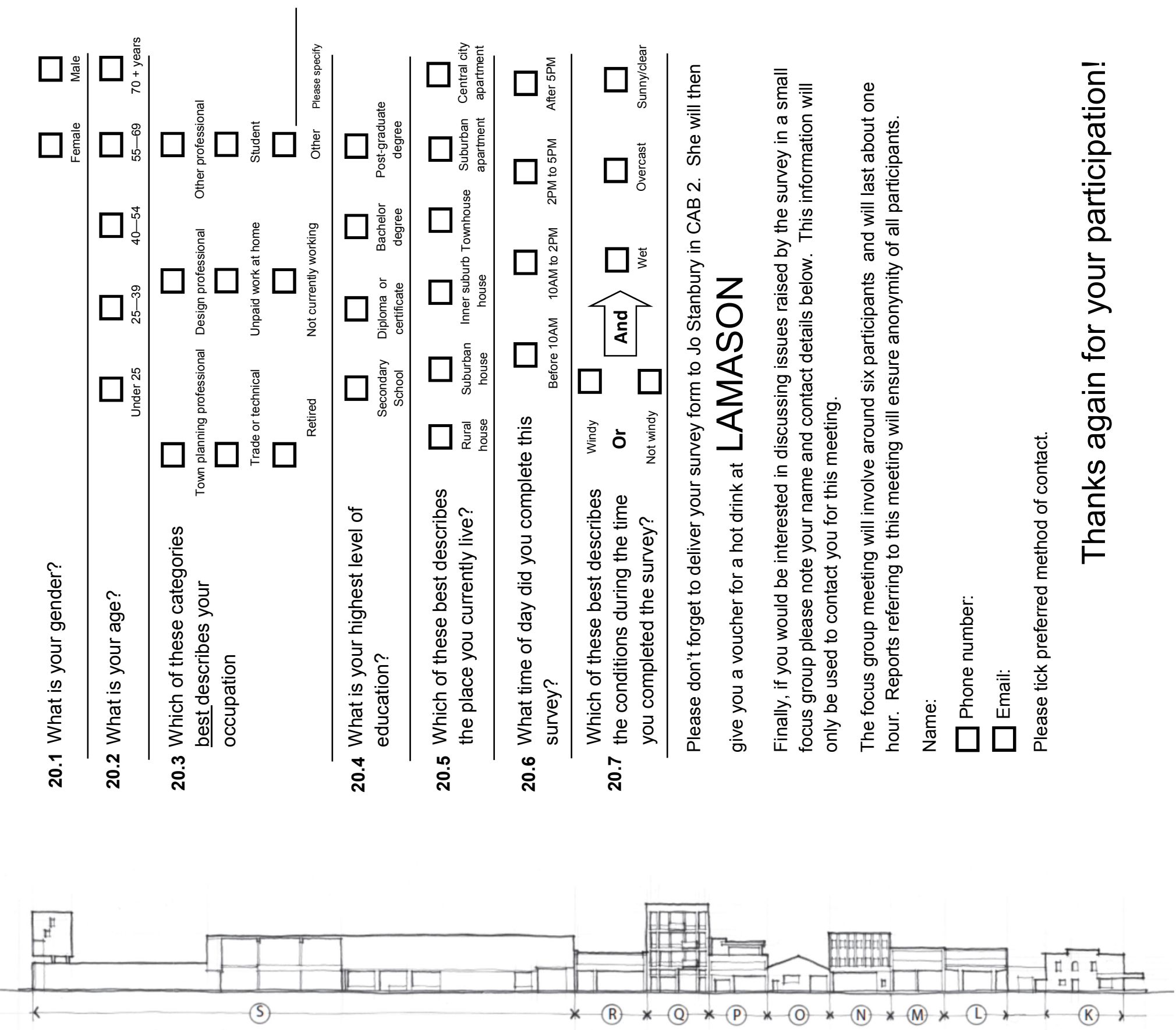

\section{Overall Block-North Side}

19.1 How do you feel about the relationship between building heights along this side of the street?

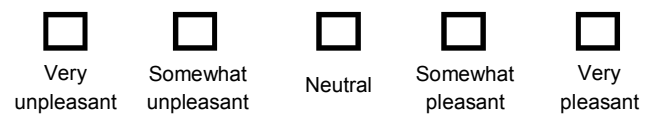

19.2 Please state briefly the principal reason for your response to question 19.1

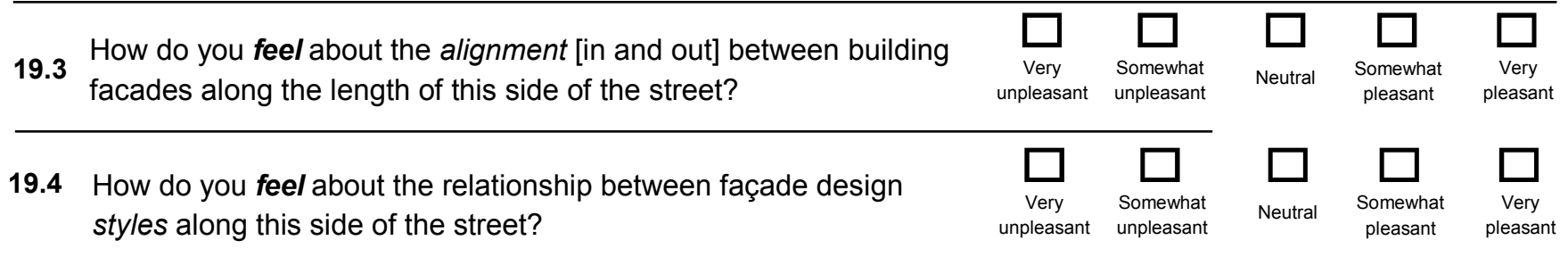



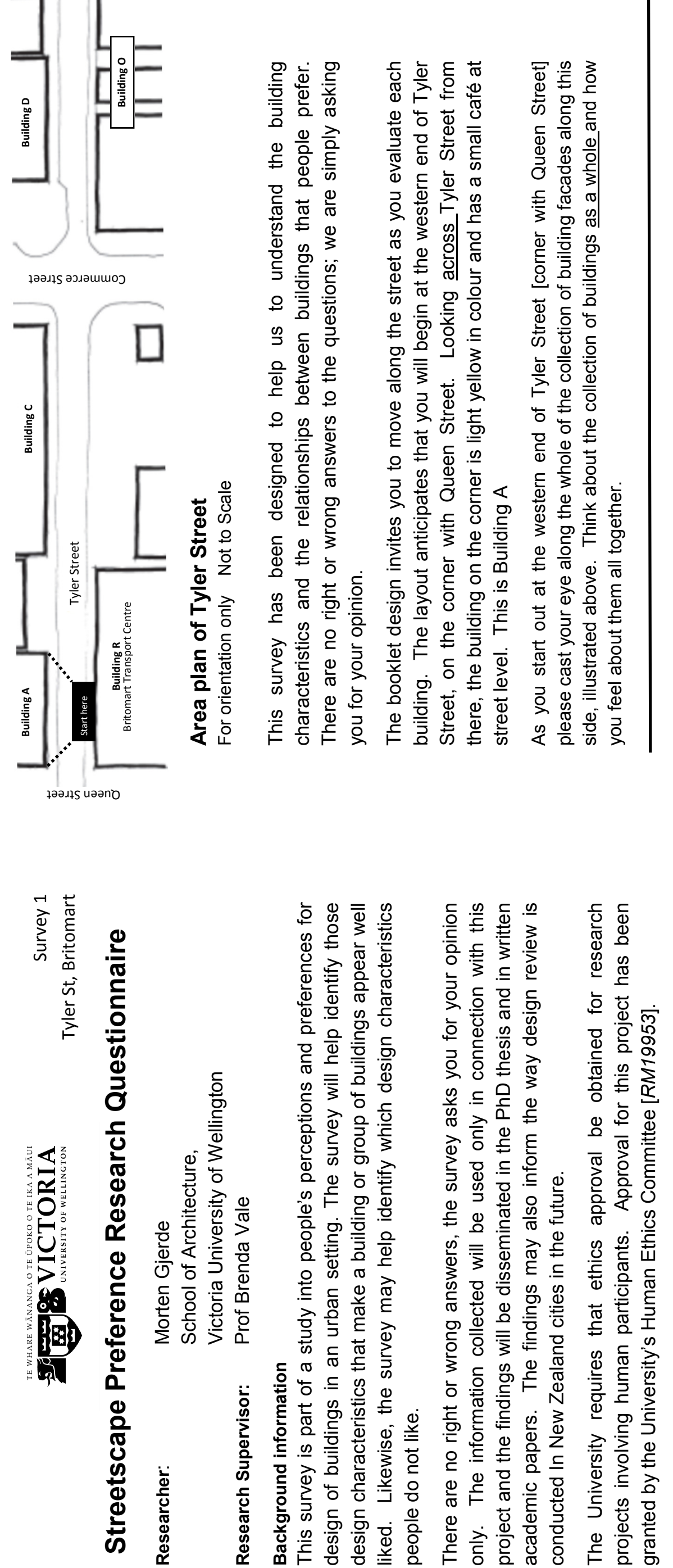

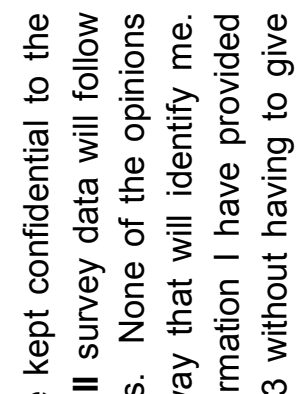

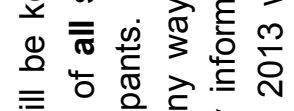

$\stackrel{\dddot{Q}}{\stackrel{\pi}{0}}$

产 0 은 $\frac{1}{\sigma}$

ब

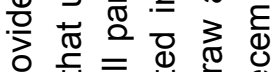

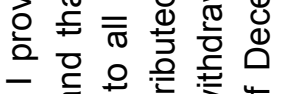

ธ

旁

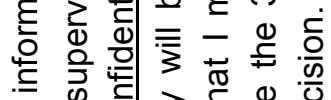

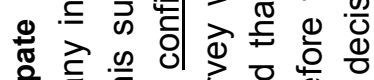

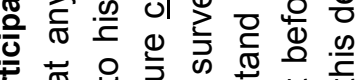

뚜워

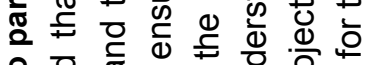

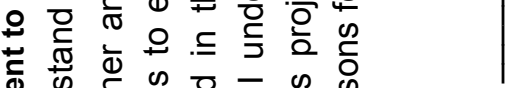

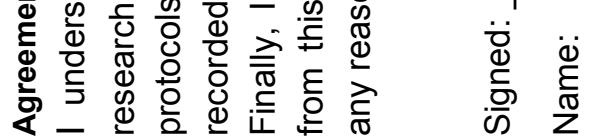




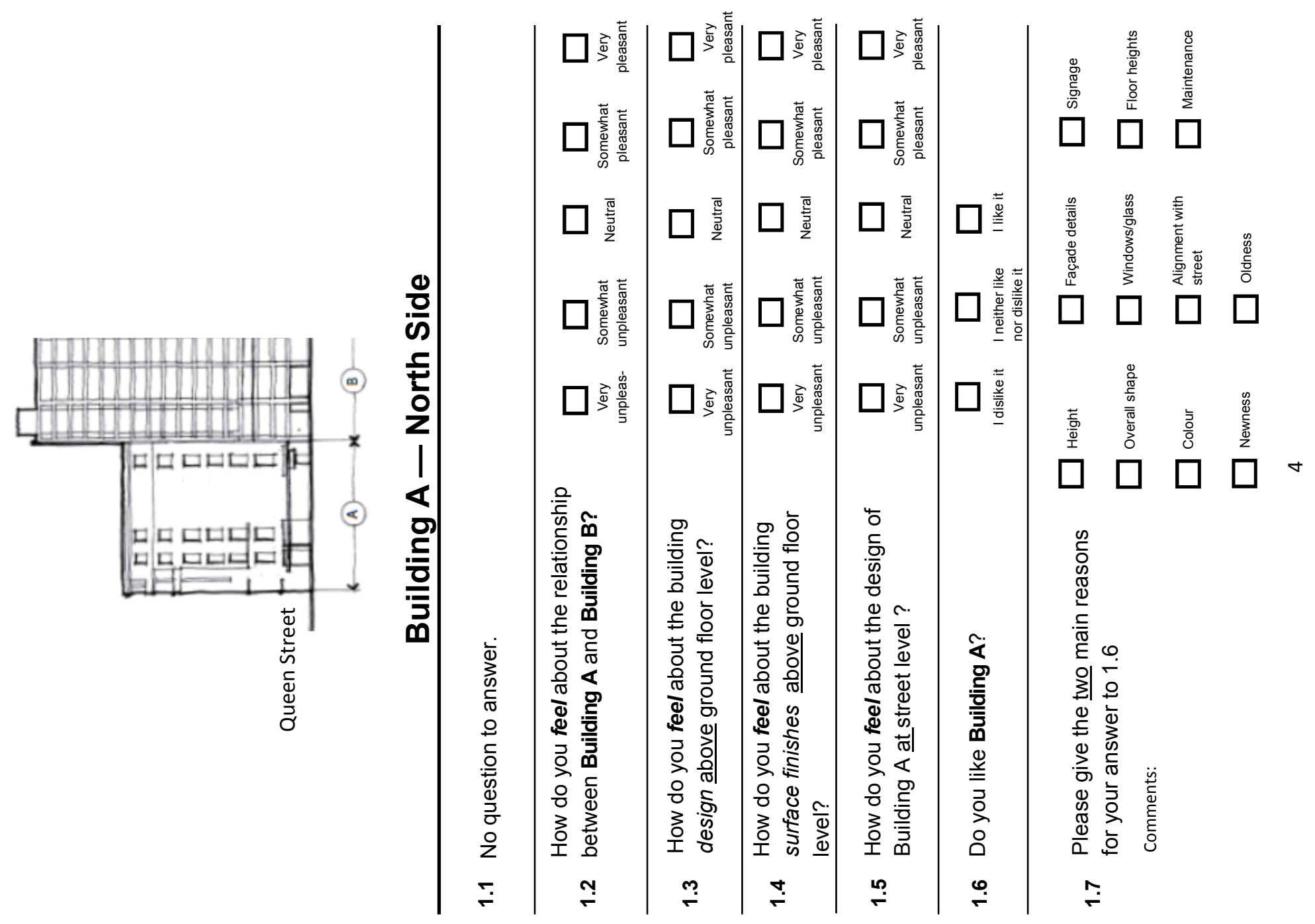

әэе|d นеسо!ำ
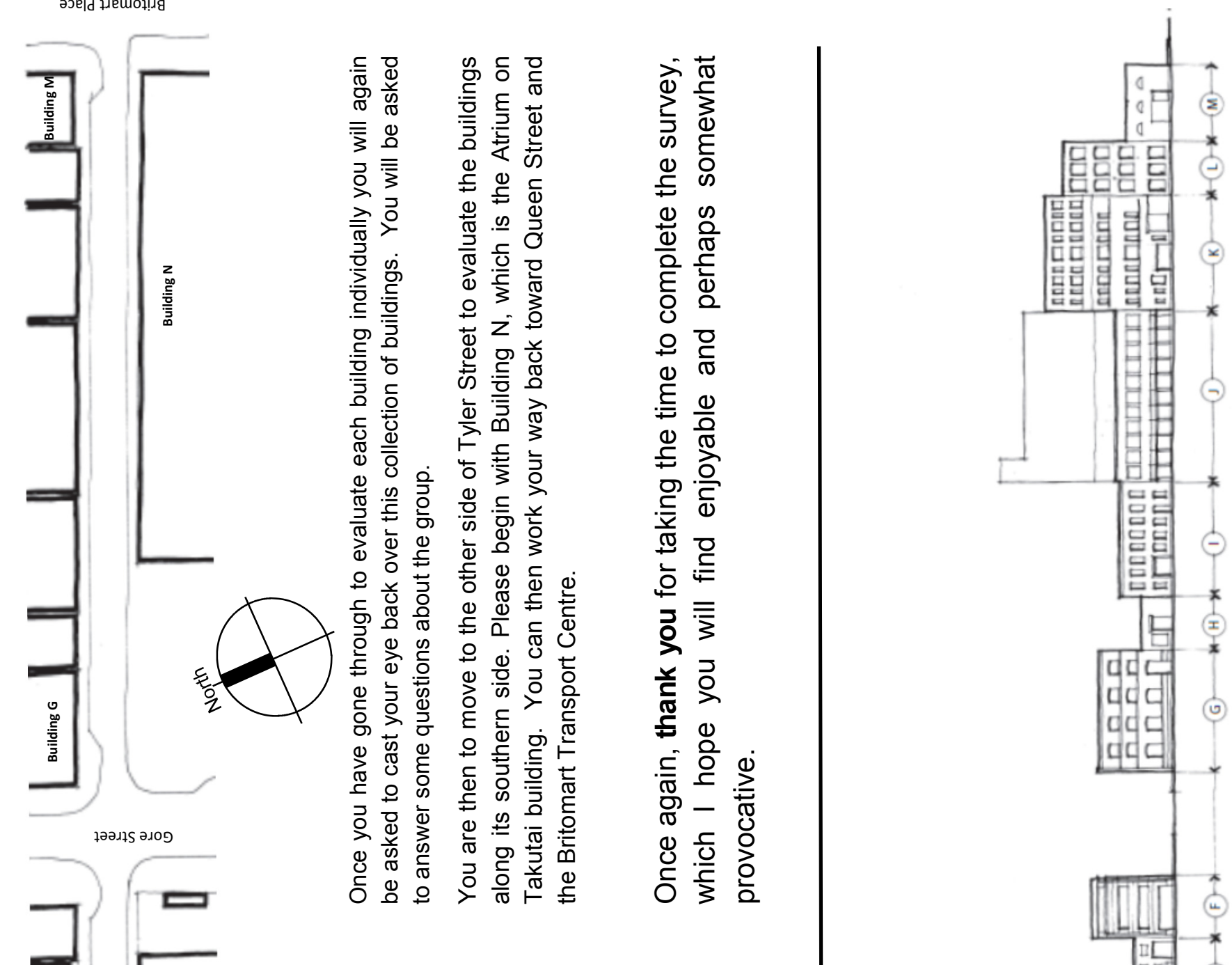


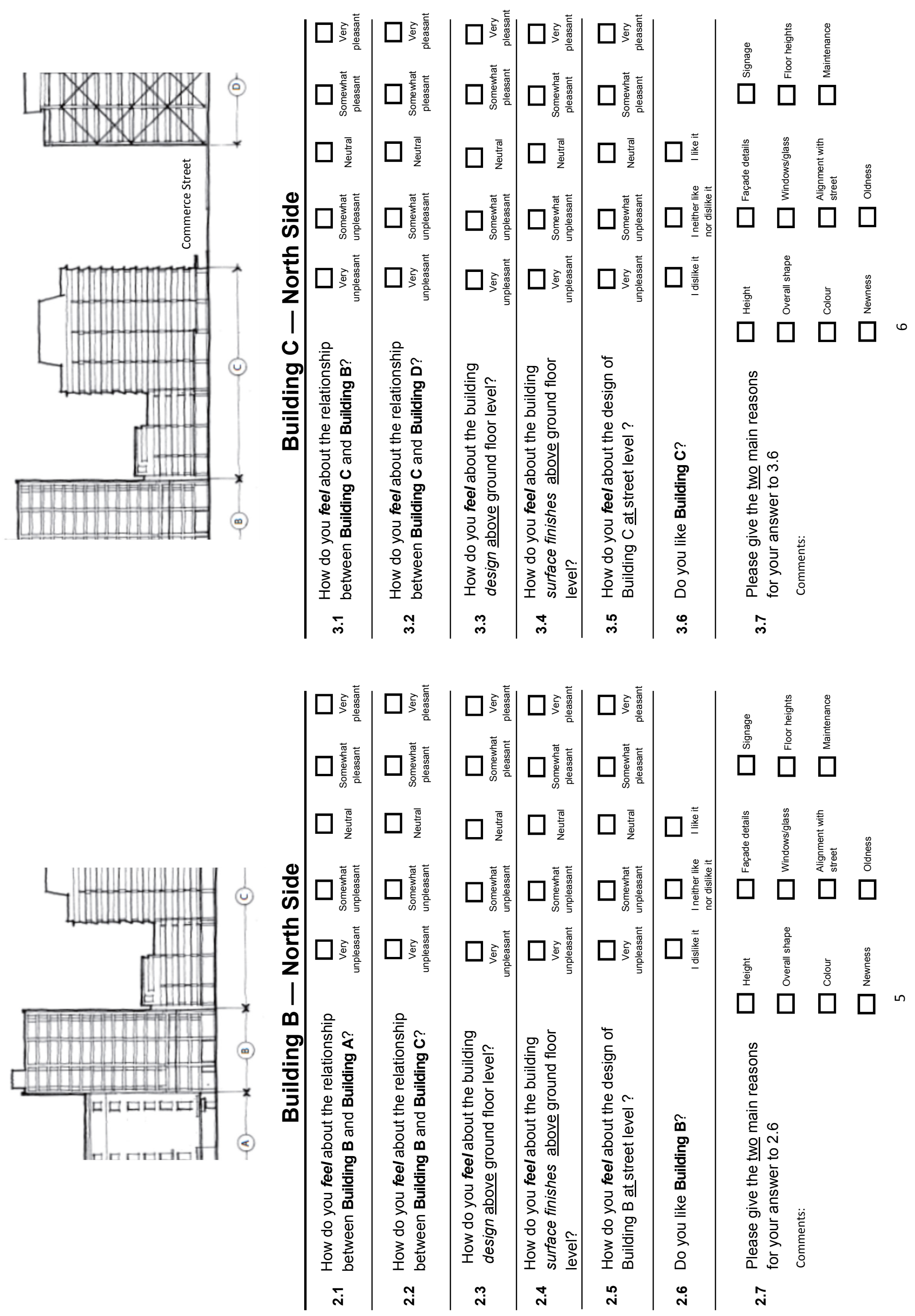




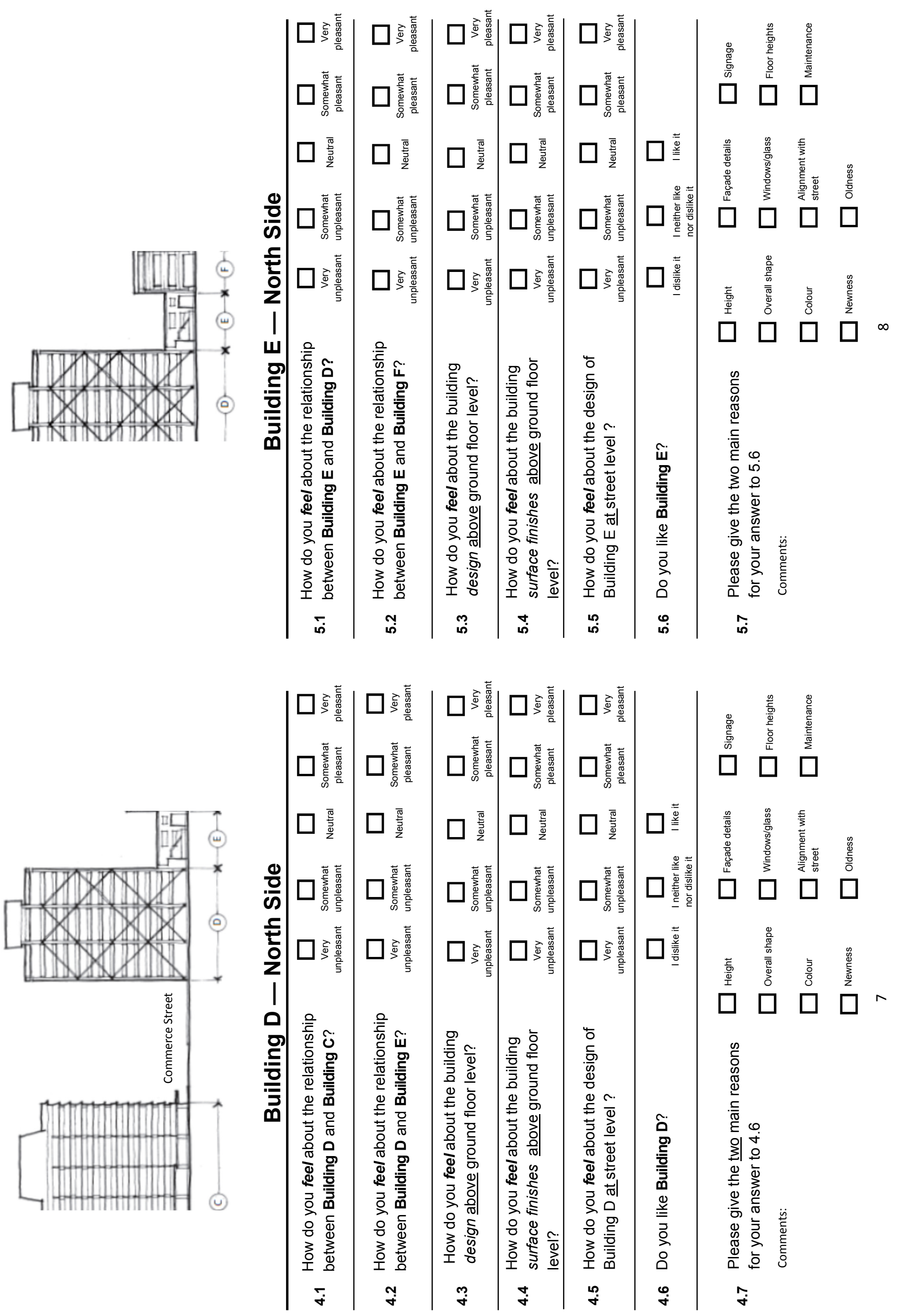




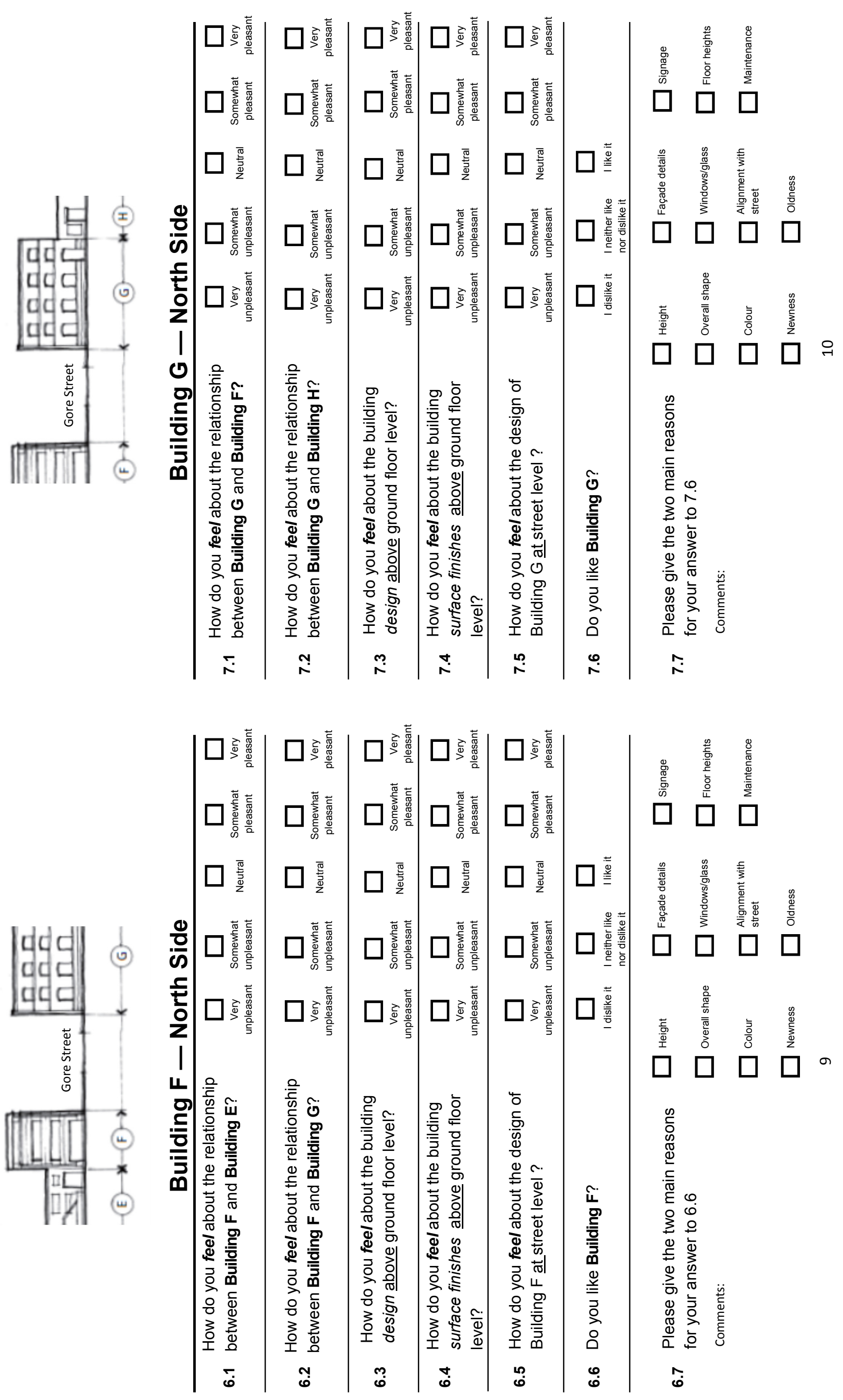




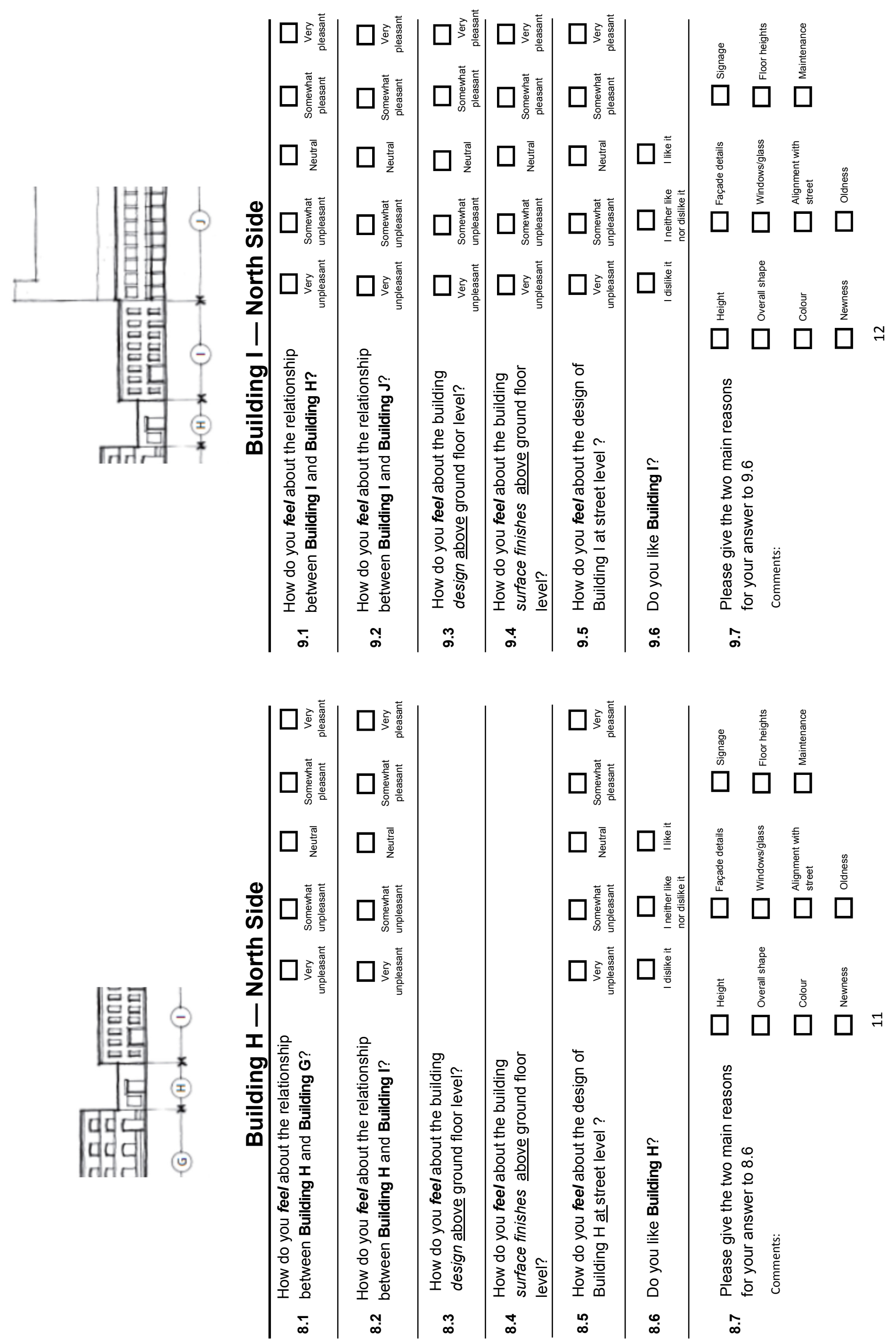




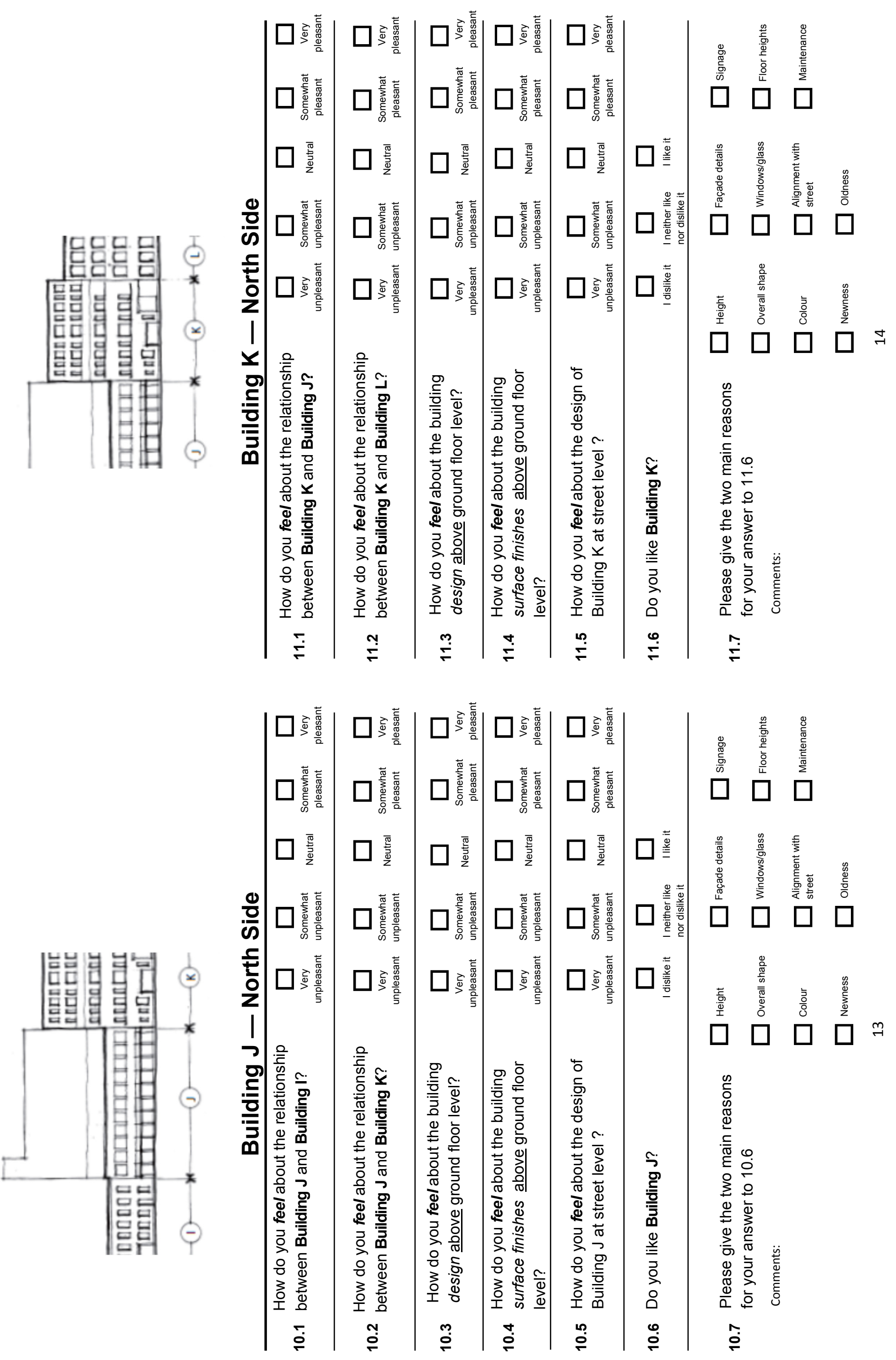



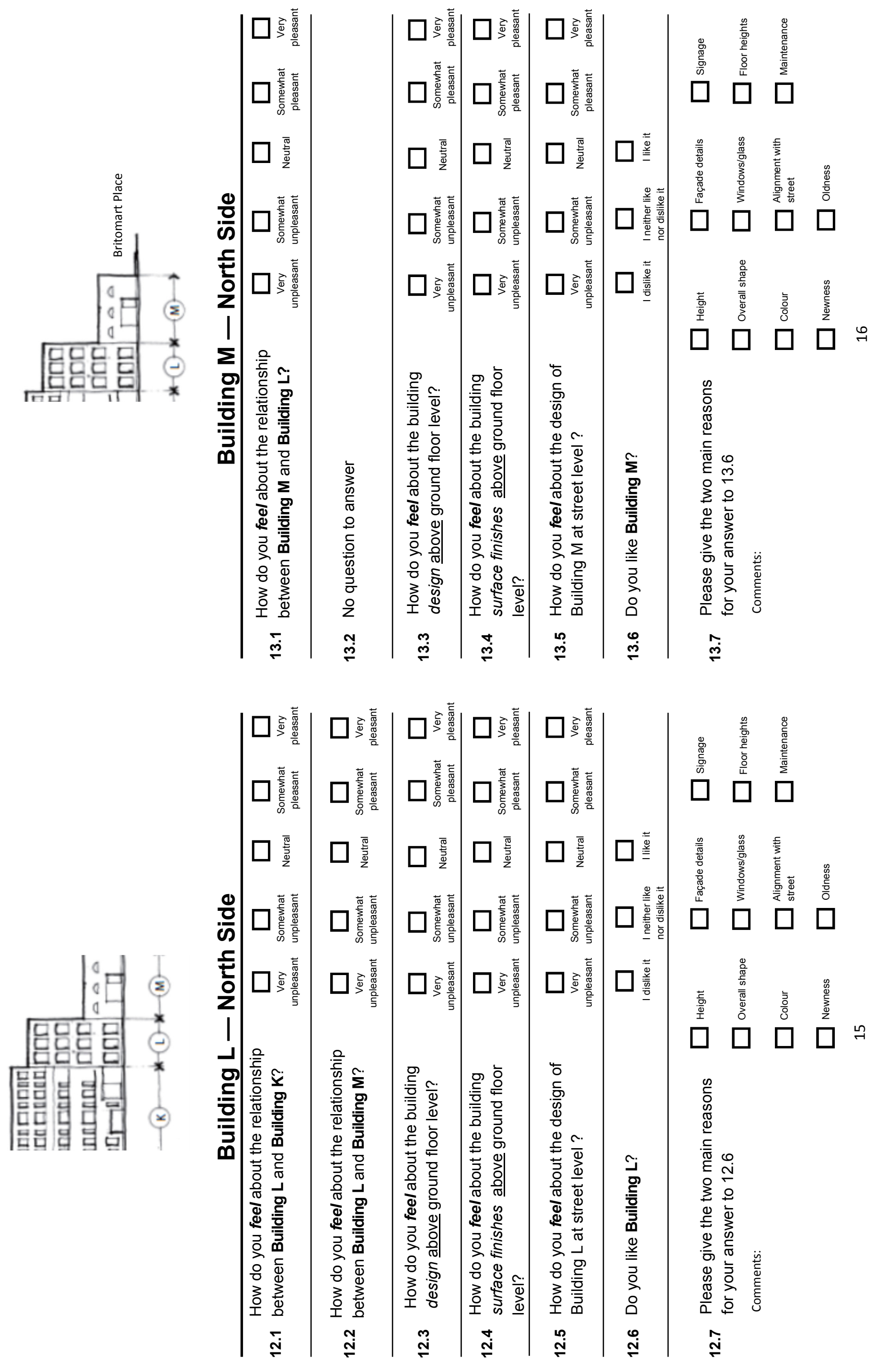


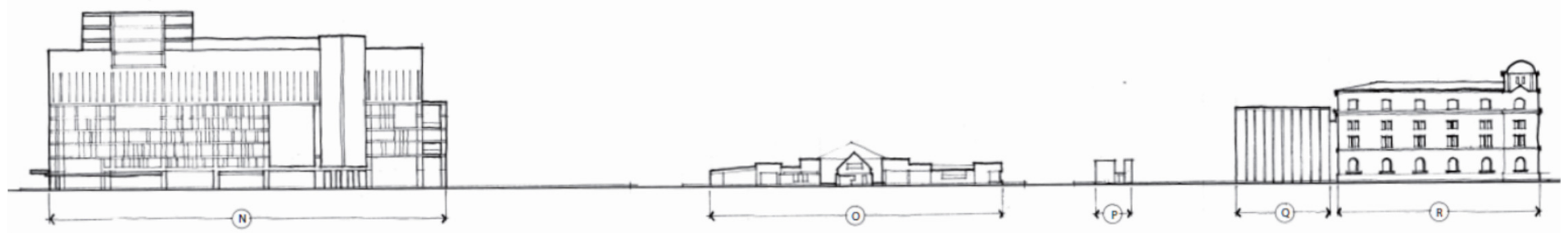

\section{Tyler Street —South Side}

As you now begin your return journey along to evaluate the buildings on the southern side of Tyler Street please cast your eye over the whole of the collection of building facades along this side. Think about the collection of buildings as a whole and how you feel about them all together.

Once you have gone through to evaluate each building individually you will be asked to cast your eye back over these buildings and to then answer some questions about them as a group.

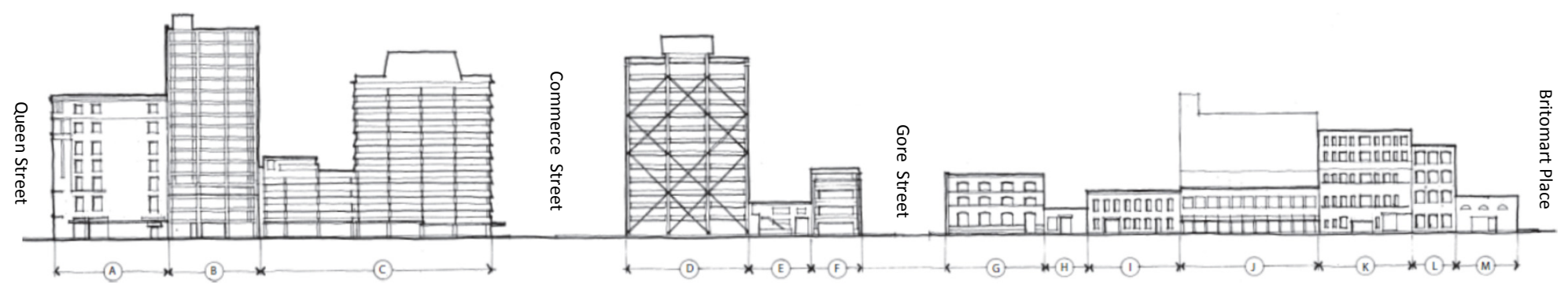

Tyler Street Block — North Side

14.1 How do you feel about the relationship between building heights along this side of the street?
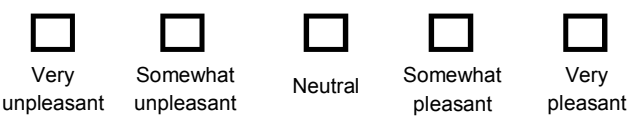

14.2 Please state briefly the principal reason for your response to question 7.1

14.3

How do you feel about the alignment [in and out] between building facades along the length of this side of the street?

\section{$\square$ Very unpleasant unpleasant}

\section{$\square$}

$\square$

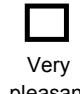

pleasant

pleasant

14.4 How do you feel about the relationship between façade design styles along this side of the street?

$\underset{\substack{\text { Very } \\ \text { unpleasant }}}{\square}$



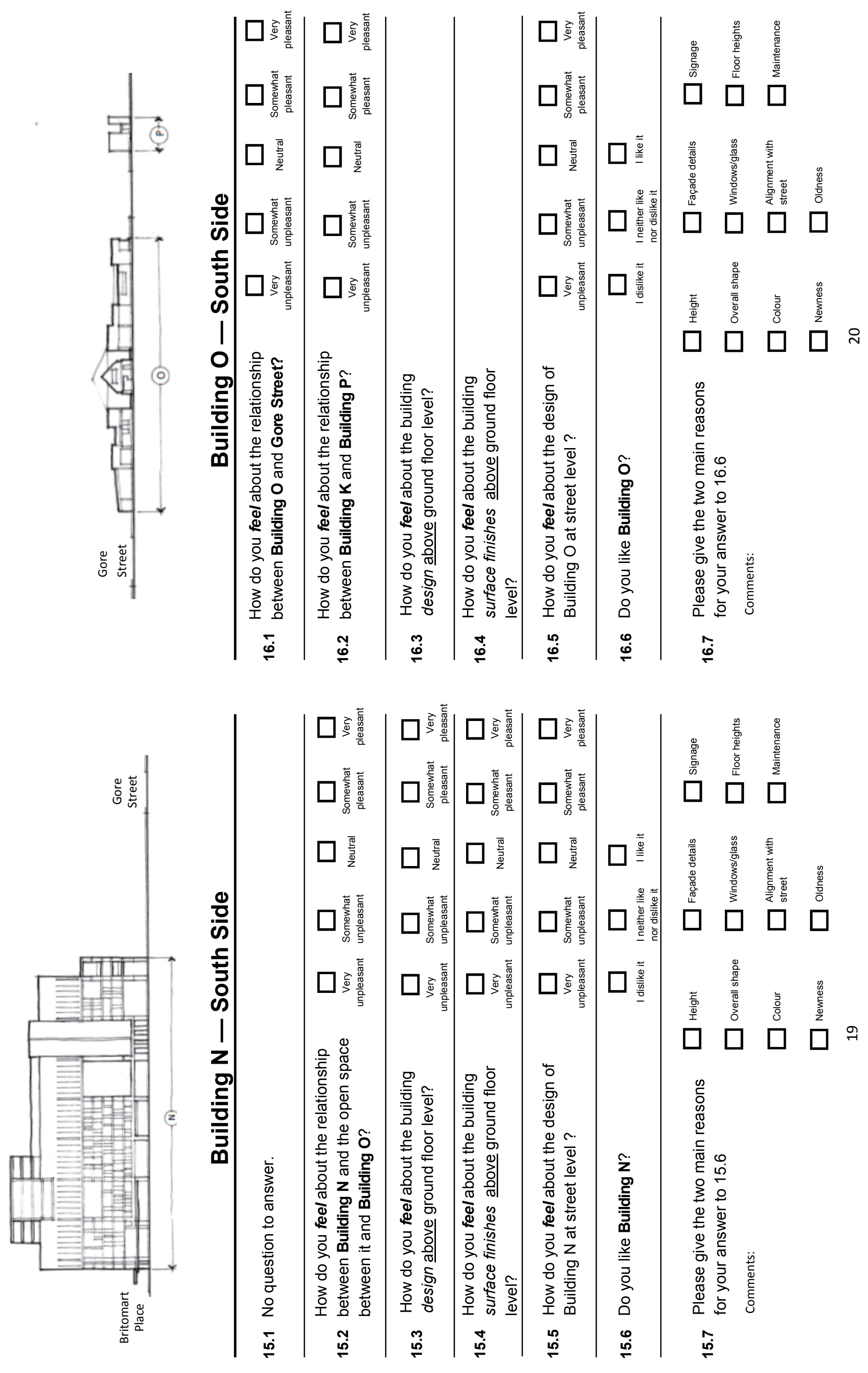


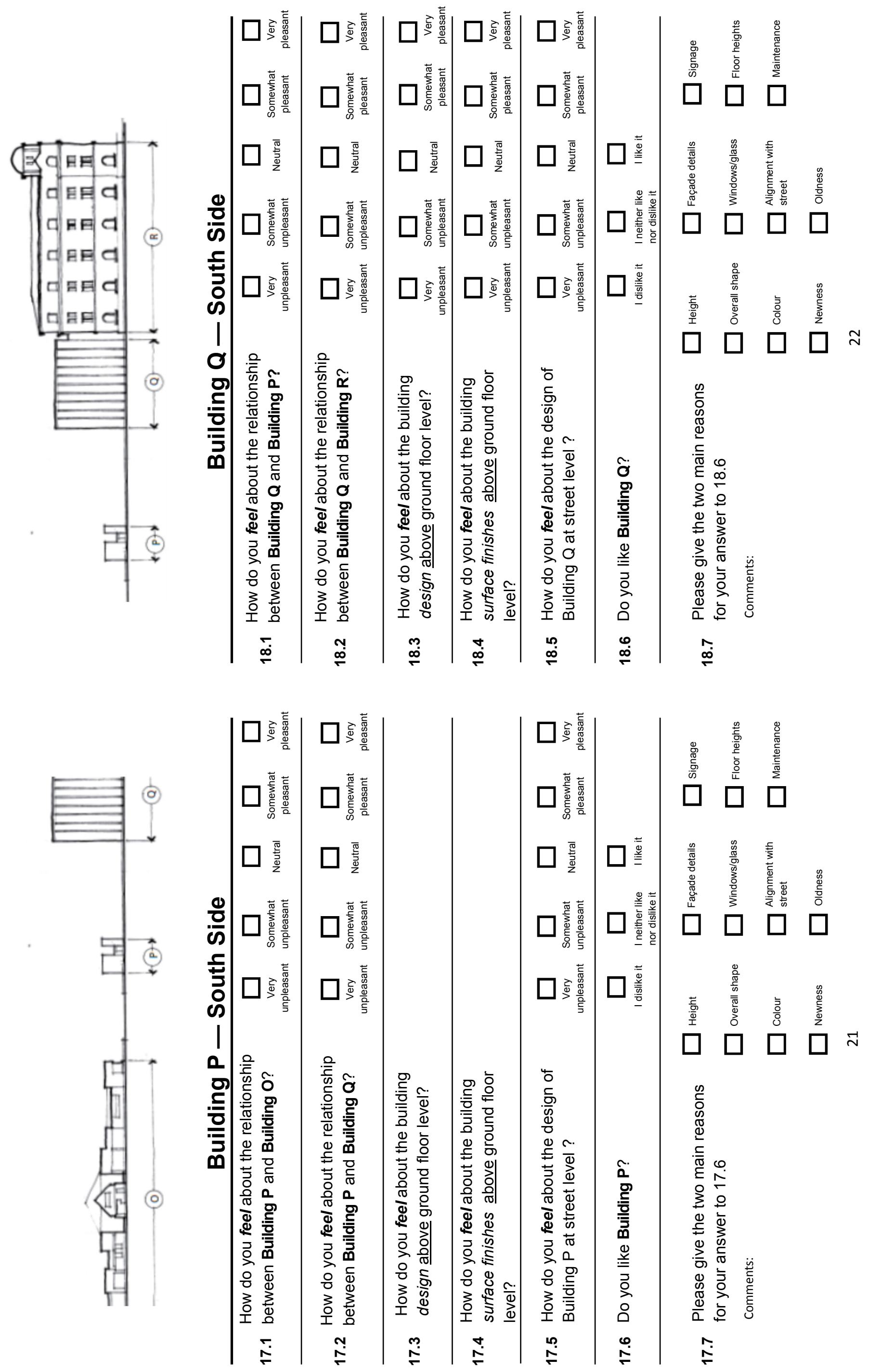




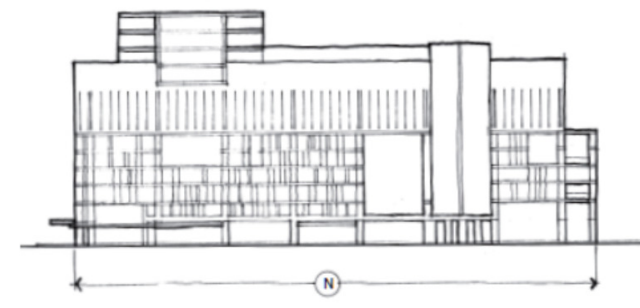

Tyler Street —South Side

20.1 How do you feel about the relationship between building heights

along this side of the street?

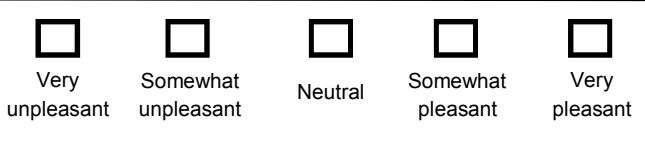

20.2 Please state briefly the principal reason for your response to question 10.1

How do you feel about the alignment [in and out] between building facades along the length of this side of the street?

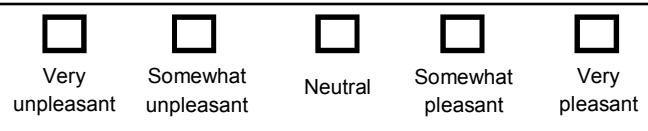

20.4 How do you feel about the relationship between façade design styles along this side of the street?

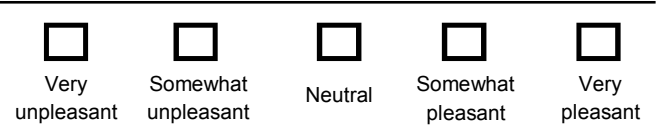
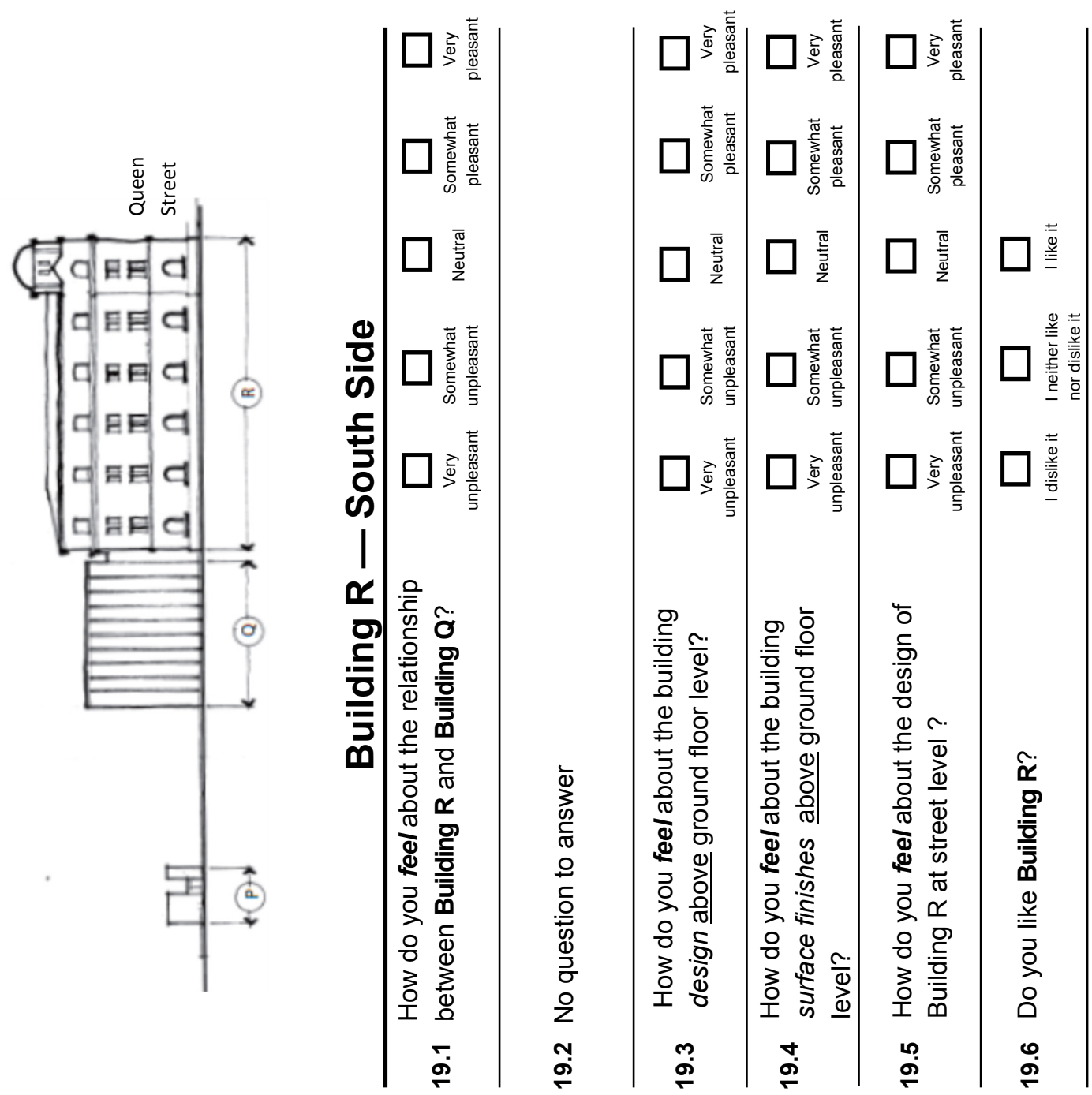
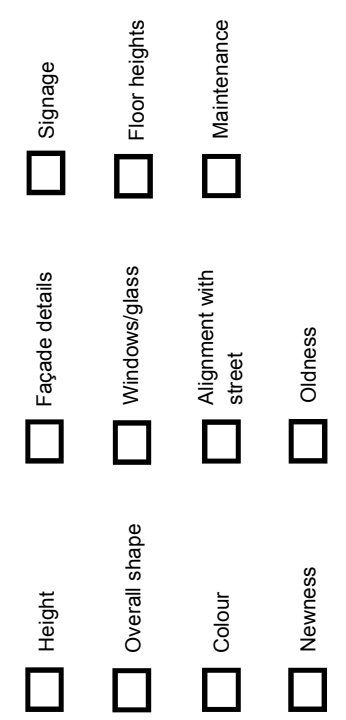

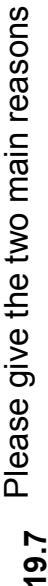

 


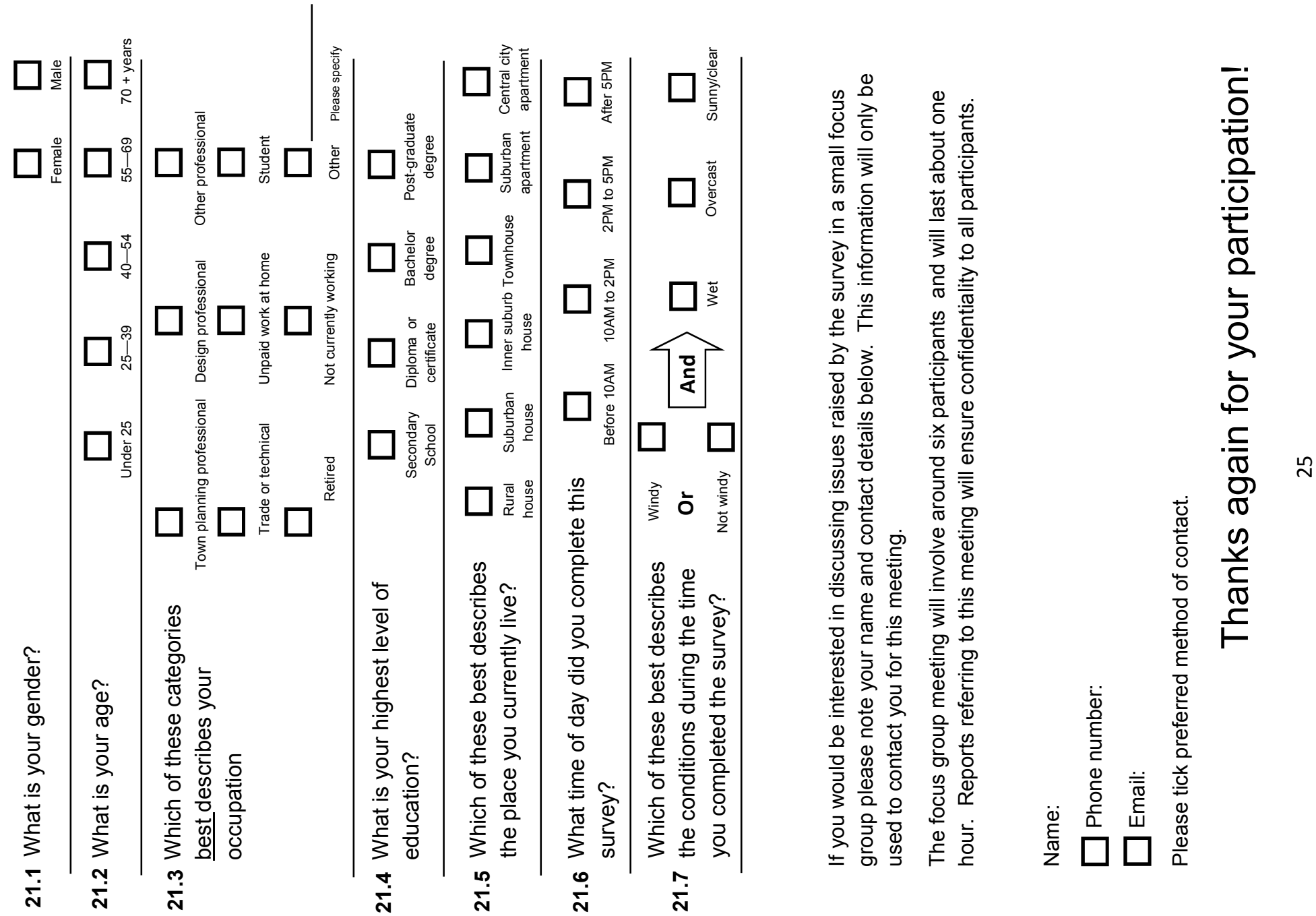




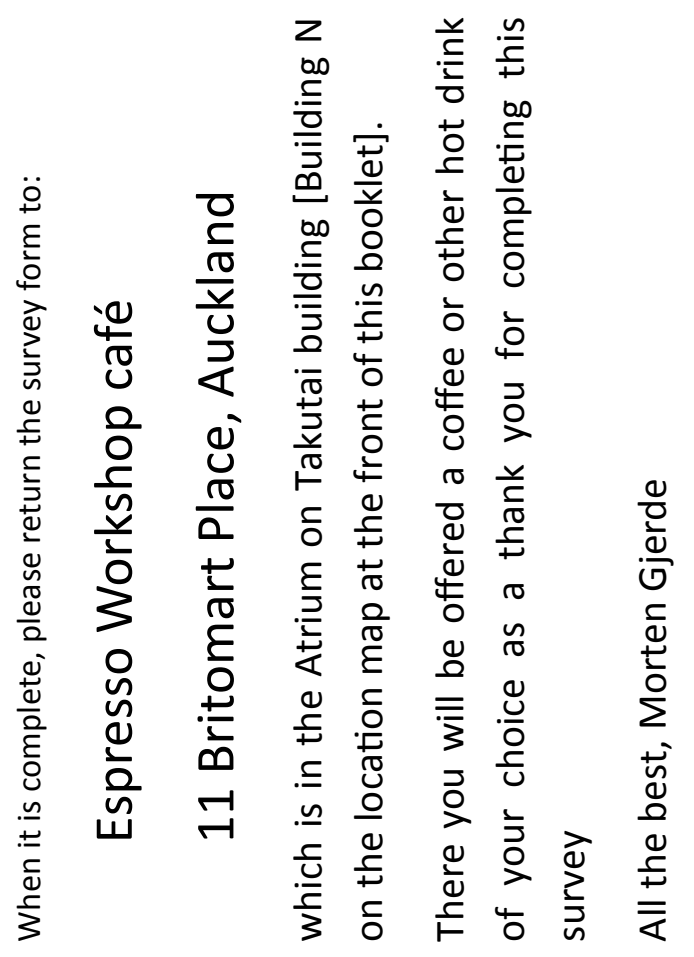



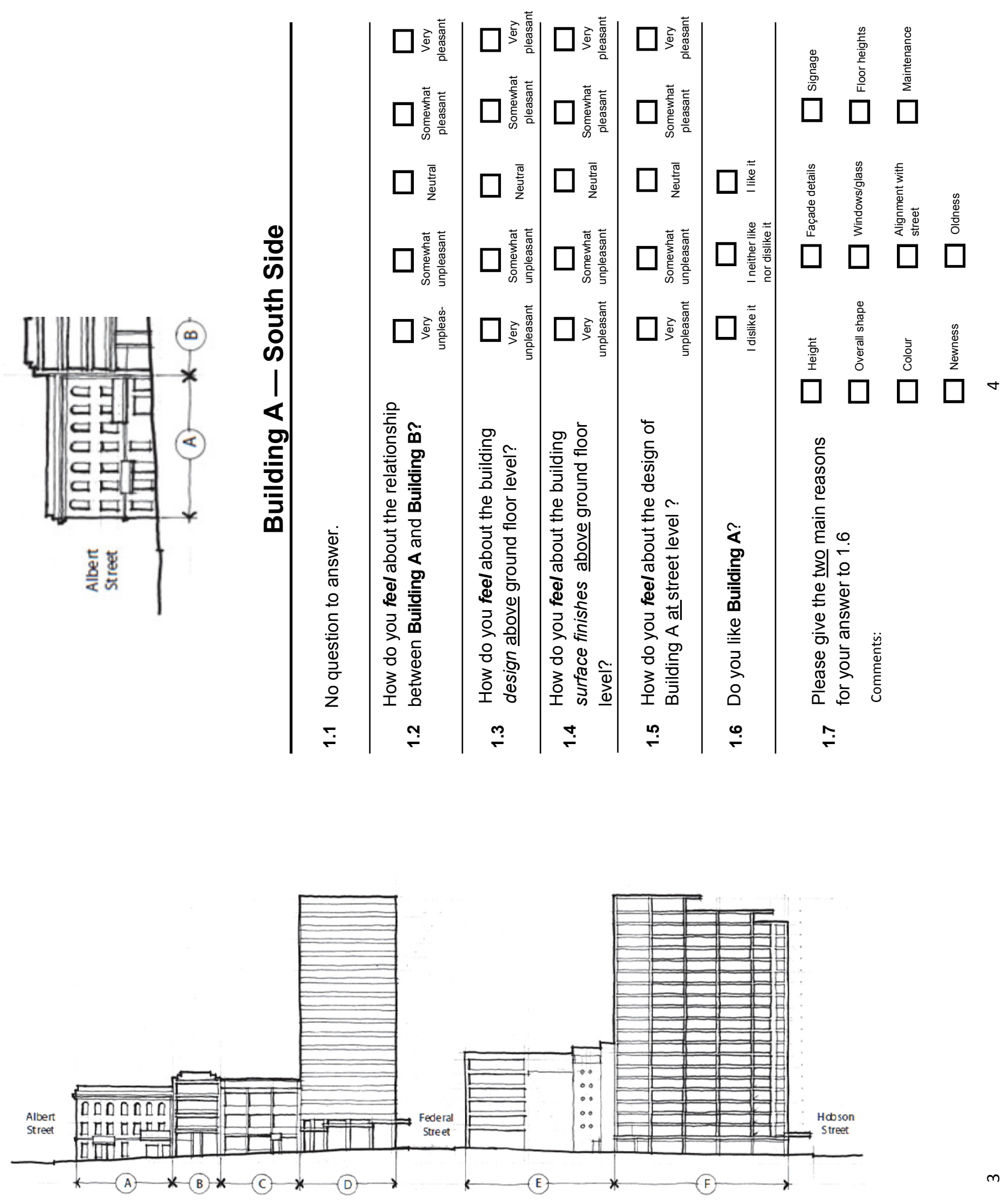

Kingston Street Block-South Side

As you start out at the eastern end of the Kingston Street block [corner with Albert Street] please cast your eye over the whole of the collection of building facades along the southern side, illustrated above. Think about the collection of buildings as a whole and how you feel about them all together.

Once you have gone through to evaluate each building individually you will again be asked to cast your eye back over this collection of buildings. You will be asked to answer some questions about the group. 


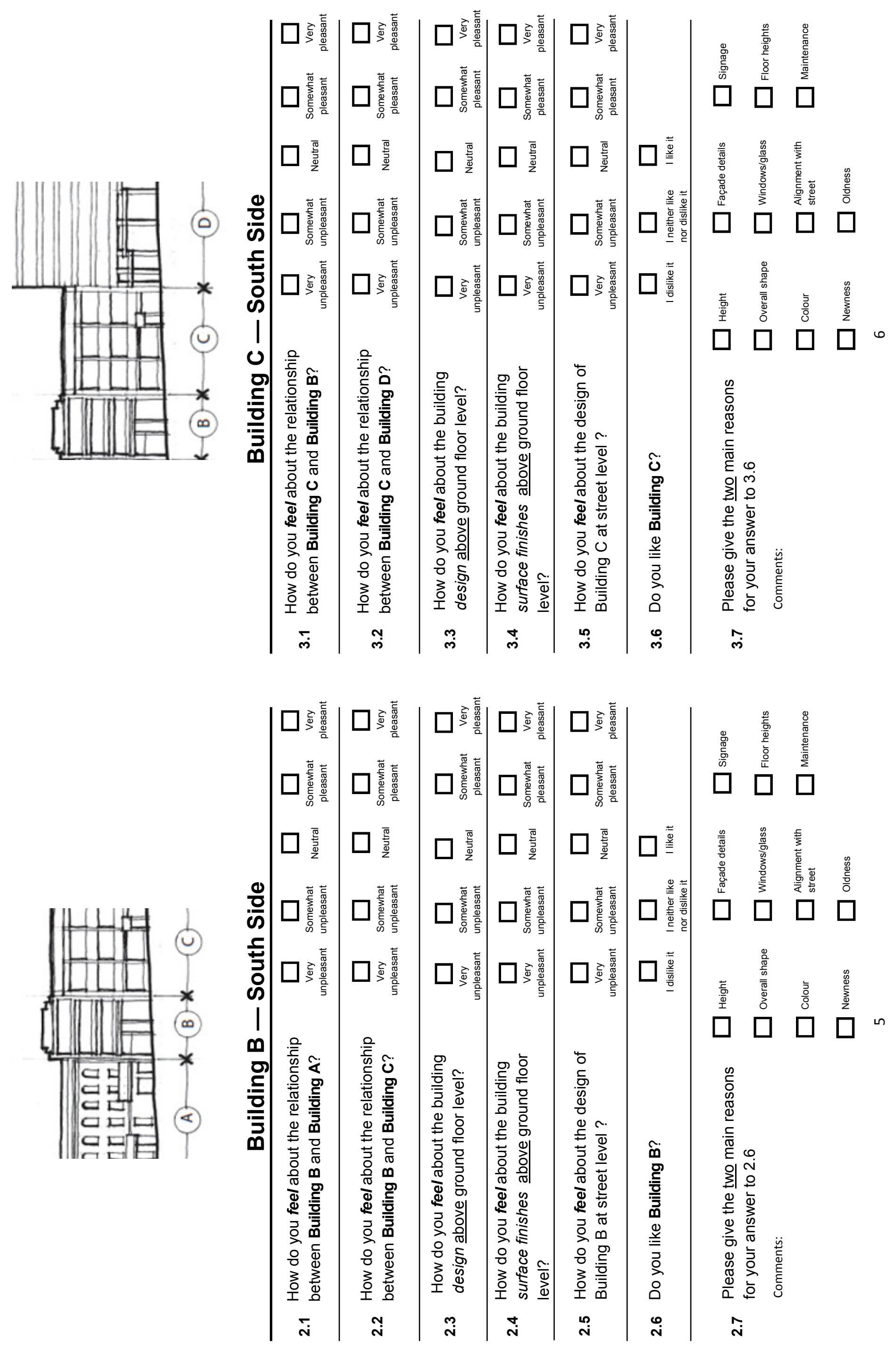




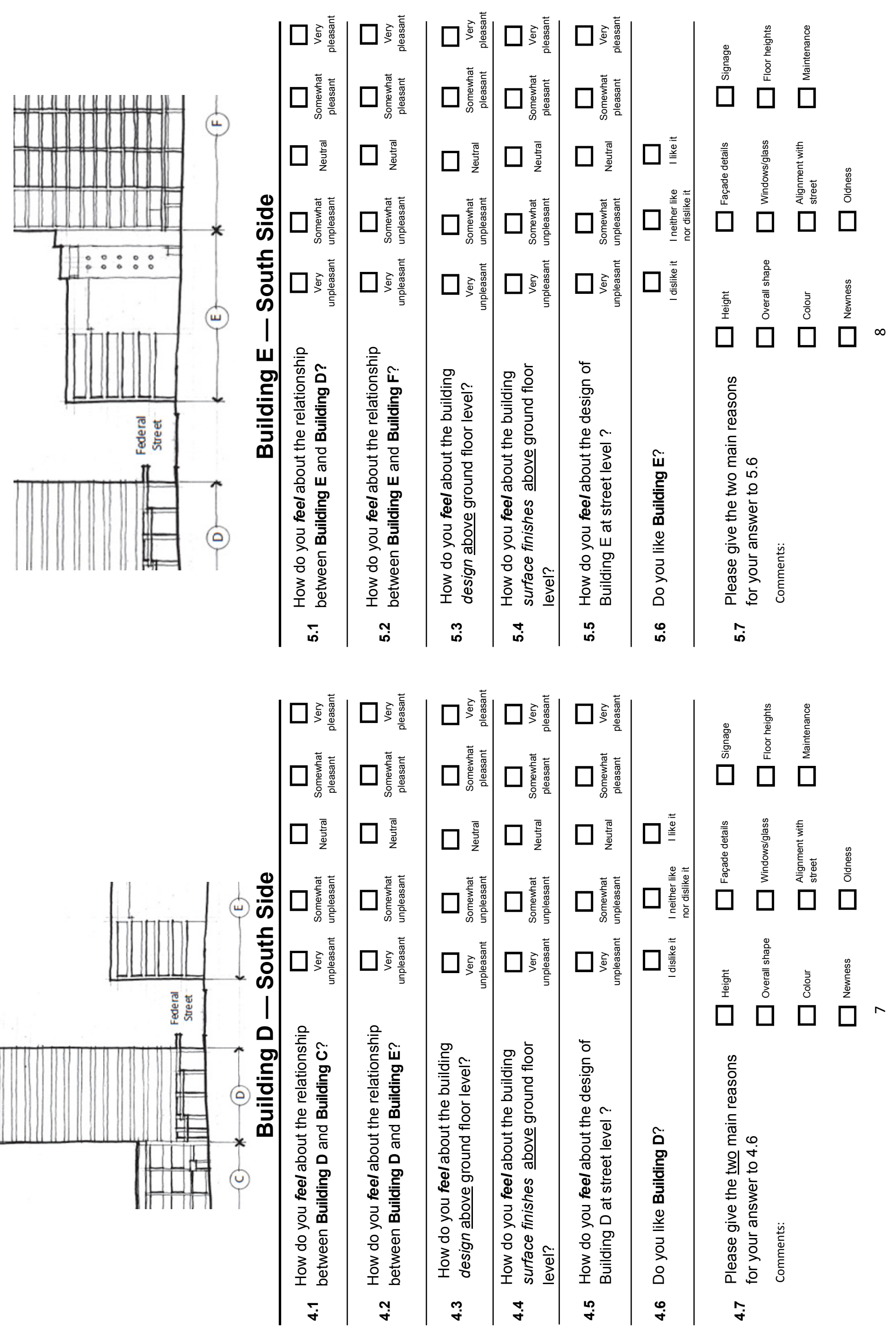




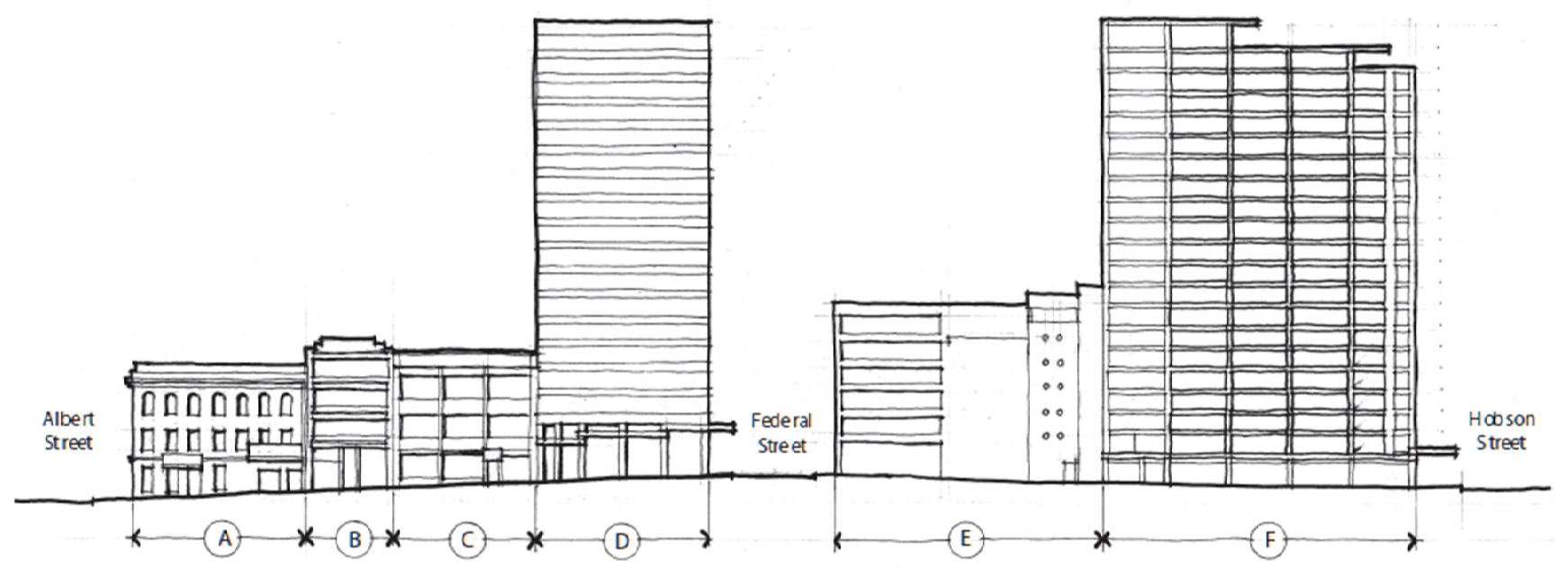

\section{Kingston Street Block - South Side}

7.1 How do you feel about the relationship between building heights along this side of the street?

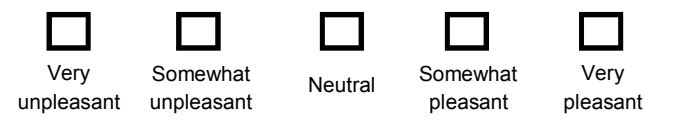

7.2 Please state briefly the principal reason for your response to question 7.1

7.3 How do you feel about the alignment [in and out] between building facades along the length of this side of the street?

Very
unpleasant
$\begin{gathered}\text { Somewhat } \\ \text { unpleasant }\end{gathered}$

7.4 How do you feel about the relationship between façade design styles along this side of the street?

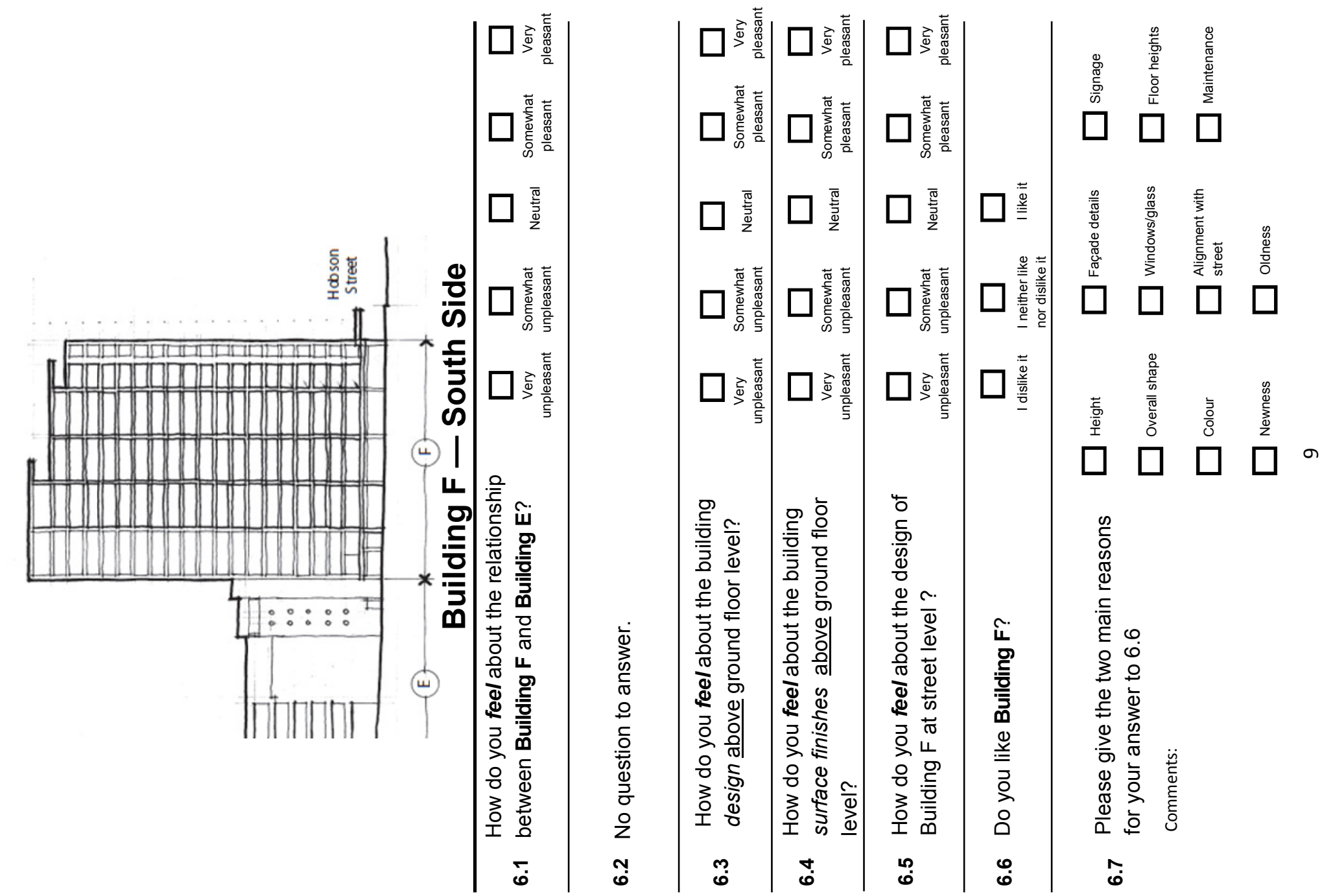



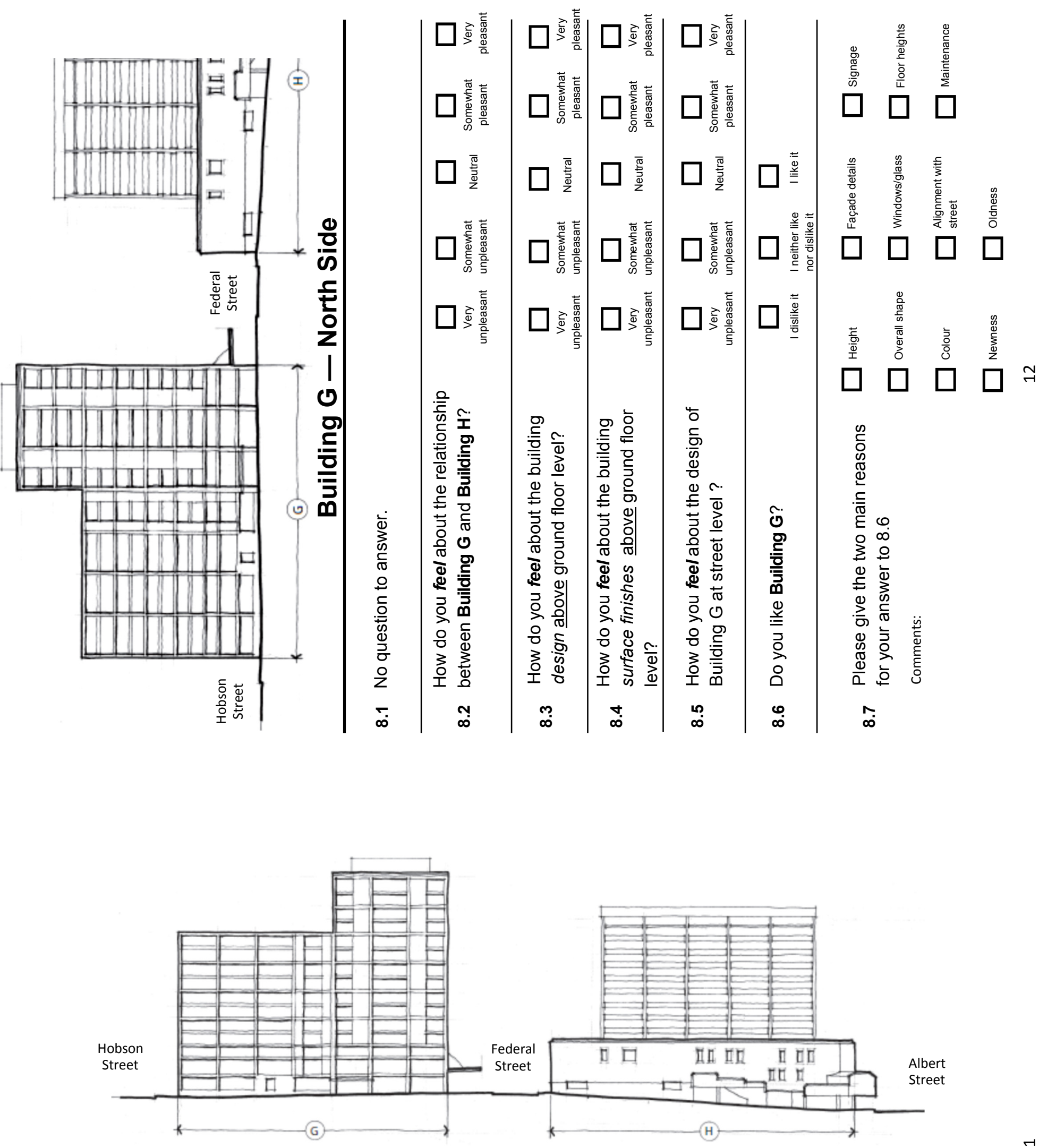

Kingston Street Block Block-North Side

As you now begin your return journey along to evaluate the buildings on the northern side of Kingston Street please cast your eye over the whole of the collection of building facades along this side. Think about the collection of buildings as a whole and how you feel about them all together.

Once you have gone through to evaluate each building individually you will be asked to cast your eye back over these buildings and to then answer some questions about them as a group. 


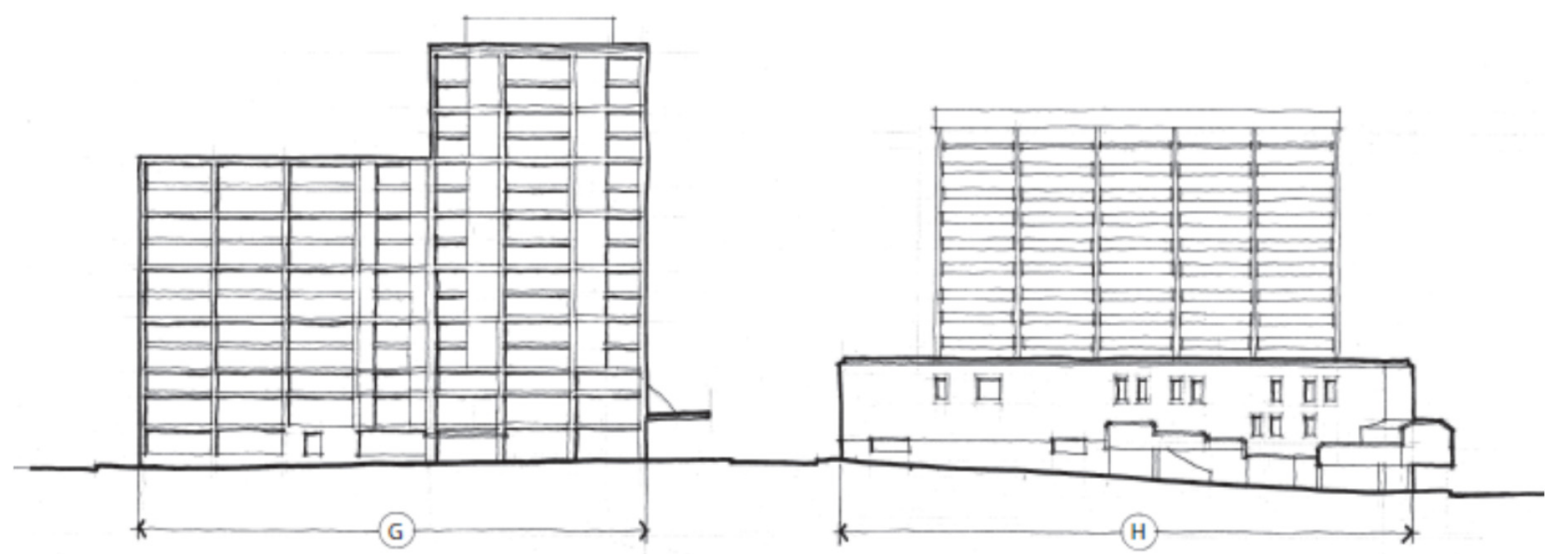

Kingston Street Block-North Side

10.1 How do you feel about the relationship between building heights along this side of the street?

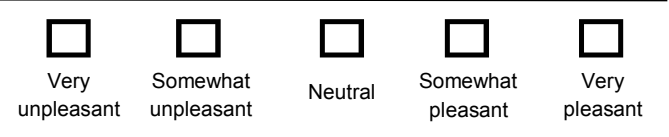

10.2 Please state briefly the principal reason for your response to question 10.1

10.3 How do you feel about the alignment [in and out] between building facades along the length of this side of the street?

\begin{tabular}{|c|c|c|c|c|}
\hline$\square$ & $\square$ & $\square$ & $\square$ & $\square$ \\
\hline $\begin{array}{c}\text { Very } \\
\text { unpleasant }\end{array}$ & $\begin{array}{l}\text { Somewhat } \\
\text { unpleasant }\end{array}$ & Neutral & $\begin{array}{l}\text { Somewhat } \\
\text { pleasant }\end{array}$ & $\begin{array}{c}\text { Very } \\
\text { pleasant }\end{array}$ \\
\hline$\square$ & $\square$ & $\square$ & $\square$ & $\square$ \\
\hline $\begin{array}{l}\text { Very } \\
\text { unpleasant }\end{array}$ & $\begin{array}{l}\text { Somewhat } \\
\text { unpleasant }\end{array}$ & Neutral & $\begin{array}{c}\text { Somewhat } \\
\text { pleasant }\end{array}$ & $\begin{array}{c}\text { Very } \\
\text { pleasant }\end{array}$ \\
\hline
\end{tabular}

10.4 How do you feel about the relationship between façade design styles along this side of the street?
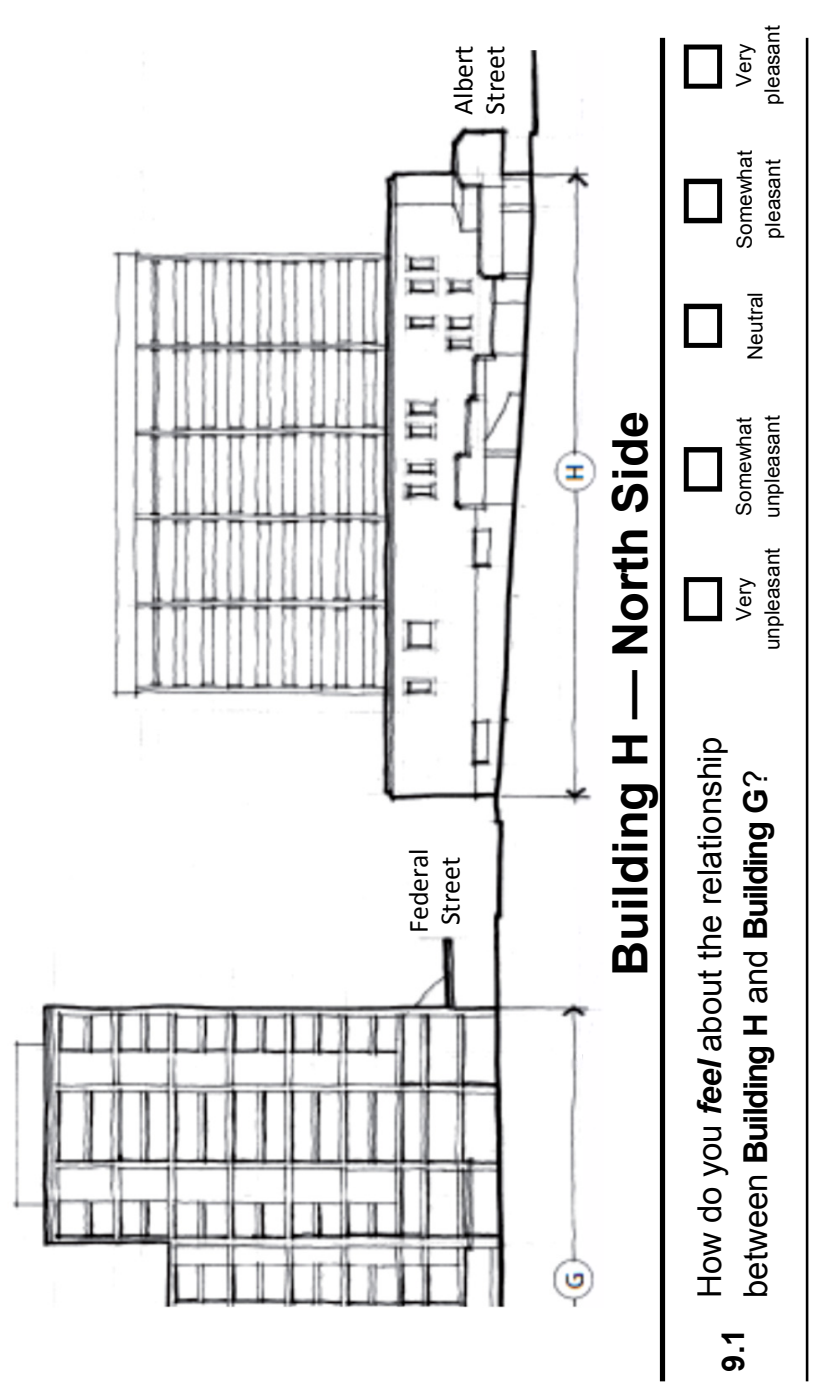

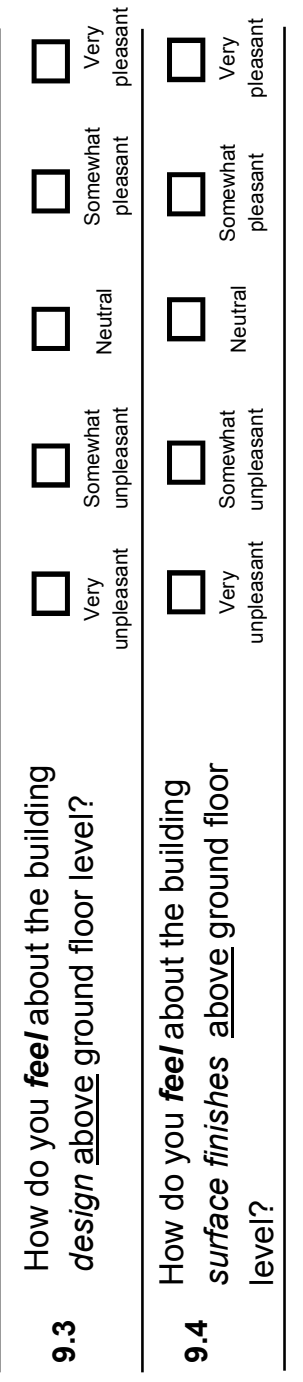

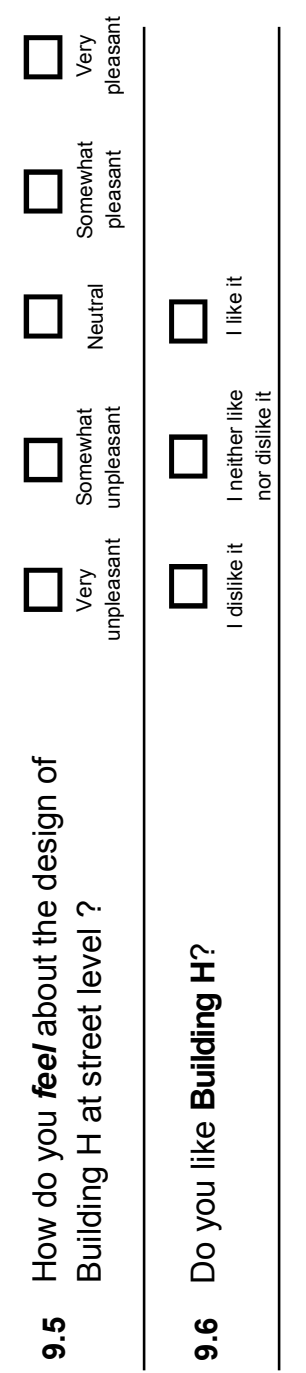

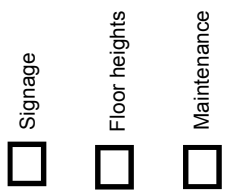

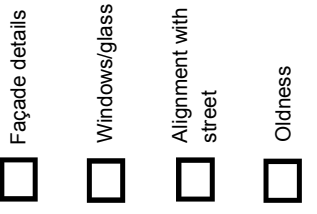

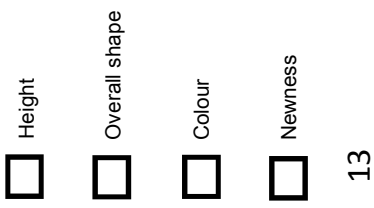

¿ू

(ల)

है

ஸे

인

()

1)

言

ฌ)

음 으

ตั 


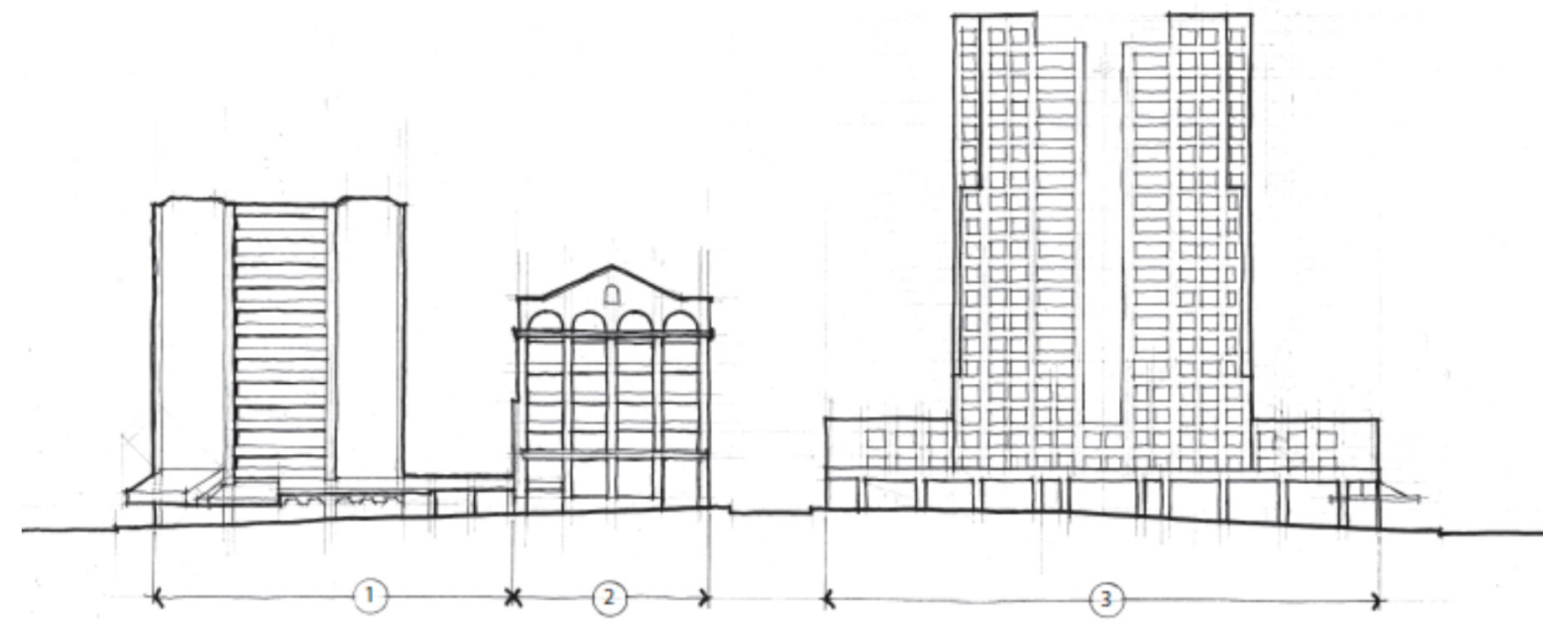

\section{Wyndham Street Block-South Side}

As you start out at the eastern end of the Wyndham Street block [corner with Albert Street] please cast your eye over the whole of the collection of building facades along the southern side, illustrated above. Think about the collection of buildings as a whole and how you feel about them all together.

Once you have gone through to evaluate each building individually you will again be asked to cast your eye back over this collection of buildings. You will be asked to answer some questions about the group.
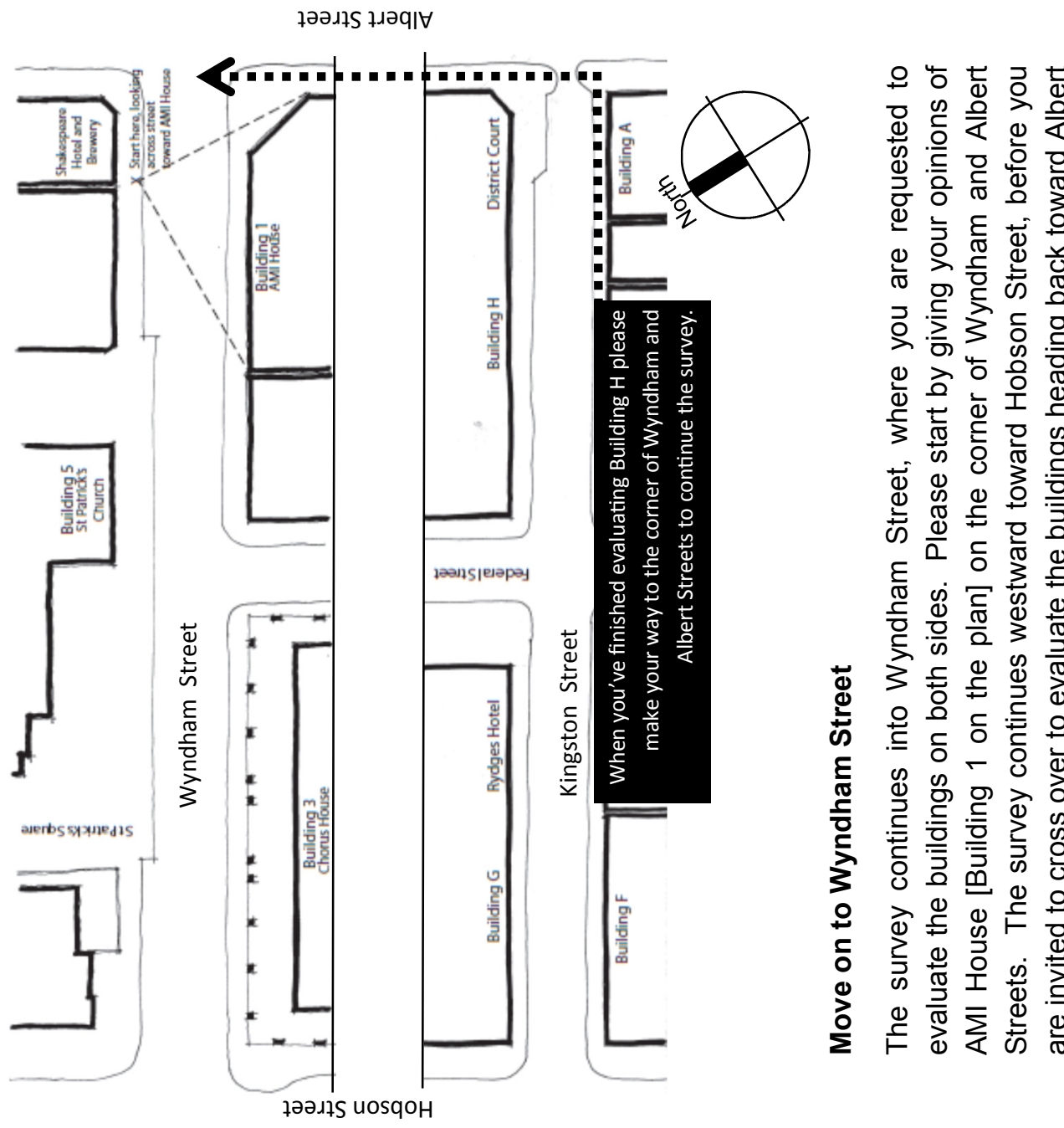

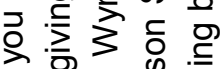

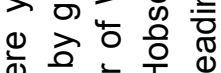

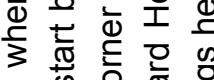

बं क ठ 己

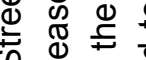

ब 등

हैं के

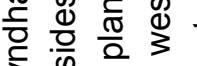

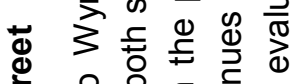

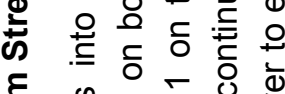

ह

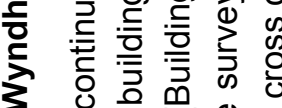

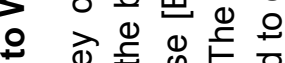

व

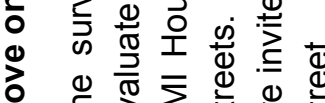

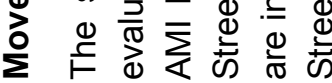




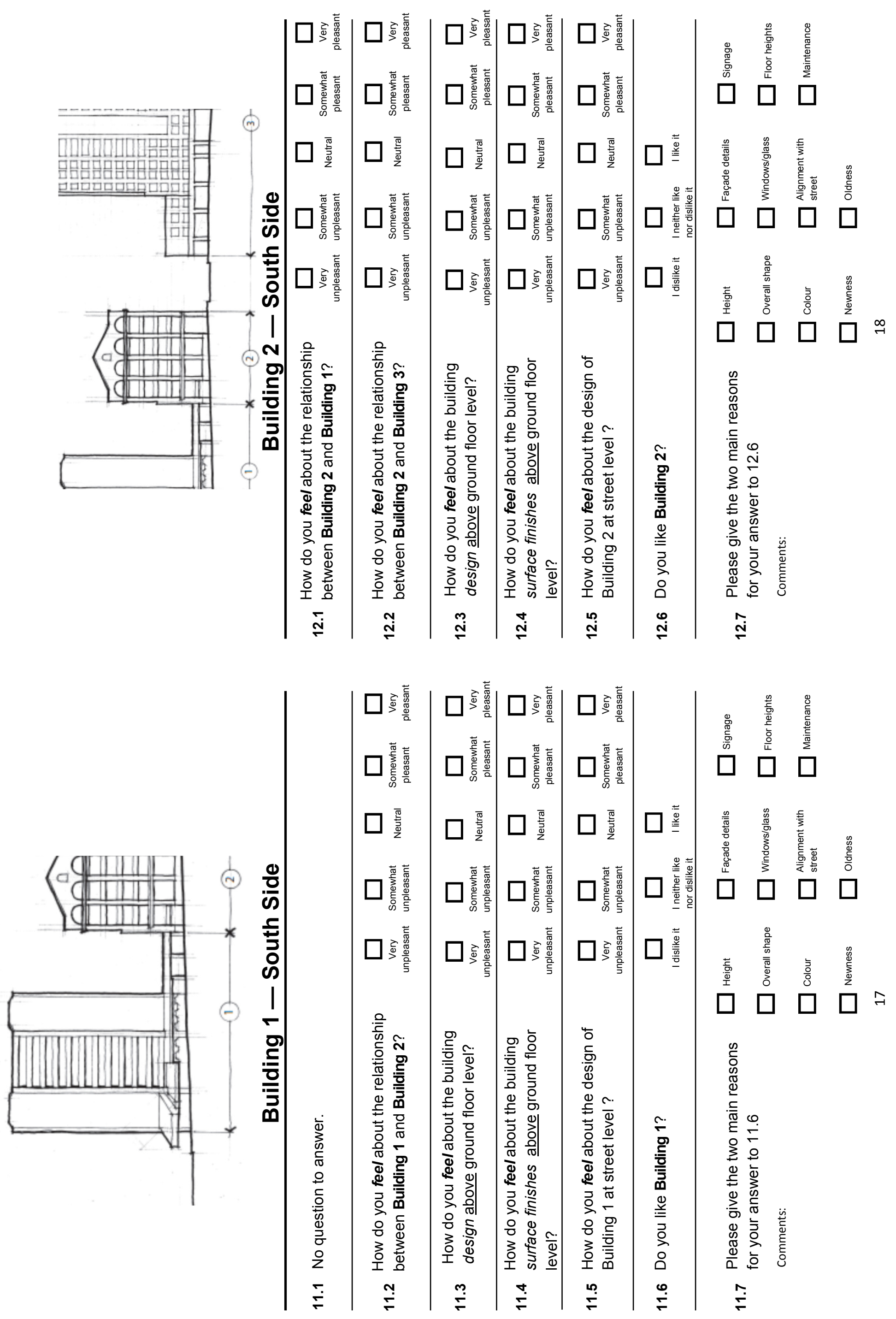




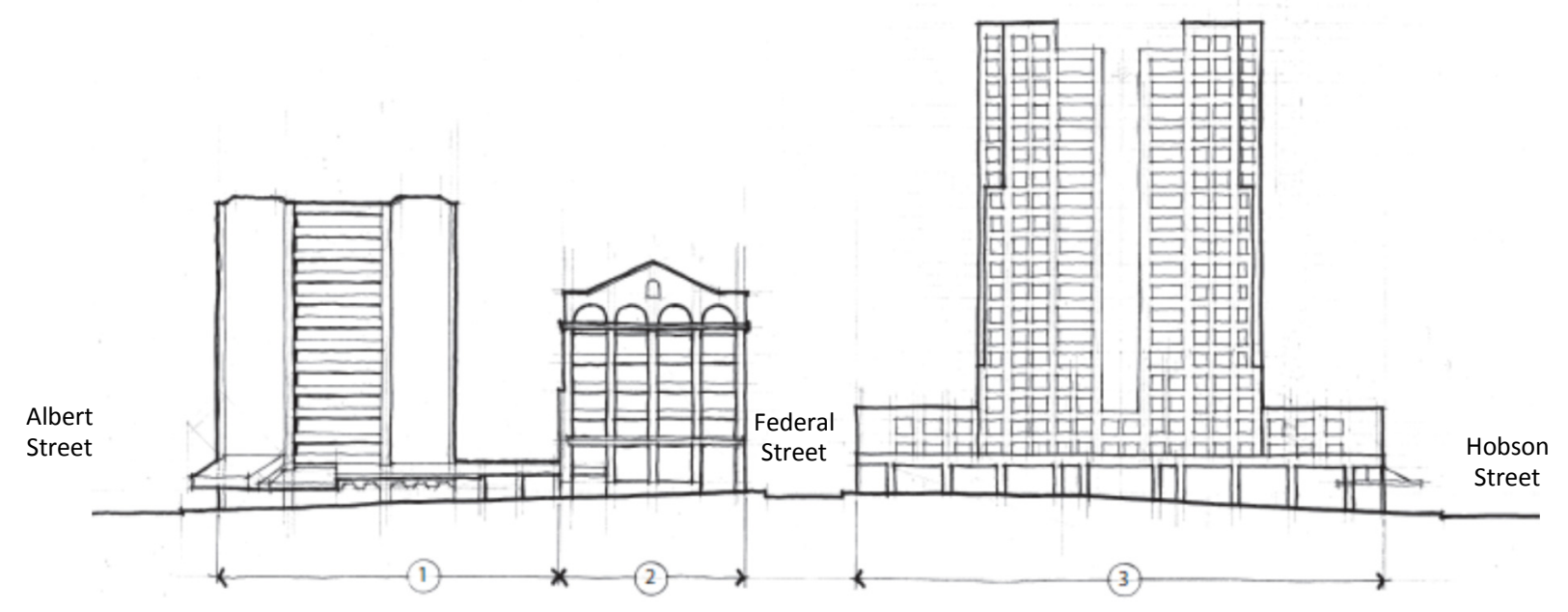

Wyndham Street Block-South Side

14.1 How do you feel about the relationship between building heights along this side of the street?

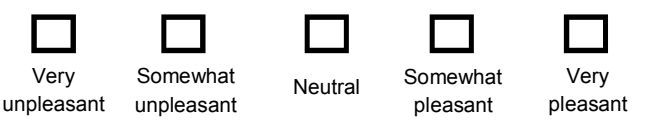

14.2 Please state briefly the principal reason

for your response to question 14.1

How do you feel about the alignment [in and out] between building

14.3 facades along the length of this side of the street?
How do you feel about the relationship between façade design styles along this side of the street?

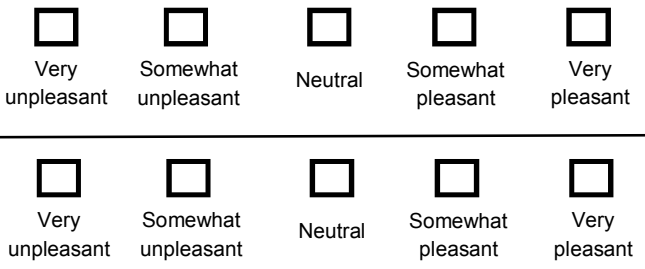

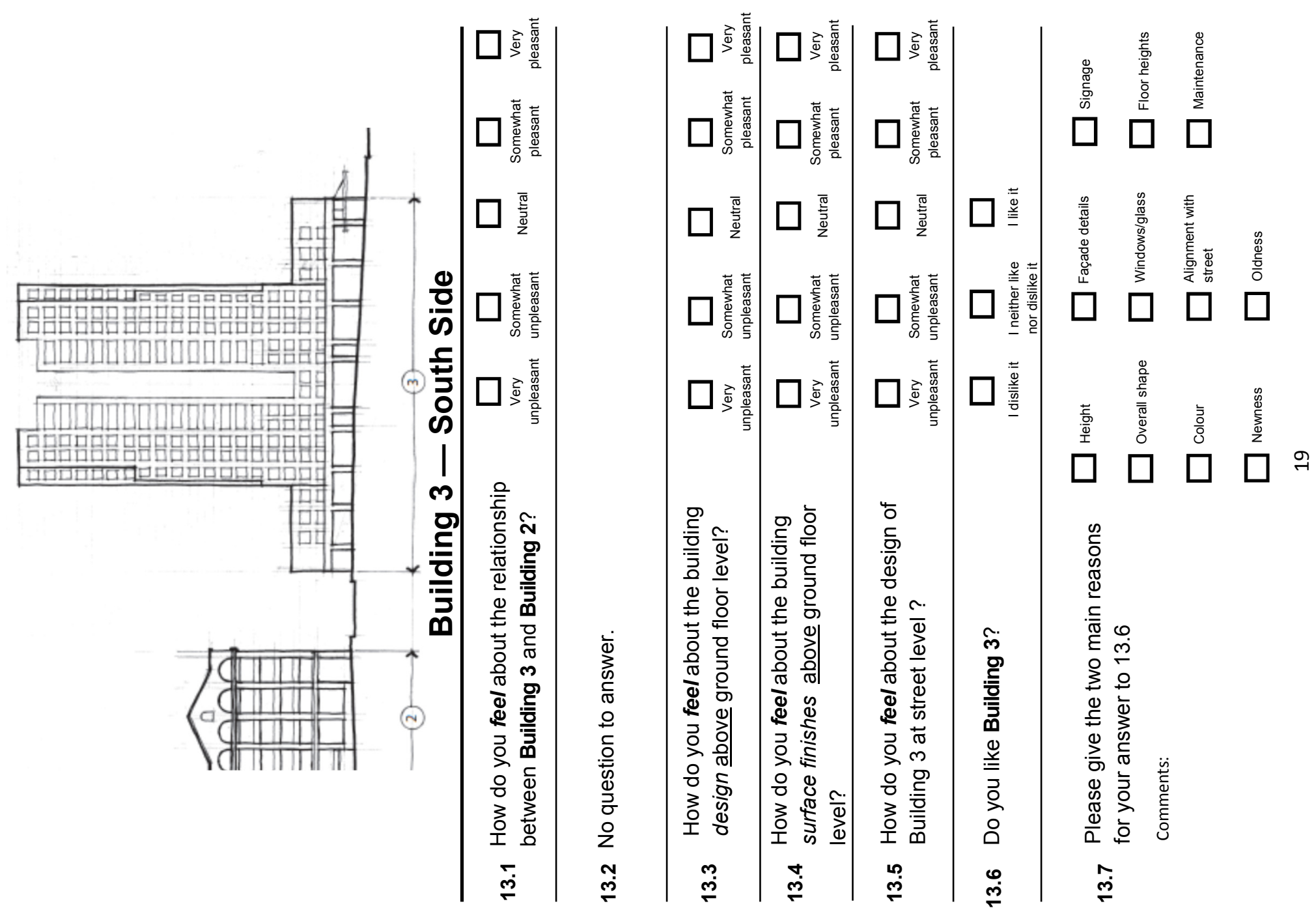




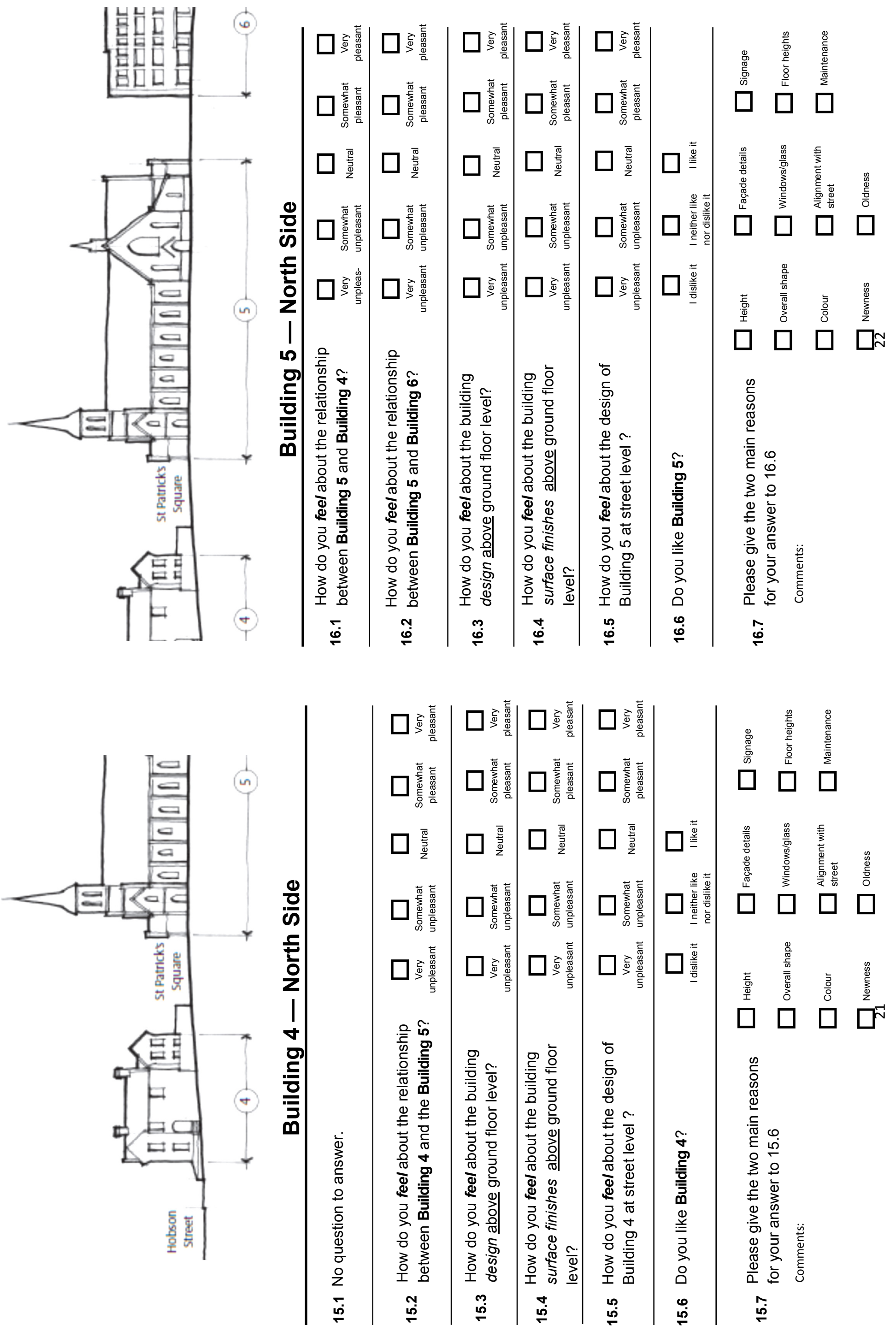



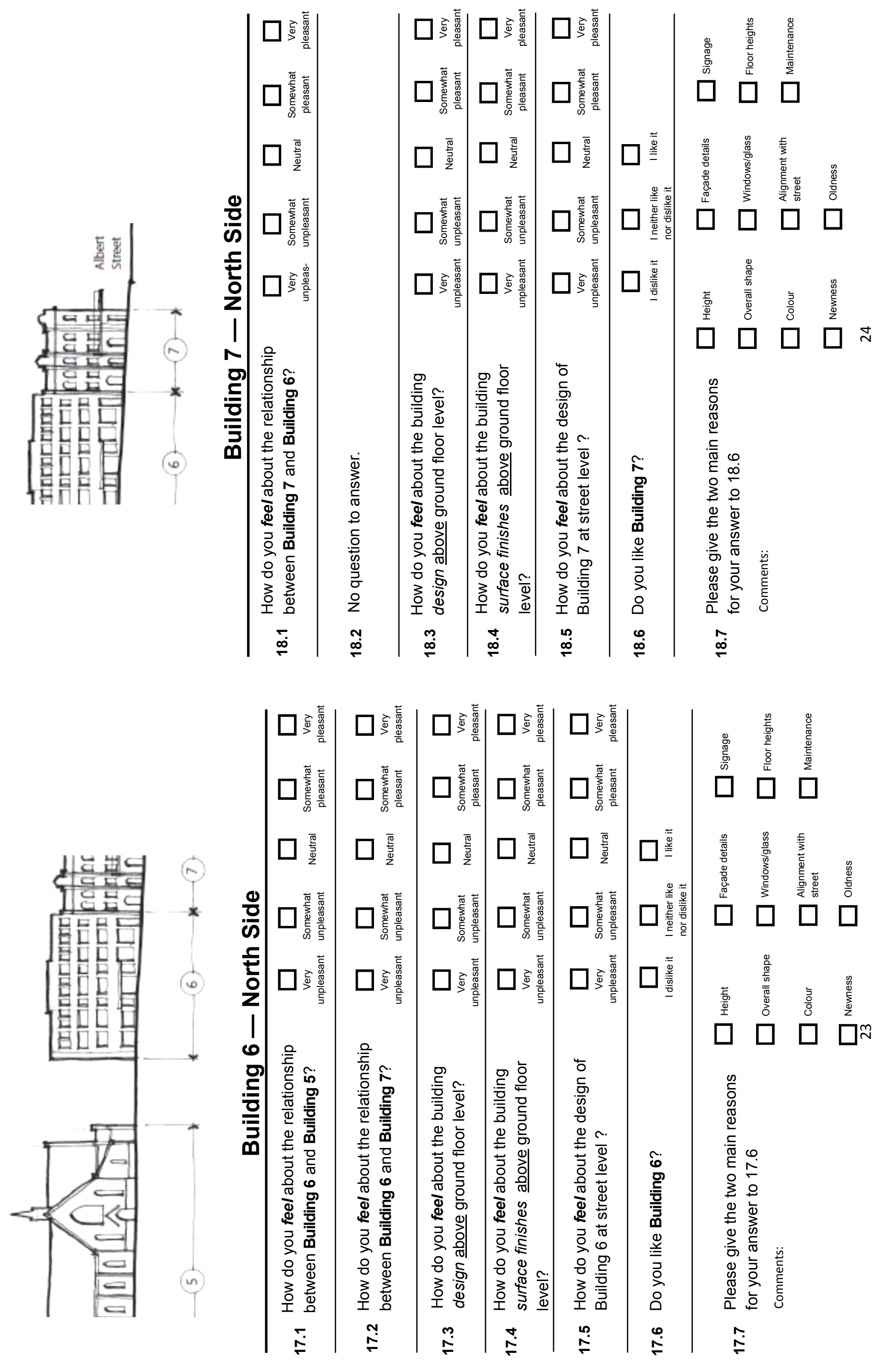

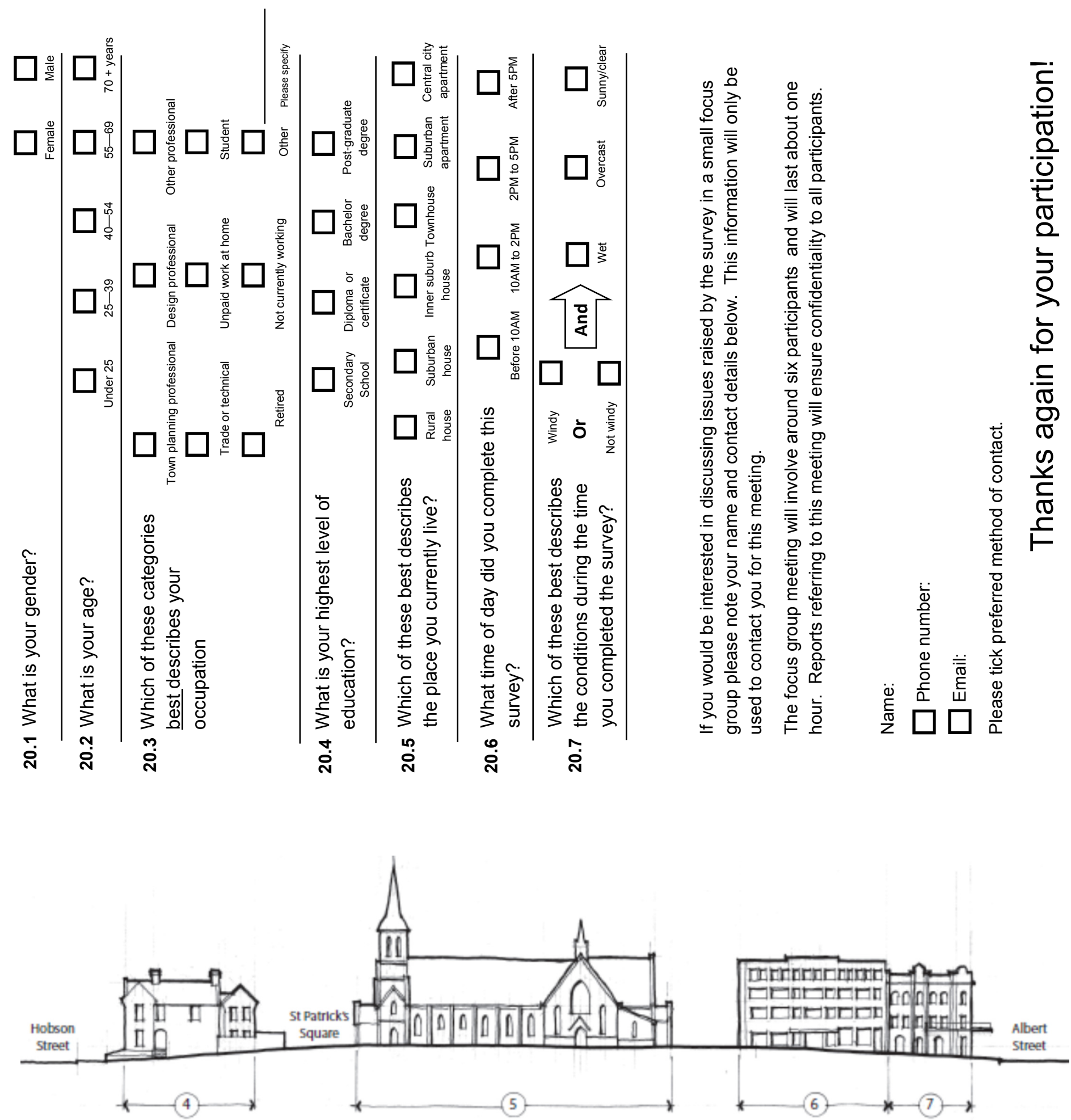

\section{Overall Block-North Side}

19.1 How do you feel about the relationship between building heights along this side of the street?

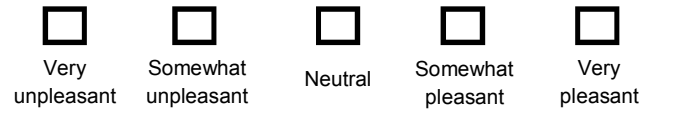

19.2 Please state briefly the principal reason for your response to question 19.1

19.3 How do you feel about the alignment [in and out] between building facades along the length of this side of the street?

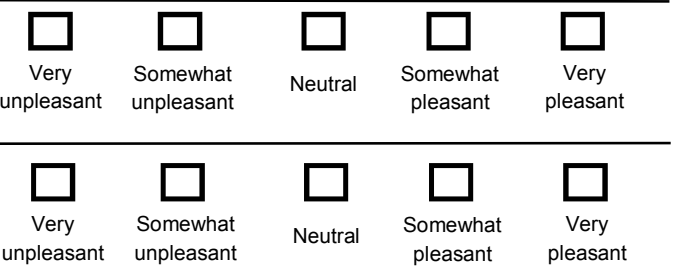

19.4 How do you feel about the relationship between façade design styles along this side of the street? 
Finally, if you would like to go into the draw to win one of two $\$ 100$ vouchers to be used at Portofino Restaurant in the Viaduct Basin precinct, please list your name and contact details below. This information will be used only for the draw and will not be used in any other way.

The prizes will be drawn 10 December 2013. The winners will be notified and a notice placed in the New Zealand Herald advising the result on Friday 13 December.

Name:

Contact details for prize if name drawn:

\section{Good luck !}

\section{Please return the completed survey booklet to:}

\section{, Takapuna Service Centre}




\section{Appendix C}

List of individual findings from Studies One and Two, sorted by principal theme, on which the research findings are based 


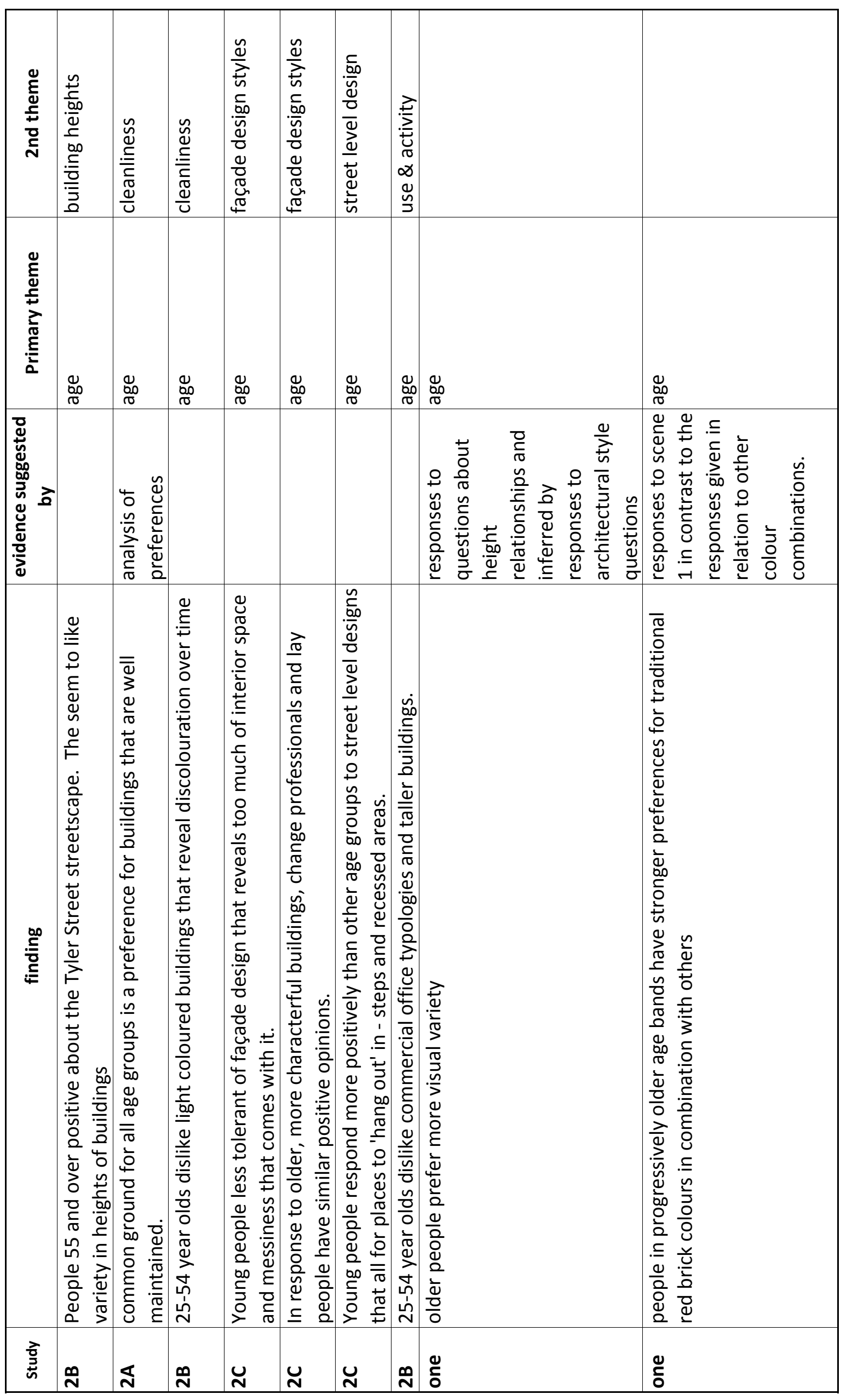




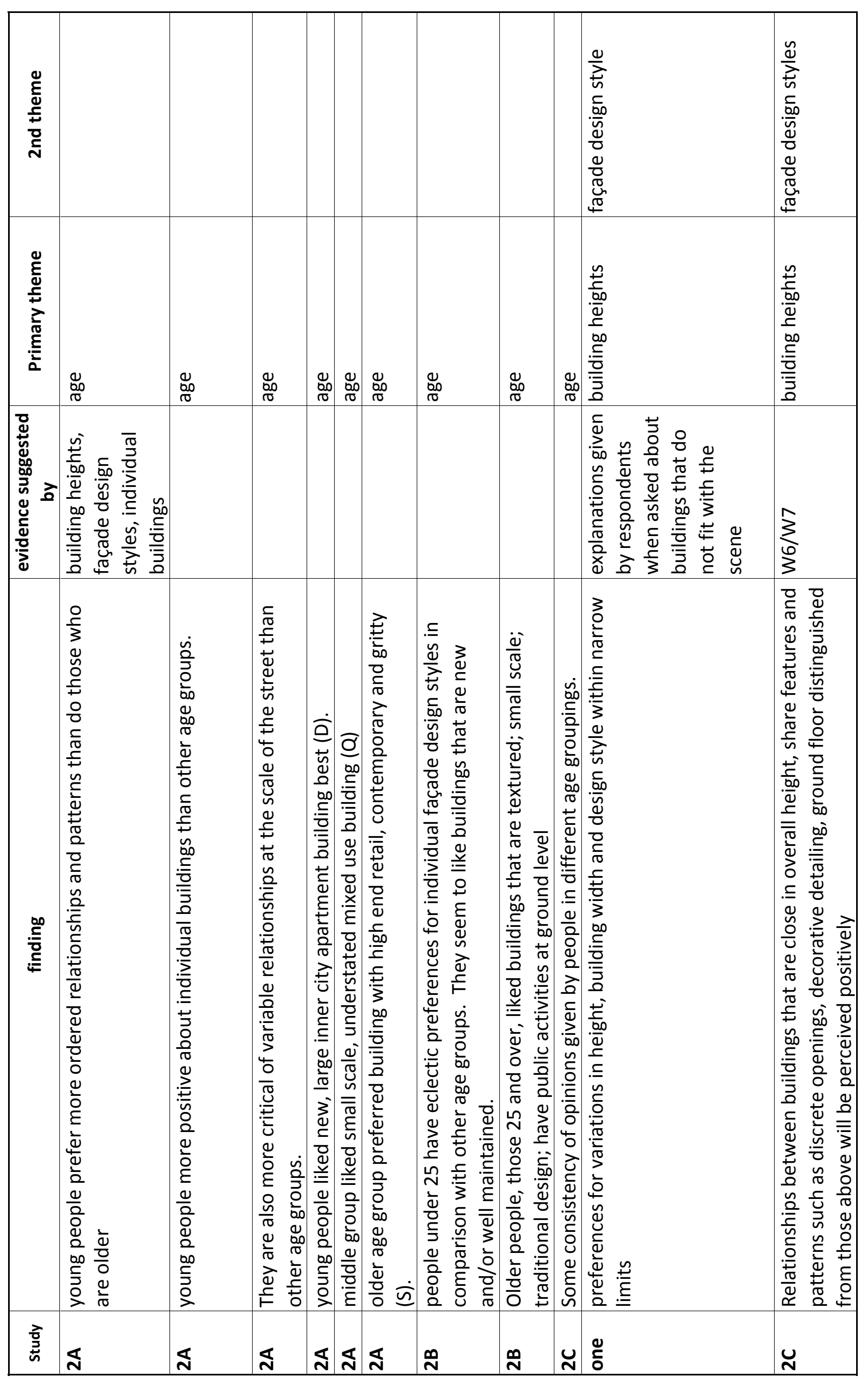




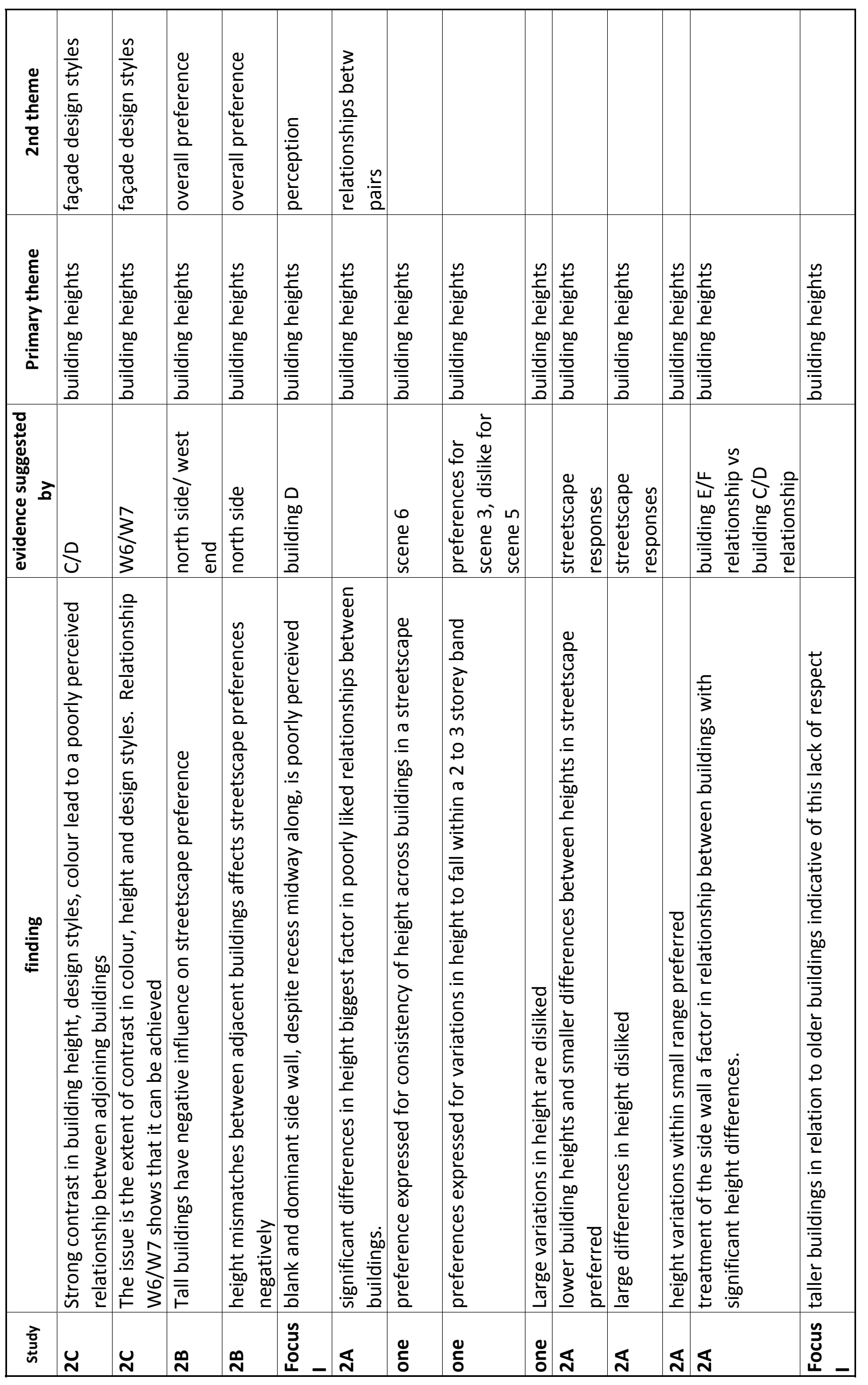




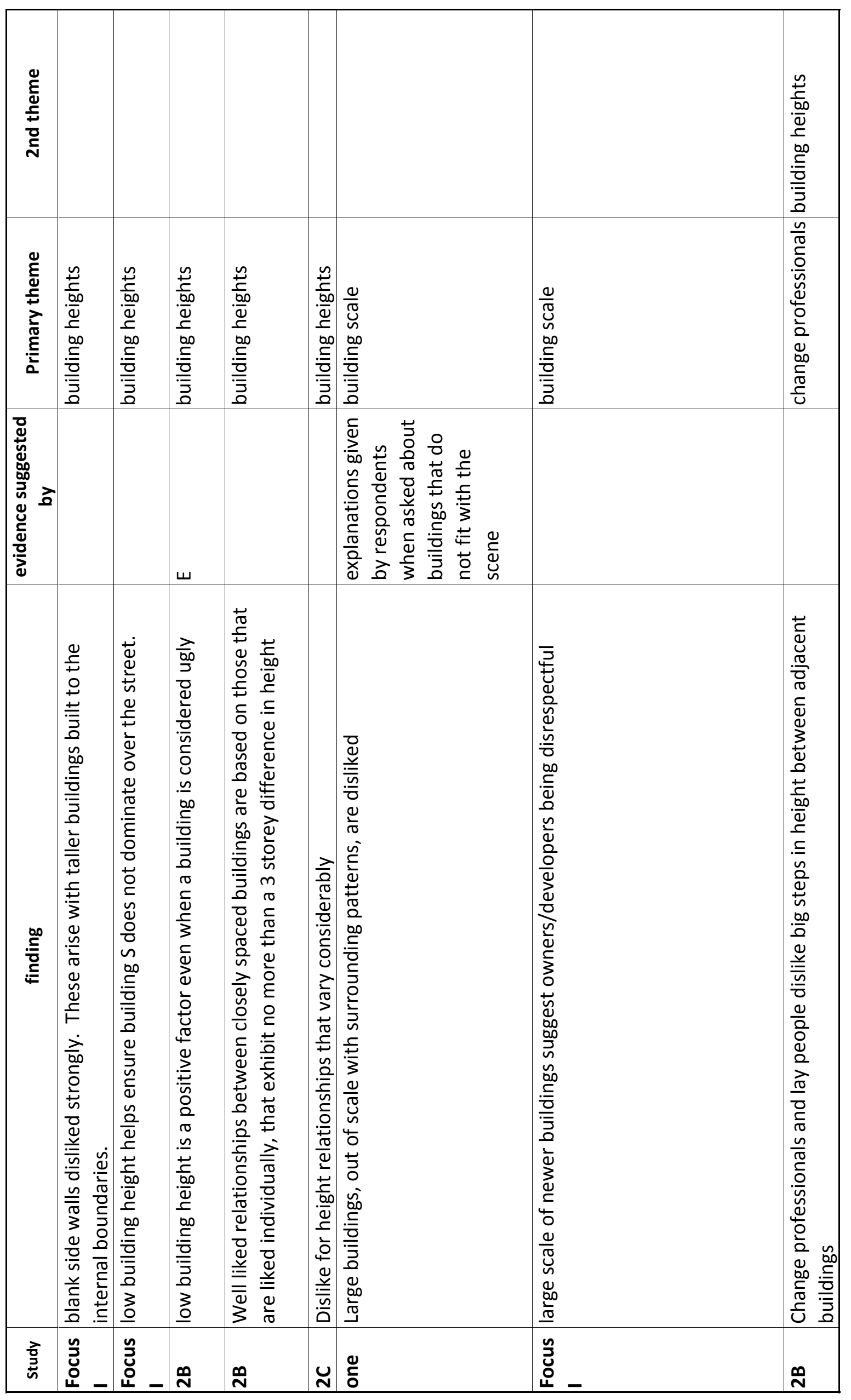




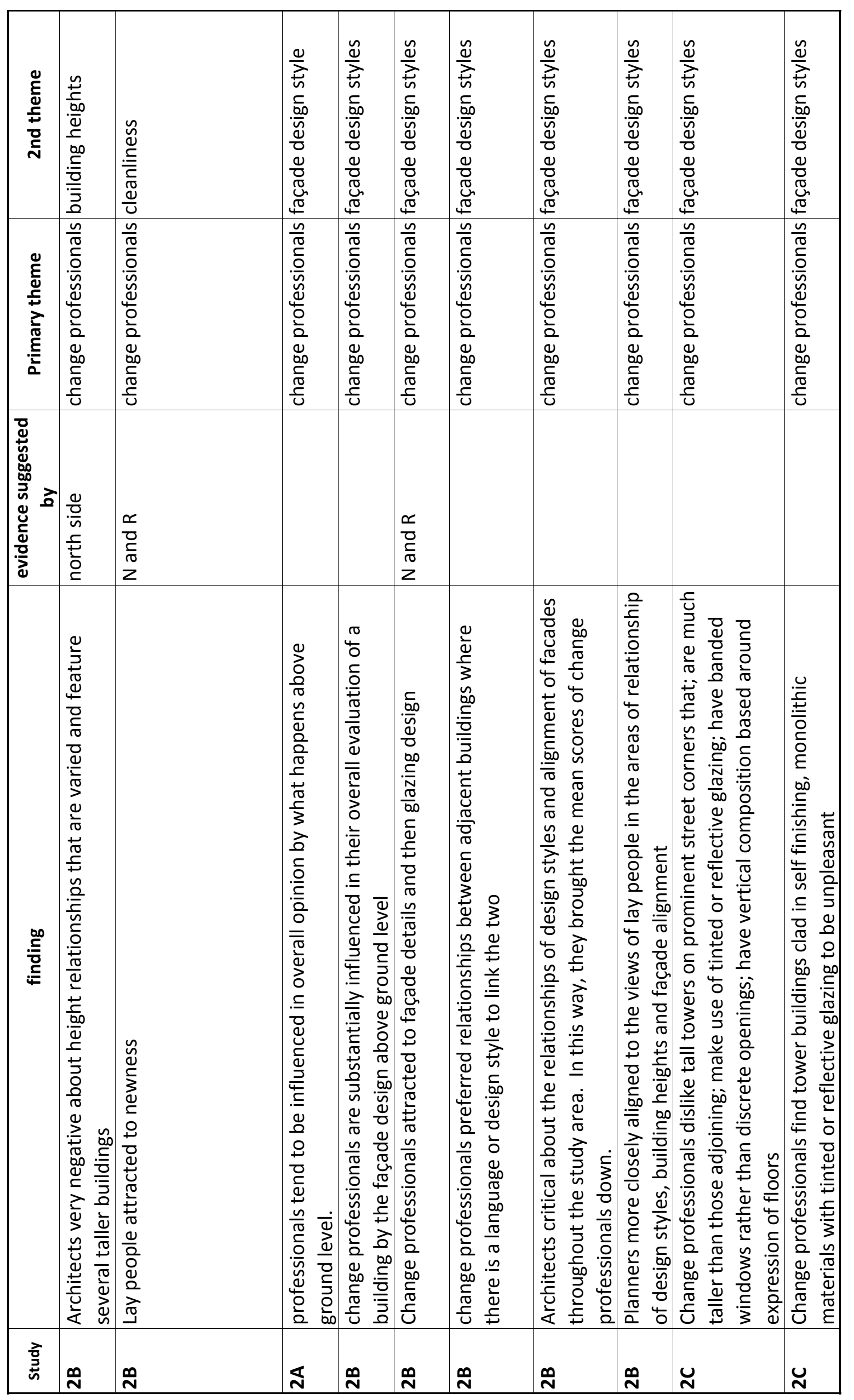




\begin{tabular}{|c|c|c|c|c|c|c|c|c|c|c|c|c|c|c|}
\hline 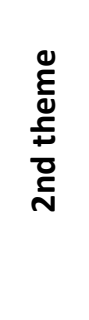 & 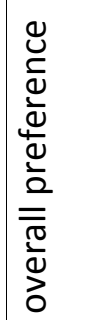 & 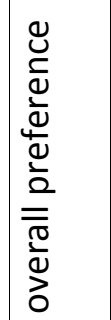 & 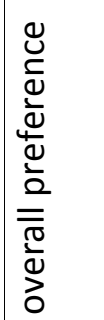 & 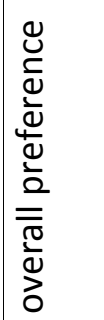 & 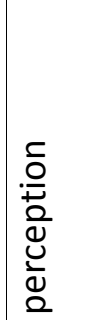 & 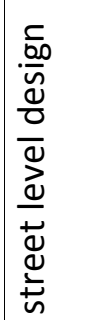 & 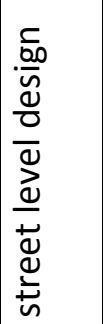 & 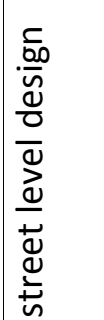 & 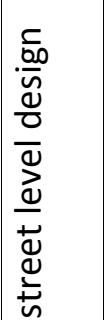 & 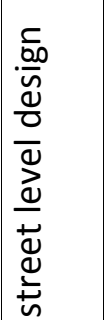 & 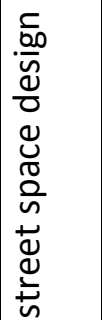 & & & \\
\hline 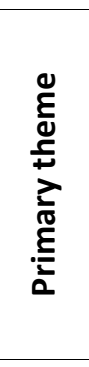 & 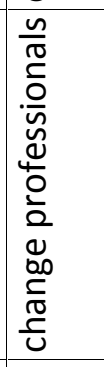 & 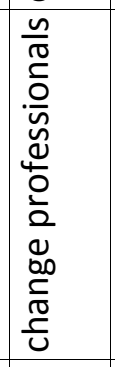 & 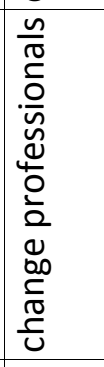 & 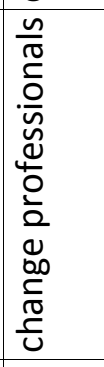 & 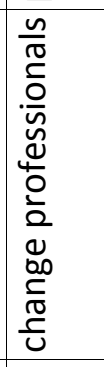 & 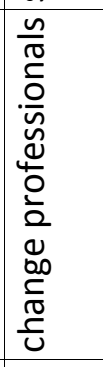 & 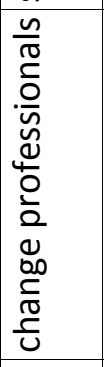 & 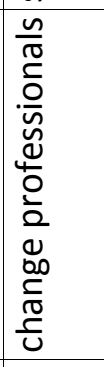 & 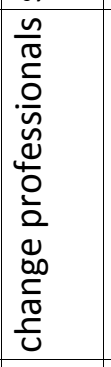 & 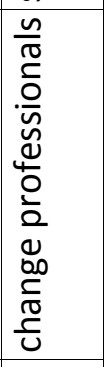 & 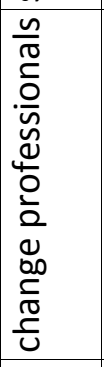 & 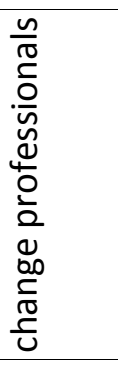 & 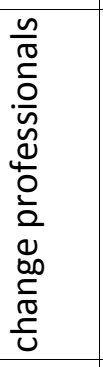 & 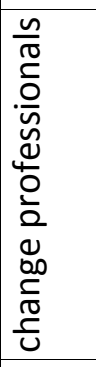 \\
\hline 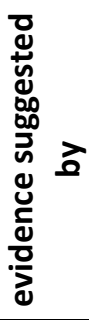 & & & & 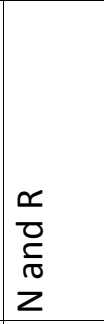 & & & & $\begin{array}{l}\frac{\alpha}{0} \\
\frac{0}{\pi} \\
\tilde{z} \\
z\end{array}$ & L & $>$ & & & & \\
\hline 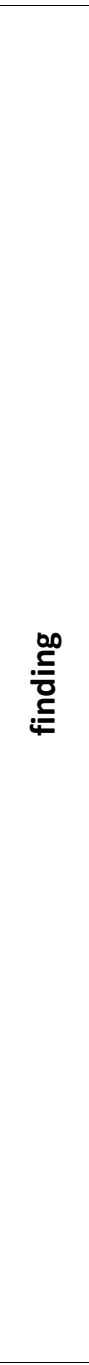 & 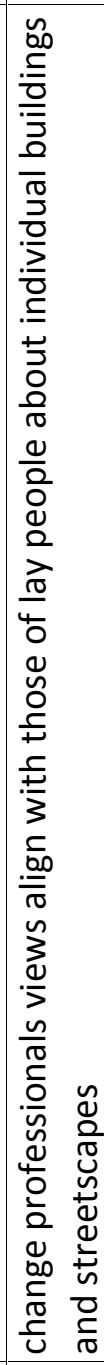 & 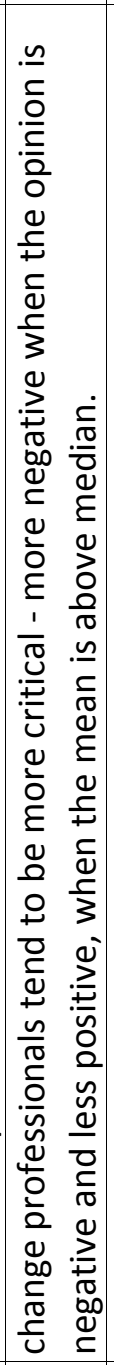 & 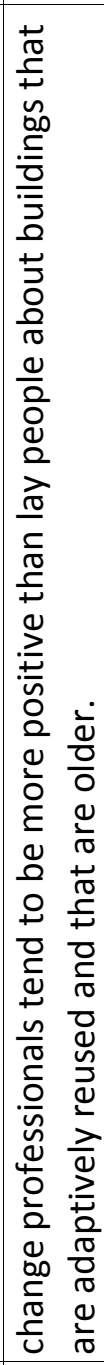 & 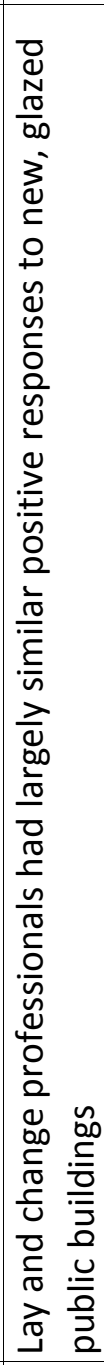 & 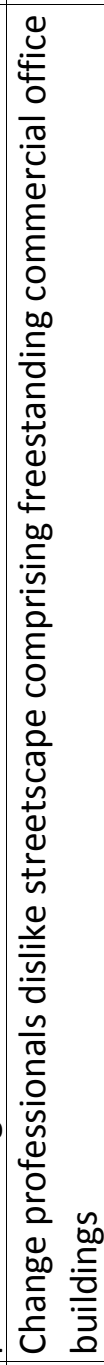 & 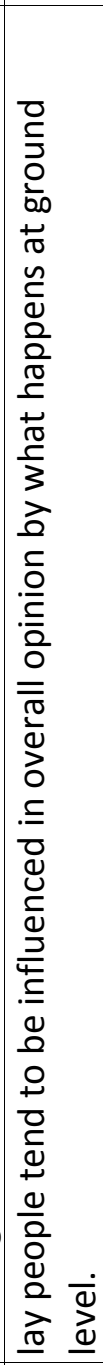 & 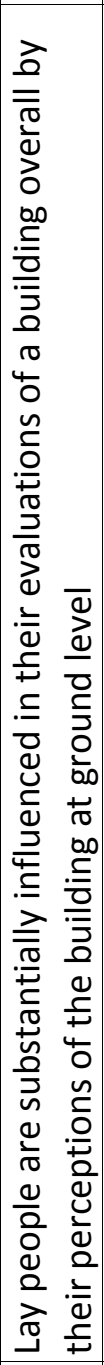 & 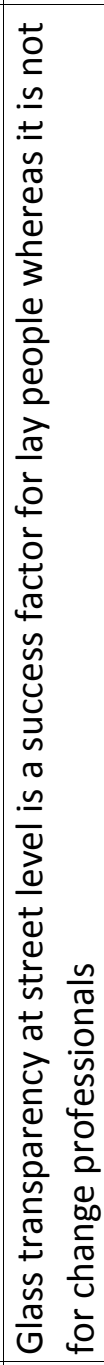 & 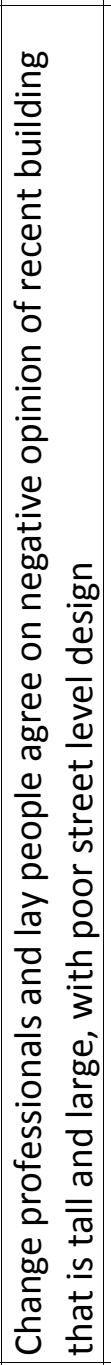 & 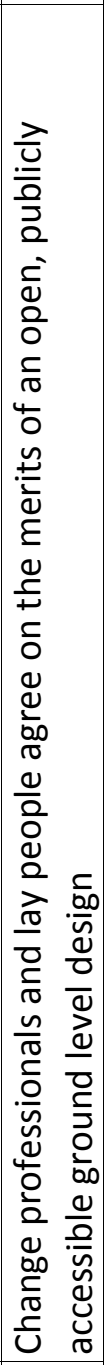 & 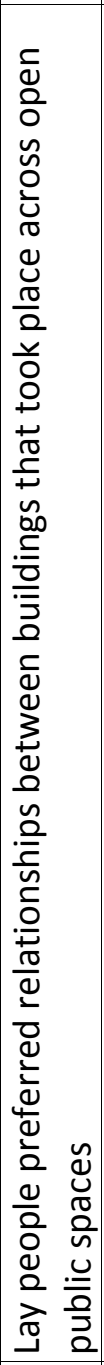 & 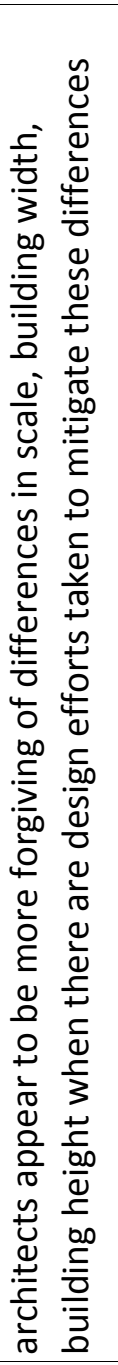 & 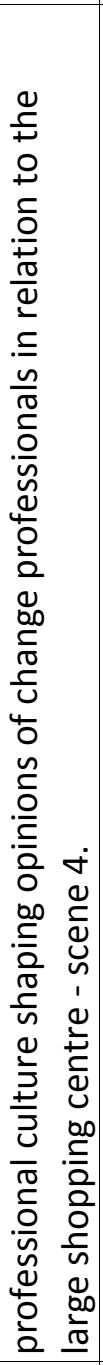 & 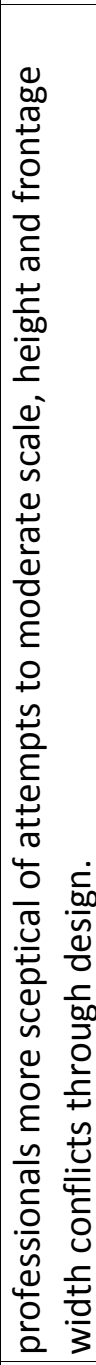 \\
\hline 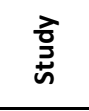 & $\mathbf{N}$ & $\ddot{\sim}$ & $\mathbf{\sim}$ & 崩 & N & $\boldsymbol{N}$ & 崩 & 崩 & $\boldsymbol{N}$ & $\boldsymbol{N}$ & 㸃 & $\overline{0}$ & ธ์ & ؛ั \\
\hline
\end{tabular}




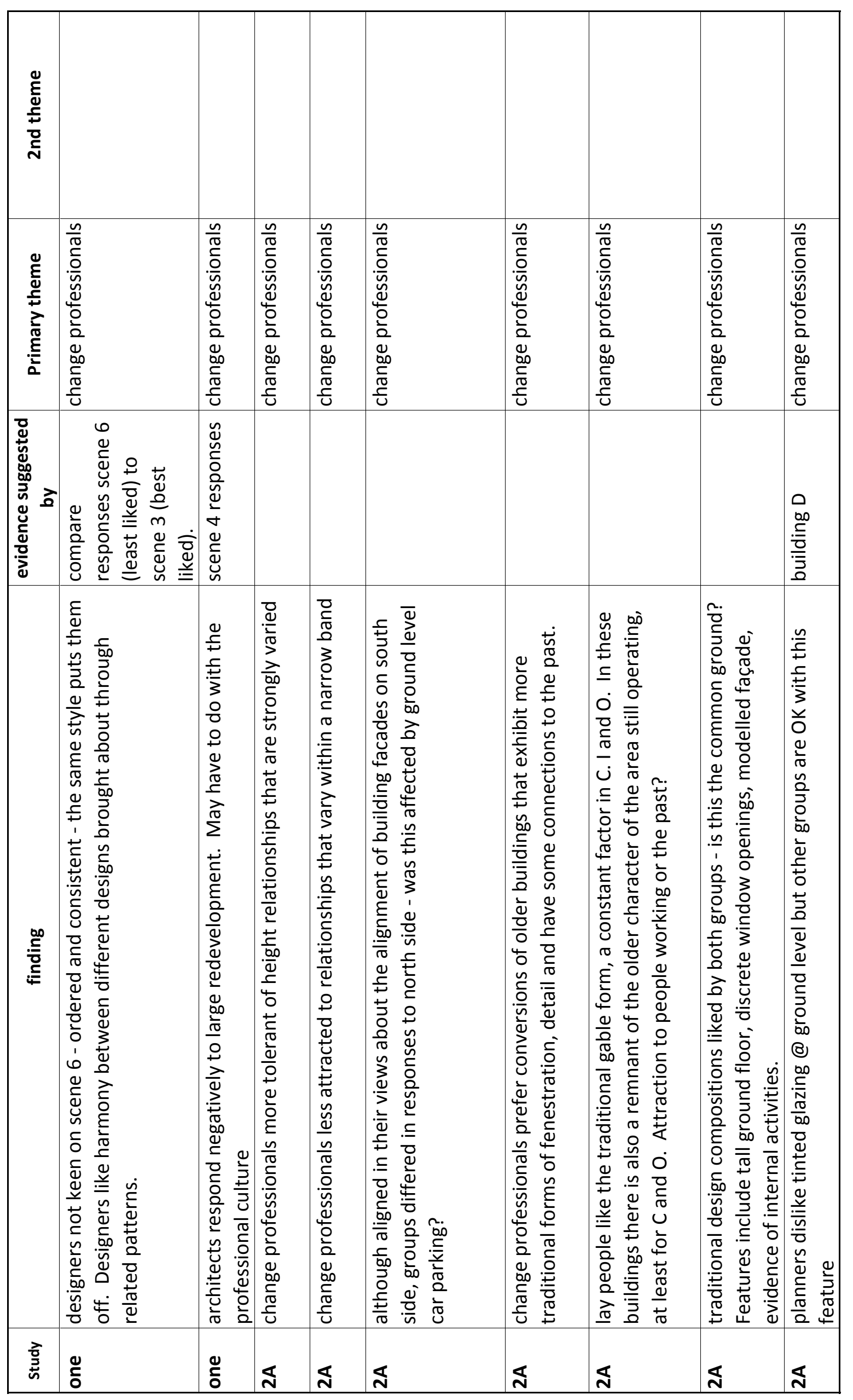




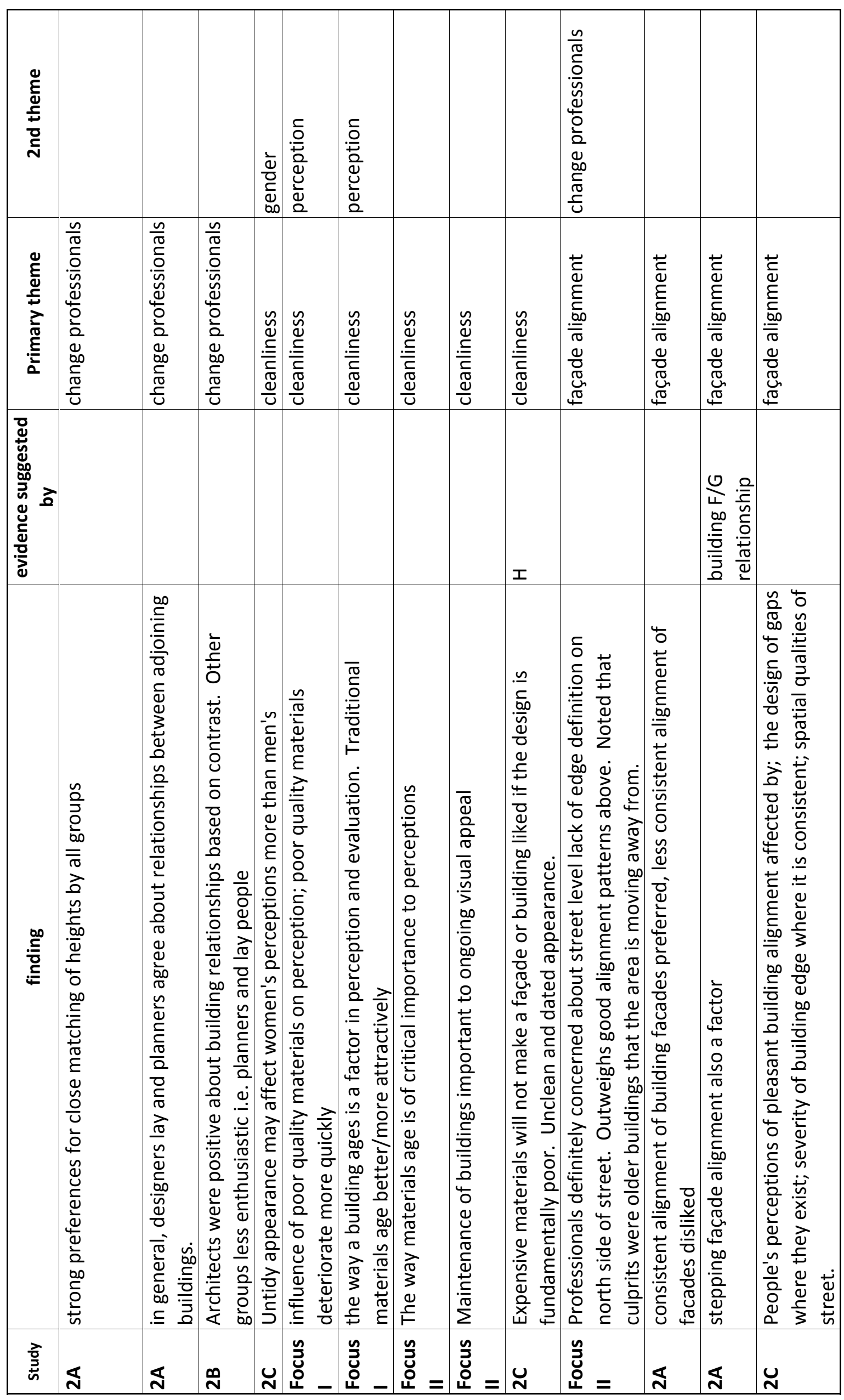




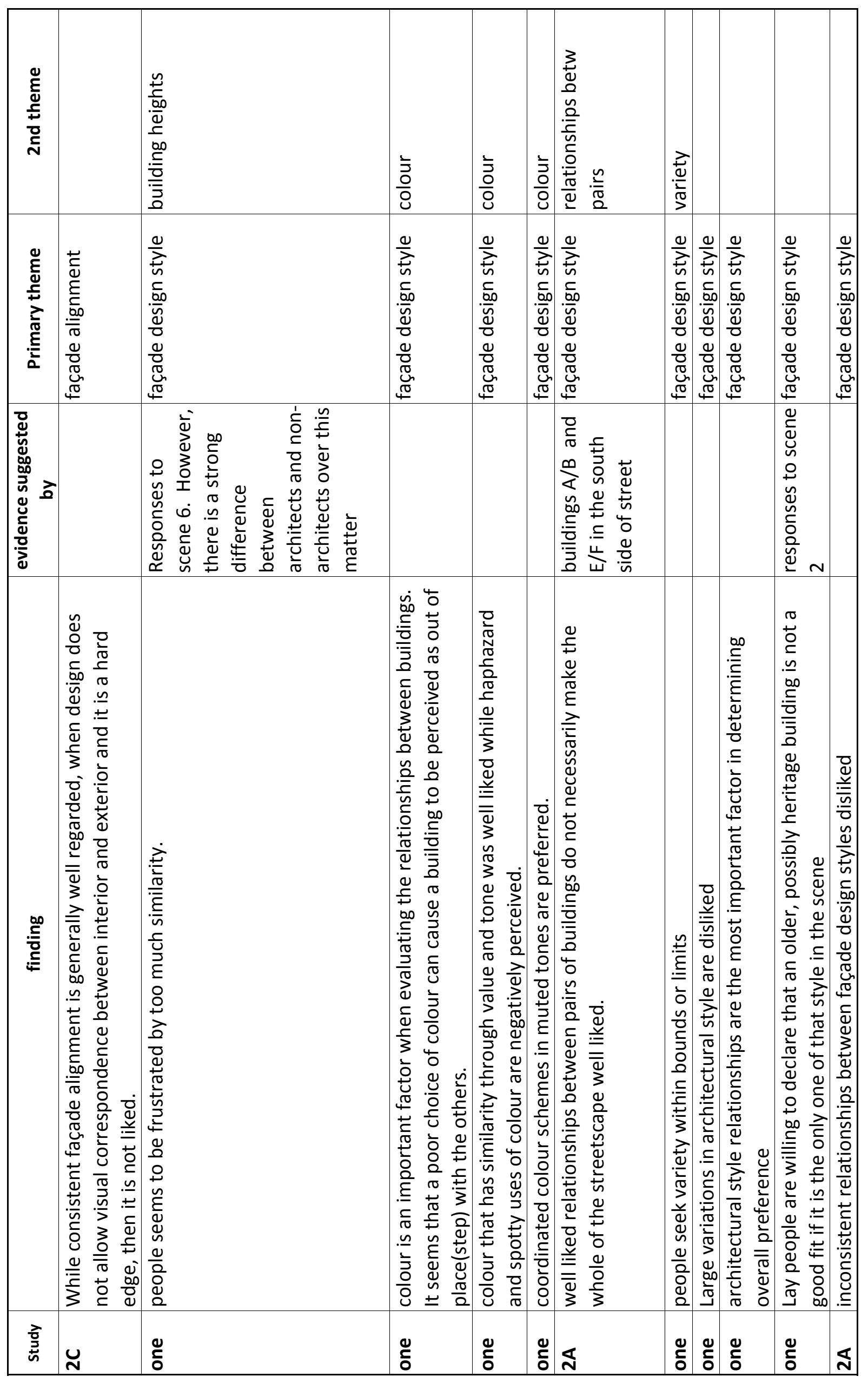




\begin{tabular}{|c|c|c|c|c|c|c|c|c|c|c|c|c|}
\hline 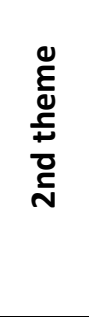 & & & & & & & & & 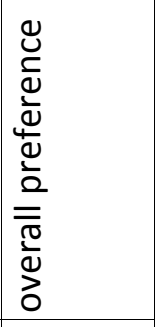 & 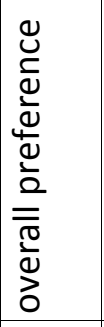 & & \\
\hline 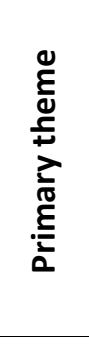 & 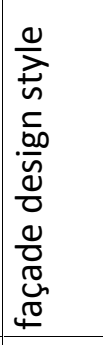 & 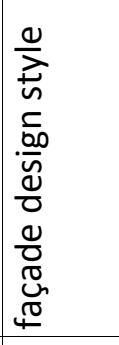 & 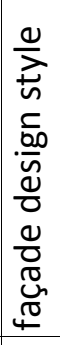 & 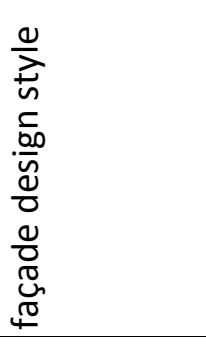 & 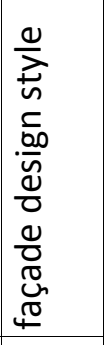 & 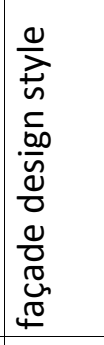 & 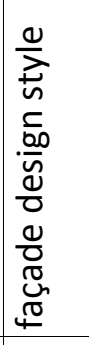 & 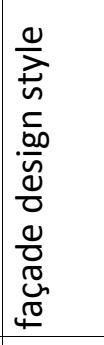 & 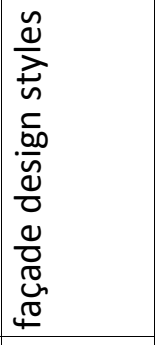 & 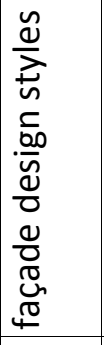 & 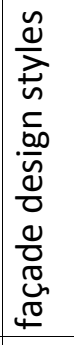 & 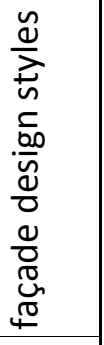 \\
\hline 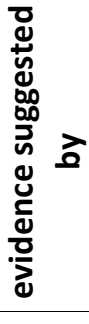 & & 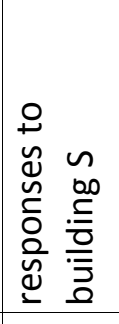 & 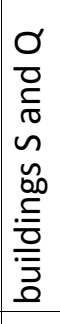 & 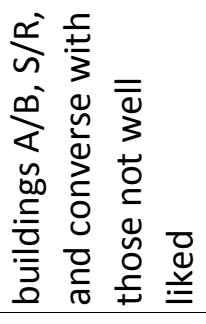 & & & & & $\begin{array}{l}\overline{\bar{\sigma}} \\
\frac{\bar{\sigma}}{\sigma} \\
0 \\
0\end{array}$ & 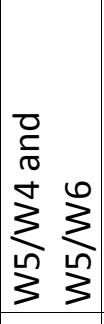 & $\begin{array}{l}0 \\
0 \\
\frac{0}{0} \\
\frac{1}{0} \\
0 \\
\Sigma \\
\Sigma\end{array}$ & 0 \\
\hline & 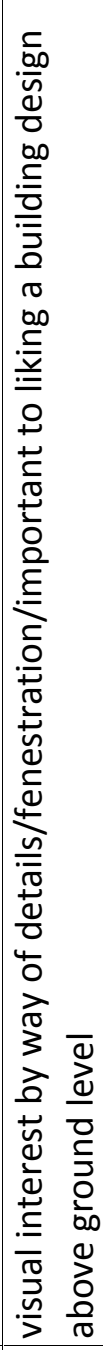 & 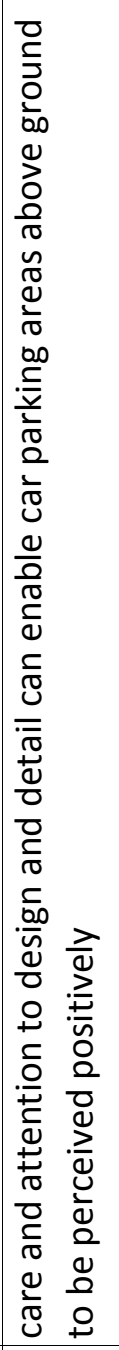 & 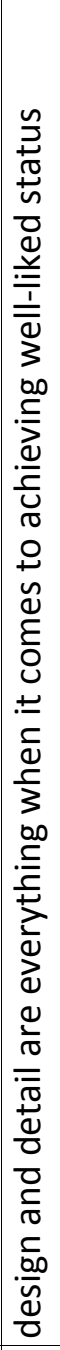 & 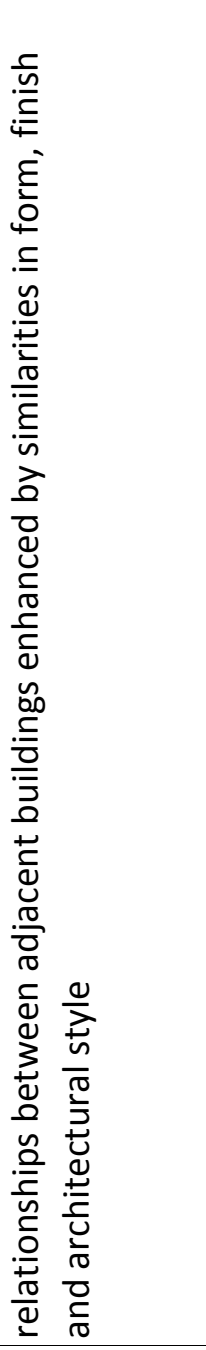 & 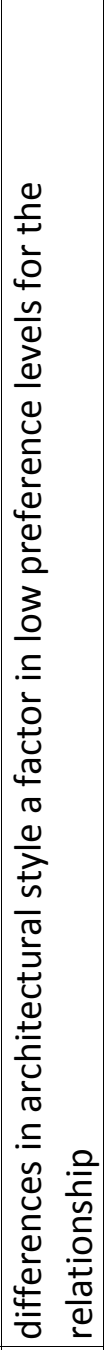 & 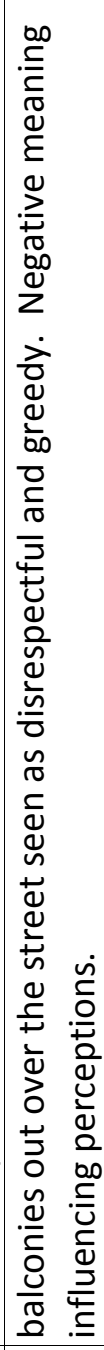 & 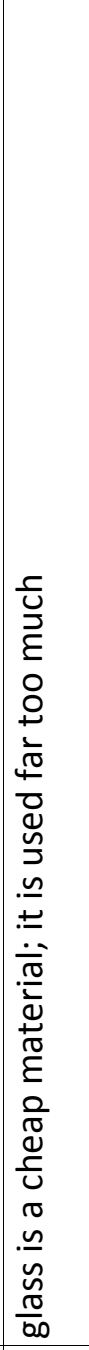 & 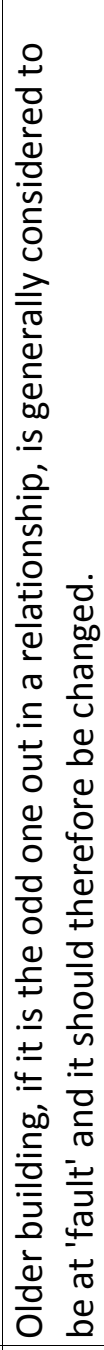 & 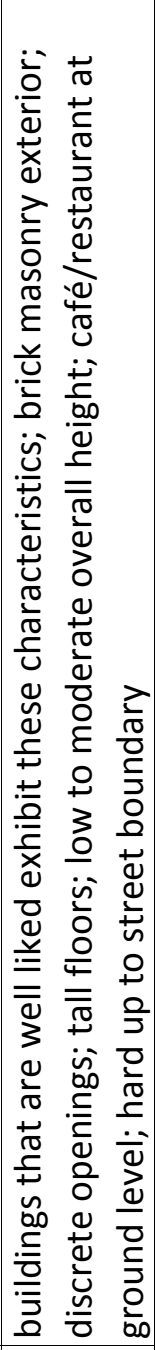 & 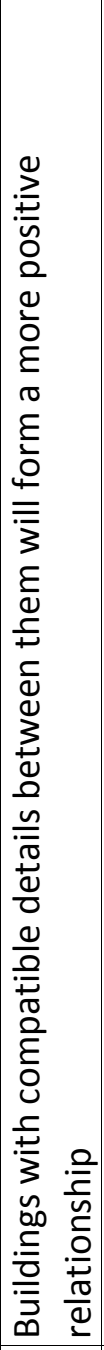 & 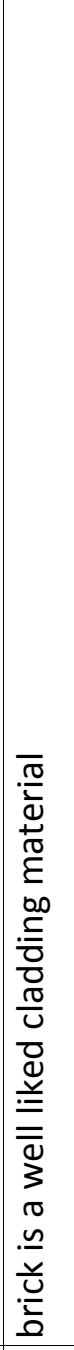 & 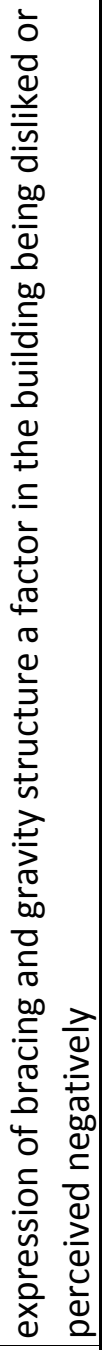 \\
\hline 产 & న & s & న & $\mathbb{N}$ & ง & 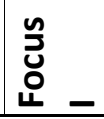 & $\begin{array}{l}n \\
\mathcal{Z} \\
0 \\
\end{array}$ & 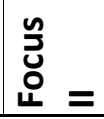 & $\vec{\sim}$ & N & $\vec{\sim}$ & $\vec{\sim}$ \\
\hline
\end{tabular}




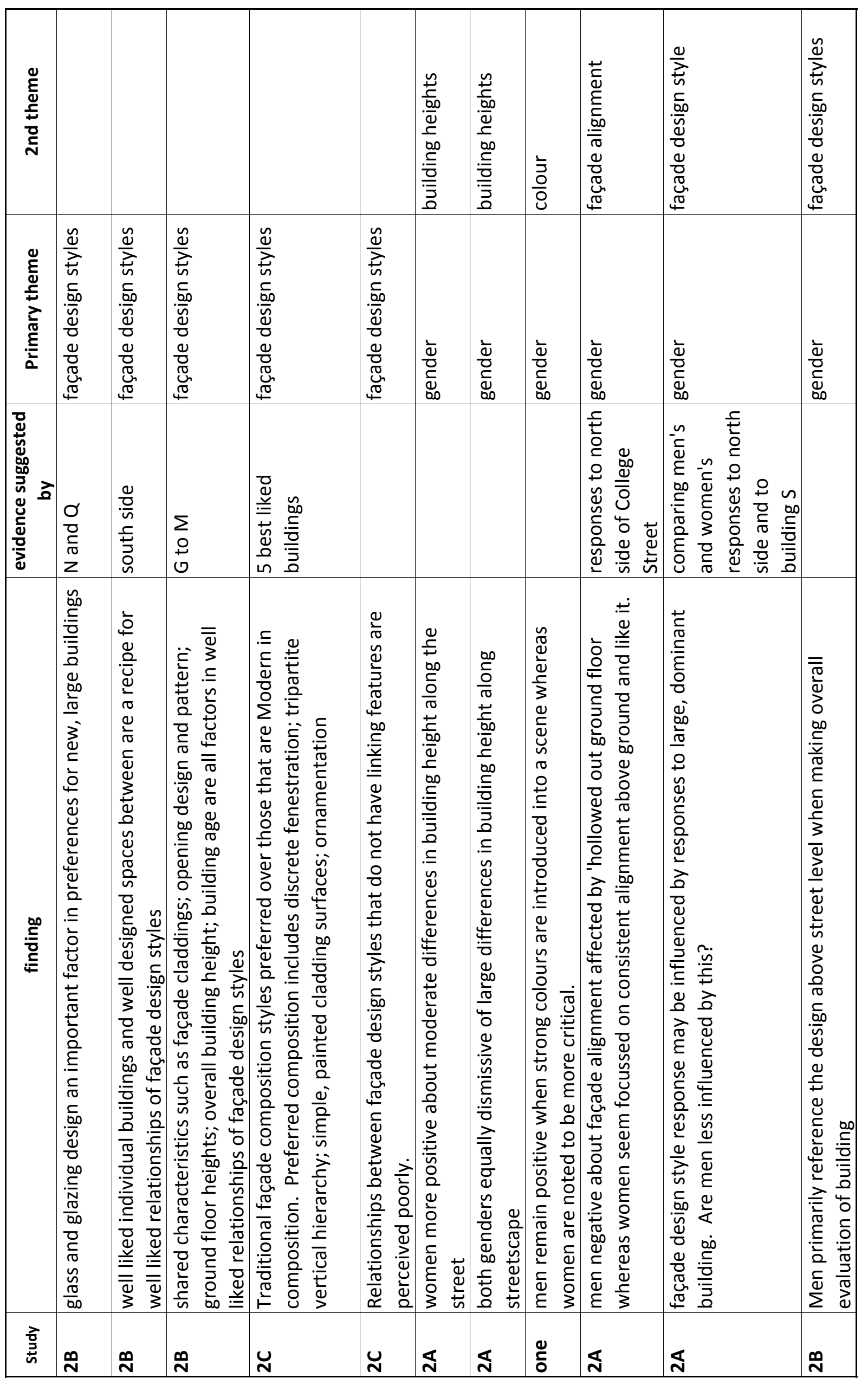




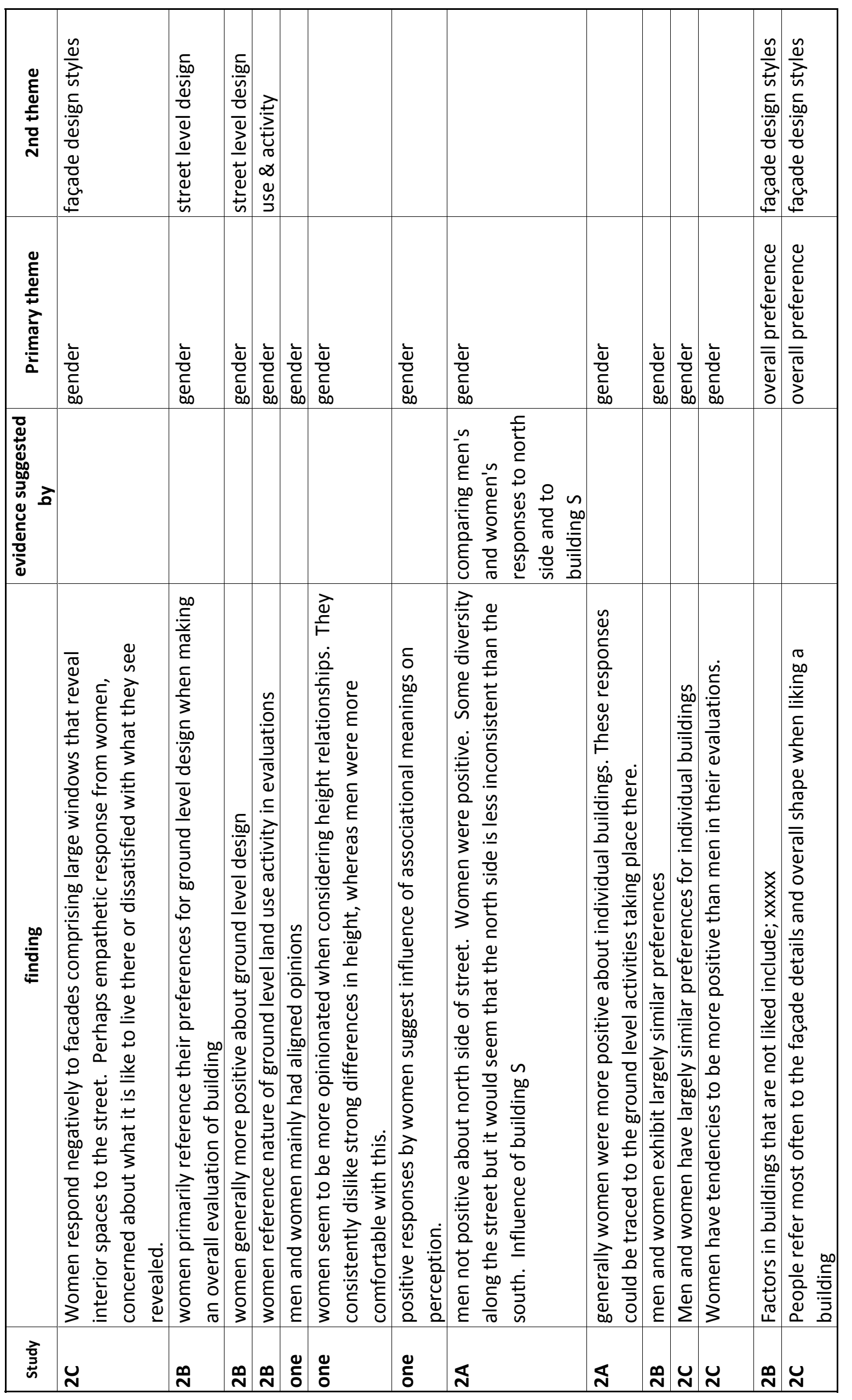




\begin{tabular}{|c|c|c|c|c|c|c|c|c|c|c|}
\hline 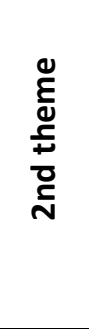 & 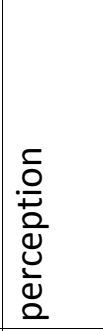 & 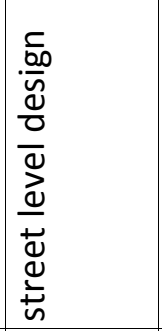 & 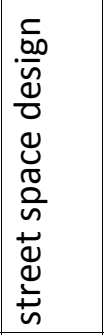 & 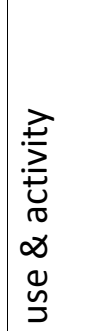 & & & & & & \\
\hline 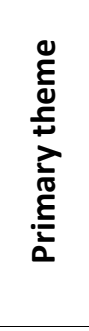 & 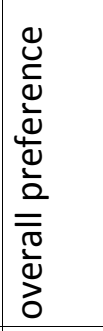 & 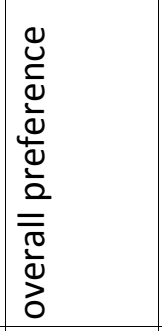 & 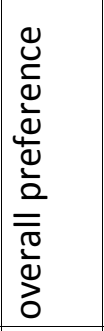 & 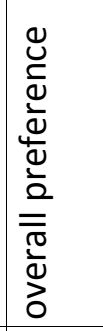 & 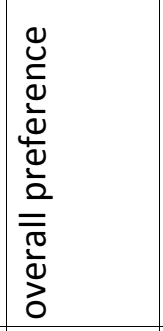 & 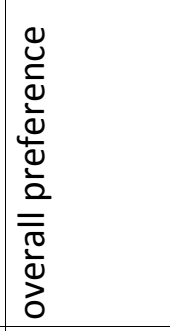 & 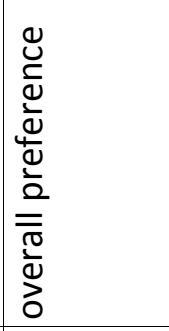 & 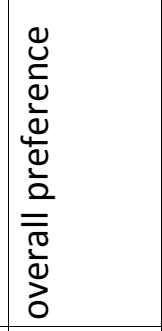 & 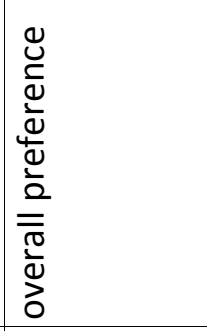 & 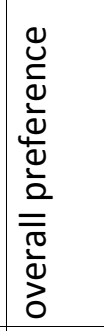 \\
\hline 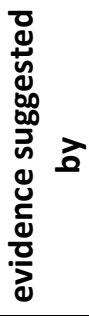 & 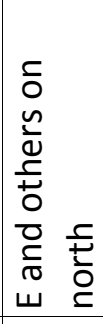 & & $\begin{array}{l}\frac{0}{0} \\
\frac{0}{n} \\
\frac{1}{5} \\
\frac{1}{2} \\
0 \\
w\end{array}$ & ш & 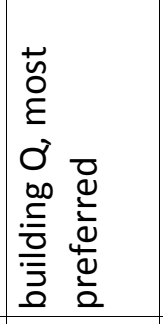 & 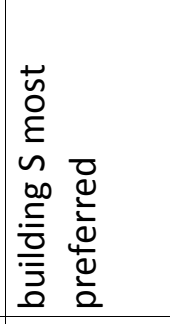 & 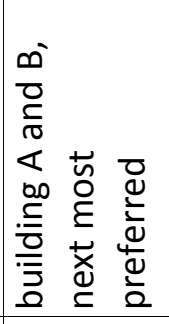 & 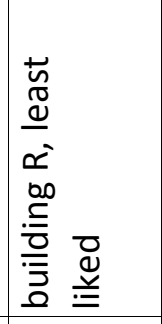 & 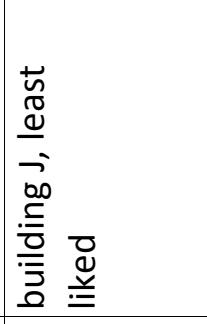 & $\frac{0}{0}$ \\
\hline 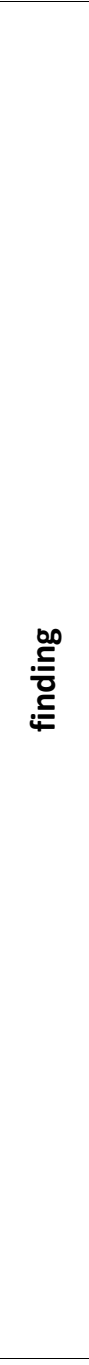 & 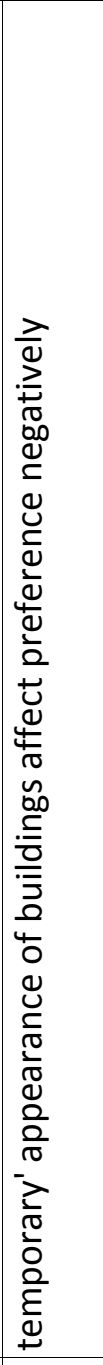 & 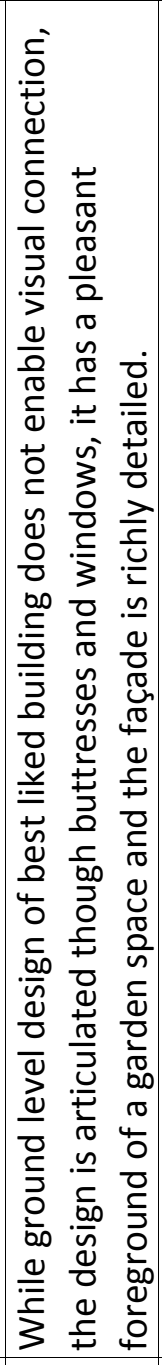 & 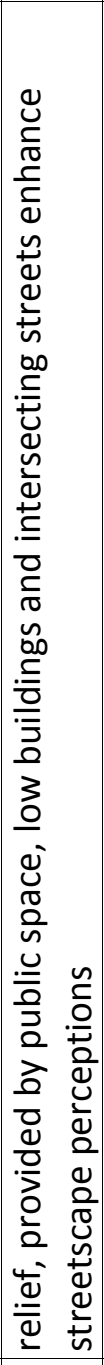 & 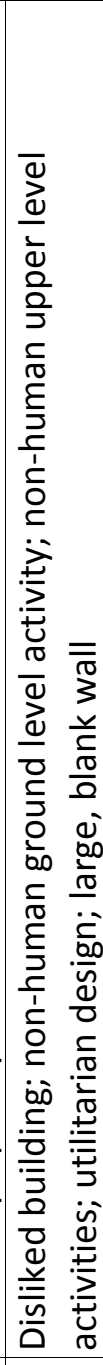 & 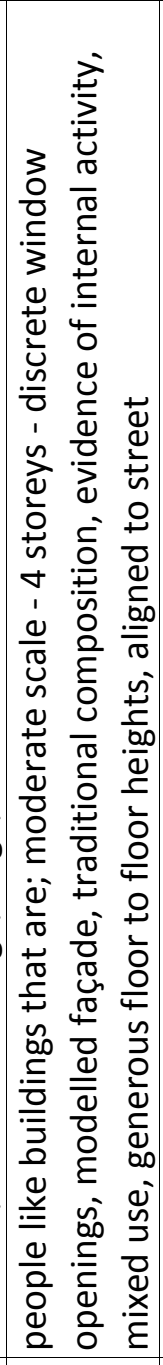 & 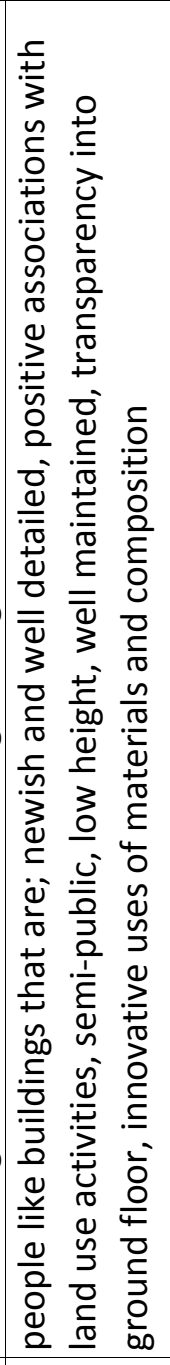 & 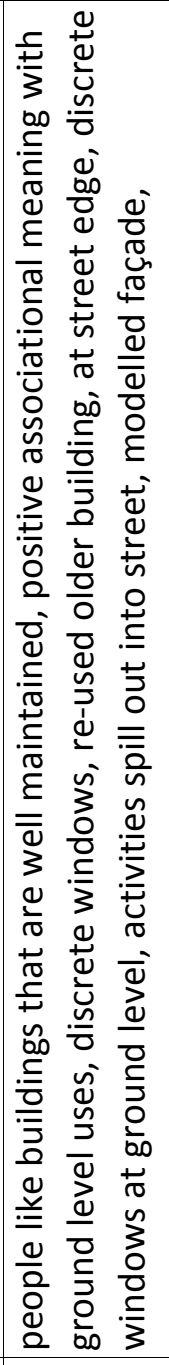 & 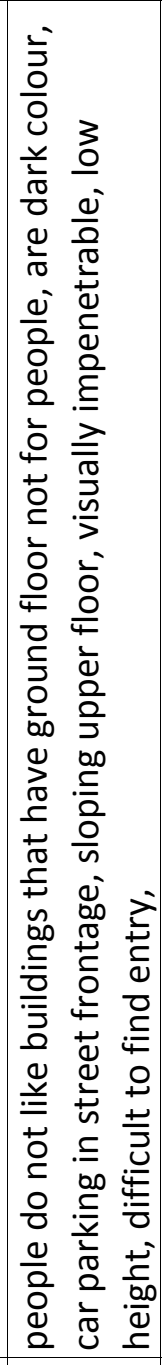 & 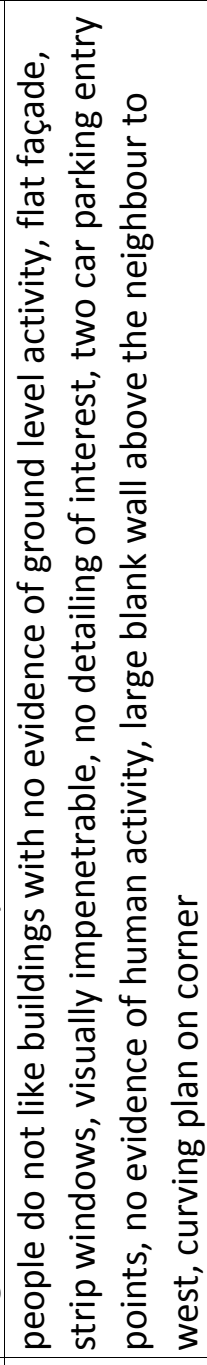 & 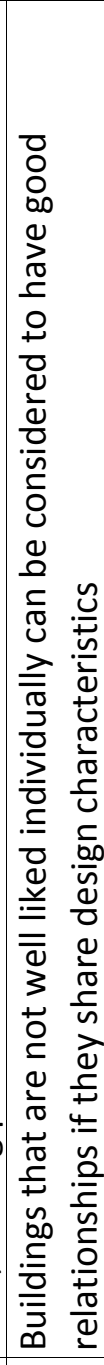 \\
\hline 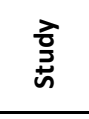 & $\stackrel{\infty}{\mathbf{N}}$ & U & $\dot{\mathbf{N}}$ & U & 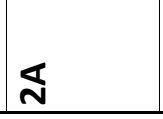 & $\pi$ & N & 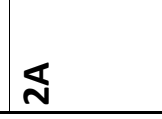 & $\nwarrow$ & $\mathbf{\sim}$ \\
\hline
\end{tabular}




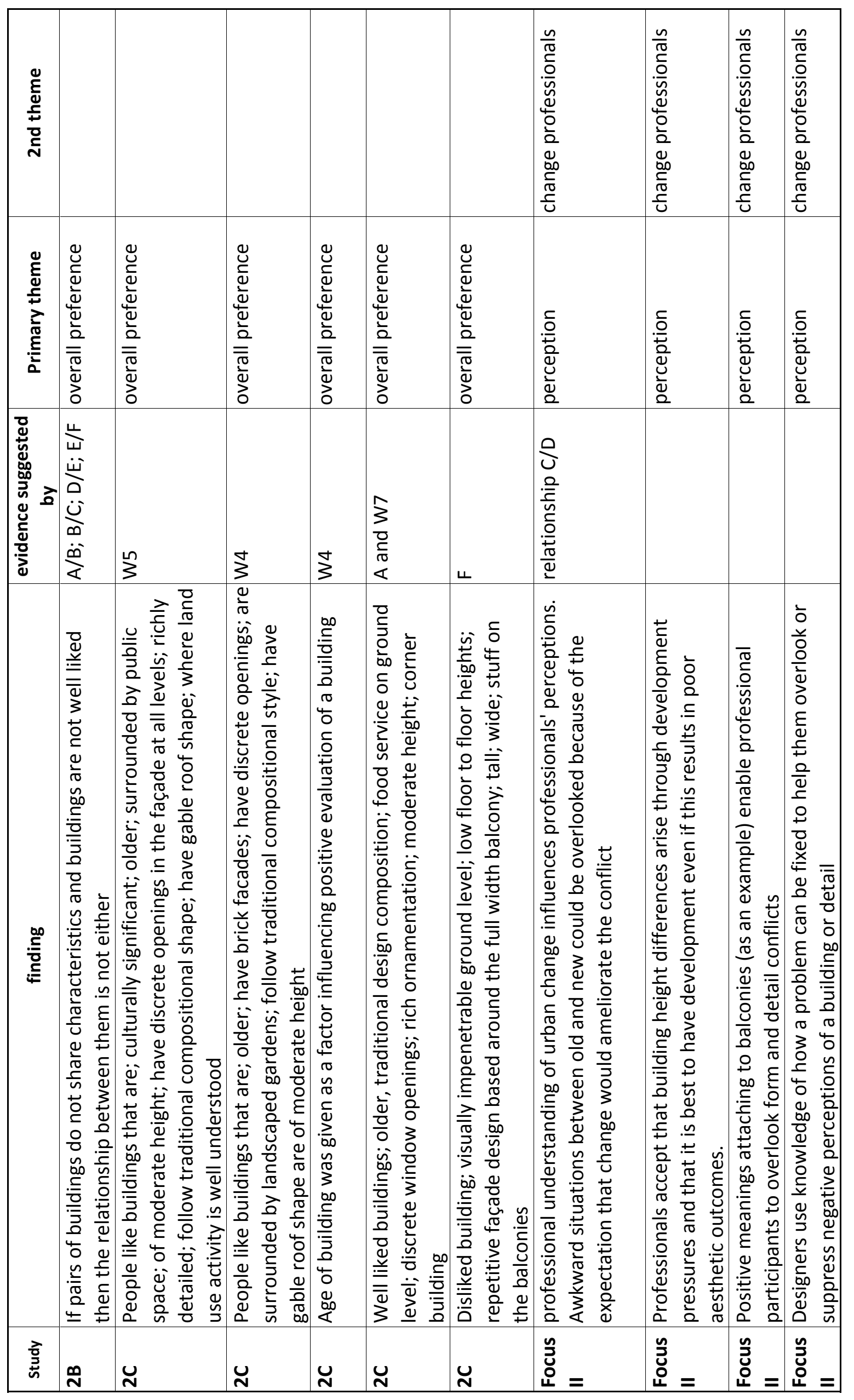




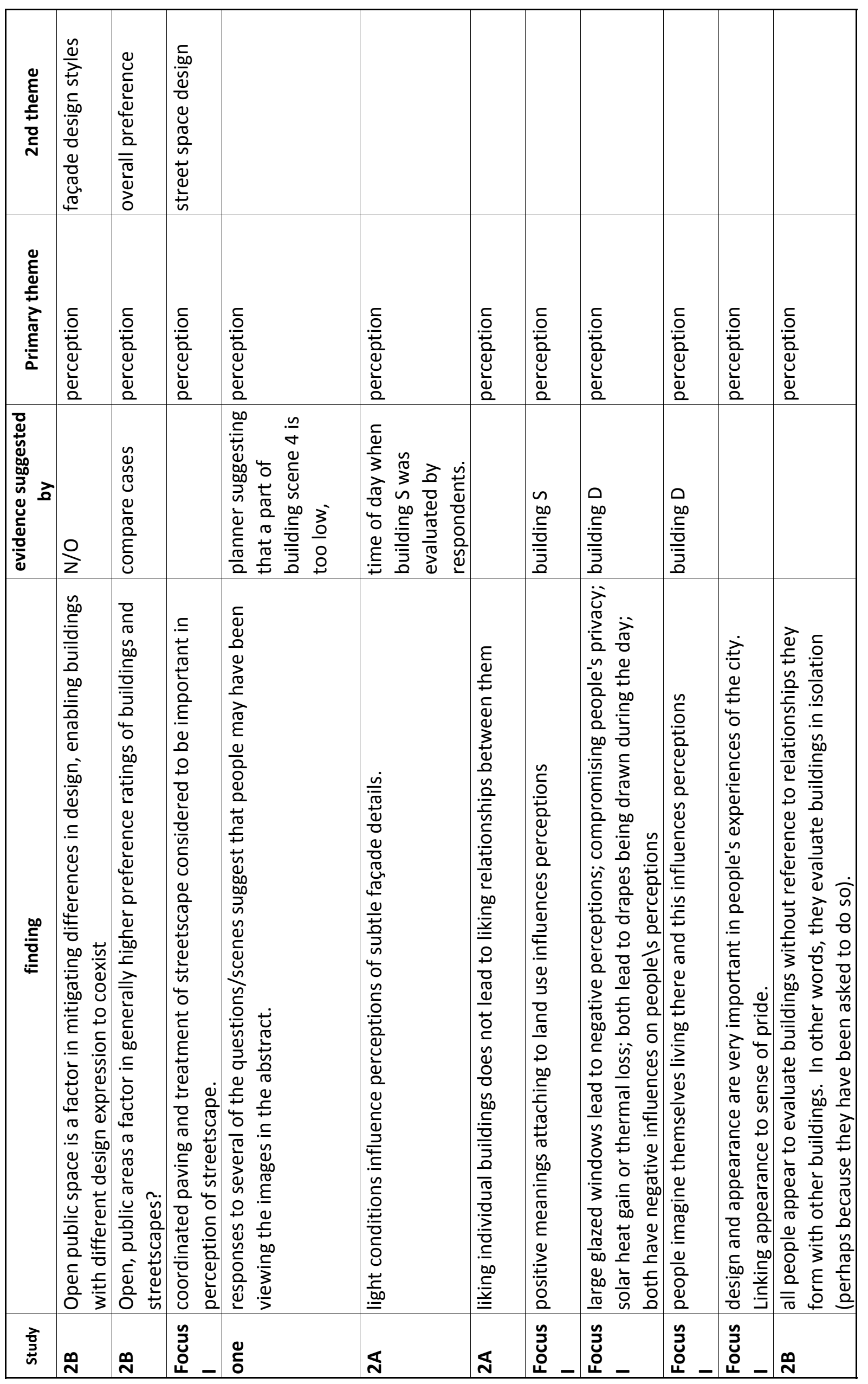




\begin{tabular}{|c|c|c|c|c|c|c|c|c|c|c|c|c|}
\hline 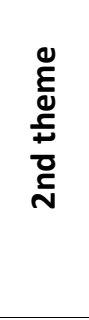 & & & 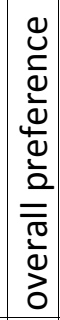 & 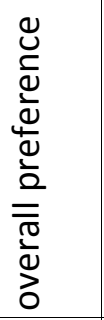 & 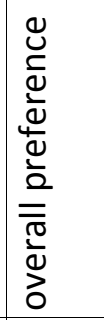 & 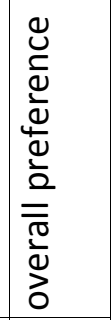 & 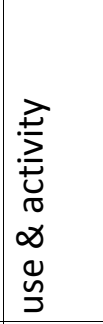 & & & & & \\
\hline 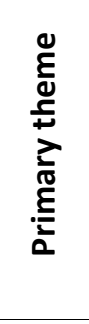 & 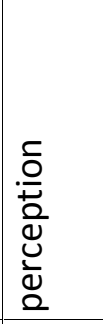 & 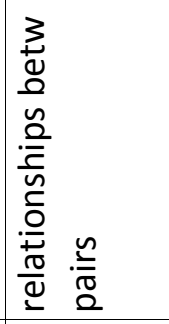 & 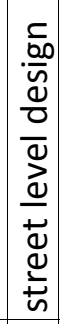 & 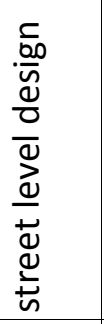 & 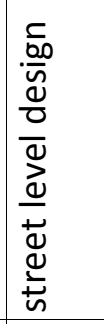 & 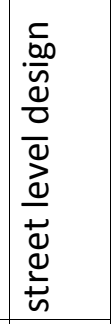 & 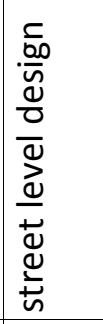 & 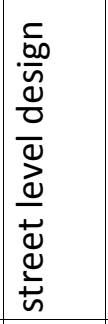 & 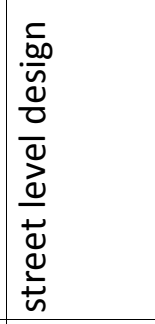 & 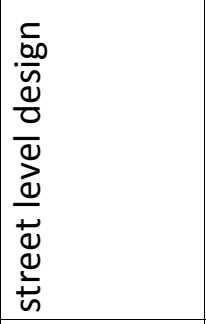 & 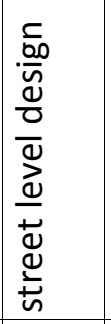 & 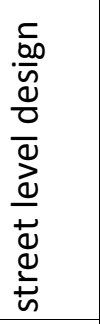 \\
\hline 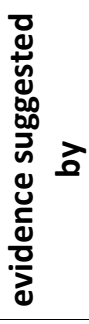 & & 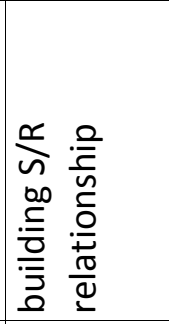 & & $\hat{3}$ & $\hat{3}$ & $\varangle$ & $\stackrel{m}{3}$ & 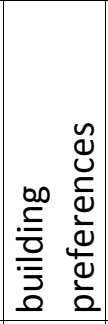 & 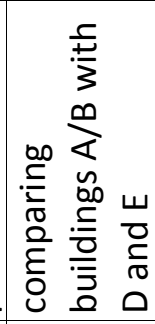 & 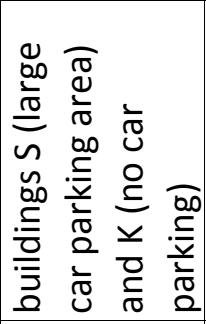 & & $\Sigma$ \\
\hline 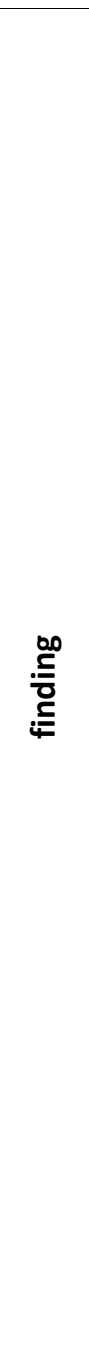 & 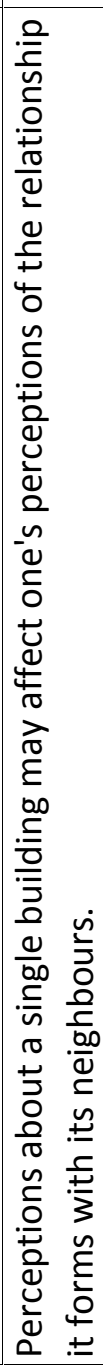 & 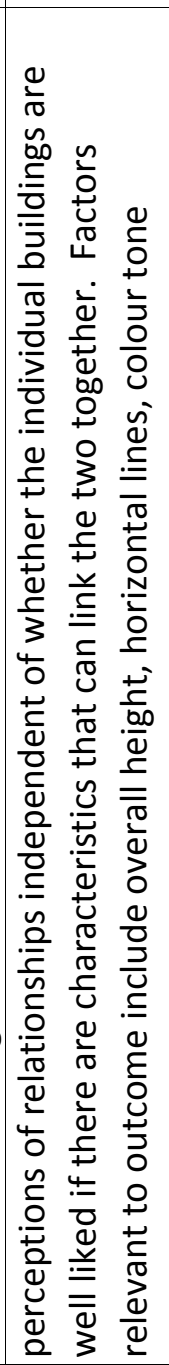 & 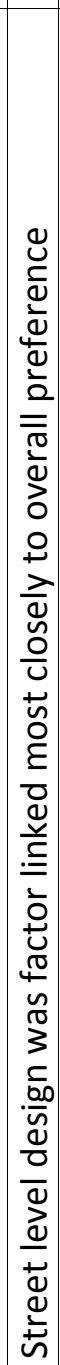 & 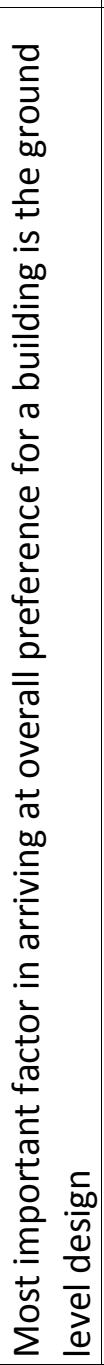 & 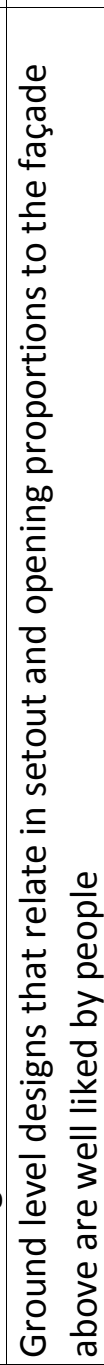 & 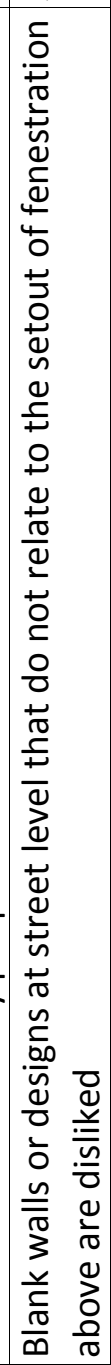 & 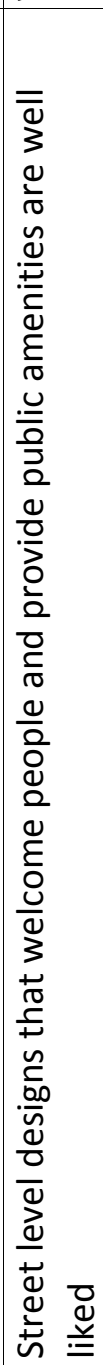 & 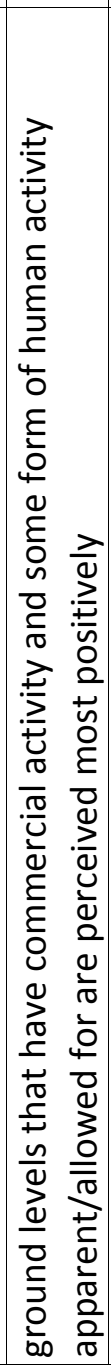 & 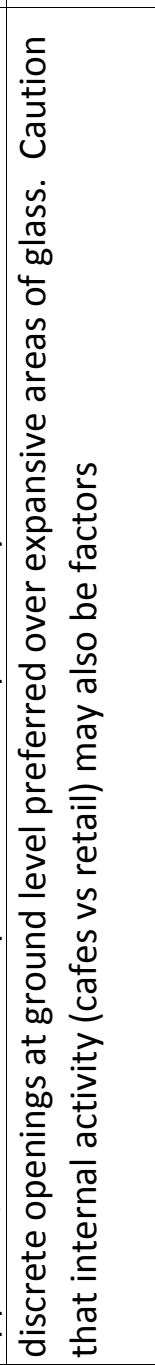 & 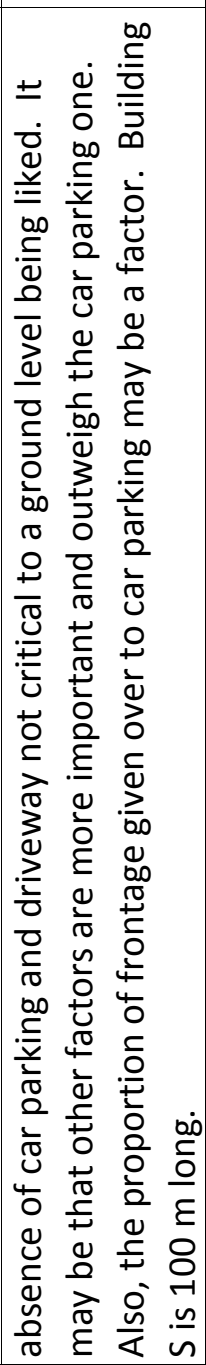 & 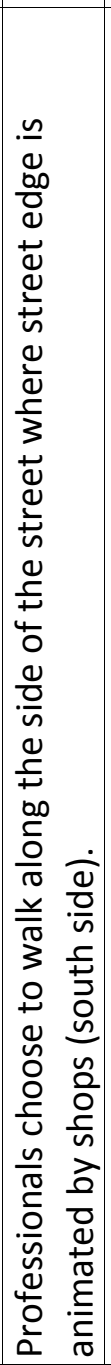 & 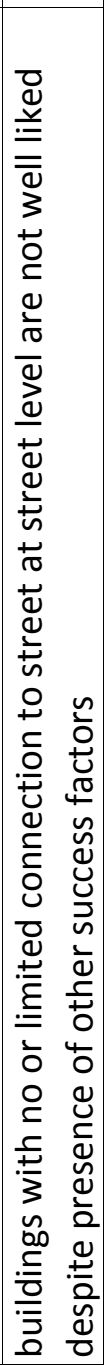 \\
\hline $\begin{array}{l}\text { ते } \\
\overrightarrow{\tilde{H}}\end{array}$ & U & $\lesssim$ & N & N & U & U & N & 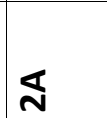 & 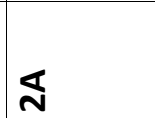 & 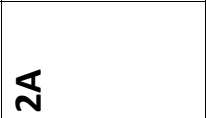 & $\begin{array}{l}n \\
\breve{z} \\
0 \\
L\end{array}=$ & $\stackrel{\infty}{\mathbf{N}}$ \\
\hline
\end{tabular}




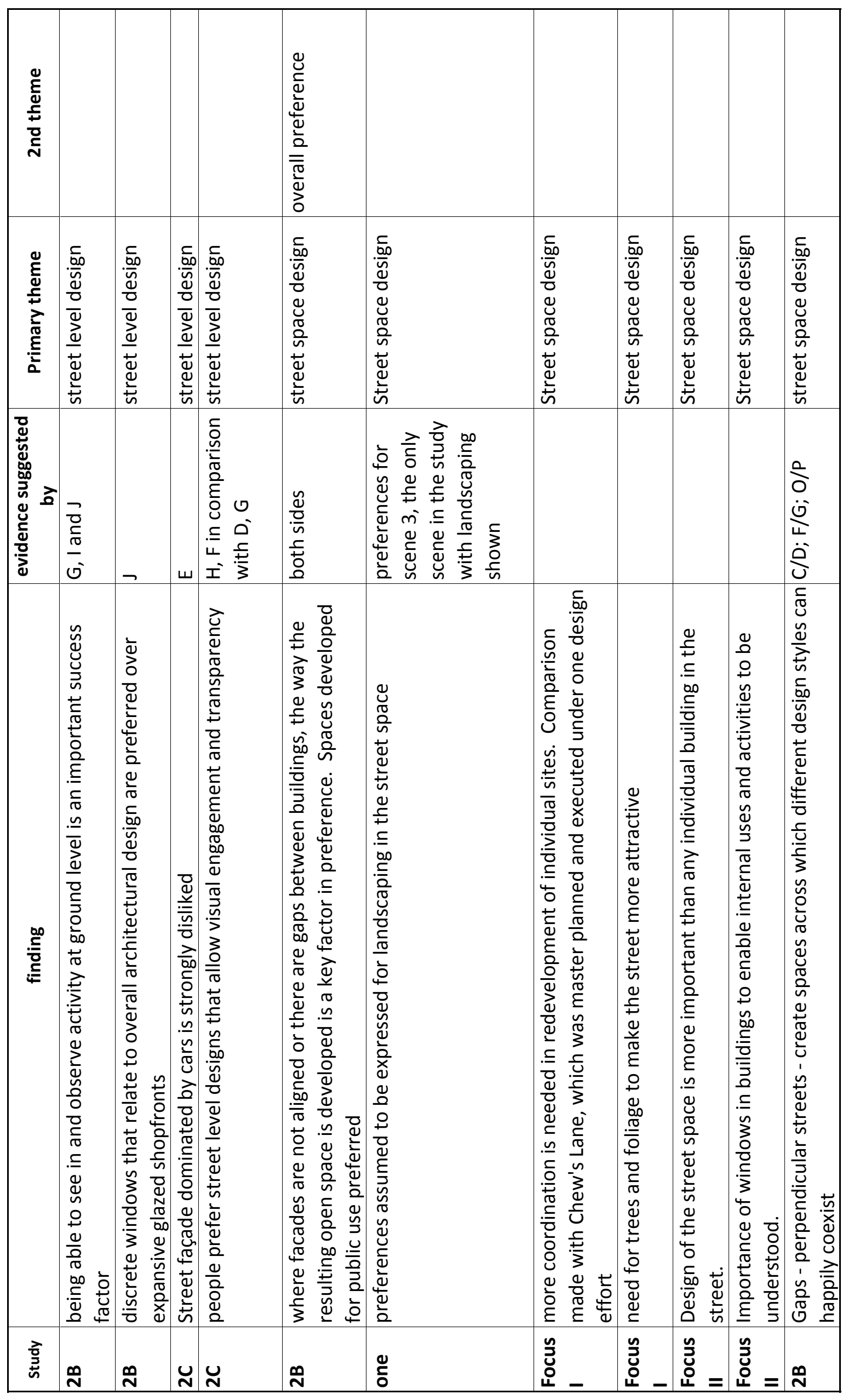




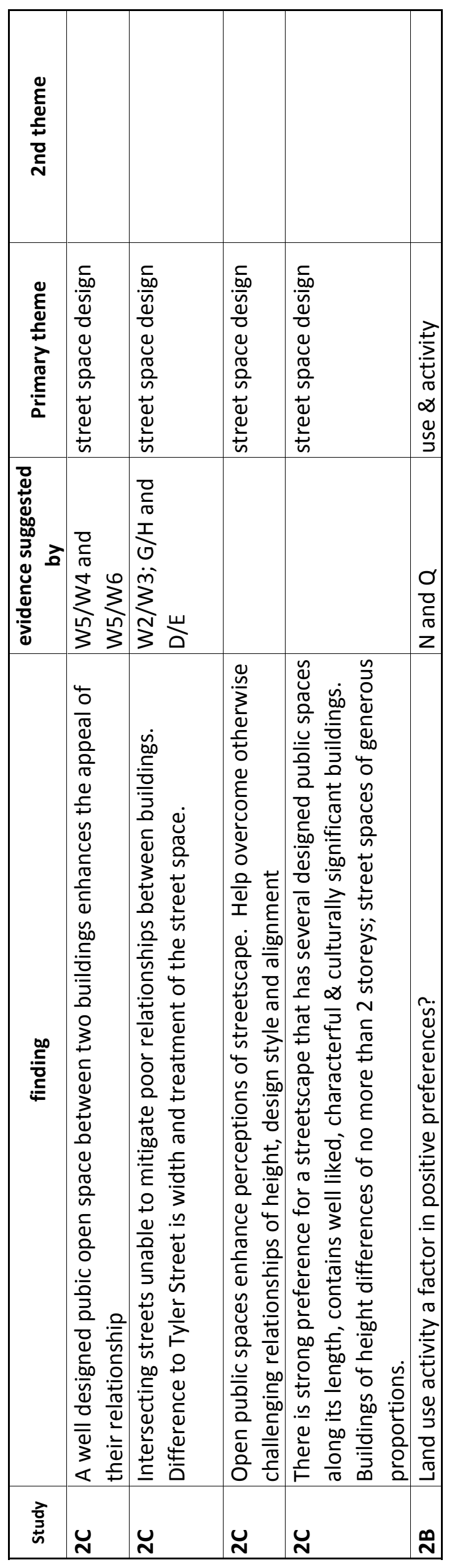

